\title{
Highly Diastereoselective Strain-Increase Allylborations: Rapid Access to Alkylidenecyclopropanes and Alkylidenecyclobutanes
}

Durga Prasad Hari, Rudrakshula Madhavachary, Valerio Fasano, Jack Haire and Varinder K.

$$
\text { Aggarwal* }
$$

School of Chemistry, University of Bristol, Cantock's Close, Bristol BS8 1TS, U.K.

$$
\text { v.aggarwal@bristol.ac.uk. }
$$

Table of Contents

$\begin{array}{ll}\text { 1. General methods } & \text { S2 }\end{array}$

$\begin{array}{ll}\text { 2. General procedures } & \text { S3 }\end{array}$

$\begin{array}{ll}\text { 3. Characterization data } & \text { S7 }\end{array}$

4. Determination of relative stereochemistry $\quad$ S44

5. ${ }^{11} \mathrm{~B}$ NMR analysis of reaction: evidence for borinic ester intermediates $\quad$ S47

$\begin{array}{lr}\text { 6. DFT calculations } & \text { S49 }\end{array}$

$\begin{array}{ll}\text { 7. References } & \text { S98 }\end{array}$

$\begin{array}{ll}\text { 8. Spectra for new compounds } & \text { S99 }\end{array}$ 


\section{General Methods}

\subsection{Solvents, Reagents, Glassware and Reaction Setup}

Unless otherwise stated, all reactions were conducted under an inert atmosphere of nitrogen in flame dried glassware using standard Schlenk techniques. Air- and moisture-sensitive liquids and solutions were transferred via syringe into the reaction vessels through a rubber septum. Unless otherwise specified, all reagents were purchased at highest commercial quality and used as received. Non-anhydrous solvents were purchased (unless specified) at the highest commercial quality and used as received. DCM, $\mathrm{Et}_{2} \mathrm{O}$ and THF were dried on an Anhydrous Engineering alumina column drying system. Temperatures described below $-10{ }^{\circ} \mathrm{C}$ were achieved using Thermo Scientific EK-90 or Huber TC100E cryostats or appropriate solvent/dry ice baths.

\subsection{Analytical methods}

Chromatography: Flash column chromatography was carried out using Sigma-Aldrich silica

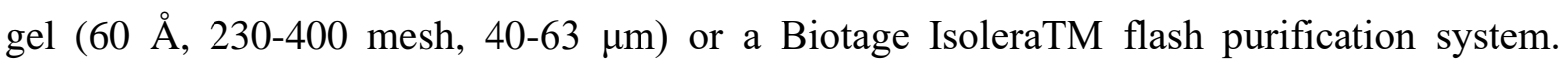
Reactions were followed by thin-layer chromatography (TLC) where practical, using aluminium-backed Merck Kieselgel 60 F254 fluorescent treated silica gel plates, which were visualised under UV light or by staining with aqueous basic $\mathrm{KMnO}_{4}$, acidic $p$-anisaldehyde solution in ethanol, or phosphomolybdic acid solution in ethanol. NMR yields: Following work up, dibromomethane (1 equiv relative to limiting SM) was added to the crude residue. The resultant mixture was dissolved in $\mathrm{CDCl}_{3}(5 \mathrm{~mL})$, and a $0.6 \mathrm{~mL}$ sample of the resultant solution taken for ${ }^{1} \mathrm{H}$ NMR analysis. Yields were calculated based on the integrals of known product resonances relative to dibromomethane $\left(2 \mathrm{H}\right.$, at $4.94 \mathrm{ppm}$ in $\left.\mathrm{CDCl}_{3}\right)$. IR: IR spectra were recorded on neat compounds using a Perkin Elmer (Spectrum One) FT-IR spectrometer (ATR sampling accessory). Selected absorbances (vmax, expressed in $\mathrm{cm}^{-1}$ ) are reported. ${ }^{\mathbf{1}} \mathbf{H}$ NMR: Spectra were recorded on Jeol ECS (400 MHz), Jeol ECZ (400 MHz or Bruker Avance (400 $\mathrm{MHz}$ or $500 \mathrm{MHz}$ ) instruments. Chemical shifts $(\delta)$ are quoted in parts per million (ppm) and referenced to the appropriate NMR solvent peak(s) and are assigned in accordance with numbered diagrams; with resonances described as s (singlets), $\mathrm{d}$ (doublets), $\mathrm{t}$ (triplets), $\mathrm{q}$ (quartets), $\mathrm{p}$ (pentets), combinations thereof (i.e. td indicates a triplet of doublets) or $\mathrm{m}$ (multiplets) and br s (broad singlet). ${ }^{13} \mathbf{C}$ NMR: Spectra were recorded on a Bruker Avance $(101 \mathrm{MHz}$ or $126 \mathrm{MHz})$ and Jeol ECZ (101 MHz) instruments. Chemical shifts $(\delta)$ are quoted in parts per million ( $\mathrm{ppm}$ ) and referenced to the appropriate NMR solvent peak(s) and are 
assigned in accordance with numbered diagrams. HRMS: High resolution mass spectra were recorded on a Bruker Daltronics MicroTOF II (ESI), Thermo Scientific Orbitrap (ESI, APCI) or Thermo Scientific QExactive (EI). Only molecular ion $\left([\mathrm{M}+\mathrm{H}]^{+}\right.$or $[\mathrm{M}+\mathrm{Na}]^{+}$for ESI and $\mathrm{APCI} ; \mathrm{M}^{+}$or $[\mathrm{M}-\mathrm{H}]^{+}$for EI peaks are reported.

\section{General Procedures}

The synthesis of vinylcyclopropyl boronic esters $(\mathbf{1 a}, \mathbf{1 b}, \mathbf{1 d}, \mathbf{1 e}, \mathbf{1 g}, \mathbf{1 h}$ and $\mathbf{1 j})$ had been already described before. ${ }^{1}$ The procedure is taken here from the indicated publication to facilitate reproduction of the results by having all the data in the same file.

\section{General Procedure A: Synthesis of vinylcyclopropyl boronic esters}

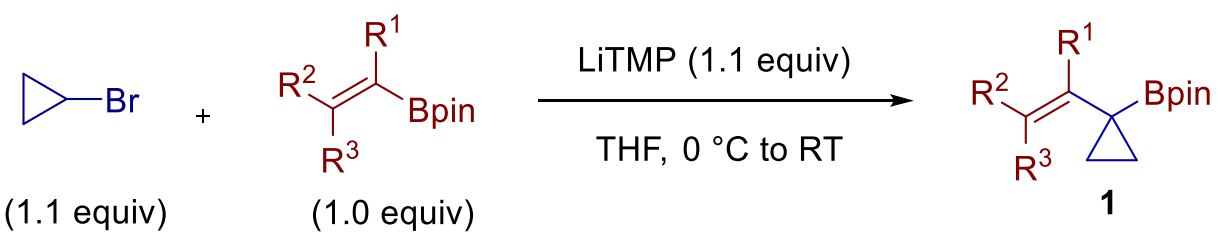

To a stirred solution of 2,2,6,6-tetramethylpiperidine ( $778 \mathrm{mg}, 5.50 \mathrm{mmol}, 1.10$ equiv) in THF (1.5M) under $\mathrm{N}_{2}$ at $0{ }^{\circ} \mathrm{C}$ was added $n \operatorname{BuLi}(3.50 \mathrm{~mL}, 1.6 \mathrm{M}, 5.50 \mathrm{mmol}, 1.10$ equiv) dropwise over $15 \mathrm{~min}$. The solution was then stirred for $30 \mathrm{~min}$ at $0{ }^{\circ} \mathrm{C}$, warmed to $\mathrm{RT}$ and stirred for a further $30 \mathrm{~min}$. In another Schlenk flask, a solution of cyclopropyl bromide (666 mg, 5.50 mmol, 1.10 equiv) and vinylboronic ester (5.00 mmol, 1.00 equiv) was prepared in THF $(0.37 \mathrm{M})$ and cooled to $0{ }^{\circ} \mathrm{C}$. To this solution was added the freshly prepared LiTMP over 20 min. The reaction was allowed to stir at $0{ }^{\circ} \mathrm{C}$ for $2 \mathrm{~h}$, then allowed to warm slowly to $\mathrm{RT}$ overnight. A saturated solution of $\mathrm{NaHCO}_{3}(15 \mathrm{~mL})$ was added to quench the reaction, and the mixture was allowed to warm to $\mathrm{RT}$. $\mathrm{Et}_{2} \mathrm{O}(15 \mathrm{~mL})$ was added, and the layers were separated. The aqueous phase was extracted with $\mathrm{Et}_{2} \mathrm{O}(3 \times 15 \mathrm{~mL})$ and the combined organic layers were washed with water $(20 \mathrm{~mL})$ and brine $(20 \mathrm{~mL})$, dried over $\mathrm{MgSO}_{4}$, filtered and concentrated to afford the crude product. Crude material was purified by flash column chromatography (DCM/pentane or $\mathrm{Et}_{2} \mathrm{O}$ :pentane) to afford the desired product. 


\section{General Procedure B: Synthesis of vinylcyclobutyl boronic esters}

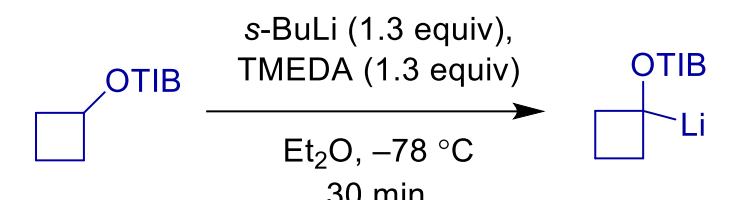

(1.0 equiv)

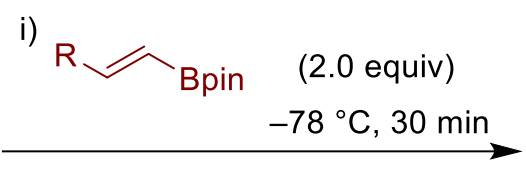

ii) $\mathrm{CHCl}_{3}, 60{ }^{\circ} \mathrm{C}$, overnight

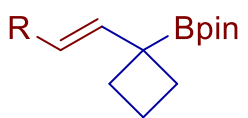

14

Under a nitrogen atmosphere, TMEDA $(0.80 \mathrm{~mL}, 5.2 \mathrm{mmol}, 1.3$ equiv) was added to cyclobutyl 2,4,6- triisopropylbenzoate $(1.2 \mathrm{~g}, 4.0 \mathrm{mmol}, 1.0$ equiv) dissolved in anhydrous $\mathrm{Et}_{2} \mathrm{O}(15 \mathrm{~mL})$ in a flame dried Schlenk tube. After cooling to $-78^{\circ} \mathrm{C}, s-\mathrm{BuLi}(1.3 \mathrm{M}, 4.0 \mathrm{~mL}$, $5.2 \mathrm{mmol}, 1.3$ equiv) was added dropwise and the brown/orange reaction mixture stirred for 30 min at $-78^{\circ} \mathrm{C}$. A solution of vinylboronic ester $\left(8.0 \mathrm{mmol}, 2.0\right.$ equiv) in dry $\mathrm{Et}_{2} \mathrm{O}(10 \mathrm{~mL})$ was added dropwise to the reaction mixture at the same temperature. After $30 \mathrm{~min}$, the reaction was warmed to room temperature and the solvent exchanged to $\mathrm{CHCl}_{3}(25 \mathrm{~mL})$. The resultant mixture was heated to $60{ }^{\circ} \mathrm{C}$ and stirred at that temperature for overnight. The crude material was filtered through a silica plug and washed with $\mathrm{Et}_{2} \mathrm{O}(50 \mathrm{~mL})$, concentrated under reduced pressure. The residue was either distilled using Kugelrohr or purified by flash column chromatography to afford the desired product.

\section{General Procedure C: Strain-increase allylboration of vinylcyclopropyl boronic esters.}

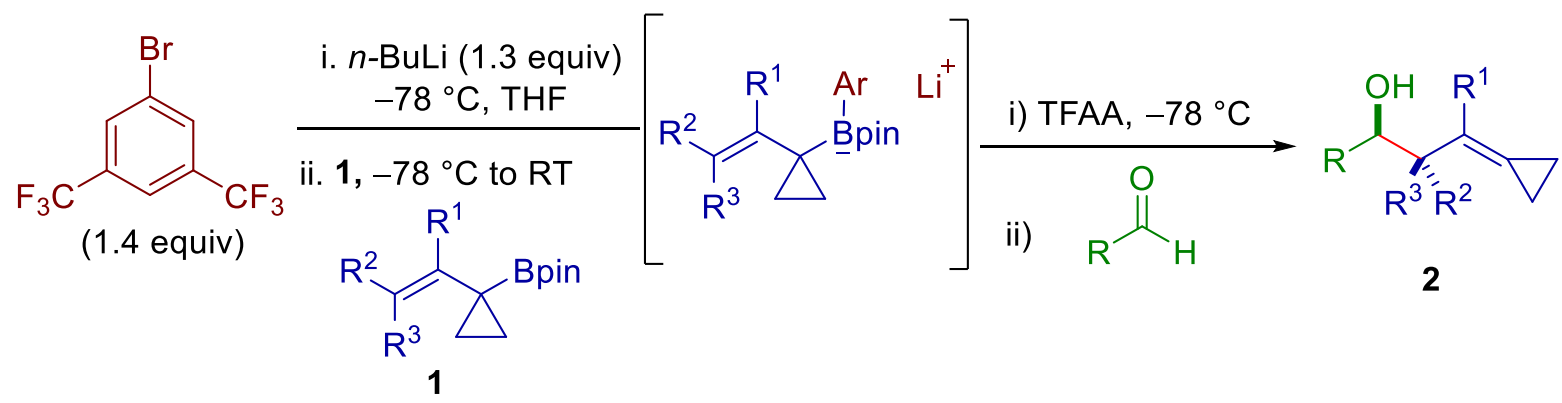

To a stirred solution of 1-bromo-3,5-bis(trifluoromethyl)benzene (73 $\mu \mathrm{L}, 0.42 \mathrm{mmol}, 1.4$ equiv) in THF ( $2 \mathrm{~mL})$ under $\mathrm{N}_{2}$ at $-78{ }^{\circ} \mathrm{C}$ was added $n$-BuLi $(0.245 \mathrm{~mL}, 1.6 \mathrm{M}, 0.390 \mathrm{mmol}$, 1.30 equiv) dropwise. The solution was then stirred for $1 \mathrm{~h}$ at $-78^{\circ} \mathrm{C}$, at which point a solution of vinylcyclopropyl boronic ester $1(0.30 \mathrm{mmol}, 1.0$ equiv $)$ in THF $(2 \mathrm{~mL})$ was added dropwise. The reaction mixture was stirred at $-78{ }^{\circ} \mathrm{C}$ for $1 \mathrm{~h}$ and then warmed to $\mathrm{RT}$ and stirred for another $1 \mathrm{~h}$. The reaction mixture was cooled back to $-78{ }^{\circ} \mathrm{C}$ then added TFAA $(60 \mu \mathrm{L}, 0.42$ mmol, 1.4 equiv) and stirred for $30 \mathrm{~min}$. Aldehyde ( $0.45 \mathrm{mmol}, 1.5$ equiv) was then added at $-78^{\circ} \mathrm{C}$. The reaction was allowed to stir at $-78^{\circ} \mathrm{C}$ for $2 \mathrm{~h}$, then allowed to warm slowly to RT. 
After completion of reaction, $0.5 \mathrm{~N} \mathrm{NaOMe}$ solution $(3 \mathrm{~mL})$ in methanol was added and stirred for $30 \mathrm{~min}$. The mixture was diluted with $0.5 \mathrm{~N}$ aq. $\mathrm{NaOH}(15 \mathrm{~mL})$ and the mixture was extracted with $\mathrm{Et}_{2} \mathrm{O}(3 \times 20 \mathrm{~mL})$. The combined organic phases were dried over $\mathrm{MgSO}_{4}$, filtered, and concentrated under reduced pressure. The crude material was purified by flash chromatography using EtOAc/pentane or $\mathrm{Et}_{2} \mathrm{O} /$ pentane as mobile phase to afford 2.

General Procedure D: Strain-increase allylic functionalizations using non-carbonylbased electrophiles.<smiles>FC(F)(F)c1cc(Br)cc(C(F)(F)F)c1</smiles>

(1.4 equiv)
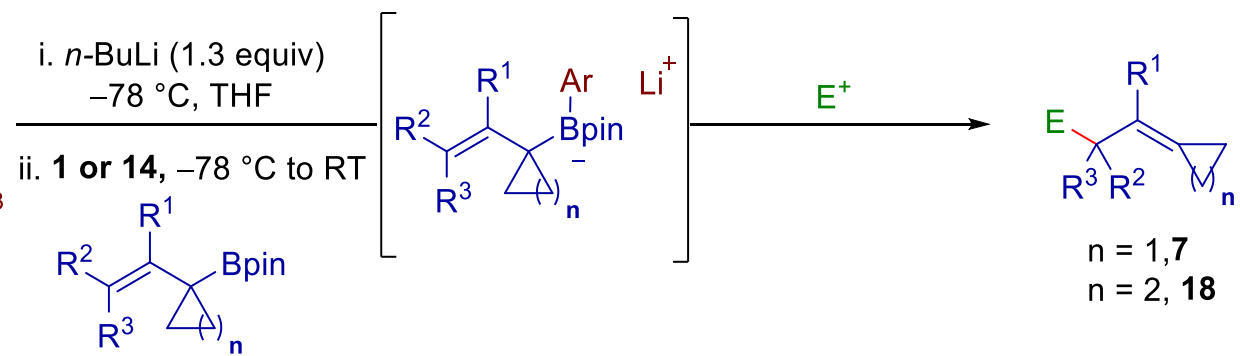

To a stirred solution of 1-bromo-3,5-bis(trifluoromethyl)benzene ( $73 \mu \mathrm{L}, 0.42 \mathrm{mmol}, 1.4$ equiv) in THF ( $2 \mathrm{~mL})$ under $\mathrm{N}_{2}$ at $-78{ }^{\circ} \mathrm{C}$ was added $n$-BuLi $(0.245 \mathrm{~mL}, 1.6 \mathrm{M}, 0.390 \mathrm{mmol}$, 1.30 equiv) dropwise. The solution was then stirred for $1 \mathrm{~h}$ at $-78{ }^{\circ} \mathrm{C}$, at which point a solution of vinylcycloalkyl boronic ester ( $0.30 \mathrm{mmol}, 1.0$ equiv) in THF ( $2 \mathrm{~mL})$ was added dropwise. The reaction mixture was stirred at $-78{ }^{\circ} \mathrm{C}$ for $1 \mathrm{~h}$ and then warmed to $\mathrm{RT}$ and stirred for another $1 \mathrm{~h}$. The reaction mixture was cooled back to $-78^{\circ} \mathrm{C}$ and then electrophile $(0.45 \mathrm{mmol}$, 1.5 equiv) was added. The reaction was allowed to stir at $-78{ }^{\circ} \mathrm{C}$ for $2 \mathrm{~h}$, then allowed to warm slowly to RT. After completion of reaction, the mixture was diluted with $0.5 \mathrm{~N}$ aq. $\mathrm{NaOH}(15$ $\mathrm{mL})$ and extracted with $\mathrm{Et}_{2} \mathrm{O}(3 \times 20 \mathrm{~mL})$. The combined organic phases were dried over $\mathrm{MgSO}_{4}$, filtered, and concentrated under reduced pressure. The crude material was purified by flash chromatography using EtOAc/pentane or $\mathrm{Et}_{2} \mathrm{O} /$ pentane as mobile phase to afford the corresponding product. 
General Procedure E: Strain-increase allylic functionalizations using radical electrophiles.

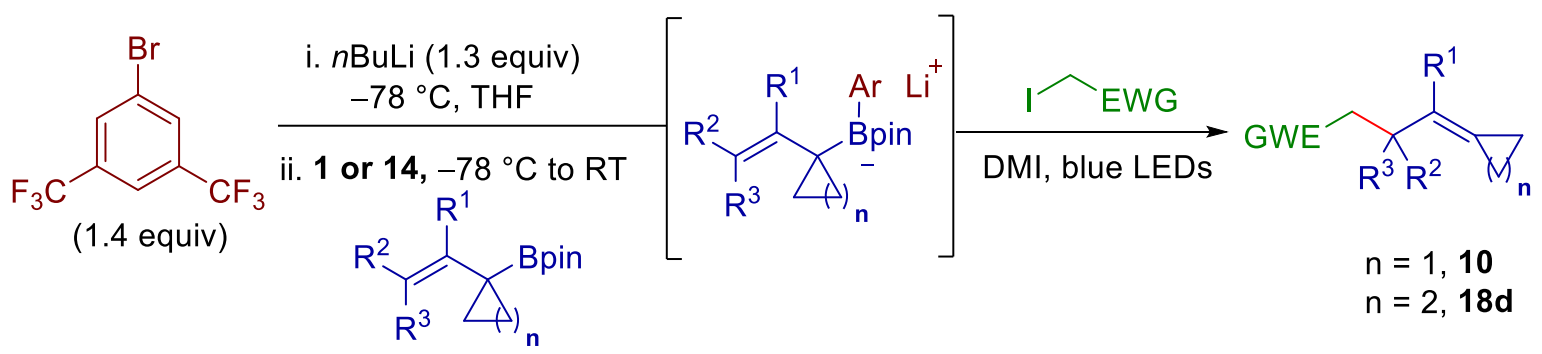

To a stirred solution of 1-bromo-3,5-bis(trifluoromethyl)benzene (73 $\mu \mathrm{L}, 0.42 \mathrm{mmol}, 1.4$ equiv) in THF $(0.8 \mathrm{~mL})$ under $\mathrm{N}_{2}$ at $-78^{\circ} \mathrm{C}$ was added $n$-BuLi $(0.245 \mathrm{~mL}, 1.6 \mathrm{M}, 0.390 \mathrm{mmol}$, 1.30 equiv) dropwise. The solution was then stirred for $1 \mathrm{~h}$ at $-78^{\circ} \mathrm{C}$, at which point a solution of vinylcycloalkyl boronic ester $(0.30 \mathrm{mmol}, 1.0$ equiv $)$ in THF $(0.8 \mathrm{~mL})$ was added dropwise. The reaction mixture was stirred at $-78{ }^{\circ} \mathrm{C}$ for $1 \mathrm{~h}$ and then warmed to $\mathrm{RT}$ and stirred for another $1 \mathrm{~h}$. The reaction tube was placed into a crystallizing basin equipped with a $1 \mathrm{~m}$ length blue LED strip. To this, a solution of alkyl halide $(0.45 \mathrm{mmol}, 1.5$ equiv) in DMI (3.0 $\mathrm{mL})$ was added under irradiation, and the mixture was stirred vigorously overnight under constant irradiation. The reaction mixture was diluted with water $(20 \mathrm{~mL})$ and extracted with $\mathrm{Et}_{2} \mathrm{O}(3 \mathrm{x}$ $15 \mathrm{~mL}$ ). The combined organic phases were dried over $\mathrm{MgSO}_{4}$, filtered, and concentrated under reduced pressure. The crude material was purified by flash chromatography using EtOAc/pentane or $\mathrm{Et}_{2} \mathrm{O} /$ pentane as mobile phase to afford the corresponding product.

Optimization conditions: Strain-increase allylboration of vinylcyclobutyl boronic esters.<smiles>FC(F)(F)c1cc(Br)cc(C(F)(F)F)c1</smiles>
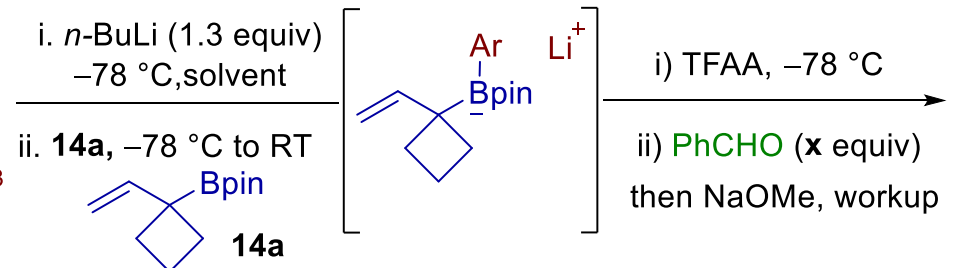

(1.4 equiv)

$14 a$

then $\mathrm{NaOMe}$, workup<smiles>OC(CC=C1CCC1)c1ccccc1</smiles>

$17 a$

\begin{tabular}{|c|c|c|c|}
\hline S.No. & Aldehyde (x equiv) & Solvent $(x \mathrm{M})$ & Yield (NMR) \\
\hline 1 & 1.5 & $\operatorname{THF}(0.1 \mathrm{M})$ & $32 \%$ \\
2 & 1.5 & $\operatorname{THF}(0.2 \mathrm{M})$ & $44 \%$ \\
3 & 3.0 & $\operatorname{THF}(0.1 \mathrm{M})$ & $33 \%$ \\
4 & 3.0 & $\operatorname{THF}(0.2 \mathrm{M})$ & $70 \%$ \\
$5^{\mathrm{a}}$ & 3.0 & $\operatorname{THF}(0.2 \mathrm{M})$ & $<5 \%$ \\
6 & 3.0 & $\operatorname{THF}(0.3 \mathrm{M})$ & $41 \%$ \\
7 & 3.0 & $\operatorname{Et} \mathrm{C}_{2}(0.2 \mathrm{M})$ & $80 \%$ \\
8 & 1.5 & $\operatorname{Et}_{\mathbf{2}} \mathrm{O}(0.2 \mathrm{M})$ & $\mathbf{8 0} \%$ \\
\hline
\end{tabular}

${ }^{a}$ No TFAA 


\section{General Procedure F: Strain-increase allylboration of vinylcyclobutyl boronic esters.}

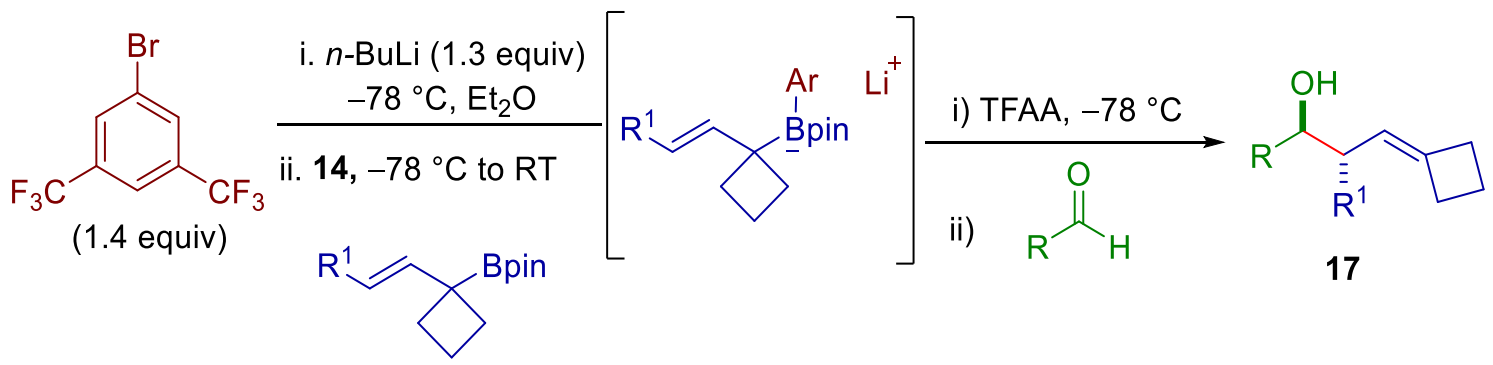

14

To a stirred solution of 1-bromo-3,5-bis(trifluoromethyl)benzene (73 $\mu \mathrm{L}, 0.42 \mathrm{mmol}, 1.4$ equiv) in $\mathrm{Et}_{2} \mathrm{O}(1 \mathrm{~mL})$ under $\mathrm{N}_{2}$ at $-78{ }^{\circ} \mathrm{C}$ was added $n$-BuLi $(0.245 \mathrm{~mL}, 1.6 \mathrm{M}, 0.390 \mathrm{mmol}$, 1.30 equiv) dropwise. The solution was then stirred for $1 \mathrm{~h}$ at $-78{ }^{\circ} \mathrm{C}$, at which point a solution of vinylcyclobutyl boronic ester $(0.30 \mathrm{mmol}, 1.0$ equiv $)$ in $\mathrm{Et}_{2} \mathrm{O}(0.5 \mathrm{~mL})$ was added dropwise. The reaction mixture was stirred at $-78{ }^{\circ} \mathrm{C}$ for $1 \mathrm{~h}$ and then warmed to $\mathrm{RT}$ and stirred for another $1 \mathrm{~h}$. The reaction mixture was cooled back to $-78{ }^{\circ} \mathrm{C}$ then added TFAA $(60 \mu \mathrm{L}, 0.42$ mmol, 1.4 equiv) and stirred for $30 \mathrm{~min}$. Aldehyde ( $0.45 \mathrm{mmol}, 1.5$ equiv) was then added at $-78^{\circ} \mathrm{C}$. The reaction was allowed to stir at $-78^{\circ} \mathrm{C}$ for $2 \mathrm{~h}$, then allowed to warm slowly to RT overnight. After completion of reaction, 0.5M NaOMe solution ( $3 \mathrm{~mL})$ in methanol was added and stirred for $30 \mathrm{~min}$. The mixture was diluted with $0.5 \mathrm{~N}$ aq. $\mathrm{NaOH}(15 \mathrm{~mL})$ and the mixture was extracted with $\mathrm{Et}_{2} \mathrm{O}(3 \times 20 \mathrm{~mL})$. The combined organic phases were dried over $\mathrm{MgSO}_{4}$, filtered, and concentrated under reduced pressure. The crude material was purified by flash chromatography using EtOAc/pentane or $\mathrm{Et}_{2} \mathrm{O} /$ pentane as mobile phase to afford $\mathbf{1 7}$.

\section{Characterization data}

\section{4,4,5,5-Tetramethyl-2-(1-vinylcyclopropyl)-1,3,2-dioxaborolane (1a)}

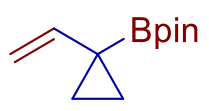

$1 \mathrm{a}$

Following general procedure A, using 2,2,6,6-tetramethylpiperidine (770 mg, $5.50 \mathrm{mmol}, 1.10$ equiv), $n$-BuLi (3.44 mL, $5.50 \mathrm{mmol}, 1.6 \mathrm{M}$ in hexanes, 1.10 equiv), cyclopropyl bromide (665 $\mathrm{mg}, 5.50 \mathrm{mmol}, 1.00$ equiv) and 4,4,5,5-tetramethyl-2-vinyl-1,3,2-dioxaborolane (770 mg, $5.00 \mathrm{mmol}, 1.00$ equiv). The crude reaction mixture was purified by flash chromatography using DCM:pentane 1:10 as mobile phase to afford $1 \mathbf{a}(704 \mathrm{mg}, 3.63 \mathrm{mmol}, 72 \%)$ as a colorless liquid (volatile, solvents removed at RT). TLC (DCM:pentane, 1:3 v/v): $\mathrm{R}_{\mathrm{f}}=0.36, \mathrm{KMnO}_{4} ;{ }^{1} \mathrm{H}$ 
NMR (400 MHz, $\left.\mathrm{CDCl}_{3}\right): \delta 5.78(\mathrm{dd}, J=17.3,10.5 \mathrm{~Hz}, 1 \mathrm{H}), 5.10(\mathrm{dd}, J=17.3,1.7 \mathrm{~Hz}, 1 \mathrm{H})$, $4.87(\mathrm{dd}, J=10.5,1.7 \mathrm{~Hz}, 1 \mathrm{H}), 1.22(\mathrm{~s}, 12 \mathrm{H}), 0.99-0.90(\mathrm{~m}, 2 \mathrm{H}), 0.72-0.62(\mathrm{~m}, 2 \mathrm{H}) ;{ }^{13} \mathrm{C} \mathrm{NMR}$ $\left(101 \mathrm{MHz}, \mathrm{CDCl}_{3}\right): \delta 142.4,111.6,83.1,24.7,14.0$. The characterization data corresponded to the reported values. ${ }^{1}$

\section{4,4,5,5-Tetramethyl-2-(1-(prop-1-en-2-yl)cyclopropyl)-1,3,2-dioxaborolane (1b)}

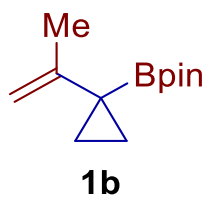

Following general procedure A, using 2,2,6,6-tetramethylpiperidine (770 mg, $5.50 \mathrm{mmol}, 1.10$ equiv), $n$-BuLi (3.44 mL, $5.50 \mathrm{mmol}, 1.6 \mathrm{M}$ in hexanes, 1.10 equiv), cyclopropyl bromide (665 mg, $5.50 \mathrm{mmol}, 1.00$ equiv) and 4,4,5,5-tetramethyl-2-(prop-1-en-2-yl)-1,3,2-dioxaborolane (840 mg, $5.00 \mathrm{mmol}, 1.00$ equiv). The crude reaction mixture was purified by flash chromatography using DCM:pentane 1:10 as mobile phase to afford $\mathbf{1 b}$ (852 $\mathrm{mg}, 4.09 \mathrm{mmol}$, $82 \%$ ) as a white semi solid. TLC (DCM:pentane, $1: 3 \mathrm{v} / \mathrm{v}$ ): $\mathrm{R}_{\mathrm{f}}=0.38, \mathrm{KMnO}_{4} ;{ }^{1} \mathrm{H}$ NMR (400 $\left.\mathrm{MHz}, \mathrm{CDCl}_{3}\right): \delta 4.73(\mathrm{p}, J=1.4 \mathrm{~Hz}, 1 \mathrm{H}), 4.68-4.69(\mathrm{~m}, 1 \mathrm{H}), 1.83-1.80(\mathrm{~m}, 3 \mathrm{H}), 1.21(\mathrm{~s}$, $12 \mathrm{H}), 0.86-0.77(\mathrm{~m}, 2 \mathrm{H}), 0.69-0.55(\mathrm{~m}, 2 \mathrm{H}) ;{ }^{13} \mathrm{C} \mathrm{NMR}\left(101 \mathrm{MHz}, \mathrm{CDCl}_{3}\right): \delta 148.6,109.7$, $83.1,24.55,23.0,11.7$. The characterization data corresponded to the reported values. ${ }^{1}$

\section{4,4,5,5-Tetramethyl-2-(1-(1-phenylvinyl)cyclopropyl)-1,3,2-dioxaborolane (1c)}

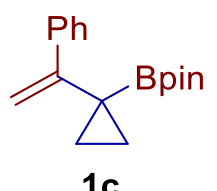

Following general procedure A, using 2,2,6,6-tetramethylpiperidine (770 mg, $5.50 \mathrm{mmol}, 1.10$ equiv), $n$-BuLi (3.44 mL, $5.50 \mathrm{mmol}, 1.6 \mathrm{M}$ in hexanes, 1.10 equiv), cyclopropyl bromide (665 $\mathrm{mg}, 5.50 \mathrm{mmol}, 1.00$ equiv) and 4,4,5,5-tetramethyl-2-(1-phenylvinyl)-1,3,2-dioxaborolane (1.15 g, $5.00 \mathrm{mmol}, 1.00$ equiv). The crude reaction mixture was purified by flash chromatography using DCM:pentane 1:5 as mobile phase to afford 1c (1.05 g, $3.89 \mathrm{mmol}$, $77 \%$ ) as a white semi solid. TLC (DCM:pentane, $1: 3 \mathrm{v} / \mathrm{v}): \mathrm{R}_{\mathrm{f}}=0.32, \mathrm{KMnO}_{4} ;{ }^{1} \mathrm{H} \mathrm{NMR}$ (400 $\left.\mathrm{MHz}, \mathrm{CDCl}_{3}\right): \delta 7.62-7.56(\mathrm{~m}, 2 \mathrm{H}), 7.32-7.27(\mathrm{~m}, 2 \mathrm{H}), 7.25-7.20(\mathrm{~m}, 1 \mathrm{H}), 5.49(\mathrm{~d}, J=$ $1.1 \mathrm{~Hz}, 1 \mathrm{H}), 5.06(\mathrm{~d}, J=1.1 \mathrm{~Hz}, 1 \mathrm{H}), 1.11(\mathrm{~s}, 12 \mathrm{H}), 1.09-1.05(\mathrm{~m}, 2 \mathrm{H}), 0.83-0.79(\mathrm{~m}, 2 \mathrm{H})$; ${ }^{13} \mathrm{C} \mathrm{NMR}\left(101 \mathrm{MHz}, \mathrm{CDCl}_{3}\right): \delta$ 150.2, 141.4, 127.9, 127.1, 126.2, 111.4, 83.3, 24.3, 12.8; IR 
$v$ 3082, 2977, 1622, 1425, 1385, 1371, 1134, 960, 853; HRMS (ESI) calcd. for $\mathrm{C}_{17} \mathrm{H}_{23} \mathrm{BNaO}_{2}$ $[\mathrm{M}+\mathrm{Na}]^{+}$293.1686; found: 293.1693. Carbon attached to boron was not observed due to quadrupolar relaxation. See Spectra

(E)-4,4,5,5-Tetramethyl-2-(1-(prop-1-en-1-yl)cyclopropyl)-1,3,2-dioxaborolane (1d)

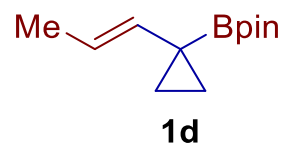

Following general procedure A on a $2.5 \mathrm{mmol}$ scale, using 2,2,6,6-tetramethylpiperidine (385 mg, $2.75 \mathrm{mmol}, 1.10$ equiv), $n$-BuLi (1.75 mL, $2.75 \mathrm{mmol}, 1.6 \mathrm{M}$ in hexanes, 1.10 equiv), cyclopropyl bromide (333 mg, $2.75 \mathrm{mmol}, 1.00$ equiv) and (E)-4,4,5,5-tetramethyl-2-(prop-1en-1-yl)-1,3,2-dioxaborolane (420 mg, $2.50 \mathrm{mmol}, 1.00$ equiv). The crude reaction mixture was purified by flash chromatography using DCM:pentane 1:10 as mobile phase to afford 1d (289 $\mathrm{mg}, 1.39 \mathrm{mmol}, 56 \%$ ) as a colorless liquid. Extensive exposure to vacuum was avoided due to product volatility. TLC (DCM:pentane, $1: 3 \mathrm{v} / \mathrm{v}): \mathrm{R}_{\mathrm{f}}=0.38, \mathrm{KMnO}_{4} ;{ }^{1} \mathrm{H}$ NMR (400 $\left.\mathrm{MHz}, \mathrm{CDCl}_{3}\right): \delta 5.53(\mathrm{dq}, J=15.4,6.4 \mathrm{~Hz}, 1 \mathrm{H}), 5.37(\mathrm{dd}, J=15.4,6.4 \mathrm{~Hz}, 1 \mathrm{H}), 1.63(\mathrm{dd}$, $J=6.4,1.5 \mathrm{~Hz}, 3 \mathrm{H}), 1.22$ (s, 12H), 0.88 (ddd, $J=3.4,3.4,3.4 \mathrm{~Hz}, 2 \mathrm{H}), 0.60$ (ddd, $J=3.4,3.4$, $3.4 \mathrm{~Hz}, 2 \mathrm{H}) ;{ }^{13} \mathrm{C}$ NMR $\left(101 \mathrm{MHz}, \mathrm{CDCl}_{3}\right): \delta$ 135.0, 122.4, 83.2, 24.8, 18.2, 13.9. The characterization data corresponded to the reported values. ${ }^{1}$

(Z)-4,4,5,5-Tetramethyl-2-(1-(prop-1-en-1-yl)cyclopropyl)-1,3,2-dioxaborolane (1d')

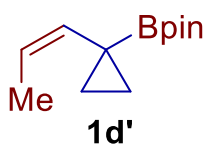

Following general procedure A on a $2.38 \mathrm{mmol}$ scale, using 2,2,6,6-tetramethylpiperidine (370 $\mathrm{mg}, 2.62 \mathrm{mmol}, 1.10$ equiv), $n$-BuLi (1.64 mL, $2.62 \mathrm{mmol}, 1.6 \mathrm{M}$ in hexanes, 1.10 equiv), cyclopropyl bromide (317 mg, $2.62 \mathrm{mmol}, 1.10$ equiv) and (Z)-4,4,5,5-tetramethyl-2-(prop-1en-1-yl)-1,3,2-dioxaborolane (400 $\mathrm{mg}, 2.38 \mathrm{mmol}, 1.00$ equiv). The crude reaction mixture was purified by flash chromatography using DCM:pentane 1:10 as mobile phase to afford $\mathbf{1 d}^{\prime}$ (93 $\mathrm{mg}, 0.45 \mathrm{mmol}, 19 \%$ ) as a colorless liquid. The poor isolation is due to the difficult separation from the over-homologated product/starting material. Extensive exposure to vacuum was avoided due to product volatility. TLC (DCM:pentane, 1:3 v/v): $\mathrm{R}_{\mathrm{f}}=0.38$, $\mathrm{KMnO}_{4} ;{ }^{1} \mathrm{H} \mathrm{NMR}\left(400 \mathrm{MHz}, \mathrm{CDCl}_{3}\right): \delta 5.52(\mathrm{dq}, J=10.3,6.6 \mathrm{~Hz}, 1 \mathrm{H}), 5.43(\mathrm{dq}, J=10.3,1.6$ 
$\mathrm{Hz}, 1 \mathrm{H}), 1.67(\mathrm{dd}, J=6.6,1.6 \mathrm{~Hz}, 3 \mathrm{H}), 1.21(\mathrm{~s}, 12 \mathrm{H}), 0.96(\mathrm{q}, J=3.3 \mathrm{~Hz}, 2 \mathrm{H}), 0.56(\mathrm{q}, J=3.2$ $\mathrm{Hz}, 2 \mathrm{H}) ;{ }^{13} \mathrm{C} \mathrm{NMR}\left(101 \mathrm{MHz}, \mathrm{CDCl}_{3}\right): \delta 133.8,127.3,83.1,24.6,14.1$; IR v 3081, 1432, 1388 , 1307, 1140, 968, 856, 707; HRMS (ESI) calcd. for $\mathrm{C}_{12} \mathrm{H}_{21} \mathrm{BO}_{2}[\mathrm{M}]^{+}$208.1629; found 208.1628. One carbon was not resolved at $101 \mathrm{MHz}$. See spectra

(E)-2-(1-(Hex-1-en-1-yl)cyclopropyl)-4,4,5,5-tetramethyl-1,3,2-dioxaborolane (1e)

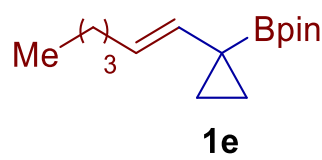

Following general procedure A, using 2,2,6,6-tetramethylpiperidine (770 mg, $5.50 \mathrm{mmol}, 1.10$ equiv), $n$-BuLi (3.44 mL, $5.50 \mathrm{mmol}, 1.6 \mathrm{M}$ in hexanes, 1.10 equiv), cyclopropyl bromide (665 $\mathrm{mg}, \quad 5.50 \mathrm{mmol}, \quad 1.00$ equiv) and (E)-2-(hex-1-en-1-yl)-4,4,5,5-tetramethyl-1,3,2dioxaborolane ( $1.05 \mathrm{~g}, 5.00 \mathrm{mmol}, 1.00$ equiv). The crude reaction mixture was purified by flash chromatography using DCM:pentane 1:10 as mobile phase to afford 1e (924 mg, 3.70 mmol, $74 \%$ ) as a colourless liquid. TLC (DCM:pentane, 1:3 v/v): $\mathrm{R}_{\mathrm{f}}=0.4, \mathrm{KMnO}_{4} ;{ }^{1} \mathrm{H} \mathrm{NMR}$ (400 MHz, $\left.\mathrm{CDCl}_{3}\right): \delta 5.48(\mathrm{dt}, J=15.4,6.5 \mathrm{~Hz}, 1 \mathrm{H}), 5.38(\mathrm{~d}, J=15.5 \mathrm{~Hz}, 1 \mathrm{H}), 2.01-1.87$ (m, $2 \mathrm{H}), 1.34-1.24(\mathrm{~m}, 4 \mathrm{H}), 1.22(\mathrm{~s}, 12 \mathrm{H}), 0.89-0.84(\mathrm{~m}, 5 \mathrm{H}), 0.67-0.53(\mathrm{~m}, 2 \mathrm{H}) ;{ }^{13} \mathrm{C}$ NMR $\left(101 \mathrm{MHz}, \mathrm{CDCl}_{3}\right): \delta 133.6,127.7,83.0,32.4,32.0,24.7,22.2,14.0,14.0$. The characterization data corresponded to the reported values. ${ }^{1}$

(E)-2-(1-(6-Chlorohex-1-en-1-yl)cyclopropyl)-4,4,5,5-tetramethyl-1,3-dioxolane (1f)

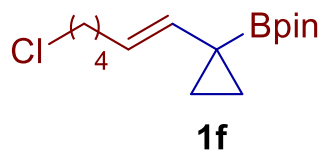

Following general procedure A, using 2,2,6,6-tetramethylpiperidine (770 mg, $5.50 \mathrm{mmol}, 1.10$ equiv), $n$-BuLi (3.44 mL, $5.50 \mathrm{mmol}, 1.6 \mathrm{M}$ in hexanes, 1.10 equiv), cyclopropyl bromide (665 $\mathrm{mg}, 5.50 \mathrm{mmol}, 1.00$ equiv) and (E)-2-(6-chlorohex-1-en-1-yl)-4,4,5,5-tetramethyl-1,3,2dioxaborolane $(1.22 \mathrm{~g}, 5.00 \mathrm{mmol}, 1.00$ equiv). The crude reaction mixture was purified by flash chromatography using DCM:pentane 1:10 as mobile phase to afford 1f (950 mg, 3.34 mmol, 67\%) as a colourless liquid. TLC (DCM:pentane, 1:3 v/v): $\mathrm{Rf}=0.38, \mathrm{KMnO}_{4} ;{ }^{1} \mathrm{H}$ NMR (400 MHz, CDCl3): $\delta 5.46(\mathrm{dt}, J=15.4,6.3 \mathrm{~Hz}, 1 \mathrm{H}), 5.38(\mathrm{~d}, J=15.4 \mathrm{~Hz}, 1 \mathrm{H}), 3.51(\mathrm{t}, J=6.8$ $\mathrm{Hz}, 2 \mathrm{H}), 2.05-1.92(\mathrm{~m}, 2 \mathrm{H}), 1.74(\mathrm{dq}, J=8.5,6.8 \mathrm{~Hz}, 2 \mathrm{H}), 1.46(\mathrm{tt}, J=9.6,6.5 \mathrm{~Hz}, 2 \mathrm{H}), 1.21$ (s, 12H), $0.93-0.83(\mathrm{~m}, 2 \mathrm{H}), 0.61-0.56(\mathrm{~m}, 2 \mathrm{H}) ;{ }^{13} \mathrm{C} \mathrm{NMR}\left(101 \mathrm{MHz}, \mathrm{CDCl}_{3}\right): \delta 134.5$, 
126.7, 83.0, 45.0, 32.1, 31.8, 26.8, 24.6, 13.9; IR v 2978, 2932, 1661, 1389, 1404, 1315, 1136, 967, 854; HRMS (ESI) calcd. for $\mathrm{C}_{15} \mathrm{H}_{26} \mathrm{BClNaO}_{2}$ [M+Na $]^{+}$307.1609; found: 307.1614. Carbon attached to boron was not observed due to quadrupolar relaxation. See spectra

\section{(E)-Tert-butyldimethyl((4-(1-(4,4,5,5-tetramethyl-1,3,2-dioxaborolan-2-} yl)cyclopropyl)but-3-en-1-yl)oxy)silane (1g)

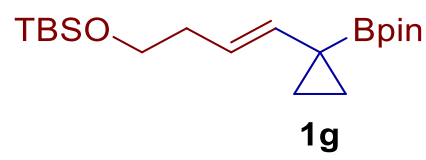

Following general procedure A on a $2.5 \mathrm{mmol}$ scale, using 2,2,6,6-tetramethylpiperidine (385 $\mathrm{mg}, 2.75 \mathrm{mmol}, 1.10$ equiv), $n$-BuLi (1.75 mL, $2.75 \mathrm{mmol}, 1.6 \mathrm{M}$ in hexanes, 1.10 equiv), cyclopropyl bromide (333 mg, $2.75 \mathrm{mmol}, 1.00$ equiv) and $(E)$-tert-butyldimethyl((4-(4,4,5,5tetramethyl-1,3,2-dioxaborolan-2-yl)but-3-en-1-yl)oxy)silane (781 mg, $2.50 \mathrm{mmol}, 1.00$ equiv). The crude reaction mixture was purified by flash chromatography using 1:10 DCM:pentane to $100 \%$ DCM as mobile phase to afford $1 \mathrm{~g}$ (646 $\mathrm{mg}, 1.82 \mathrm{mmol}, 73 \%$ ) as a colourless liquid. TLC (DCM:pentane, 1:3 v/v): $\mathrm{R}_{\mathrm{f}}=0.35, \mathrm{KMnO}_{4} ;{ }^{1} \mathrm{H}$ NMR (400 MHz, $\left.\mathrm{CDCl}_{3}\right): \delta 5.52(\mathrm{dt}, J=15.5,6.9 \mathrm{~Hz}, 1 \mathrm{H}), 5.40(\mathrm{dt}, J=15.5,1.1 \mathrm{~Hz}, 1 \mathrm{H}), 3.58(\mathrm{t}, J=6.9 \mathrm{~Hz}$, 2H), 2.19 (qd, $J=6.9,1.1 \mathrm{~Hz}, 2 \mathrm{H}), 1.21$ (s, 12H), 0.89 (s, 9H), 0.89 (ddd, $J=3.3,3.3,3.3 \mathrm{~Hz}$, 2H) $0.60(\mathrm{ddd}, J=3.3,3.3,3.3 \mathrm{~Hz}, 2 \mathrm{H}), 0.04(\mathrm{~s}, 6 \mathrm{H}) ;{ }^{13} \mathrm{C} \mathrm{NMR}\left(101 \mathrm{MHz}, \mathrm{CDCl}_{3}\right): \delta 136.2$, $123.9,83.2,63.6,36.6,26.1,24.8,18.5,14.2,-5.06$. The characterization data corresponded to the reported values. ${ }^{1}$

(E)-4,4,5,5-Tetramethyl-2-(1-styrylcyclopropyl)-1,3,2-dioxaborolane (1h)

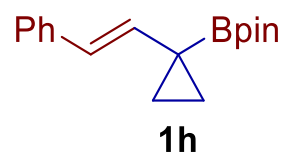

Following general procedure $\mathbf{A}$, on a $2.5 \mathrm{mmol}$ scale, using 2,2,6,6-tetramethylpiperidine (385 $\mathrm{mg}, 2.75 \mathrm{mmol}, 1.10$ equiv), $n$-BuLi (1.75 mL, $2.75 \mathrm{mmol}, 1.6 \mathrm{M}$ in hexanes, 1.10 equiv), cyclopropyl bromide (333 mg, $2.75 \mathrm{mmol}, 1.00$ equiv) and (E)-4,4,5,5-tetramethyl-2-styryl1,3,2-dioxaborolane ( $575 \mathrm{mg}, 2.50 \mathrm{mmol}, 1.00$ equiv). The crude reaction mixture was purified by flash chromatography using 1:5 DCM:pentane as mobile phase to afford $\mathbf{1 h}$ (377 $\mathrm{mg}, 1.40$ mmol, 56\%) as a white solid. TLC (DCM:pentane, 1:3 v/v): $\mathrm{R}_{\mathrm{f}}=0.35, \mathrm{KMnO}_{4} ;$ M.p. $45-50$ ${ }^{\circ} \mathrm{C} ;{ }^{1} \mathrm{H}$ NMR $\left(400 \mathrm{MHz}, \mathrm{CDCl}_{3}\right): \delta 7.34-7.29$ (m, 2H), $7.29-7.22(\mathrm{~m}, 2 \mathrm{H}), 7.14(\mathrm{tt}, J=7.2$, 
$1.3 \mathrm{~Hz}, 1 \mathrm{H}), 6.50(\mathrm{~d}, J=16.0 \mathrm{~Hz}, 1 \mathrm{H}), 6.16(\mathrm{~d}, J=16.0 \mathrm{~Hz}, 1 \mathrm{H}), 1.25(\mathrm{~s}, 12 \mathrm{H}), 1.06$ (ddd, $J=$ 3.5, 3.5, $3.5 \mathrm{~Hz}, 2 \mathrm{H}), 0.80$ (ddd, $J=3.5,3.5,3.5 \mathrm{~Hz}, 2 \mathrm{H}) ;{ }^{13} \mathrm{C} \mathrm{NMR}\left(101 \mathrm{MHz}, \mathrm{CDCl}_{3}\right): \delta$ $138.5,135.4,128.5,127.1,126.5,125.9,83.4,24.9,14.9$. The characterization data corresponded to the reported values. ${ }^{1}$

(Z)-4,4,5,5-Tetramethyl-2-(1-styrylcyclopropyl)-1,3,2-dioxaborolane (1h')

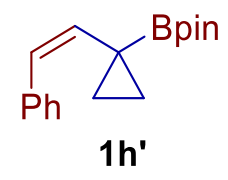

Following general procedure A on a $2.5 \mathrm{mmol}$ scale, using 2,2,6,6-tetramethylpiperidine (385 mg, $2.75 \mathrm{mmol}, 1.10$ equiv), $n$-BuLi (1.75 mL, $2.75 \mathrm{mmol}, 1.6 \mathrm{M}$ in hexanes, 1.10 equiv), cyclopropyl bromide (333 mg, $2.75 \mathrm{mmol}, 1.00$ equiv) and (Z)-4,4,5,5-tetramethyl-2-styryl1,3,2-dioxaborolane (575 $\mathrm{mg}, 2.50 \mathrm{mmol}, 1.00$ equiv). The crude reaction mixture was purified by flash chromatography using 1:5 DCM:pentane as mobile phase to afford $\mathbf{1 h}$ ' $(377 \mathrm{mg}, 1.40$ mmol, 56\%) as a pale yellow oil. TLC (DCM:pentane, 1:3 v/v): $\mathrm{R}_{\mathrm{f}}=0.35, \mathrm{KMnO}_{4} ;{ }^{1} \mathrm{H}$ NMR (400 MHz, $\left.\mathrm{CDCl}_{3}\right): 7.60$ - 7.41 (m, 2H), 7.39 - 7.27 (m, 2H), 7.22 - 7.13 (m, 1H), 6.41 (d, J $=11.4 \mathrm{~Hz}, 1 \mathrm{H}), 5.72(\mathrm{~d}, J=11.3 \mathrm{~Hz}, 1 \mathrm{H}), 1.09-1.05(\mathrm{~m}, 14 \mathrm{H}), 0.66(\mathrm{q}, J=3.4 \mathrm{~Hz}, 2 \mathrm{H}) ;{ }^{13} \mathrm{C}$ NMR (101 MHz, $\left.\mathrm{CDCl}_{3}\right): \delta 137.9,135.2,130.9,128.9,127.9,126.4,83.2,24.4,16.1$; IR v 3080, 2994, 2978, 1480, 1401, 1381, 1135, 968, 863, 777, 694; HRMS (ESI) calcd. for $\mathrm{C}_{17} \mathrm{H}_{24} \mathrm{BO}_{2}[\mathrm{M}+\mathrm{H}]^{+}$271.1867; found 271.1873. See spectra

2-(1-(Cyclopent-1-en-1-yl)cyclopropyl)-4,4,5,5-tetramethyl-1,3,2-dioxaborolane (1i)

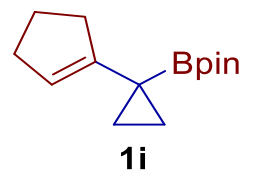

Following general procedure A, using 2,2,6,6-tetramethylpiperidine (770 mg, $5.50 \mathrm{mmol}, 1.10$ equiv), $n$-BuLi (3.44 mL, $5.50 \mathrm{mmol}, 1.6 \mathrm{M}$ in hexanes, 1.10 equiv), cyclopropyl bromide (665 $\mathrm{mg}, \quad 5.50 \mathrm{mmol}, \quad 1.00$ equiv) and 2-(cyclopent-1-en-1-yl)-4,4,5,5-tetramethyl-1,3,2dioxaborolane ( $970 \mathrm{mg}, 5.00 \mathrm{mmol}, 1.00$ equiv). The crude reaction mixture was purified by flash chromatography using DCM:pentane 1:10 as mobile phase to afford $\mathbf{1 i}(820 \mathrm{mg}, 3.50$ mmol, 70\%) as a colorless liquid. TLC (DCM:pentane, 1:3 v/v): $\mathrm{R}_{\mathrm{f}}=0.4, \mathrm{KMnO}_{4} ;{ }^{1} \mathrm{H}$ NMR (400 MHz, $\left.\mathrm{CDCl}_{3}\right): \delta 5.46(\mathrm{p}, J=2.0 \mathrm{~Hz}, 1 \mathrm{H}), 2.28(\mathrm{tq}, J=7.4,2.2 \mathrm{~Hz}, 2 \mathrm{H}), 2.21(\mathrm{tq}, J=6.6$, $2.1 \mathrm{~Hz}, 2 \mathrm{H}), 1.81(\mathrm{dt}, J=8.6,7.0 \mathrm{~Hz}, 2 \mathrm{H}), 1.20(\mathrm{~s}, 12 \mathrm{H}), 0.87-0.78(\mathrm{~m}, 2 \mathrm{H}), 0.74-0.65(\mathrm{~m}$, 
$2 \mathrm{H}) ;{ }^{13} \mathrm{C} \mathrm{NMR}\left(101 \mathrm{MHz}, \mathrm{CDCl}_{3}\right): \delta 146.9,122.9,83.0,33.7,32.4,24.6,23.2,11.9 ;$ IR v 2978, 2931, 1643, 1428, 1396, 1312, 1164, 1142, 1076, 923, 855; HRMS (ESI) calcd. for $\mathrm{C}_{14} \mathrm{H}_{23} \mathrm{BNaO}_{2}[\mathrm{M}+\mathrm{Na}]^{+}$257.1685; found 257.1690. Carbon attached to boron was not observed due to quadrupolar relaxation. See spectra

\section{2-(1-(Cyclohex-1-en-1-yl)cyclopropyl)-4,4,5,5-tetramethyl-1,3,2-dioxaborolane (1j)}

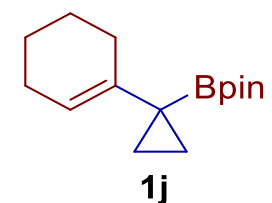

Following general procedure A, using 2,2,6,6-tetramethylpiperidine (770 mg, $5.50 \mathrm{mmol}, 1.10$ equiv), $n$-BuLi (3.44 mL, $5.50 \mathrm{mmol}, 1.6 \mathrm{M}$ in hexanes, 1.10 equiv), cyclopropyl bromide (665 mg, 5.50 mmol, 1.00 equiv) and 4-(4,4,5,5-tetramethyl-1,3,2-dioxaborolan-2-yl)cyclohex-3en-1-ylium (770 mg, $5.00 \mathrm{mmol}, 1.00$ equiv). The crude reaction mixture was purified by flash chromatography using DCM:pentane 1:10 as mobile phase to afford $\mathbf{1 j}$ ( $882 \mathrm{mg}, 3.55 \mathrm{mmol}$, $71 \%$ ) as a colorless liquid. TLC (DCM:pentane, $1: 3 \mathrm{v} / \mathrm{v}$ ): $\mathrm{R}_{\mathrm{f}}=0.4, \mathrm{KMnO}_{4} ;{ }^{1} \mathrm{H}$ NMR (400 $\left.\mathrm{MHz}, \mathrm{CDCl}_{3}\right): \delta 5.37(\mathrm{tt}, J=3.7,1.7 \mathrm{~Hz}, 1 \mathrm{H}), 2.07-2.02(\mathrm{~m}, 2 \mathrm{H}), 1.99-1.93(\mathrm{~m}, 2 \mathrm{H}), 1.63$ - $1.56(\mathrm{~m}, 2 \mathrm{H}), 1.55-1.50(\mathrm{~m}, 2 \mathrm{H}), 1.20(\mathrm{~s}, 12 \mathrm{H}), 0.78-0.69(\mathrm{~m}, 2 \mathrm{H}), 0.61-0.51(\mathrm{~m}, 2 \mathrm{H})$;

${ }^{13} \mathrm{C}$ NMR (101 MHz, $\left.\mathrm{CDCl}_{3}\right): \delta 140.4,120.2,82.9,29.1,25.2,24.6,23.3,22.7,11.0$. The characterization data corresponded to the reported values. ${ }^{1}$

\section{4,4,5,5-Tetramethyl-2-((1r,6s,7r)-7-vinylbicyclo[4.1.0]heptan-7-yl)-1,3,2-dioxaborolane} $(1 \mathrm{k})$

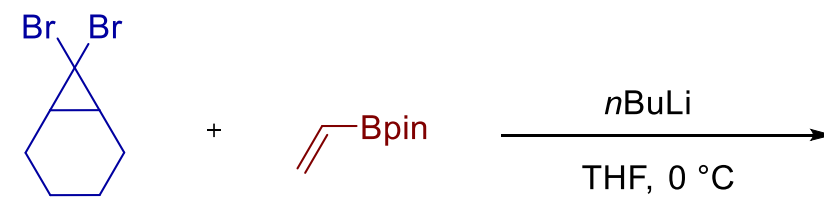

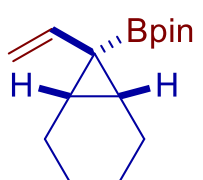

$1 \mathrm{k}$

Following a slightly modified procedure, ${ }^{2}$ to a stirred solution of ,7dibromobicyclo[4.1.0]heptane (838 mg, $3.30 \mathrm{mmol}, 1.10$ equiv) and vinylboronic acid pinacol ester (462 mg, $3.00 \mathrm{mmol}, 1.00$ equiv) in THF $(9 \mathrm{~mL})$ under $\mathrm{N}_{2}$ at $-78{ }^{\circ} \mathrm{C}$ was added $n \mathrm{BuLi}$ (2.06 mL, 1.6M, $3.30 \mathrm{mmol}, 1.10$ equiv) dropwise over $30 \mathrm{~min}$. The reaction was allowed to stir at $-78{ }^{\circ} \mathrm{C}$ for $2 \mathrm{~h}$, then allowed to warm slowly to RT overnight. The reaction mixture was quenched by $\mathrm{H}_{2} \mathrm{O}(10 \mathrm{~mL})$ at $0{ }^{\circ} \mathrm{C}$ and extracted with $\mathrm{Et}_{2} \mathrm{O}(3 \times 15 \mathrm{~mL})$. The combined organic layers were dried over $\mathrm{MgSO}_{4}$ and concentrated in vacuo. The crude reaction mixture was 
purified by flash chromatography using DCM:pentane 1:10 as mobile phase to afford $\mathbf{1 k}$ (150 $\mathrm{mg}, 0.604 \mathrm{mmol}, 20 \%$ ) as a colorless thick liquid. TLC (DCM:pentane, 1:3 v/v): $\mathbf{R}_{\mathrm{f}}=0.41$, $\mathrm{KMnO}_{4} ;{ }^{1} \mathrm{H} \mathrm{NMR}\left(500 \mathrm{MHz}, \mathrm{CDCl}_{3}\right): \delta 5.64(\mathrm{dd}, J=17.2,10.9 \mathrm{~Hz}, 1 \mathrm{H}), 5.30-5.18(\mathrm{~m}, 2 \mathrm{H})$, $2.09-1.96(\mathrm{~m}, 1 \mathrm{H}), 1.92-1.81(\mathrm{~m}, 2 \mathrm{H}), 1.58-1.45(\mathrm{~m}, 3 \mathrm{H}), 1.38-1.32(\mathrm{~m}, 2 \mathrm{H}), 1.31-1.26$ $(\mathrm{m}, 2 \mathrm{H}), 1.20(\mathrm{~s}, 12 \mathrm{H}) ;{ }^{13} \mathrm{C} \mathrm{NMR}\left(126 \mathrm{MHz} \mathrm{CDCl}_{3}\right): \delta 135.3,118.4,83.0,24.6,22.0,20.1$, 19.5; IR v 2977, 2932, 2858, 1713, 1473, 1446, 1371, 1325, 1142, 981, 850; HRMS (EI) calcd. for $\mathrm{C}_{15} \mathrm{H}_{25} \mathrm{BO}_{2}{ }^{+}[\mathrm{M}]^{+}$248.1942; found 248.1941. Carbon attached to boron was not observed due to quadrupolar relaxation. We were failed to remove unknown impurities from the product 1k. See spectra

\section{4,4,5,5-Tetramethyl-2-(1-vinylcyclobutyl)-1,3,2-dioxaborolane (14a)}

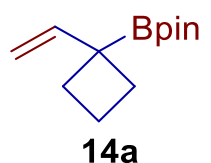

Following general procedure B, using TMEDA ( $0.80 \mathrm{~mL}, 5.2 \mathrm{mmol}, 1.3$ equiv), cyclobutyl 2,4,6- triisopropylbenzoate (1.2 g, $4.0 \mathrm{mmol}, 1.0$ equiv), sec-BuLi (4.0 mL, 1.3M in hexane, $5.2 \mathrm{mmol}, 1.3$ equiv), and vinylboronic acid pinacol ester (1.36 mL, $8.00 \mathrm{mmol}, 2.00$ equiv). The crude reaction mixture was distilled using Kugelrohr at $145{ }^{\circ} \mathrm{C}$ under high vacuum to afford 14a (524 mg, $2.52 \mathrm{mmol}, 63 \%)$ as a colourless liquid. ${ }^{1} \mathrm{H}$ NMR (500 $\left.\mathrm{MHz}, \mathrm{CDCl}_{3}\right): \delta$ $6.04(\mathrm{dd}, J=17.3,10.4 \mathrm{~Hz}, 1 \mathrm{H}), 5.01-4.86(\mathrm{~m}, 2 \mathrm{H}), 2.28-2.16(\mathrm{~m}, 2 \mathrm{H}), 2.04-1.91(\mathrm{~m}, 3 \mathrm{H})$, $1.89-1.78(\mathrm{~m}, 1 \mathrm{H}), 1.27$ (s, 12H); ${ }^{13} \mathrm{C}$ NMR (126 MHz, $\left.\mathrm{CDCl}_{3}\right): \delta 144.5,110.2,83.2,29.9$, 24.6, 18.3; IR $\vee 2975,2932,1473,1453,1371,1327,1145,982,860$, and 674; HRMS (ESI) calcd. for $\mathrm{C}_{12} \mathrm{H}_{22} \mathrm{BO}_{2}[\mathrm{M}+\mathrm{H}]^{+}$209.1707; found 209.1701. Carbon attached to boron was not observed due to quadrupolar relaxation. See spectra

(E)-4,4,5,5-Tetramethyl-2-(1-(prop-1-en-1-yl)cyclobutyl)-1,3,2-dioxaborolane (14b)

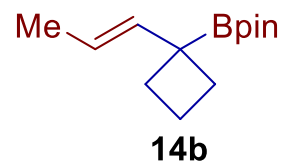

Following general procedure B, using TMEDA ( $0.80 \mathrm{~mL}, 5.2 \mathrm{mmol}, 1.3$ equiv), cyclobutyl 2,4,6- triisopropylbenzoate (1.2 g, $4.0 \mathrm{mmol}, 1.0$ equiv), sec-BuLi (4.0 mL, 1.3M in hexane, $5.2 \mathrm{mmol}, 1.3$ equiv), and trans-1-propenylboronic acid pinacol ester (1.5 mL, $8.0 \mathrm{mmol}, 2.0$ equiv). The crude reaction mixture was distilled using Kugelrohr at $160{ }^{\circ} \mathrm{C}$ under high vacuum 
to afford 14b (508 mg, $2.28 \mathrm{mmol}, 57 \%$ ) as a colourless liquid; ${ }^{1} \mathrm{H}$ NMR (500 $\mathrm{MHz}, \mathrm{CDCl}_{3}$ ): $\delta 5.65(\mathrm{dq}, J=15.5,1.7 \mathrm{~Hz}, 1 \mathrm{H}), 5.34(\mathrm{dq}, J=15.5,6.4 \mathrm{~Hz}, 1 \mathrm{H}), 2.27-2.15(\mathrm{~m}, 2 \mathrm{H}), 2.02-$ $1.77(\mathrm{~m}, 4 \mathrm{H}), 1.69$ (dd, $J=6.4,1.6 \mathrm{~Hz}, 3 \mathrm{H}), 1.28(\mathrm{~s}, 12 \mathrm{H}) ;{ }^{13} \mathrm{C} \mathrm{NMR}\left(126 \mathrm{MHz}, \mathrm{CDCl}_{3}\right): \delta$ 137.3, 121.1, 83.1, 30.7, 24.6, 18.4, 18.1; IR v 2975, 2932, 1473, 1453, 1371, 1327, 1145, 982, 860, and 674; HRMS (ESI) calcd. for $\mathrm{C}_{13} \mathrm{H}_{24} \mathrm{BO}_{2}[\mathrm{M}+\mathrm{H}]^{+}$223.1864; found 223.1859. Carbon attached to boron was not observed due to quadrupolar relaxation. See spectra

(E)-2-(1-(Hex-1-en-1-yl)cyclobutyl)-4,4,5,5-tetramethyl-1,3,2-dioxaborolane (14c)

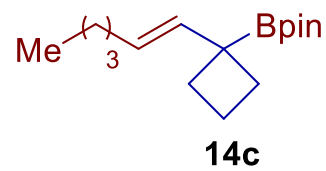

Following general procedure B, using TMEDA $(0.80 \mathrm{~mL}, 5.2 \mathrm{mmol}, 1.3$ equiv), cyclobutyl 2,4,6- triisopropylbenzoate (1.2 g, $4.0 \mathrm{mmol}, 1.0$ equiv), sec-BuLi (4.0 mL, 1.3M in hexane, $5.2 \mathrm{mmol}, 1.3$ equiv), and trans-1-hexen-1-ylboronic acid pinacol ester (1.9 mL, $8.0 \mathrm{mmol}, 2.0$ equiv). The crude reaction mixture was purified by flash chromatography using $\mathrm{Et}_{2} \mathrm{O}$ :pentane 1:9 as mobile phase to afford $\mathbf{1 4 c}(657.0 \mathrm{mg}, 2.48 \mathrm{mmol}, 62 \%)$ as a colourless liquid. TLC $\left(\mathrm{Et}_{2} \mathrm{O}\right.$ :pentane, $\left.1: 6 \mathrm{v} / \mathrm{v}\right): \mathrm{R}_{\mathrm{f}}=0.42, \mathrm{KMnO}_{4} ;{ }^{1} \mathrm{H} \mathrm{NMR}\left(500 \mathrm{MHz}, \mathrm{CDCl}_{3}\right): \delta 5.62(\mathrm{dt}, J=15.5$, $1.4 \mathrm{~Hz}, 1 \mathrm{H}), 5.31(\mathrm{dt}, J=15.5,6.8 \mathrm{~Hz}, 1 \mathrm{H}), 2.31-2.15(\mathrm{~m}, 2 \mathrm{H}), 2.11-1.99(\mathrm{~m}, 2 \mathrm{H}), 1.99-$ $1.77(\mathrm{~m}, 4 \mathrm{H}), 1.39-1.30(\mathrm{~m}, 4 \mathrm{H}), 1.28(\mathrm{~s}, 12 \mathrm{H}), 0.90(\mathrm{t}, J=7.2 \mathrm{~Hz}, 3 \mathrm{H}) ;{ }^{13} \mathrm{C} \mathrm{NMR}(126 \mathrm{MHz}$, $\left.\mathrm{CDCl}_{3}\right): \delta 136.1,126.8,83.1,32.3,31.9,30.8,24.6,22.1,18.5,13.9 ;$ IR $\vee 2976,1723,1472$, 1449, 1371, 1327, 1145, 981, 850, 698; HRMS (ESI) calcd. for $\mathrm{C}_{16} \mathrm{H}_{30} \mathrm{BO}_{2}[\mathrm{M}+\mathrm{H}]^{+} 265.2333$; found 265.2331. Carbon attached to boron was not observed due to quadrupolar relaxation. See $\underline{\text { spectra }}$

\section{Tert-butyl-4-(1-(4,4,5,5-tetramethyl-1,3,2-dioxaborolan-2-yl)cyclobutyl)-3,6-} dihydropyridine-1(2H)-carboxylate (14d)

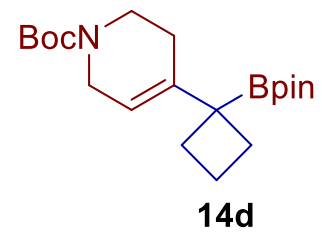

Following general procedure B, using TMEDA $(0.80 \mathrm{~mL}, 5.2 \mathrm{mmol}, 1.3$ equiv), cyclobutyl 2,4,6- triisopropylbenzoate (1.2 g, $4.0 \mathrm{mmol}, 1.0$ equiv), sec-BuLi (4.0 mL, 1.3M in hexane, 5.2 mmol, 1.3 equiv), and $N$-Boc-1,2,3,6-tetrahydropyridine-4-boronic acid pinacol ester (2.9 
g, $8.0 \mathrm{mmol}, 2.0$ equiv). The crude reaction mixture was purified by flash chromatography using EtOAc:pentane 1:4 as mobile phase to afford $\mathbf{1 4 d}(902.0 \mathrm{mg}, 62 \%)$ as a semi white solid. ${ }^{1} \mathrm{H} \mathrm{NMR}\left(400 \mathrm{MHz}, \mathrm{CDCl}_{3}\right): \delta 4.72(\mathrm{~s}, 1 \mathrm{H}), 3.46-3.33(\mathrm{~m}, 2 \mathrm{H}), 2.93(\mathrm{t}, J=5.9 \mathrm{~Hz}, 2 \mathrm{H}), 1.75$ $-1.63(\mathrm{~m}, 2 \mathrm{H}), 1.56-1.36(\mathrm{~m}, 5 \mathrm{H}), 1.29-1.16(\mathrm{~m}, 1 \mathrm{H}), 0.96(\mathrm{~s}, 9 \mathrm{H}), 0.74(\mathrm{~s}, 12 \mathrm{H}) ;{ }^{13} \mathrm{C} \mathrm{NMR}$ (101 $\mathrm{MHz}, \mathrm{CDCl}_{3}$ ) (mixture of rotamers): $\delta 155.1,154.9,141.8,114.5,113.9,83.4,83.3,79.4$, 79.2, 43.6, 39.9, 29.5, 28.5, 25.9, 24.8, 24.5, 17.9; IR v 2976, 1638, 1445, 1371, 1350, 1308, 1148, 1124, 906, 868, 729; HRMS (ESI) calcd. for $\mathrm{C}_{20} \mathrm{H}_{34} \mathrm{BNNaO}_{4}[\mathrm{M}+\mathrm{Na}]^{+}$386.2479; found 386.2458. Carbon attached to boron was not observed due to quadrupolar relaxation. See spectra

\section{3-Cyclopropylidene-1-phenylpropan-1-ol (2a)}

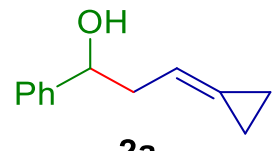

$2 a$

Following general procedure $\mathbf{C}$, using 1-bromo-3,5-bis(trifluoromethyl)benzene (73 $\mu \mathrm{L}, 0.42$ mmol, 1.4 equiv), $n$-BuLi (0.245 mL, 1.6M in hexanes, $0.390 \mathrm{mmol}, 1.30$ equiv), 4,4,5,5tetramethyl-2-(1-vinylcyclopropyl)-1,3,2-dioxaborolane (1a) (59.0 mg, $0.300 \mathrm{mmol}, 1.00$ equiv), TFAA ( $60 \mu \mathrm{L}, 0.42 \mathrm{mmol}, 1.4$ equiv) and benzaldehyde ( $46 \mu \mathrm{L}, 0.45 \mathrm{mmol}, 1.5$ equiv). The crude reaction mixture was purified by flash chromatography using $\mathrm{Et}_{2} \mathrm{O}$ :pentane $1: 12$ as mobile phase to afford $\mathbf{2 a}(45.0 \mathrm{mg}, 0.258 \mathrm{mmol}, 86 \%)$ as a colourless liquid. TLC TLC $\left(\mathrm{Et}_{2} \mathrm{O}\right.$ :pentane, $\left.1: 10 \mathrm{v} / \mathrm{v}\right): \mathrm{R}_{\mathrm{f}}=0.22, \mathrm{KMnO}_{4} ;{ }^{1} \mathrm{H}$ NMR $\left(500 \mathrm{MHz}, \mathrm{CDCl}_{3}\right): \delta 7.43-7.34(\mathrm{~m}$, $4 \mathrm{H}), 7.33-7.26(\mathrm{~m}, 1 \mathrm{H}), 5.82(\mathrm{tt}, J=7.0,2.1 \mathrm{~Hz}, 1 \mathrm{H}), 4.83(\mathrm{t}, J=6.5 \mathrm{~Hz}, 1 \mathrm{H}), 2.71-2.56$ $(\mathrm{m}, 2 \mathrm{H}), 2.15(\mathrm{~s}, 1 \mathrm{H}), 1.19-0.91(\mathrm{~m}, 4 \mathrm{H}),{ }^{13} \mathrm{C} \mathrm{NMR}\left(126 \mathrm{MHz}, \mathrm{CDCl}_{3}\right): \delta 144.1,128.3,127.4$, 125.8, 125.7, 113.7, 73.7, 41.8, 2.7, 1.8; IR v 3379, 2979, 1765, 1494, 1453, 1278, 1025; HRMS (ESI) calcd. for $\mathrm{C}_{12} \mathrm{H}_{14} \mathrm{ONa}[\mathrm{M}+\mathrm{Na}]^{+}$197.0937; found 197.0935. See spectra

\section{3-Cyclopropylidene-1-phenylbutan-1-ol (2b)}

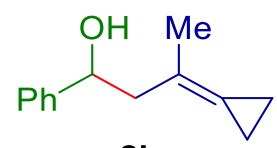

2b

Following general procedure $\mathbf{F}$, using 1-bromo-3,5-bis(trifluoromethyl)benzene (73 $\mu \mathrm{L}, 0.42$ mmol, 1.4 equiv), $n$-BuLi (0.245 mL, 1.6M in hexanes, $0.390 \mathrm{mmol}, 1.30$ equiv), 4,4,5,5tetramethyl-2-(1-(prop-1-en-2-yl)cyclopropyl)-1,3,2-dioxaborolane (1b) (62.5 mg, 0.300 
mmol, 1.00 equiv), TFAA ( $60 \mu \mathrm{L}, 0.42 \mathrm{mmol}, 1.4$ equiv) and benzaldehyde ( $46 \mu \mathrm{L}, 0.45 \mathrm{mmol}$, 1.5 equiv). The crude reaction mixture was purified by flash chromatography using $\mathrm{Et}_{2} \mathrm{O}$ :pentane 1:12 as mobile phase to afford $\mathbf{2 b}(46.0 \mathrm{mg}, 0.244 \mathrm{mmol}, 82 \%)$ as a colourless liquid. TLC (Et $2 \mathrm{O}$ :pentane, 1:10 v/v): $\mathrm{R}_{\mathrm{f}}=0.24, \mathrm{KMnO}_{4} ;{ }^{1} \mathrm{H}$ NMR $\left(500 \mathrm{MHz}, \mathrm{CDCl}_{3}\right): \delta 7.41$ $-7.37(\mathrm{~m}, 2 \mathrm{H}), 7.34(\mathrm{dd}, J=8.5,6.7 \mathrm{~Hz}, 2 \mathrm{H}), 7.26$ (tt, $J=6.5,1.5 \mathrm{~Hz}, 1 \mathrm{H}), 4.93(\mathrm{dd}, J=9.2$, $4.4 \mathrm{~Hz}, 1 \mathrm{H}), 2.66-2.58(\mathrm{~m}, 1 \mathrm{H}), 2.57-2.51(\mathrm{~m}, 1 \mathrm{H}) 2.24-1.95$ (br s, 1H), 1.89 (p, J= 1.8 $\mathrm{Hz}, 3 \mathrm{H}), 1.14-0.87(\mathrm{~m}, 4 \mathrm{H}) ;{ }^{13} \mathrm{C}$ NMR $\left(126 \mathrm{MHz}, \mathrm{CDCl}_{3}\right): \delta 144.3,128.3,127.3,125.8$, 120.9, 119.5, 72.0, 47.0, 20.9, 2.9, 2.2; IR v 3404, 2972, 1771, 1495, 1450, 1393, 1278, 1176, 1066, 891, 756; HRMS (ESI) calcd. for $\mathrm{C}_{13} \mathrm{H}_{16} \mathrm{ONa}[\mathrm{M}+\mathrm{Na}]^{+}$211.1093; found 211.1094. See spectra

\section{3-Cyclopropylidene-1,3-diphenylpropan-1-ol (2c)}

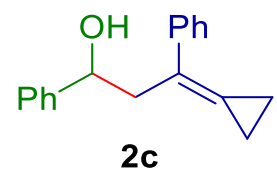

Following general procedure $\mathbf{F}$, using 1-bromo-3,5-bis(trifluoromethyl)benzene (73 $\mu \mathrm{L}, 0.42$ mmol, 1.4 equiv), $n$-BuLi ( $0.245 \mathrm{~mL}, 1.6 \mathrm{M}$ in hexanes, $0.390 \mathrm{mmol}, 1.30$ equiv), 4,4,5,5tetramethyl-2-(1-(1-phenylvinyl)cyclopropyl)-1,3,2-dioxaborolane (1c) (81.1 mg, 0.300 mmol, 1.00 equiv), TFAA ( $60 \mu \mathrm{L}, 0.42 \mathrm{mmol}, 1.4$ equiv) and benzaldehyde ( $46 \mu \mathrm{L}, 0.45 \mathrm{mmol}$, 1.5 equiv). The crude reaction mixture was purified by flash chromatography using $\mathrm{Et}_{2} \mathrm{O}$ :pentane 1:12 as mobile phase to afford $2 \mathrm{c}(46.5 \mathrm{mg}, 0.186 \mathrm{mmol}, 62 \%)$ as a colourless liquid. TLC (Et $2 \mathrm{O}$ :pentane, 1:10 v/v): $\mathrm{R}_{\mathrm{f}}=0.22, \mathrm{KMnO}_{4} ;{ }^{1} \mathrm{H} \mathrm{NMR}\left(500 \mathrm{MHz}, \mathrm{CDCl}_{3}\right): \delta 7.69$ $-7.64(\mathrm{~m}, 2 \mathrm{H}), 7.44-7.33(\mathrm{~m}, 6 \mathrm{H}), 7.32-7.24(\mathrm{~m}, 2 \mathrm{H}), 4.96(\mathrm{dd}, J=7.6,6.0 \mathrm{~Hz}, 1 \mathrm{H}), 3.12$ (ddt, $J=7.5,2.2,1.1 \mathrm{~Hz}, 2 \mathrm{H}), 2.13$ (br s, $1 \mathrm{H}), 1.54-1.46(\mathrm{~m}, 1 \mathrm{H}), 1.45-1.33(\mathrm{~m}, 1 \mathrm{H}), 1.20$ - $1.12(\mathrm{~m}, 1 \mathrm{H}), 0.95-0.85(\mathrm{~m}, 1 \mathrm{H}) ;{ }^{13} \mathrm{C} \mathrm{NMR}\left(126 \mathrm{MHz}, \mathrm{CDCl}_{3}\right): \delta 144.2,139.4,128.4$, 128.3, 127.4, 126.9, 126.0, 125.9, 124.6, 123.8, 72.7, 44.2, 5.3, 1.3; IR v 3458, 2988, 2903, 1276, 1066, 751, 700; HRMS (ESI) calcd. for $\mathrm{C}_{18} \mathrm{H}_{18} \mathrm{ONa}[\mathrm{M}+\mathrm{Na}]^{+} 273.1250$; found 273.1254.

\section{$\underline{\text { See spectra }}$}

(1s,2s)-3-Cyclopropylidene-2-methyl-1-phenylpropan-1-ol (2d)

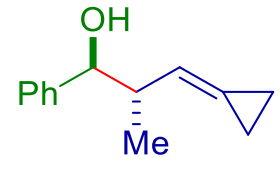


Following general procedure $\mathbf{C}$, using 1-bromo-3,5-bis(trifluoromethyl)benzene (73 $\mu \mathrm{L}, 0.42$ mmol, 1.4 equiv), $n$-BuLi ( $0.245 \mathrm{~mL}, 1.6 \mathrm{M}$ in hexanes, $0.390 \mathrm{mmol}, 1.30$ equiv), $(E)-4,4,5,5-$ tetramethyl-2-(1-(prop-1-en-1-yl)cyclopropyl)-1,3,2-dioxaborolane (1d) (62.5 mg, 0.300 mmol, 1.00 equiv), TFAA ( $60 \mu \mathrm{L}, 0.42 \mathrm{mmol}, 1.4$ equiv) and benzaldehyde ( $46 \mu \mathrm{L}, 0.45 \mathrm{mmol}$, 1.5 equiv). The crude reaction mixture was purified by flash chromatography using EtOA:pentane 1:10 as mobile phase to afford $2 \mathbf{d}(52.0 \mathrm{mg}, 0.276 \mathrm{mmol}, 92 \%,>20: 1 \mathrm{dr})$ as a colourless liquid. TLC (EtOAc:pentane, 1:9 v/v): $\mathrm{R}_{\mathrm{f}}=0.28, \mathrm{KMnO}_{4} ;{ }^{1} \mathrm{H} \mathrm{NMR}(500 \mathrm{MHz}$, $\left.\mathrm{CDCl}_{3}\right): \delta 7.36-7.32(\mathrm{~m}, 4 \mathrm{H}), 7.29-7.23(\mathrm{~m}, 1 \mathrm{H}), 5.75(\mathrm{dt}, J=8.3,2.0 \mathrm{~Hz}, 1 \mathrm{H}), 4.44(\mathrm{dd}, J$ = 7.9, 2.4 Hz, 1H), $2.69(\mathrm{dp}, J=14.7,7.0 \mathrm{~Hz}, 1 \mathrm{H}), 2.18(\mathrm{~d}, J=2.5 \mathrm{~Hz}, 1 \mathrm{H}), 1.13-1.01$ (m, $4 \mathrm{H}), 0.92(\mathrm{~d}, J=6.9 \mathrm{~Hz}, 3 \mathrm{H}) ;{ }^{13} \mathrm{C}$ NMR $\left(126 \mathrm{MHz}, \mathrm{CDCl}_{3}\right): \delta 142.7,128.1,127.5,126.9$, 124.6, 119.9, 78.6, 45.0, 16.9, 2.4, 2.3; IR v 3414, 2976, 2901, 1604, 1494, 1453, 1373, 1278, 1179, 1137, 1067, 761; HRMS (ESI) calcd. for $\mathrm{C}_{13} \mathrm{H}_{16} \mathrm{ONa}[\mathrm{M}+\mathrm{Na}]^{+}$211.1093; found 211.1102. See spectra

\section{(1s,2r)-3-Cyclopropylidene-2-methyl-1-phenylpropan-1-ol (2d')}

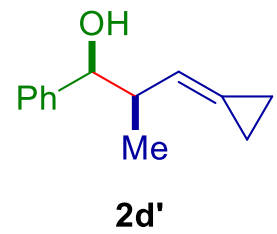

Following general procedure $\mathbf{C}$, using 1-bromo-3,5-bis(trifluoromethyl)benzene (73 $\mu \mathrm{L}, 0.42$ mmol, 1.4 equiv), $n$-BuLi (0.245 mL, 1.6M in hexanes, $0.390 \mathrm{mmol}, 1.30$ equiv), (Z)-4,4,5,5tetramethyl-2-(1-(prop-1-en-1-yl)cyclopropyl)-1,3,2-dioxaborolane (1d') (62.5 mg, 0.300 mmol, 1.00 equiv), TFAA ( $60 \mu \mathrm{L}, 0.42 \mathrm{mmol}, 1.4$ equiv) and benzaldehyde ( $46 \mu \mathrm{L}, 0.45 \mathrm{mmol}$, 1.5 equiv). The crude reaction mixture was purified by flash chromatography using EtOAc:pentane 1:10 as mobile phase to afford $\mathbf{2 d}$ ' $(36.0 \mathrm{mg}, 0.19 \mathrm{mmol}, 64 \%,>20: 1 \mathrm{dr})$ as a colourless liquid. TLC (EtOAc:pentane, $1: 9 \mathrm{v} / \mathrm{v}): \mathrm{R}_{\mathrm{f}}=0.28, \mathrm{KMnO}_{4} ;{ }^{1} \mathrm{H} \mathrm{NMR}(500 \mathrm{MHz}$, $\left.\mathrm{CDCl}_{3}\right): \delta 7.33-7.27(\mathrm{~m}, 4 \mathrm{H}), 7.25-7.17(\mathrm{~m}, 1 \mathrm{H}), 5.66(\mathrm{dp}, J=7.3,2.0 \mathrm{~Hz}, 1 \mathrm{H}), 4.66(\mathrm{~d}, J$ $=5.8 \mathrm{~Hz}, 1 \mathrm{H}), 2.77(\mathrm{dtt}, J=8.1,7.0,5.8 \mathrm{~Hz}, 1 \mathrm{H}), 2.00(\mathrm{brs}, 1 \mathrm{H}), 1.07$ (d, $J=6.9 \mathrm{~Hz}, 3 \mathrm{H}), 1.05$ $-0.80(\mathrm{~m}, 4 \mathrm{H}) ;{ }^{13} \mathrm{C} \mathrm{NMR}\left(126 \mathrm{MHz}, \mathrm{CDCl}_{3}\right): \delta 142.9,127.9,127.1,126.5,123.2,119.8,77.7$, $43.8,14.9,2.5,1.7 ; \mathrm{IR} v 3401,2977,1452,1279,1138,1014,749,699 ; \mathrm{C}_{13} \mathrm{H}_{16} \mathrm{ONa}[\mathrm{M}+\mathrm{Na}]^{+}$ 211.1093; found 211.1102. See spectra 


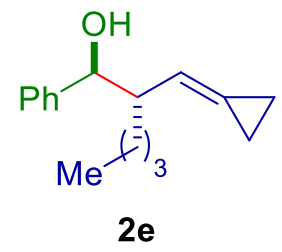

Following general procedure $\mathbf{C}$, using 1-bromo-3,5-bis(trifluoromethyl)benzene (73 $\mu \mathrm{L}, 0.42$ mmol, 1.4 equiv), $n$-BuLi $(0.245 \mathrm{~mL}, 1.6 \mathrm{M}$ in hexanes, $0.390 \mathrm{mmol}, 1.30$ equiv), $(E)-2-(1-$ (hex-1-en-1-yl)cyclopropyl)-4,4,5,5-tetramethyl-1,3,2-dioxaborolane (1e) (75.1 mg, 0.300 mmol, 1.00 equiv), TFAA ( $60 \mu \mathrm{L}, 0.42 \mathrm{mmol}, 1.4$ equiv) and benzaldehyde ( $46 \mu \mathrm{L}, 0.45 \mathrm{mmol}$, 1.5 equiv). The crude reaction mixture was purified by flash chromatography using $\mathrm{Et}_{2} \mathrm{O}$ :pentane 1:15 as mobile phase to afford $2 \mathrm{e}(58.5 \mathrm{mg}, 0.254 \mathrm{mmol}, 84 \%,>20: 1 \mathrm{dr})$ as a colourless liquid. TLC $\left(\mathrm{Et}_{2} \mathrm{O}\right.$ :pentane, $\left.1: 10 \mathrm{v} / \mathrm{v}\right): \mathrm{R}_{\mathrm{f}}=0.24, \mathrm{KMnO}_{4} ;{ }^{1} \mathrm{H} \mathrm{NMR}(500 \mathrm{MHz}$, $\left.\mathrm{CDCl}_{3}\right): \delta 7.34-7.30(\mathrm{~m}, 4 \mathrm{H}), 7.29-7.22(\mathrm{~m}, 1 \mathrm{H}), 5.63(\mathrm{dt}, J=9.1,2.0 \mathrm{~Hz}, 1 \mathrm{H}), 4.48(\mathrm{~d}, J=$ $7.6 \mathrm{~Hz}, 1 \mathrm{H}), 2.53$ (tdd, $J=9.2,7.5,3.9 \mathrm{~Hz}, 1 \mathrm{H}), 2.07$ (s, 1H), $1.32-1.16(\mathrm{~m}, 4 \mathrm{H}), 1.16-1.08$ $(\mathrm{m}, 4 \mathrm{H}), 1.07-0.90(\mathrm{~m}, 2 \mathrm{H}), 0.83-0.71(\mathrm{~m}, 3 \mathrm{H}) ;{ }^{13} \mathrm{C} \mathrm{NMR}\left(126 \mathrm{MHz}, \mathrm{CDCl}_{3}\right): \delta 142.9$, 128.1, 127.4, 126.9, 126.1, 118.6, 77.4, 50.8, 30.8, 29.5, 22.5, 14.0, 2.7, 2.4; IR v 3433, 3030, 2956, 2929, 2858, 1603, 1494, 1453, 1378, 1277, 1178, 1026, 968, 749; HRMS (ESI) calcd. for $\mathrm{C}_{16} \mathrm{H}_{22} \mathrm{ONa}[\mathrm{M}+\mathrm{Na}]^{+} 253.1563$; found 253.1559. See spectra

\section{(1s,2s)-6-Chloro-2-(cyclopropylidenemethyl)-1-phenylhexan-1-ol (2f)}

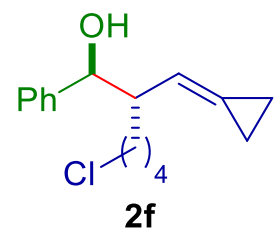

Following general procedure $\mathbf{C}$, using 1-bromo-3,5-bis(trifluoromethyl)benzene (73 $\mu \mathrm{L}, 0.42$ mmol, 1.4 equiv), $n$-BuLi ( $0.245 \mathrm{~mL}, 1.6 \mathrm{M}$ in hexanes, $0.390 \mathrm{mmol}, 1.30$ equiv), (E)-2-(1-(6chlorohex-1-en-1-yl)cyclopropyl)-4,4,5,5-tetramethyl-1,3-dioxolane (1f) (85.5 mg, 0.300 mmol, 1.00 equiv), TFAA ( $60 \mu \mathrm{L}, 0.42 \mathrm{mmol}, 1.4$ equiv) and benzaldehyde ( $46 \mu \mathrm{L}, 0.45 \mathrm{mmol}$, 1.5 equiv). The crude reaction mixture was purified by flash chromatography using $\mathrm{Et}_{2} \mathrm{O}$ :pentane 1:10 as mobile phase to afford $2 \mathbf{f}(78.5 \mathrm{mg}, 0.285 \mathrm{mmol}, 95 \%,>20: 1 \mathrm{dr})$ as a colourless liquid. TLC (Et $2 \mathrm{O}$ :pentane, 1:10 v/v): $\mathrm{R}_{\mathrm{f}}=0.20, \mathrm{KMnO}_{4} ;{ }^{1} \mathrm{H}$ NMR $(500 \mathrm{MHz}$, $\left.\mathrm{CDCl}_{3}\right): \delta 7.38-7.30(\mathrm{~m}, 4 \mathrm{H}), 7.29-7.24(\mathrm{~m}, 1 \mathrm{H}), 5.64(\mathrm{dt}, J=9.0,2.0 \mathrm{~Hz}, 1 \mathrm{H}), 4.50(\mathrm{~d}, J=$ 
7.6 Hz, 1H), $3.50-3.31(\mathrm{~m}, 2 \mathrm{H}), 2.55$ (tdd, $J=9.5,7.6,3.8 \mathrm{~Hz}, 1 \mathrm{H}), 1.98$ (br s, 1H), 1.79 $1.55(\mathrm{~m}, 2 \mathrm{H}), 1.45-1.38(\mathrm{~m}, 1 \mathrm{H}), 1.36-1.22(\mathrm{~m}, 3 \mathrm{H}), 1.16-1.10(\mathrm{~m}, 2 \mathrm{H}), 1.07-1.01(\mathrm{~m}$, $1 \mathrm{H}), 1.00-0.94(\mathrm{~m}, 1 \mathrm{H}) ;{ }^{13} \mathrm{C} \mathrm{NMR}\left(126 \mathrm{MHz}, \mathrm{CDCl}_{3}\right): \delta 142.7,128.2,127.5,126.9,126.5$, 118.2, 77.4, 50.7, 44.9, 32.4, 30.3, 24.6, 2.8, 2.4; IR v 3434, 2977, 1494, 1452, 1393, 1279 , 1192, 1066, 1047, 936, 750; HRMS (ESI) calcd. for $\mathrm{C}_{16} \mathrm{H}_{21} \mathrm{OClNa}[\mathrm{M}+\mathrm{Na}]^{+}$287.1173; found 287.1184. See spectra

(1s,2s)-4-((Tert-butyldimethylsilyl)oxy)-2-(cyclopropylidenemethyl)-1-phenylbutan-1-ol $(2 \mathrm{~g})$

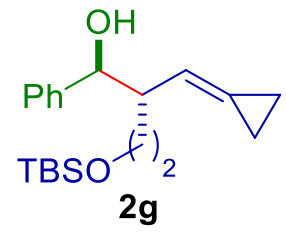

Following general procedure $\mathbf{C}$, using 1-bromo-3,5-bis(trifluoromethyl)benzene (73 $\mu \mathrm{L}, 0.42$ mmol, 1.4 equiv), $n$-BuLi ( $0.245 \mathrm{~mL}, 1.6 \mathrm{M}$ in hexanes, $0.390 \mathrm{mmol}, 1.30$ equiv), $(E)$-tertbutyldimethyl((4-(1-(4,4,5,5-tetramethyl-1,3,2-dioxaborolan-2-yl)cyclopropyl)but-3-en-1yl)oxy)silane (1g) (106 mg, $0.300 \mathrm{mmol}, 1.00$ equiv), TFAA (60 $\mu \mathrm{L}, 0.42 \mathrm{mmol}, 1.4$ equiv) and benzaldehyde ( $46 \mu \mathrm{L}, 0.45 \mathrm{mmol}, 1.5$ equiv). The crude reaction mixture was purified by flash chromatography using $\mathrm{Et}_{2} \mathrm{O}$ :pentane 1:10 as mobile phase to afford $\mathbf{2 g}$ (72.0 $\mathrm{mg}, 0.216$ mmol, $72 \%,>20: 1 \mathrm{dr})$ as a colourless liquid. TLC $\left(\mathrm{Et}_{2} \mathrm{O}\right.$ :pentane, 1:10 v/v): $\mathrm{R}_{\mathrm{f}}=0.22, \mathrm{KMnO}_{4}$; ${ }^{1} \mathrm{H}$ NMR $\left(500 \mathrm{MHz}, \mathrm{CDCl}_{3}\right): \delta 7.37-7.30(\mathrm{~m}, 4 \mathrm{H}), 7.30-7.24(\mathrm{~m}, 1 \mathrm{H}), 5.71(\mathrm{dt}, J=8.8,2.0$ $\mathrm{Hz}, 1 \mathrm{H}), 4.64$ (d, $J=6.6 \mathrm{~Hz}, 1 \mathrm{H}), 3.65$ (ddd, $J=10.2,6.4,5.5 \mathrm{~Hz}, 1 \mathrm{H}), 3.53$ (ddd, $J=10.2$, 7.6, $6.1 \mathrm{~Hz}, 1 \mathrm{H}), 2.79$ (tdd, $J=8.8,6.6,5.0 \mathrm{~Hz}, 1 \mathrm{H}), 2.36$ (br s, 1H), $1.81-1.54$ (m, 2H), 1.13 $-1.05(\mathrm{~m}, 2 \mathrm{H}), 1.04-0.97(\mathrm{~m}, 1 \mathrm{H}), 0.90-0.83(\mathrm{~m}, 10 \mathrm{H}), 0.01(\mathrm{~s}, 3 \mathrm{H}), 0.00(\mathrm{~s}, 3 \mathrm{H}) ;{ }^{13} \mathrm{C} \mathrm{NMR}$ $\left(126 \mathrm{MHz}, \mathrm{CDCl}_{3}\right): \delta 142.8,128.0,127.2,126.8,125.9,117.7,77.0,61.1,47.4,34.5,25.9$, 18.2, 2.6, 2.3, -5.4, -5.5; IR v 3368, 2954, 2928, 2885, 2857, 1723, 1494, 1452, 1277, 1253, 1087, 1065, 834, 773, 750; HRMS (ESI) calcd. for $\mathrm{C}_{20} \mathrm{H}_{32} \mathrm{NaO}_{2} \mathrm{Si}[\mathrm{M}+\mathrm{Na}]^{+}$355.2063; found 355.2074. See spectra

(1s,2r)-3-Cyclopropylidene-1,2-diphenylpropan-1-ol (2h)<smiles>OC(c1ccccc1)[C@H](C=C1CC1)c1ccccc1</smiles>

$2 \mathrm{~h}$ 
Following general procedure $\mathbf{C}$, using 1-bromo-3,5-bis(trifluoromethyl)benzene (73 $\mu \mathrm{L}, 0.42$ mmol, 1.4 equiv), $n$-BuLi ( $0.245 \mathrm{~mL}, 1.6 \mathrm{M}$ in hexanes, $0.390 \mathrm{mmol}, 1.30$ equiv), $(E)-4,4,5,5-$ tetramethyl-2-(1-styrylcyclopropyl)-1,3,2-dioxaborolane (1h) $(81.1 \mathrm{mg}, 0.300 \mathrm{mmol}, 1.00$ equiv), TFAA ( $60 \mu \mathrm{L}, 0.42 \mathrm{mmol}, 1.4$ equiv) and benzaldehyde ( $46 \mu \mathrm{L}, 0.45 \mathrm{mmol}, 1.5$ equiv). The crude reaction mixture was purified by flash chromatography using $\mathrm{Et}_{2} \mathrm{O}$ :pentane 1:10 as mobile phase to afford $\mathbf{2 h}(71.5 \mathrm{mg}, 0.286 \mathrm{mmol}, 95 \%,>20: 1 \mathrm{dr})$ as a colourless liquid. TLC $\left(\mathrm{Et}_{2} \mathrm{O}\right.$ :pentane, 1:10 v/v): $\mathrm{R}_{\mathrm{f}}=0.33, \mathrm{KMnO}_{4} ;{ }^{1} \mathrm{H} \mathrm{NMR}\left(500 \mathrm{MHz}, \mathrm{CDCl}_{3}\right): \delta 7.25-7.13(\mathrm{~m}$, $8 \mathrm{H}), 7.11(\mathrm{tt}, J=5.9,1.4 \mathrm{~Hz}, 2 \mathrm{H}), 6.22(\mathrm{dp}, J=9.1,2.0 \mathrm{~Hz}, 1 \mathrm{H}), 4.94(\mathrm{~d}, J=7.7 \mathrm{~Hz}, 1 \mathrm{H}), 3.77$ $(\mathrm{dd}, J=9.1,7.7 \mathrm{~Hz}, 1 \mathrm{H}), 2.31(\mathrm{~s}, 1 \mathrm{H}), 1.26-0.80(\mathrm{~m}, 4 \mathrm{H}) ;{ }^{13} \mathrm{C} \mathrm{NMR}\left(126 \mathrm{MHz}, \mathrm{CDCl}_{3}\right): \delta$ 142.0, 141.2, 128.3, 128.3, 127.8, 127.3, 126.7, 126.4, 126.4, 117.3, 77.9, 57.7, 2.9, 2.2; IR v 3438, 3065, 3028, 1682, 1600, 1494, 1451, 1277, 1176, 1135, 1027, 760; HRMS (ESI) calcd. for $\mathrm{C}_{18} \mathrm{H}_{18} \mathrm{NaO}[\mathrm{M}+\mathrm{Na}]^{+} 273.1249$; found 273.1251. See spectra

(1s,2s)-3-Cyclopropylidene-1,2-diphenylpropan-1-ol (2h')

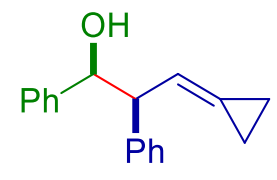

2h'

Following general procedure $\mathbf{C}$, using 1-bromo-3,5-bis(trifluoromethyl)benzene ( $73 \mu \mathrm{L}, 0.42$ mmol, 1.4 equiv), $n$-BuLi (0.245 mL, 1.6M in hexanes, $0.390 \mathrm{mmol}, 1.30$ equiv), (Z)-4,4,5,5tetramethyl-2-(1-styrylcyclopropyl)-1,3,2-dioxaborolane (1h') $(81.1 \mathrm{mg}, 0.300 \mathrm{mmol}, 1.00$ equiv), TFAA ( $60 \mu \mathrm{L}, 0.42 \mathrm{mmol}, 1.4$ equiv) and benzaldehyde ( $46 \mu \mathrm{L}, 0.45 \mathrm{mmol}, 1.5$ equiv). The crude reaction mixture was purified by flash chromatography using $\mathrm{Et}_{2} \mathrm{O}$ :pentane 1:10 as mobile phase to afford $\mathbf{2 h}$ ' (51.0 $\mathrm{mg}, 0.204 \mathrm{mmol}, 68 \%,>20: 1 \mathrm{dr}$ ) as a colourless liquid. TLC $\left(\mathrm{Et}_{2} \mathrm{O}\right.$ :pentane, 1:10 v/v): $\mathrm{R}_{\mathrm{f}}=0.33$, Anisaldehyde; ${ }^{1} \mathrm{H} \mathrm{NMR}\left(500 \mathrm{MHz}, \mathrm{CDCl}_{3}\right): \delta 7.42-7.19$ $(\mathrm{m}, 10 \mathrm{H}), 5.89(\mathrm{dq}, J=8.3,2.0 \mathrm{~Hz}, 1 \mathrm{H}), 4.95(\mathrm{~d}, J=8.6 \mathrm{~Hz}, 1 \mathrm{H}), 3.82(\mathrm{t}, J=8.5 \mathrm{~Hz}, 1 \mathrm{H}), 2.01$ (brs, $1 \mathrm{H}), 0.98-0.72(\mathrm{~m}, 3 \mathrm{H}), 0.43$ (dddd, $J=9.9,8.3,5.7,2.2 \mathrm{~Hz}, 1 \mathrm{H}) ;{ }^{13} \mathrm{C}$ NMR $(126 \mathrm{MHz}$, $\left.\mathrm{CDCl}_{3}\right): \delta$ 142.2, 141.2, 128.6, 127.9, 127.4, 127.1, 126.8, 125.1, 117.5, 77.8, 57.3, 2.4, 1.9; IR $v$ 3444, 3028, 2910, 1668, 1608, 1451, 1278, 1178, 1135, 975, 756, 699; HRMS (ESI) calcd. for $\mathrm{C}_{18} \mathrm{H}_{18} \mathrm{NaO}[\mathrm{M}+\mathrm{Na}]^{+} 273.1249$; found 273.1251. One carbon was not resolved at $126 \mathrm{MHz}$. $\underline{\text { See spectra }}$ 


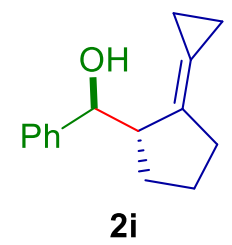

Following general procedure $\mathbf{C}$, using 1-bromo-3,5-bis(trifluoromethyl)benzene (73 $\mu \mathrm{L}, 0.42$ mmol, 1.4 equiv), $n$-BuLi (0.245 mL, $1.6 \mathrm{M}$ in hexanes, $0.390 \mathrm{mmol}, 1.30$ equiv), 2-(1(cyclopent-1-en-1-yl)cyclopropyl)-4,4,5,5-tetramethyl-1,3,2-dioxaborolane (1i) (70.5 mg, $0.300 \mathrm{mmol}, 1.00$ equiv), TFAA ( $60 \mu \mathrm{L}, 0.42 \mathrm{mmol}, 1.4$ equiv) and benzaldehyde ( $46 \mu \mathrm{L}, 0.45$ mmol, 1.5 equiv). The crude reaction mixture was purified by flash chromatography using $\mathrm{Et}_{2} \mathrm{O}$ :pentane 1:10 as mobile phase to afford $2 \mathbf{i}(46.0 \mathrm{mg}, 0.215 \mathrm{mmol}, 72 \%,>20: 1 \mathrm{dr}$ ) as a colourless liquid. TLC (Et $2 \mathrm{O}$ :pentane, 1:10 v/v): $\mathrm{R}_{\mathrm{f}}=0.24, \mathrm{KMnO}_{4} ;{ }^{1} \mathrm{H}$ NMR $(400 \mathrm{MHz}$, $\left.\mathrm{CDCl}_{3}\right): \delta 7.39-7.30(\mathrm{~m}, 4 \mathrm{H}), 7.29-7.23(\mathrm{~m}, 1 \mathrm{H}), 4.51(\mathrm{~d}, J=8.7 \mathrm{~Hz}, 1 \mathrm{H}), 2.95-2.80(\mathrm{~m}$, $1 \mathrm{H}), 2.75(\mathrm{~s}, 1 \mathrm{H}), 2.49-2.35(\mathrm{~m}, 2 \mathrm{H}), 1.75-1.64(\mathrm{~m}, 1 \mathrm{H}), 1.64-1.51(\mathrm{~m}, 2 \mathrm{H}), 1.48-1.34$ $(\mathrm{m}, 1 \mathrm{H}), 1.27-1.00(\mathrm{~m}, 4 \mathrm{H}) ;{ }^{13} \mathrm{C} \mathrm{NMR}\left(101 \mathrm{MHz}, \mathrm{CDCl}_{3}\right): \delta 142.8,133.7,128.1,127.5,127.0$, 113.6, 77.0, 51.6, 32.8, 29.5, 24.3, 3.7, 2.8; IR v 3414, 2953, 1712, 1494, 1451, 1382, 1278, 1174, 1133, 1056, 948, 913, 753; HRMS (ESI) calcd. for $\mathrm{C}_{15} \mathrm{H}_{18} \mathrm{NaO}[\mathrm{M}+\mathrm{Na}]^{+} 237.1250$; found 237.1246. See spectra

(s)-((s)-2-Cyclopropylidenecyclohexyl)(phenyl)methanol $(2 \mathbf{j})$

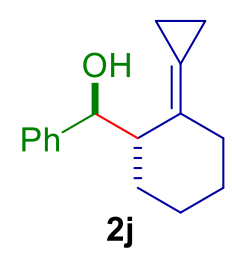

Following general procedure $\mathbf{C}$, using 1-bromo-3,5-bis(trifluoromethyl)benzene ( $73 \mu \mathrm{L}, 0.42$ mmol, 1.4 equiv), $n$-BuLi (0.245 mL, 1.6M in hexanes, $0.390 \mathrm{mmol}, 1.30$ equiv), 2-(1(cyclohex-1-en-1-yl)cyclopropyl)-4,4,5,5-tetramethyl-1,3,2-dioxaborolane (1j) $\quad 74.5 \mathrm{mg}$, $0.300 \mathrm{mmol}, 1.00$ equiv), TFAA ( $60 \mu \mathrm{L}, 0.42 \mathrm{mmol}, 1.4$ equiv) and benzaldehyde ( $46 \mu \mathrm{L}, 0.45$ mmol, 1.5 equiv). The crude reaction mixture was purified by flash chromatography using $\mathrm{Et}_{2} \mathrm{O}$ :pentane 1:10 as mobile phase to afford $\mathbf{2 \mathbf { j }}(60.0 \mathrm{mg}, 0.263 \mathrm{mmol}, 88 \%,>20: 1 \mathrm{dr})$ as a white semi-solid. TLC (Et $2 \mathrm{O}$ :pentane, 1:10 v/v): $\mathrm{R}_{\mathrm{f}}=0.24, \mathrm{KMnO}_{4} ;{ }^{1} \mathrm{H}$ NMR $(500 \mathrm{MHz}$, $\left.\mathrm{CDCl}_{3}\right): \delta 7.42-7.33(\mathrm{~m}, 4 \mathrm{H}), 7.32-7.27(\mathrm{~m}, 1 \mathrm{H}), 4.85(\mathrm{~d}, J=10.2 \mathrm{~Hz}, 1 \mathrm{H}), 2.62(\mathrm{dt}, J=$ 
9.9, $4.8 \mathrm{~Hz}, 1 \mathrm{H}), 2.47-2.33(\mathrm{~m}, 2 \mathrm{H}), 2.27(\mathrm{~s}, 1 \mathrm{H}), 1.79-1.70(\mathrm{~m}, 1 \mathrm{H}), 1.70-1.60(\mathrm{~m}, 1 \mathrm{H})$, $1.53-1.42(\mathrm{~m}, 2 \mathrm{H}), 1.37-1.18(\mathrm{~m}, 4 \mathrm{H}), 1.17-1.07(\mathrm{~m}, 2 \mathrm{H}) ;{ }^{13} \mathrm{C} \mathrm{NMR}\left(126 \mathrm{MHz}, \mathrm{CDCl}_{3}\right)$ : $\delta 142.7,128.2,127.6,127.2,116.5,73.8,50.8,31.5,28.9,27.7,23.0,3.0,1.7$; IR v 3458, 2974, 2928, 2855, 1494, 1447, 1273, 1191, 1037, 907, 762; HRMS (ESI) calcd. for $\mathrm{C}_{16} \mathrm{H}_{20} \mathrm{NaO}$ $[\mathrm{M}+\mathrm{Na}]^{+}$251.1406; found 251.1402. One carbon was not resolved at $126 \mathrm{MHz}$. See spectra

(E)-3-((1r,6s)-bicyclo[4.1.0]heptan-7-ylidene)-1-phenylpropan-1-ol (2k)

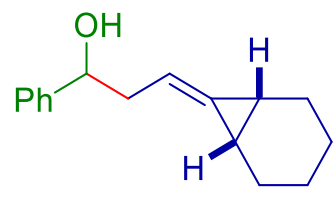

2k

Following general procedure $\mathbf{C}$, using 1-bromo-3,5-bis(trifluoromethyl)benzene ( $73 \mu \mathrm{L}, 0.42$ mmol, 1.4 equiv), $n$-BuLi (0.245 mL, 1.6M in hexanes, $0.390 \mathrm{mmol}, 1.30$ equiv), 4,4,5,5tetramethyl-2-((1r,6s,7r)-7-vinylbicyclo[4.1.0]heptan-7-yl)-1,3,2-dioxaborolane (1k) (74.5 $\mathrm{mg}, 0.300 \mathrm{mmol}, 1.00$ equiv), TFAA ( $60 \mu \mathrm{L}, 0.42 \mathrm{mmol}, 1.4$ equiv) and benzaldehyde ( $46 \mu \mathrm{L}$, $0.45 \mathrm{mmol}, 1.5$ equiv). The crude reaction mixture was purified by flash chromatography using $\mathrm{Et}_{2} \mathrm{O}$ :pentane $1: 10$ as mobile phase to afford $\mathbf{2 k}(26.0 \mathrm{mg}, 0.114 \mathrm{mmol}, 40 \%)$ as a thick colorless oil. TLC $\left(\mathrm{Et}_{2} \mathrm{O}\right.$ :pentane, 1:10 v/v): $\mathrm{R}_{\mathrm{f}}=0.25, \mathrm{KMnO}_{4} ;{ }^{1} \mathrm{H} \mathrm{NMR}\left(500 \mathrm{MHz}, \mathrm{CDCl}_{3}\right)$ : $\delta 7.41-7.37(\mathrm{~m}, 2 \mathrm{H}), 7.37-7.32(\mathrm{~m}, 2 \mathrm{H}), 7.29-7.24(\mathrm{~m}, 1 \mathrm{H}), 5.72(\mathrm{td}, J=7.2,3.6 \mathrm{~Hz}, 1 \mathrm{H})$, 4.77 (dd, $J=7.6,5.6 \mathrm{~Hz}, 1 \mathrm{H}), 2.69-2.51$ (m, 2H), 1.90 (br s, 1H), 1.75 (dq, $J=12.9,6.4 \mathrm{~Hz}$, 2H), $1.68-1.50(\mathrm{~m}, 4 \mathrm{H}), 1.24-1.05(\mathrm{~m}, 4 \mathrm{H}) ;{ }^{13} \mathrm{C} \mathrm{NMR}\left(126 \mathrm{MHz}, \mathrm{CDCl}_{3}\right): \delta 144.2,137.1$, 128.3, 127.4, 125.9, 112.8, 74.0, 41.8, 22.9, 22.4, 21.2, 21.1, 13.0, 12.3; IR v 3310, 2934, 2868 , $1738,1613,1450,1391,1277,1171,1131,945,877,758$; HRMS (ESI) calcd. for $\mathrm{C}_{16} \mathrm{H}_{20} \mathrm{NaO}$ $[\mathrm{M}+\mathrm{Na}]^{+}$251.1406; found 251.1404. See spectra

(1s,2s)-3-Cyclopropylidene-1-(4-methoxyphenyl)-2-methylpropan-1-ol (2l)

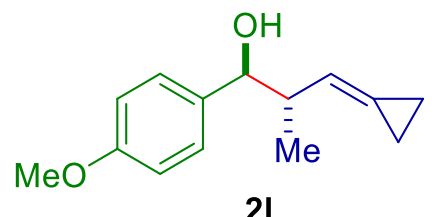

Following general procedure $\mathbf{C}$, using 1-bromo-3,5-bis(trifluoromethyl)benzene ( $73 \mu \mathrm{L}, 0.42$ mmol, 1.4 equiv), $n$-BuLi ( $0.245 \mathrm{~mL}, 1.6 \mathrm{M}$ in hexanes, $0.390 \mathrm{mmol}, 1.30$ equiv), $(E)-4,4,5,5-$ tetramethyl-2-(1-(prop-1-en-1-yl)cyclopropyl)-1,3,2-dioxaborolane (1d) (62.5 mg, 0.300 
mmol, 1.00 equiv), TFAA ( $60 \mu \mathrm{L}, 0.42 \mathrm{mmol}, 1.4$ equiv) and 4-methoxybenzaldehyde $(55 \mu \mathrm{L}$, $0.45 \mathrm{mmol}, 1.5$ equiv). The crude reaction mixture was purified by flash chromatography using $\mathrm{Et}_{2} \mathrm{O}$ :pentane 1:8 as mobile phase to afford $2 \mathrm{I}(59.5 \mathrm{mg}, 0.273 \mathrm{mmol}, 91 \%,>20: 1 \mathrm{dr})$ as a colourless liquid. TLC (Et $2 \mathrm{O}$ :pentane, 1:8 v/v): $\mathrm{R}_{\mathrm{f}}=0.3, \mathrm{KMnO}_{4} ;{ }^{1} \mathrm{H} \mathrm{NMR}\left(500 \mathrm{MHz}, \mathrm{CDCl}_{3}\right)$ : $\delta 7.33-7.24(\mathrm{~m}, 2 \mathrm{H}), 6.95-6.80(\mathrm{~m}, 2 \mathrm{H}), 5.79(\mathrm{dq}, J=8.2,2.1 \mathrm{~Hz}, 1 \mathrm{H}), 4.41(\mathrm{~d}, J=8.1 \mathrm{~Hz}$, 1H), 3.83 (s, 3H), 2.69 (h, $J=7.3 \mathrm{~Hz}, 1 \mathrm{H}), 2.17$ (br s, 1H), 1.12 (dq, $J=7.2,4.8,3.4 \mathrm{~Hz}, 4 \mathrm{H}$ ), $0.92(\mathrm{~d}, J=6.9 \mathrm{~Hz}, 3 \mathrm{H}) ;{ }^{13} \mathrm{C}$ NMR $\left(126 \mathrm{MHz}, \mathrm{CDCl}_{3}\right): \delta 159.0,134.9,128.0,124.3,120.2$, 113.5, 78.2, 55.2, 45.0, 17.0, 2.4, 2.3; IR v 2975, 2835, 1611, 1585, 1510, 1457, 1302, 1246, 1172, 1036, 967, 830, 750; HRMS (ESI) calcd. for $\mathrm{C}_{14} \mathrm{H}_{18} \mathrm{NaO}_{2}[\mathrm{M}+\mathrm{Na}]^{+}$241.1199; found 241.1205. See spectra

(1s,2s)-1-(3,5-Bis(trifluoromethyl)phenyl)-3-cyclopropylidene-2-methylpropan-1-ol (2m)<smiles>CC(C=C1CC1)C(O)c1cc(C(F)(F)F)cc(C(F)(F)F)c1</smiles>

Following general procedure $\mathbf{C}$, using 1-bromo-3,5-bis(trifluoromethyl)benzene (73 $\mu \mathrm{L}, 0.42$ mmol, 1.4 equiv), $n$-BuLi ( $0.245 \mathrm{~mL}, 1.6 \mathrm{M}$ in hexanes, $0.390 \mathrm{mmol}, 1.30$ equiv), $(E)-4,4,5,5-$ tetramethyl-2-(1-(prop-1-en-1-yl)cyclopropyl)-1,3,2-dioxaborolane (1d) (62.5 mg, 0.300 mmol, $\quad 1.00$ equiv), TFAA $(60 \mu \mathrm{L}, \quad 0.42 \quad \mathrm{mmol}, \quad 1.4$ equiv $)$ and 3,5bis(trifluoromethyl)benzaldehyde $(75 \mu \mathrm{L}, 0.45 \mathrm{mmol}, 1.5$ equiv). The crude reaction mixture was purified by flash chromatography using $\mathrm{Et}_{2} \mathrm{O}$ :pentane $1: 15$ as mobile phase to afford $\mathbf{2 m}$ (95.5 mg, $0.294 \mathrm{mmol}, 98 \%$, >20:1 dr) as a colourless liquid. TLC (Et $2 \mathrm{O}:$ pentane, 1:10 v/v): $\mathrm{R}_{\mathrm{f}}=0.35, \mathrm{KMnO}_{4} ;{ }^{1} \mathrm{H} \mathrm{NMR}\left(500 \mathrm{MHz}, \mathrm{CDCl}_{3}\right): \delta 7.80(\mathrm{~s}, 2 \mathrm{H}), 7.79(\mathrm{~s}, 1 \mathrm{H}), 5.70(\mathrm{dt}, J=8.4$, $2.1 \mathrm{~Hz}, 1 \mathrm{H}), 4.63(\mathrm{dd}, J=7.1,2.4 \mathrm{~Hz}, 1 \mathrm{H}), 2.67$ (p, $J=7.2 \mathrm{~Hz}, 1 \mathrm{H}), 2.34(\mathrm{~d}, J=2.7 \mathrm{~Hz}, 1 \mathrm{H})$, $1.12-1.03(\mathrm{~m}, 3 \mathrm{H}), 1.01(\mathrm{~d}, J=6.9 \mathrm{~Hz}, 3 \mathrm{H}), 0.93(\mathrm{qd}, J=7.5,2.1 \mathrm{~Hz}, 1 \mathrm{H}) ;{ }^{13} \mathrm{C} \mathrm{NMR}(126$ $\left.\mathrm{MHz}_{\mathrm{CDCl}}\right): \delta 145.4,131.3(\mathrm{q}, J=33.3 \mathrm{~Hz}), 127.0(\mathrm{q}, J=4.0 \mathrm{~Hz}), 126.2,123.4(\mathrm{q}, J=272.7$ Hz), 121.3 (p, $J=3.9$ Hz), 118.2, 77.3, 45.1, 16.7, 2.4, 2.4; IR v 3433, 2981, 1777, 1623, 1464 , $1362,1275,1168,1124,969,899,843,731$; HRMS (ESI) calcd. for $\mathrm{C}_{15} \mathrm{H}_{14} \mathrm{~F}_{6} \mathrm{NaO}[\mathrm{M}+\mathrm{Na}]^{+}$ 347.0841; found 347.0832. See spectra 


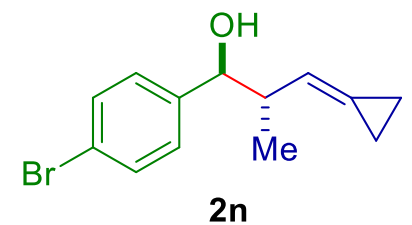

Following general procedure $\mathbf{C}$, using 1-bromo-3,5-bis(trifluoromethyl)benzene (73 $\mu \mathrm{L}, 0.42$ mmol, 1.4 equiv), $n$-BuLi ( $0.245 \mathrm{~mL}, 1.6 \mathrm{M}$ in hexanes, $0.390 \mathrm{mmol}, 1.30$ equiv), $(E)-4,4,5,5-$ tetramethyl-2-(1-(prop-1-en-1-yl)cyclopropyl)-1,3,2-dioxaborolane (1d) (62.5 mg, 0.300 mmol, 1.00 equiv), TFAA (60 $\mu \mathrm{L}, 0.42 \mathrm{mmol}, 1.4$ equiv) and 4-bromobenzaldehyde ( $83.5 \mathrm{mg}$, $0.45 \mathrm{mmol}, 1.5$ equiv). The crude reaction mixture was purified by flash chromatography using $\mathrm{Et}_{2} \mathrm{O}$ :pentane 1:10 as mobile phase to afford $\mathbf{2 n}(74.4 \mathrm{mg}, 0.279 \mathrm{mmol}, 93 \%,>20: 1 \mathrm{dr})$ as a colourless liquid. TLC $\left(\mathrm{Et}_{2} \mathrm{O}\right.$ :pentane, $\left.1: 10 \mathrm{v} / \mathrm{v}\right): \mathrm{R}_{\mathrm{f}}=0.22, \mathrm{KMnO}_{4} ;{ }^{1} \mathrm{H} \mathrm{NMR}(500 \mathrm{MHz}$, $\left.\mathrm{CDCl}_{3}\right): \delta 7.49-7.44(\mathrm{~m}, 2 \mathrm{H}), 7.25-7.18(\mathrm{~m}, 2 \mathrm{H}), 5.72(\mathrm{dt}, J=8.2,2.1 \mathrm{~Hz}, 1 \mathrm{H}), 4.41(\mathrm{~d}, J=$ $7.7 \mathrm{~Hz}, 1 \mathrm{H}), 2.63$ (h, $J=7.3 \mathrm{~Hz}, 1 \mathrm{H}), 2.20$ (br s, 1H), $1.16-0.99$ (m, 4H), 0.92 (d, $J=6.9 \mathrm{~Hz}$, $3 \mathrm{H}) ;{ }^{13} \mathrm{C}$ NMR (126 MHz, $\left.\mathrm{CDCl}_{3}\right): \delta 141.7,131.2,128.6,125.0,121.2,119.5,77.8,45.0,16.8$, 2.5, 2.3; IR v 3413, 2976, 2901, 1592, 1485, 1278, 1137, 1071, 1009, 820, 750; HRMS (ESI) calcd. for $\mathrm{C}_{13} \mathrm{H}_{15} \mathrm{BrNaO}[\mathrm{M}+\mathrm{Na}]^{+} 289.0198$; found 289.0202. See spectra

(1s,2s)-3-Cyclopropylidene-2-methyl-1-(pyridin-4-yl)propan-1-ol (2o)

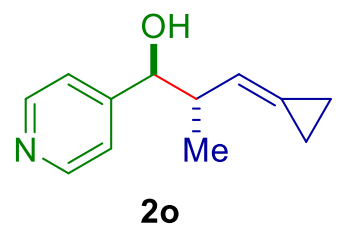

Following general procedure $\mathbf{C}$, using 1-bromo-3,5-bis(trifluoromethyl)benzene ( $73 \mu \mathrm{L}, 0.42$ mmol, 1.4 equiv), $n$-BuLi ( $0.245 \mathrm{~mL}, 1.6 \mathrm{M}$ in hexanes, $0.390 \mathrm{mmol}, 1.30$ equiv), $(E)-4,4,5,5-$ tetramethyl-2-(1-(prop-1-en-1-yl)cyclopropyl)-1,3,2-dioxaborolane (1d) (62.5 mg, 0.300 mmol, 1.00 equiv), TFAA ( $60 \mu \mathrm{L}, 0.42 \mathrm{mmol}, 1.4$ equiv) and isonicotinaldehyde ( $43 \mu \mathrm{L}, 0.45$ mmol, 1.5 equiv). The crude reaction mixture was purified by flash chromatography using EtOAc:pentane 1:1 as mobile phase to afford $20(36.8 \mathrm{mg}, 0.195 \mathrm{mmol}, 65 \%,>20: 1 \mathrm{dr})$ as a colourless liquid. TLC (EtOAc:pentane, 1:1 v/v): $\mathrm{R}_{\mathrm{f}}=0.28, \mathrm{KMnO}_{4} ;{ }^{1} \mathrm{H} \mathrm{NMR}(500 \mathrm{MHz}$, $\left.\mathrm{CDCl}_{3}\right): \delta 8.55-8.42(\mathrm{~m}, 2 \mathrm{H}), 7.33-7.14(\mathrm{~m}, 2 \mathrm{H}), 5.68(\mathrm{dt}, J=8.1,2.1 \mathrm{~Hz}, 1 \mathrm{H}), 4.51(\mathrm{~d}, J=$ $6.7 \mathrm{~Hz}, 1 \mathrm{H}), 2.93$ (br s, 1H), $2.72-2.63(\mathrm{~m}, 1 \mathrm{H}), 1.10-0.98(\mathrm{~m}, 6 \mathrm{H}), 0.97-0.88(\mathrm{~m}, 1 \mathrm{H})$; ${ }^{13} \mathrm{C} \mathrm{NMR}\left(126 \mathrm{MHz}, \mathrm{CDCl}_{3}\right): \delta 152.4,149.1,125.3,122.0,118.5,76.9,44.5,16.7,2.5,2.3$; IR 
$v$ 3204, 2975, 2901, 1603, 1561, 1453, 1413, 1219, 1065, 1001, 935, 797, 750; HRMS (ESI) calcd. for $\mathrm{C}_{12} \mathrm{H}_{15} \mathrm{NNaO}[\mathrm{M}+\mathrm{Na}]^{+} 212.1046$; found 212.1043. See spectra

(1s,2s)-3-Cyclopropylidene-2-methyl-1-(thiophen-2-yl)propan-1-ol (2p)

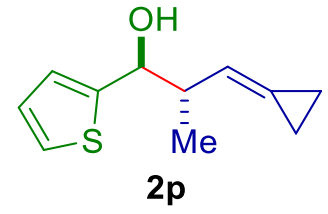

Following general procedure $\mathbf{C}$, using 1-bromo-3,5-bis(trifluoromethyl)benzene (73 $\mu \mathrm{L}, 0.42$ mmol, 1.4 equiv), $n$-BuLi ( $0.245 \mathrm{~mL}, 1.6 \mathrm{M}$ in hexanes, $0.390 \mathrm{mmol}, 1.30$ equiv), $(E)-4,4,5,5-$ tetramethyl-2-(1-(prop-1-en-1-yl)cyclopropyl)-1,3,2-dioxaborolane (1d) (62.5 mg, 0.300 mmol, 1.00 equiv), TFAA (60 $\mu \mathrm{L}, 0.42 \mathrm{mmol}, 1.4$ equiv) and thiophene-2-carbaldehyde (42 $\mu \mathrm{L}, 0.45 \mathrm{mmol}, 1.5$ equiv). The crude reaction mixture was purified by flash chromatography using $\mathrm{Et}_{2} \mathrm{O}$ :pentane 1:10 as mobile phase to afford $\mathbf{2 p}$ (44.6 mg, $0.230 \mathrm{mmol}, 76 \%,>20: 1 \mathrm{dr}$ ) as a colourless liquid. TLC (Et $2 \mathrm{O}$ :pentane, $1: 10 \mathrm{v} / \mathrm{v}): \mathrm{R}_{\mathrm{f}}=0.24, \mathrm{KMnO}_{4} ;{ }^{1} \mathrm{H} \mathrm{NMR}(400 \mathrm{MHz}$, $\left.\mathrm{CDCl}_{3}\right): \delta 7.28-7.23(\mathrm{~m}, 1 \mathrm{H}), 6.99(\mathrm{ddd}, J=3.4,1.3,0.6 \mathrm{~Hz}, 1 \mathrm{H}), 6.98-6.95(\mathrm{~m}, 1 \mathrm{H}), 5.78$ (dp, $J=8.1,2.0 \mathrm{~Hz}, 1 \mathrm{H}), 4.91-4.53(\mathrm{~m}, 1 \mathrm{H}), 2.83-2.58$ (m, 1H), 2.30 (br s, 1H), 1.11 (tdt, $J$ $=4.2,1.9,0.9 \mathrm{~Hz}, 4 \mathrm{H}), 1.01(\mathrm{~d}, J=6.8 \mathrm{~Hz}, 3 \mathrm{H}) ;{ }^{13} \mathrm{C} \mathrm{NMR}\left(101 \mathrm{MHz}, \mathrm{CDCl}_{3}\right): \delta 146.6,126.3$, 125.0, 124.7, 124.7, 119.4, 74.5, 45.4, 17.0, 2.6, 2.3; IR v 3050, 2976, 2928, 1718, 1664, 1437 , 1227, 1178, 1048, 967, 827; HRMS (ESI) calcd. for $\mathrm{C}_{11} \mathrm{H}_{14} \mathrm{NaOS}[\mathrm{M}+\mathrm{Na}]^{+} 217.0657$; found 217.0654. See spectra

(3r,4s,E)-5-Cyclopropylidene-4-methyl-1-phenylpent-1-en-3-ol (2q)

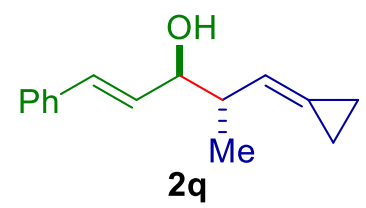

Following general procedure $\mathbf{C}$, using 1-bromo-3,5-bis(trifluoromethyl)benzene ( $73 \mu \mathrm{L}, 0.42$ mmol, 1.4 equiv), $n$-BuLi ( $0.245 \mathrm{~mL}, 1.6 \mathrm{M}$ in hexanes, $0.390 \mathrm{mmol}, 1.30$ equiv), $(E)-4,4,5,5-$ tetramethyl-2-(1-(prop-1-en-1-yl)cyclopropyl)-1,3,2-dioxaborolane (1d) (62.5 mg, 0.300 mmol, 1.00 equiv), TFAA (60 $\mu \mathrm{L}, 0.42 \mathrm{mmol}, 1.4$ equiv) and cinnamaldehyde (56 $\mu \mathrm{L}, 0.45$ mmol, 1.5 equiv). The crude reaction mixture was purified by flash chromatography using $\mathrm{Et}_{2} \mathrm{O}$ :pentane 1:10 as mobile phase to afford $\mathbf{2 q}(58.5 \mathrm{mg}, 0.273 \mathrm{mmol}, 91 \%,>20: 1 \mathrm{dr})$ as a colourless liquid. TLC (Et $2 \mathrm{O}$ :pentane, 1:10 v/v): $\mathrm{R}_{\mathrm{f}}=0.22, \mathrm{KMnO}_{4} ;{ }^{1} \mathrm{H}$ NMR $(400 \mathrm{MHz}$, 
$\left.\mathrm{CDCl}_{3}\right): \delta 7.41-7.35(\mathrm{~m}, 2 \mathrm{H}), 7.34-7.28(\mathrm{~m}, 2 \mathrm{H}), 7.27-7.19(\mathrm{~m}, 1 \mathrm{H}), 6.61(\mathrm{dd}, J=15.9$, $1.1 \mathrm{~Hz}, 1 \mathrm{H}), 6.23(\mathrm{dd}, J=15.9,6.9 \mathrm{~Hz}, 1 \mathrm{H}), 5.75(\mathrm{dp}, J=8.1,2.0 \mathrm{~Hz}, 1 \mathrm{H}), 4.11(\mathrm{td}, J=7.0$, $1.2 \mathrm{~Hz}, 1 \mathrm{H}), 2.58(\mathrm{ttd}, J=7.9,6.9,6.0 \mathrm{~Hz}, 1 \mathrm{H}), 1.88(\mathrm{br} \mathrm{s}, 1 \mathrm{H}), 1.17-1.00(\mathrm{~m}, 7 \mathrm{H}) ;{ }^{13} \mathrm{C} \mathrm{NMR}$ (101 MHz, $\left.\mathrm{CDCl}_{3}\right): \delta 136.8,131.5,130.5,128.5,127.5,126.4,124.3,119.6,76.8,43.4,16.5$, 2.5, 2.2; IR v 3398, 2923, 1673, 1492, 1146, 1392, 1278, 1175, 1133, 1027, 946, 763; HRMS (ESI) calcd. for $\mathrm{C}_{15} \mathrm{H}_{18} \mathrm{NaO}[\mathrm{M}+\mathrm{Na}]^{+} 237.1250$; found 237.1253. See spectra

\section{(2s,3r)-1-Cyclopropylidene-2-methyl-5-phenylpentan-3-ol (2r)}

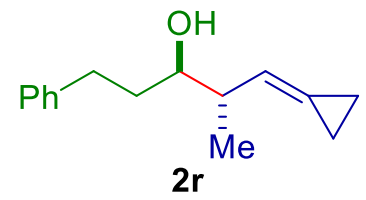

Following general procedure $\mathbf{C}$, using 1-bromo-3,5-bis(trifluoromethyl)benzene (73 $\mu \mathrm{L}, 0.42$ mmol, 1.4 equiv), $n$-BuLi ( $0.245 \mathrm{~mL}, 1.6 \mathrm{M}$ in hexanes, $0.390 \mathrm{mmol}, 1.30$ equiv), $(E)-4,4,5,5-$ tetramethyl-2-(1-(prop-1-en-1-yl)cyclopropyl)-1,3,2-dioxaborolane (1d) (62.5 mg, 0.300 mmol, 1.00 equiv), TFAA (60 $\mu \mathrm{L}, 0.42 \mathrm{mmol}, 1.4$ equiv) and 3-phenylpropanal (60 $\mu \mathrm{L}, 0.45$ mmol, 1.5 equiv). The crude reaction mixture was purified by flash chromatography using $\mathrm{Et}_{2} \mathrm{O}$ :pentane 1:10 as mobile phase to afford $2 \mathbf{r}(62.4 \mathrm{mg}, 0.288 \mathrm{mmol}, 96 \%,>20: 1 \mathrm{dr})$ as a colourless liquid. TLC $\left(\mathrm{Et}_{2} \mathrm{O}\right.$ :pentane, 1:10 v/v): $\mathrm{R}_{\mathrm{f}}=0.25, \mathrm{KMnO}_{4} ;{ }^{1} \mathrm{H} \mathrm{NMR}(400 \mathrm{MHz}$, $\left.\mathrm{CDCl}_{3}\right): \delta 7.32-7.26(\mathrm{~m}, 2 \mathrm{H}), 7.25-7.14(\mathrm{~m}, 3 \mathrm{H}), 5.71(\mathrm{dp}, J=8.2,2.0 \mathrm{~Hz}, 1 \mathrm{H}), 3.50$ (ddd, $J=9.2,6.1,3.4 \mathrm{~Hz}, 1 \mathrm{H}), 2.86(\mathrm{ddd}, J=13.8,10.2,5.2 \mathrm{~Hz}, 1 \mathrm{H}), 2.69$ (ddd, $J=13.7,10.0,6.6$ $\mathrm{Hz}, 1 \mathrm{H}), 2.57-2.32(\mathrm{~m}, 1 \mathrm{H}), 1.86$ (dddd, $J=13.6,10.0,6.5,3.3 \mathrm{~Hz}, 1 \mathrm{H}), 1.72$ (dddd, $J=13.9$, 10.0, 9.0, 5.3 Hz, 1H), 1.63 (br s, $1 \mathrm{H}), 1.15-1.02$ (m, 7H); $\left.{ }^{13} \mathrm{C} \mathrm{NMR} \mathrm{(101} \mathrm{MHz,} \mathrm{CDCl}_{3}\right): \delta$ $142.4,128.5,128.3,125.7,123.9,119.6,74.8,42.9,36.2,32.2,16.7,2.5,2.2$; IR v 3415, 2972 , 1714, 1603, 1496, 1454, 1278, 1177, 1066, 966, 749; HRMS (ESI) calcd. for $\mathrm{C}_{15} \mathrm{H}_{20} \mathrm{NaO}$ $[\mathrm{M}+\mathrm{Na}]^{+}$239.1406; found 239.1417. See procedure

(2s,3s)-4-Cyclopropylidene-3-methyl-1,1-diphenylbutan-2-ol (2s)

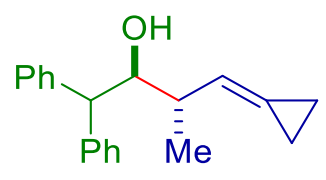

$2 s$

Following general procedure $\mathbf{C}$, using 1-bromo-3,5-bis(trifluoromethyl)benzene (73 $\mu \mathrm{L}, 0.42$ mmol, 1.4 equiv), $n$-BuLi ( $0.245 \mathrm{~mL}, 1.6 \mathrm{M}$ in hexanes, $0.390 \mathrm{mmol}, 1.30$ equiv), $(E)-4,4,5,5-$ 
tetramethyl-2-(1-(prop-1-en-1-yl)cyclopropyl)-1,3,2-dioxaborolane (1d) (62.5 mg, 0.300 mmol, 1.00 equiv), TFAA (60 $\mu \mathrm{L}, 0.42 \mathrm{mmol}, 1.4$ equiv) and 2,2-diphenylacetaldehyde (80 $\mu \mathrm{L}, 0.45 \mathrm{mmol}, 1.5$ equiv). The crude reaction mixture was purified by flash chromatography using $\mathrm{Et}_{2} \mathrm{O}$ :pentane 1:10 as mobile phase to afford $2 \mathrm{~s}(65.2 \mathrm{mg}, 0.234 \mathrm{mmol}, 78 \%,>20: 1 \mathrm{dr})$ as a colourless liquid. TLC (Et $2 \mathrm{O}$ :pentane, $1: 10 \mathrm{v} / \mathrm{v}): \mathrm{R}_{\mathrm{f}}=0.22, \mathrm{KMnO}_{4} ;{ }^{1} \mathrm{H} \mathrm{NMR}(400 \mathrm{MHz}$, $\left.\mathrm{CDCl}_{3}\right): \delta 7.44-7.35(\mathrm{~m}, 2 \mathrm{H}), 7.33-7.27(\mathrm{~m}, 2 \mathrm{H}), 7.27-7.14(\mathrm{~m}, 6 \mathrm{H}), 5.89(\mathrm{dt}, J=8.5,2.1$ $\mathrm{Hz}, 1 \mathrm{H}), 4.31(\mathrm{dd}, J=9.1,3.5 \mathrm{~Hz}, 1 \mathrm{H}), 3.98(\mathrm{~d}, J=9.2 \mathrm{~Hz}, 1 \mathrm{H}), 2.49(\mathrm{pd}, J=7.1,3.3 \mathrm{~Hz}, 1 \mathrm{H})$, $1.16(\mathrm{dd}, J=7.0,0.8 \mathrm{~Hz}, 3 \mathrm{H}), 1.05(\mathrm{ddtd}, J=13.7,9.9,6.7,5.5,1.8 \mathrm{~Hz}, 2 \mathrm{H}), 0.89$ (tdd, $J=$ 8.5, 5.7, 2.2 Hz, 1H), $0.82-0.73(\mathrm{~m}, 1 \mathrm{H}) ;{ }^{13} \mathrm{C} \mathrm{NMR}\left(101 \mathrm{MHz}, \mathrm{CDCl}_{3}\right): \delta 142.4,141.8,128.8$, 128.7, 128.5, 128.3, 126.7, 126.4, 123.5, 118.0, 77.7, 56.4, 38.9, 18.4, 2.3, 2.1; IR v 3559, 3026, 2975, 2925, 1659, 1597, 1494, 1450, 1277, 1141, 988, 846, 746; HRMS (ESI) calcd. for $\mathrm{C}_{20} \mathrm{H}_{22} \mathrm{NaO}[\mathrm{M}+\mathrm{Na}]^{+}$301.1563; found 301.1554. See spectra

\section{(2s,3s)-1-Cyclopropylidene-2,4,4-trimethylpentan-3-ol (2t)}

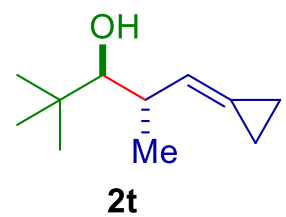

Following general procedure $\mathbf{C}$, using 1-bromo-3,5-bis(trifluoromethyl)benzene (73 $\mu \mathrm{L}, 0.42$ mmol, 1.4 equiv), $n$-BuLi ( $0.245 \mathrm{~mL}, 1.6 \mathrm{M}$ in hexanes, $0.390 \mathrm{mmol}, 1.30$ equiv), $(E)-4,4,5,5-$ tetramethyl-2-(1-(prop-1-en-1-yl)cyclopropyl)-1,3,2-dioxaborolane (1d) (62.5 mg, 0.300 mmol, 1.00 equiv), TFAA (60 $\mu \mathrm{L}, 0.42 \mathrm{mmol}, 1.4$ equiv) and pivalaldehyde ( $49 \mu \mathrm{L}, 0.45$ mmol, 1.5 equiv). The crude reaction mixture was purified by flash chromatography using $\mathrm{Et}_{2} \mathrm{O}$ :pentane 1:10 as mobile phase to afford $\mathbf{2 t}(22.4 \mathrm{mg}, 0.133 \mathrm{mmol}, 44 \%,>20: 1 \mathrm{dr})$ as a colourless liquid. Extensive exposure to vacuum was avoided due to product volatility. TLC $\left(\mathrm{Et}_{2} \mathrm{O}\right.$ :pentane, 1:10 v/v): $\mathrm{R}_{\mathrm{f}}=0.28, \mathrm{KMnO}_{4} ;{ }^{1} \mathrm{H} \mathrm{NMR}\left(500 \mathrm{MHz}, \mathrm{CDCl}_{3}\right): \delta 5.85(\mathrm{dt}, J=8.6$, $2.1 \mathrm{~Hz}, 1 \mathrm{H}), 3.18(\mathrm{~d}, J=2.5 \mathrm{~Hz}, 1 \mathrm{H}), 2.81-2.66(\mathrm{~m}, 1 \mathrm{H}), 1.16(\mathrm{~d}, J=7.0 \mathrm{~Hz}, 3 \mathrm{H}), 1.13-0.98$ (m, 4H), 0.91 (s, 9H); $\left.{ }^{13} \mathrm{C} \mathrm{NMR} \mathrm{(126} \mathrm{MHz,} \mathrm{CDCl}_{3}\right): \delta 122.4,119.6,83.5,38.1,35.7,26.6$, 20.9, 2.4, 2.1; IR v 3436, 2963, 2871, 1699, 1465, 1392, 1279, 1178, 1137, 984; HRMS (ESI) calcd. for $\mathrm{C}_{11} \mathrm{H}_{20} \mathrm{NaO}[\mathrm{M}+\mathrm{Na}]^{+}$191.1406; found 191.1402. $\underline{\text { See spectra }}$

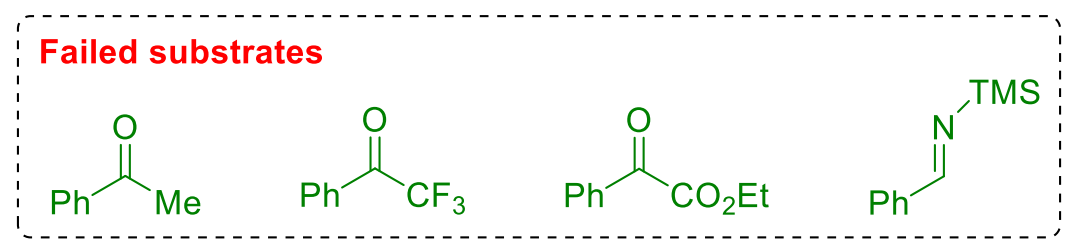




\section{3-Cyclopropylidene- $N, N$-dimethyl-3-phenylpropan-1-amine (7a)}

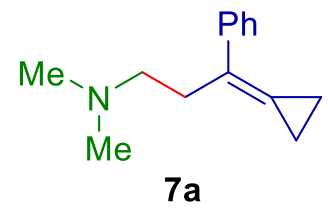

Following general procedure $\mathbf{D}$, using 1-bromo-3,5-bis(trifluoromethyl)benzene (73 $\mu \mathrm{L}, 0.42$ mmol, 1.4 equiv), $n$-BuLi ( $0.245 \mathrm{~mL}, 1.6 \mathrm{M}$ in hexanes, $0.390 \mathrm{mmol}, 1.30$ equiv), 4,4,5,5tetramethyl-2-(1-(1-phenylvinyl)cyclopropyl)-1,3,2-dioxaborolane (1c) (81.1 mg, 0.300 mmol, 1.00 equiv), and Eschenmoser's salt ( $83.5 \mathrm{mg}, 0.450 \mathrm{mmol}, 1.50$ equiv). The crude reaction mixture was purified by flash chromatography using DCM:MeOH 1:15 as mobile phase to afford 7a (59.2 $\mathrm{mg}, 0.294 \mathrm{mmol}, 98 \%)$ as a pale yellow liquid. TLC (DCM:MeOH, $1: 10 \mathrm{v} / \mathrm{v}): \mathrm{R}_{\mathrm{f}}=0.22, \mathrm{KMnO}_{4} ;{ }^{1} \mathrm{H}$ NMR $\left(400 \mathrm{MHz}, \mathrm{CDCl}_{3}\right): \delta 7.62-7.55(\mathrm{~m}, 2 \mathrm{H}), 7.39-7.30$ $(\mathrm{m}, 2 \mathrm{H}), 7.26-7.20(\mathrm{~m}, 1 \mathrm{H}), 3.09-3.01(\mathrm{~m}, 2 \mathrm{H}), 2.89-2.75(\mathrm{~m}, 2 \mathrm{H}), 2.56(\mathrm{~s}, 6 \mathrm{H}), 1.49-$ $1.32(\mathrm{~m}, 2 \mathrm{H}), 1.31-1.05(\mathrm{~m}, 2 \mathrm{H}) ;{ }^{13} \mathrm{C} \mathrm{NMR}\left(101 \mathrm{MHz}, \mathrm{CDCl}_{3}\right): \delta 138.9,128.5,126.9,125.8$, 123.7, 123.1, 57.5, 44.1, 30.5, 5.0, 1.3; IR v 3412, 2970, 2466, 1668, 1597, 1445, 1394, 1250, 1066, 964, 763; HRMS (ESI) calcd. for $\mathrm{C}_{14} \mathrm{H}_{20} \mathrm{~N}[\mathrm{M}+\mathrm{H}]^{+}$202.1590; found 202.1599. See spectra

\section{1-(3-Cyclopropylidene-3-phenylpropyl)piperidine (7b)}

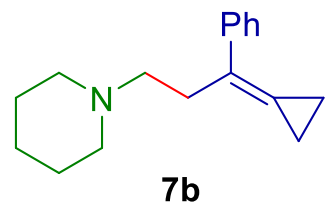

Following general procedure D, using 1-bromo-3,5-bis(trifluoromethyl)benzene ( $73 \mu \mathrm{L}, 0.42$ mmol, 1.4 equiv), $n$-BuLi (0.245 mL, 1.6M in hexanes, $0.390 \mathrm{mmol}, 1.30$ equiv), 4,4,5,5tetramethyl-2-(1-(1-phenylvinyl)cyclopropyl)-1,3,2-dioxaborolane (1c) (81.1 mg, 0.300 mmol, 1.00 equiv), and piperidine derived iminium salt $^{2}$ (102 $\mathrm{mg}, 0.450 \mathrm{mmol}, 1.50$ equiv). The crude reaction mixture was purified by flash chromatography using DCM:MeOH 1:15 as mobile phase to afford $7 \mathbf{b}(66.2 \mathrm{mg}, 0.274 \mathrm{mmol}, 91 \%)$ as a pale yellow liquid. TLC (DCM:MeOH, 1:10 v/v): $\mathrm{R}_{\mathrm{f}}=0.24, \mathrm{KMnO}_{4} ;{ }^{1} \mathrm{H} \mathrm{NMR}\left(500 \mathrm{MHz}, \mathrm{CDCl}_{3}\right): \delta 7.64-7.57(\mathrm{~m}$, 2H), $7.33(\mathrm{t}, J=7.8 \mathrm{~Hz}, 2 \mathrm{H}), 7.25-7.19(\mathrm{~m}, 1 \mathrm{H}), 2.99-2.84(\mathrm{~m}, 2 \mathrm{H}), 2.67-2.45(\mathrm{~m}, 6 \mathrm{H})$, $1.67(\mathrm{p}, J=5.7 \mathrm{~Hz}, 4 \mathrm{H}), 1.52-1.43(\mathrm{~m}, 2 \mathrm{H}), 1.43-1.36(\mathrm{~m}, 2 \mathrm{H}), 1.19-1.13(\mathrm{~m}, 2 \mathrm{H}) ;{ }^{13} \mathrm{C}$ NMR (126 MHz, $\left.\mathrm{CDCl}_{3}\right): \delta 139.6,128.2,126.6,125.8,125.1,121.9,58.1,54.4,30.9,25.5$, 
24.1, 5.0, 1.1; IR v 2938, 1609, 1496, 1445, 1377, 1277, 1127, 1074, 762, 697; HRMS (ESI) calcd. for $\mathrm{C}_{17} \mathrm{H}_{24} \mathrm{~N}[\mathrm{M}+\mathrm{H}]^{+} 242.1903$; found 242.1905. See spectra

7-(2-Cyclopropylidene-2-phenylethyl)cyclohepta-1,3,5-triene (7c)

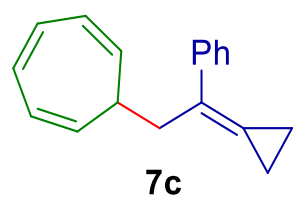

Following general procedure $\mathbf{D}$, using 1-bromo-3,5-bis(trifluoromethyl)benzene (73 $\mu \mathrm{L}, 0.42$ mmol, 1.4 equiv), $n$-BuLi ( $0.245 \mathrm{~mL}, 1.6 \mathrm{M}$ in hexanes, $0.390 \mathrm{mmol}, 1.30$ equiv), 4,4,5,5tetramethyl-2-(1-(1-phenylvinyl)cyclopropyl)-1,3,2-dioxaborolane (1c) (81.1 $\mathrm{mg}, \quad 0.300$ mmol, 1.00 equiv), and tropylium tetrafluoroborate salt $(80.1 \mathrm{mg}, 0.450 \mathrm{mmol}, 1.50$ equiv). The crude reaction mixture was purified by flash chromatography using pentane to afford $\mathbf{7 c}$ (55.8 mg, $0.238 \mathrm{mmol}, 79 \%)$ as a colourless liquid. TLC (pentane): $\mathrm{R}_{\mathrm{f}}=0.34, \mathrm{KMnO}_{4} ;{ }^{1} \mathrm{H}$ NMR (500 MHz, $\left.\mathrm{CDCl}_{3}\right): \delta 7.60-7.53(\mathrm{~m}, 2 \mathrm{H}), 7.31(\mathrm{dd}, J=8.4,7.1 \mathrm{~Hz}, 2 \mathrm{H}), 7.23-7.16$ (m, 1H), $6.67-6.49$ (m, 2H), 6.13 (dddd, $J=9.0,3.9,2.6,1.5 \mathrm{~Hz}, 2 \mathrm{H}), 5.23(\mathrm{dd}, J=9.2,5.4$ $\mathrm{Hz}, 2 \mathrm{H}), 3.08$ (dp, $J=8.0,1.4 \mathrm{~Hz}, 2 \mathrm{H}), 2.06-1.99(\mathrm{~m}, 1 \mathrm{H}), 1.47-1.40$ (m, 2H), $1.25-1.04$ $(\mathrm{m}, 2 \mathrm{H}) ;{ }^{13} \mathrm{C} \mathrm{NMR}\left(126 \mathrm{MHz}, \mathrm{CDCl}_{3}\right): \delta 140.0,130.8,128.2,126.7,126.5,125.9,125.4,124.6$, 122.5, 37.7, 37.5, 5.0, 2.0; IR v 2988, 2901, 1720, 1494, 1448, 1394, 1278, 1172, 1066, 760, 700; HRMS (EI) calcd. for $\mathrm{C}_{18} \mathrm{H}_{17}[\mathrm{M}-\mathrm{H}]^{+} 233.1325$; found 233.1324. See spectra

\section{7-(2-Cyclopropylidenepropyl)cyclohepta-1,3,5-triene (7d)}

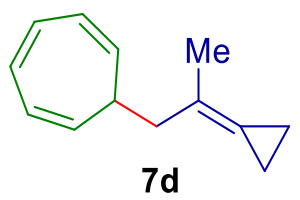

Following general procedure D, using 1-bromo-3,5-bis(trifluoromethyl)benzene (73 $\mu \mathrm{L}, 0.42$ mmol, 1.4 equiv), $n$-BuLi (0.245 mL, 1.6M in hexanes, $0.390 \mathrm{mmol}, 1.30$ equiv), 4,4,5,5tetramethyl-2-(1-(prop-1-en-2-yl)cyclopropyl)-1,3,2-dioxaborolane (1b) (62.5 mg, 0.300 mmol, 1.00 equiv), and tropylium tetrafluoroborate $(80.1 \mathrm{mg}, 0.450 \mathrm{mmol}, 1.50$ equiv). The crude reaction mixture was purified by flash chromatography using pentane to afford 7d (44.0 $\mathrm{mg}, 0.244 \mathrm{mmol}, 85 \%$ ) as a colourless liquid. TLC (pentane): $\mathrm{R}_{\mathrm{f}}=0.33, \mathrm{KMnO}_{4} ;{ }^{1} \mathrm{H} \mathrm{NMR}$ $\left(500 \mathrm{MHz}, \mathrm{CDCl}_{3}\right): \delta 6.73-6.48(\mathrm{~m}, 2 \mathrm{H}), 6.19-6.10(\mathrm{~m}, 2 \mathrm{H}), 5.17(\mathrm{dd}, J=9.2,5.3 \mathrm{~Hz}, 2 \mathrm{H})$, $2.59(\mathrm{dt}, J=8.1,1.5 \mathrm{~Hz}, 2 \mathrm{H}), 1.96-1.87(\mathrm{~m}, 1 \mathrm{H}), 1.78$ (p, $J=1.7 \mathrm{~Hz}, 3 \mathrm{H}), 1.14-0.93$ (m, 
$4 \mathrm{H}) ;{ }^{13} \mathrm{C} \mathrm{NMR}\left(126 \mathrm{MHz}, \mathrm{CDCl}_{3}\right): \delta 130.8,126.9,124.4,122.3,117.3,40.5,37.2,20.7,3.3$, 1.9; IR $v$ 3011, 2974, 2912, 1444, 1397, 1210, 1066, 994, 874, 742; HRMS (EI) calcd. for $\mathrm{C}_{13} \mathrm{H}_{16}[\mathrm{M}]^{+}$172.1247; found 172.1244. See spectra

\section{2-(2-Cyclopropylidene-2-phenylethyl)benzo[d][1,3]dithiole (7f)}

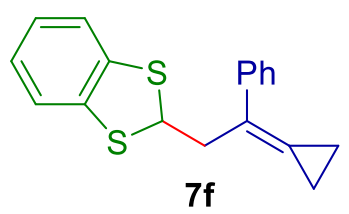

Following general procedure $\mathbf{D}$, using 1-bromo-3,5-bis(trifluoromethyl)benzene (73 $\mu \mathrm{L}, 0.42$ mmol, 1.4 equiv), $n$-BuLi ( $0.245 \mathrm{~mL}, 1.6 \mathrm{M}$ in hexanes, $0.390 \mathrm{mmol}, 1.30$ equiv), 4,4,5,5tetramethyl-2-(1-(1-phenylvinyl)cyclopropyl)-1,3,2-dioxaborolane (1c) (81.1 $\mathrm{mg}, \quad 0.300$ mmol, 1.00 equiv), and benzo[d][1,3]dithiol-1-ium tetrafluoroborate (108 $\mathrm{mg}, 0.450 \mathrm{mmol}$, 1.50 equiv). The crude reaction mixture was purified by flash chromatography using $\mathrm{Et}_{2} \mathrm{O}$ :pentane $(1: 20)$ to afford $7 \mathbf{f}(69.0 \mathrm{mg}, 0.233 \mathrm{mmol}, 78 \%)$ as a colourless liquid. TLC $\left(\mathrm{Et}_{2} \mathrm{O}\right.$ :pentane, $\left.1: 15 \mathrm{v} / \mathrm{v}\right): \mathrm{R}_{\mathrm{f}}=0.25, \mathrm{KMnO}_{4} ;{ }^{1} \mathrm{H} \mathrm{NMR}\left(500 \mathrm{MHz}, \mathrm{CDCl}_{3}\right): \delta 7.60-7.55(\mathrm{~m}$, 2H), $7.42-7.34(\mathrm{~m}, 2 \mathrm{H}), 7.30-7.25(\mathrm{~m}, 1 \mathrm{H}), 7.23(\mathrm{dd}, J=5.7,3.2 \mathrm{~Hz}, 2 \mathrm{H}), 7.09-6.96(\mathrm{~m}$, 2H), 5.21 (t, $J=7.5 \mathrm{~Hz}, 1 \mathrm{H}), 3.32(\mathrm{dt}, J=7.6,1.3 \mathrm{~Hz}, 2 \mathrm{H}), 1.52-1.46(\mathrm{~m}, 2 \mathrm{H}), 1.30-1.23$ $(\mathrm{m}, 2 \mathrm{H}) ;{ }^{13} \mathrm{C} \mathrm{NMR}\left(126 \mathrm{MHz}, \mathrm{CDCl}_{3}\right): \delta 139.0,137.4,128.4,126.9,125.9,125.4,124.9,123.7$, 122.5, 53.6, 42.7, 4.9, 2.2; IR v 3055, 2973, 1778, 1683, 1598, 1444, 1309, 1278, 1179, 1136, 1038, 906, 744; HRMS (EI) calcd. for $\mathrm{C}_{18} \mathrm{H}_{15} \mathrm{~S}_{2}[\mathrm{M}-\mathrm{H}]^{+}$295.0610; found 295.0609. See spectra (1-Cyclopropylidene-3,3,3-trifluoropropyl)benzene (7g)

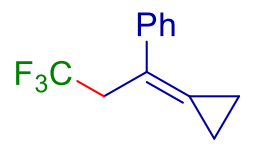

$7 g$

Following general procedure D, using 1-bromo-3,5-bis(trifluoromethyl)benzene ( $73 \mu \mathrm{L}, 0.42$ mmol, 1.4 equiv), $n$-BuLi ( $0.245 \mathrm{~mL}, 1.6 \mathrm{M}$ in hexanes, $0.390 \mathrm{mmol}, 1.30$ equiv), 4,4,5,5tetramethyl-2-(1-(1-phenylvinyl)cyclopropyl)-1,3,2-dioxaborolane (1c) (81.1 mg, 0.300 mmol, $\quad 1.00 \quad$ equiv), and 3,3-dimethyl-1-(trifluoromethyl)-1,3-dihydro-113benzo[d][1,2]iodaoxole (149 $\mathrm{mg}, 0.450 \mathrm{mmol}, 1.50$ equiv). The crude reaction mixture was purified by flash chromatography using pentane to afford $7 \mathrm{~g}(25.8 \mathrm{mg}, 0.122 \mathrm{mmol}, 41 \%)$ as a colourless liquid. Extensive exposure to vacuum was avoided due to product volatility. TLC 
(pentane): $\mathrm{R}_{\mathrm{f}}=0.55, \mathrm{KMnO}_{4} ;{ }^{1} \mathrm{H} \mathrm{NMR}\left(500 \mathrm{MHz}, \mathrm{CDCl}_{3}\right): \delta 7.60-7.54(\mathrm{~m}, 2 \mathrm{H}), 7.39-7.32$ $(\mathrm{m}, 2 \mathrm{H}), 7.28-7.24(\mathrm{~m}, 1 \mathrm{H}), 3.43(\mathrm{qt}, J=10.8,1.0 \mathrm{~Hz}, 2 \mathrm{H}), 1.55-1.50(\mathrm{~m}, 2 \mathrm{H}), 1.28-1.21$ $(\mathrm{m}, 2 \mathrm{H}) ;{ }^{13} \mathrm{C} \mathrm{NMR}\left(126 \mathrm{MHz}, \mathrm{CDCl}_{3}\right): \delta 138.7,128.8,128.3,127.1,125.8,117.7,37.9$ (q, $J=$ $29.6 \mathrm{~Hz}$ ), 6.0, 1.8; IR $\vee 2988,2906,1393,1255,1129$, 1066, 892; HRMS (EI) calcd. for $\mathrm{C}_{12} \mathrm{H}_{11} \mathrm{~F}_{3}[\mathrm{M}]^{+}$212.0807; found 212.0806. One carbon was not resolved at $500 \mathrm{MHz}$. See $\underline{\text { spectra }}$

\section{(1-Cyclopropylideneethyl)benzene (7h)}

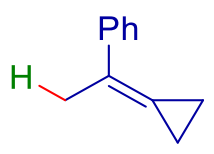

$7 \mathrm{~h}$

Following general procedure $\mathbf{D}$, using 1-bromo-3,5-bis(trifluoromethyl)benzene (73 $\mu \mathrm{L}, 0.42$ mmol, 1.4 equiv), $n$-BuLi ( $0.245 \mathrm{~mL}, 1.6 \mathrm{M}$ in hexanes, $0.390 \mathrm{mmol}, 1.30$ equiv), 4,4,5,5tetramethyl-2-(1-(1-phenylvinyl)cyclopropyl)-1,3,2-dioxaborolane (1c) (81.1 mg, 0.300 mmol, 1.00 equiv), and tetrafluoroboric acid diethyl ether complex (62 $\mu \mathrm{L}, 0.450 \mathrm{mmol}, 1.50$ equiv). The crude reaction mixture was purified by flash chromatography using pentane to afford $7 \mathrm{~h}$ (35.2 $\mathrm{mg}, 0.244 \mathrm{mmol}, 81 \%)$ as a colourless liquid. Extensive exposure to vacuum was avoided due to product volatility. TLC (pentane): $\mathrm{R}_{\mathrm{f}}=0.6, \mathrm{KMnO}_{4} ;{ }^{1} \mathrm{H} \mathrm{NMR}(500 \mathrm{MHz}$, $\left.\mathrm{CDCl}_{3}\right): \delta 7.68-7.63(\mathrm{~m}, 2 \mathrm{H}), 7.39-7.31(\mathrm{~m}, 2 \mathrm{H}), 7.25-7.16(\mathrm{~m}, 1 \mathrm{H}), 2.28-2.21(\mathrm{~m}, 3 \mathrm{H})$, $1.49-1.42$ (m, 2H), $1.14-1.08$ (m, 2H); $\left.{ }^{13} \mathrm{C} \mathrm{NMR} \mathrm{(101} \mathrm{MHz,} \mathrm{CDCl}_{3}\right): \delta 140.5,128.1,126.5$, $125.4,122.4,120.5,19.7,5.8,0.4$. The characterization data corresponded to the reported values. ${ }^{3}$

\section{(1-Cyclopropylidene-2-fluoroethyl)benzene (7i)}

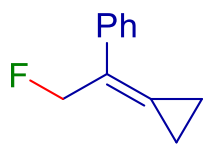

$7 \mathbf{i}$

Following general procedure $\mathbf{D}$, using 1-bromo-3,5-bis(trifluoromethyl)benzene (73 $\mu \mathrm{L}, 0.42$ mmol, 1.4 equiv), $n$-BuLi ( $0.245 \mathrm{~mL}, 1.6 \mathrm{M}$ in hexanes, $0.390 \mathrm{mmol}, 1.30$ equiv), 4,4,5,5tetramethyl-2-(1-(1-phenylvinyl)cyclopropyl)-1,3,2-dioxaborolane (1c) (81.1 mg, 0.300 mmol, $\quad 1.00 \quad$ equiv), and $\quad N$-Chloromethyl- $N$-fluorotriethylenediammonium bis(tetrafluoroborate) (160 mg, $0.450 \mathrm{mmol}, 1.50$ equiv). The crude reaction mixture was 
purified by flash chromatography using pentane to afford $7 \mathbf{i}(26.0 \mathrm{mg}, 0.160 \mathrm{mmol}, 53 \%)$ as a colourless liquid. Extensive exposure to vacuum was avoided due to product volatility. TLC (pentane): $\mathrm{R}_{\mathrm{f}}=0.48, \mathrm{KMnO}_{4} ;{ }^{1} \mathrm{H} \mathrm{NMR}\left(500 \mathrm{MHz}, \mathrm{CDCl}_{3}\right): \delta 7.73-7.66(\mathrm{~m}, 2 \mathrm{H}), 7.38(\mathrm{t}, J=$ $7.7 \mathrm{~Hz}, 2 \mathrm{H}), 7.31-7.25(\mathrm{~m}, 1 \mathrm{H}), 5.42$ (d, $J=48.3 \mathrm{~Hz}, 2 \mathrm{H}), 1.59-1.50$ (m, 2H), $1.33-1.21$ $(\mathrm{m}, 2 \mathrm{H}) ;{ }^{13} \mathrm{C}$ NMR $\left(126 \mathrm{MHz}, \mathrm{CDCl}_{3}\right): \delta 137.3,129.1(\mathrm{~d}, J=11.2 \mathrm{~Hz}), 128.4,127.2,125.8$, $123.3(\mathrm{~d}, J=15.2 \mathrm{~Hz}), 84.7(\mathrm{~d}, J=165.2 \mathrm{~Hz}), 5.2(\mathrm{~d}, J=1.9 \mathrm{~Hz}), 0.7(\mathrm{~d}, J=2.2 \mathrm{~Hz})$; IR v 2971, 1598, 1492, 1445, 1393, 1241, 1066, 1027, 902, 762; HRMS (EI) calcd. for $\mathrm{C}_{11} \mathrm{H}_{11} \mathrm{~F}$ $[\mathrm{M}]^{+}$162.0839; found 162.0838. See spectra

\section{(2-Chloro-1-cyclopropylideneethyl)benzene (7j)}

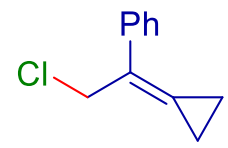

$7 \mathbf{j}$

Following general procedure $\mathbf{D}$, using 1-bromo-3,5-bis(trifluoromethyl)benzene (73 $\mu \mathrm{L}, 0.42$ mmol, 1.4 equiv), $n$-BuLi ( $0.245 \mathrm{~mL}, 1.6 \mathrm{M}$ in hexanes, $0.390 \mathrm{mmol}, 1.30$ equiv), 4,4,5,5tetramethyl-2-(1-(1-phenylvinyl)cyclopropyl)-1,3,2-dioxaborolane (1c) (81.1 mg, 0.300 mmol, 1.00 equiv), and 1,3-dichloro-5,5-dimethylimidazolidine-2,4-dione (89.0 mg, 0.450 mmol, 1.50 equiv). The crude reaction mixture was purified by flash chromatography using pentane to afford $7 \mathbf{j}$ ( $44.2 \mathrm{mg}, 0.247 \mathrm{mmol}, 82 \%$ ) as a colourless liquid. Extensive exposure to vacuum was avoided due to product volatility. TLC (pentane): $\mathrm{R}_{\mathrm{f}}=0.45, \mathrm{KMnO}_{4} ;{ }^{1} \mathrm{H} \mathrm{NMR}$ (500 MHz, $\left.\mathrm{CDCl}_{3}\right): \delta 7.76$ - $7.64(\mathrm{~m}, 2 \mathrm{H}), 7.45$ - $7.34(\mathrm{~m}, 2 \mathrm{H}), 7.34-7.20(\mathrm{~m}, 1 \mathrm{H}), 4.67$ (s, $2 \mathrm{H}), 1.61-1.52(\mathrm{~m}, 2 \mathrm{H}), 1.32-1.23(\mathrm{~m}, 2 \mathrm{H}) ;{ }^{13} \mathrm{C} \mathrm{NMR}\left(126 \mathrm{MHz}, \mathrm{CDCl}_{3}\right): \delta 136.7,128.4$, 127.9, 127.3, 125.8, 124.3, 46.2, 5.8, 0.9; IR v 2973, 2901, 1599, 1496, 1449, 1394, 1260, 1066, 1046, 909, 768; HRMS (EI) calcd. for $\mathrm{C}_{11} \mathrm{H}_{11} \mathrm{Cl}[\mathrm{M}]^{+}$178.0544; found 178.0543. See spectra

\section{(1-(1-Bromocyclopropyl)vinyl)benzene (8a)}

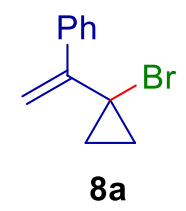

Following general procedure $\mathbf{D}$, using 1-bromo-3,5-bis(trifluoromethyl)benzene (73 $\mu \mathrm{L}, 0.42$ mmol, 1.4 equiv), $n$-BuLi (0.245 mL, 1.6M in hexanes, $0.390 \mathrm{mmol}, 1.30$ equiv), 4,4,5,5- 
tetramethyl-2-(1-(1-phenylvinyl)cyclopropyl)-1,3,2-dioxaborolane (1c) (81.1 $\mathrm{mg}, \quad 0.300$ mmol, 1.00 equiv), and 1,3-dibromo-5,5-dimethylimidazolidine-2,4-dione (129 mg, 0.450 mmol, 1.50 equiv). The crude reaction mixture was purified by flash chromatography using pentane to afford $\mathbf{8 a}(40.4 \mathrm{mg}, 0.181 \mathrm{mmol}, 60 \%)$ as a colourless liquid. TLC (pentane): $\mathrm{R}_{\mathrm{f}}=$ 0.44, $\mathrm{KMnO}_{4} ;{ }^{1} \mathrm{H}$ NMR (500 MHz, $\left.\mathrm{CDCl}_{3}\right): \delta 7.73-7.63(\mathrm{~m}, 2 \mathrm{H}), 7.41-7.35(\mathrm{~m}, 2 \mathrm{H}), 7.35$ $-7.30(\mathrm{~m}, 1 \mathrm{H}), 5.57(\mathrm{~s}, 1 \mathrm{H}), 5.46(\mathrm{~s}, 1 \mathrm{H}), 1.51-1.46(\mathrm{~m}, 2 \mathrm{H}), 1.31-1.20(\mathrm{~m}, 2 \mathrm{H}) ;{ }^{13} \mathrm{C} \mathrm{NMR}$ $\left(126 \mathrm{MHz}, \mathrm{CDCl}_{3}\right): \delta 148.8,137.9,128.2,128.1,126.7,114.9,32.9,17.1 ; \mathrm{IR} v 2931,2852$, 1381, 1278, 1179, 1137, 901; HRMS (EI) calcd. for $\mathrm{C}_{11} \mathrm{H}_{11} \mathrm{Br}[\mathrm{M}]^{+} 222.0039$; found 222.0037 . $\underline{\text { See spectra }}$

\section{(1-(1-Iodocyclopropyl)vinyl)benzene (8b)}

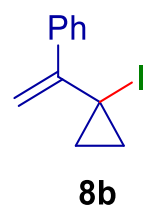

Following general procedure $\mathbf{D}$, using 1-bromo-3,5-bis(trifluoromethyl)benzene (73 $\mu \mathrm{L}, 0.42$ mmol, 1.4 equiv), $n$-BuLi ( $0.245 \mathrm{~mL}, 1.6 \mathrm{M}$ in hexanes, $0.390 \mathrm{mmol}, 1.30$ equiv), 4,4,5,5tetramethyl-2-(1-(1-phenylvinyl)cyclopropyl)-1,3,2-dioxaborolane (1c) (81.1 mg, 0.300 mmol, 1.00 equiv), and 1,3-diiodo-5,5-dimethylimidazolidine-2,4-dione (171 mg, $0.450 \mathrm{mmol}$, 1.50 equiv). The crude reaction mixture was purified by flash chromatography using pentane to afford $\mathbf{8 b}$ (50.0 mg, $0.185 \mathrm{mmol}, 62 \%)$ as a colourless liquid. TLC (pentane): $\mathrm{R}_{\mathrm{f}}=0.45$, $\mathrm{KMnO}_{4} ;{ }^{1} \mathrm{H}$ NMR (500 MHz, $\left.\mathrm{CDCl}_{3}\right): \delta 7.73-7.66(\mathrm{~m}, 2 \mathrm{H}), 7.41-7.36(\mathrm{~m}, 2 \mathrm{H}), 7.36-7.30$ $(\mathrm{m}, 1 \mathrm{H}), 5.50(\mathrm{~s}, 1 \mathrm{H}), 5.47(\mathrm{~s}, 1 \mathrm{H}), 1.44-1.38(\mathrm{~m}, 2 \mathrm{H}), 1.29-1.24(\mathrm{~m}, 2 \mathrm{H}) ;{ }^{13} \mathrm{C} \mathrm{NMR}(126$ $\left.\mathrm{MHz}, \mathrm{CDCl}_{3}\right): \delta 151.5,138.3,128.2,128.1,126.8,113.2,18.8,2.8 ;$ IR $v 3083,1612,1574$, 1493, 1340, 1278, 1133, 1029, 905, 779; HRMS (EI) calcd. for $\mathrm{C}_{11} \mathrm{H}_{11} \mathrm{I}[\mathrm{M}]^{+}$269.9900; found 269.9901. See spectra

\section{Phenyl(1-(1-phenylvinyl)cyclopropyl)selane (8c)}

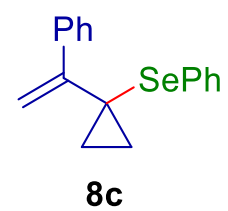

Following general procedure $\mathbf{D}$, using 1-bromo-3,5-bis(trifluoromethyl)benzene (73 $\mu \mathrm{L}, 0.42$ mmol, 1.4 equiv), $n$-BuLi (0.245 mL, 1.6 M in hexanes, $0.390 \mathrm{mmol}, 1.30$ equiv), 4,4,5,5- 
tetramethyl-2-(1-(1-phenylvinyl)cyclopropyl)-1,3,2-dioxaborolane (1c) (81.1 $\mathrm{mg}, \quad 0.300$ mmol, 1.00 equiv), and phenylselenyl chloride ( $86.2 \mathrm{mg}, 0.450 \mathrm{mmol}, 1.50$ equiv). The crude reaction mixture was purified by flash chromatography using pentane to afford $\mathbf{8 c}(88.1 \mathrm{mg}$, $0.294 \mathrm{mmol}, 98 \%$ ) as a pale-yellow liquid. TLC (pentane): $\mathrm{R}_{\mathrm{f}}=0.3, \mathrm{KMnO}_{4} ;{ }^{1} \mathrm{H} \mathrm{NMR}(400$ $\left.\mathrm{MHz} \mathrm{CDCl}_{3}\right): \delta 7.63-7.57(\mathrm{~m}, 2 \mathrm{H}), 7.51-7.44(\mathrm{~m}, 2 \mathrm{H}), 7.38-7.30(\mathrm{~m}, 3 \mathrm{H}), 7.30-7.22(\mathrm{~m}$, $3 \mathrm{H}), 5.34(\mathrm{~d}, J=1.1 \mathrm{~Hz}, 1 \mathrm{H}), 4.94(\mathrm{~d}, J=1.1 \mathrm{~Hz}, 1 \mathrm{H}), 1.38-1.33(\mathrm{~m}, 2 \mathrm{H}), 1.32-1.26(\mathrm{~m}$, $2 \mathrm{H}) ;{ }^{13} \mathrm{C}$ NMR $\left(101 \mathrm{MHz}, \mathrm{CDCl}_{3}\right): \delta$ 149.3, 139.8, 134.1, 130.8, 128.7, 128.1, 127.7, 127.5, 126.8, 113.9, 26.6, 15.9; IR v 3055, 2988, 1574, 1474, 1436, 1065, 1020, 732; HRMS (EI) calcd. for $\mathrm{C}_{17} \mathrm{H}_{16} \mathrm{Se}[\mathrm{M}]^{+}$300.0412; found 300.0410. See spectra

\section{4-Cyclopropylidene-1,4-diphenylbutan-1-one (10a)}

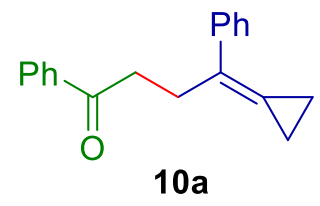

Following general procedure E, using 1-bromo-3,5-bis(trifluoromethyl)benzene (73 $\mu \mathrm{L}, 0.42$ mmol, 1.4 equiv), $n$-BuLi ( $0.245 \mathrm{~mL}, 1.6 \mathrm{M}$ in hexanes, $0.390 \mathrm{mmol}, 1.30$ equiv), 4,4,5,5tetramethyl-2-(1-(1-phenylvinyl)cyclopropyl)-1,3,2-dioxaborolane (1c) (81.1 $\mathrm{mg}, \quad 0.300$ mmol, 1.00 equiv), and 2-iodo-1-phenylethan-1-one (111 mg, $0.450 \mathrm{mmol}, 1.50$ equiv). The crude reaction mixture was purified by flash chromatography using DCM:pentane (1:10) to afford 10a (48.2 mg, $0.294 \mathrm{mmol}, 62 \%$ ) as a colourless liquid. TLC (DCM:pentane, 1:10 v/v): $\mathrm{R}_{\mathrm{f}}=0.24, \mathrm{KMnO}_{4} ;{ }^{1} \mathrm{H}$ NMR $\left(500 \mathrm{MHz}, \mathrm{CDCl}_{3}\right): \delta 7.98-7.93(\mathrm{~m}, 2 \mathrm{H}), 7.67-7.62(\mathrm{~m}, 2 \mathrm{H})$, $7.59-7.53(\mathrm{~m}, 1 \mathrm{H}), 7.46(\mathrm{dd}, J=8.4,7.1 \mathrm{~Hz}, 2 \mathrm{H}), 7.42-7.35(\mathrm{~m}, 2 \mathrm{H}), 7.30-7.23(\mathrm{~m}, 1 \mathrm{H})$, $3.35-3.21(\mathrm{~m}, 2 \mathrm{H}), 3.17-3.11(\mathrm{~m}, 2 \mathrm{H}), 1.45-1.35(\mathrm{~m}, 2 \mathrm{H}), 1.27-1.11(\mathrm{~m}, 2 \mathrm{H}) ;{ }^{13} \mathrm{C} \mathrm{NMR}$ $\left(126 \mathrm{MHz}, \mathrm{CDCl}_{3}\right): \delta 200.0,139.6,136.9,132.9,128.5,128.3,128.0,126.7,126.1,125.9$, 121.3, 37.4, 28.3, 4.6, 1.3; IR v 2971, 1682, 1597, 1580, 1447, 1308, 1278, 1001, 910, 762; HRMS (ESI) calcd. for $\mathrm{C}_{19} \mathrm{H}_{18} \mathrm{NaO}[\mathrm{M}+\mathrm{Na}]^{+} 285.1250$; found 285.1256. See spectra

\section{4-Cyclopropylidene-1-phenylpentan-1-one (10b)}

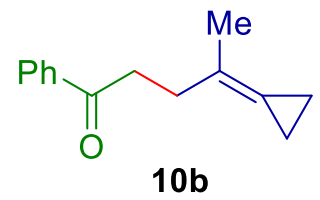


Following general procedure $\mathbf{E}$, using 1-bromo-3,5-bis(trifluoromethyl)benzene (73 $\mu \mathrm{L}, 0.42$ mmol, 1.4 equiv), $n$-BuLi ( $0.245 \mathrm{~mL}, 1.6 \mathrm{M}$ in hexanes, $0.390 \mathrm{mmol}, 1.30$ equiv), 4,4,5,5tetramethyl-2-(1-(prop-1-en-2-yl)cyclopropyl)-1,3,2-dioxaborolane (1b) (62.5 mg, 0.300 mmol, 1.00 equiv), and 2-iodo-1-phenylethan-1-one (111 mg, $0.450 \mathrm{mmol}, 1.50$ equiv). The crude reaction mixture was purified by flash chromatography using DCM:pentane (1:10) to afford 10b (20.6 mg, $0.103 \mathrm{mmol}, 34 \%)$ as a colourless liquid. TLC (DCM:pentane, 1:10 v/v): $\mathrm{R}_{\mathrm{f}}=0.24, \mathrm{KMnO}_{4} ;{ }^{1} \mathrm{H} \mathrm{NMR}\left(500 \mathrm{MHz}, \mathrm{CDCl}_{3}\right): \delta 8.05-7.85(\mathrm{~m}, 2 \mathrm{H}), 7.59-7.52(\mathrm{~m}, 1 \mathrm{H})$, $7.50-7.43(\mathrm{~m}, 2 \mathrm{H}), 3.28-3.16(\mathrm{~m}, 2 \mathrm{H}), 2.67-2.50(\mathrm{~m}, 2 \mathrm{H}), 1.87(\mathrm{~m}, 3 \mathrm{H}), 1.09-0.97(\mathrm{~m}$, 2H), $0.96-0.88(\mathrm{~m}, 2 \mathrm{H}) ;{ }^{13} \mathrm{C} \mathrm{NMR}\left(126 \mathrm{MHz}, \mathrm{CDCl}_{3}\right): \delta 200.2,137.0,132.9,128.5,128.0$, 123.0, 115.5, 36.6, 31.0, 21.20, 3.2, 1.0; IR v 2974, 1771, 1715, 1687, 1598, 1449, 1373, 1279 , 1177, 1068, 745; HRMS (ESI) calcd. for $\mathrm{C}_{14} \mathrm{H}_{16} \mathrm{NaO}[\mathrm{M}+\mathrm{Na}]^{+} 223.1093$; found 223.1098. See spectra

\section{4-Cyclopropylidene-4-phenylbutanenitrile (10d)}

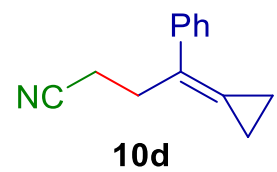

Following general procedure E, using 1-bromo-3,5-bis(trifluoromethyl)benzene ( $73 \mu \mathrm{L}, 0.42$ mmol, 1.4 equiv), $n$-BuLi (0.245 mL, 1.6M in hexanes, $0.390 \mathrm{mmol}, 1.30$ equiv), 4,4,5,5tetramethyl-2-(1-(1-phenylvinyl)cyclopropyl)-1,3,2-dioxaborolane (1c) (81.1 $\mathrm{mg}, \quad 0.300$ mmol, 1.00 equiv), and 2-iodoacetonitrile ( $33 \mu \mathrm{L}, 0.45 \mathrm{mmol}, 1.5$ equiv). The crude reaction mixture was purified by flash chromatography using DCM:pentane (1:10) to afford 10d (34.7 $\mathrm{mg}, 0.103 \mathrm{mmol}, 63 \%)$ as a colourless liquid. TLC (DCM:pentane, 1:10 v/v): $\mathrm{R}_{\mathrm{f}}=0.26$, $\mathrm{KMnO}_{4} ;{ }^{1} \mathrm{H}$ NMR $\left(500 \mathrm{MHz}, \mathrm{CDCl}_{3}\right): \delta 7.59-7.50(\mathrm{~m}, 2 \mathrm{H}), 7.42-7.35(\mathrm{~m}, 2 \mathrm{H}), 7.33-7.25$ (m, 1H), 3.05 (tt, $J=7.4,1.4 \mathrm{~Hz}, 2 \mathrm{H}), 2.62(\mathrm{t}, J=7.4 \mathrm{~Hz}, 2 \mathrm{H}), 1.51-1.42(\mathrm{~m}, 2 \mathrm{H}), 1.35-1.27$ $(\mathrm{m}, 2 \mathrm{H}) ;{ }^{13} \mathrm{C} \mathrm{NMR}\left(126 \mathrm{MHz}, \mathrm{CDCl}_{3}\right): \delta 138.5,128.5,127.1,125.7,123.9,123.7,119.7,29.9$, 16.4, 4.6, 1.7; IR v 2974, 2246, 1598, 1495, 1445, 1393, 1279, 1176, 1134, 1066, 879, 761; HRMS (ESI) calcd. for $\mathrm{C}_{13} \mathrm{H}_{13} \mathrm{NNa}[\mathrm{M}+\mathrm{Na}]^{+}$206.0940; found 206.0947. See spectra

\section{3-Cyclobutylidene-1-phenylpropan-1-ol (17a)}

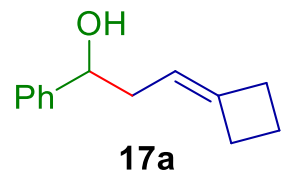


Following general procedure $\mathbf{F}$, using 1-bromo-3,5-bis(trifluoromethyl)benzene (73 $\mu \mathrm{L}, 0.42$ mmol, 1.4 eq.), $n$-BuLi (0.245 mL, $0.390 \mathrm{mmol}, 1.6 \mathrm{M}$ in hexanes, 1.30 equiv), 4,4,5,5tetramethyl-2-(1-vinylcyclobutyl)-1,3,2-dioxaborolane 14a $(62.4 \mathrm{mg}, 0.300 \mathrm{mmol}, 1.00$ equiv), TFAA (60 $\mu \mathrm{L}, 0.42 \mathrm{mmol}, 1.4$ equiv) and benzaldehyde ( $46 \mu \mathrm{L}, 0.45 \mathrm{mmol}, 1.5$ eq.). The crude reaction mixture was purified by flash chromatography using EtOAc:pentane 1:9 as mobile phase to afford $17 \mathbf{a}(45.1 \mathrm{mg}, 0.240 \mathrm{mmol}, 80 \%)$ as a colourless liquid. TLC (EtOAc:pentane, 1:9 v/v): $\mathrm{R}_{\mathrm{f}}=0.32, p$-anisaldehyde stain; ${ }^{1} \mathrm{H} \mathrm{NMR}\left(500 \mathrm{MHz}, \mathrm{CDCl}_{3}\right): \delta 7.42$ $-7.33(\mathrm{~m}, 4 \mathrm{H}), 7.32-7.26(\mathrm{~m}, 1 \mathrm{H}), 5.17-5.04(\mathrm{~m}, 1 \mathrm{H}), 4.69(\mathrm{td}, J=6.5,2.9 \mathrm{~Hz}, 1 \mathrm{H}), 2.78-$ $2.51(\mathrm{~m}, 4 \mathrm{H}), 2.36(\mathrm{td}, J=7.4,1.4 \mathrm{~Hz}, 2 \mathrm{H}), 2.10(\mathrm{~d}, J=3.1 \mathrm{~Hz}, 1 \mathrm{H}), 2.02-1.86(\mathrm{~m}, 2 \mathrm{H}) ;{ }^{13} \mathrm{C}$ NMR (126 MHz, $\left.\mathrm{CDCl}_{3}\right): \delta$ 144.7, 144.2, 128.3, 127.3, 125.8, 115.3, 73.7, 38.2, 31.0, 29.5, 16.9; IR v 3427, 2933, 2854, 1651, 1464, 1451, 1325, 1278, 1137, 1022, 975, 855, 758, 694; HRMS (ESI) calcd. for $\mathrm{C}_{13} \mathrm{H}_{16} \mathrm{NaO}[\mathrm{M}+\mathrm{Na}]^{+} 211.1099$; found 211.1099. See spectra

\section{(1s,2s)-3-Cyclobutylidene-2-methyl-1-phenylpropan-1-ol (17b)}

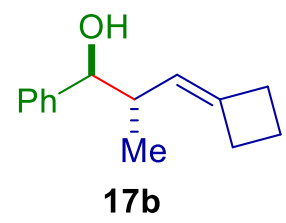

Following general procedure E, using 1-bromo-3,5-bis(trifluoromethyl)benzene ( $73 \mu \mathrm{L}, 0.42$ mmol, 1.4 eq.), $n$-BuLi (0.245 mL, $0.390 \mathrm{mmol}, 1.6 \mathrm{M}$ in hexanes, 1.30 equiv), $(E)-4,4,5,5-$ tetramethyl-2-(1-(prop-1-en-1-yl)cyclobutyl)-1,3,2-dioxaborolane 14b (66.6 mg, $0.300 \mathrm{mmol}$, 1.00 equiv), TFAA (60 $\mu \mathrm{L}, 0.42 \mathrm{mmol}, 1.4$ equiv) and benzaldehyde ( $46 \mu \mathrm{L}, 0.45 \mathrm{mmol}, 1.5$ equiv). The crude reaction mixture was purified by flash chromatography using EtOAc:pentane 1:10 as mobile phase to afford $\mathbf{1 7 b}(55 \mathrm{mg}, 0.273 \mathrm{mmol}, 91 \%)$ as a colourless liquid. TLC (EtOAc:pentane, 1:10 v/v): $\mathrm{R}_{\mathrm{f}}=0.32, p$-anisaldehyde stain; ${ }^{1} \mathrm{H} \mathrm{NMR}\left(500 \mathrm{MHz}, \mathrm{CDCl}_{3}\right): \delta$ $7.40-7.32(\mathrm{~d}, J=4.5 \mathrm{~Hz}, 4 \mathrm{H}), 7.33-7.26(\mathrm{~m}, 1 \mathrm{H}), 5.02(\mathrm{dp}, J=9.5,2.3 \mathrm{~Hz}, 1 \mathrm{H}), 4.25(\mathrm{~d}, J$ $=8.5 \mathrm{~Hz}, 1 \mathrm{H}), 2.81-2.65(\mathrm{~m}, 4 \mathrm{H}), 2.45-2.35(\mathrm{~m}, 1 \mathrm{H}), 2.04$ (brs, $1 \mathrm{H}), 1.99$ (p, J= $7.9 \mathrm{~Hz}$, $2 \mathrm{H}), 0.81(\mathrm{~d}, J=6.8 \mathrm{~Hz}, 3 \mathrm{H}) ;{ }^{13} \mathrm{C} \mathrm{NMR}\left(126 \mathrm{MHz}, \mathrm{CDCl}_{3}\right): \delta 144.2,142.6,128.1,127.5$, 127.0, 122.2 , 78.6, 41.8, 31.1, 29.8, 17.1, 17.0; IR v 3438, 2955, 2925, 1693, 1494, 1453, 1278, 1193, 1016, 995, 859, 760, 698; HRMS (ESI) calcd. for $\mathrm{C}_{14} \mathrm{H}_{18} \mathrm{NaO}[\mathrm{M}+\mathrm{Na}]^{+}$225.1255; found 225.1244. See spectra 


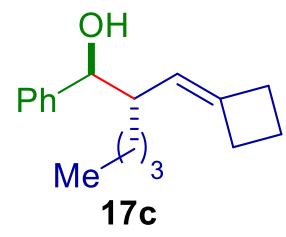

Following general procedure $\mathbf{F}$, using 1-bromo-3,5-bis(trifluoromethyl)benzene (73 $\mu \mathrm{L}, 0.42$ mmol, 1.4 equiv), $n$-BuLi (0.245 mL, $0.395 \mathrm{mmol}, 1.6 \mathrm{M}$ in hexanes, 1.30 equiv), (E)-2-(1(hex-1-en-1-yl)cyclobutyl)-4,4,5,5-tetramethyl-1,3,2-dioxaborolane $\mathbf{1 4 c}$ (79.2 $\mathrm{mg}, 0.300$ mmol, 1.00 equiv), TFAA ( $60 \mu \mathrm{L}, 0.42 \mathrm{mmol}, 1.4$ equiv) and benzaldehyde ( $46 \mu \mathrm{L}, 0.45 \mathrm{mmol}$, 1.5 equiv). The crude reaction mixture was purified by flash chromatography using EtOAc:pentane 1:10 as mobile phase to afford 17c (66 mg, $0.270 \mathrm{mmol}, 90 \%)$ as a colourless liquid. TLC (EtOAc:pentane, 1:9 v/v): $\mathrm{R}_{\mathrm{f}}=0.32$, $p$-anisaldehyde stain; ${ }^{1} \mathrm{H}$ NMR $(500 \mathrm{MHz}$, $\left.\mathrm{CDCl}_{3}\right): \delta 7.39-7.32(\mathrm{~m}, 4 \mathrm{H}), 7.31-7.26(\mathrm{~m}, 1 \mathrm{H}), 4.94(\mathrm{dp}, J=10.0,2.3 \mathrm{~Hz}, 1 \mathrm{H}), 4.31(\mathrm{~d}, J$ $=8.1 \mathrm{~Hz}, 1 \mathrm{H}), 2.80-2.72(\mathrm{~m}, 2 \mathrm{H}), 2.71-2.55(\mathrm{~m}, 2 \mathrm{H}), 2.35(\mathrm{~s}, 1 \mathrm{H}), 2.30-2.20(\mathrm{~m}, 1 \mathrm{H})$, $2.03-1.91(\mathrm{~m}, 2 \mathrm{H}), 1.33-1.03(\mathrm{~m}, 6 \mathrm{H}), 0.82(\mathrm{t}, J=7.2 \mathrm{~Hz}, 3 \mathrm{H}) ;{ }^{13} \mathrm{C} \mathrm{NMR}\left(126 \mathrm{MHz}, \mathrm{CDCl}_{3}\right)$ : $\delta 145.4,142.9,128.1,127.4,127.1,120.7,77.5,47.4,31.1,30.9,29.9,29.5,22.6,17.0,14.0$; IR $v$ 3427, 2933, 2853, 1702, 1495, 1451, 1278, 1137, 975, 855, 758, 695; HRMS (ESI) calcd. for $\mathrm{C}_{17} \mathrm{H}_{24} \mathrm{NaBO}[\mathrm{M}+\mathrm{Na}]^{+} 267.1725$; found 267.1726. See spectra

Tert-butyl (r)-4-cyclobutylidene-3-((r)-hydroxy(phenyl)methyl)piperidine-1-carboxylate (17d)

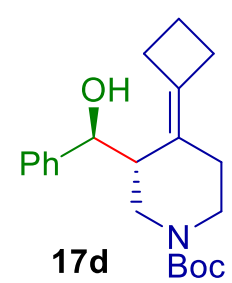

Following general procedure E, using 1-bromo-3,5-bis(trifluoromethyl)benzene (73 $\mu \mathrm{L}, 0.42$ mmol, 1.4 equiv), $n$-BuLi ( $0.245 \mathrm{~mL}, 0.390 \mathrm{mmol}, 1.6 \mathrm{M}$ in hexanes, 1.30 equiv), tert-butyl 4(1-(4,4,5,5-tetramethyl-1,3,2-dioxaborolan-2-yl)cyclobutyl)-3,6-dihydropyridine-1(2H)carboxylate 14d (109 mg, $0.300 \mathrm{mmol}, 1.00$ equiv), TFAA (60 $\mu \mathrm{L}, 0.42 \mathrm{mmol}, 1.4$ equiv) and benzaldehyde ( $46 \mu \mathrm{L}, 0.45 \mathrm{mmol}, 1.5$ equiv). The crude reaction mixture was purified by flash chromatography using EtOAc:pentane 1:1 as mobile phase to afford 17d (58 mg, $0.171 \mathrm{mmol}$, $57 \%$ ) as a colourless liquid. TLC (EtOAc:pentane, 1:1 v/v): $\mathbf{R}_{\mathbf{f}}=0.2, p$-anisaldehyde stain; ${ }^{1} \mathrm{H}$ $\operatorname{NMR}\left(500 \mathrm{MHz}, \mathrm{CDCl}_{3}\right): \delta 7.46(\mathrm{~d}, J=7.5 \mathrm{~Hz}, 2 \mathrm{H}), 7.38(\mathrm{t}, J=7.5 \mathrm{~Hz}, 2 \mathrm{H}), 7.32(\mathrm{t}, J=7.3$ 
Hz, 1H), 4.60 (d, $J=9.9 \mathrm{~Hz}, 1 \mathrm{H}), 4.29$ (brs, 1H), 3.81 (brs, 1H), 2.78 (t, $J=8.1 \mathrm{~Hz}, 4 \mathrm{H}), 2.71$ $-2.52(\mathrm{~m}, 2 \mathrm{H}), 2.45(\mathrm{dd}, J=9.6,3.5 \mathrm{~Hz}, 1 \mathrm{H}), 2.36-2.11(\mathrm{~m}, 3 \mathrm{H}), 2.03$ (p, $J=7.8 \mathrm{~Hz}, 2 \mathrm{H})$, $1.48(\mathrm{~s}, 9 \mathrm{H}) ;{ }^{13} \mathrm{C} \mathrm{NMR}\left(126 \mathrm{MHz}, \mathrm{CDCl}_{3}\right): \delta 155.0,142.6,138.8,128.2,127.7,127.5,124.7$, 79.7, 72.3, 46.0, 45.0, 44.3, 29.1, 29.0, 28.4, 24.9, 16.1; IR v 3395, 2975, 2933, 1701, 1473, $1453,1371,1327,1145,982,851,674$; HRMS (ESI) calcd. for $\mathrm{C}_{21} \mathrm{H}_{29} \mathrm{NNaBO}_{3}[\mathrm{M}+\mathrm{Na}]^{+}$ 366.2045; found 366.2041. See spectra

(1s,2s)-1-(3,5-Bis(trifluoromethyl)phenyl)-2-(cyclobutylidenemethyl)hexan-1-ol (17e)

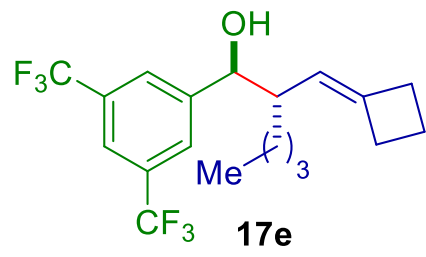

Following general procedure $\mathbf{F}$, using 1-bromo-3,5-bis(trifluoromethyl)benzene ( $73 \mu \mathrm{L}, 0.42$ mmol, 1.4 equiv), $n$-BuLi (0.245 mL, $0.390 \mathrm{mmol}, 1.6 \mathrm{M}$ in hexanes, 1.30 equiv), (E)-2-(1(hex-1-en-1-yl)cyclobutyl)-4,4,5,5-tetramethyl-1,3,2-dioxaborolane $\mathbf{1 4 c}$ (79.2 $\mathrm{mg}, 0.300$ mmol, 1.00 equiv), TFAA $(60 \mu \mathrm{L}, \quad 0.42 \quad \mathrm{mmol}, 1.4$ equiv $)$ and 3,5bis(trifluoromethyl)benzaldehyde ( $74 \mu \mathrm{L}, 0.45 \mathrm{mmol}, 1.5$ equiv). The crude reaction mixture was purified by flash chromatography using $\mathrm{Et}_{2} \mathrm{O}$ :pentane 1:9 as mobile phase to afford 17e (104 mg, $0.273 \mathrm{mmol}, 91 \%$ ) as a colourless liquid. After the column chromatography purification, the product was washed with saturated aq. sodium bisulfite solution to remove starting aldehyde. The yield was calculated after excluding the aldehyde impurities. TLC $\left(\mathrm{Et}_{2} \mathrm{O}\right.$ :pentane, $\left.1: 9 \mathrm{v} / \mathrm{v}\right): \mathrm{R}_{\mathrm{f}}=0.31, p$-anisaldehyde stain; ${ }^{1} \mathrm{H} \mathrm{NMR}\left(500 \mathrm{MHz}, \mathrm{CDCl}_{3}\right): \delta 7.85$ - $7.73(\mathrm{~m}, 3 \mathrm{H}), 4.93(\mathrm{dp}, J=9.7,2.3 \mathrm{~Hz}, 1 \mathrm{H}), 4.54(\mathrm{dd}, J=6.8,1.9 \mathrm{~Hz}, 1 \mathrm{H}), 2.77$ - 2.65 (m, 2H), $2.62-2.50(\mathrm{~m}, 1 \mathrm{H}), 2.47(\mathrm{~d}, J=2.1 \mathrm{~Hz}, 1 \mathrm{H}), 2.36-2.25(\mathrm{~m}, 1 \mathrm{H}), 2.25-2.12(\mathrm{~m}, 1 \mathrm{H})$, $2.01-1.81(\mathrm{~m}, 2 \mathrm{H}), 1.38-1.09(\mathrm{~m}, 6 \mathrm{H}), 0.85(\mathrm{t}, J=7.1 \mathrm{~Hz}, 3 \mathrm{H}) ;{ }^{13} \mathrm{C} \mathrm{NMR}\left(126 \mathrm{MHz}, \mathrm{CDCl}_{3}\right)$ : $\delta 146.46,145.83,131.21(\mathrm{q}, J=33.2 \mathrm{~Hz}), 127.18(\mathrm{~m}), 123.4(J=272.6 \mathrm{MHz}), 121.23(\mathrm{p}, J=$ $3.9 \mathrm{~Hz}), 118.86,76.41,47.31,31.03,31.02,29.68,29.45,24.81,22.57,16.83,13.90$; IR v 3434, 2958, 2932, 2860, 1716, 1623, 1374, 1276, 1170, 1131, 899, 708, 682; HRMS (ESI) calcd. for $\mathrm{C}_{19} \mathrm{H}_{22} \mathrm{~F}_{6} \mathrm{NaO}[\mathrm{M}+\mathrm{Na}]^{+}$403.1473; found 404.1484. See spectra 


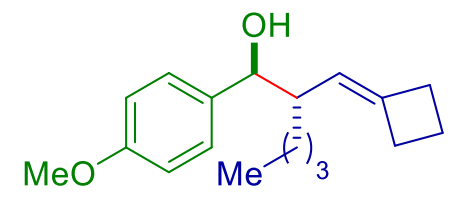

$17 f$

Following general procedure $\mathbf{F}$, using 1-bromo-3,5-bis(trifluoromethyl)benzene (73 $\mu \mathrm{L}, 0.42$ mmol, 1.4 equiv), $n$-BuLi (0.245 mL, $0.390 \mathrm{mmol}, 1.6 \mathrm{M}$ in hexanes, 1.30 equiv), (E)-2-(1(hex-1-en-1-yl)cyclobutyl)-4,4,5,5-tetramethyl-1,3,2-dioxaborolane $\mathbf{1 4 c}$ (79.2 mg, 0.300 mmol, 1.00 equiv), TFAA (60 $\mu \mathrm{L}, 0.42 \mathrm{mmol}, 1.4$ equiv) and 4-anisaldehyde ( $55 \mu \mathrm{L}, 0.45$ mmol, 1.5 equiv). The crude reaction mixture was purified by flash chromatography using EtOAc:pentane 1:9 as mobile phase to afford $\mathbf{1 7 f}(76 \mathrm{mg}, 0.276 \mathrm{mmol}, 92 \%)$ as a colourless liquid. TLC (EtOAc:pentane, 1:9 v/v): $\mathrm{R}_{\mathrm{f}}=0.3, p$-anisaldehyde stain; ${ }^{1} \mathrm{H}$ NMR $(500 \mathrm{MHz}$, $\left.\mathrm{CDCl}_{3}\right): \delta 7.32-7.21(\mathrm{~m}, 2 \mathrm{H}), 6.95-6.80(\mathrm{~m}, 2 \mathrm{H}), 5.01-4.86(\mathrm{~m}, 1 \mathrm{H}), 4.24(\mathrm{~d}, J=8.5 \mathrm{~Hz}$, $1 \mathrm{H}), 3.83(\mathrm{~s}, 3 \mathrm{H}), 2.83-2.70(\mathrm{~m}, 2 \mathrm{H}), 2.70-2.59(\mathrm{~m}, 2 \mathrm{H}), 2.33(\mathrm{~s}, 1 \mathrm{H}), 2.27-2.11(\mathrm{~m}, 1 \mathrm{H})$, $1.98(\mathrm{p}, J=8.0 \mathrm{~Hz}, 2 \mathrm{H}), 1.34-0.96(\mathrm{~m}, 6 \mathrm{H}), 0.82(\mathrm{t}, J=7.1 \mathrm{~Hz}, 3 \mathrm{H}) ;{ }^{13} \mathrm{C} \mathrm{NMR}(126 \mathrm{MHz}$, $\left.\mathrm{CDCl}_{3}\right): \delta 159.0,145.3,135.0,128.2,121.0,113.5,77.0,55.2,47.5,31.1,30.9,29.9,29.5$, 22.6, 17.0, 14.0; IR $v 3425,2934,2862,1612,1516,1438,1253,1182,1034,831$; HRMS (ESI) calcd. for $\mathrm{C}_{18} \mathrm{H}_{26} \mathrm{NaO}_{2}[\mathrm{M}+\mathrm{Na}]^{+} 297.1830$; found 297.1831. See spectra

(1s,2s)-2-(Cyclobutylidenemethyl)-1-(pyridin-4-yl)hexan-1-ol (17g)

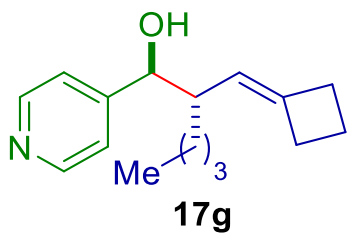

Following general procedure $\mathbf{F}$, using 1-bromo-3,5-bis(trifluoromethyl)benzene (73 $\mu \mathrm{L}, 0.42$ mmol, 1.4 equiv), $n$-BuLi (0.245 mL, $0.390 \mathrm{mmol}, 1.6 \mathrm{M}$ in hexanes, 1.30 equiv), (E)-2-(1(hex-1-en-1-yl)cyclobutyl)-4,4,5,5-tetramethyl-1,3,2-dioxaborolane $\mathbf{1 4 c}$ (79.2 $\mathrm{mg}, 0.300$ mmol, 1.00 equiv), TFAA ( $60 \mu \mathrm{L}, 0.42 \mathrm{mmol}, 1.4$ equiv) and isonicotinaldehyde ( $42 \mu \mathrm{L}, 0.45$ mmol, 1.5 equiv). The crude reaction mixture was purified by flash chromatography using EtOAc:pentane 1:1 as mobile phase to afford $\mathbf{1 7 g}(45 \mathrm{mg}, 0.186 \mathrm{mmol}, 62 \%)$ as a colourless liquid. TLC (EtOAc:pentane, 1:1 v/v): $\mathrm{R}_{\mathrm{f}}=0.30, p$-anisaldehyde stain; ${ }^{1} \mathrm{H}$ NMR $(500 \mathrm{MHz}$, $\left.\mathrm{CDCl}_{3}\right): \delta 8.59(\mathrm{~d}, J=4.7 \mathrm{~Hz}, 2 \mathrm{H}), 7.32(\mathrm{~d}, J=6.1 \mathrm{~Hz}, 2 \mathrm{H}), 4.91(\mathrm{dt}, J=10.1,2.3 \mathrm{~Hz}, 1 \mathrm{H})$, 
$4.39(\mathrm{~d}, J=7.1 \mathrm{~Hz}, 1 \mathrm{H}), 2.72(\mathrm{t}, J=8.0 \mathrm{~Hz}, 2 \mathrm{H}), 2.65-2.56(\mathrm{~m}, 1 \mathrm{H}), 2.52-2.40(\mathrm{~m}, 2 \mathrm{H})$, $2.25-2.16(\mathrm{~m}, 1 \mathrm{H}), 2.00-1.86(\mathrm{~m}, 2 \mathrm{H}), 1.36-1.07(\mathrm{~m}, 6 \mathrm{H}), 0.84(\mathrm{t}, J=7.2 \mathrm{~Hz}, 3 \mathrm{H}) ;{ }^{13} \mathrm{C}$ NMR (126 MHz, $\left.\mathrm{CDCl}_{3}\right): \delta 152.7,149.0,146.2,122.2,119.3,76.1,47.1,31.1,30.8,29.8$, 29.5, 22.6, 16.9, 14.0; IR v 3196, 2953, 2926, 2856, 1603, 1414, 1277, 1133, 1054, 1003, 822, 729; HRMS (ESI) calcd. for $\mathrm{C}_{16} \mathrm{H}_{24} \mathrm{NO}[\mathrm{M}+\mathrm{H}]^{+}$246.1858; found 246.1865. See spectra

(3r,4s)-4-(Cyclobutylidenemethyl)-1-phenyloctan-3-ol (17h)

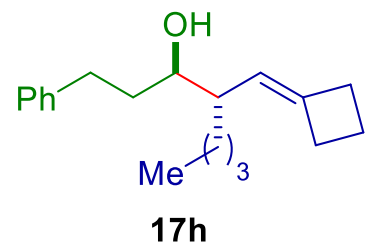

Following general procedure $\mathbf{F}$, using 1-bromo-3,5-bis(trifluoromethyl)benzene ( $73 \mu \mathrm{L}, 0.42$ mmol, 1.4 equiv), $n$-BuLi (0.245 mL, $0.390 \mathrm{mmol}, 1.60 \mathrm{M}$ in hexanes, 1.30 equiv), (E)-2-(1(hex-1-en-1-yl)cyclobutyl)-4,4,5,5-tetramethyl-1,3,2-dioxaborolane $\mathbf{1 4 c}$ (79.2 $\mathrm{mg}, 0.300$ mmol, 1.00 equiv), TFAA (60 $\mu \mathrm{L}, 0.42 \mathrm{mmol}, 1.4$ equiv) and 3-phenylpropanal (60 $\mu \mathrm{L}, 0.45$ mmol, 1.5 equiv). The crude reaction mixture was purified by flash chromatography using EtOAc:pentane 1:9 as mobile phase to afford $\mathbf{1 7 h}$ (45 $\mathrm{mg}, 0.240 \mathrm{mmol}, 80 \%)$ as a colourless liquid. TLC (EtOAc:pentane, 1:9 v/v): $\mathrm{R}_{\mathrm{f}}=0.32$, $p$-anisaldehyde stain; ${ }^{1} \mathrm{H}$ NMR $(500 \mathrm{MHz}$, $\left.\mathrm{CDCl}_{3}\right): \delta 7.34-7.27(\mathrm{~m}, 2 \mathrm{H}), 7.27-7.18(\mathrm{~m}, 3 \mathrm{H}), 4.95-4.83(\mathrm{~m}, 1 \mathrm{H}), 3.42(\mathrm{ddd}, J=9.3$, 6.1, 3.5 Hz, 1H), 2.86 (ddd, $J=13.5,10.3,5.3 \mathrm{~Hz}, 1 \mathrm{H}), 2.76-2.59(\mathrm{~m}, 5 \mathrm{H}), 2.07-1.92$ (m, $3 \mathrm{H}), 1.85$ (tdt, $J=10.1,6.4,3.4 \mathrm{~Hz}, 1 \mathrm{H}), 1.79-1.65(\mathrm{~m}, 2 \mathrm{H}), 1.52-1.39(\mathrm{~m}, 1 \mathrm{H}), 1.36-1.13$ $(\mathrm{m}, 5 \mathrm{H}), 0.91(\mathrm{t}, J=7.2 \mathrm{~Hz}, 3 \mathrm{H}) ;{ }^{13} \mathrm{C} \mathrm{NMR}\left(126 \mathrm{MHz}, \mathrm{CDCl}_{3}\right): \delta 144.0,142.5,128.5,128.3$, 125.7, 120.4, 73.8, 45.0, 36.5, 32.2, 31.2, 31.1, 30.0, 29.6, 22.8, 17.0, 14.1; IR v 3401, 3026, 2928, 2857, 1686, 1495, 1453, 1278, 1134, 1030, 906, 731, 698; HRMS (ESI) calcd. for $\mathrm{C}_{19} \mathrm{H}_{28} \mathrm{NaO}[\mathrm{M}+\mathrm{Na}]^{+}$295.2038; found 295.2033. See spectra

\section{1-(3-Cyclobutylidenepropyl)piperidine (18a)}

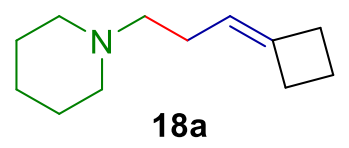

Following general procedure $\mathbf{D}$, using 1-bromo-3,5-bis(trifluoromethyl)benzene ( $73 \mu \mathrm{L}, 0.42$ mmol, 1.4 equiv), $n$-BuLi ( $0.245 \mathrm{~mL}, 0.390 \mathrm{mmol}, 1.6 \mathrm{M}$ in hexanes, 1.30 equiv), 4,4,5,5tetramethyl-2-(1-vinylcyclobutyl)-1,3,2-dioxaborolane $14 \mathbf{a}(62.4 \mathrm{mg}, 0.300 \mathrm{mmol}, 1.00$ 
equiv), and piperidine derived iminium salt (102 mg, $0.450 \mathrm{mmol}, 1.50$ equiv). The crude reaction mixture was purified by flash chromatography using MeOH:DCM 1:9 as mobile phase to afford 18a (45 mg, $0.156 \mathrm{mmol}, 52 \%)$ as a colourless liquid. TLC (MeOH:DCM, 1:9 v/v): $\mathrm{R}_{\mathrm{f}}=0.32, \mathrm{KMnO}_{4} ;{ }^{1} \mathrm{H}$ NMR $\left(500 \mathrm{MHz}, \mathrm{CDCl}_{3}\right): \delta 5.09-4.85(\mathrm{~m}, 1 \mathrm{H}), 3.54$ (brs, 2H), $2.98-$ $2.82(\mathrm{~m}, 2 \mathrm{H}), 2.77-2.58(\mathrm{~m}, 5 \mathrm{H}), 2.58-2.43(\mathrm{~m}, 2 \mathrm{H}), 2.04-1.79(\mathrm{~m}, 4 \mathrm{H}), 1.64$ (brs, 6H); ${ }^{13} \mathrm{C} \mathrm{NMR}\left(126 \mathrm{MHz}, \mathrm{CDCl}_{3}\right): \delta 145.3,113.4,56.9,53.1,30.9,29.3,22.6,22.6,22.5,16.8$; IR $v$ 2921, 2635, 2541, 1459, 1278, 1133, 964, 841; HRMS (ESI) calcd. for $\mathrm{C}_{12} \mathrm{H}_{22} \mathrm{~N}[\mathrm{M}+\mathrm{H}]^{+}$ 180.1752; found 180.1755 . See spectra

\section{7-(2-Cyclobutylideneethyl)cyclohepta-1,3,5-triene (18b)}

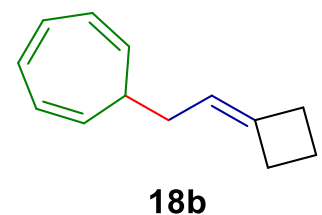

Following general procedure $\mathbf{D}$, using 1-bromo-3,5-bis(trifluoromethyl)benzene (73 $\mu \mathrm{L}, 0.42$ mmol, 1.4 equiv), $n$-BuLi ( $0.245 \mathrm{~mL}, 0.390 \mathrm{mmol}, 1.6 \mathrm{M}$ in hexanes, 1.30 equiv), 4,4,5,5tetramethyl-2-(1-vinylcyclobutyl)-1,3,2-dioxaborolane 14a $(62.4 \mathrm{mg}, 0.300 \mathrm{mmol}, 1.00$ equiv), and tropylium tetrafluoroborate ( $80.1 \mathrm{mg}, 0.450 \mathrm{mmol}, 1.50$ equiv). The crude reaction mixture was purified by flash chromatography using pentane to afford $\mathbf{1 8 b}$ (45 $\mathrm{mg}, 0.258$ mmol, $86 \%$ ) as a colourless liquid. TLC (pentane): $\mathrm{R}_{\mathrm{f}}=0.31, p$-anisaldehyde stain; ${ }^{1} \mathrm{H}$ NMR $\left(500 \mathrm{MHz}, \mathrm{CDCl}_{3}\right): \delta 6.69-6.61(\mathrm{~m}, 2 \mathrm{H}), 6.24-6.14(\mathrm{~m}, 2 \mathrm{H}), 5.24-5.14(\mathrm{~m}, 3 \mathrm{H}), 2.76-$ $2.64(\mathrm{~m}, 4 \mathrm{H}), 2.35-2.27(\mathrm{~m}, 2 \mathrm{H}), 1.98(\mathrm{p}, J=7.9 \mathrm{~Hz}, 2 \mathrm{H}), 1.71-1.63(\mathrm{~m}, 1 \mathrm{H}) ;{ }^{13} \mathrm{C} \mathrm{NMR}$ $\left(126 \mathrm{MHz}, \mathrm{CDCl}_{3}\right): \delta 141.9,130.9,126.7,124.7,117.7,39.1,31.5,31.0,29.5,17.0$; IR v 3011, 2911, 1693, 1436, 1398, 1177, 1015, 846, 741, 703; HRMS (ESI) calcd. for $\mathrm{C}_{13} \mathrm{H}_{17}[\mathrm{M}+\mathrm{H}]^{+}$ 173.1330; found 173.1327. See spectra

\section{7-(2-Cyclobutylideneethyl)cyclohepta-1,3,5-triene (18c)}

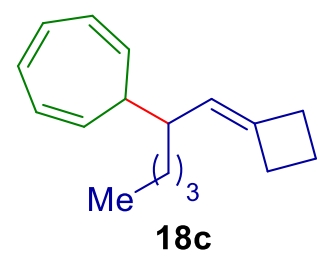

Following general procedure D, using 1-bromo-3,5-bis(trifluoromethyl)benzene (73 $\mu \mathrm{L}, 0.42$ mmol, 1.4 equiv), $n$-BuLi ( $0.245 \mathrm{~mL}, 0.390 \mathrm{mmol}, 1.6 \mathrm{M}$ in hexanes, 1.30 equiv), (E)-2-(1- 
(hex-1-en-1-yl)cyclobutyl)-4,4,5,5-tetramethyl-1,3,2-dioxaborolane $\mathbf{1 4 c}$ (79.2 $\mathrm{mg}, 0.300$ mmol, 1.0 equiv), and tropylium tetrafluoroborate $(80.1 \mathrm{mg}, 0.450 \mathrm{mmol}, 1.50$ equiv). The crude reaction mixture was purified by flash chromatography using pentane to afford $\mathbf{1 8 c}$ (62 $\mathrm{mg}, 0.273 \mathrm{mmol}, 91 \%$ ) as a colourless liquid. TLC (pentane): $\mathrm{R}_{\mathrm{f}}=0.34, p$-anisaldehyde stain; ${ }^{1} \mathrm{H}$ NMR $\left(500 \mathrm{MHz}, \mathrm{CDCl}_{3}\right): \delta 6.72-6.60(\mathrm{~m}, 2 \mathrm{H}), 6.29-6.12(\mathrm{~m}, 2 \mathrm{H}), 5.32-5.16(\mathrm{~m}, 2 \mathrm{H})$, $4.94-4.88$ (m, 1H), $2.78-2.63(\mathrm{~m}, 4 \mathrm{H}), 2.40-2.23(\mathrm{~m}, 1 \mathrm{H}), 1.99$ (p, J= $7.7 \mathrm{~Hz}, 2 \mathrm{H}), 1.68-$ $1.58(\mathrm{~m}, 1 \mathrm{H}), 1.48-1.12(\mathrm{~m}, 6 \mathrm{H}), 0.92(\mathrm{t}, J=7.1 \mathrm{~Hz}, 3 \mathrm{H}) ;{ }^{13} \mathrm{C} \mathrm{NMR}\left(126 \mathrm{MHz}, \mathrm{CDCl}_{3}\right): \delta$ 141.3, 130.7, 130.7, 125.7, 125.2, 124.4, 124.4, 122.9, 43.9, 40.6, 33.1, 30.9, 29.9, 29.5, 22.9, 17.1, 14.1; IR v 3013, 2953, 2923, 2856, 1695, 1465, 1377, 1277, 1176, 1138, 856, 741, 696; HRMS (ESI) calcd. for $\mathrm{C}_{17} \mathrm{H}_{25}[\mathrm{M}+\mathrm{H}]^{+} 229.1956$; found 229.1952. See spectra

\section{7-(2-Cyclobutylideneethyl)cyclohepta-1,3,5-triene (18d)}

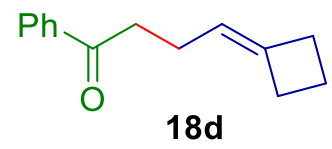

Following general procedure E, using 1-bromo-3,5-bis(trifluoromethyl)benzene ( $73 \mu \mathrm{L}, 0.42$ mmol, 1.4 equiv), $n$-BuLi (0.245 mL, $0.390 \mathrm{mmol}, 1.6 \mathrm{M}$ in hexanes, 1.30 equiv), 4,4,5,5tetramethyl-2-(1-vinylcyclobutyl)-1,3,2-dioxaborolane 14a $(62.4 \mathrm{mg}, 0.300 \mathrm{mmol}, 1.00$ equiv), and 2-iodo-1-phenylethan-1-one (111 mg, $0.450 \mathrm{mmol}, 1.50$ equiv). The crude reaction mixture was purified by flash chromatography using DCM:pentane 1:9 as mobile phase to afford 18d (28 mg, 47\%) as a colourless liquid. TLC (DCM:pentane, 1:9 v/v): $\mathrm{R}_{\mathrm{f}}=0.26, p$ anisaldehyde stain; ${ }^{1} \mathrm{H}$ NMR $\left(500 \mathrm{MHz}, \mathrm{CDCl}_{3}\right): \delta 8.05-7.89(\mathrm{~m}, 2 \mathrm{H}), 7.67-7.52(\mathrm{~m}, 1 \mathrm{H})$, $7.52-7.41(\mathrm{~m}, 2 \mathrm{H}), 5.21-5.06(\mathrm{~m}, 1 \mathrm{H}), 3.02(\mathrm{t}, J=7.4 \mathrm{~Hz}, 2 \mathrm{H}), 2.74-2.57(\mathrm{~m}, 4 \mathrm{H}), 2.39-$ $2.25(\mathrm{~m}, 2 \mathrm{H}), 1.95$ (p, $J=7.9 \mathrm{~Hz}, 2 \mathrm{H}) ;{ }^{13} \mathrm{C} \mathrm{NMR}\left(126 \mathrm{MHz}, \mathrm{CDCl}_{3}\right): \delta 200.1,141.4,137.0$, 132.9, 128.5, 128.1, 118.6, 38.7, 30.9, 29.2, 22.9, 17.0; IR v 3026, 2931, 1686, 1495, 1453, 1278, 1134, 1029, 906, 731, 607; HRMS (ESI) calcd. for $\mathrm{C}_{14} \mathrm{H}_{16} \mathrm{NaO}[\mathrm{M}+\mathrm{Na}]^{+} 223.1099$; found 223.1096. See spectra 


\section{Determination of relative stereochemistry}

(1s,2s)-3-Cyclopropylidene-2-methyl-1-phenylpropyl (4-bromophenyl)carbamate (20)

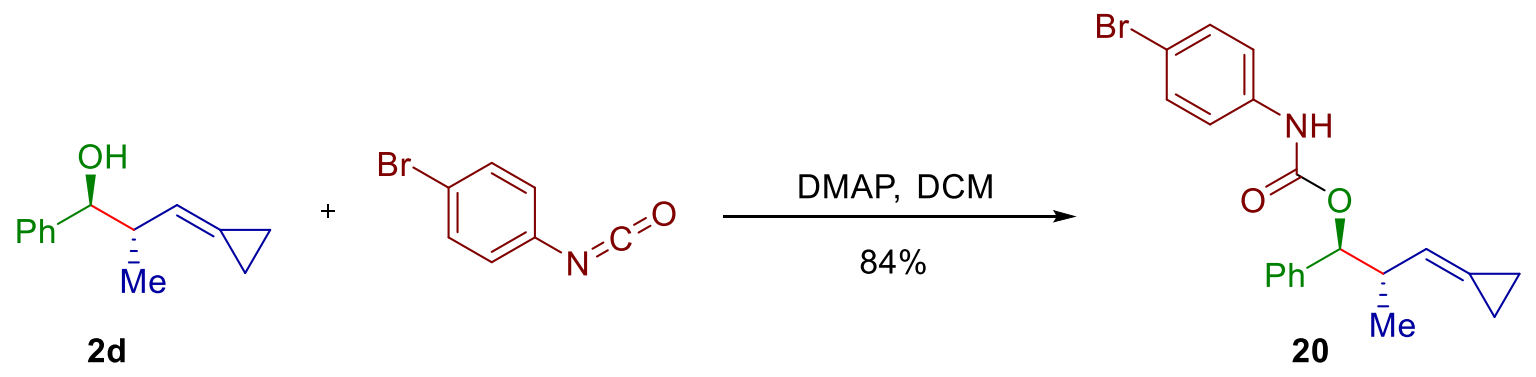

Following a slightly modified procedure ${ }^{4}$, to a solution of alcohol $\mathbf{2 d}(28.5 \mathrm{mg}, 0.150 \mathrm{mmol}$, 1.00 equiv) and DMAP (55 mg, $0.45 \mathrm{mmol}, 3.0$ equiv) in DCM (1 mL) under $\mathrm{N}_{2}$ was added $p$ bromophenyl isocyanate (90 $\mathrm{mg}, 0.45 \mathrm{mmol}, 3.0$ equiv) forming a yellow suspension. The reaction mixture was stirred at room temperature overnight and then quenched by addition of aq. $\mathrm{NaHCO}_{3}(5 \mathrm{~mL})$. The reaction was extracted with $\mathrm{DCM}(3 \times 5 \mathrm{~mL})$ and the combined organic layers were dried over $\mathrm{MgSO}_{4}$, filtered and concentrated in vacuo. The crude reaction mixture was purified by flash column chromatography using EtOAc:hexane 1:8 as a mobile phase to afford product 20 as a white solid $(48.6 \mathrm{mg}, 0.126 \mathrm{mmol}, 84 \%)$ which was crystallised from $\mathrm{CHCl}_{3} /$ pentane. Mp: $122.4-126.5^{\circ} \mathrm{C}$ TLC (EtOAc:hexane, 1:5 v/v): $\mathrm{R}_{\mathrm{f}}=0.35, \mathrm{KMnO}_{4}$; ${ }^{1} \mathrm{H}$ NMR (500 MHz, $\mathrm{CDCl}_{3} \delta 7.40-7.38$ (m, 2H), 7.35 - 7.29 (m, 5H), $7.27-7.25(\mathrm{~m}, 2 \mathrm{H})$, $6.60(\mathrm{~s}, 1 \mathrm{H}), 5.76(\mathrm{dp}, J=8.0,2.1 \mathrm{~Hz}, 1 \mathrm{H}), 5.67$ (d, $J=7.4 \mathrm{~Hz}, 1 \mathrm{H}), 2.90$ (hept, $J=6.9 \mathrm{~Hz}$, 1H), $1.06-0.91(\mathrm{~m}, 6 \mathrm{H}), 0.92-0.78(\mathrm{~m}, 1 \mathrm{H}) ;{ }^{13} \mathrm{C} \mathrm{NMR}\left(126 \mathrm{MHz}, \mathrm{CDCl}_{3}\right): 152.7,139.3$, 137.0, 131.9, 128.2, 127.8, 127.0, 123.3, 120.1, 119.0, 115.8, 81.0, 42.1, 16.8, 2.3, 2.0; IR v 3320, 2926, 1702, 1594, 1531, 1490, 1397, 1306, 1220, 1075, 1048, 824, 762; HRMS (ESI) calcd. for $\mathrm{C}_{20} \mathrm{H}_{20} \mathrm{BrNNaO}_{2}[\mathrm{M}+\mathrm{Na}]^{+}$408.0569; found 408.0565. See spectra

\section{Crystallography}

X-ray diffraction experiments on 20 were carried out at 100(2) K on a Bruker APEX II diffractometer using Mo-K $\mathrm{K}_{\alpha}$ radiation $(\lambda=0.71073 \AA$ ), Data collections were performed using a CCD area detector. Intensities were integrated in $\mathrm{SAINT}^{5}$ and absorption corrections based on equivalent reflections were applied using SADABS. ${ }^{6}$ The structure was solved using ShelXT ${ }^{7}$ and refined by full matrix least squares against $F^{6}$ in ShelXL ${ }^{8,9}$ using Olex $2 .{ }^{10}$ All of the non-hydrogen atoms were refined anisotropically. While all of the hydrogen atoms were located geometrically and refined using a riding model, apart from the N-H proton which was located in the difference map. Absolute configuration was not determined, and the Flack 
parameter has been removed from the CIF. Crystal structure and refinement data are given in Table 1. Crystallographic data has been deposited with the Cambridge Crystallographic Data Centre as supplementary publication CCDC 2061386. Copies of the data can be obtained free of charge on application to CCDC, 12 Union Road, Cambridge CB2 1EZ, UK [fax(+44) 1223 336033, e-mail: deposit@ccdc.cam.ac.uk].

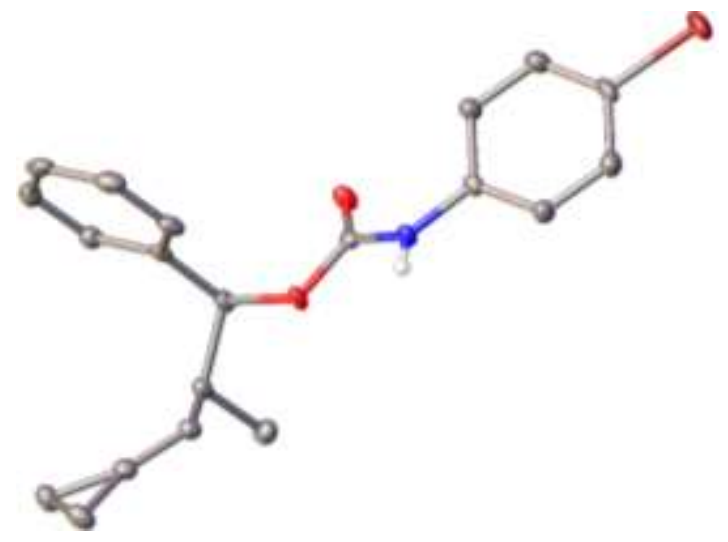

Figure 1. Crystal structure of 20. Thermal ellipsoids are shown at 50\% probability and hydrogen atoms, not on heteroatoms, have been omitted for clarity.

Table 1: Crystal data and structure refinement for 20.

CCDC number

Empirical formula

Formula weight

Temperature/K

Crystal system

Space group

$\mathrm{a} / \AA$

b/Å

c/Å

$\alpha /{ }^{\circ}$

$\beta /{ }^{\circ}$

$\gamma /{ }^{\circ}$

Volume $/ \AA^{3}$

Z

$\rho_{\text {calc } g / \mathrm{cm}^{3}}$

$\mu / \mathrm{mm}^{-1}$
2061386

$\mathrm{C}_{20} \mathrm{H}_{20} \mathrm{NO}_{2} \mathrm{Br}$

386.28

99.95

orthorhombic

$\mathrm{P} 2{ }_{1} 2_{1} 2_{1}$

$5.1676(2)$

14.2304(4)

23.7653(6)

90

90

90

1747.63(9)

4

1.468

2.364 
$\mathrm{F}(000)$

792.0

Crystal size $/ \mathrm{mm}^{3}$

$0.537 \times 0.169 \times 0.06$

Radiation

$\operatorname{MoK} \alpha(\lambda=0.71073)$

$2 \Theta$ range for data collection/ ${ }^{\circ}$

3.336 to 54.19

Index ranges

$-6 \leq \mathrm{h} \leq 6,-18 \leq \mathrm{k} \leq 18,-30 \leq 1 \leq$

30

Reflections collected

27898

Independent reflections

$3859\left[\mathrm{R}_{\text {int }}=0.0490, \mathrm{R}_{\mathrm{sigma}}=\right.$

$0.0285]$

Data/restraints/parameters

$3859 / 0 / 222$

Goodness-of-fit on $\mathrm{F}^{2}$

1.031

Final R indexes [I $>=2 \sigma(\mathrm{I})]$

$\mathrm{R}_{1}=0.0293, \mathrm{wR}_{2}=0.0679$

Final $\mathrm{R}$ indexes [all data]

$\mathrm{R}_{1}=0.0325, \mathrm{wR}_{2}=0.0691$

Largest diff. peak/hole / e $\AA^{-3}$

$0.32 /-0.49$ 


\section{5. ${ }^{11 B}$ NMR analysis of reaction: evidence for borinic ester intermediates}

Evidence for formation of a borinic ester intermediate was obtained by following the course of the allylboration reaction monitored by ${ }^{11} \mathrm{~B}$ NMR. Following addition of $n$ BuLi to a solution of vinylcyclopropyl boronic ester 1a in THF, a signal at 5 ppm was observed by ${ }^{11} \mathrm{~B}$ NMR analysis conducted at $-78^{\circ} \mathrm{C}$. This indicates formation of ate complex 5a. Following addition of TFAA at $-78{ }^{\circ} \mathrm{C}$, a new signal appeared at $47 \mathrm{ppm}$, and indicates the formation of borinic ester intermediate 6a. Following addition of benzaldehyde, a new signal appeared at $30 \mathrm{ppm}$, indicating the formation of boronic ester 19.

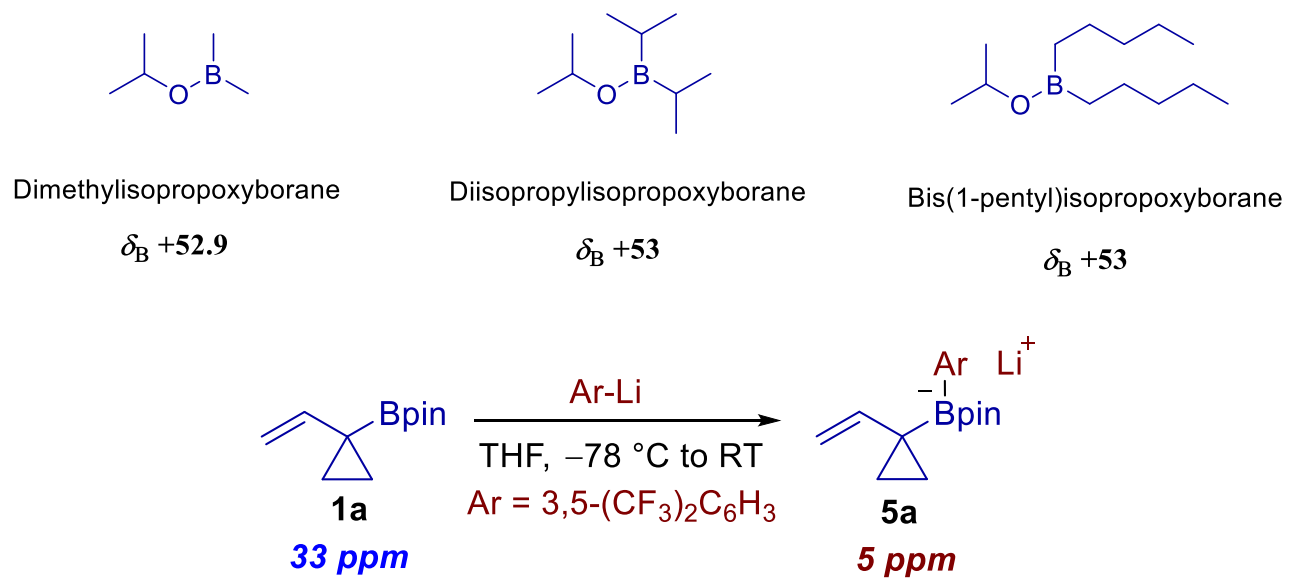

${ }^{11} \mathrm{~B}$ NMR (400 MHz), measured at RT, signal at $33 \mathrm{ppm}$
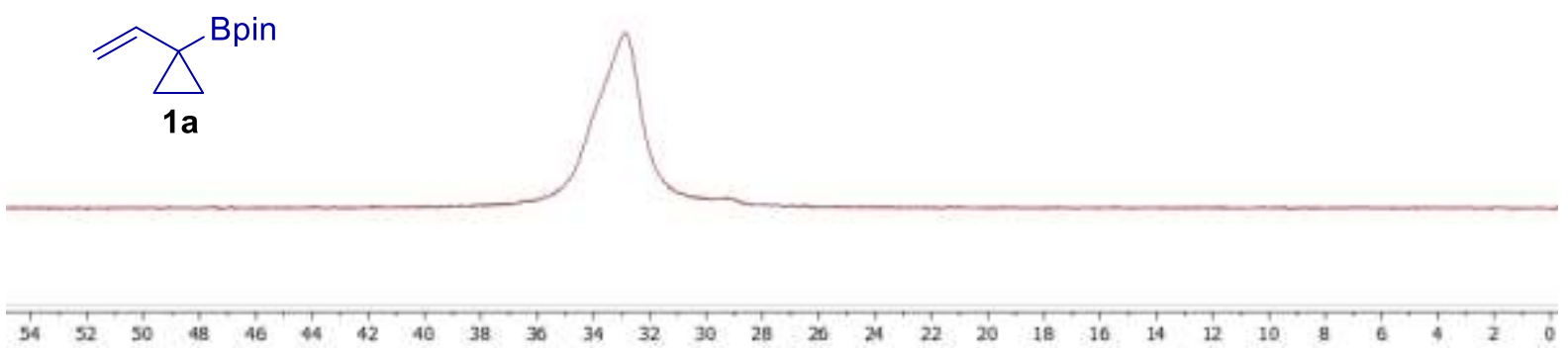

${ }^{11} \mathrm{~B}$ NMR (400 MHz), measured at RT, signal at $5 \mathrm{ppm}$

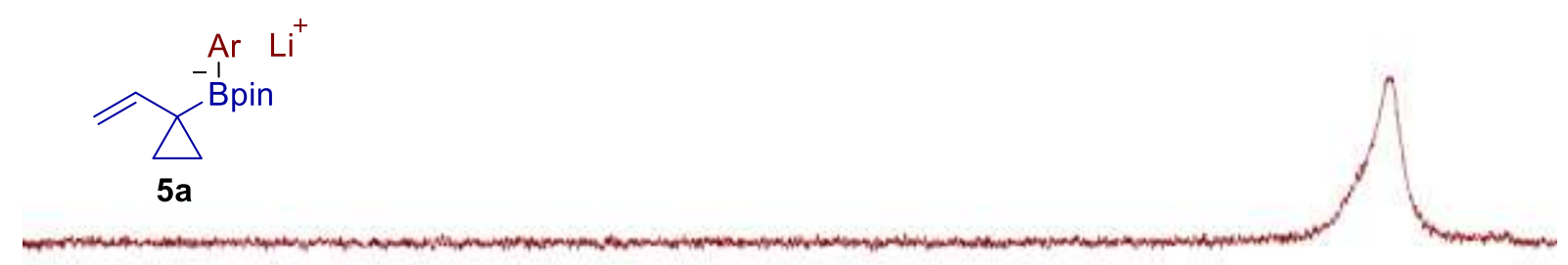




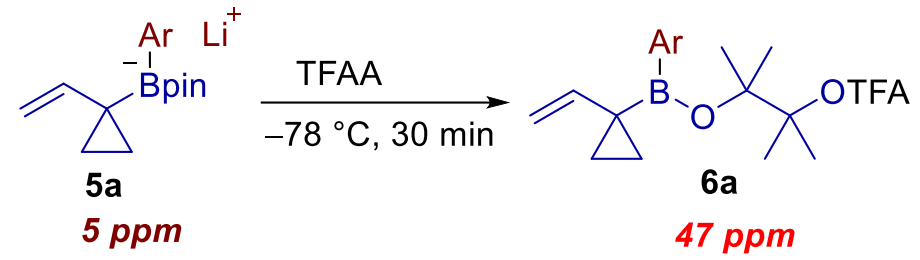

${ }^{11} \mathrm{~B}$ NMR (400 MHz), measured at RT, signal at $47 \mathrm{ppm}$<smiles>C=CC1(B(Br)OC(C)(C)C(C)(C)O[I+][Na])CC1</smiles>

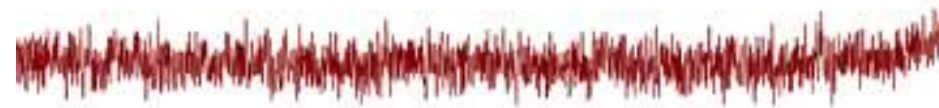

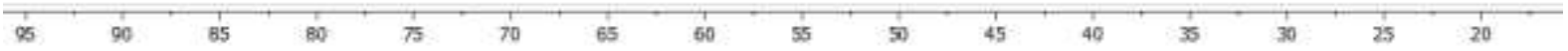

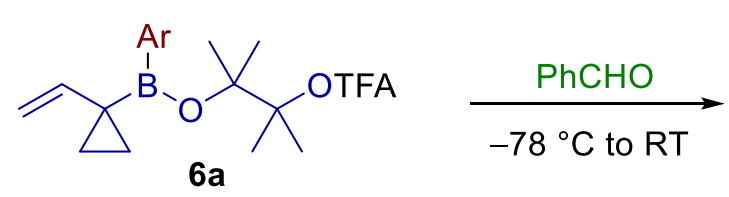

47 ppm

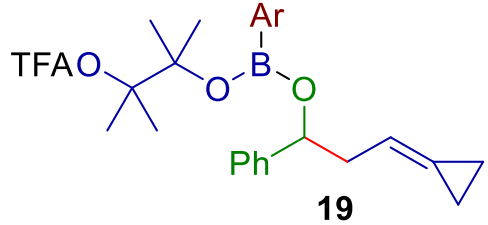

30 ppm

${ }^{11} \mathrm{~B}$ NMR (400 MHz), measured at RT, signal at $30 \mathrm{ppm}$
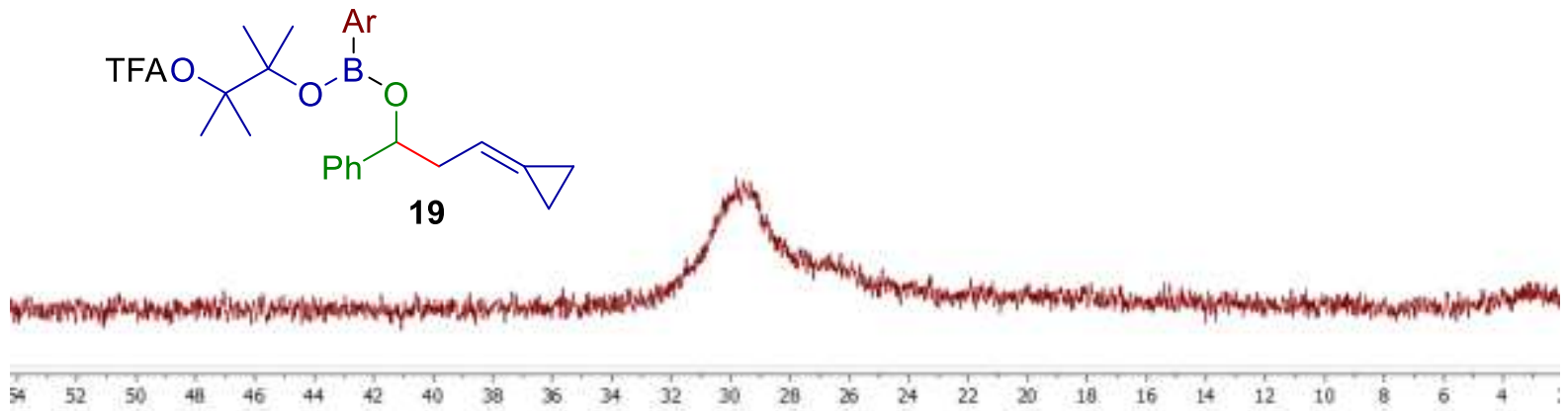


\section{DFT Calculations}

Calculations were performed using the Gaussian09 ${ }^{11}$ suite of programmes. Geometries were optimized with the DFT method using M06-2X functional as implemented in Gaussian, using 6-311G(d,p) as basis set. All calculations were carried out using the PCM model of solvation with tetrahydrofuran as the solvent. ${ }^{12}$ All geometry optimizations were full, with no restrictions. All stationary points located in the potential energy hypersurface were characterized as minima (no imaginary frequencies) or as transition states (one and only one imaginary frequency) by vibrational analysis. The analysis also provided zero-point vibrational energy corrections and thermal corrections to various thermodynamic properties.

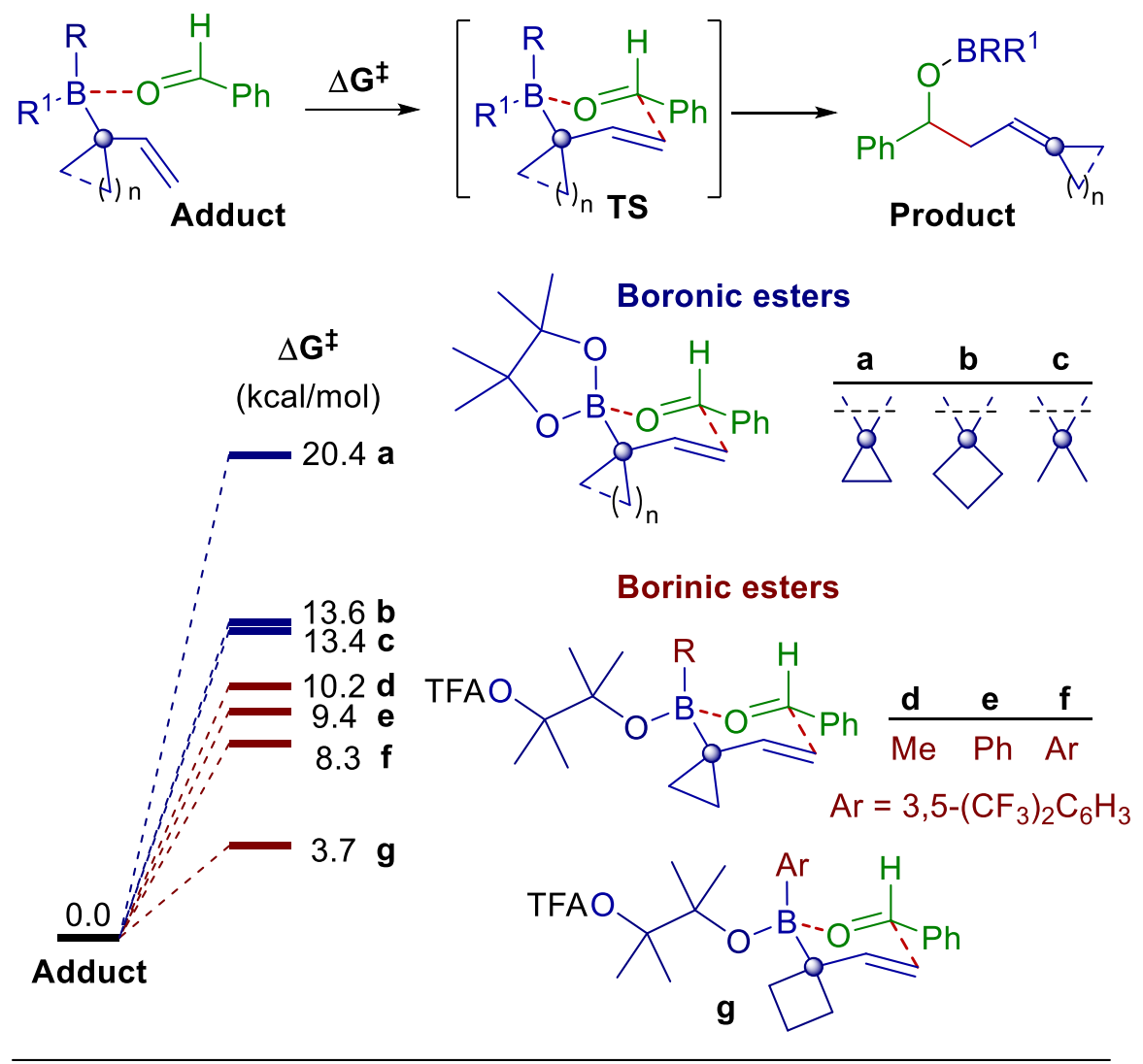

Ring-strain consideration. The series reports the calculated transition states for the concerted allyboration. The activation energies $\left(\Delta \mathrm{G}^{*}\right)$ are relative to the corresponding adducts since the latter are the energy minima (physically stable chemical species) directly connected with the TSs.

In order to evaluate the ring-strain increase, the $\Delta \mathrm{G}^{*}$ and the $\Delta \mathrm{G}$ were calculated for the following equations. 

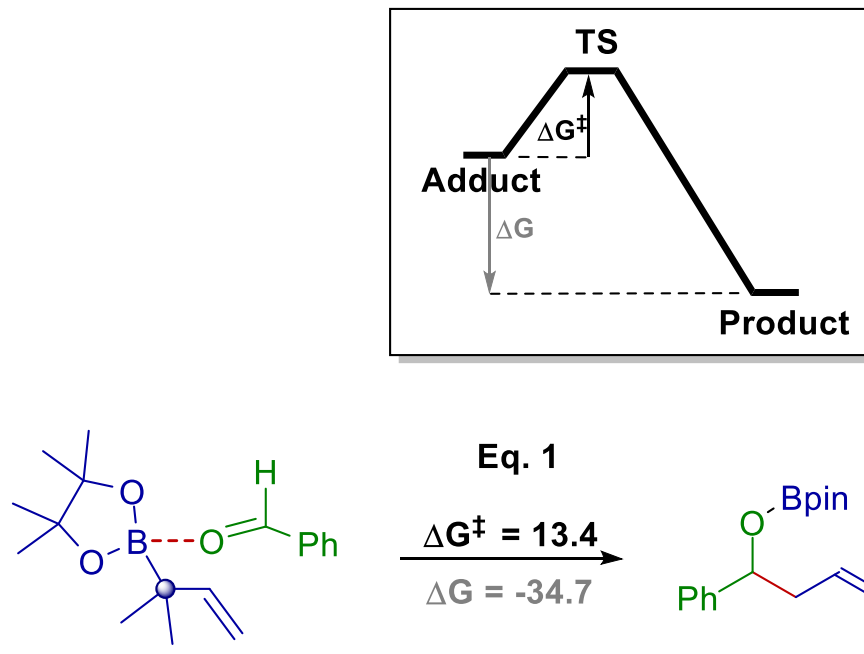

Adduct-c

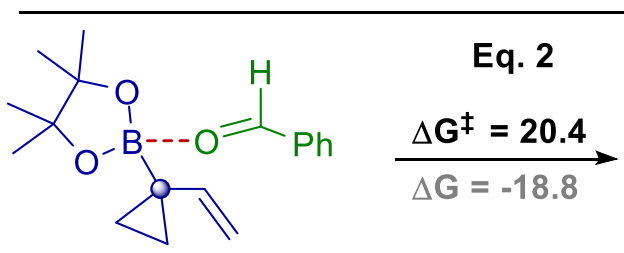

Adduct-a

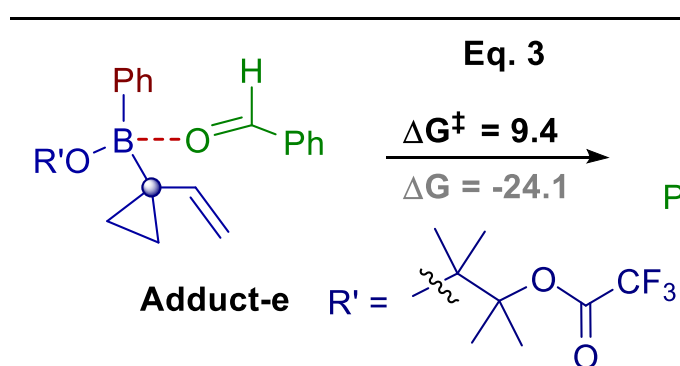<smiles>CC(C)=CCC(Oc1ccccc1)c1ccccc1</smiles>

Product-c

$\Delta \Delta \mathbf{G}^{\ddagger}=7.0$

$\Delta \Delta \mathrm{G}=15.9$

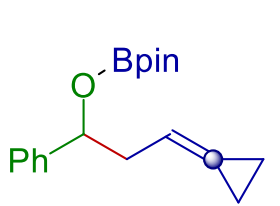

Product-a

$\Delta \Delta \mathbf{G}^{\ddagger}=-11.0$

$\Delta \Delta \mathrm{G}=-5.3$

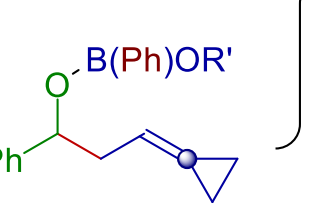

Product-e

In all the equations, the $\Delta \mathrm{G}$ is negative, thus they are all thermodynamically feasible. However, Eq. 2 has the highest kinetic barrier $\left(\Delta \mathrm{G}^{\ddagger}\right)$, preventing it from happening in the lab. The ringstrain increase can be appreciated comparing Eq. 1 and Eq. 2. In particular, replacing the gemdimethyl with a cyclopropyl, both $\Delta \mathrm{G}^{\ddagger}$ and $\Delta \mathrm{G}$ increase (by 7 and $15.9 \mathrm{kcal} / \mathrm{mol}$, respectively). The increment of the kinetic barrier $\left(\Delta \Delta \mathrm{G}^{*}=7\right)$ is less marked than the overall thermodynamic $(\Delta \Delta \mathrm{G}=15.9)$, in agreement with the Hammond postulate. Indeed, in these exergonic reactions the TS resembles more the starting material, therefore the $\alpha$-carbon is $\mathrm{sp}^{3}$-like in TS and $\mathrm{sp}^{2}$ in the product, thus in the latter the ring-strain is more evident.

Axial vs equatorial preference. For borinic ester, depending on the orientation of the boron substituents, two 6-membered chair-like TSs are possible: one with the alkoxide in axial (labelled as ') and the other having the alkoxide in equatorial. The substituents also affect the energies of the starting adducts. To avoid energy differences relative to different conformations 
of the aliphatic chain of $-\mathrm{OCH}_{2} \mathrm{CH}_{2} \mathrm{OTFA}$, the alkoxide substituent was initially modelled as $\mathrm{O} t \mathrm{Bu}$ (species h' and h).
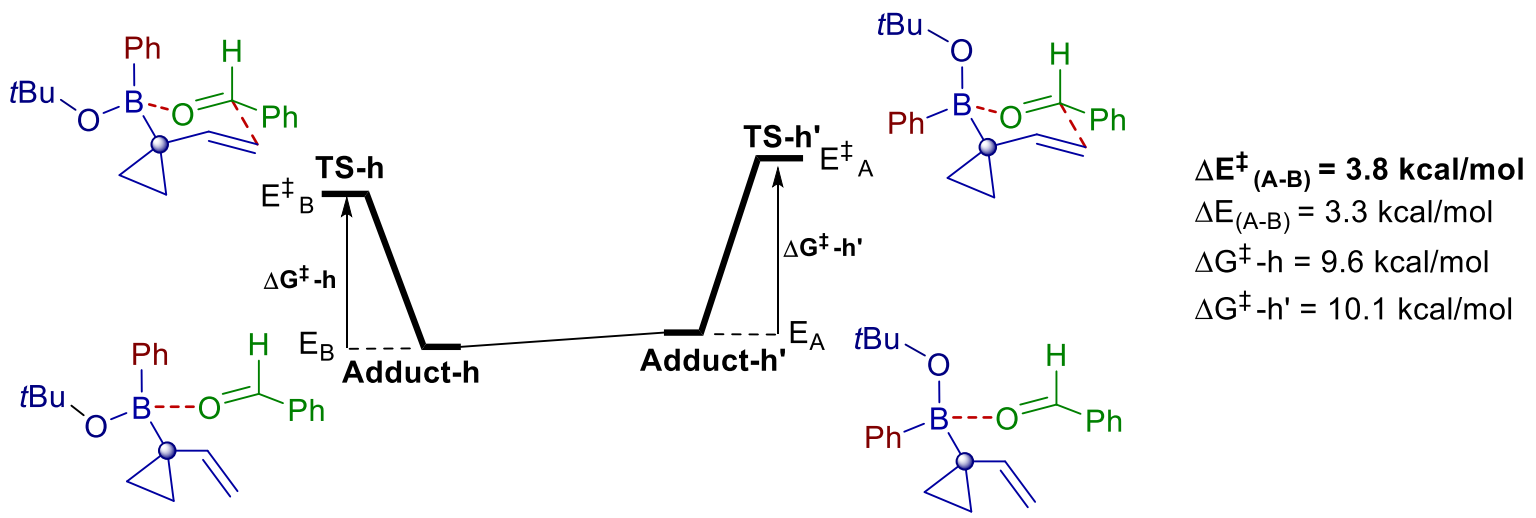

For the Curtin-Hammett principle, the product distribution reflects the difference in energy between the two rate-limiting transition states $\left(\Delta \mathrm{E}^{\ddagger}=3.8 \mathrm{kcal} / \mathrm{mol}\right)$, therefore the pathway with $-\mathrm{O} t \mathrm{Bu}$ equatorial should be the most favourable. For the borinic esters reported in the manuscript (-OR' $=-\mathrm{OCH}_{2} \mathrm{CH}_{2} \mathrm{OTFA}$ ), the $-\mathrm{O} t \mathrm{Bu}$ equatorial preference is still valid when $\mathrm{R}$ is an aryl group, whereas for $\mathrm{R}=\mathrm{Me}$ both pathways may be operative since $\Delta \mathrm{E}^{\ddagger}$ is within the error in DFT calculations.

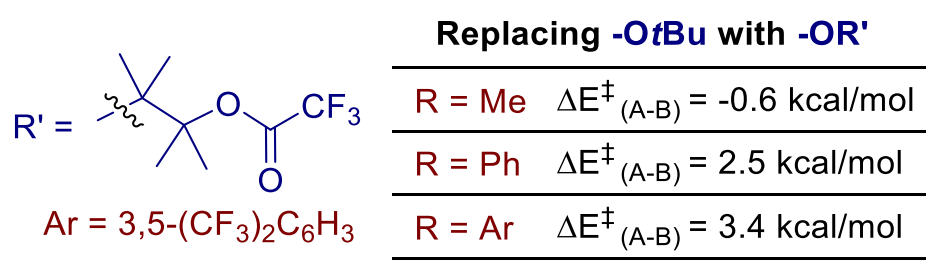

Full Cartesian coordinates for the optimised geometries are reported below. 
Adduct-a<smiles>C=CC1([B-]2(OC=Cc3ccccc3)OC(C)(C)C(C)(C)O2)CC1</smiles>

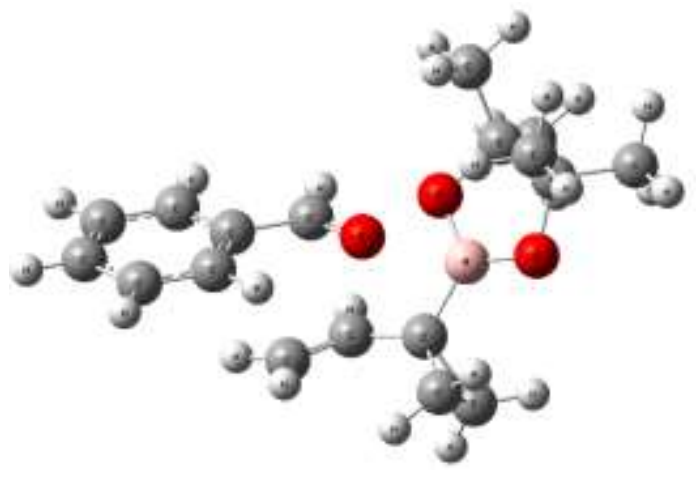

$\begin{array}{lrrr}\text { B } & 2.52683 & 0.58538 & -0.23089 \\ \mathrm{C} & 1.48124 & 2.36779 & -1.18249 \\ \mathrm{C} & 2.03246 & 2.75515 & 0.23118 \\ \mathrm{C} & 0.95857 & 2.74116 & 1.31448 \\ \mathrm{H} & 1.44523 & 2.84545 & 2.28611 \\ \mathrm{H} & 0.25666 & 3.56809 & 1.18818 \\ \mathrm{H} & 0.41362 & 1.79575 & 1.30338 \\ \mathrm{C} & 2.81142 & 4.05849 & 0.27211 \\ \mathrm{H} & 3.68168 & 4.02756 & -0.38280 \\ \mathrm{H} & 2.17182 & 4.89217 & -0.02911 \\ \mathrm{H} & 3.15393 & 4.24190 & 1.29213 \\ \mathrm{C} & 2.36344 & 2.86752 & -2.32228 \\ \mathrm{H} & 2.29971 & 3.95223 & -2.42898 \\ \mathrm{H} & 3.40684 & 2.59132 & -2.15474 \\ \mathrm{H} & 2.02933 & 2.40484 & -3.25253 \\ \mathrm{C} & 0.03062 & 2.74748 & -1.42114 \\ \mathrm{H} & -0.09824 & 3.82993 & -1.34008 \\ \mathrm{H} & -0.26583 & 2.44125 & -2.42617 \\ \mathrm{H} & -0.62645 & 2.25946 & -0.70129 \\ \mathrm{O} & 2.93487 & 1.65825 & 0.51995 \\ \mathrm{O} & 1.59079 & 0.92287 & -1.17541 \\ \mathrm{C} & 2.64333 & -1.88170 & -1.01708 \\ \mathrm{C} & 2.12770 & -3.06229 & -0.68497 \\ \mathrm{H} & 1.80153 & -3.76635 & -1.44198 \\ \mathrm{H} & 1.99734 & -3.35835 & 0.35105 \\ \mathrm{H} & 2.72092 & -1.62223 & -2.07174 \\ \mathrm{C} & -0.25893 & -1.15620 & 0.26106 \\ \mathrm{O} & 0.22098 & -0.61974 & 1.23282 \\ \mathrm{H} & -0.15997 & -0.69553 & -0.73692 \\ \mathrm{C} & -2.37237 & -4.85063 & 0.35975 \\ \mathrm{C} & -1.86643 & -4.31375 & 1.54417\end{array}$




\begin{tabular}{cccr}
$\mathrm{C}$ & -1.18125 & -3.10805 & 1.52004 \\
$\mathrm{C}$ & -1.00103 & -2.43848 & 0.30861 \\
$\mathrm{C}$ & -1.50690 & -2.97575 & -0.87362 \\
$\mathrm{C}$ & -2.19435 & -4.18377 & -0.84895 \\
$\mathrm{H}$ & -2.90770 & -5.79254 & 0.38141 \\
$\mathrm{H}$ & -2.00961 & -4.83874 & 2.48079 \\
$\mathrm{H}$ & -0.77687 & -2.67049 & 2.42551 \\
$\mathrm{H}$ & -1.35354 & -2.44837 & -1.80981 \\
$\mathrm{H}$ & -2.58843 & -4.60497 & -1.76577 \\
$\mathrm{C}$ & 3.12307 & -0.83652 & -0.07146 \\
$\mathrm{C}$ & 3.55110 & -1.22417 & 1.32530 \\
$\mathrm{C}$ & 4.59891 & -0.86662 & 0.32514 \\
$\mathrm{H}$ & 3.45951 & -2.26599 & 1.60268 \\
$\mathrm{H}$ & 3.33642 & -0.51648 & 2.11536 \\
$\mathrm{H}$ & 5.10844 & 0.08309 & 0.42695 \\
$\mathrm{H}$ & 5.18660 & -1.67431 & -0.09285 \\
\multicolumn{4}{c}{$\mathrm{G}=-951.06659$ Hartree } \\
\end{tabular}

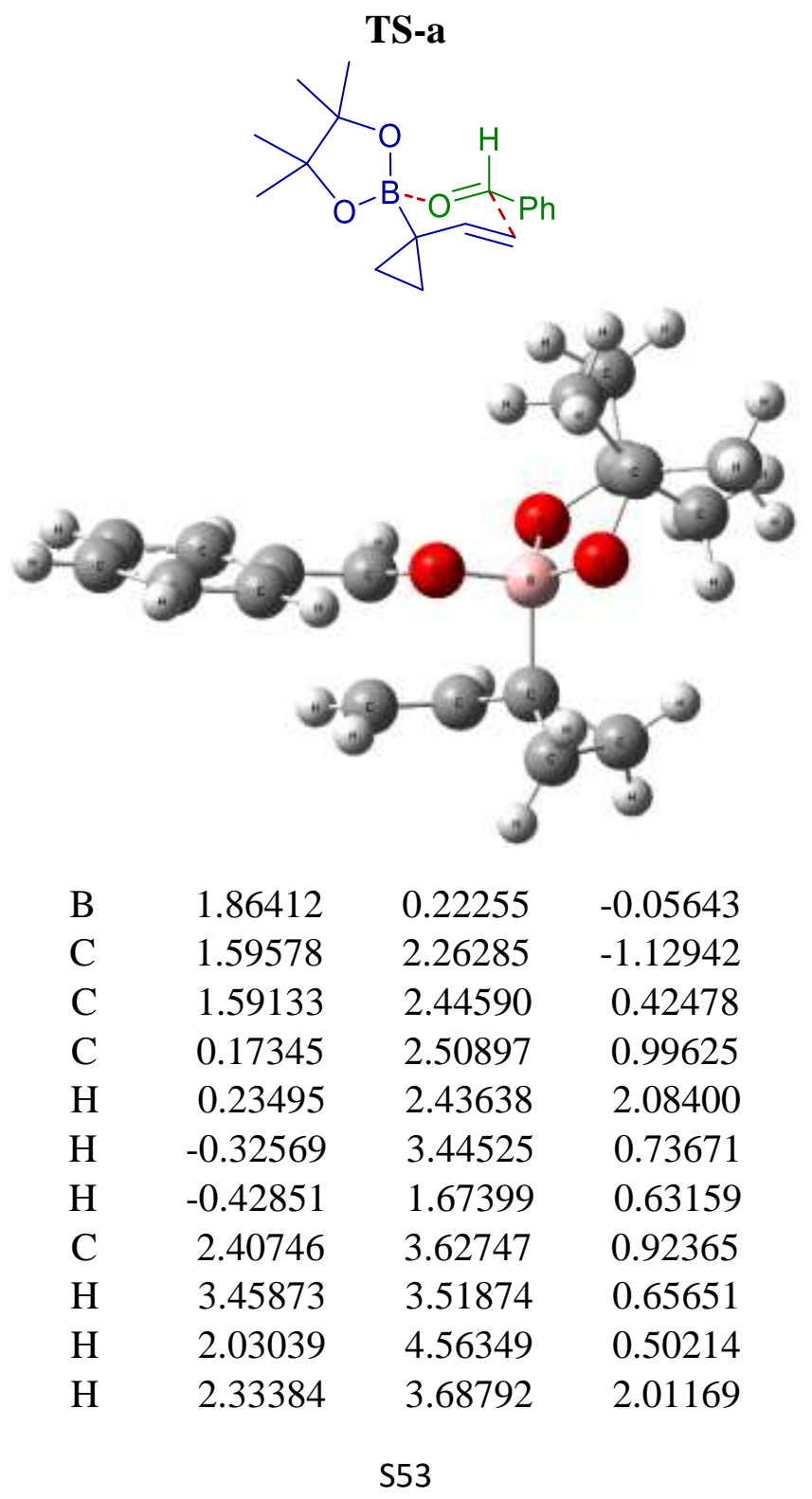




\begin{tabular}{|c|c|c|c|}
\hline $\mathrm{C}$ & 2.92896 & 2.66276 & -1.76203 \\
\hline $\mathrm{H}$ & 3.07843 & 3.74490 & -1.74079 \\
\hline $\mathrm{H}$ & 3.76110 & 2.18118 & -1.24631 \\
\hline $\mathrm{H}$ & 2.93262 & 2.33133 & -2.80266 \\
\hline $\mathrm{C}$ & 0.45524 & 2.97131 & -1.84702 \\
\hline $\mathrm{H}$ & 0.49407 & 4.04928 & -1.66688 \\
\hline $\mathrm{H}$ & 0.54180 & 2.80165 & -2.92249 \\
\hline $\mathrm{H}$ & -0.51185 & 2.59279 & -1.51672 \\
\hline $\mathrm{O}$ & 2.20087 & 1.23293 & 0.88441 \\
\hline $\mathrm{O}$ & 1.43682 & 0.84892 & -1.27983 \\
\hline $\mathrm{C}$ & 2.67537 & -1.80720 & -1.21667 \\
\hline $\mathrm{C}$ & 1.82501 & -2.83114 & -0.86448 \\
\hline $\mathrm{H}$ & 1.39130 & -3.47096 & -1.62426 \\
\hline $\mathrm{H}$ & 1.88193 & -3.23619 & 0.14040 \\
\hline $\mathrm{H}$ & 2.74396 & -1.52163 & -2.26542 \\
\hline $\mathrm{C}$ & 0.28293 & -1.54501 & -0.39577 \\
\hline $\mathrm{O}$ & 0.79117 & -0.71545 & 0.45417 \\
\hline $\mathrm{H}$ & 0.11502 & -1.17819 & -1.40881 \\
\hline $\mathrm{C}$ & -2.55786 & -4.37969 & 1.04093 \\
\hline $\mathrm{C}$ & -1.70677 & -3.73768 & 1.93813 \\
\hline $\mathrm{C}$ & -0.77223 & -2.81938 & 1.47861 \\
\hline $\mathrm{C}$ & -0.68890 & -2.54072 & 0.11369 \\
\hline $\mathrm{C}$ & -1.54111 & -3.18312 & -0.78425 \\
\hline $\mathrm{C}$ & -2.47518 & -4.10140 & -0.3204 \\
\hline $\mathrm{H}$ & -3.28648 & -5.09442 & 1.40450 \\
\hline $\mathrm{H}$ & -1.77547 & -3.95194 & 2.9977 \\
\hline $\mathrm{H}$ & -0.10915 & -2.30715 & 2.16548 \\
\hline $\mathrm{H}$ & -1.47335 & -2.95934 & -1.8440 \\
\hline $\mathrm{H}$ & -3.13981 & -4.59554 & -1.01842 \\
\hline $\mathrm{C}$ & 3.16616 & -0.88789 & -0.2483 \\
\hline $\mathrm{C}$ & 3.94694 & -1.21777 & 0.9780 \\
\hline $\mathrm{C}$ & 4.54671 & -0.34094 & -0.1074 \\
\hline $\mathrm{H}$ & 4.33627 & -2.22413 & 1.08310 \\
\hline $\mathrm{H}$ & 3.66680 & -0.72389 & 1.90046 \\
\hline $\mathrm{H}$ & 4.64608 & 0.71170 & 0.12510 \\
\hline $\mathrm{H}$ & 5.33374 & -0.76639 & -0.7181 \\
\hline
\end{tabular}




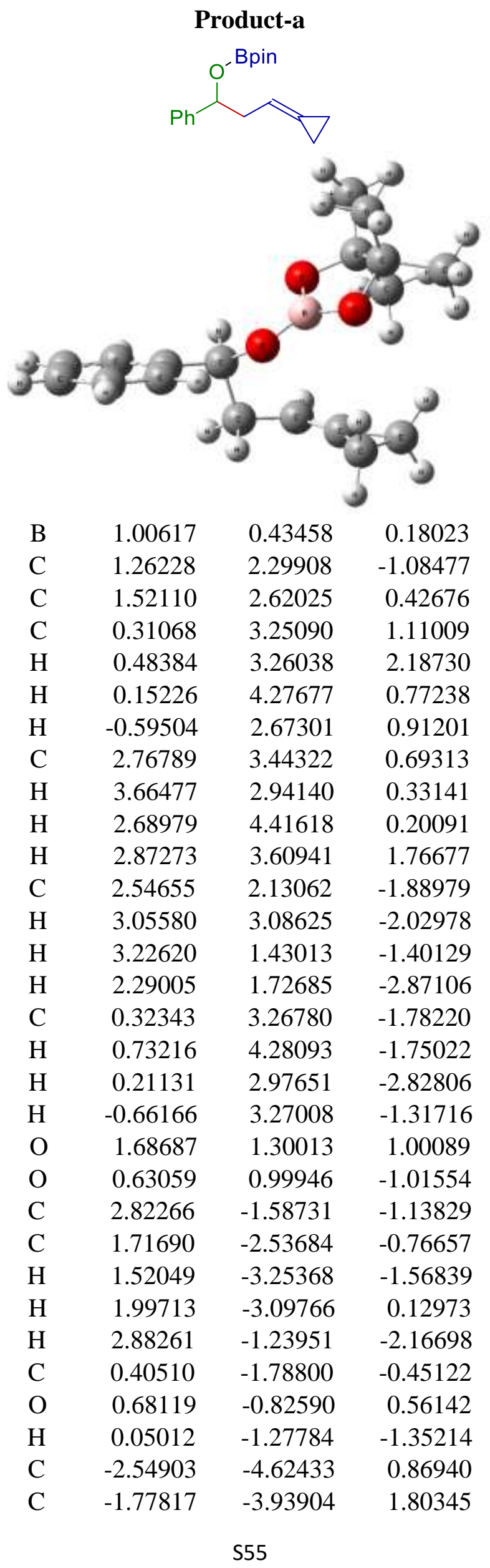




$\begin{array}{lllr}\mathrm{C} & -0.83363 & -3.00667 & 1.38539 \\ \mathrm{C} & -0.65604 & -2.74890 & 0.02743 \\ \mathrm{C} & -1.43242 & -3.43600 & -0.90500 \\ \mathrm{C} & -2.37373 & -4.37088 & -0.48797 \\ \mathrm{H} & -3.28514 & -5.34846 & 1.19784 \\ \mathrm{H} & -1.91376 & -4.12953 & 2.86177 \\ \mathrm{H} & -0.23652 & -2.46501 & 2.10881 \\ \mathrm{H} & -1.30254 & -3.23297 & -1.96367 \\ \mathrm{H} & -2.97455 & -4.89558 & -1.22140 \\ \mathrm{C} & 3.66573 & -1.12451 & -0.23250 \\ \mathrm{C} & 4.06002 & -1.15004 & 1.17755 \\ \mathrm{C} & 4.79166 & -0.27345 & 0.15489 \\ \mathrm{H} & 4.58289 & -2.02037 & 1.55835 \\ \mathrm{H} & 3.41247 & -0.64269 & 1.88427 \\ \mathrm{H} & 4.63383 & 0.79714 & 0.22587 \\ \mathrm{H} & 5.79195 & -0.57078 & -0.13893 \\ & \mathrm{G}=-951.096538 \text { Hartree }\end{array}$

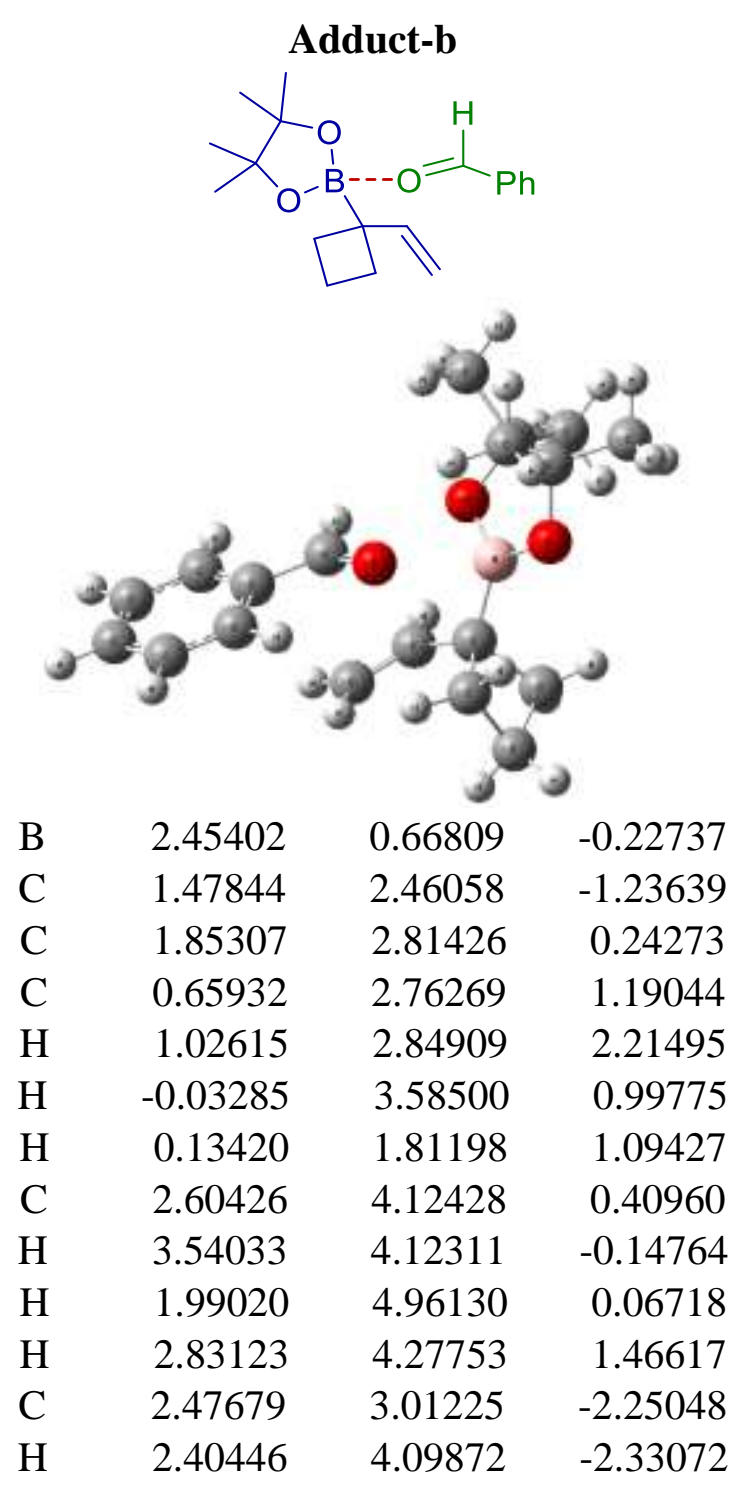




$\begin{array}{lrrr}\mathrm{H} & 3.49981 & 2.74713 & -1.97304 \\ \mathrm{H} & 2.26196 & 2.57534 & -3.22721 \\ \mathrm{C} & 0.05866 & 2.82140 & -1.63657 \\ \mathrm{H} & -0.10251 & 3.89874 & -1.54654 \\ \mathrm{H} & -0.10842 & 2.53653 & -2.67705 \\ \mathrm{H} & -0.67009 & 2.30290 & -1.01388 \\ \mathrm{O} & 2.73306 & 1.72183 & 0.60496 \\ \mathrm{O} & 1.61950 & 1.01909 & -1.25995 \\ \mathrm{C} & 2.69333 & -1.70918 & -1.11924 \\ \mathrm{C} & 2.21782 & -2.93680 & -0.92484 \\ \mathrm{H} & 1.91934 & -3.55766 & -1.76212 \\ \mathrm{H} & 2.10234 & -3.36557 & 0.06520 \\ \mathrm{H} & 2.77500 & -1.34389 & -2.14286 \\ \mathrm{C} & -0.17988 & -1.16567 & 0.15711 \\ \mathrm{O} & 0.33727 & -0.57094 & 1.07545 \\ \mathrm{H} & -0.13687 & -0.75680 & -0.86724 \\ \mathrm{C} & -2.23337 & -4.86803 & 0.58566 \\ \mathrm{C} & -1.66898 & -4.25746 & 1.70612 \\ \mathrm{C} & -1.00016 & -3.04999 & 1.57187 \\ \mathrm{C} & -0.89657 & -2.45235 & 0.31433 \\ \mathrm{C} & -1.45937 & -3.06384 & -0.80449 \\ \mathrm{C} & -2.13007 & -4.27395 & -0.66888 \\ \mathrm{H} & -2.75453 & -5.81204 & 0.69305 \\ \mathrm{H} & -1.75466 & -4.72701 & 2.67861 \\ \mathrm{H} & -0.55222 & -2.55445 & 2.42550 \\ \mathrm{H} & -1.36508 & -2.59162 & -1.77734 \\ \mathrm{H} & -2.56936 & -4.75333 & -1.53501 \\ \mathrm{C} & 3.15454 & -0.72908 & -0.08170 \\ \mathrm{C} & 3.29479 & -1.29215 & 1.36077 \\ \mathrm{C} & 4.73119 & -0.63671 & -0.09829 \\ \mathrm{H} & 2.61531 & -2.09021 & 1.66048 \\ \mathrm{H} & 3.21418 & -0.47867 & 2.08395 \\ \mathrm{H} & 5.06705 & 0.35418 & 0.21333 \\ \mathrm{H} & 5.22381 & -0.91344 & -1.03196 \\ \mathrm{C} & 4.76784 & -1.64513 & 1.06832 \\ \mathrm{H} & 4.86645 & -2.66794 & 0.70146 \\ \mathrm{H} & 5.49932 & -1.47474 & 1.85865 \\ & \mathrm{G}=-990.335564 \mathrm{Hartr} \\ & & & \end{array}$

TS-b<smiles></smiles> 


\begin{tabular}{|c|c|c|c|}
\hline B & 1.92032 & 0.26828 & -0.04386 \\
\hline $\mathrm{C}$ & 1.55807 & 2.27895 & -1.15809 \\
\hline $\mathrm{C}$ & 1.52674 & 2.48190 & 0.39443 \\
\hline $\mathrm{C}$ & 0.10204 & 2.47862 & 0.95413 \\
\hline $\mathrm{H}$ & 0.15931 & 2.42483 & 2.04323 \\
\hline $\mathrm{H}$ & -0.44202 & 3.38462 & 0.67769 \\
\hline $\mathrm{H}$ & -0.45586 & 1.61016 & 0.59875 \\
\hline $\mathrm{C}$ & 2.26907 & 3.71553 & 0.88312 \\
\hline $\mathrm{H}$ & 3.32752 & 3.66263 & 0.62815 \\
\hline $\mathrm{H}$ & 1.84409 & 4.62250 & 0.44434 \\
\hline $\mathrm{H}$ & 2.18042 & 3.78690 & 1.96940 \\
\hline $\mathrm{C}$ & 2.85675 & 2.77778 & -1.79152 \\
\hline $\mathrm{H}$ & 2.91748 & 3.86866 & -1.78535 \\
\hline $\mathrm{H}$ & 3.72419 & 2.37395 & -1.26762 \\
\hline $\mathrm{H}$ & 2.89163 & 2.43373 & -2.82745 \\
\hline $\mathrm{C}$ & 0.36912 & 2.88366 & -1.89261 \\
\hline $\mathrm{H}$ & 0.32267 & 3.96416 & -1.73030 \\
\hline $\mathrm{H}$ & 0.47566 & 2.70443 & -2.96473 \\
\hline $\mathrm{H}$ & -0.56841 & 2.43718 & -1.56159 \\
\hline $\mathrm{O}$ & 2.20102 & 1.31386 & 0.87735 \\
\hline $\mathrm{O}$ & 1.51990 & 0.85387 & -1.29501 \\
\hline $\mathrm{C}$ & 2.72804 & -1.75777 & -1.16413 \\
\hline $\mathrm{C}$ & 1.89917 & -2.81241 & -0.90560 \\
\hline $\mathrm{H}$ & 1.48315 & -3.40287 & -1.71350 \\
\hline $\mathrm{H}$ & 1.85891 & -3.24850 & 0.08598 \\
\hline $\mathrm{H}$ & 2.85185 & -1.43614 & -2.19752 \\
\hline $\mathrm{C}$ & 0.24563 & -1.43679 & -0.35639 \\
\hline $\mathrm{O}$ & 0.80818 & -0.65384 & 0.47930 \\
\hline $\mathrm{H}$ & 0.12552 & -1.08839 & -1.38201 \\
\hline $\mathrm{C}$ & -2.51097 & -4.35444 & 1.04678 \\
\hline $\mathrm{C}$ & -1.66879 & -3.70947 & 1.95096 \\
\hline C & -0.75957 & -2.76192 & 1.50238 \\
\hline $\mathrm{C}$ & -0.69223 & -2.45815 & 0.14075 \\
\hline $\mathrm{C}$ & -1.53586 & -3.10396 & -0.76406 \\
\hline $\mathrm{C}$ & -2.44495 & -4.05115 & -0.31035 \\
\hline $\mathrm{H}$ & -3.22004 & -5.09248 & 1.40231 \\
\hline $\mathrm{H}$ & -1.72521 & -3.94546 & 3.00664 \\
\hline
\end{tabular}




$\begin{array}{lrrr}\mathrm{H} & -0.10166 & -2.24793 & 2.19307 \\ \mathrm{H} & -1.47778 & -2.86141 & -1.82016 \\ \mathrm{H} & -3.10220 & -4.54976 & -1.01198 \\ \mathrm{C} & 3.21090 & -0.85480 & -0.15492 \\ \mathrm{C} & 3.71220 & -1.41858 & 1.19524 \\ \mathrm{C} & 4.59340 & -0.18779 & -0.36196 \\ \mathrm{H} & 3.44618 & -2.44783 & 1.44294 \\ \mathrm{H} & 3.43474 & -0.76361 & 2.02307 \\ \mathrm{H} & 4.58355 & 0.83995 & 0.00293 \\ \mathrm{H} & 5.02593 & -0.22559 & -1.36335 \\ \mathrm{C} & 5.15595 & -1.15246 & 0.70950 \\ \mathrm{H} & 5.58335 & -2.04499 & 0.24833 \\ \mathrm{H} & 5.86294 & -0.74029 & 1.43043 \\ & \mathrm{G}=-990.313818 \text { Hartree }\end{array}$

Adduct-c<smiles>C=CC(C)(C)[B-]1(OC=Cc2ccccc2)OC(C)(C)C(C)(C)O1</smiles>

$\begin{array}{llll} & & & \\ \mathrm{C} & & & \\ \mathrm{C} & & & \\ \mathrm{C} & 1.53483 & 2.30593 & -1.16795 \\ \mathrm{C} & 0.73080 & 2.67195 & 1.25824 \\ \mathrm{H} & 1.10448 & 2.77041 & 2.27913 \\ \mathrm{H} & 0.06025 & 3.50851 & 1.05083 \\ \mathrm{H} & 0.18012 & 1.73407 & 1.18436 \\ \mathrm{C} & 2.70459 & 3.96955 & 0.44750 \\ \mathrm{H} & 3.64015 & 3.93614 & -0.10968 \\ \mathrm{H} & 2.11095 & 4.81514 & 0.09059 \\ \mathrm{H} & 2.93615 & 4.13602 & 1.50106 \\ \mathrm{C} & 2.53495 & 2.83182 & -2.19386 \\ \mathrm{H} & 2.47983 & 3.91840 & -2.28509 \\ \mathrm{H} & 3.55544 & 2.55324 & -1.91961 \\ \mathrm{H} & 2.30763 & 2.38786 & -3.16459 \\ \mathrm{C} & 0.11688 & 2.67512 & -1.56658 \\ \mathrm{H} & -0.03261 & 3.75552 & -1.49595\end{array}$




$\begin{array}{lrrr}\mathrm{H} & -0.05897 & 2.37247 & -2.60061 \\ \mathrm{H} & -0.61371 & 2.17604 & -0.93014 \\ \mathrm{O} & 2.77787 & 1.56960 & 0.68490 \\ \mathrm{O} & 1.66063 & 0.86243 & -1.17579 \\ \mathrm{C} & 2.58062 & -1.90302 & -0.91176 \\ \mathrm{C} & 2.10820 & -3.08520 & -0.52471 \\ \mathrm{H} & 1.64803 & -3.76293 & -1.23508 \\ \mathrm{H} & 2.14946 & -3.41341 & 0.50871 \\ \mathrm{H} & 2.49686 & -1.62773 & -1.96285 \\ \mathrm{C} & -0.28482 & -1.16454 & 0.20055 \\ \mathrm{O} & 0.23084 & -0.63906 & 1.16118 \\ \mathrm{H} & -0.17172 & -0.72032 & -0.80342 \\ \mathrm{C} & -2.57079 & -4.74970 & 0.36254 \\ \mathrm{C} & -2.02390 & -4.22735 & 1.53527 \\ \mathrm{C} & -1.28305 & -3.05583 & 1.49097 \\ \mathrm{C} & -1.08760 & -2.40649 & 0.27064 \\ \mathrm{C} & -1.63477 & -2.92889 & -0.89992 \\ \mathrm{C} & -2.37842 & -4.10256 & -0.85460 \\ \mathrm{H} & -3.14924 & -5.66532 & 0.39936 \\ \mathrm{H} & -2.17876 & -4.73709 & 2.47842 \\ \mathrm{H} & -0.84779 & -2.62979 & 2.38754 \\ \mathrm{H} & -1.47034 & -2.41722 & -1.84294 \\ \mathrm{H} & -2.80438 & -4.51272 & -1.76204 \\ \mathrm{C} & 3.25097 & -0.86974 & -0.04546 \\ \mathrm{C} & 3.41902 & -1.31698 & 1.40781 \\ \mathrm{C} & 4.65011 & -0.58661 & -0.65464 \\ \mathrm{H} & 4.02880 & -2.22497 & 1.46174 \\ \mathrm{H} & 2.45404 & -1.51785 & 1.87722 \\ \mathrm{H} & 5.16702 & 0.19035 & -0.08599 \\ \mathrm{H} & 4.57400 & -0.25576 & -1.69477 \\ \mathrm{H} & 3.91750 & -0.53432 & 1.98355 \\ \mathrm{H} & 5.25799 & -1.49599 & -0.63218 \\ & \mathrm{G}=-952.271785 \mathrm{Hartr} \\ \mathrm{H} & & & \end{array}$

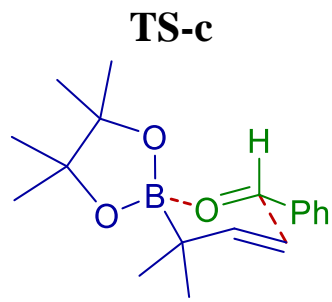




\begin{tabular}{|c|c|c|c|}
\hline B & 1.97760 & 0.19889 & -0.07916 \\
\hline $\mathrm{C}$ & 1.61406 & 2.23454 & -1.15041 \\
\hline $\mathrm{C}$ & 1.59735 & 2.40004 & 0.40798 \\
\hline $\mathrm{C}$ & 0.17864 & 2.37994 & 0.98394 \\
\hline $\mathrm{H}$ & 0.24908 & 2.29549 & 2.07037 \\
\hline $\mathrm{H}$ & -0.36580 & 3.29504 & 0.73988 \\
\hline $\mathrm{H}$ & -0.38710 & 1.52450 & 0.61189 \\
\hline $\mathrm{C}$ & 2.33511 & 3.62749 & 0.92025 \\
\hline $\mathrm{H}$ & 3.39240 & 3.58971 & 0.65885 \\
\hline $\mathrm{H}$ & 1.90048 & 4.54141 & 0.50596 \\
\hline $\mathrm{H}$ & 2.25202 & 3.67221 & 2.00838 \\
\hline $\mathrm{C}$ & 2.89080 & 2.78851 & -1.78202 \\
\hline $\mathrm{H}$ & 2.89732 & 3.88107 & -1.77544 \\
\hline $\mathrm{H}$ & 3.77610 & 2.43018 & -1.25624 \\
\hline $\mathrm{H}$ & 2.94310 & 2.44717 & -2.81836 \\
\hline $\mathrm{C}$ & 0.40195 & 2.82754 & -1.85670 \\
\hline $\mathrm{H}$ & 0.32725 & 3.90147 & -1.66430 \\
\hline $\mathrm{H}$ & 0.50327 & 2.68084 & -2.93444 \\
\hline $\mathrm{H}$ & -0.52005 & 2.34637 & -1.53047 \\
\hline $\mathrm{O}$ & 2.28575 & 1.22558 & 0.85536 \\
\hline $\mathrm{O}$ & 1.60666 & 0.81096 & -1.32515 \\
\hline $\mathrm{C}$ & 2.68417 & -1.86680 & -1.18081 \\
\hline $\mathrm{C}$ & 1.84052 & -2.90515 & -0.90501 \\
\hline $\mathrm{H}$ & 1.38573 & -3.46923 & -1.71106 \\
\hline $\mathrm{H}$ & 1.81147 & -3.35948 & 0.07813 \\
\hline $\mathrm{H}$ & 2.76488 & -1.54758 & -2.21934 \\
\hline $\mathrm{C}$ & 0.24805 & -1.45873 & -0.38651 \\
\hline $\mathrm{O}$ & 0.82526 & -0.67690 & 0.44020 \\
\hline $\mathrm{H}$ & 0.14426 & -1.12494 & -1.41907 \\
\hline $\mathrm{C}$ & -2.62403 & -4.24410 & 1.05618 \\
\hline $\mathrm{C}$ & -1.77113 & -3.60406 & 1.95360 \\
\hline $\mathrm{C}$ & -0.82468 & -2.70031 & 1.49150 \\
\hline $\mathrm{C}$ & -0.73061 & -2.43605 & 0.12335 \\
\hline $\mathrm{C}$ & -1.58561 & -3.07623 & -0.77469 \\
\hline $\mathrm{C}$ & -2.53221 & -3.97906 & -0.30750 \\
\hline $\mathrm{H}$ & -3.36180 & -4.94829 & 1.42185 \\
\hline $\mathrm{H}$ & -1.84824 & -3.80925 & 3.01435 \\
\hline $\mathrm{H}$ & -0.16015 & -2.18852 & 2.17753 \\
\hline
\end{tabular}




\begin{tabular}{lllr}
$\mathrm{H}$ & -1.50709 & -2.86427 & -1.83604 \\
$\mathrm{H}$ & -3.19841 & -4.47314 & -1.00389 \\
$\mathrm{C}$ & 3.25519 & -0.97018 & -0.20350 \\
$\mathrm{C}$ & 3.51233 & -1.55069 & 1.18665 \\
$\mathrm{C}$ & 4.47559 & -0.20738 & -0.72068 \\
$\mathrm{H}$ & 4.31154 & -2.29915 & 1.15084 \\
$\mathrm{H}$ & 2.62426 & -2.01414 & 1.61744 \\
$\mathrm{H}$ & 4.69834 & 0.63018 & -0.05625 \\
$\mathrm{H}$ & 4.29766 & 0.18728 & -1.72322 \\
$\mathrm{H}$ & 3.82361 & -0.75111 & 1.86232 \\
$\mathrm{H}$ & 5.35321 & -0.86070 & -0.75463 \\
\multicolumn{4}{c}{$\mathrm{G}=-952.250417$ Hartree }
\end{tabular}

\section{Product-c}

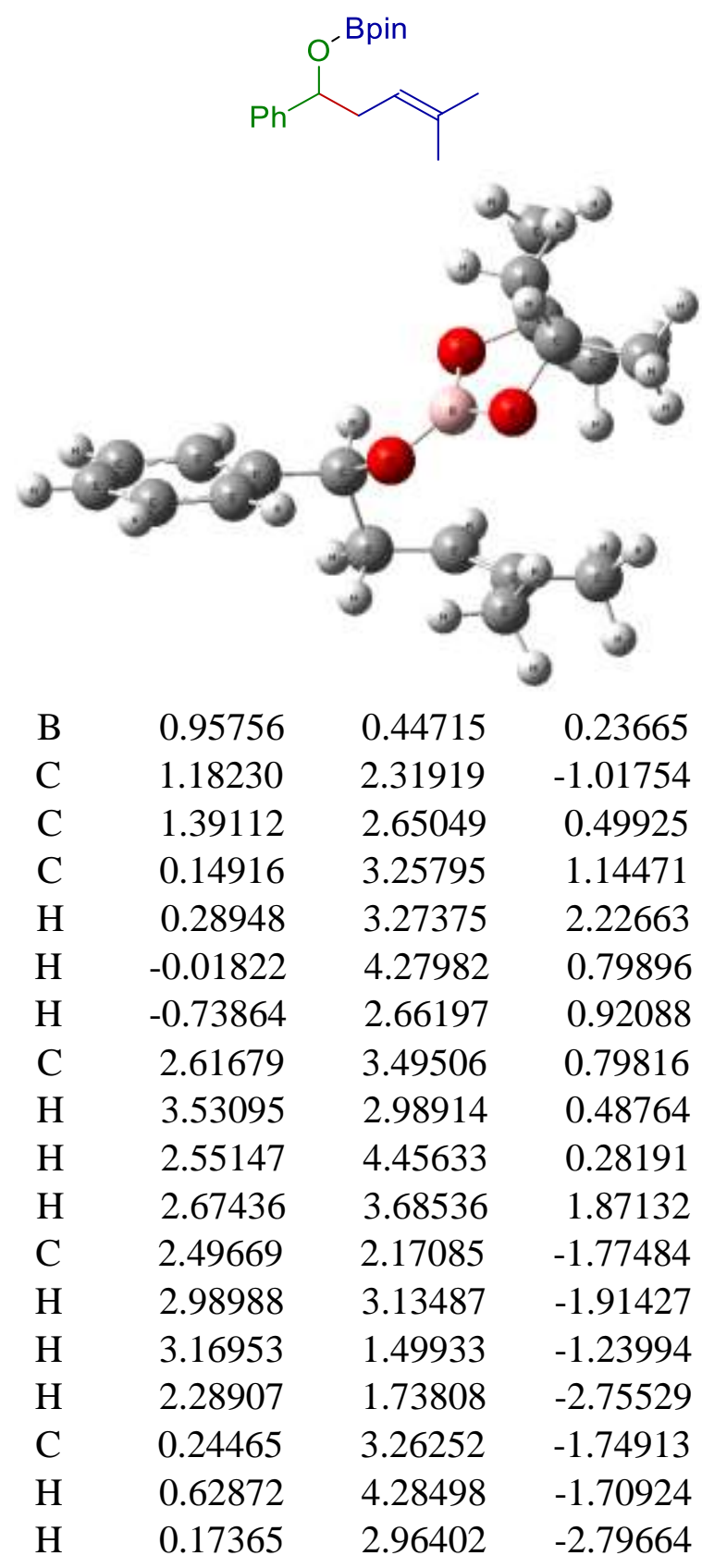




$\begin{array}{lrrr}\mathrm{H} & -0.75463 & 3.24367 & -1.31569 \\ \mathrm{O} & 1.57192 & 1.33705 & 1.08146 \\ \mathrm{O} & 0.58129 & 1.00379 & -0.96259 \\ \mathrm{C} & 2.86929 & -1.73429 & -1.08862 \\ \mathrm{C} & 1.71250 & -2.60108 & -0.67261 \\ \mathrm{H} & 1.49593 & -3.33331 & -1.45548 \\ \mathrm{H} & 1.93821 & -3.16263 & 0.23737 \\ \mathrm{H} & 2.95266 & -1.52998 & -2.15459 \\ \mathrm{C} & 0.42650 & -1.79445 & -0.41274 \\ \mathrm{O} & 0.70689 & -0.83368 & 0.60010 \\ \mathrm{H} & 0.13103 & -1.27835 & -1.33146 \\ \mathrm{C} & -2.70976 & -4.47696 & 0.81173 \\ \mathrm{C} & -1.97740 & -3.78312 & 1.76974 \\ \mathrm{C} & -0.97344 & -2.90013 & 1.38285 \\ \mathrm{C} & -0.69671 & -2.70080 & 0.03184 \\ \mathrm{C} & -1.43537 & -3.39681 & -0.92513 \\ \mathrm{C} & -2.43583 & -4.28173 & -0.53921 \\ \mathrm{H} & -3.49282 & -5.16157 & 1.11512 \\ \mathrm{H} & -2.18913 & -3.92779 & 2.82281 \\ \mathrm{H} & -0.40766 & -2.35175 & 2.12600 \\ \mathrm{H} & -1.22927 & -3.23875 & -1.97946 \\ \mathrm{H} & -3.00596 & -4.81270 & -1.29247 \\ \mathrm{C} & 3.78834 & -1.19918 & -0.27721 \\ \mathrm{C} & 3.79291 & -1.33998 & 1.22089 \\ \mathrm{C} & 4.94096 & -0.39761 & -0.82263 \\ \mathrm{H} & 4.77228 & -1.68360 & 1.56742 \\ \mathrm{H} & 3.02582 & -2.01639 & 1.59147 \\ \mathrm{H} & 5.02227 & 0.56765 & -0.31167 \\ \mathrm{H} & 4.84437 & -0.22092 & -1.89491 \\ \mathrm{H} & 3.61189 & -0.35842 & 1.67142 \\ \mathrm{H} & 5.88248 & -0.92607 & -0.64113 \\ & \mathrm{G}=-952.32701 \mathrm{Hartree} \\ & & & \end{array}$

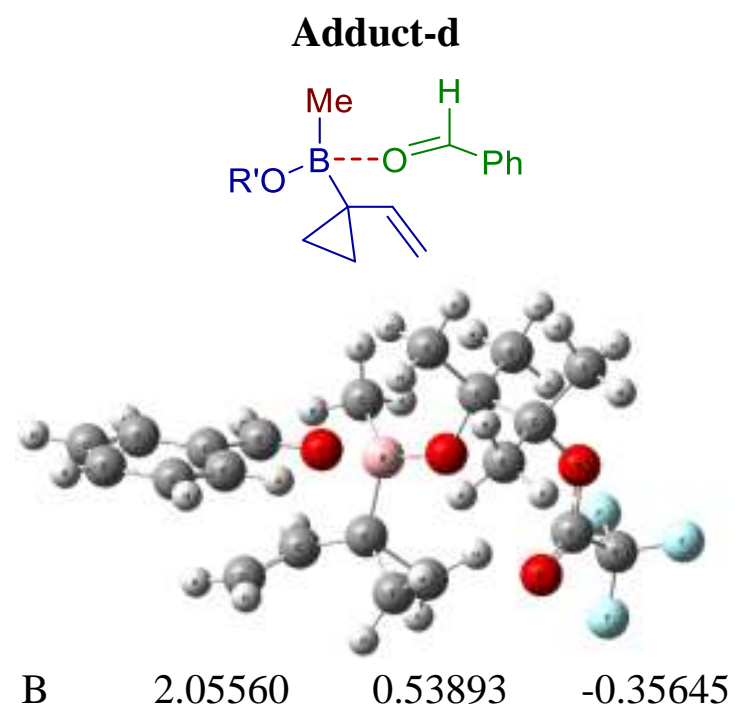




\begin{tabular}{|c|c|c|c|}
\hline $\mathrm{C}$ & 1.18007 & 3.59636 & 1.60823 \\
\hline $\mathrm{C}$ & 1.13153 & 2.83022 & 0.24716 \\
\hline $\mathrm{C}$ & 1.68828 & 3.70524 & -0.87983 \\
\hline $\mathrm{H}$ & 1.69145 & 3.14790 & -1.81412 \\
\hline $\mathrm{H}$ & 1.07285 & 4.59443 & -1.02696 \\
\hline $\mathrm{H}$ & 2.70996 & 4.01190 & -0.65194 \\
\hline $\mathrm{C}$ & -0.31199 & 2.42203 & -0.06543 \\
\hline $\mathrm{H}$ & -0.70241 & 1.73609 & 0.68586 \\
\hline $\mathrm{H}$ & -0.96065 & 3.29771 & -0.12427 \\
\hline $\mathrm{H}$ & -0.35079 & 1.91763 & -1.03112 \\
\hline $\mathrm{C}$ & 0.59054 & 2.77991 & 2.75323 \\
\hline $\mathrm{H}$ & -0.49744 & 2.84430 & 2.68855 \\
\hline $\mathrm{H}$ & 0.89601 & 1.73705 & 2.70362 \\
\hline $\mathrm{H}$ & 0.89660 & 3.19856 & 3.71208 \\
\hline $\mathrm{C}$ & 0.53949 & 4.97838 & 1.57441 \\
\hline $\mathrm{H}$ & -0.50704 & 4.91401 & 1.27650 \\
\hline $\mathrm{H}$ & 0.58287 & 5.41238 & 2.57503 \\
\hline $\mathrm{H}$ & 1.06749 & 5.64209 & 0.89127 \\
\hline $\mathrm{O}$ & 1.96458 & 1.69959 & 0.45718 \\
\hline $\mathrm{C}$ & 3.29396 & -1.77742 & -0.48691 \\
\hline $\mathrm{C}$ & 2.84976 & -2.95419 & -0.04408 \\
\hline $\mathrm{H}$ & 2.94874 & -3.85948 & -0.63278 \\
\hline $\mathrm{H}$ & 2.38507 & -3.04526 & 0.93395 \\
\hline $\mathrm{H}$ & 3.75199 & -1.73218 & -1.47496 \\
\hline $\mathrm{C}$ & 0.21218 & -1.34027 & -0.52979 \\
\hline $\mathrm{O}$ & 0.61472 & -0.36546 & 0.0983 \\
\hline $\mathrm{H}$ & 0.59147 & -1.53381 & -1.53875 \\
\hline $\mathrm{C}$ & -2.66528 & -4.04734 & 0.9752 \\
\hline $\mathrm{C}$ & -2.26231 & -2.96874 & 1.76466 \\
\hline $\mathrm{C}$ & -1.31939 & -2.07530 & 1.2841 \\
\hline $\mathrm{C}$ & -0.78311 & -2.26370 & $0.0052^{\prime}$ \\
\hline $\mathrm{C}$ & -1.18573 & -3.34413 & -0.7830 \\
\hline $\mathrm{C}$ & -2.12960 & -4.23803 & -0.2953 \\
\hline $\mathrm{H}$ & -3.40150 & -4.74464 & 1.3572 \\
\hline $\mathrm{H}$ & -2.68572 & -2.83348 & 2.7520 \\
\hline $\mathrm{H}$ & -0.98815 & -1.23271 & 1.8796 \\
\hline $\mathrm{H}$ & -0.75624 & -3.47984 & -1.7700 \\
\hline $\mathrm{H}$ & -2.44522 & -5.07934 & -0.8991 \\
\hline $\mathrm{C}$ & 3.15718 & -0.48489 & 0.2342 \\
\hline $\mathrm{C}$ & 3.39911 & -0.47621 & 1.7196 \\
\hline $\mathrm{C}$ & 4.41694 & 0.13380 & 0.7971 \\
\hline $\mathrm{H}$ & 3.66994 & -1.41327 & 2.1917 \\
\hline $\mathrm{H}$ & 2.80903 & 0.20091 & 2.3223 \\
\hline $\mathrm{H}$ & 4.49087 & 1.21327 & 0.7583 \\
\hline $\mathrm{H}$ & 5.35002 & -0.40007 & 0.6578 \\
\hline $\mathrm{C}$ & 2.01191 & 0.63748 & -1.9620 \\
\hline
\end{tabular}




$\begin{array}{llrr}\mathrm{H} & 2.86684 & 1.23865 & -2.29016 \\ \mathrm{H} & 2.12988 & -0.33674 & -2.44527 \\ \mathrm{H} & 1.11334 & 1.09869 & -2.38075 \\ \mathrm{O} & 2.60539 & 3.91034 & 1.80379 \\ \mathrm{C} & 3.46734 & 3.17234 & 2.46095 \\ \mathrm{C} & 4.88240 & 3.67715 & 2.10009 \\ \mathrm{O} & 3.31082 & 2.29740 & 3.25690 \\ \mathrm{~F} & 5.05933 & 4.93213 & 2.52326 \\ \mathrm{~F} & 5.07610 & 3.66600 & 0.77640 \\ \mathrm{~F} & 5.81099 & 2.91303 & 2.66010 \\ & \mathrm{G}=-1441.816575 \text { Hartree }\end{array}$

TS-d

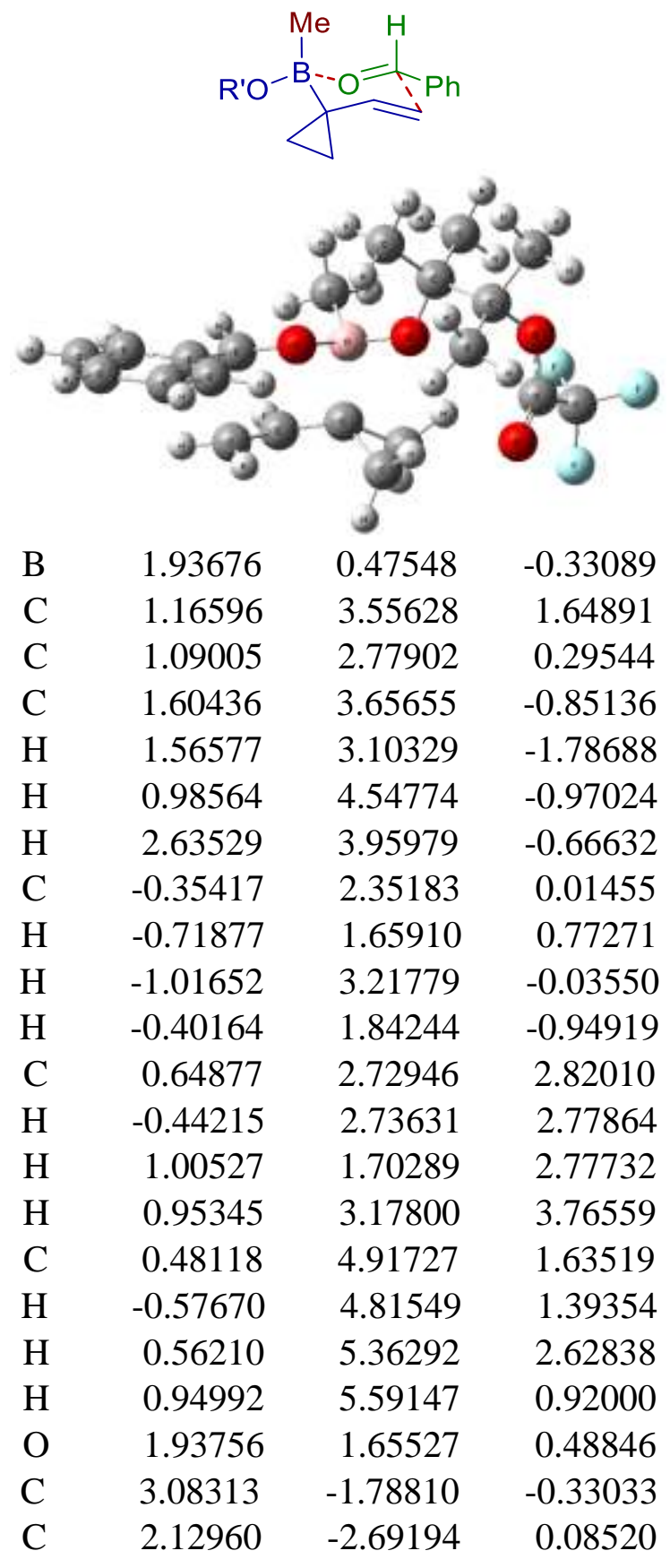




$\begin{array}{lrrr}\mathrm{H} & 1.97995 & -3.62043 & -0.45404 \\ \mathrm{H} & 1.81489 & -2.67700 & 1.12346 \\ \mathrm{H} & 3.53687 & -1.92806 & -1.31087 \\ \mathrm{C} & 0.55374 & -1.52452 & -0.53114 \\ \mathrm{O} & 0.74540 & -0.38446 & 0.04128 \\ \mathrm{H} & 0.75387 & -1.60492 & -1.59989 \\ \mathrm{C} & -2.67210 & -3.94824 & 0.84968 \\ \mathrm{C} & -2.20481 & -2.90297 & 1.64356 \\ \mathrm{C} & -1.15079 & -2.11062 & 1.20692 \\ \mathrm{C} & -0.56312 & -2.36366 & -0.03333 \\ \mathrm{C} & -1.03307 & -3.40871 & -0.82847 \\ \mathrm{C} & -2.08557 & -4.20066 & -0.38726 \\ \mathrm{H} & -3.49395 & -4.56423 & 1.19432 \\ \mathrm{H} & -2.66493 & -2.70640 & 2.60448 \\ \mathrm{H} & -0.77846 & -1.29345 & 1.81365 \\ \mathrm{H} & -0.57305 & -3.59843 & -1.79306 \\ \mathrm{H} & -2.44979 & -5.01006 & -1.00796 \\ \mathrm{C} & 3.22315 & -0.53012 & 0.31422 \\ \mathrm{C} & 3.56194 & -0.34306 & 1.75226 \\ \mathrm{C} & 4.48201 & 0.18983 & 0.66595 \\ \mathrm{H} & 3.90709 & -1.21164 & 2.30232 \\ \mathrm{H} & 3.00175 & 0.38580 & 2.31975 \\ \mathrm{H} & 4.48961 & 1.26158 & 0.50860 \\ \mathrm{H} & 5.43100 & -0.31124 & 0.51640 \\ \mathrm{C} & 2.11120 & 0.63515 & -1.92923 \\ \mathrm{H} & 2.96336 & 1.28521 & -2.14822 \\ \mathrm{H} & 2.30789 & -0.31463 & -2.43507 \\ \mathrm{H} & 1.23235 & 1.07228 & -2.41562 \\ \mathrm{O} & 2.59044 & 3.91550 & 1.77821 \\ \mathrm{C} & 3.50001 & 3.23919 & 2.43368 \\ \mathrm{C} & 4.88551 & 3.74648 & 1.97481 \\ \mathrm{O} & 3.40352 & 2.41451 & 3.29175 \\ \mathrm{~F} & 5.07678 & 5.00960 & 2.36678 \\ \mathrm{~F} & 4.99944 & 3.71489 & 0.64273 \\ & 5.85308 & 2.99855 & 2.48944 \\ & \mathrm{G}=-1441.800286 \mathrm{H} \\ \mathrm{F} & & & \end{array}$




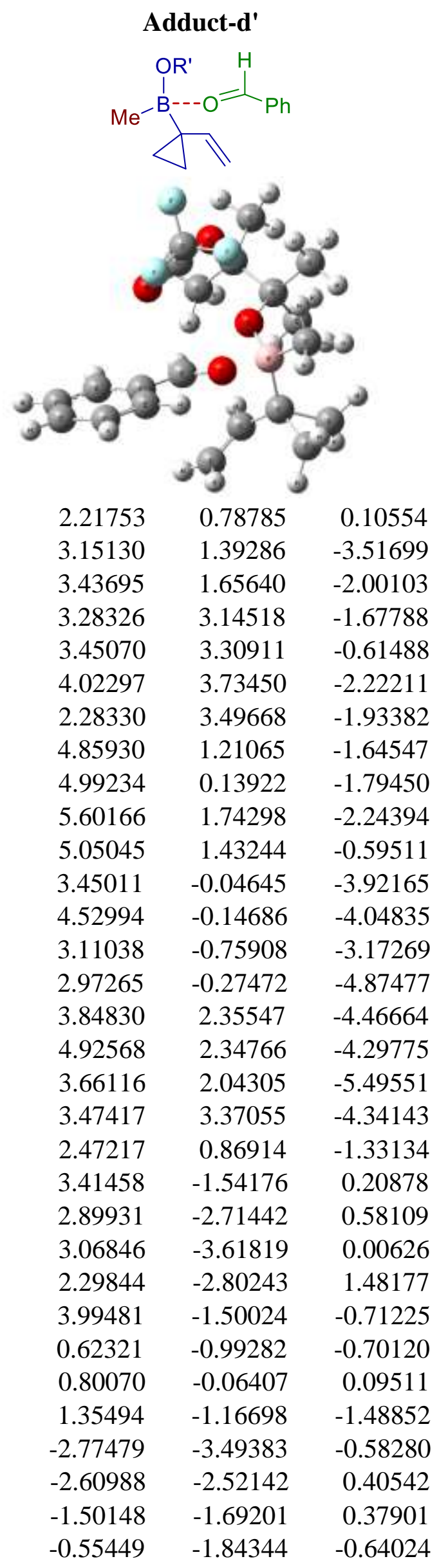




\begin{tabular}{lrrr}
$\mathrm{C}$ & -0.72222 & -2.81338 & -1.63179 \\
$\mathrm{C}$ & -1.83668 & -3.64045 & -1.60106 \\
$\mathrm{H}$ & -3.64490 & -4.13923 & -0.55856 \\
$\mathrm{H}$ & -3.34891 & -2.41572 & 1.18969 \\
$\mathrm{H}$ & -1.35238 & -0.92961 & 1.13398 \\
$\mathrm{H}$ & 0.01517 & -2.90293 & -2.42181 \\
$\mathrm{H}$ & -1.97685 & -4.39395 & -2.36571 \\
$\mathrm{C}$ & 3.19364 & -0.24446 & 0.90128 \\
$\mathrm{C}$ & 3.17032 & -0.26129 & 2.40911 \\
$\mathrm{C}$ & 4.35782 & 0.31337 & 1.68997 \\
$\mathrm{H}$ & 3.31169 & -1.21197 & 2.90930 \\
$\mathrm{H}$ & 2.50781 & 0.42617 & 2.91925 \\
$\mathrm{H}$ & 4.49775 & 1.38807 & 1.70834 \\
$\mathrm{H}$ & 5.27497 & -0.26481 & 1.70568 \\
$\mathrm{C}$ & 1.80331 & 2.11895 & 0.90681 \\
$\mathrm{O}$ & 1.72640 & 1.74069 & -3.67917 \\
$\mathrm{C}$ & 0.75047 & 0.91936 & -3.37139 \\
$\mathrm{C}$ & -0.45658 & 1.73609 & -2.86363 \\
$\mathrm{O}$ & 0.66949 & -0.26821 & -3.49398 \\
$\mathrm{~F}$ & -0.97389 & 2.47366 & -3.85042 \\
$\mathrm{~F}$ & -0.10566 & 2.56152 & -1.87732 \\
$\mathrm{~F}$ & -1.40454 & 0.92393 & -2.40606 \\
$\mathrm{H}$ & 1.23929 & 2.80671 & 0.27183 \\
$\mathrm{H}$ & 1.16243 & 1.85570 & 1.75376 \\
$\mathrm{H}$ & 2.65649 & 2.66888 & 1.31533 \\
& $\mathrm{G}=-1441.816253 \mathrm{Hartree}$ \\
\hline & & &
\end{tabular}

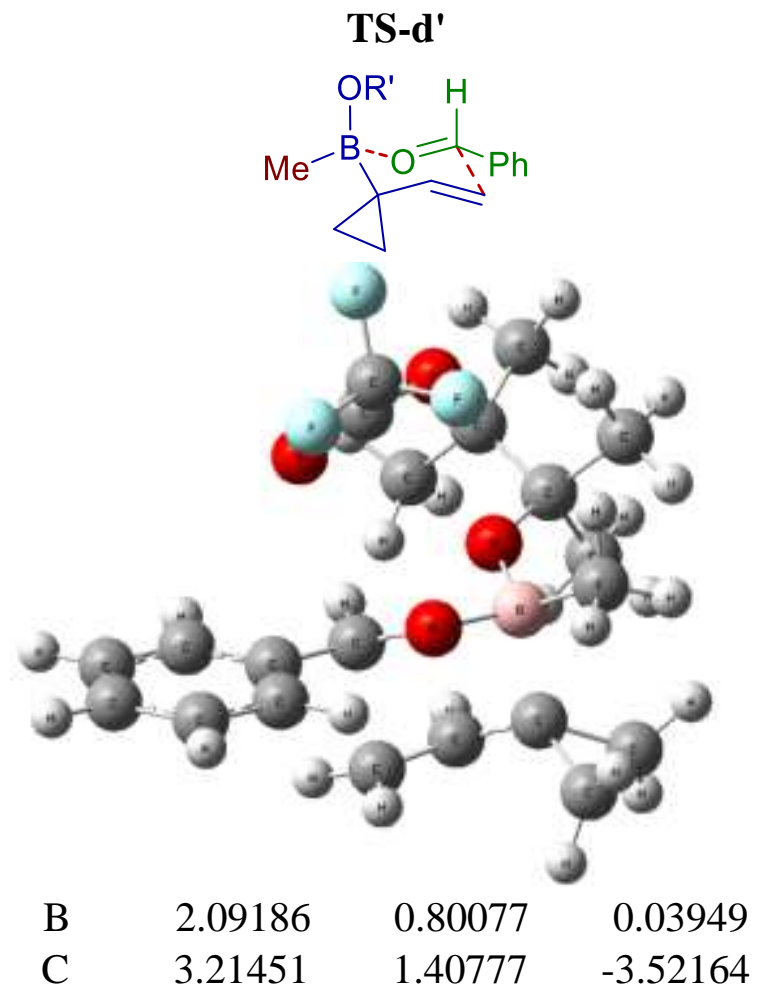




\begin{tabular}{|c|c|c|c|}
\hline $\mathrm{C}$ & 3.45588 & 1.67464 & -1.99737 \\
\hline $\mathrm{C}$ & 3.28797 & 3.16321 & -1.68440 \\
\hline $\mathrm{H}$ & 3.41704 & 3.33349 & -0.61740 \\
\hline $\mathrm{H}$ & 4.04760 & 3.74948 & -2.20434 \\
\hline $\mathrm{H}$ & 2.29846 & 3.51370 & -1.97979 \\
\hline $\mathrm{C}$ & 4.86816 & 1.23123 & -1.60104 \\
\hline $\mathrm{H}$ & 4.97705 & 0.14812 & -1.66455 \\
\hline $\mathrm{H}$ & 5.62422 & 1.69680 & -2.23698 \\
\hline $\mathrm{H}$ & 5.06488 & 1.53218 & -0.57242 \\
\hline $\mathrm{C}$ & 3.54939 & -0.02719 & -3.91369 \\
\hline $\mathrm{H}$ & 4.63480 & -0.11266 & -3.99728 \\
\hline $\mathrm{H}$ & 3.18995 & -0.74391 & -3.17752 \\
\hline $\mathrm{H}$ & 3.11194 & -0.26370 & -4.88359 \\
\hline $\mathrm{C}$ & 3.92012 & 2.38086 & -4.45416 \\
\hline $\mathrm{H}$ & 4.99295 & 2.39334 & -4.25851 \\
\hline $\mathrm{H}$ & 3.76485 & 2.06373 & -5.48683 \\
\hline $\mathrm{H}$ & 3.52468 & 3.38925 & -4.34032 \\
\hline $\mathrm{O}$ & 2.47641 & 0.87853 & -1.36204 \\
\hline $\mathrm{C}$ & 3.17735 & -1.46685 & 0.18820 \\
\hline $\mathrm{C}$ & 2.15944 & -2.38558 & 0.29348 \\
\hline $\mathrm{H}$ & 2.14598 & -3.26558 & -0.33952 \\
\hline $\mathrm{H}$ & 1.58791 & -2.43748 & 1.21450 \\
\hline $\mathrm{H}$ & 3.84252 & -1.53584 & -0.67142 \\
\hline $\mathrm{C}$ & 0.81046 & -1.13225 & -0.64305 \\
\hline $\mathrm{O}$ & 0.85217 & -0.05068 & 0.06485 \\
\hline $\mathrm{H}$ & 1.30874 & -1.12769 & -1.60742 \\
\hline $\mathrm{C}$ & -2.69150 & -3.54850 & -0.4515 \\
\hline $\mathrm{C}$ & -2.39534 & -2.68394 & 0.6011 \\
\hline $\mathrm{C}$ & -1.25503 & -1.89380 & 0.55446 \\
\hline $\mathrm{C}$ & -0.40533 & -1.97012 & -0.5502 \\
\hline $\mathrm{C}$ & -0.70620 & -2.82857 & -1.6072 \\
\hline $\mathrm{C}$ & -1.84824 & -3.61854 & -1.5565 \\
\hline $\mathrm{H}$ & -3.58293 & -4.16318 & $-0.4121 \mathrm{~S}$ \\
\hline $\mathrm{H}$ & -3.05718 & -2.62668 & 1.45684 \\
\hline $\mathrm{H}$ & -1.01559 & -1.21262 & 1.36257 \\
\hline $\mathrm{H}$ & -0.05131 & -2.85925 & -2.47155 \\
\hline $\mathrm{H}$ & -2.08446 & -4.28132 & -2.38003 \\
\hline $\mathrm{C}$ & 3.17698 & -0.25700 & 0.94090 \\
\hline $\mathrm{C}$ & 3.16557 & -0.20611 & 2.43439 \\
\hline $\mathrm{C}$ & 4.35361 & 0.32446 & 1.65740 \\
\hline $\mathrm{H}$ & 3.29977 & -1.13684 & $2.9738^{\circ}$ \\
\hline $\mathrm{H}$ & 2.53074 & 0.52138 & 2.9239 \\
\hline $\mathrm{H}$ & 4.49230 & 1.39893 & 1.63757 \\
\hline $\mathrm{H}$ & 5.27161 & -0.25094 & 1.68631 \\
\hline $\mathrm{C}$ & 1.77888 & 2.13247 & 0.8839 \\
\hline $\mathrm{O}$ & 1.79044 & 1.73110 & -3.7177 \\
\hline
\end{tabular}




\begin{tabular}{lrrr} 
C & 0.82347 & 0.88296 & -3.44399 \\
C & -0.39760 & 1.66050 & -2.91067 \\
O & 0.76150 & -0.29725 & -3.62610 \\
F & -0.93999 & 2.39585 & -3.88865 \\
F & -0.06122 & 2.48752 & -1.92150 \\
F & -1.32239 & 0.82193 & -2.45831 \\
H & 1.25447 & 2.86249 & 0.26183 \\
H & 1.12024 & 1.88225 & 1.72023 \\
H & 2.65997 & 2.62722 & 1.30131 \\
\multicolumn{4}{c}{$\mathrm{G}=-1441.80117$ Hartree }
\end{tabular}

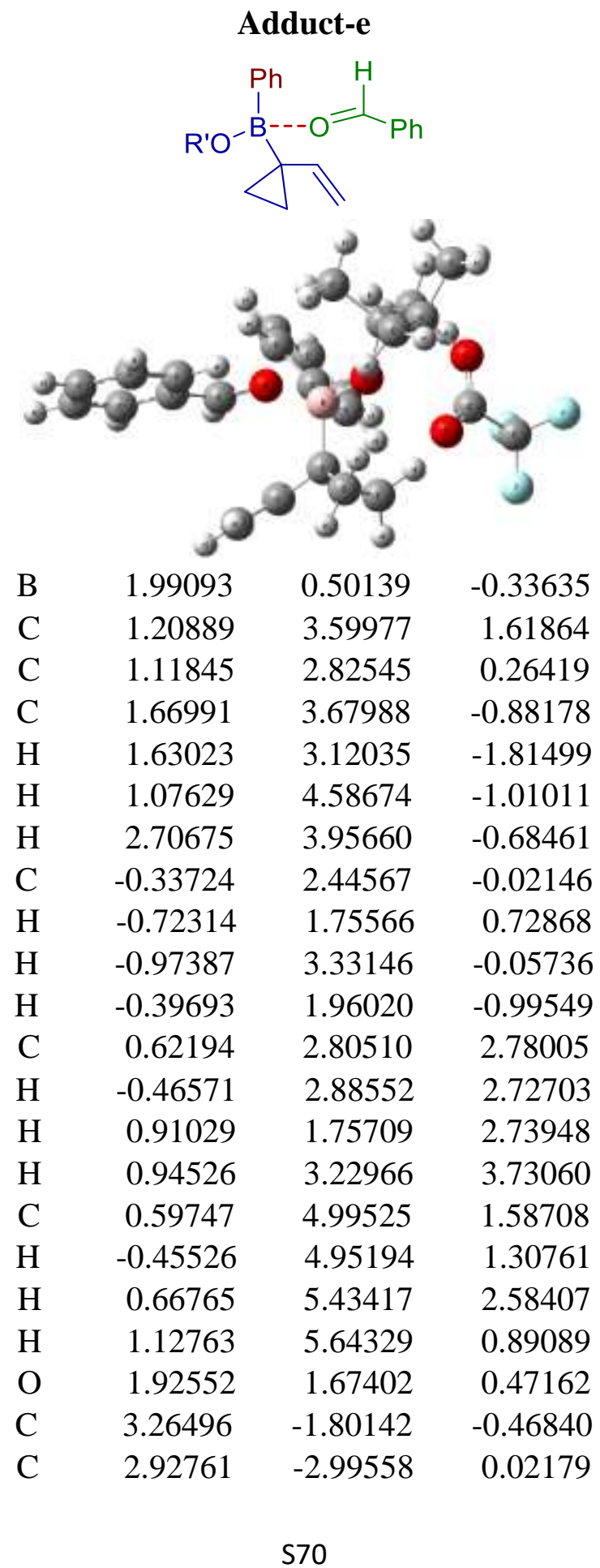




\begin{tabular}{|c|c|c|c|}
\hline $\mathrm{H}$ & 3.03267 & -3.90096 & -0.56571 \\
\hline $\mathrm{H}$ & 2.54417 & -3.10065 & 1.03258 \\
\hline $\mathrm{H}$ & 3.63157 & -1.74115 & -1.49341 \\
\hline $\mathrm{C}$ & 0.27741 & -1.41327 & -0.52399 \\
\hline $\mathrm{O}$ & 0.59373 & -0.36642 & 0.04752 \\
\hline $\mathrm{H}$ & 0.76548 & -1.68521 & -1.46419 \\
\hline $\mathrm{C}$ & -2.71165 & -4.01743 & 0.92747 \\
\hline $\mathrm{C}$ & -2.39513 & -2.86867 & 1.65535 \\
\hline $\mathrm{C}$ & -1.41455 & -2.00731 & 1.19355 \\
\hline $\mathrm{C}$ & -0.75205 & -2.29996 & -0.00461 \\
\hline $\mathrm{C}$ & -1.06800 & -3.45154 & -0.73120 \\
\hline $\mathrm{C}$ & -2.05135 & -4.31130 & -0.26232 \\
\hline $\mathrm{H}$ & -3.47873 & -4.68887 & 1.29455 \\
\hline $\mathrm{H}$ & -2.91527 & -2.65394 & 2.58024 \\
\hline $\mathrm{H}$ & -1.14888 & -1.11168 & 1.74248 \\
\hline $\mathrm{H}$ & -0.54132 & -3.66665 & -1.65478 \\
\hline $\mathrm{H}$ & -2.30256 & -5.20562 & -0.81808 \\
\hline $\mathrm{C}$ & 3.11705 & -0.50722 & 0.24530 \\
\hline $\mathrm{C}$ & 3.36883 & -0.48526 & 1.72890 \\
\hline $\mathrm{C}$ & 4.37530 & 0.12689 & 0.79649 \\
\hline $\mathrm{H}$ & 3.65203 & -1.41425 & 2.20903 \\
\hline $\mathrm{H}$ & 2.77805 & 0.19277 & 2.32988 \\
\hline $\mathrm{H}$ & 4.43723 & 1.20652 & 0.74936 \\
\hline $\mathrm{H}$ & 5.31182 & -0.39967 & 0.65289 \\
\hline $\mathrm{O}$ & 2.64418 & 3.88461 & 1.78493 \\
\hline $\mathrm{C}$ & 3.50185 & 3.14376 & 2.44321 \\
\hline $\mathrm{C}$ & 4.92029 & 3.62028 & 2.05636 \\
\hline $\mathrm{O}$ & 3.34172 & 2.28497 & 3.25626 \\
\hline $\mathrm{F}$ & 5.13005 & 4.86917 & 2.48249 \\
\hline $\mathrm{F}$ & 5.08967 & 3.61192 & 0.72952 \\
\hline $\mathrm{F}$ & 5.84391 & 2.83504 & 2.59560 \\
\hline $\mathrm{C}$ & 2.02572 & 0.66246 & -1.94725 \\
\hline $\mathrm{C}$ & 0.93539 & 0.59165 & -2.82221 \\
\hline $\mathrm{C}$ & 3.27302 & 0.96010 & -2.51800 \\
\hline $\mathrm{C}$ & 1.07113 & 0.82329 & -4.19045 \\
\hline $\mathrm{H}$ & -0.05751 & 0.36389 & -2.44394 \\
\hline $\mathrm{C}$ & 3.42235 & 1.19851 & -3.87963 \\
\hline $\mathrm{H}$ & 4.14487 & 1.01678 & -1.87194 \\
\hline $\mathrm{C}$ & 2.31628 & 1.13375 & -4.72398 \\
\hline $\mathrm{H}$ & 0.20248 & 0.76558 & -4.83696 \\
\hline $\mathrm{H}$ & 4.40008 & 1.43462 & -4.28480 \\
\hline $\mathrm{H}$ & 2.42618 & 1.31923 & -5.78616 \\
\hline \multicolumn{4}{|c|}{$\mathrm{G}=-1633.47554$ Hartree } \\
\hline
\end{tabular}

TS-e 


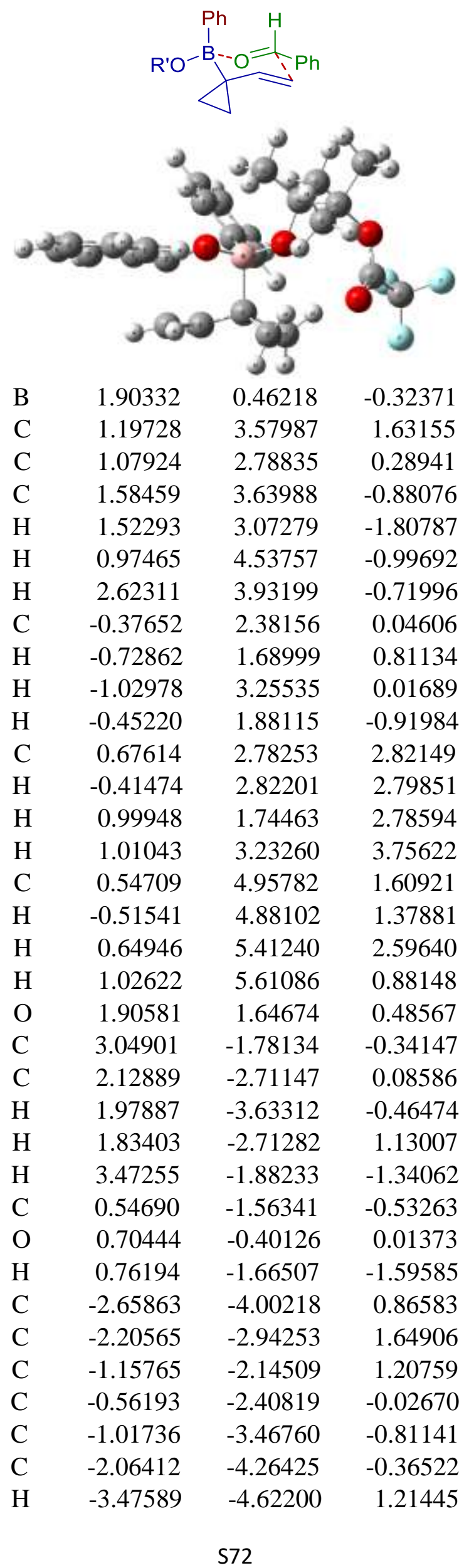




\begin{tabular}{lrrr}
$\mathrm{H}$ & -2.67235 & -2.73884 & 2.60526 \\
$\mathrm{H}$ & -0.79678 & -1.31636 & 1.80553 \\
$\mathrm{H}$ & -0.55104 & -3.66420 & -1.77158 \\
$\mathrm{H}$ & -2.41744 & -5.08488 & -0.97740 \\
$\mathrm{C}$ & 3.17206 & -0.53034 & 0.32560 \\
$\mathrm{C}$ & 3.47207 & -0.38395 & 1.77807 \\
$\mathrm{C}$ & 4.42779 & 0.16839 & 0.73813 \\
$\mathrm{H}$ & 3.79066 & -1.27000 & 2.31609 \\
$\mathrm{H}$ & 2.90437 & 0.33895 & 2.34621 \\
$\mathrm{H}$ & 4.44909 & 1.24333 & 0.61121 \\
$\mathrm{H}$ & 5.37557 & -0.33735 & 0.59729 \\
$\mathrm{O}$ & 2.63144 & 3.90560 & 1.73650 \\
$\mathrm{C}$ & 3.53481 & 3.21438 & 2.38462 \\
$\mathrm{C}$ & 4.92389 & 3.69665 & 1.90864 \\
$\mathrm{O}$ & 3.43294 & 2.39217 & 3.24445 \\
$\mathrm{~F}$ & 5.14050 & 4.95783 & 2.29150 \\
$\mathrm{~F}$ & 5.02254 & 3.65479 & 0.57533 \\
$\mathrm{~F}$ & 5.88462 & 2.93485 & 2.41663 \\
$\mathrm{C}$ & 2.07674 & 0.64999 & -1.92509 \\
$\mathrm{C}$ & 1.00607 & 0.57570 & -2.82542 \\
$\mathrm{C}$ & 3.33111 & 0.97346 & -2.46042 \\
$\mathrm{C}$ & 1.16812 & 0.82573 & -4.18680 \\
$\mathrm{H}$ & 0.01178 & 0.32854 & -2.46235 \\
$\mathrm{C}$ & 3.50733 & 1.22668 & -3.81720 \\
$\mathrm{H}$ & 4.18809 & 1.04153 & -1.79708 \\
$\mathrm{C}$ & 2.42245 & 1.15668 & -4.68761 \\
$\mathrm{H}$ & 0.31544 & 0.76566 & -4.85410 \\
$\mathrm{H}$ & 4.49028 & 1.48195 & -4.19747 \\
$\mathrm{H}$ & 2.55506 & 1.35539 & -5.74485 \\
& $\mathrm{G}=-1633.460591 \mathrm{Hartree}$ \\
\hline & & &
\end{tabular}

Product-e<smiles>[R10][Ba][Ba]C(CC=C1CC1)c1ccccc1</smiles> 


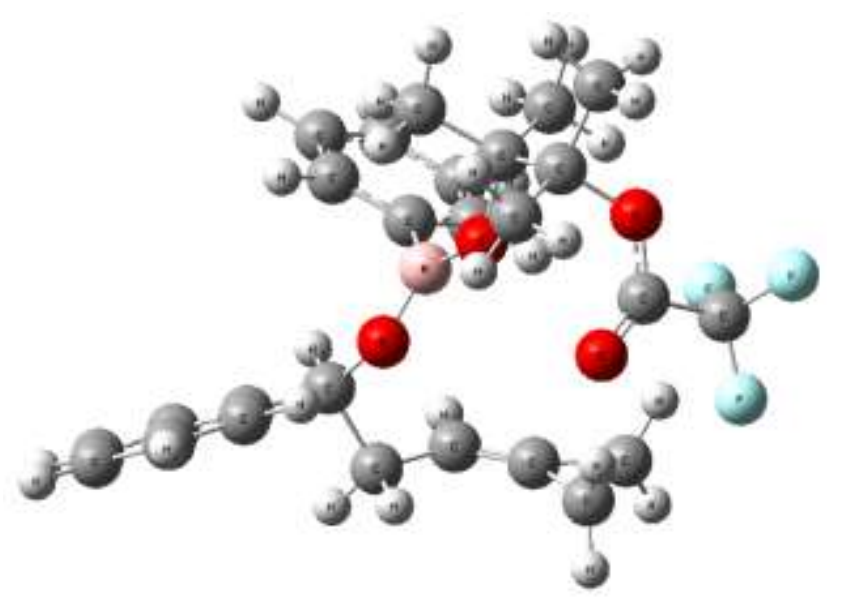

$\begin{array}{lrrr}\text { B } & 1.19423 & 0.74183 & -0.38777 \\ \mathrm{C} & 0.94851 & 3.87715 & 1.55317 \\ \mathrm{C} & 0.81880 & 3.17602 & 0.16777 \\ \mathrm{C} & 1.70553 & 3.84351 & -0.88154 \\ \mathrm{H} & 1.57860 & 3.35208 & -1.84479 \\ \mathrm{H} & 1.42419 & 4.88982 & -1.00515 \\ \mathrm{H} & 2.75591 & 3.78897 & -0.59295 \\ \mathrm{C} & -0.63616 & 3.15088 & -0.30011 \\ \mathrm{H} & -1.26285 & 2.59815 & 0.40080 \\ \mathrm{H} & -1.03494 & 4.15884 & -0.42156 \\ \mathrm{H} & -0.68505 & 2.65652 & -1.27292 \\ \mathrm{C} & 0.08047 & 3.23168 & 2.62790 \\ \mathrm{H} & -0.94596 & 3.57778 & 2.49259 \\ \mathrm{H} & 0.11082 & 2.14662 & 2.58129 \\ \mathrm{H} & 0.41551 & 3.55740 & 3.61371 \\ \mathrm{C} & 0.68890 & 5.37749 & 1.51214 \\ \mathrm{H} & -0.31099 & 5.58573 & 1.13068 \\ \mathrm{H} & 0.75534 & 5.77337 & 2.52670 \\ \mathrm{H} & 1.42443 & 5.89259 & 0.89626 \\ \mathrm{O} & 1.23815 & 1.83328 & 0.43783 \\ \mathrm{C} & 3.47397 & -1.72730 & -0.30139 \\ \mathrm{C} & 2.22530 & -2.42674 & 0.15144 \\ \mathrm{H} & 2.19860 & -3.45013 & -0.23699 \\ \mathrm{H} & 2.19349 & -2.47761 & 1.24348 \\ \mathrm{H} & 3.66932 & -1.69948 & -1.37199 \\ \mathrm{C} & 0.95849 & -1.70782 & -0.33424 \\ \mathrm{O} & 0.91069 & -0.42079 & 0.26244 \\ \mathrm{H} & 1.00237 & -1.61508 & -1.42425 \\ \mathrm{C} & -2.51694 & -4.01286 & 0.74341 \\ \mathrm{C} & -2.11222 & -2.95242 & 1.54754 \\ \mathrm{C} & -1.00027 & -2.19069 & 1.19905 \\ \mathrm{C} & -0.28496 & -2.48321 & 0.03940 \\ \mathrm{C} & -0.69627 & -3.54613 & -0.76508 \\ \mathrm{C} & -1.80515 & -4.30888 & -0.41596 \\ \mathrm{H} & -3.38371 & -4.60345 & 1.01564\end{array}$




$\begin{array}{lrrr}\mathrm{H} & -2.66377 & -2.71538 & 2.44993 \\ \mathrm{H} & -0.68571 & -1.36094 & 1.82024 \\ \mathrm{H} & -0.14709 & -3.77468 & -1.67359 \\ \mathrm{H} & -2.11702 & -5.12982 & -1.05086 \\ \mathrm{C} & 4.31419 & -1.14133 & 0.52999 \\ \mathrm{C} & 4.64705 & -0.83002 & 1.92174 \\ \mathrm{C} & 5.55616 & -0.40207 & 0.76185 \\ \mathrm{H} & 5.00006 & -1.63271 & 2.55996 \\ \mathrm{H} & 4.08092 & -0.05650 & 2.42902 \\ \mathrm{H} & 5.59072 & 0.65299 & 0.51753 \\ \mathrm{H} & 6.49665 & -0.92903 & 0.64546 \\ \mathrm{O} & 2.38254 & 3.80878 & 1.87797 \\ \mathrm{C} & 2.98267 & 2.81632 & 2.49406 \\ \mathrm{C} & 4.50281 & 3.02917 & 2.31950 \\ \mathrm{O} & 2.54878 & 1.88867 & 3.10508 \\ \mathrm{~F} & 4.87824 & 4.24970 & 2.69925 \\ \mathrm{~F} & 4.83199 & 2.88438 & 1.02692 \\ \mathrm{~F} & 5.19172 & 2.14119 & 3.02303 \\ \mathrm{C} & 1.45824 & 0.78921 & -1.94322 \\ \mathrm{C} & 0.43324 & 0.58228 & -2.87331 \\ \mathrm{C} & 2.75176 & 1.02394 & -2.42612 \\ \mathrm{C} & 0.68405 & 0.63434 & -4.24212 \\ \mathrm{H} & -0.57646 & 0.37773 & -2.52763 \\ \mathrm{C} & 3.01098 & 1.06332 & -3.79197 \\ \mathrm{H} & 3.56501 & 1.17873 & -1.72257 \\ \mathrm{C} & 1.97418 & 0.87614 & -4.70276 \\ \mathrm{H} & -0.12449 & 0.48194 & -4.94749 \\ \mathrm{H} & 4.01880 & 1.24392 & -4.14741 \\ \mathrm{H} & 2.17274 & 0.91361 & -5.76742 \\ & \mathrm{G}=-1633.513985 \mathrm{Hartr} \\ & & & \end{array}$




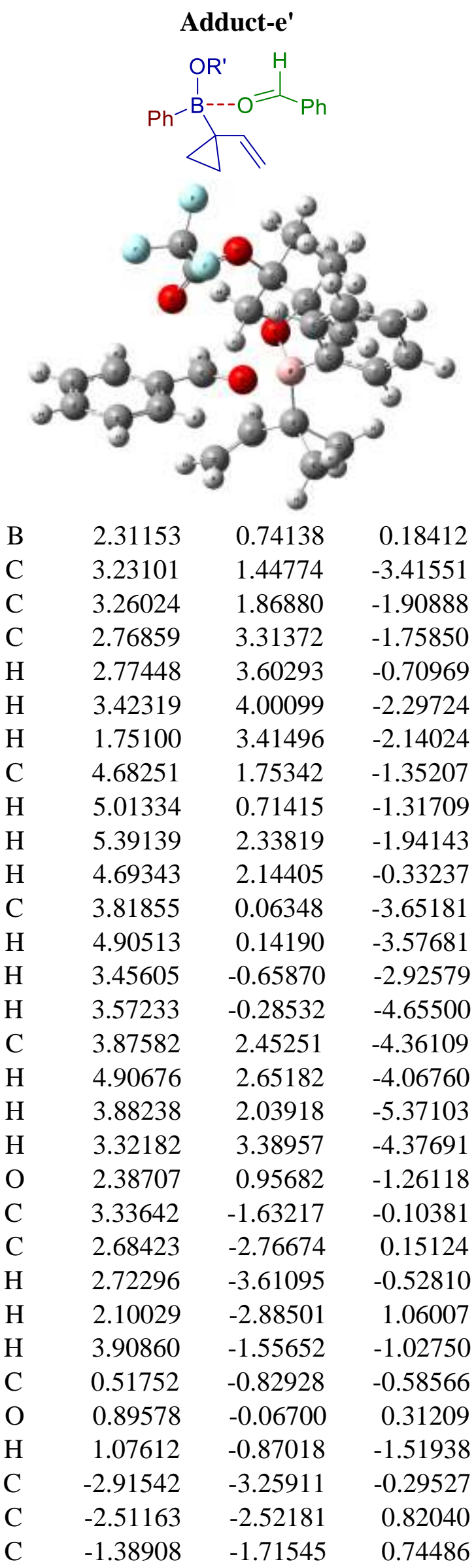




$\begin{array}{rrrr}\mathrm{C} & -0.66869 & -1.65598 & -0.45418 \\ \mathrm{C} & -1.07360 & -2.39194 & -1.57209 \\ \mathrm{C} & -2.20326 & -3.19454 & -1.48950 \\ \mathrm{H} & -3.79520 & -3.88849 & -0.23055 \\ \mathrm{H} & -3.07682 & -2.58151 & 1.74196 \\ \mathrm{H} & -1.05544 & -1.13291 & 1.59532 \\ \mathrm{H} & -0.50441 & -2.31956 & -2.49332 \\ \mathrm{H} & -2.52721 & -3.76797 & -2.34872 \\ \mathrm{C} & 3.32362 & -0.41147 & 0.75193 \\ \mathrm{C} & 3.45257 & -0.62810 & 2.24088 \\ \mathrm{C} & 4.64048 & -0.15518 & 1.45036 \\ \mathrm{H} & 3.47798 & -1.64463 & 2.61466 \\ \mathrm{H} & 2.96138 & 0.08518 & 2.89167 \\ \mathrm{H} & 5.01092 & 0.85195 & 1.57582 \\ \mathrm{H} & 5.42194 & -0.88613 & 1.27218 \\ \mathrm{O} & 1.80010 & 1.53193 & -3.78201 \\ \mathrm{C} & 0.94411 & 0.55168 & -3.69407 \\ \mathrm{C} & -0.47873 & 1.13117 & -3.53564 \\ \mathrm{O} & 1.10501 & -0.63603 & -3.70549 \\ \mathrm{~F} & -1.39297 & 0.22962 & -3.87473 \\ \mathrm{~F} & -0.67566 & 2.22905 & -4.25723 \\ \mathrm{~F} & -0.67101 & 1.44425 & -2.24476 \\ \mathrm{C} & 1.99989 & 2.06294 & 1.06780 \\ \mathrm{C} & 0.75612 & 2.68886 & 0.87890 \\ \mathrm{C} & 2.87957 & 2.68425 & 1.96067 \\ \mathrm{C} & 0.41372 & 3.86797 & 1.52947 \\ \mathrm{H} & 0.04452 & 2.25047 & 0.18570 \\ \mathrm{C} & 2.55127 & 3.86968 & 2.61868 \\ \mathrm{H} & 3.85324 & 2.25489 & 2.15835 \\ \mathrm{C} & 1.31656 & 4.46887 & 2.40409 \\ \mathrm{H} & -0.55498 & 4.32318 & 1.35379 \\ \mathrm{H} & 3.26335 & 4.32130 & 3.30063 \\ \mathrm{H} & 1.05760 & 5.38978 & 2.91379 \\ & \mathrm{G}=-1633.471275 \mathrm{Hartr} \\ & & & \end{array}$

TS-e'<smiles>[R7]OB(OC1(C2(c3ccccc3)C=CC2C=CC=O)CC1)c1ccccc1</smiles> 


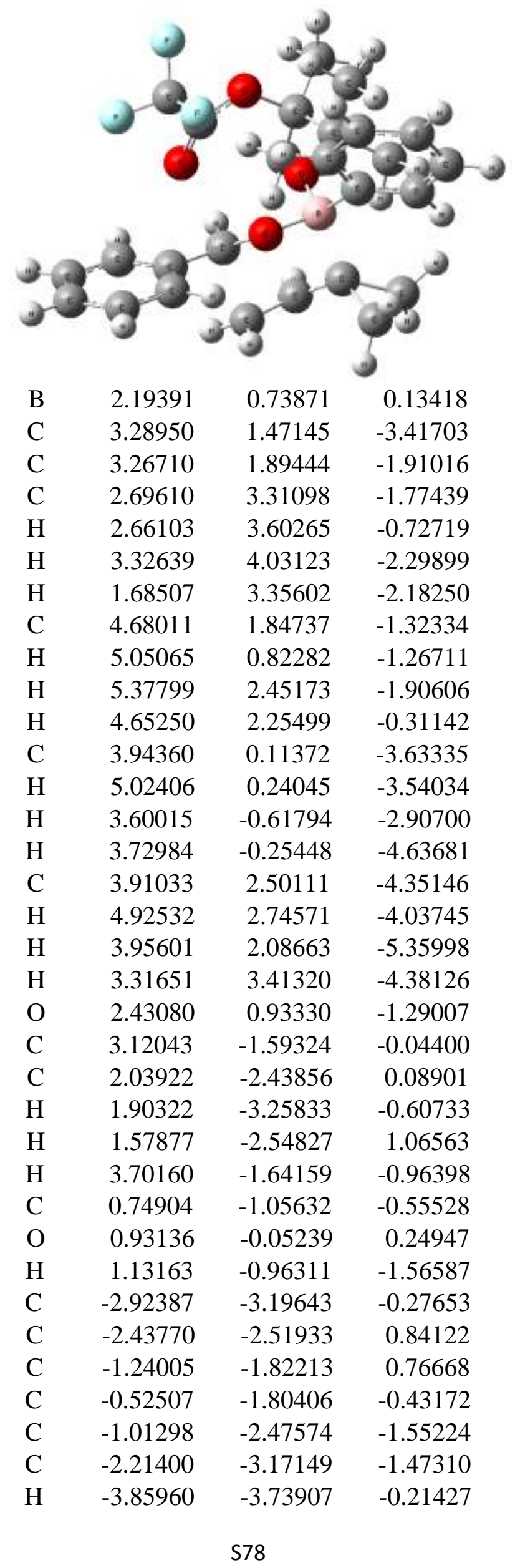




$\begin{array}{rrrr}\mathrm{H} & -2.99670 & -2.53525 & 1.76901 \\ \mathrm{H} & -0.85227 & -1.28451 & 1.62431 \\ \mathrm{H} & -0.45606 & -2.43525 & -2.48314 \\ \mathrm{H} & -2.59755 & -3.68831 & -2.34431 \\ \mathrm{C} & 3.31847 & -0.46160 & 0.80302 \\ \mathrm{C} & 3.49111 & -0.58111 & 2.28366 \\ \mathrm{C} & 4.66066 & -0.17555 & 1.40777 \\ \mathrm{H} & 3.51717 & -1.57154 & 2.72414 \\ \mathrm{H} & 3.04812 & 0.18838 & 2.90438 \\ \mathrm{H} & 5.03627 & 0.83507 & 1.46653 \\ \mathrm{H} & 5.43409 & -0.91949 & 1.25340 \\ \mathrm{O} & 1.86620 & 1.49046 & -3.81108 \\ \mathrm{C} & 1.05070 & 0.47294 & -3.72121 \\ \mathrm{C} & -0.39111 & 0.99911 & -3.55135 \\ \mathrm{O} & 1.26398 & -0.70529 & -3.75321 \\ \mathrm{~F} & -1.27410 & 0.04499 & -3.82517 \\ \mathrm{~F} & -0.64547 & 2.04659 & -4.33271 \\ \mathrm{~F} & -0.57150 & 1.37827 & -2.28062 \\ \mathrm{C} & 1.96809 & 2.05391 & 1.04990 \\ \mathrm{C} & 0.68830 & 2.63093 & 0.99773 \\ \mathrm{C} & 2.92071 & 2.71823 & 1.83052 \\ \mathrm{C} & 0.38341 & 3.81352 & 1.66260 \\ \mathrm{H} & -0.08112 & 2.14651 & 0.40599 \\ \mathrm{C} & 2.62739 & 3.90273 & 2.50491 \\ \mathrm{H} & 3.92545 & 2.32654 & 1.92378 \\ \mathrm{C} & 1.35718 & 4.46011 & 2.41888 \\ \mathrm{H} & -0.61391 & 4.23329 & 1.58880 \\ \mathrm{H} & 3.39503 & 4.38893 & 3.09665 \\ \mathrm{H} & 1.12688 & 5.38331 & 2.93804 \\ & \mathrm{G}=-1633.456641 \mathrm{H} \\ & & & \end{array}$

\section{Adduct-f}<smiles>[R]O[B-]([Se])(O/C(C=C)=C/c1ccccc1)C1(C=C)CC1</smiles>

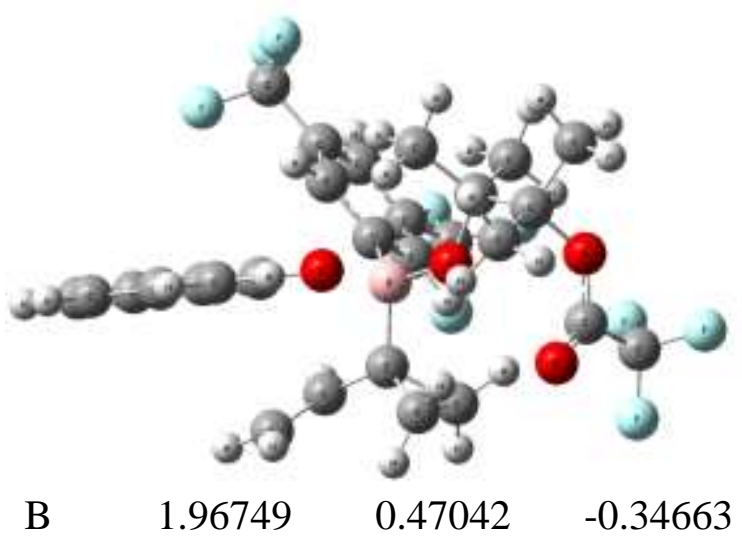




\begin{tabular}{|c|c|c|c|}
\hline $\mathrm{C}$ & 1.32046 & 3.56447 & 1.65509 \\
\hline $\mathrm{C}$ & 1.19945 & 2.82600 & 0.28305 \\
\hline $\mathrm{C}$ & 1.79136 & 3.68166 & -0.84151 \\
\hline $\mathrm{H}$ & 1.70675 & 3.16271 & -1.79504 \\
\hline $\mathrm{H}$ & 1.25290 & 4.62598 & -0.93535 \\
\hline $\mathrm{H}$ & 2.84495 & 3.88882 & -0.64774 \\
\hline $\mathrm{C}$ & -0.27045 & 2.51466 & -0.01269 \\
\hline $\mathrm{H}$ & -0.68216 & 1.81392 & 0.71334 \\
\hline $\mathrm{H}$ & -0.87076 & 3.42592 & -0.01245 \\
\hline $\mathrm{H}$ & -0.35738 & 2.07182 & -1.00526 \\
\hline $\mathrm{C}$ & 0.69171 & 2.76975 & 2.79430 \\
\hline $\mathrm{H}$ & -0.39093 & 2.89864 & 2.73556 \\
\hline $\mathrm{H}$ & 0.93516 & 1.71141 & 2.73276 \\
\hline $\mathrm{H}$ & 1.02503 & 3.15913 & 3.75624 \\
\hline $\mathrm{C}$ & 0.77340 & 4.98708 & 1.65524 \\
\hline $\mathrm{H}$ & -0.27723 & 5.00105 & 1.36426 \\
\hline $\mathrm{H}$ & 0.85223 & 5.39486 & 2.66459 \\
\hline $\mathrm{H}$ & 1.34114 & 5.62910 & 0.98349 \\
\hline $\mathrm{O}$ & 1.95998 & 1.63733 & 0.46619 \\
\hline $\mathrm{C}$ & 3.17870 & -1.85975 & -0.56104 \\
\hline $\mathrm{C}$ & 2.79889 & -3.04927 & -0.09170 \\
\hline $\mathrm{H}$ & 2.86534 & -3.94632 & -0.6972 \\
\hline $\mathrm{H}$ & 2.41921 & -3.15871 & 0.92003 \\
\hline $\mathrm{H}$ & 3.54384 & -1.79560 & -1.58671 \\
\hline $\mathrm{C}$ & 0.20772 & -1.38449 & -0.55050 \\
\hline $\mathrm{O}$ & 0.56308 & -0.34942 & 0.02541 \\
\hline $\mathrm{H}$ & 0.66723 & -1.65787 & $-1.5045 c$ \\
\hline $\mathrm{C}$ & -2.79162 & -3.95463 & 0.9258 \\
\hline $\mathrm{C}$ & -2.44746 & -2.81791 & 1.66073 \\
\hline $\mathrm{C}$ & -1.46344 & -1.96468 & 1.1919 \\
\hline $\mathrm{C}$ & -0.82683 & -2.25379 & -0.0216 \\
\hline $\mathrm{C}$ & -1.17149 & -3.39315 & -0.7558 \\
\hline $\mathrm{C}$ & -2.15696 & -4.24491 & -0.2787 \\
\hline $\mathrm{H}$ & -3.56082 & -4.61989 & 1.2996 \\
\hline $\mathrm{H}$ & -2.94889 & -2.60710 & 2.5967 \\
\hline $\mathrm{H}$ & -1.17623 & -1.07910 & 1.7463 \\
\hline $\mathrm{H}$ & -0.66406 & -3.60489 & -1.69093 \\
\hline $\mathrm{H}$ & -2.42954 & -5.12994 & -0.83920 \\
\hline $\mathrm{C}$ & 3.08209 & -0.57652 & 0.1816 \\
\hline $\mathrm{C}$ & 3.36421 & -0.58988 & 1.6595 \\
\hline $\mathrm{C}$ & 4.36693 & 0.01581 & 0.7191 \\
\hline $\mathrm{H}$ & 3.63264 & -1.53561 & 2.1143 \\
\hline $\mathrm{H}$ & 2.80150 & 0.08991 & 2.2847 \\
\hline $\mathrm{H}$ & 4.45635 & 1.09439 & 0.6934 \\
\hline $\mathrm{H}$ & 5.28618 & -0.53122 & 0.5449 \\
\hline $\mathrm{O}$ & 2.76641 & 3.77901 & 1.8307 \\
\hline
\end{tabular}




$\begin{array}{cccc}\text { C } & 3.58515 & 2.99215 & 2.48545 \\ \text { C } & 5.02767 & 3.41939 & 2.13132 \\ \text { O } & 3.37886 & 2.12272 & 3.27639 \\ \text { F } & 5.29172 & 4.63356 & 2.62161 \\ \text { F } & 5.20890 & 3.46881 & 0.80718 \\ \text { F } & 5.90718 & 2.56461 & 2.63734 \\ \text { C } & 1.99706 & 0.66924 & -1.95873 \\ \text { C } & 0.90547 & 0.63960 & -2.82697 \\ \text { C } & 3.24471 & 0.95424 & -2.52969 \\ \text { C } & 1.04630 & 0.89930 & -4.18928 \\ \text { H } & -0.09293 & 0.42718 & -2.45456 \\ \text { C } & 3.38243 & 1.22115 & -3.88529 \\ \text { H } & 4.12570 & 0.97593 & -1.89397 \\ \text { C } & 2.28293 & 1.20023 & -4.73617 \\ \text { H } & 2.39171 & 1.40626 & -5.79265 \\ \text { C } & -0.17723 & 0.82972 & -5.05223 \\ \text { C } & 4.73905 & 1.58191 & -4.41322 \\ \text { F } & -0.66390 & -0.42191 & -5.11828 \\ \text { F } & 0.05239 & 1.22886 & -6.30778 \\ \text { F } & -1.16981 & 1.59318 & -4.56626 \\ \text { F } & 4.79317 & 1.54619 & -5.75118 \\ \text { F } & 5.10024 & 2.82084 & -4.04013 \\ \text { F } & 5.68999 & 0.75444 & -3.95412 \\ & \text { G = -2307.568639Hartree } \\ & & \text { TS-f } & \end{array}$

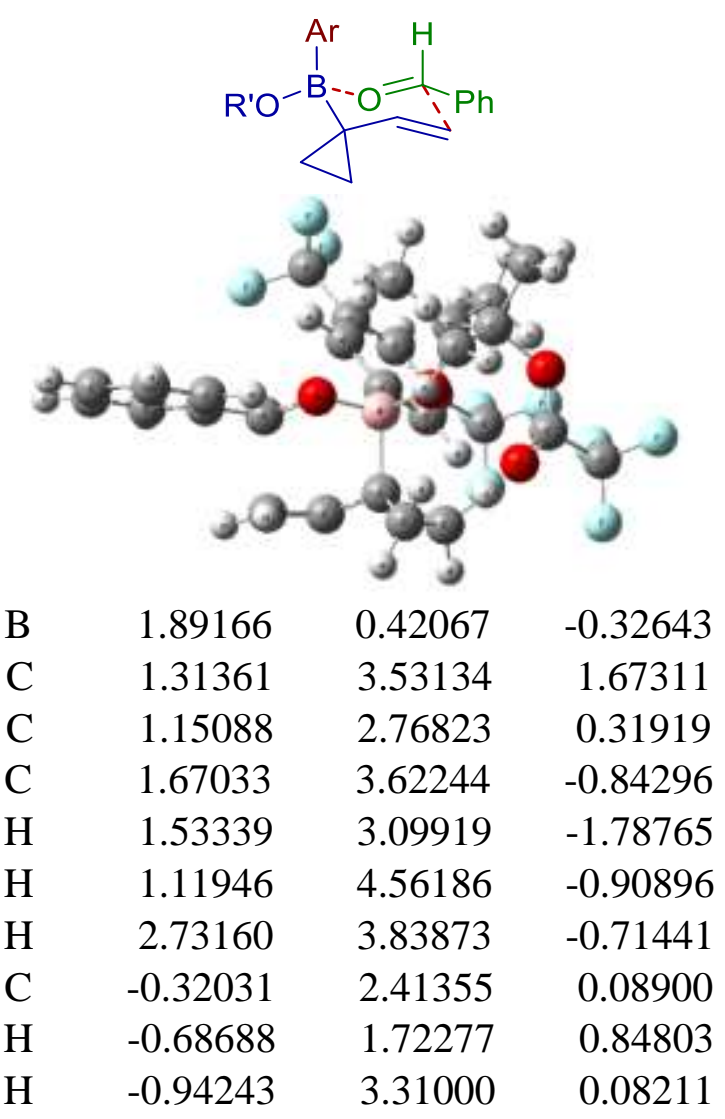




\begin{tabular}{|c|c|c|c|}
\hline $\mathrm{H}$ & -0.43040 & 1.93345 & -0.88419 \\
\hline $\mathrm{C}$ & 0.79629 & 2.72338 & 2.85767 \\
\hline $\mathrm{H}$ & -0.29360 & 2.78867 & 2.85659 \\
\hline $\mathrm{H}$ & 1.09446 & 1.67926 & 2.79847 \\
\hline $\mathrm{H}$ & 1.15817 & 3.14900 & 3.79358 \\
\hline $\mathrm{C}$ & 0.69535 & 4.92425 & 1.69226 \\
\hline $\mathrm{H}$ & -0.37269 & 4.87797 & 1.47999 \\
\hline $\mathrm{H}$ & 0.82537 & 5.35320 & 2.68748 \\
\hline $\mathrm{H}$ & 1.17682 & 5.58381 & 0.97210 \\
\hline $\mathrm{O}$ & 1.94558 & 1.59686 & 0.48572 \\
\hline $\mathrm{C}$ & 2.95327 & -1.86016 & -0.40966 \\
\hline $\mathrm{C}$ & 2.00769 & -2.75944 & 0.02811 \\
\hline $\mathrm{H}$ & 1.81652 & -3.67133 & -0.52591 \\
\hline $\mathrm{H}$ & 1.73144 & -2.75789 & 1.07736 \\
\hline $\mathrm{H}$ & 3.35713 & -1.97305 & -1.41600 \\
\hline $\mathrm{C}$ & 0.46714 & -1.55723 & -0.56056 \\
\hline $\mathrm{O}$ & 0.66712 & -0.40254 & -0.00600 \\
\hline $\mathrm{H}$ & 0.66227 & -1.65715 & -1.62826 \\
\hline $\mathrm{C}$ & -2.81435 & -3.88740 & 0.83991 \\
\hline $\mathrm{C}$ & -2.30333 & -2.86638 & 1.63880 \\
\hline $\mathrm{C}$ & -1.22953 & -2.10468 & 1.19710 \\
\hline $\mathrm{C}$ & -0.66743 & -2.36533 & -0.05349 \\
\hline $\mathrm{C}$ & -1.17997 & -3.38618 & -0.85369 \\
\hline $\mathrm{C}$ & -2.25281 & -4.14690 & -0.40696 \\
\hline $\mathrm{H}$ & -3.65197 & -4.47931 & 1.18876 \\
\hline $\mathrm{H}$ & -2.74497 & -2.66535 & 2.60734 \\
\hline $\mathrm{H}$ & -0.82266 & -1.30667 & 1.80714 \\
\hline $\mathrm{H}$ & -0.73883 & -3.57999 & -1.82619 \\
\hline $\mathrm{H}$ & -2.65232 & -4.93654 & -1.03117 \\
\hline $\mathrm{C}$ & 3.13543 & -0.61905 & 0.26670 \\
\hline $\mathrm{C}$ & 3.48027 & -0.50600 & 1.71277 \\
\hline $\mathrm{C}$ & 4.42935 & 0.02432 & 0.65658 \\
\hline $\mathrm{H}$ & 3.77695 & -1.41297 & 2.22743 \\
\hline $\mathrm{H}$ & 2.95625 & 0.22848 & 2.30719 \\
\hline $\mathrm{H}$ & 4.49025 & 1.10003 & 0.54863 \\
\hline $\mathrm{H}$ & 5.35214 & -0.51538 & 0.48192 \\
\hline $\mathrm{O}$ & 2.75634 & 3.82078 & 1.75416 \\
\hline $\mathrm{C}$ & 3.65692 & 3.08948 & 2.36174 \\
\hline $\mathrm{C}$ & 5.04502 & 3.54148 & 1.85465 \\
\hline $\mathrm{O}$ & 3.55322 & 2.24975 & 3.20406 \\
\hline $\mathrm{F}$ & 5.30229 & 4.79447 & 2.23590 \\
\hline $\mathrm{F}$ & 5.10587 & 3.50197 & 0.51824 \\
\hline $\mathrm{F}$ & 5.99924 & 2.75340 & 2.33340 \\
\hline $\mathrm{C}$ & 2.05191 & 0.64447 & -1.93014 \\
\hline $\mathrm{C}$ & 0.97770 & 0.60180 & -2.82100 \\
\hline $\mathrm{C}$ & 3.30151 & 0.97346 & -2.46760 \\
\hline
\end{tabular}




$\begin{array}{lrrr}\text { C } & 1.13780 & 0.88635 & -4.17527 \\ \text { H } & -0.01905 & 0.35840 & -2.46239 \\ \text { C } & 3.45881 & 1.26210 & -3.81770 \\ \text { H } & 4.17006 & 1.01596 & -1.81738 \\ \text { C } & 2.37854 & 1.22537 & -4.69168 \\ \text { H } & 2.50438 & 1.45001 & -5.74246 \\ \text { C } & -0.06624 & 0.80194 & -5.06382 \\ \text { C } & 4.81453 & 1.66963 & -4.31260 \\ \text { F } & -0.52406 & -0.45901 & -5.15576 \\ \text { F } & 0.18053 & 1.22142 & -6.30991 \\ \text { F } & -1.08405 & 1.53831 & -4.58957 \\ \text { F } & 4.90085 & 1.64240 & -5.64907 \\ \text { F } & 5.12744 & 2.91747 & -3.92581 \\ \text { F } & 5.78158 & 0.87090 & -3.83525 \\ & \mathrm{G}=-2307.555393 \text { Hartree }\end{array}$

Adduct-f'

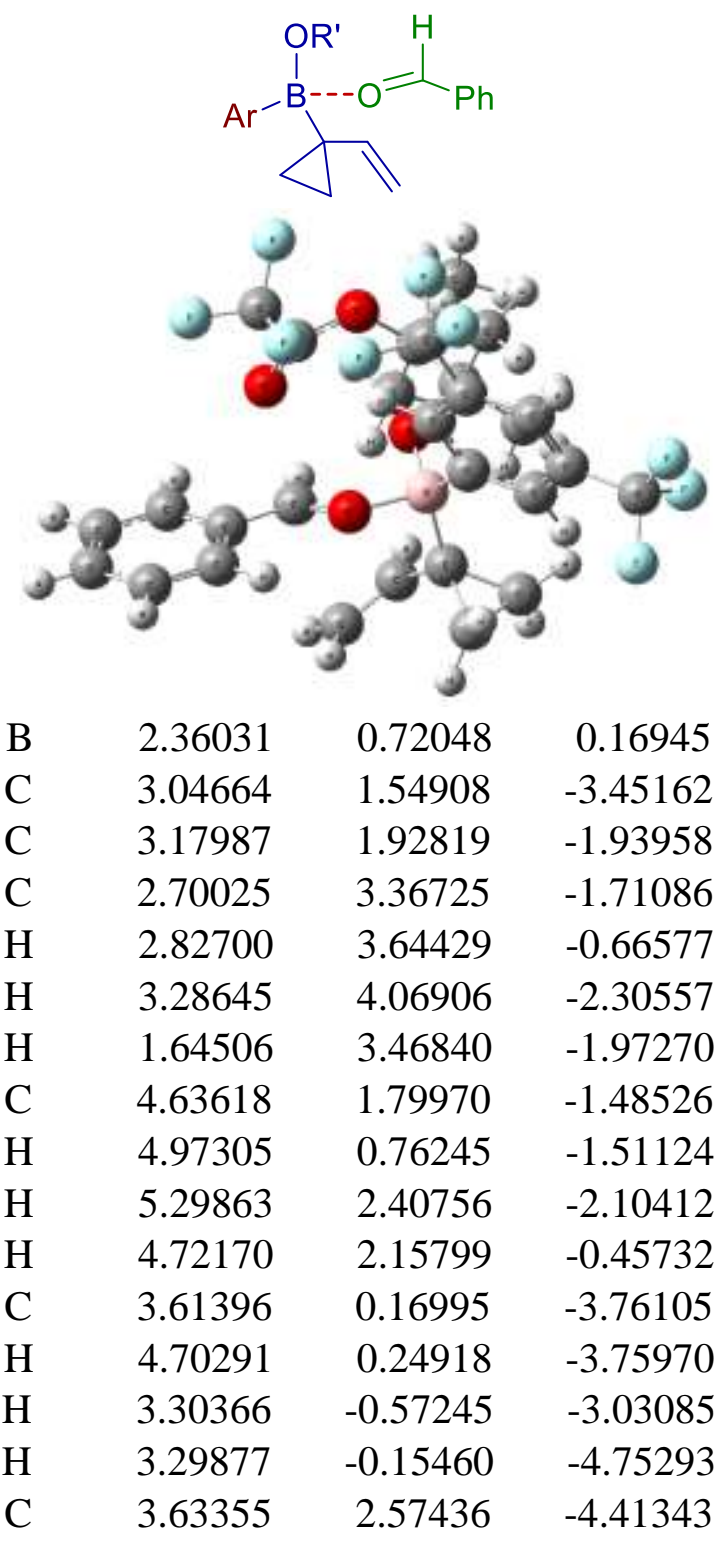




\begin{tabular}{|c|c|c|c|}
\hline $\mathrm{H}$ & 4.68322 & 2.76213 & -4.18555 \\
\hline $\mathrm{H}$ & 3.57023 & 2.18384 & -5.43041 \\
\hline $\mathrm{H}$ & 3.08506 & 3.51384 & -4.37287 \\
\hline $\mathrm{O}$ & 2.34643 & 0.99726 & -1.26206 \\
\hline $\mathrm{C}$ & 3.49938 & -1.57239 & -0.23458 \\
\hline $\mathrm{C}$ & 2.91161 & -2.74524 & 0.00184 \\
\hline $\mathrm{H}$ & 2.96965 & -3.56311 & -0.70756 \\
\hline $\mathrm{H}$ & 2.36469 & -2.92344 & 0.92375 \\
\hline $\mathrm{H}$ & 4.03629 & -1.43793 & -1.17239 \\
\hline $\mathrm{C}$ & 0.58729 & -0.88790 & -0.54340 \\
\hline $\mathrm{O}$ & 0.98945 & -0.12964 & 0.35085 \\
\hline $\mathrm{H}$ & 1.10182 & -0.89615 & -1.50332 \\
\hline $\mathrm{C}$ & -2.77473 & -3.39238 & -0.13172 \\
\hline $\mathrm{C}$ & -2.33166 & -2.66716 & 0.97703 \\
\hline $\mathrm{C}$ & -1.23001 & -1.83765 & 0.86292 \\
\hline $\mathrm{C}$ & -0.56917 & -1.74352 & -0.36855 \\
\hline $\mathrm{C}$ & -1.01379 & -2.46784 & -1.4795 \\
\hline $\mathrm{C}$ & -2.12257 & -3.29359 & -1.3575 \\
\hline $\mathrm{H}$ & -3.63851 & -4.03967 & -0.0362 \\
\hline $\mathrm{H}$ & -2.85072 & -2.75444 & 1.9231 \\
\hline $\mathrm{H}$ & -0.86825 & -1.26296 & 1.7070 \\
\hline $\mathrm{H}$ & -0.49339 & -2.36539 & -2.4260 \\
\hline $\mathrm{H}$ & -2.47775 & -3.85724 & -2.2107 \\
\hline $\mathrm{C}$ & 3.45066 & -0.38345 & 0.66410 \\
\hline $\mathrm{C}$ & 3.63654 & -0.64030 & $2.1408 ?$ \\
\hline $\mathrm{C}$ & 4.76560 & -0.06041 & 1.3355 \\
\hline $\mathrm{H}$ & 3.74180 & -1.66559 & 2.4737 \\
\hline $\mathrm{H}$ & 3.11491 & 0.00733 & 2.8351 \\
\hline $\mathrm{H}$ & 5.06577 & 0.96791 & 1.4807 \\
\hline $\mathrm{H}$ & 5.59220 & -0.72607 & 1.1130 \\
\hline $\mathrm{O}$ & 1.59606 & 1.64824 & -3.7173 \\
\hline $\mathrm{C}$ & 0.74425 & 0.66576 & -3.6128 \\
\hline $\mathrm{C}$ & -0.66841 & 1.23957 & -3.37102 \\
\hline $\mathrm{O}$ & 0.90407 & -0.52112 & -3.6575 \\
\hline $\mathrm{F}$ & -0.89349 & 2.36891 & -4.03166 \\
\hline $\mathrm{F}$ & -0.80139 & 1.49381 & -2.05828 \\
\hline $\mathrm{F}$ & -1.59822 & 0.35474 & -3.70846 \\
\hline $\mathrm{C}$ & 2.02853 & 2.02026 & 1.08813 \\
\hline $\mathrm{C}$ & 0.80753 & 2.66222 & $0.8352^{\prime}$ \\
\hline $\mathrm{C}$ & 2.84738 & 2.60548 & 2.0528 \\
\hline $\mathrm{C}$ & 0.44398 & 3.83230 & 1.4853 \\
\hline $\mathrm{H}$ & 0.13598 & 2.24876 & 0.0886 \\
\hline $\mathrm{C}$ & 2.47991 & 3.78122 & 2.7083 \\
\hline $\mathrm{H}$ & 3.80037 & 2.16448 & 2.3162 \\
\hline $\mathrm{C}$ & 1.27989 & 4.41434 & 2.4319 \\
\hline $\mathrm{H}$ & 1.00073 & 5.32867 & 2.9384 \\
\hline
\end{tabular}




$\begin{array}{lrrr}\text { C } & 3.41163 & 4.33476 & 3.74334 \\ \text { C } & -0.84708 & 4.49000 & 1.10328 \\ \text { F } & -0.78461 & 5.02244 & -0.12995 \\ \text { F } & -1.18619 & 5.48114 & 1.93819 \\ \text { F } & -1.86759 & 3.61844 & 1.08602 \\ \text { F } & 4.66264 & 4.46204 & 3.27003 \\ \text { F } & 3.49676 & 3.53060 & 4.81692 \\ \text { F } & 3.03251 & 5.54005 & 4.18633\end{array}$

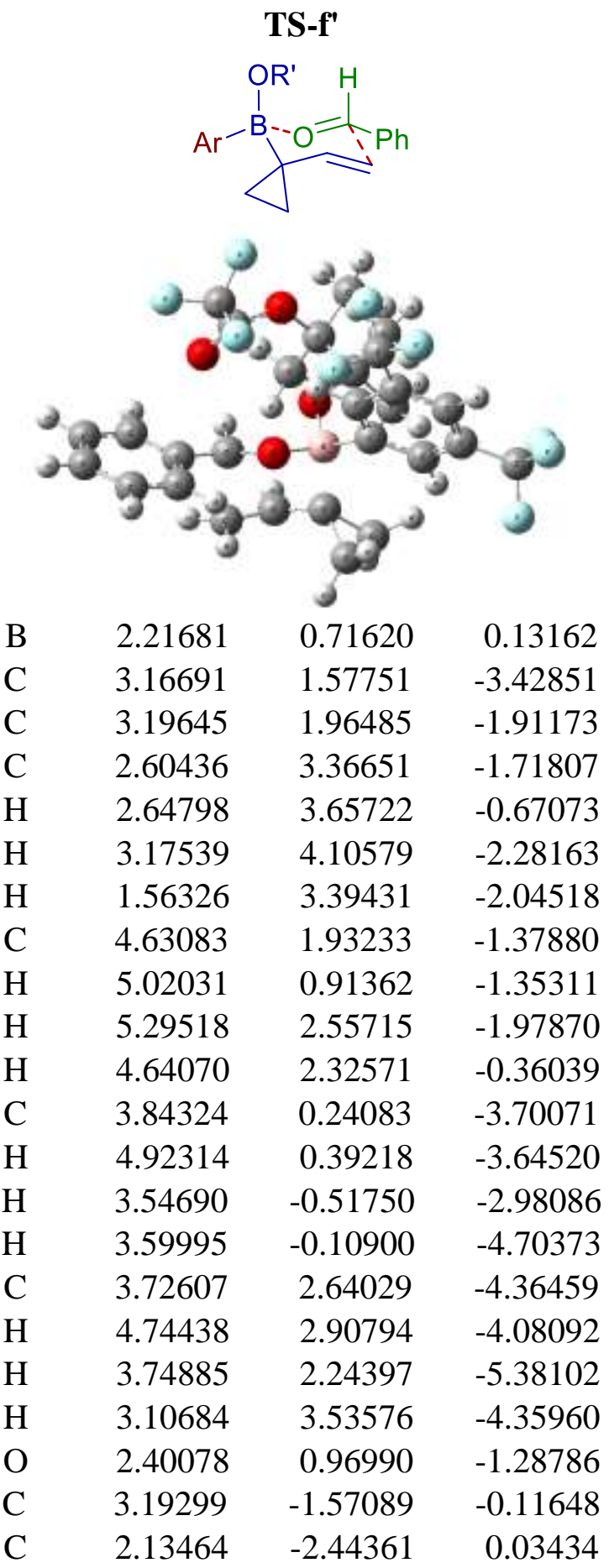




\begin{tabular}{|c|c|c|c|}
\hline $\mathrm{H}$ & 2.00158 & -3.25693 & -0.67009 \\
\hline $\mathrm{H}$ & 1.71078 & -2.58322 & 1.02364 \\
\hline $\mathrm{H}$ & 3.74825 & -1.59032 & -1.05339 \\
\hline $\mathrm{C}$ & 0.80818 & -1.09823 & -0.55211 \\
\hline $\mathrm{O}$ & 0.97639 & -0.09719 & 0.26644 \\
\hline $\mathrm{H}$ & 1.15946 & -0.96935 & -1.57016 \\
\hline $\mathrm{C}$ & -2.82969 & -3.29381 & -0.26071 \\
\hline $\mathrm{C}$ & -2.32011 & -2.65825 & 0.87092 \\
\hline $\mathrm{C}$ & -1.13149 & -1.94619 & 0.79354 \\
\hline $\mathrm{C}$ & -0.44984 & -1.87165 & -0.42169 \\
\hline $\mathrm{C}$ & -0.96079 & -2.50162 & -1.55591 \\
\hline $\mathrm{C}$ & -2.15270 & -3.21258 & -1.47357 \\
\hline $\mathrm{H}$ & -3.75871 & -3.84758 & -0.19647 \\
\hline $\mathrm{H}$ & -2.85378 & -2.71755 & 1.81172 \\
\hline $\mathrm{H}$ & -0.72697 & -1.44040 & 1.66273 \\
\hline $\mathrm{H}$ & -0.43091 & -2.41535 & -2.49950 \\
\hline $\mathrm{H}$ & -2.55543 & -3.69611 & -2.35509 \\
\hline $\mathrm{C}$ & 3.38659 & -0.44705 & 0.74466 \\
\hline $\mathrm{C}$ & 3.60719 & -0.58953 & 2.21769 \\
\hline $\mathrm{C}$ & 4.73630 & -0.12998 & 1.31646 \\
\hline $\mathrm{H}$ & 3.67892 & -1.58760 & 2.63476 \\
\hline $\mathrm{H}$ & 3.15806 & 0.14722 & 2.87299 \\
\hline $\mathrm{H}$ & 5.08221 & 0.89126 & 1.37970 \\
\hline $\mathrm{H}$ & 5.52901 & -0.84447 & 1.12699 \\
\hline $\mathrm{O}$ & 1.72679 & 1.57309 & -3.75675 \\
\hline $\mathrm{C}$ & 0.94258 & 0.53198 & -3.66069 \\
\hline $\mathrm{C}$ & -0.50135 & 0.98857 & -3.35512 \\
\hline $\mathrm{O}$ & 1.18261 & -0.63721 & -3.76484 \\
\hline $\mathrm{F}$ & -0.79273 & 2.17825 & -3.87080 \\
\hline $\mathrm{F}$ & -0.65288 & 1.06527 & -2.02551 \\
\hline $\mathrm{F}$ & -1.37839 & 0.10167 & -3.81380 \\
\hline $\mathrm{C}$ & 1.97151 & 2.01683 & 1.06879 \\
\hline $\mathrm{C}$ & 0.68954 & 2.58054 & 1.00634 \\
\hline $\mathrm{C}$ & 2.90586 & 2.68243 & 1.86199 \\
\hline $\mathrm{C}$ & 0.37542 & 3.75804 & 1.67243 \\
\hline $\mathrm{H}$ & -0.07232 & 2.09416 & 0.40542 \\
\hline $\mathrm{C}$ & 2.58763 & 3.86221 & 2.53221 \\
\hline $\mathrm{H}$ & 3.91355 & 2.30161 & 1.97169 \\
\hline $\mathrm{C}$ & 1.32268 & 4.42115 & 2.44243 \\
\hline $\mathrm{H}$ & 1.07941 & 5.33946 & 2.96001 \\
\hline $\mathrm{C}$ & 3.66480 & 4.53663 & 3.32593 \\
\hline $\mathrm{C}$ & -1.00571 & 4.31933 & 1.51366 \\
\hline $\mathrm{F}$ & -1.23795 & 4.71000 & 0.24914 \\
\hline $\mathrm{F}$ & -1.22018 & 5.38440 & 2.29719 \\
\hline $\mathrm{F}$ & -1.95008 & 3.41314 & 1.80909 \\
\hline $\mathrm{F}$ & 4.64750 & 4.99775 & 2.53177 \\
\hline
\end{tabular}




$$
\begin{array}{llrr}
F & 4.24889 & 3.69414 & 4.19349 \\
F & 3.20963 & 5.57883 & 4.03103 \\
& \text { G }=-2307.549967 \text { Hartree }
\end{array}
$$

Adduct-g

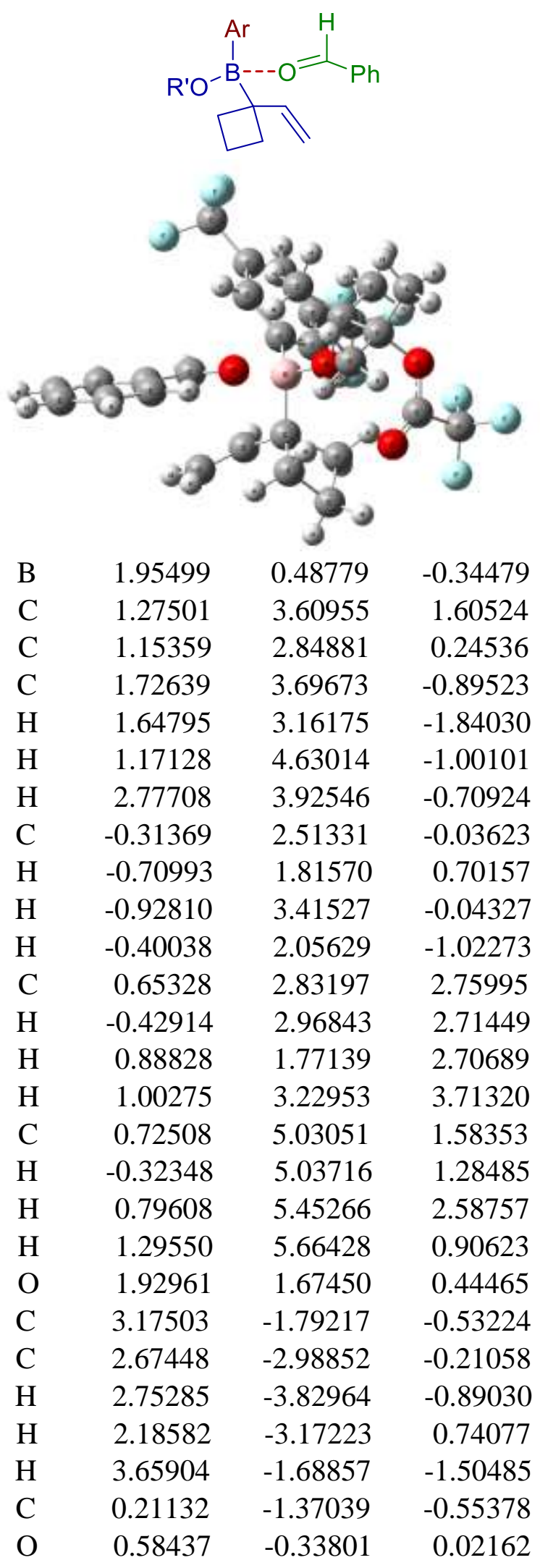




\begin{tabular}{|c|c|c|c|}
\hline $\mathrm{H}$ & 0.61955 & -1.62353 & -1.53489 \\
\hline $\mathrm{C}$ & -2.70625 & -3.97066 & 1.03084 \\
\hline $\mathrm{C}$ & -2.32189 & -2.84929 & 1.76902 \\
\hline $\mathrm{C}$ & -1.36322 & -1.98622 & 1.26581 \\
\hline $\mathrm{C}$ & -0.79328 & -2.25050 & 0.01395 \\
\hline $\mathrm{C}$ & -1.17785 & -3.37498 & -0.72338 \\
\hline $\mathrm{C}$ & -2.13713 & -4.23637 & -0.21173 \\
\hline $\mathrm{H}$ & -3.45495 & -4.64360 & 1.43155 \\
\hline $\mathrm{H}$ & -2.77241 & -2.65807 & 2.73478 \\
\hline $\mathrm{H}$ & -1.04619 & -1.11173 & 1.8219 \\
\hline $\mathrm{H}$ & -0.72072 & -3.56772 & -1.68813 \\
\hline $\mathrm{H}$ & -2.43947 & -5.11014 & -0.7746 \\
\hline $\mathrm{C}$ & 3.08698 & -0.53478 & 0.26468 \\
\hline $\mathrm{C}$ & 3.00515 & -0.75372 & 1.80400 \\
\hline $\mathrm{C}$ & 4.50223 & 0.09240 & 0.49432 \\
\hline $\mathrm{H}$ & 2.59867 & -1.70054 & 2.16313 \\
\hline $\mathrm{H}$ & 2.47645 & 0.07134 & 2.27987 \\
\hline $\mathrm{H}$ & 4.43949 & 1.18057 & 0.54292 \\
\hline $\mathrm{H}$ & 5.29085 & -0.19294 & -0.20684 \\
\hline $\mathrm{O}$ & 2.72059 & 3.83123 & 1.77810 \\
\hline $\mathrm{C}$ & 3.54809 & 3.01910 & 2.38992 \\
\hline $\mathrm{C}$ & 4.98648 & 3.45883 & 2.03317 \\
\hline $\mathrm{O}$ & 3.35253 & 2.12277 & 3.15315 \\
\hline $\mathrm{F}$ & 5.26681 & 4.63996 & 2.59097 \\
\hline $\mathrm{F}$ & 5.14565 & 3.58581 & 0.71229 \\
\hline $\mathrm{F}$ & 5.87056 & 2.57093 & 2.47229 \\
\hline $\mathrm{C}$ & 2.01593 & 0.67802 & -1.95726 \\
\hline $\mathrm{C}$ & 0.93409 & 0.62676 & -2.83725 \\
\hline $\mathrm{C}$ & 3.26132 & 0.98995 & -2.51908 \\
\hline $\mathrm{C}$ & 1.08269 & 0.88208 & -4.1995 \\
\hline $\mathrm{H}$ & -0.06545 & 0.40445 & -2.4742 \\
\hline $\mathrm{C}$ & 3.40799 & 1.24872 & -3.8752 \\
\hline $\mathrm{H}$ & 4.13776 & 1.04669 & -1.8804 \\
\hline $\mathrm{C}$ & 2.31884 & 1.20005 & -4.73774 \\
\hline $\mathrm{H}$ & 2.43459 & 1.40084 & -5.79448 \\
\hline $\mathrm{C}$ & -0.13276 & 0.79050 & -5.07181 \\
\hline $\mathrm{C}$ & 4.76373 & 1.63214 & -4.3892 \\
\hline $\mathrm{F}$ & -0.59975 & -0.46862 & -5.1377 \\
\hline $\mathrm{F}$ & 0.10034 & 1.18911 & -6.3268 \\
\hline $\mathrm{F}$ & -1.14038 & 1.54038 & -4.5957 \\
\hline $\mathrm{F}$ & 4.83601 & 1.58526 & -5.7257 \\
\hline $\mathrm{F}$ & 5.09607 & 2.88087 & -4.0224 \\
\hline $\mathrm{F}$ & 5.72453 & 0.82735 & $-3.9096^{\prime}$ \\
\hline $\mathrm{C}$ & 4.53381 & -0.55718 & 1.8940 \\
\hline $\mathrm{H}$ & 5.06445 & -1.51105 & 1.8727 \\
\hline $\mathrm{H}$ & 4.90580 & 0.05518 & 2.7148 \\
\hline
\end{tabular}




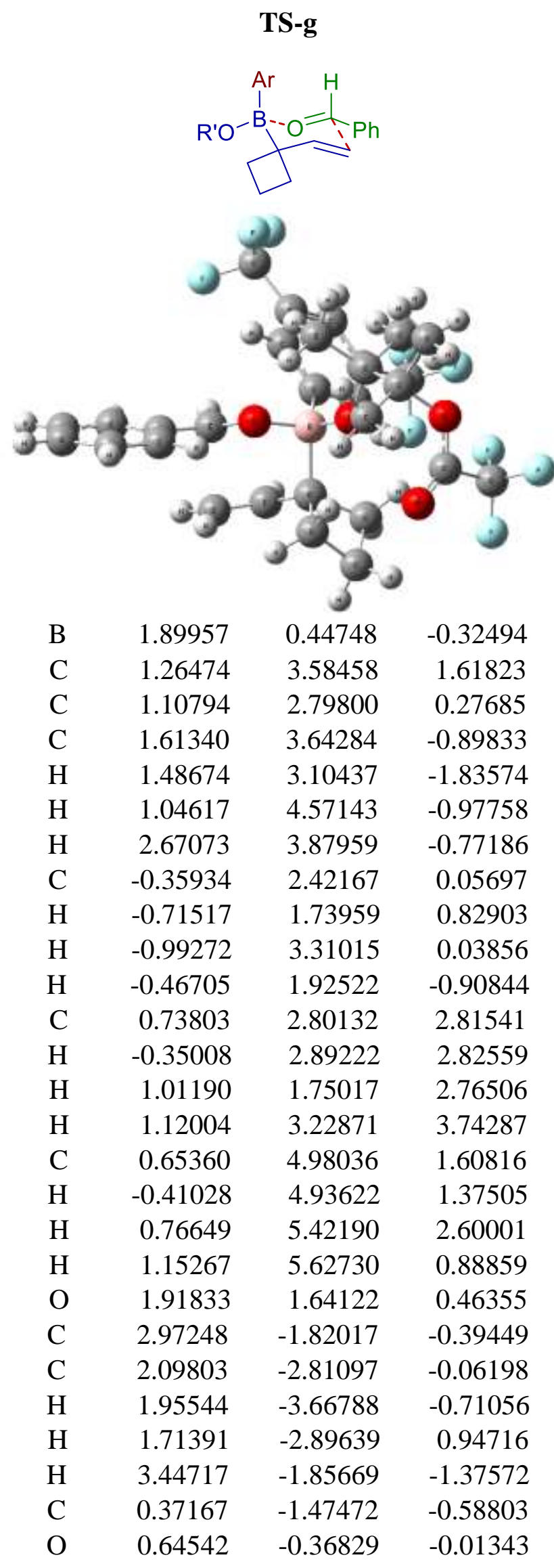




\begin{tabular}{|c|c|c|c|}
\hline $\mathrm{H}$ & 0.61102 & -1.60664 & -1.64167 \\
\hline $\mathrm{C}$ & -2.74373 & -3.91967 & 0.94475 \\
\hline $\mathrm{C}$ & -2.24069 & -2.87874 & 1.72377 \\
\hline $\mathrm{C}$ & -1.21927 & -2.07511 & 1.23759 \\
\hline $\mathrm{C}$ & -0.70149 & -2.31574 & -0.03771 \\
\hline $\mathrm{C}$ & -1.20692 & -3.35682 & -0.81820 \\
\hline $\mathrm{C}$ & -2.22837 & -4.15813 & -0.3264 \\
\hline $\mathrm{H}$ & -3.53952 & -4.54563 & $1.3299^{\circ}$ \\
\hline $\mathrm{H}$ & -2.64749 & -2.69724 & 2.71109 \\
\hline $\mathrm{H}$ & -0.81605 & -1.26273 & 1.83110 \\
\hline $\mathrm{H}$ & -0.79674 & -3.53468 & -1.80699 \\
\hline $\mathrm{H}$ & -2.62244 & -4.96494 & -0.9316 \\
\hline $\mathrm{C}$ & 3.10414 & -0.59051 & 0.34936 \\
\hline $\mathrm{C}$ & 3.07342 & -0.68756 & 1.89508 \\
\hline $\mathrm{C}$ & 4.52459 & 0.03483 & 0.44782 \\
\hline $\mathrm{H}$ & 2.68173 & -1.60762 & 2.33139 \\
\hline $\mathrm{H}$ & 2.57264 & 0.17335 & 2.3338 \\
\hline $\mathrm{H}$ & 4.47266 & 1.12385 & 0.42528 \\
\hline $\mathrm{H}$ & 5.27585 & -0.31242 & -0.26481 \\
\hline $\mathrm{O}$ & 2.70727 & 3.87000 & 1.70890 \\
\hline $\mathrm{C}$ & 3.60325 & 3.09207 & 2.26531 \\
\hline $\mathrm{C}$ & 4.99195 & 3.54009 & 1.75613 \\
\hline $\mathrm{O}$ & 3.49512 & 2.21737 & 3.07013 \\
\hline $\mathrm{F}$ & 5.27860 & 4.77160 & 2.18374 \\
\hline $\mathrm{F}$ & 5.03292 & 3.55377 & 0.41880 \\
\hline $\mathrm{F}$ & 5.93931 & 2.71630 & 2.18854 \\
\hline $\mathrm{C}$ & 2.06866 & 0.66079 & -1.92970 \\
\hline $\mathrm{C}$ & 1.00211 & 0.60887 & -2.83009 \\
\hline $\mathrm{C}$ & 3.31454 & 1.01590 & -2.46087 \\
\hline $\mathrm{C}$ & 1.16694 & 0.89600 & -4.1832 \\
\hline $\mathrm{H}$ & 0.00203 & 0.36387 & -2.48324 \\
\hline $\mathrm{C}$ & 3.47773 & 1.30403 & -3.81017 \\
\hline $\mathrm{H}$ & 4.17827 & 1.09065 & -1.80894 \\
\hline $\mathrm{C}$ & 2.40609 & 1.24902 & -4.69316 \\
\hline $\mathrm{H}$ & 2.53610 & 1.47434 & -5.74327 \\
\hline $\mathrm{C}$ & -0.03289 & 0.80251 & -5.07667 \\
\hline $\mathrm{C}$ & 4.83101 & 1.73457 & -4.29237 \\
\hline $\mathrm{F}$ & -0.48080 & -0.46176 & -5.17107 \\
\hline $\mathrm{F}$ & 0.21548 & 1.22477 & -6.32127 \\
\hline $\mathrm{F}$ & -1.05796 & 1.53086 & -4.6053 \\
\hline $\mathrm{F}$ & 4.93237 & 1.70135 & -5.62747 \\
\hline $\mathrm{F}$ & 5.11584 & 2.99010 & -3.90917 \\
\hline $\mathrm{F}$ & 5.80706 & 0.95660 & -3.7993 \\
\hline $\mathrm{C}$ & 4.60793 & -0.50578 & 1.8933 \\
\hline $\mathrm{H}$ & 5.12324 & -1.46802 & 1.9193 \\
\hline $\mathrm{H}$ & 5.02611 & 0.16439 & 2.6431 \\
\hline
\end{tabular}




\section{Adduct-h}

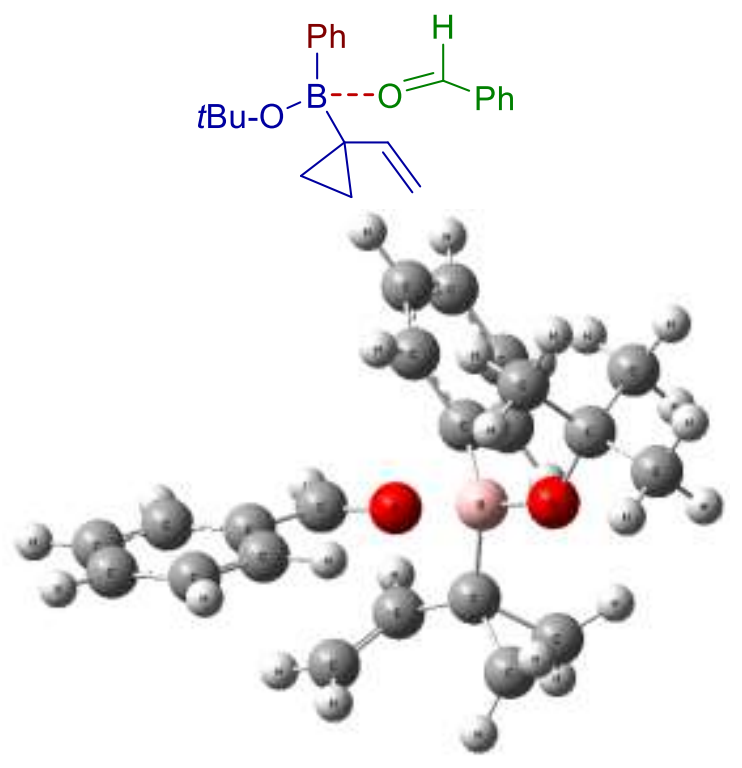

$\begin{array}{lrrr}\text { B } & 1.96751 & 0.49916 & -0.31820 \\ \text { C } & 1.25983 & 3.61857 & 1.58894 \\ \text { C } & 1.13833 & 2.84426 & 0.27691 \\ \text { C } & 1.75478 & 3.66991 & -0.85653 \\ \text { H } & 1.60135 & 3.20344 & -1.83030 \\ \text { H } & 1.30632 & 4.66674 & -0.87601 \\ \text { H } & 2.82988 & 3.77633 & -0.68949 \\ \text { C } & -0.33467 & 2.54772 & -0.00745 \\ \text { H } & -0.75738 & 1.93695 & 0.79347 \\ \text { H } & -0.89919 & 3.48143 & -0.07231 \\ \text { H } & -0.45197 & 2.01462 & -0.95183 \\ \text { O } & 1.87125 & 1.64259 & 0.50104 \\ \text { C } & 3.24620 & -1.81100 & -0.39550 \\ \text { C } & 2.90258 & -2.99798 & 0.10729 \\ \text { H } & 3.01758 & -3.91207 & -0.46461 \\ \text { H } & 2.50321 & -3.08806 & 1.11340 \\ \text { H } & 3.62763 & -1.76588 & -1.41602 \\ \text { C } & 0.25539 & -1.44877 & -0.55276 \\ \text { O } & 0.54399 & -0.40239 & 0.02892 \\ \text { H } & 0.76132 & -1.70674 & -1.48866 \\ \text { C } & -2.70996 & -4.10609 & 0.86060 \\ \text { C } & -2.41617 & -2.95480 & 1.59344 \\ \text { C } & -1.44447 & -2.07838 & 1.14117 \\ \text { C } & -0.76699 & -2.35792 & -0.05131 \\ \text { C } & -1.05995 & -3.51170 & -0.78304 \\ \text { C } & -2.03523 & -4.38674 & -0.32433 \\ \text { H } & -3.47036 & -4.78954 & 1.21941\end{array}$




\begin{tabular}{rrrr}
$\mathrm{H}$ & -2.94697 & -2.74899 & 2.51435 \\
$\mathrm{H}$ & -1.19683 & -1.17952 & 1.69289 \\
$\mathrm{H}$ & -0.52283 & -3.71712 & -1.70285 \\
$\mathrm{H}$ & -2.26926 & -5.28306 & -0.88444 \\
$\mathrm{C}$ & 3.08356 & -0.50538 & 0.29233 \\
$\mathrm{C}$ & 3.31928 & -0.45139 & 1.77840 \\
$\mathrm{C}$ & 4.33272 & 0.14851 & 0.84357 \\
$\mathrm{H}$ & 3.59778 & -1.36852 & 2.28323 \\
$\mathrm{H}$ & 2.70722 & 0.23310 & 2.35138 \\
$\mathrm{H}$ & 4.38804 & 1.22900 & 0.79133 \\
$\mathrm{H}$ & 5.27214 & -0.37894 & 0.72286 \\
$\mathrm{C}$ & 2.01561 & 0.66149 & -1.92648 \\
$\mathrm{C}$ & 0.91993 & 0.63224 & -2.79769 \\
$\mathrm{C}$ & 3.26976 & 0.92057 & -2.49993 \\
$\mathrm{C}$ & 1.05785 & 0.87025 & -4.16421 \\
$\mathrm{H}$ & -0.07662 & 0.43083 & -2.41477 \\
$\mathrm{C}$ & 3.42161 & 1.16543 & -3.86060 \\
$\mathrm{H}$ & 4.14611 & 0.93962 & -1.85781 \\
$\mathrm{C}$ & 2.31067 & 1.14538 & -4.70039 \\
$\mathrm{H}$ & 0.18547 & 0.84479 & -4.80790 \\
$\mathrm{H}$ & 4.40518 & 1.37062 & -4.26879 \\
$\mathrm{H}$ & 2.42249 & 1.33601 & -5.76150 \\
$\mathrm{H}$ & 0.72715 & 4.57073 & 1.53228 \\
$\mathrm{H}$ & 2.31214 & 3.81630 & 1.80610 \\
$\mathrm{H}$ & 0.84289 & 3.02907 & 2.40866 \\
& $\mathrm{G}=-1029.347172 \mathrm{Hartree}$ \\
\hline & & &
\end{tabular}

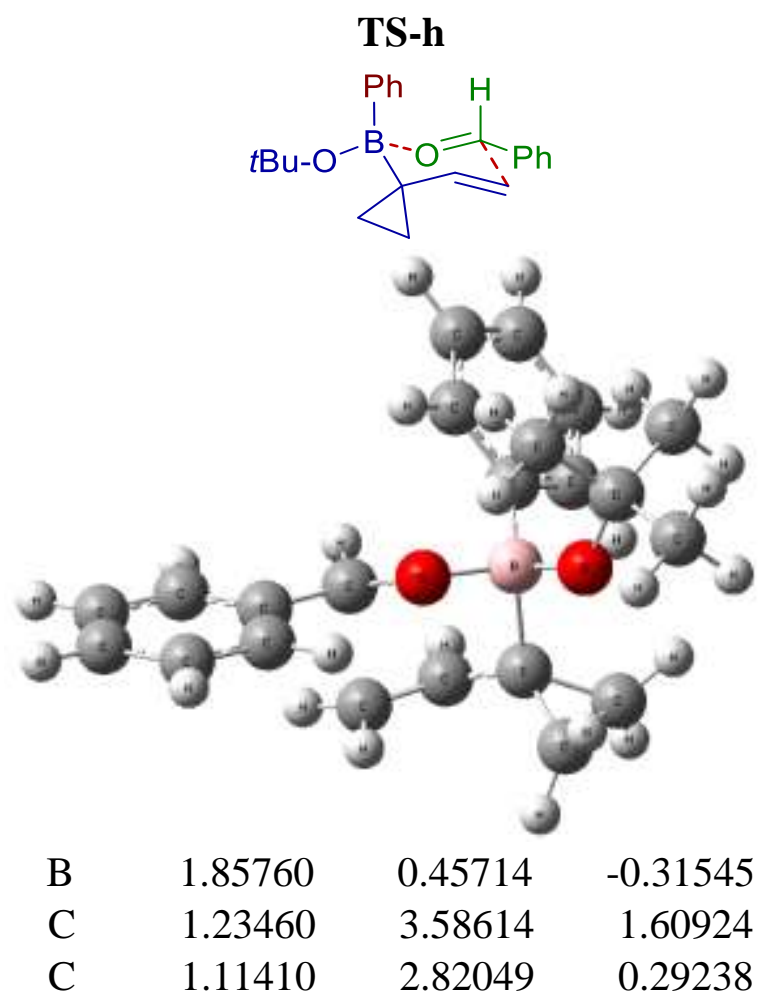




\begin{tabular}{|c|c|c|c|}
\hline $\mathrm{C}$ & 1.73895 & 3.65005 & -0.83404 \\
\hline $\mathrm{H}$ & 1.59038 & 3.18636 & -1.80995 \\
\hline $\mathrm{H}$ & 1.29247 & 4.64787 & -0.85337 \\
\hline $\mathrm{H}$ & 2.81348 & 3.75420 & -0.66100 \\
\hline $\mathrm{C}$ & -0.35792 & 2.53021 & -0.00254 \\
\hline $\mathrm{H}$ & -0.78438 & 1.90858 & 0.78808 \\
\hline $\mathrm{H}$ & -0.92201 & 3.46473 & -0.05962 \\
\hline $\mathrm{H}$ & -0.47020 & 2.00624 & -0.95307 \\
\hline $\mathrm{O}$ & 1.83868 & 1.61236 & 0.51046 \\
\hline $\mathrm{C}$ & 3.05017 & -1.77853 & -0.28854 \\
\hline $\mathrm{C}$ & 2.14071 & -2.71474 & 0.1446 \\
\hline $\mathrm{H}$ & 2.01549 & -3.65058 & -0.3878 \\
\hline $\mathrm{H}$ & 1.81939 & -2.69453 & 1.1807 \\
\hline $\mathrm{H}$ & 3.48971 & -1.89719 & -1.27906 \\
\hline $\mathrm{C}$ & 0.53922 & -1.59729 & -0.53488 \\
\hline $\mathrm{O}$ & 0.66767 & -0.43403 & 0.00862 \\
\hline $\mathrm{H}$ & 0.78687 & -1.70368 & -1.59114 \\
\hline $\mathrm{C}$ & -2.63956 & -4.09473 & 0.8198 \\
\hline $\mathrm{C}$ & -2.22971 & -3.01454 & 1.5988 \\
\hline $\mathrm{C}$ & -1.19045 & -2.19889 & $1.1707^{\prime}$ \\
\hline $\mathrm{C}$ & -0.55934 & -2.46389 & -0.0453 \\
\hline $\mathrm{C}$ & -0.97207 & -3.54362 & -0.8263 \\
\hline $\mathrm{C}$ & -2.01041 & -4.35852 & -0.39359 \\
\hline $\mathrm{H}$ & -3.45053 & -4.72862 & 1.15768 \\
\hline $\mathrm{H}$ & -2.72370 & -2.80831 & 2.5406 \\
\hline $\mathrm{H}$ & -0.86465 & -1.35266 & 1.7639 \\
\hline $\mathrm{H}$ & -0.47944 & -3.74105 & -1.7730 \\
\hline $\mathrm{H}$ & -2.33050 & -5.19519 & -1.0024 \\
\hline $\mathrm{C}$ & 3.15199 & -0.50894 & 0.3456 \\
\hline $\mathrm{C}$ & 3.47153 & -0.30485 & 1.7887 \\
\hline $\mathrm{C}$ & 4.40145 & 0.22405 & 0.7124 \\
\hline $\mathrm{H}$ & 3.80759 & -1.16459 & 2.3575 \\
\hline $\mathrm{H}$ & 2.89149 & 0.43014 & 2.33065 \\
\hline $\mathrm{H}$ & 4.40494 & 1.29711 & 0.56452 \\
\hline $\mathrm{H}$ & 5.35365 & -0.27511 & 0.57584 \\
\hline $\mathrm{C}$ & 2.02557 & 0.64416 & -1.91780 \\
\hline $\mathrm{C}$ & 0.93715 & 0.59927 & -2.79933 \\
\hline $\mathrm{C}$ & 3.27906 & 0.92507 & -2.47789 \\
\hline $\mathrm{C}$ & 1.08093 & 0.84433 & -4.1633 \\
\hline $\mathrm{H}$ & -0.05486 & 0.37309 & -2.4169 \\
\hline $\mathrm{C}$ & 3.43774 & 1.17231 & -3.8382 \\
\hline $\mathrm{H}$ & 4.15161 & 0.95952 & -1.8328 \\
\hline $\mathrm{C}$ & 2.33500 & 1.13852 & -4.6878 \\
\hline $\mathrm{H}$ & 0.21464 & 0.80772 & -4.8148 \\
\hline $\mathrm{H}$ & 4.42157 & 1.39269 & -4.2379 \\
\hline $\mathrm{H}$ & 2.45385 & 1.33311 & -5.7475 \\
\hline
\end{tabular}




\begin{tabular}{llrl}
$\mathrm{H}$ & 0.71140 & 4.54374 & 1.55455 \\
$\mathrm{H}$ & 2.28764 & 3.77333 & 1.83300 \\
$\mathrm{H}$ & 0.80738 & 2.99624 & 2.42331 \\
\multicolumn{4}{l}{$\mathrm{G}=-1029.331912$ Hartree }
\end{tabular}

\section{Adduct-h'}<smiles>C=CC1([B-](OC=Cc2ccccc2)(OC(C)C)c2ccccc2)CC1</smiles>

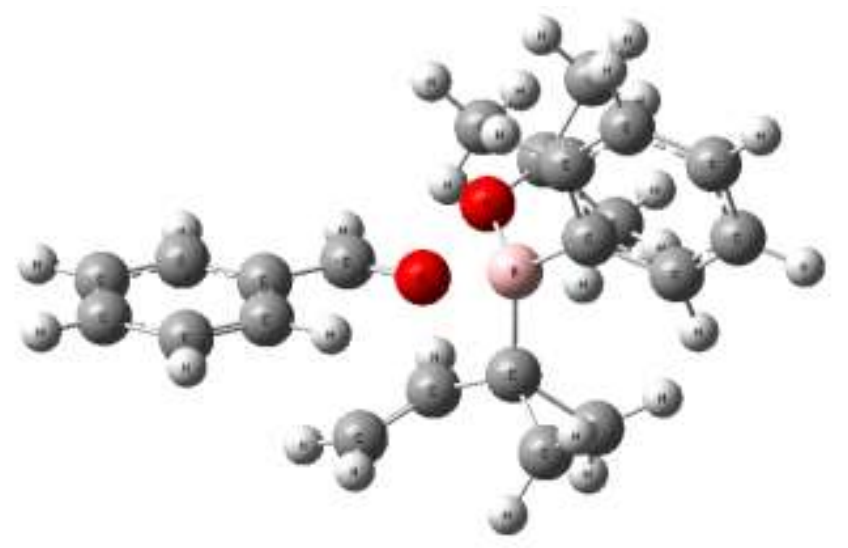

$\begin{array}{lrrr}\text { B } & 2.27306 & 0.79803 & 0.19757 \\ \mathrm{C} & 3.25659 & 1.35255 & -3.34955 \\ \mathrm{C} & 3.22616 & 1.87136 & -1.91285 \\ \mathrm{C} & 2.72551 & 3.31894 & -1.89930 \\ \mathrm{H} & 2.75374 & 3.73502 & -0.89118 \\ \mathrm{H} & 3.34843 & 3.93884 & -2.54985 \\ \mathrm{H} & 1.69480 & 3.35810 & -2.26011 \\ \mathrm{C} & 4.62870 & 1.80262 & -1.30658 \\ \mathrm{H} & 4.99497 & 0.77315 & -1.29250 \\ \mathrm{H} & 5.31756 & 2.41292 & -1.89593 \\ \mathrm{H} & 4.62470 & 2.18166 & -0.28169 \\ \mathrm{O} & 2.31235 & 1.02242 & -1.22646 \\ \mathrm{C} & 3.24314 & -1.55341 & -0.23970 \\ \mathrm{C} & 2.63149 & -2.72088 & -0.04067 \\ \mathrm{H} & 2.64739 & -3.50636 & -0.78818 \\ \mathrm{H} & 2.10357 & -2.92781 & 0.88617 \\ \mathrm{H} & 3.74301 & -1.37813 & -1.19209 \\ \mathrm{C} & 0.40077 & -0.70224 & -0.58866 \\ \mathrm{O} & 0.82608 & -0.00885 & 0.33987 \\ \mathrm{H} & 0.88181 & -0.62067 & -1.56636 \\ \mathrm{C} & -2.86941 & -3.34196 & -0.17248 \\ \mathrm{C} & -2.45606 & -2.58946 & 0.92866 \\ \mathrm{C} & -1.38782 & -1.71687 & 0.80722 \\ \mathrm{C} & -0.73430 & -1.59814 & -0.42497\end{array}$




$\begin{array}{rrrr}\mathrm{C} & -1.14989 & -2.35046 & -1.52661 \\ \mathrm{C} & -2.22007 & -3.22517 & -1.39821 \\ \mathrm{H} & -3.70378 & -4.02573 & -0.07107 \\ \mathrm{H} & -2.96951 & -2.69029 & 1.87655 \\ \mathrm{H} & -1.04762 & -1.12375 & 1.64757 \\ \mathrm{H} & -0.63068 & -2.24876 & -2.47348 \\ \mathrm{H} & -2.54603 & -3.81400 & -2.24619 \\ \mathrm{C} & 3.25521 & -0.40226 & 0.70664 \\ \mathrm{C} & 3.39257 & -0.70687 & 2.17714 \\ \mathrm{C} & 4.57950 & -0.19659 & 1.40804 \\ \mathrm{H} & 3.41508 & -1.74249 & 2.49559 \\ \mathrm{H} & 2.90853 & -0.02800 & 2.86953 \\ \mathrm{H} & 4.95437 & 0.80195 & 1.58238 \\ \mathrm{H} & 5.35481 & -0.92101 & 1.18319 \\ \mathrm{C} & 2.00051 & 2.11235 & 1.10252 \\ \mathrm{C} & 0.80521 & 2.81180 & 0.86656 \\ \mathrm{C} & 2.86326 & 2.65981 & 2.05796 \\ \mathrm{C} & 0.48977 & 3.98929 & 1.53297 \\ \mathrm{H} & 0.10881 & 2.42746 & 0.12659 \\ \mathrm{C} & 2.56304 & 3.84386 & 2.73260 \\ \mathrm{H} & 3.79821 & 2.16896 & 2.29652 \\ \mathrm{C} & 1.37536 & 4.51509 & 2.47184 \\ \mathrm{H} & -0.44262 & 4.50111 & 1.32102 \\ \mathrm{H} & 3.26038 & 4.23883 & 3.46329 \\ \mathrm{H} & 1.13873 & 5.43478 & 2.99451 \\ \mathrm{H} & 2.25159 & 1.38712 & -3.77732 \\ \mathrm{H} & 3.60472 & 0.31684 & -3.36478 \\ \mathrm{H} & 3.92153 & 1.95721 & -3.97072 \\ & \mathrm{G}=-1029.341959 \mathrm{Hartree} \\ \mathrm{H} & & & \end{array}$

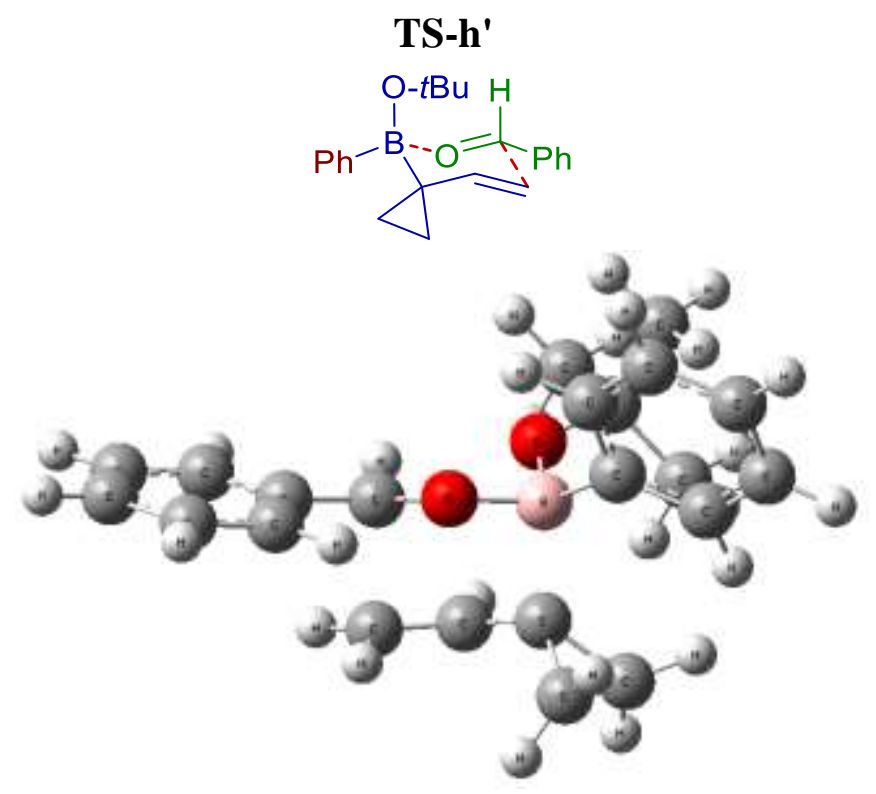




\begin{tabular}{|c|c|c|c|}
\hline B & 2.17166 & 0.77307 & 0.14349 \\
\hline $\mathrm{C}$ & 3.34619 & 1.43142 & -3.35049 \\
\hline $\mathrm{C}$ & 3.27091 & 1.89550 & -1.89609 \\
\hline $\mathrm{C}$ & 2.70924 & 3.32001 & -1.84288 \\
\hline $\mathrm{H}$ & 2.70773 & 3.71036 & -0.82427 \\
\hline $\mathrm{H}$ & 3.31236 & 3.98332 & -2.46882 \\
\hline $\mathrm{H}$ & 1.68235 & 3.32490 & -2.21613 \\
\hline $\mathrm{C}$ & 4.66522 & 1.85310 & -1.27017 \\
\hline $\mathrm{H}$ & 5.05171 & 0.83064 & -1.26544 \\
\hline $\mathrm{H}$ & 5.35104 & 2.48560 & -1.83941 \\
\hline $\mathrm{H}$ & 4.63755 & 2.21768 & -0.24189 \\
\hline $\mathrm{O}$ & 2.38857 & 0.97728 & -1.26532 \\
\hline $\mathrm{C}$ & 3.11336 & -1.51493 & -0.16737 \\
\hline $\mathrm{C}$ & 2.05478 & -2.39340 & -0.09357 \\
\hline $\mathrm{H}$ & 1.92224 & -3.15439 & -0.85443 \\
\hline $\mathrm{H}$ & 1.60959 & -2.59301 & 0.87578 \\
\hline $\mathrm{H}$ & 3.67665 & -1.45907 & -1.09804 \\
\hline $\mathrm{C}$ & 0.69653 & -0.98355 & -0.59667 \\
\hline $\mathrm{O}$ & 0.90212 & -0.02744 & 0.25393 \\
\hline $\mathrm{H}$ & 1.03262 & -0.81859 & -1.61899 \\
\hline $\mathrm{C}$ & -2.88401 & -3.26277 & -0.18186 \\
\hline $\mathrm{C}$ & -2.41909 & -2.50521 & 0.89134 \\
\hline $\mathrm{C}$ & -1.25186 & -1.76347 & 0.76812 \\
\hline $\mathrm{C}$ & -0.54658 & -1.77698 & -0.43602 \\
\hline $\mathrm{C}$ & -1.01366 & -2.53336 & -1.51069 \\
\hline $\mathrm{C}$ & -2.18148 & -3.27529 & -1.38352 \\
\hline $\mathrm{H}$ & -3.79573 & -3.83957 & -0.08246 \\
\hline $\mathrm{H}$ & -2.97033 & -2.49258 & 1.82380 \\
\hline $\mathrm{H}$ & -0.88140 & -1.16451 & 1.59156 \\
\hline $\mathrm{H}$ & -0.46167 & -2.53616 & -2.44511 \\
\hline $\mathrm{H}$ & -2.54445 & -3.85943 & -2.22037 \\
\hline $\mathrm{C}$ & 3.29068 & -0.45428 & 0.77070 \\
\hline $\mathrm{C}$ & 3.43121 & -0.67310 & 2.24186 \\
\hline $\mathrm{C}$ & 4.61710 & -0.20105 & 1.42330 \\
\hline $\mathrm{H}$ & 3.45492 & -1.68928 & 2.61960 \\
\hline $\mathrm{H}$ & 2.96870 & 0.05272 & 2.90080 \\
\hline $\mathrm{H}$ & 4.98630 & 0.80410 & 1.56082 \\
\hline $\mathrm{H}$ & 5.39779 & -0.92941 & 1.23507 \\
\hline $\mathrm{C}$ & 1.95625 & 2.08046 & 1.07322 \\
\hline $\mathrm{C}$ & 0.68576 & 2.67728 & 1.01479 \\
\hline $\mathrm{C}$ & 2.91176 & 2.72881 & 1.8635 \\
\hline $\mathrm{C}$ & 0.39082 & 3.85993 & 1.6839 \\
\hline $\mathrm{H}$ & -0.08779 & 2.20580 & 0.4175 \\
\hline $\mathrm{C}$ & 2.62912 & 3.91307 & $2.5432^{\prime}$ \\
\hline $\mathrm{H}$ & 3.91051 & 2.32373 & 1.9607 \\
\hline $\mathrm{C}$ & 1.36735 & 4.48834 & 2.4518 \\
\hline
\end{tabular}




\begin{tabular}{llll}
$\mathrm{H}$ & -0.60031 & 4.29342 & 1.60512 \\
$\mathrm{H}$ & 3.39903 & 4.38559 & 3.14315 \\
$\mathrm{H}$ & 1.14571 & 5.41145 & 2.97502 \\
$\mathrm{H}$ & 2.34786 & 1.44241 & -3.79427 \\
$\mathrm{H}$ & 3.73410 & 0.41073 & -3.39535 \\
$\mathrm{H}$ & 3.99822 & 2.08206 & -3.93774 \\
\multicolumn{4}{l}{$\mathrm{G}=-1029.325927$ Hartree }
\end{tabular}




\section{References}

1. Hari, D. P.; Abell, J. C.; Fasano, V.; Aggarwal, V. K. Ring-Expansion Induced 1,2-Metalate Rearrangements: Highly Diastereoselective Synthesis of Cyclobutyl Boronic Esters. J. Am. Chem. Soc. 2020, 142, 5515.

2. Synthesized using the procedure reported in the reference: Fabre, J. M.; Garín, J.; Uriel, S. The Synthesis of Primary, Secondary and Tertiary Aminomethyltetrathiafulvalenes. Tetrahedron 1992, 48, 3983.

3. Maercker, A.; Daub, V. E. E. Polylithiumorganic Compounds - 19. Regioselective CarbonCarbon $\sigma$-Bond Scission Followed by a 1,6-Proton Shift upon the Reductive Metalation of Benzylidenecyclopropane Derivatives with Lithium Metal. Tetrahedron 1994, 50, 2439. 4. Chen, J. L. Y.; Scott, H. K.; Hesse, M. J.; Willis, C. L.; Aggarwal, V. K. Highly Diastereoand Enantioselective Allylboration of Aldehydes Using $\alpha$-Substituted Allyl/Crotyl Pinacol Boronic Esters via in Situ Generated Borinic Esters. J. Am. Chem. Soc. 2013, 135, 5316. 5. Bruker, SAINT + v8.38A Integration Engine, Data Reduction Software, Bruker Analytical X-ray Instruments Inc., Madison, WI, USA 2015.

6. Bruker, SADABS 2014/5, Bruker AXS area detector scaling and absorption correction, Bruker Analytical X-ray Instruments Inc., Madison, Wisconsin, USA 2014/5.

7. Sheldrick, G. M., SHELXT - Integrated space-group and crystal-structure determination. Acta Crystallogr., Sect. A 2015, 71, 3.

8. Sheldrick, G. M., A short history of SHELX. Acta Crystallogr., Sect. A 2008, 64, 112 9. Sheldrick, G. M., Crystal structure refinement with SHELXL. Acta Crystallogr., Sect. C 2015, 71, 3 .

10. Dolomanov, O. V.; Bourhis, L. J.; Gildea, R. J.; Howard, J. A. K.; Puschmann, H., OLEX2: a complete structure solution, refinement and analysis program. J. Appl. Crystallogr. 2009, 42, 339.

11.Gaussian 09, Revision C1, Frisch, M. J.; Trucks, G. W.; Schlegel, H. B.; Scuseria, G. E.; Robb, M. A.; Cheeseman, J. R.; Scalmani, G.; Barone, V.; Mennucci, B.; Petersson, G. A.; Nakatsuji, H.; Caricato, M.; Li, X.; Hratchian, H. P.; Izmaylov, A. F.; Bloino, J.; Zheng, G.; Sonnenberg, J. L.; Hada, M.; Ehara, M.; Toyota, K.; Fukuda, R.; Hasegawa, J.; Ishida, M.; Nakajima, T.; Honda, Y.; Kitao, O.; Nakai, H.; Vreven, T.; Montgomery, Jr., J. A.; Peralta, J. E.; Ogliaro, F.; Bearpark, M.; Heyd, J. J.; Brothers, E.; Kudin, K. N.; Staroverov, V. N.; Kobayashi, R.; Normand, J.; Raghavachari, K.; Rendell, A.; Burant, J. C.; Iyengar, S. S.; Tomasi, J.; Cossi, M.; Rega, N.; Millam, J. M.; Klene, M.; Knox, J. E.; Cross, J. B.; Bakken, V.; Adamo, C.; Jaramillo, J.; Gomperts, R.; Stratmann, R. E.; Yazyev, O.; Austin, A. J.; Cammi, R.; Pomelli, C.; Ochterski, J. W.; Martin, R. L.; Morokuma, K.; Zakrzewski, V. G.; Voth, G. A.; Salvador, P.; Dannenberg, J. J.; Dapprich, S.; Daniels, A. D.; Farkas, Ö.; Foresman, J. B.; Ortiz, J. V.; Cioslowski, J.; Fox, D. J. Gaussian, Inc., Wallingford CT, 2009. 12. http://comp.chem.umn.edu/info/DFT.htm. 


\section{NMR spectra of new compounds}

${ }^{1} \mathbf{H}-\mathrm{NMR}\left(400 \mathrm{MHz}, \mathrm{CDCl}_{3}\right)$ of compound 1c see procedure

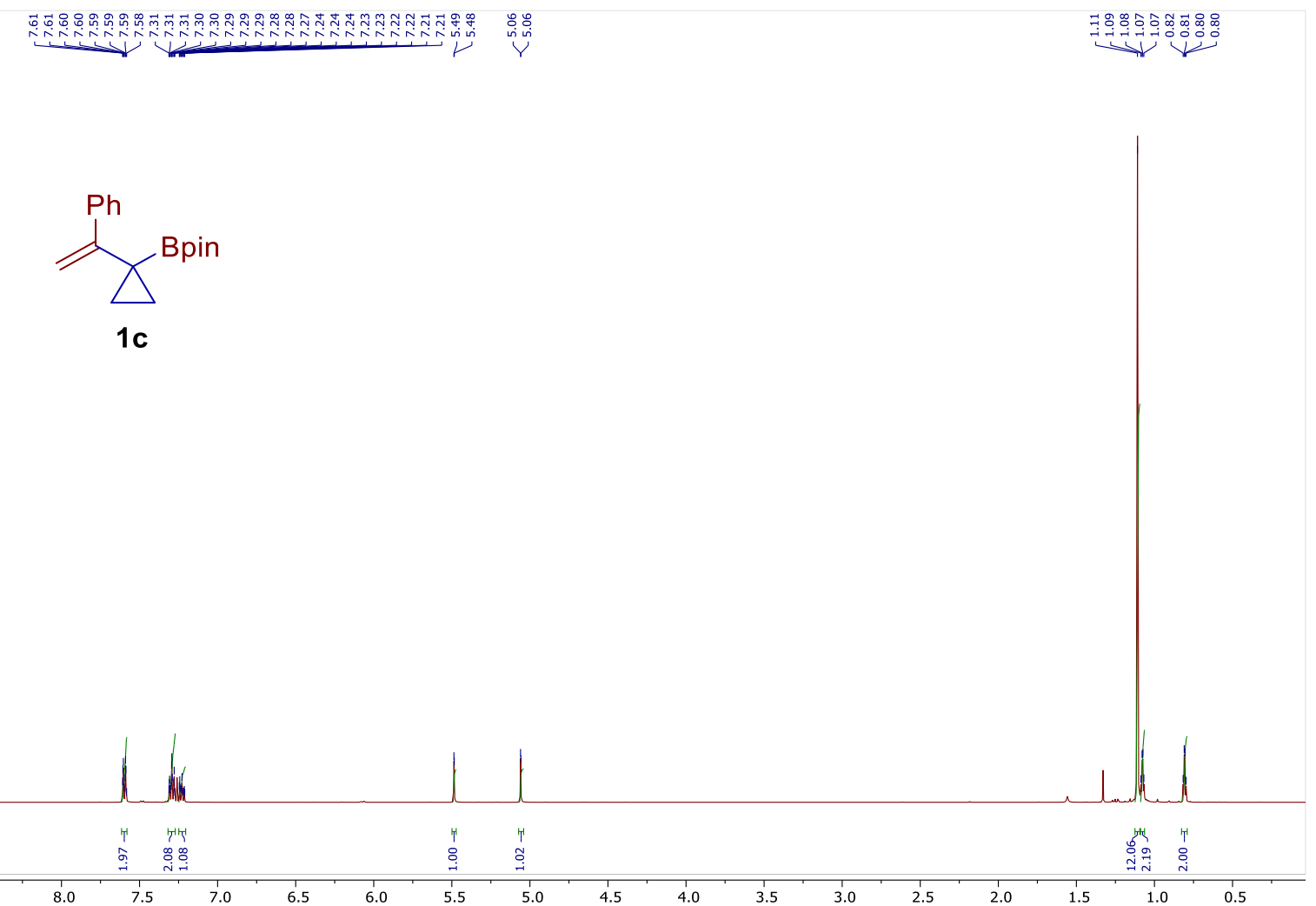

${ }^{\mathbf{1 3}} \mathbf{C}-\mathbf{N M R}\left(101 \mathrm{MHz}, \mathrm{CDCl}_{3}\right)$ of compound 1c

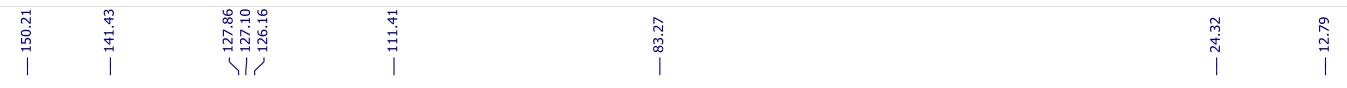

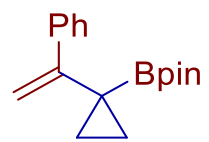

$1 \mathrm{c}$

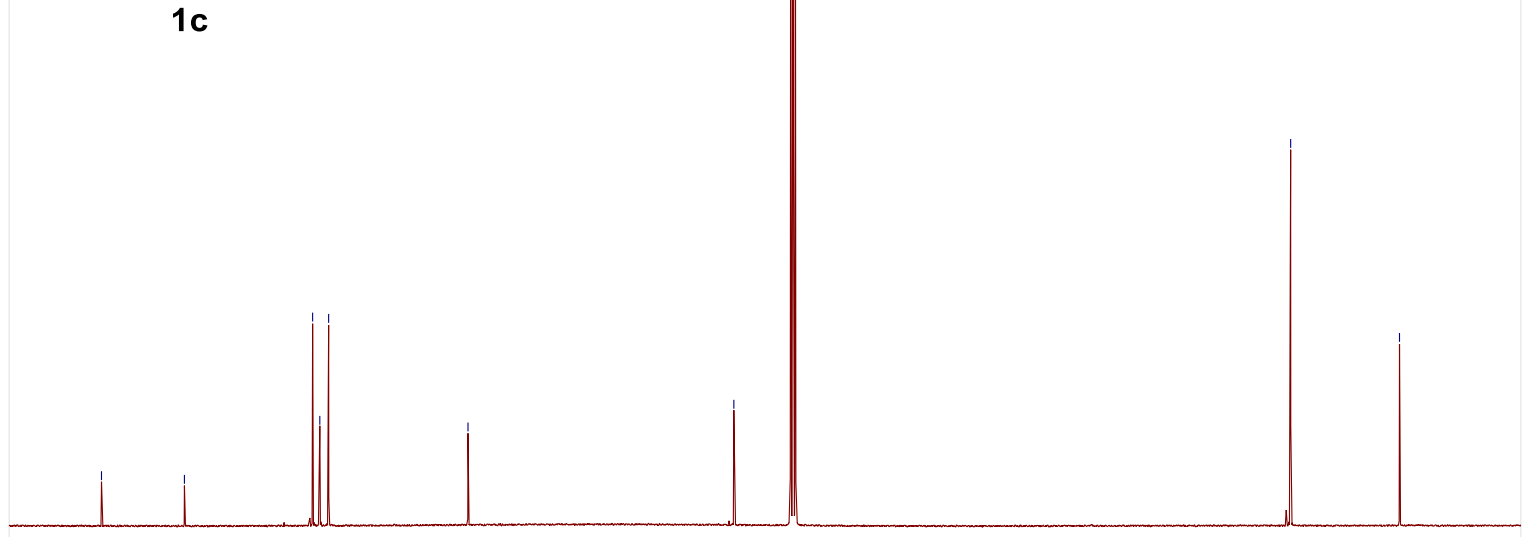

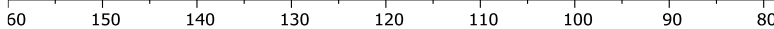


${ }^{\mathbf{1}} \mathbf{H}-\mathrm{NMR}\left(400 \mathrm{MHz}, \mathrm{CDCl}_{3}\right)$ of compound 1d' see procedure

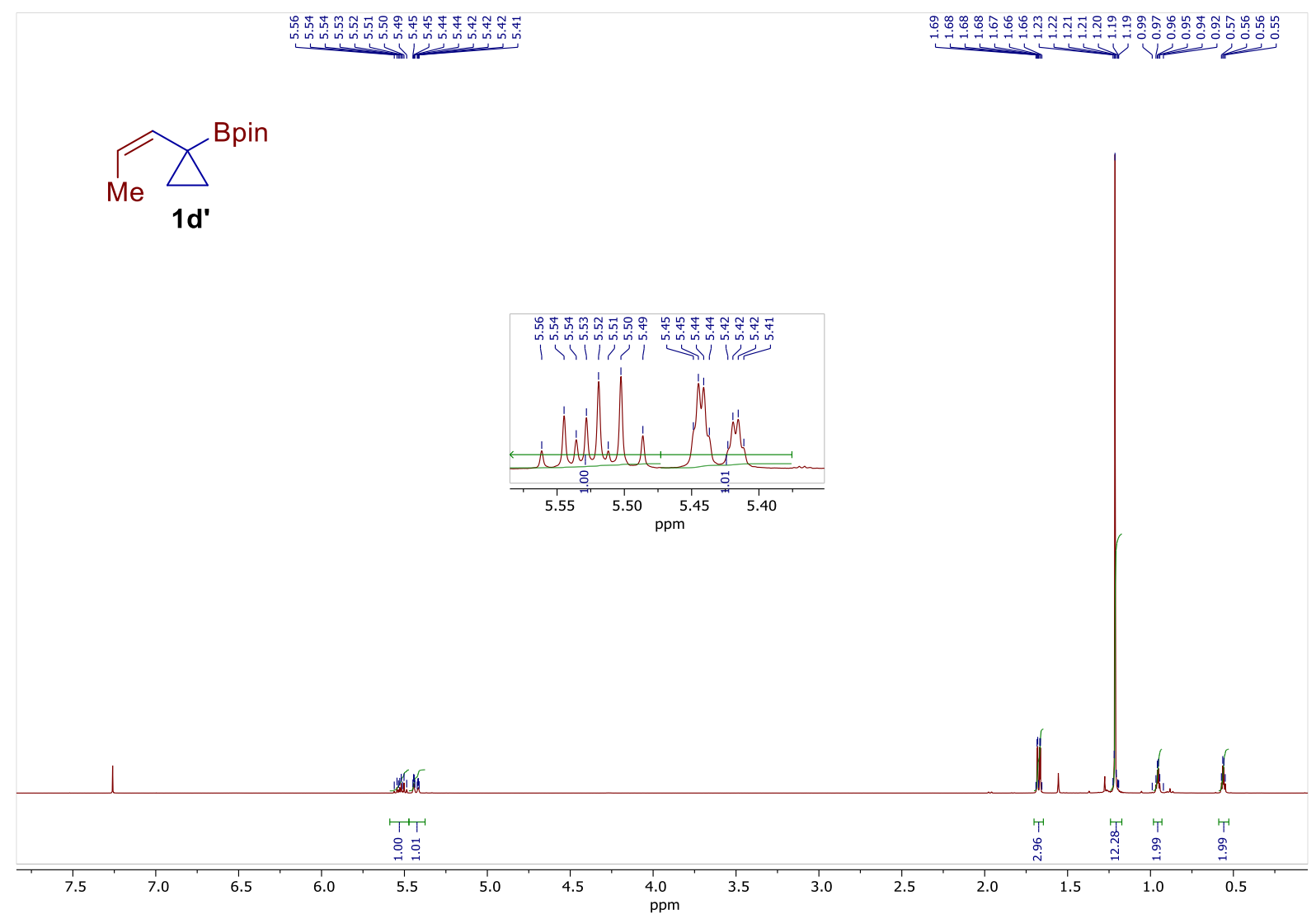

${ }^{13} \mathbf{C}-\mathbf{N M R}\left(101 \mathrm{MHz}, \mathrm{CDCl}_{3}\right)$ of compound 1d'

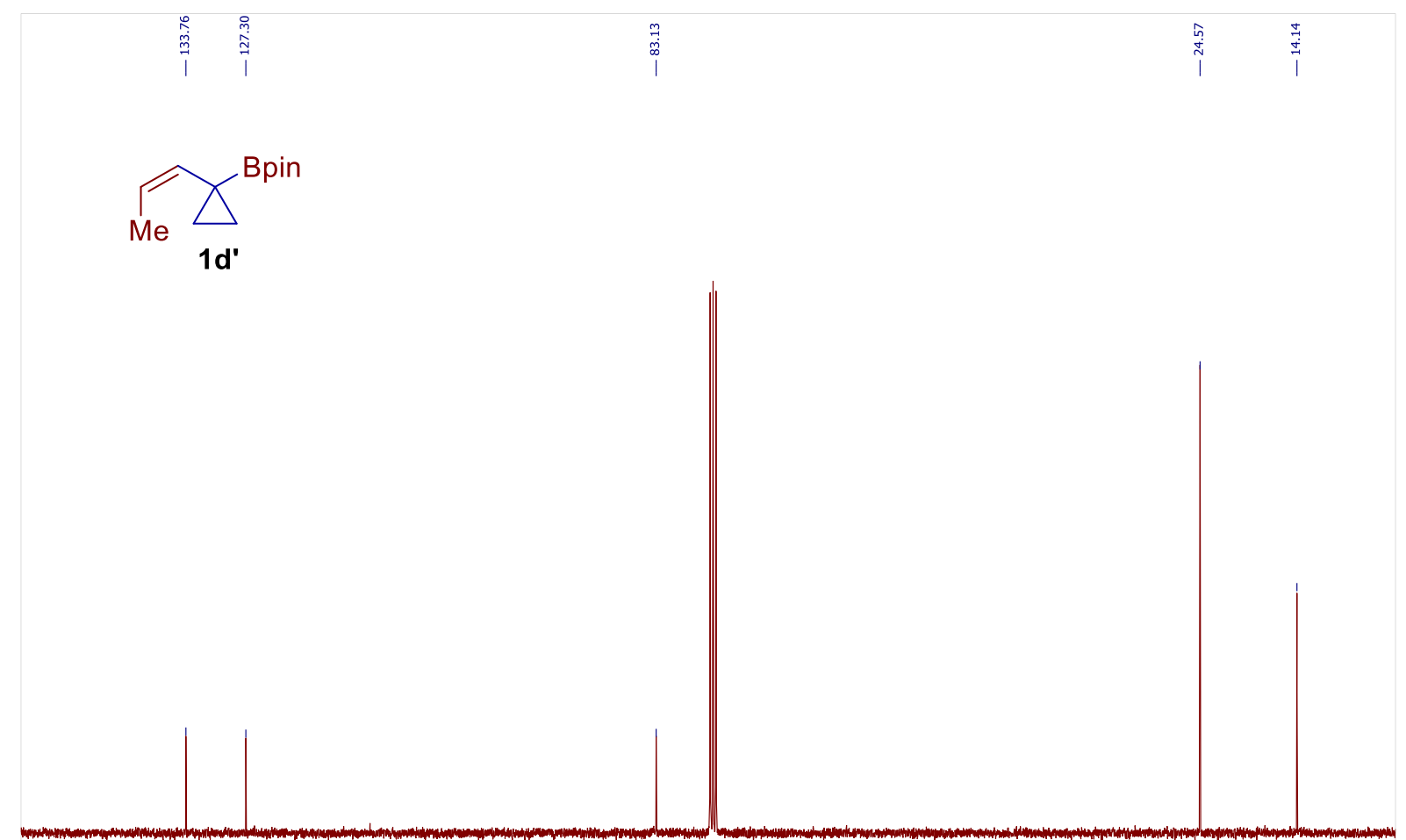

$\begin{array}{lllllllllllllllllllllllllllllllllllllllllllllllll}150 & 145 & 140 & 135 & 130 & 125 & 120 & 115 & 110 & 105 & 100 & 95 & 90 & 85 & 80 & 75 & 70 & 65 & 60 & 55 & 50 & 45 & 40 & 35 & 30 & 25 & 20 & 15 & 10 & 5\end{array}$ 
${ }^{\mathbf{1}} \mathbf{H}-\mathrm{NMR}\left(400 \mathrm{MHz}, \mathrm{CDCl}_{3}\right)$ of compound $\mathbf{1 f}$ see procedure

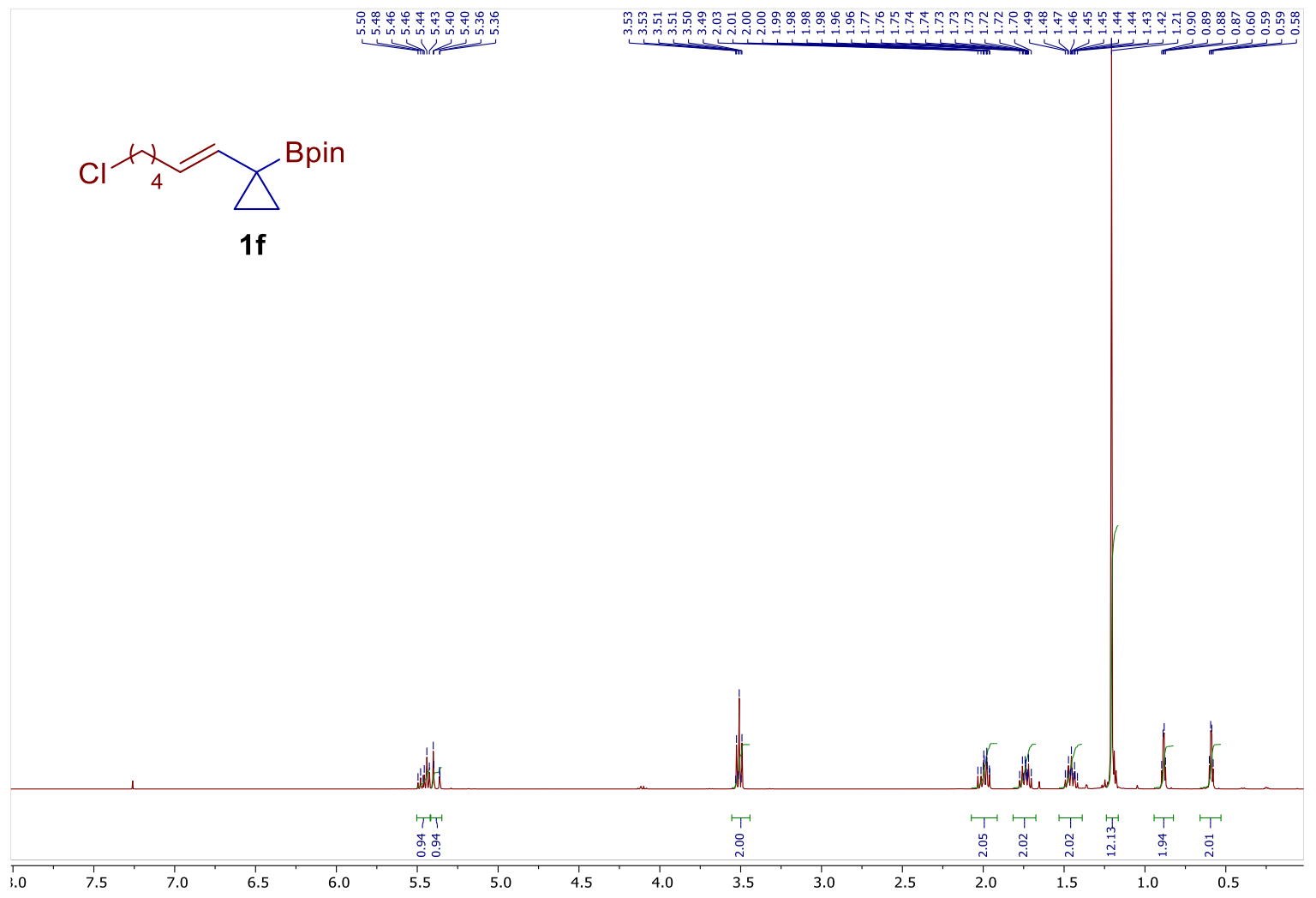

${ }^{\mathbf{1 3}} \mathbf{C}-\mathbf{N M R}\left(101 \mathrm{MHz}, \mathrm{CDCl}_{3}\right)$ of compound $\mathbf{1 f}$

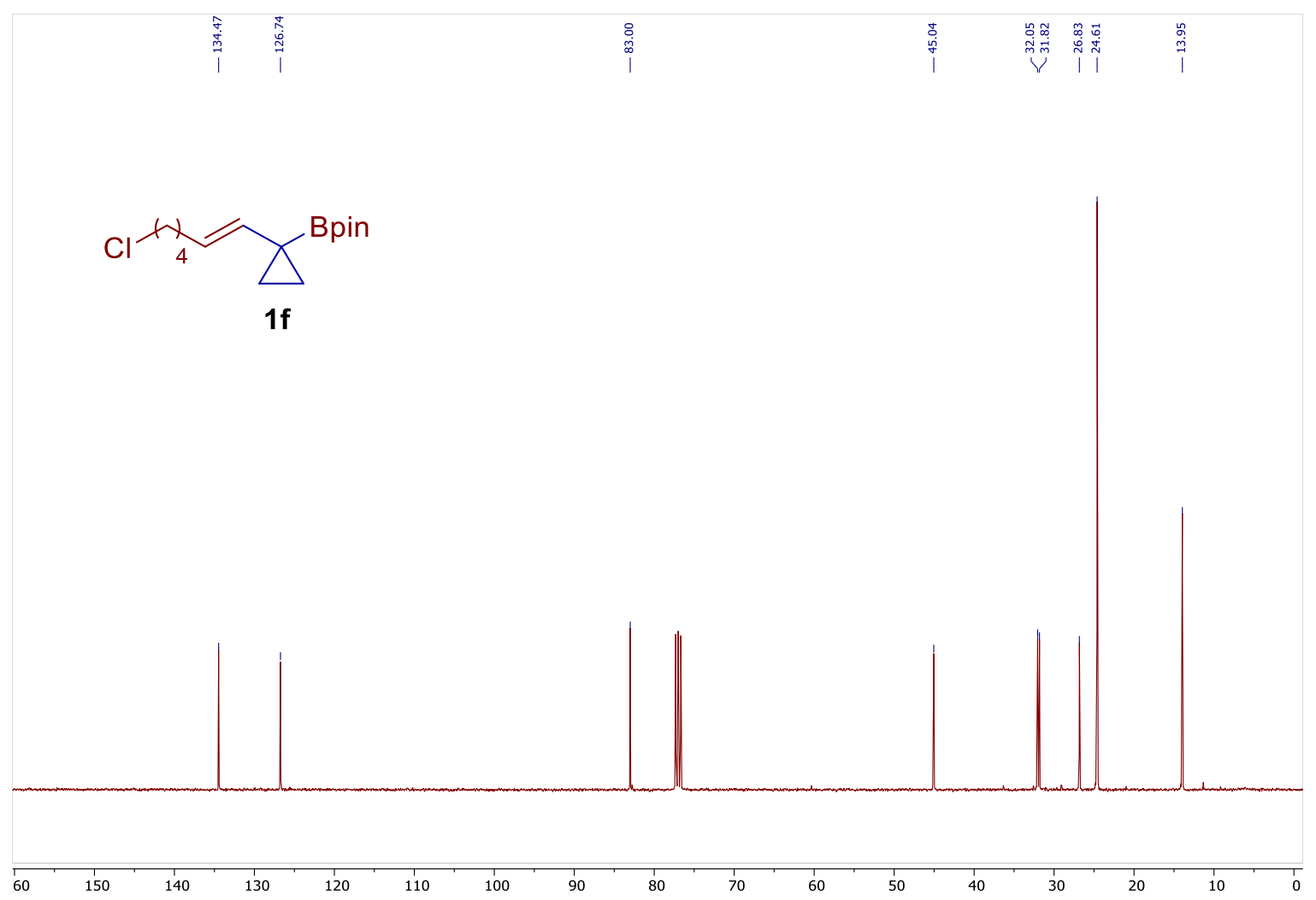


${ }^{\mathbf{1}} \mathbf{H}-\mathrm{NMR}\left(400 \mathrm{MHz}, \mathrm{CDCl}_{3}\right)$ of compound $\mathbf{1 h}$ ' see procedure

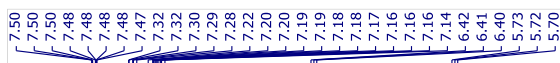

$\overbrace{\mathrm{Ph}}^{\mathrm{Bpin}}$

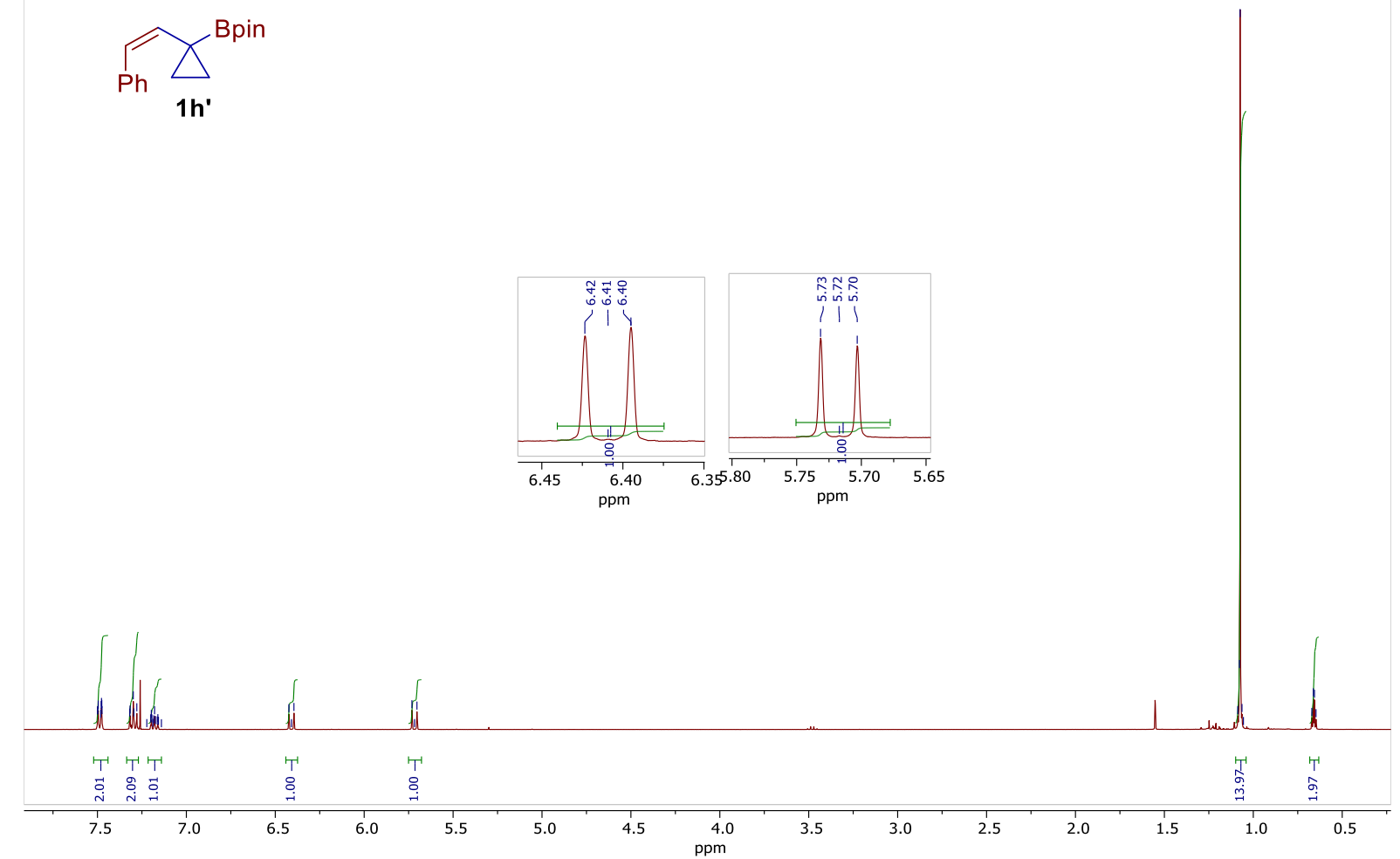

${ }^{13} \mathbf{C}-\mathbf{N M R}\left(101 \mathrm{MHz}, \mathrm{CDCl}_{3}\right)$ of compound $\mathbf{1 h}$

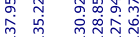

।

$\triangle_{\mathrm{Ph}}^{\mathrm{Bpin}}$
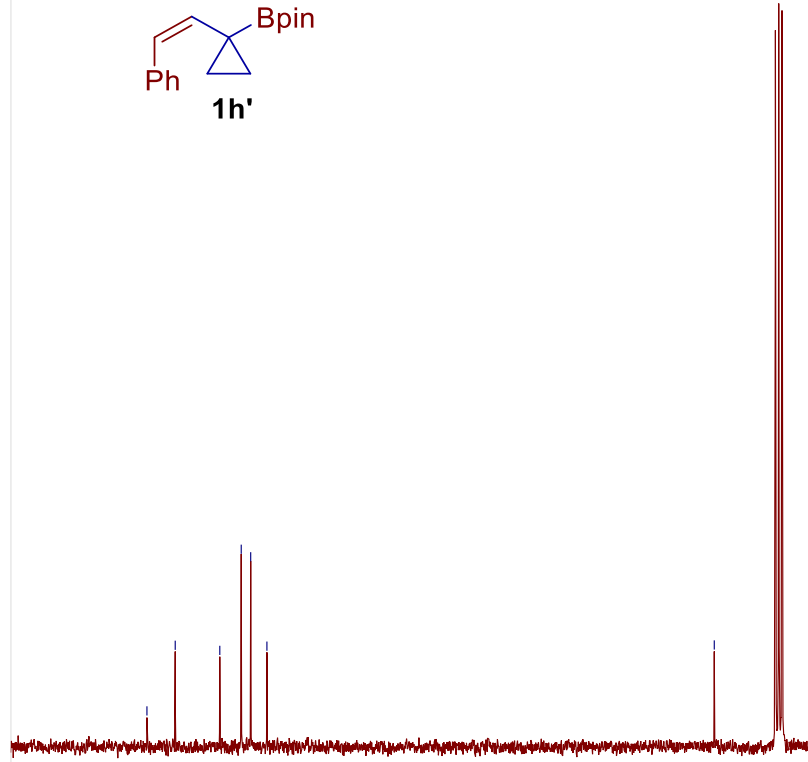

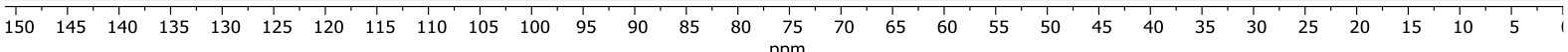


${ }^{\mathbf{1}} \mathbf{H}-\mathrm{NMR}\left(400 \mathrm{MHz}, \mathrm{CDCl}_{3}\right)$ of compound $\mathbf{1 i}$ see procedure

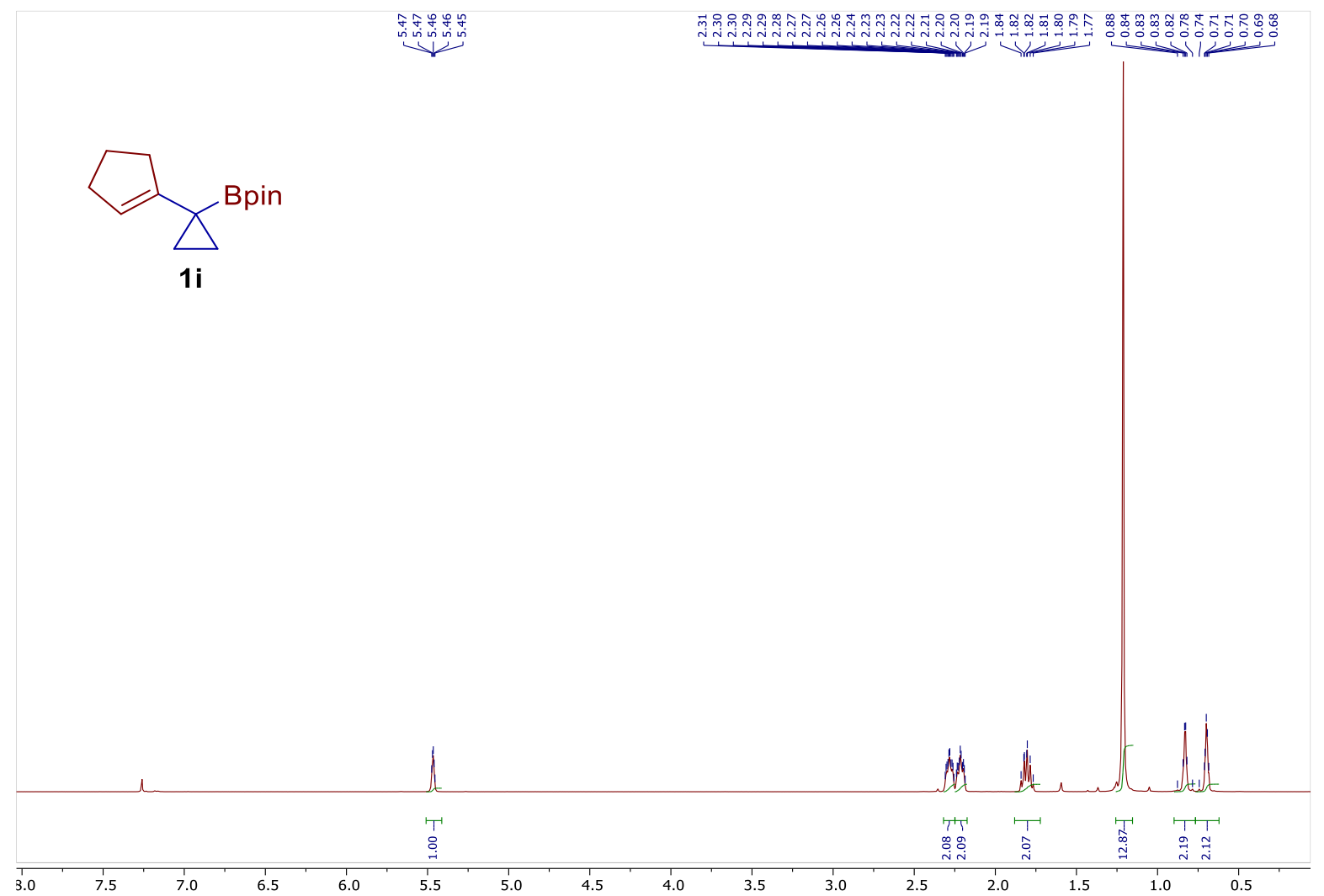

${ }^{13} \mathbf{C}-\mathbf{N M R}\left(101 \mathrm{MHz}, \mathrm{CDCl}_{3}\right)$ of compound $\mathbf{1 i}$

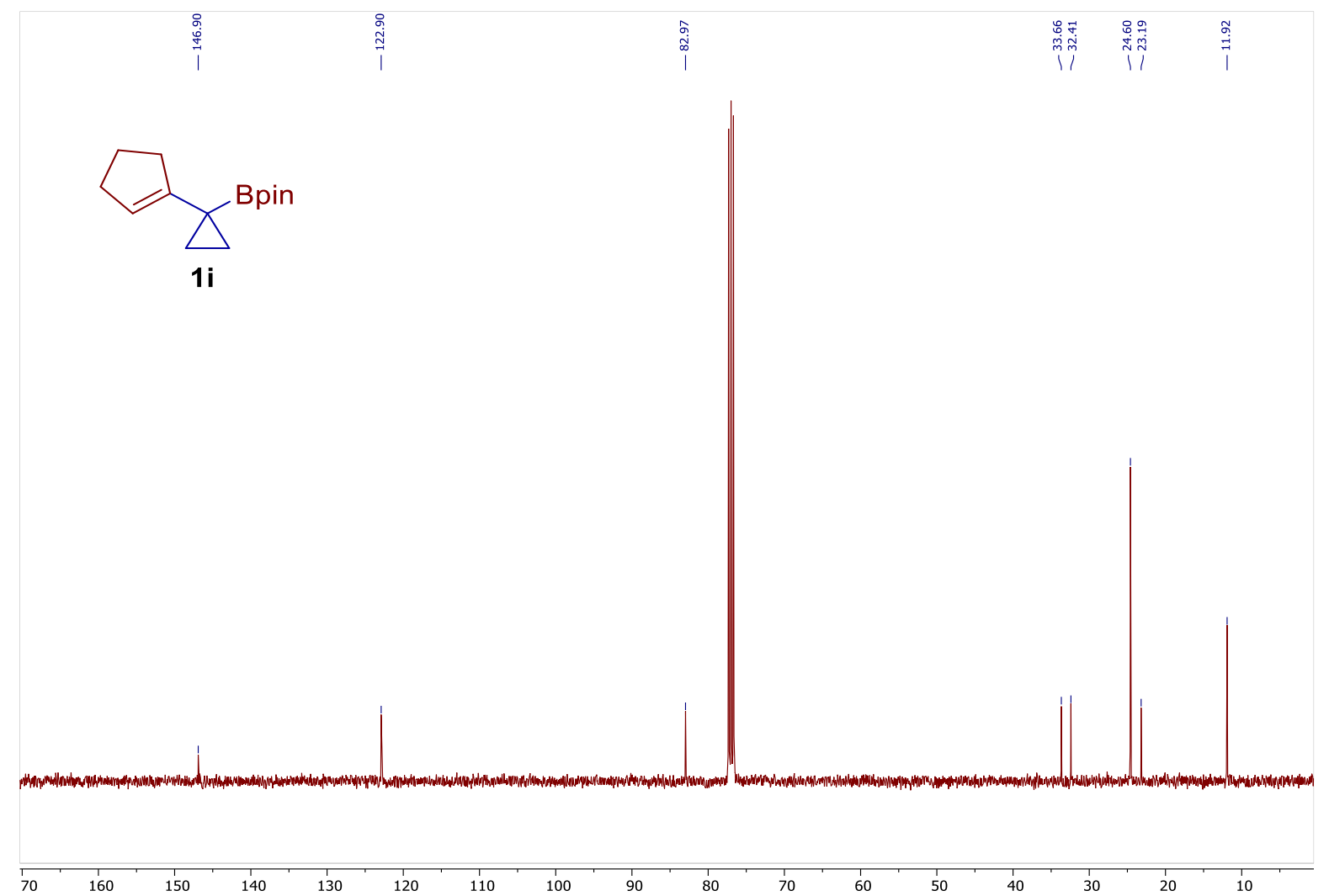


${ }^{1} \mathbf{H}-\mathrm{NMR}\left(500 \mathrm{MHz}, \mathrm{CDCl}_{3}\right)$ of compound $1 \mathbf{k}$ see procedure

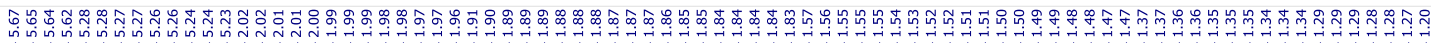<smiles>C=C[C@H]1CCCCC1[Pb]</smiles>

$1 \mathrm{k}$
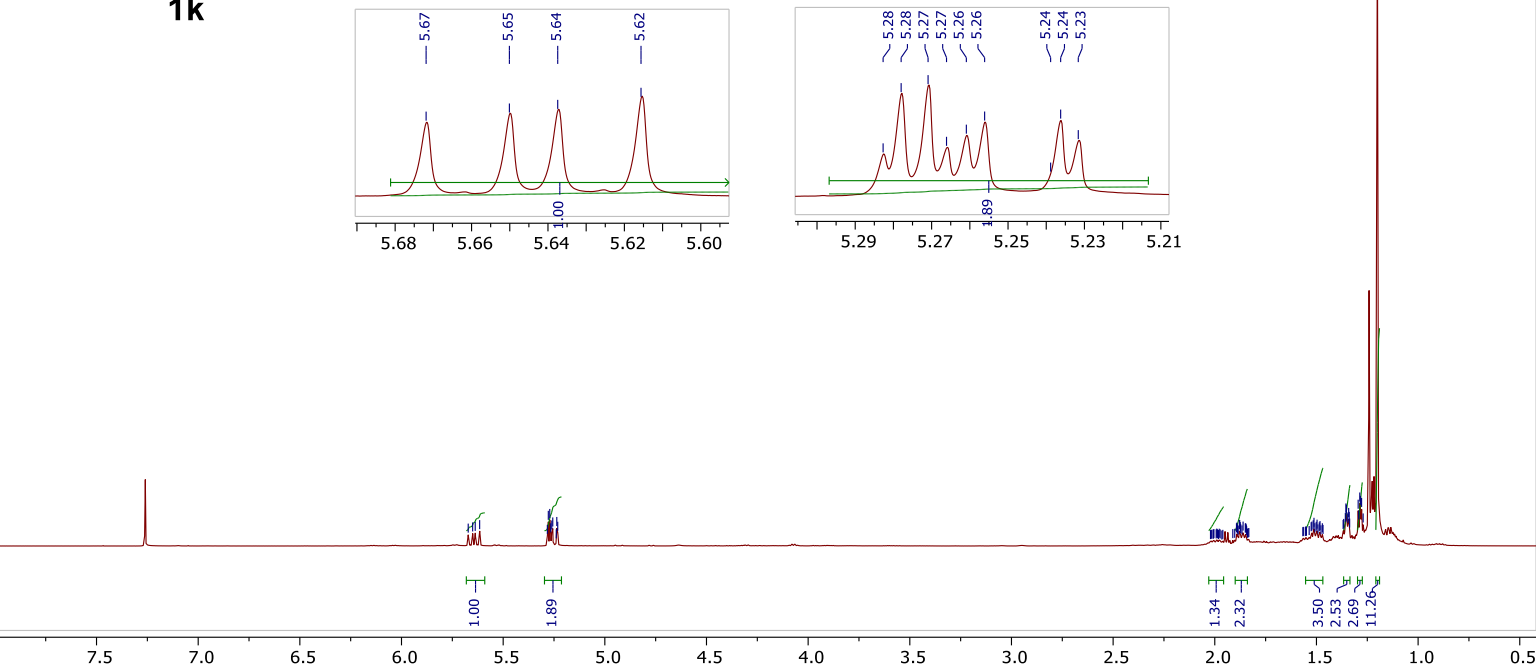

${ }^{13} \mathbf{C}-\mathbf{N M R}\left(126 \mathrm{MHz}, \mathrm{CDCl}_{3}\right)$ of compound $\mathbf{1 k}$

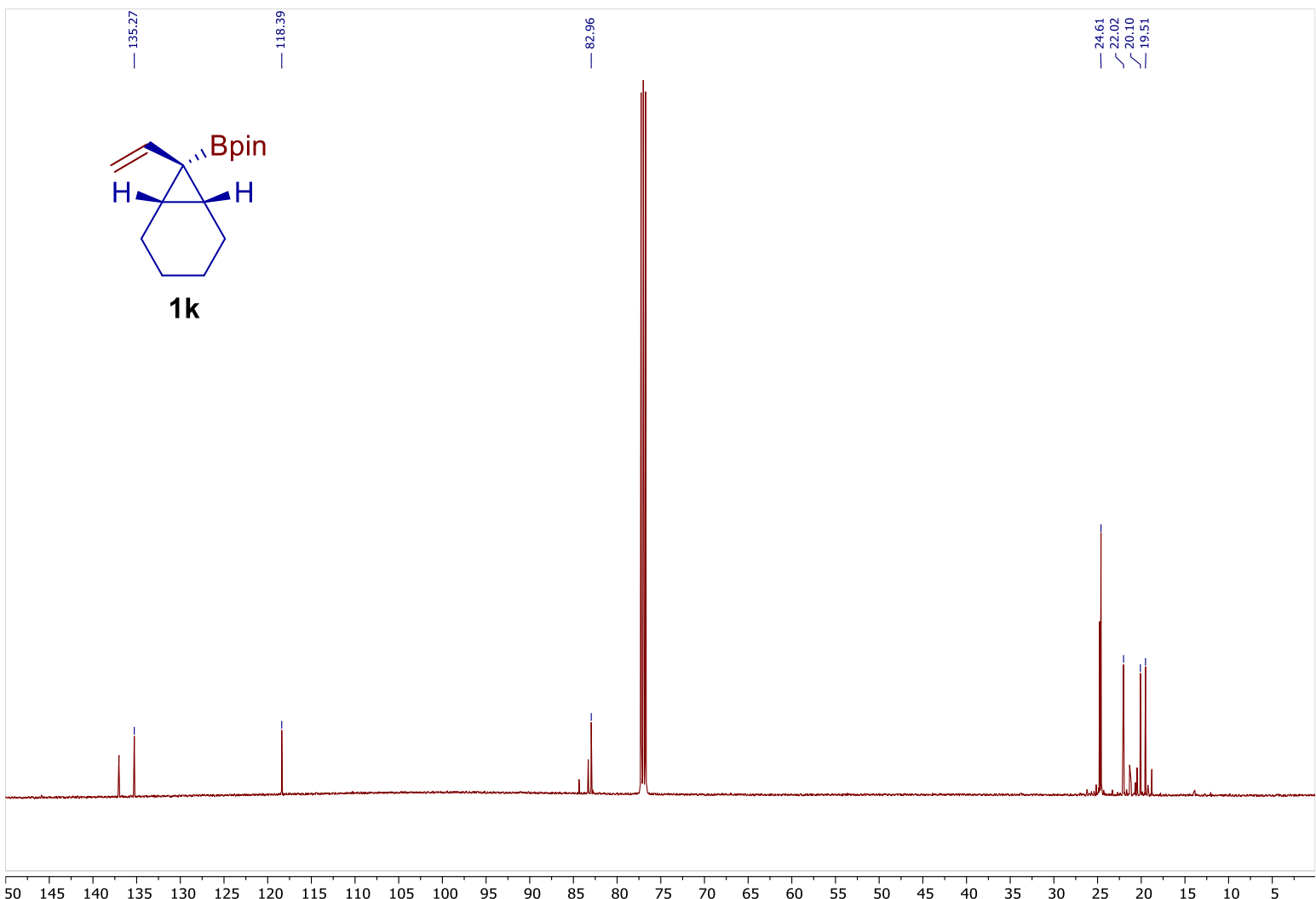


${ }^{1} \mathbf{H}-\mathrm{NMR}\left(500 \mathrm{MHz}, \mathrm{CDCl}_{3}\right)$ of compound 14a see procedure

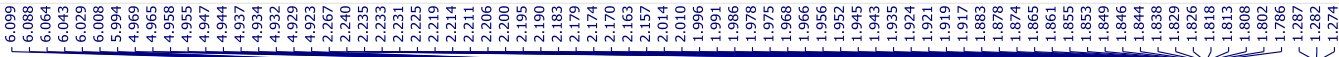
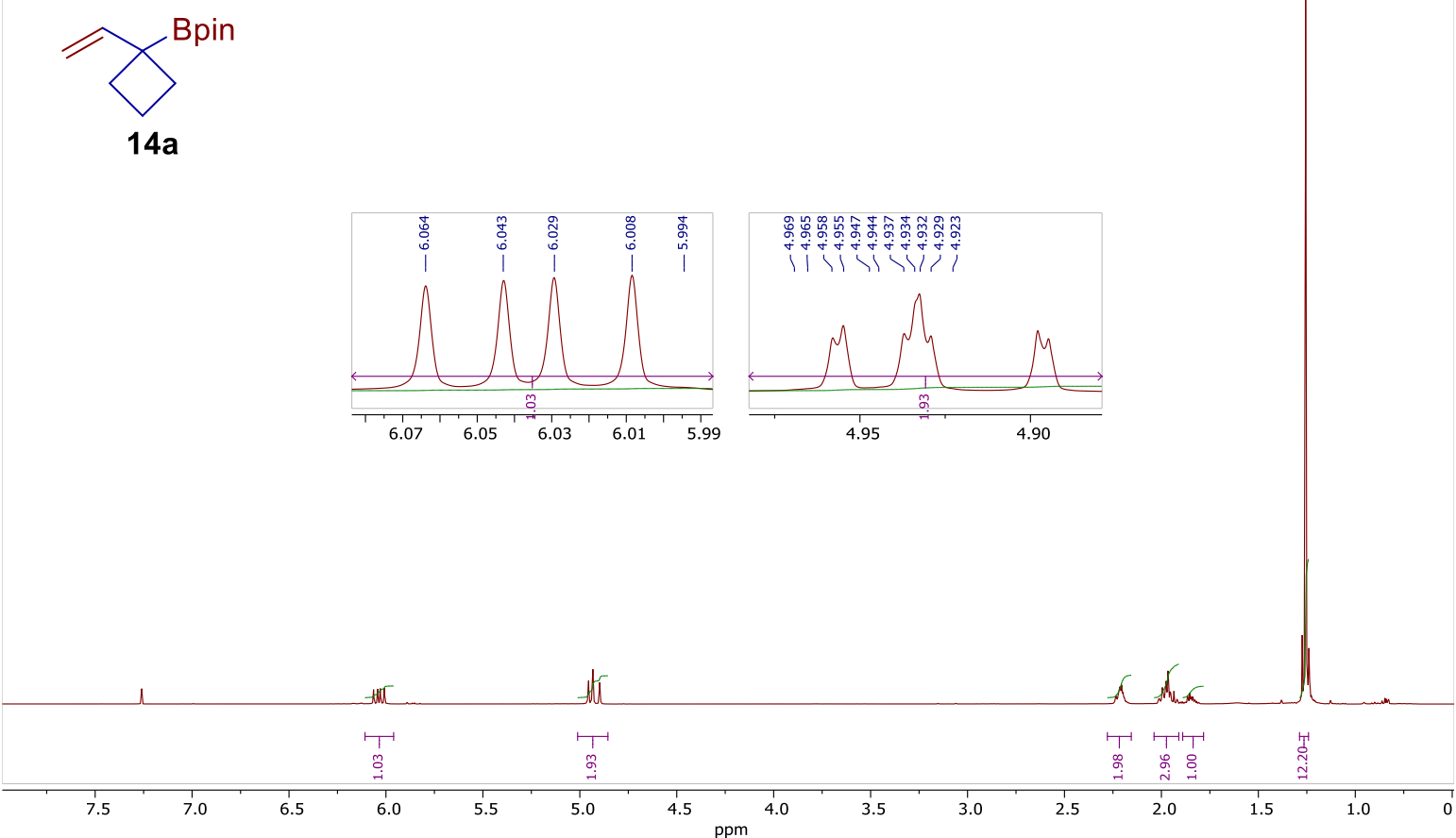

${ }^{13} \mathbf{C}-\mathbf{N M R}\left(126 \mathrm{MHz}, \mathrm{CDCl}_{3}\right)$ of compound $\mathbf{1 4 a}$

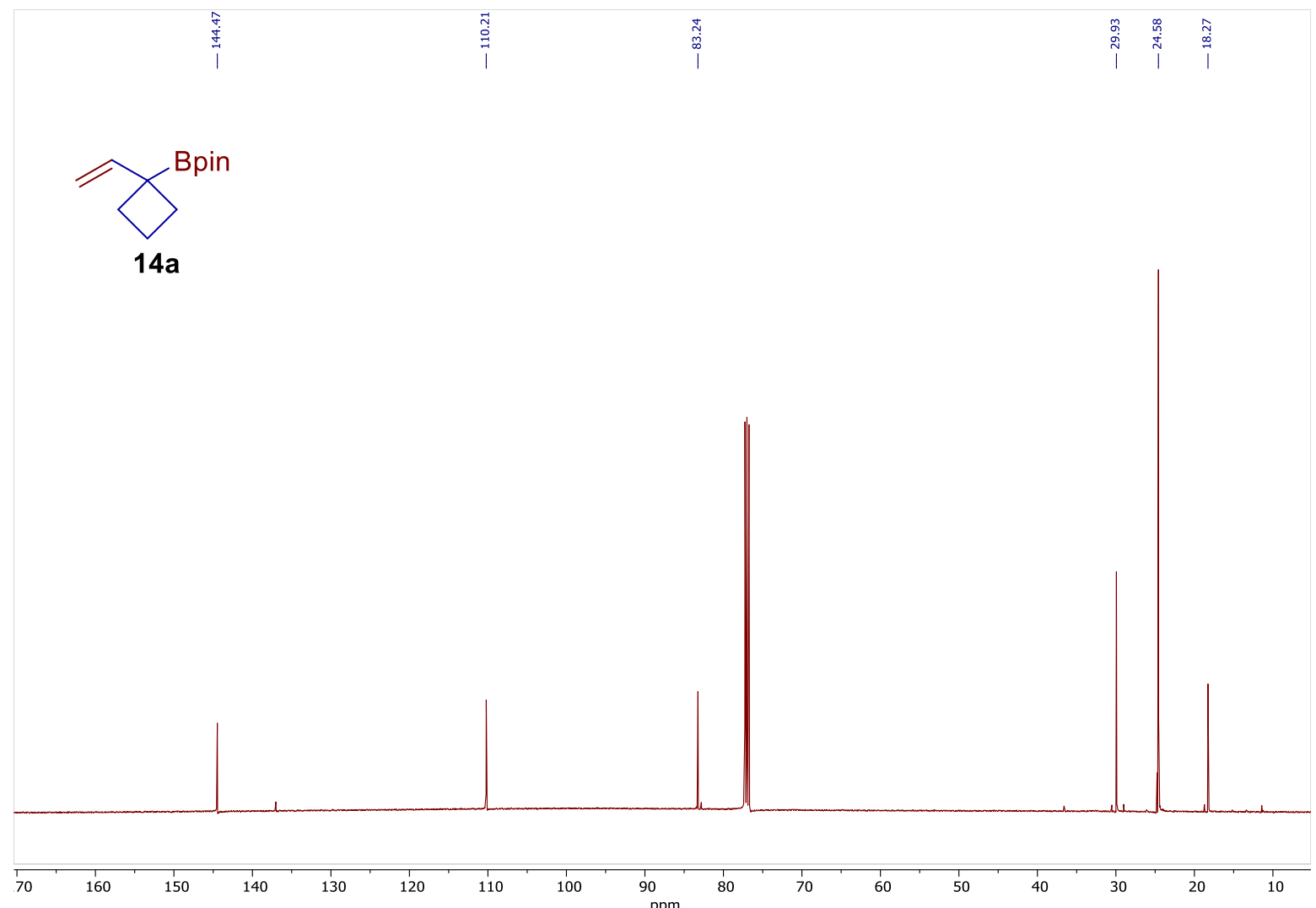


${ }^{1} \mathbf{H}-\mathrm{NMR}\left(500 \mathrm{MHz}, \mathrm{CDCl}_{3}\right)$ of compound $\mathbf{1 4 b}$ see procedure

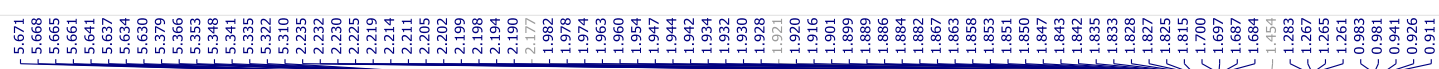<smiles>C/C=C/C1(Cc2ccccc2)CCC1</smiles>
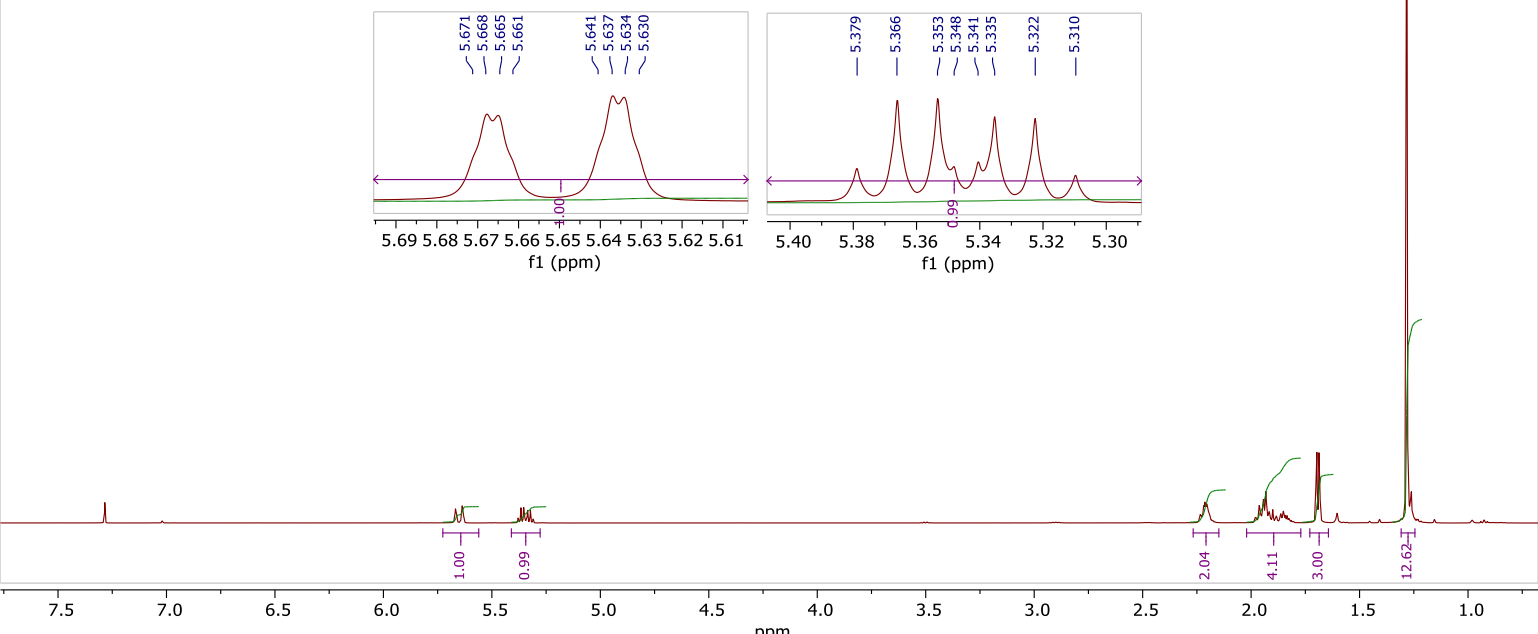

${ }^{13} \mathbf{C}-\mathbf{N M R}\left(126 \mathrm{MHz}, \mathrm{CDCl}_{3}\right)$ of compound $\mathbf{1 4 b}$

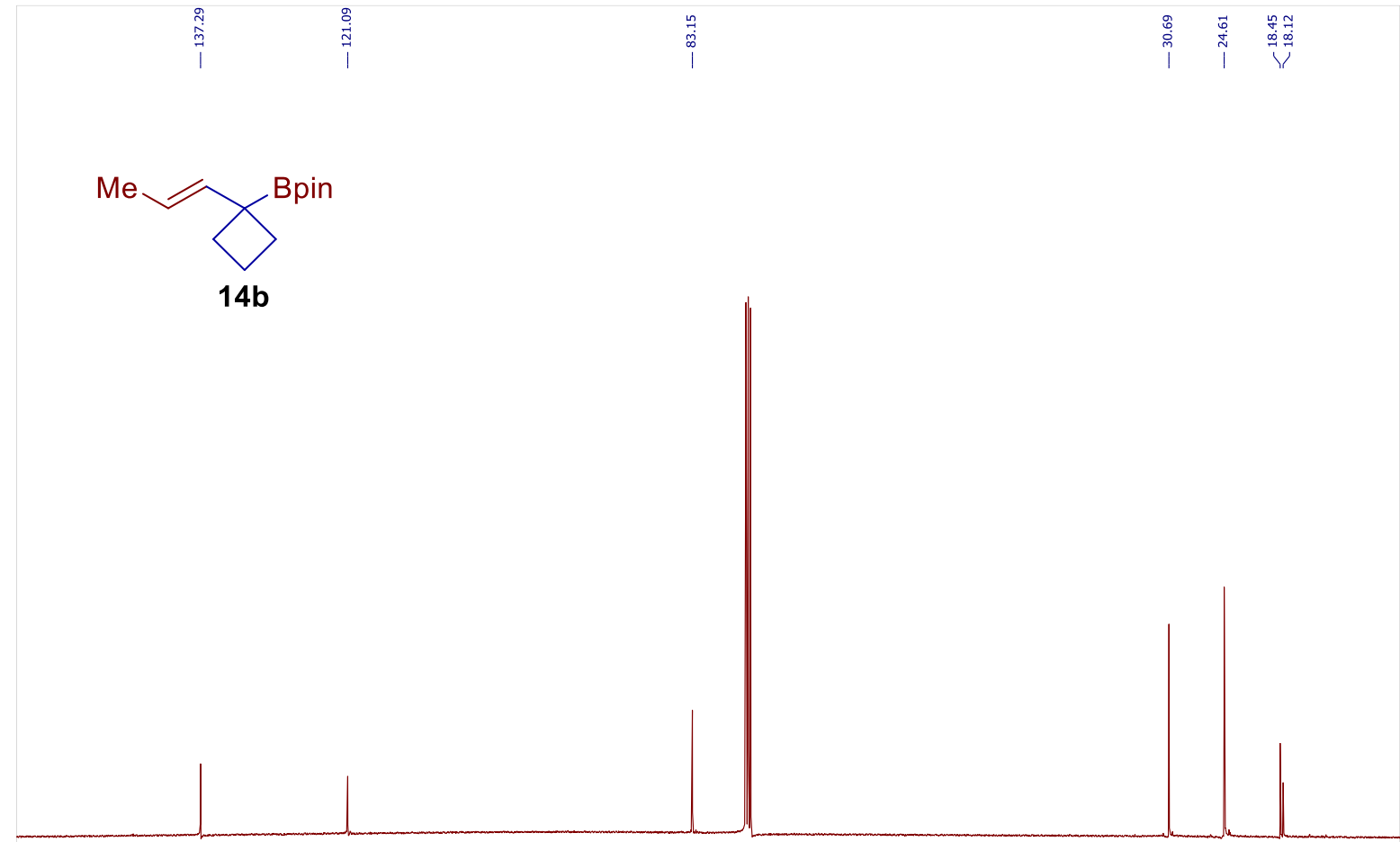

$\begin{array}{lllllllllllllllllllllllllllllllllllllllllllllll}155 & 150 & 145 & 140 & 135 & 130 & 125 & 120 & 115 & 110 & 105 & 100 & 95 & 90 & 85 & 80 & 75 & 70 & 65 & 60 & 55 & 50 & 45 & 40 & 35 & 30 & 25 & 20 & 15 & 10\end{array}$ 
${ }^{\mathbf{1}} \mathbf{H}-\mathrm{NMR}\left(500 \mathrm{MHz}, \mathrm{CDCl}_{3}\right)$ of compound $\mathbf{1 4 c}$ see procedure

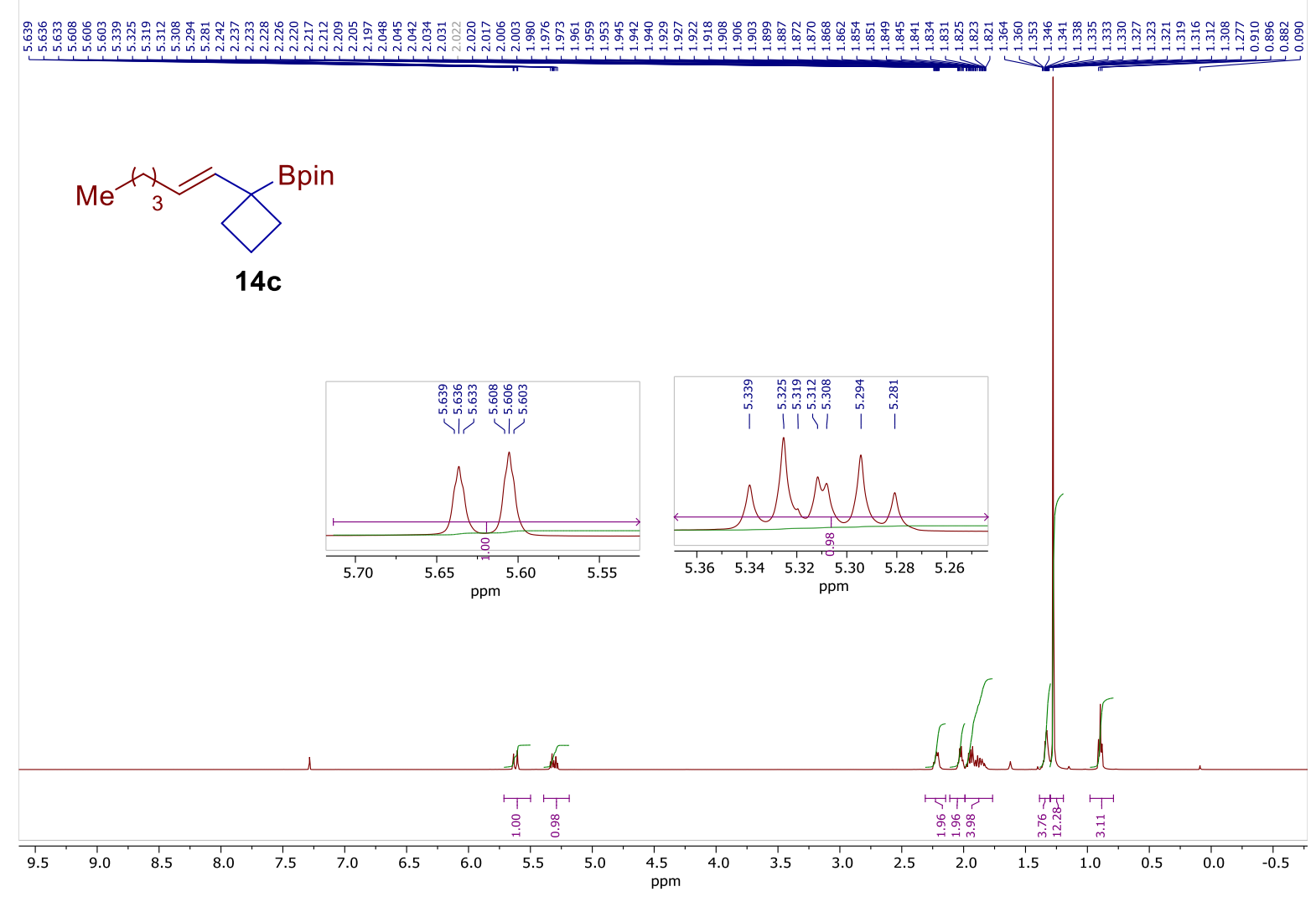

${ }^{13} \mathbf{C}-\mathbf{N M R}\left(126 \mathrm{MHz}, \mathrm{CDCl}_{3}\right)$ of compound $\mathbf{1 4 c}$

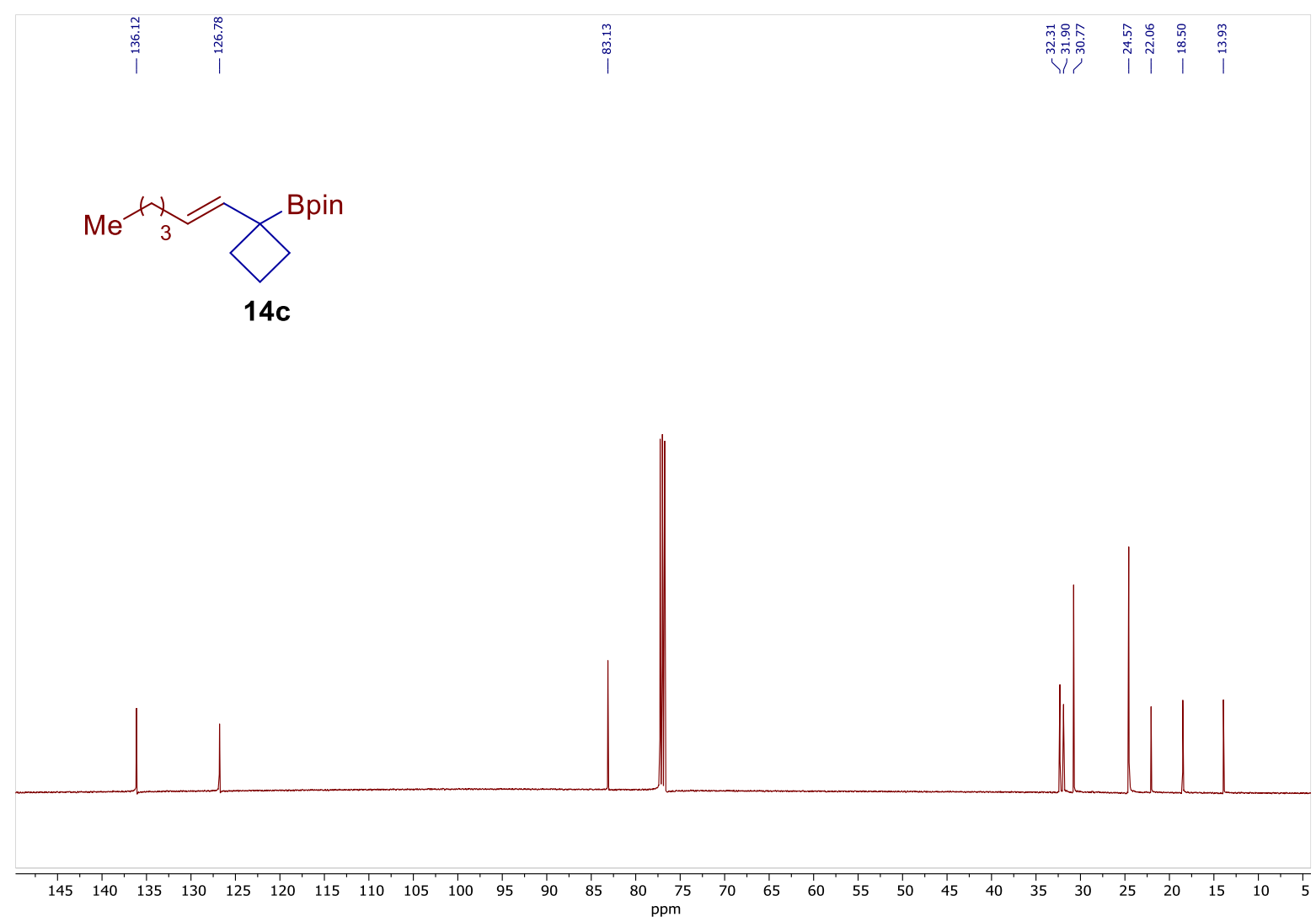


${ }^{\mathbf{1}} \mathbf{H}$-NMR (400 MHz, $\mathrm{CDCl}_{3}$ ) of compound $\mathbf{1 4 d}$ see procedure

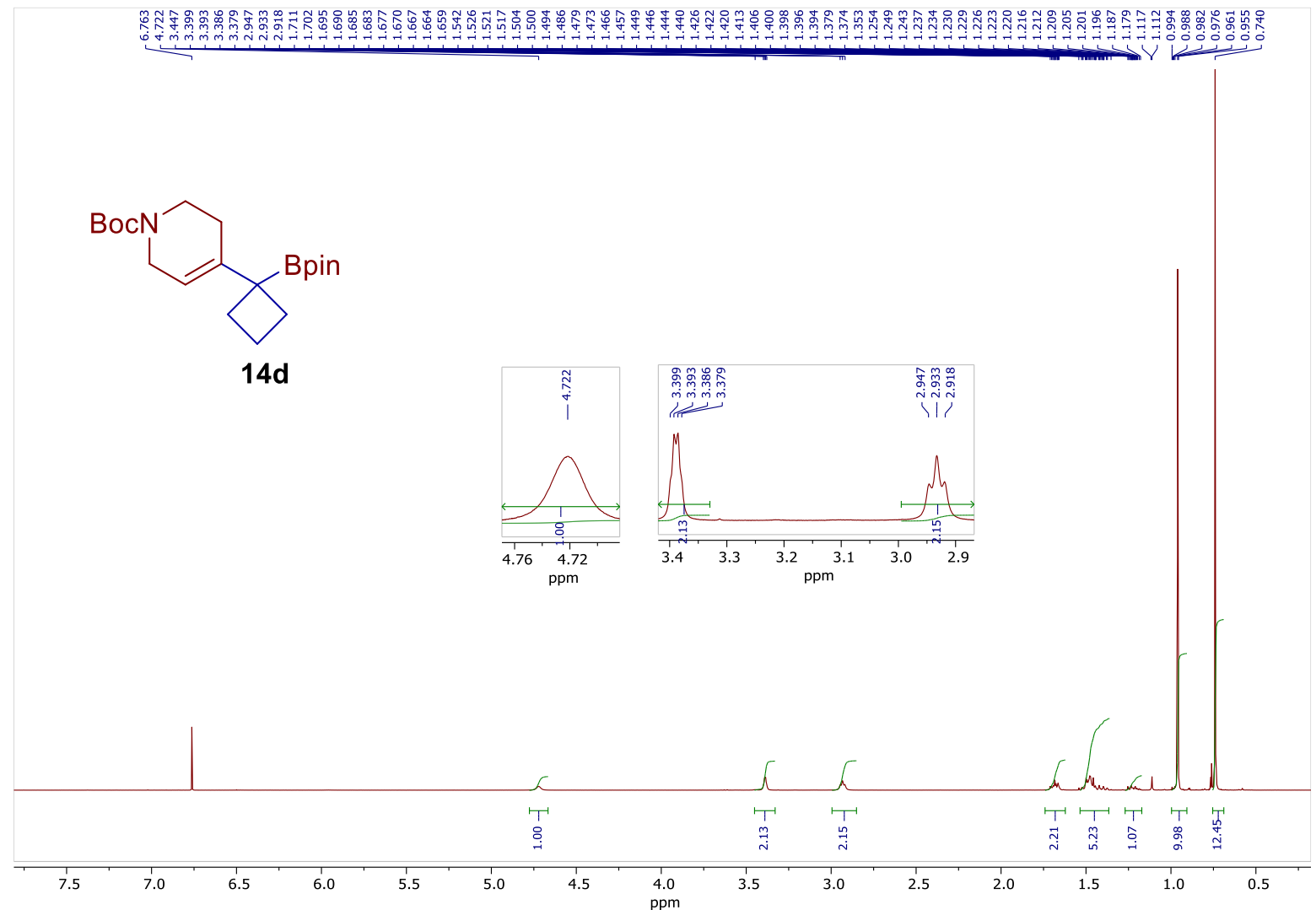

${ }^{13} \mathbf{C}-\mathbf{N M R}\left(101 \mathrm{MHz}, \mathrm{CDCl}_{3}\right)$ of compound 14d

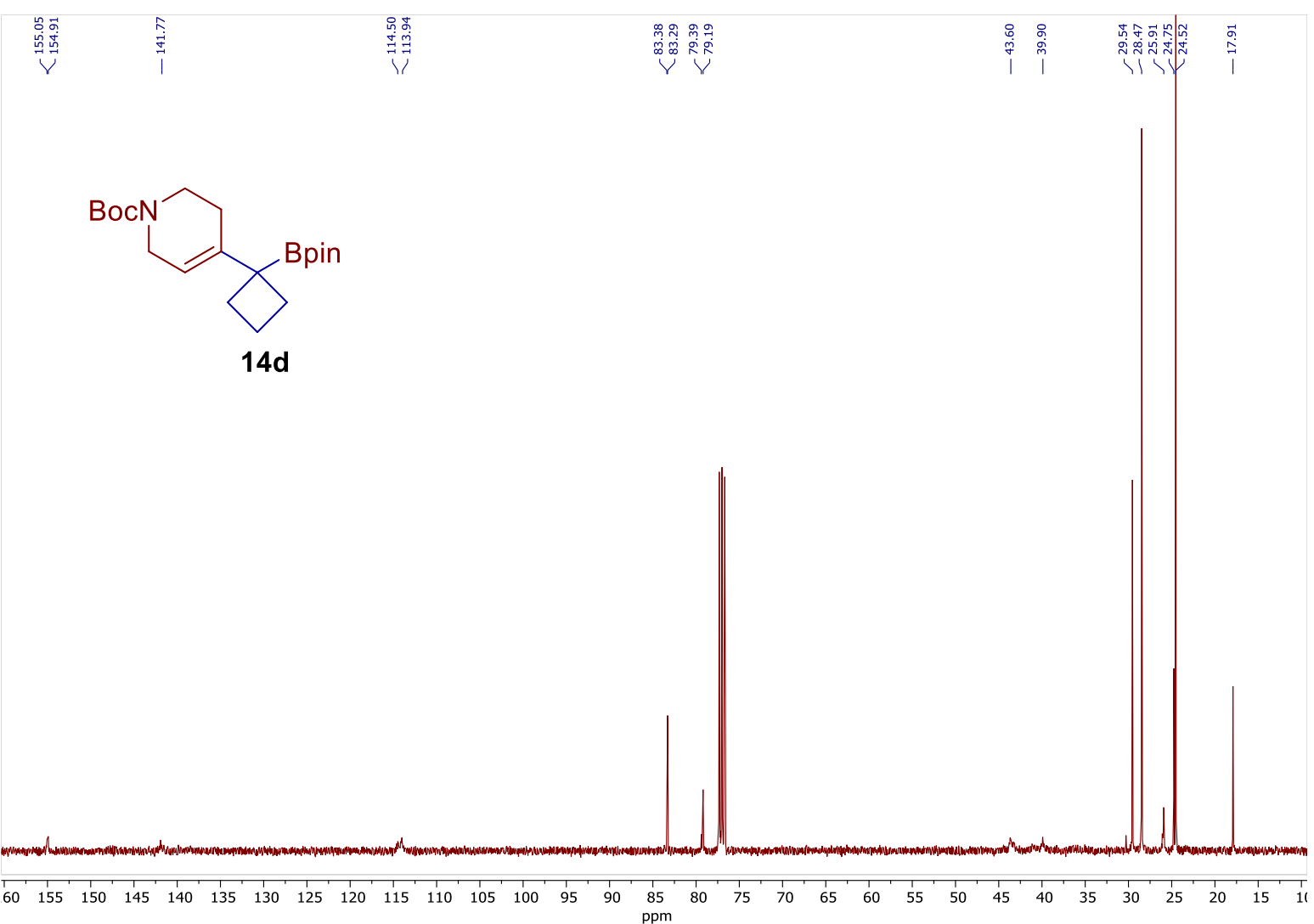


${ }^{1} \mathbf{H}$-NMR $\left(500 \mathrm{MHz}, \mathrm{CDCl}_{3}\right)$ of compound 2a see procedure

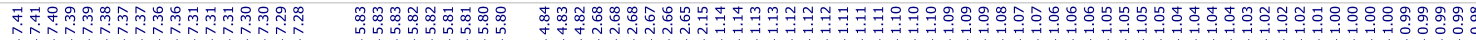

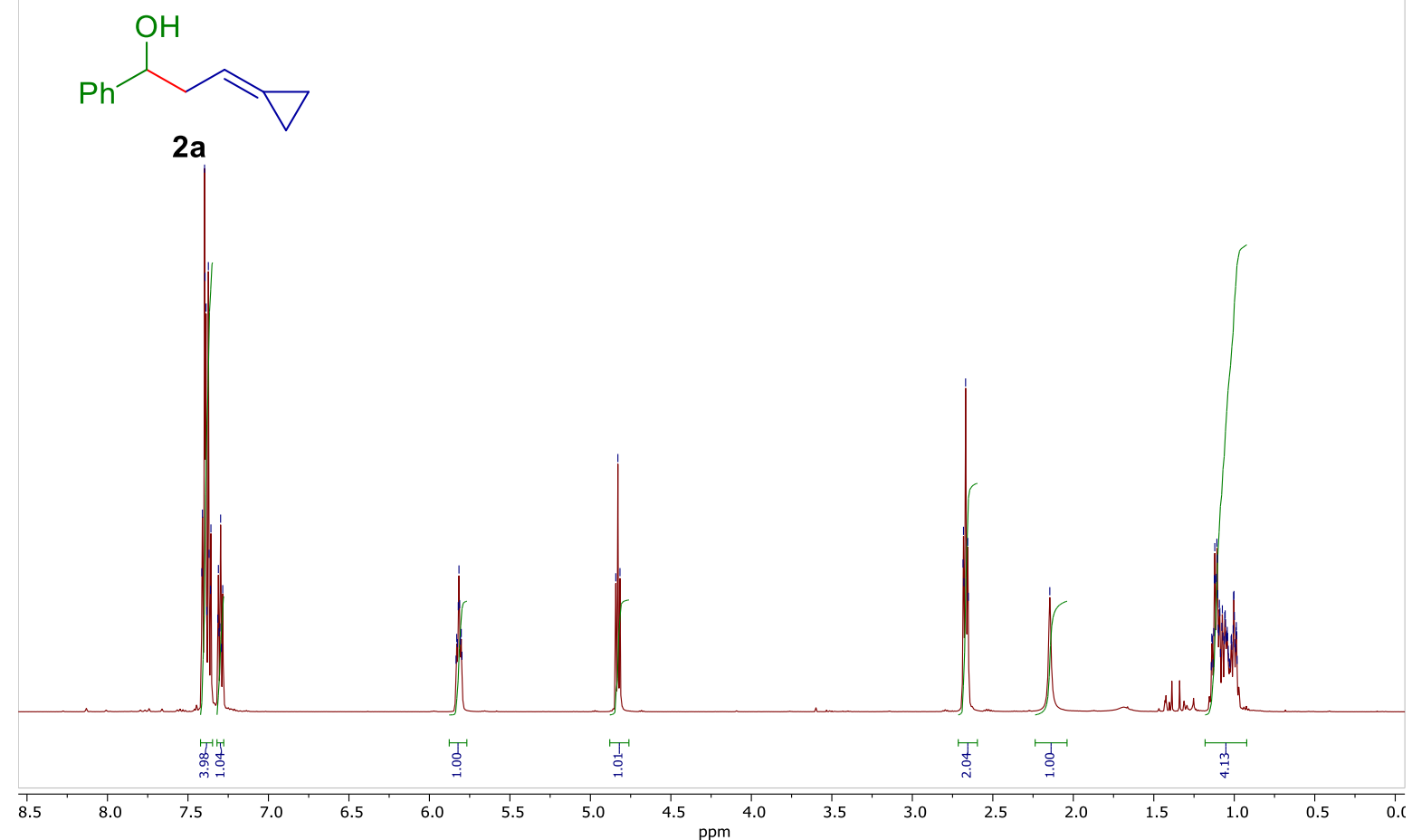

${ }^{13} \mathbf{C}-\mathbf{N M R}\left(126 \mathrm{MHz}, \mathrm{CDCl}_{3}\right)$ of compound 2a

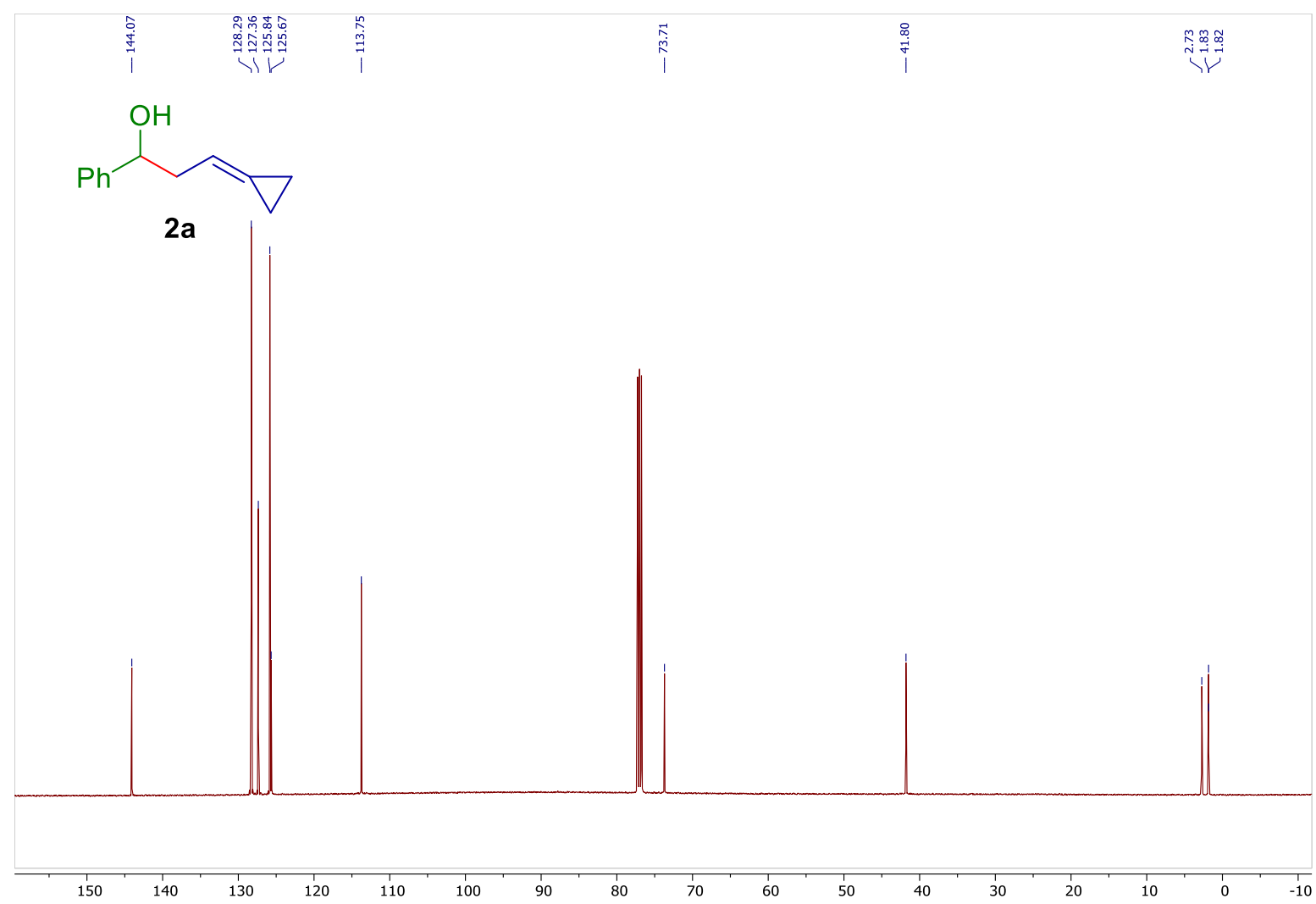


${ }^{1} \mathbf{H}$-NMR $\left(500 \mathrm{MHz}, \mathrm{CDCl}_{3}\right)$ of compound $\mathbf{2 b}$ see procedure

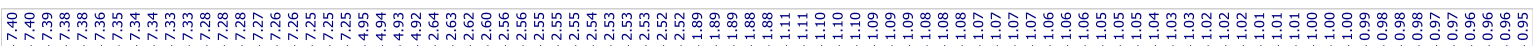<smiles>CC(CC(O)c1ccccc1)=C1CC1</smiles>

2b

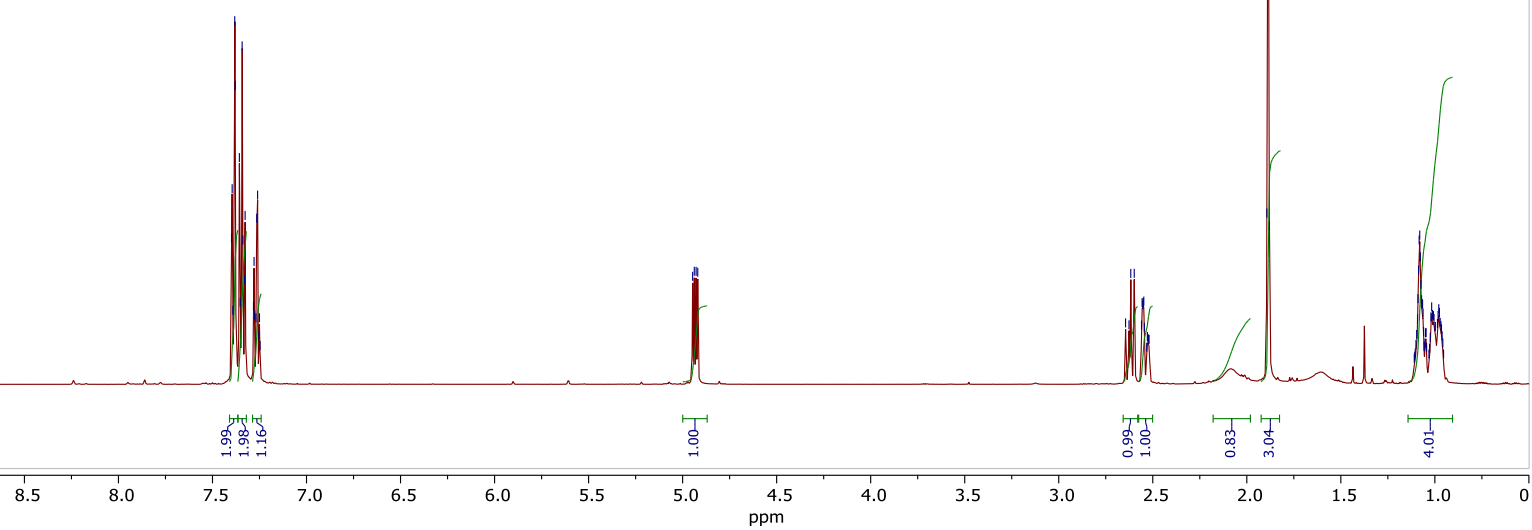

${ }^{13} \mathbf{C}-\mathbf{N M R}\left(126 \mathrm{MHz}, \mathrm{CDCl}_{3}\right)$ of compound $\mathbf{2 b}$

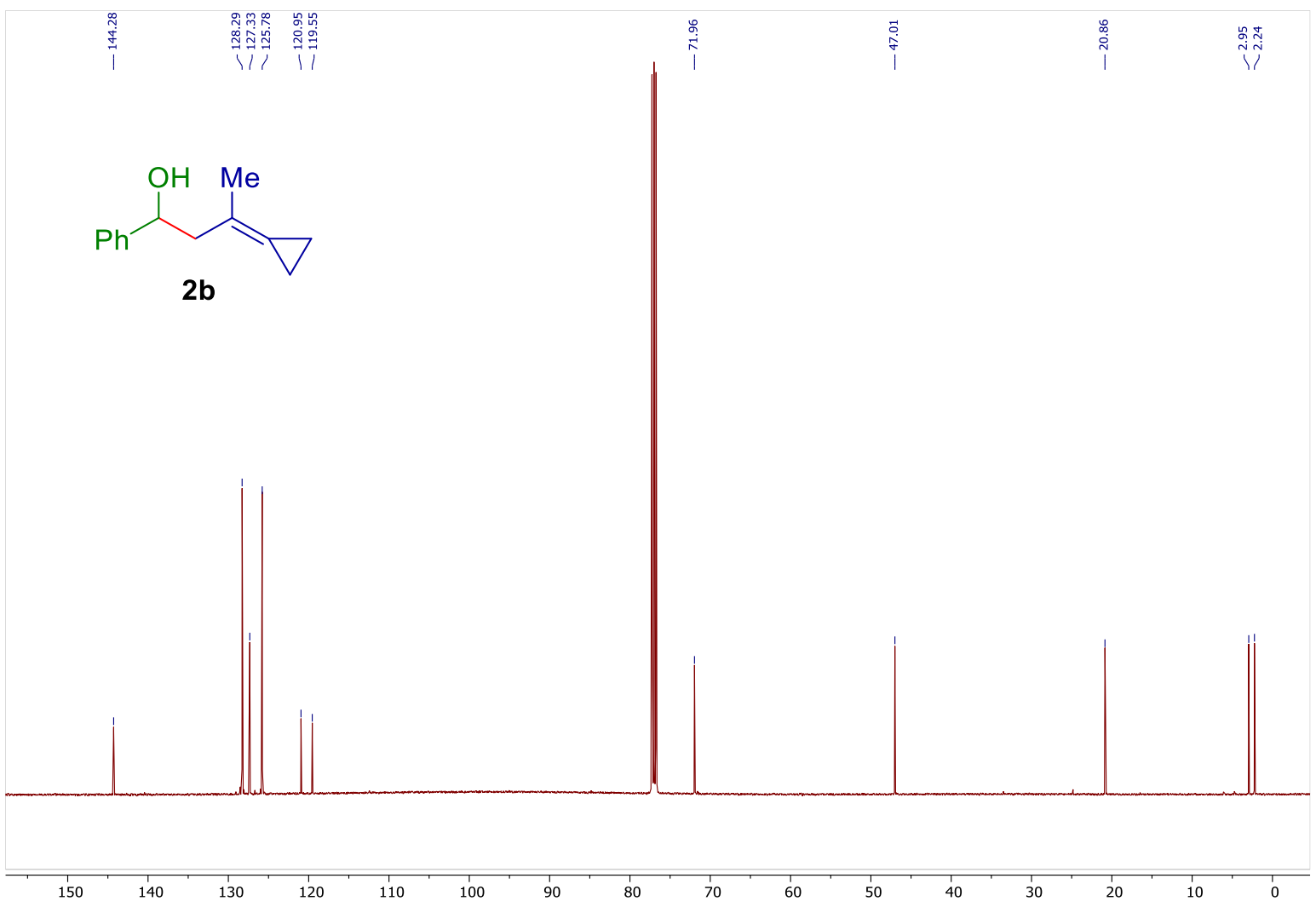


${ }^{1} \mathbf{H}-\mathbf{N M R}\left(500 \mathrm{MHz}, \mathrm{CDCl}_{3}\right)$ of compound $\mathbf{2 c}$ see procedure

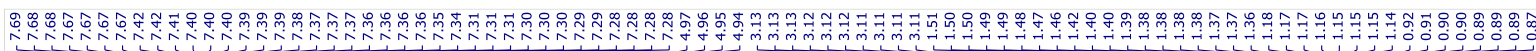

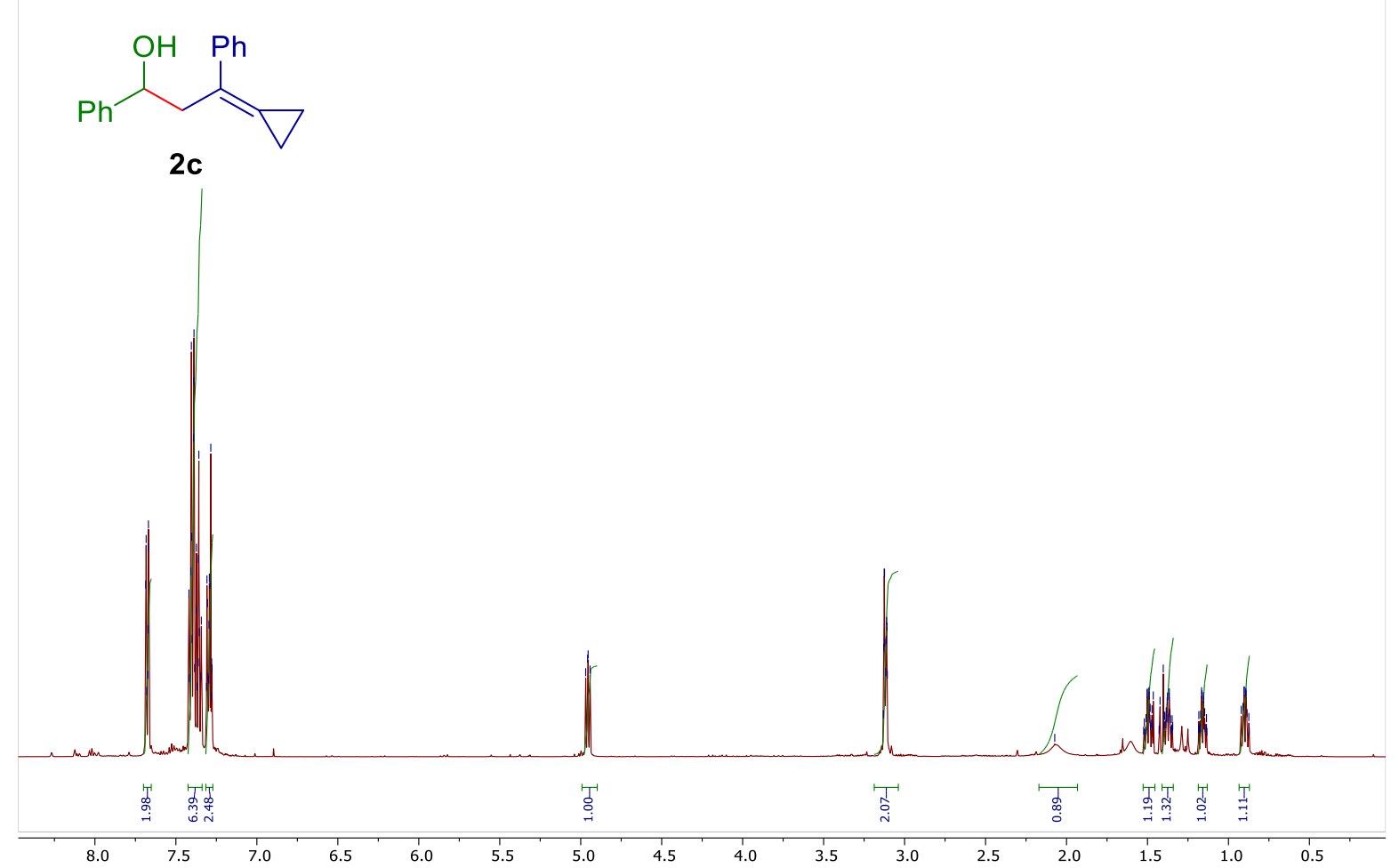

${ }^{13} \mathbf{C}$-NMR $\left(126 \mathrm{MHz}, \mathrm{CDCl}_{3}\right)$ of compound $\mathbf{2 c}$

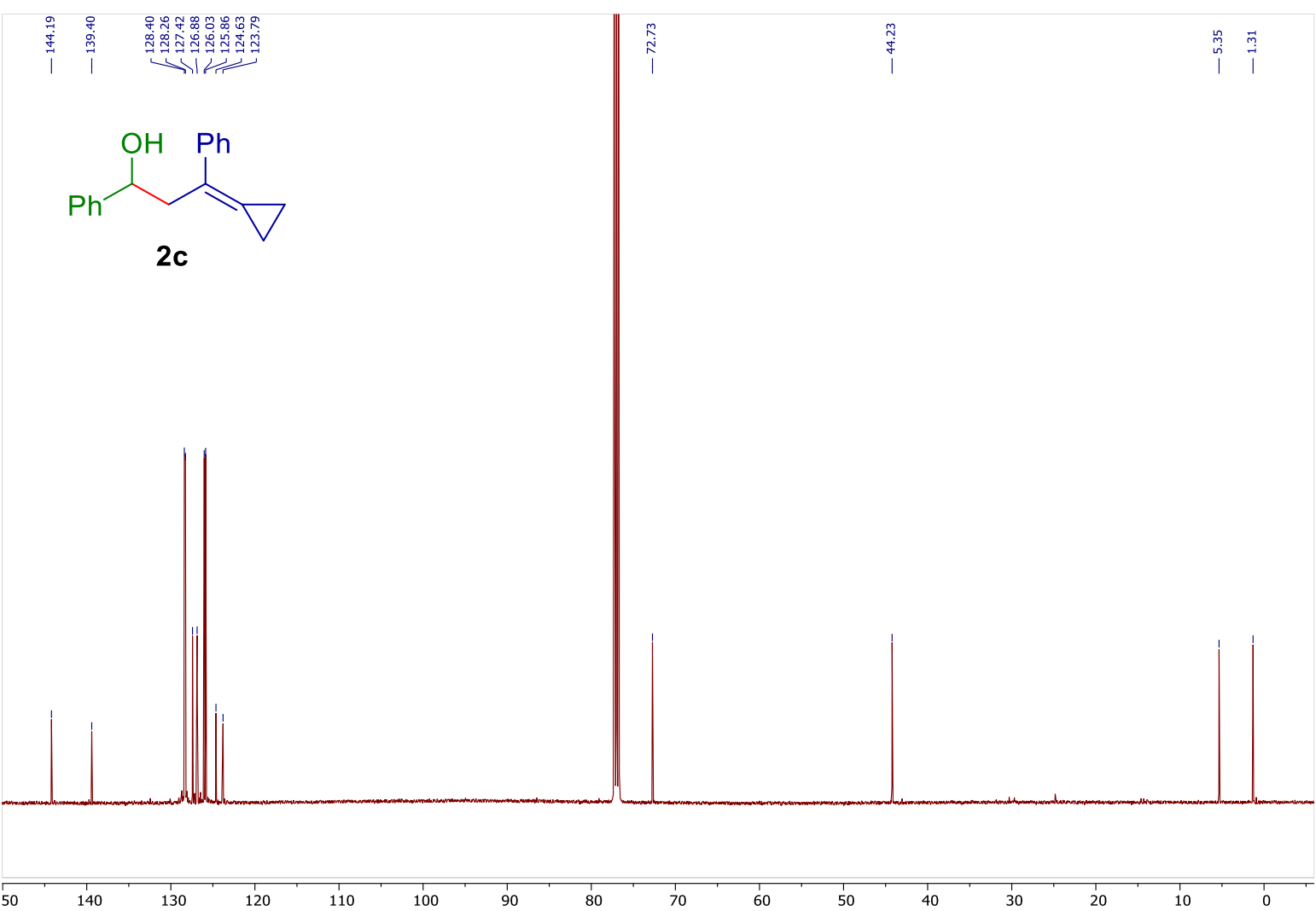


${ }^{1} \mathbf{H}-\mathrm{NMR}\left(500 \mathrm{MHz}, \mathrm{CDCl}_{3}\right)$ of compound $\mathbf{2 d}$ see procedure

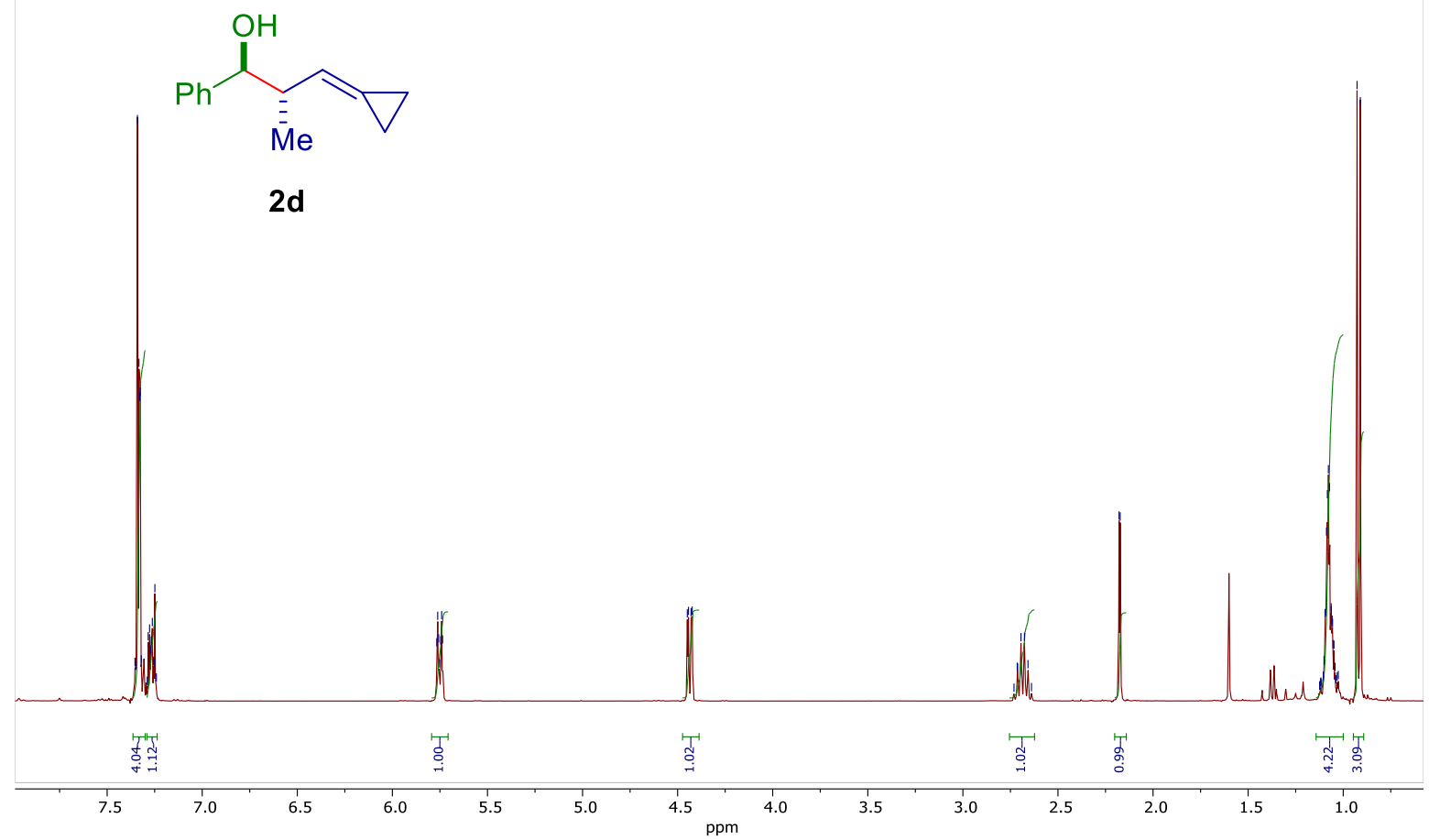

${ }^{13}$ C-NMR $\left(126 \mathrm{MHz}, \mathrm{CDCl}_{3}\right)$ of compound 2d

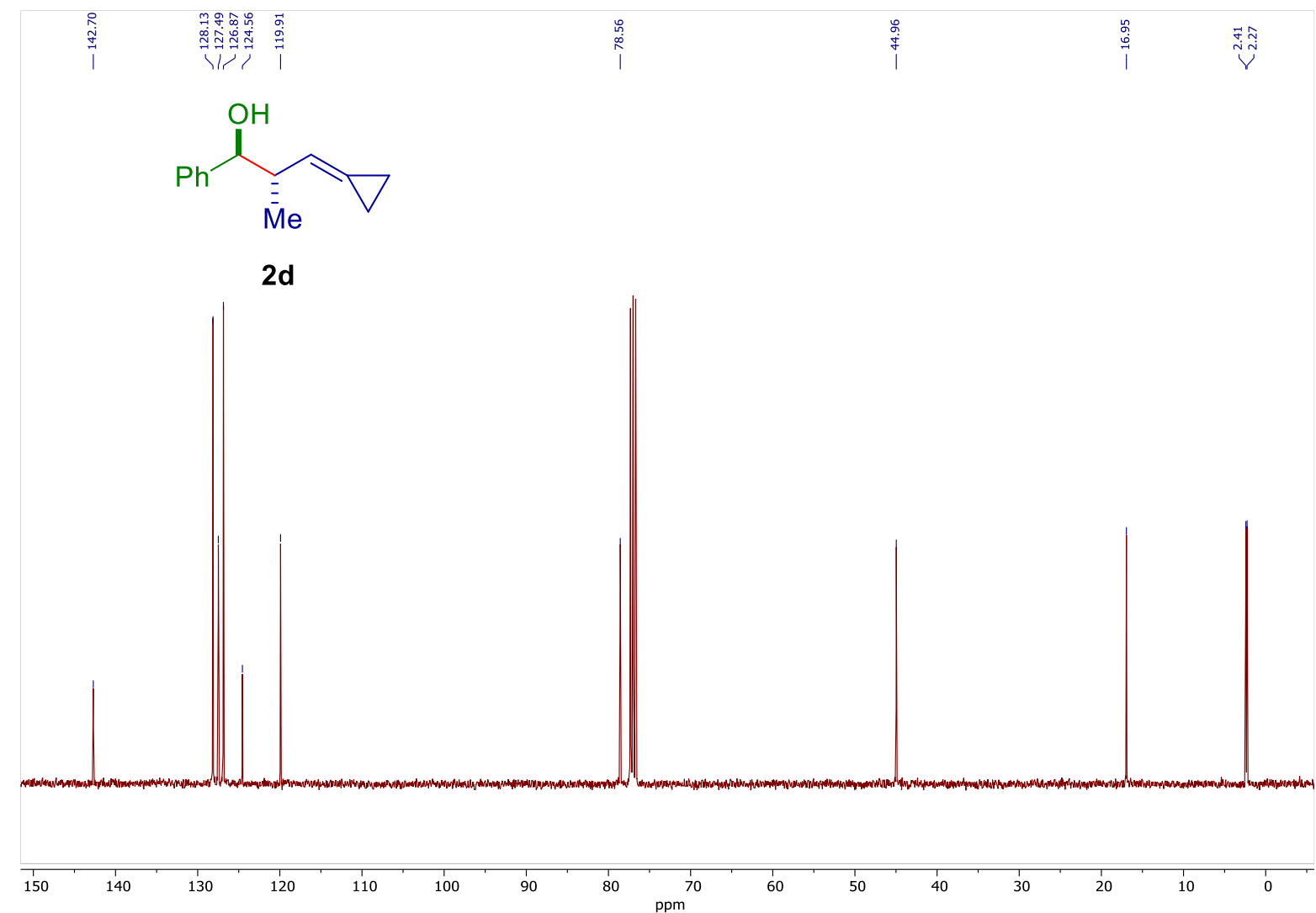


${ }^{1} \mathbf{H}-\mathrm{NMR}\left(500 \mathrm{MHz}, \mathrm{CDCl}_{3}\right)$ of compound $\mathbf{2 d}$ ' see procedure

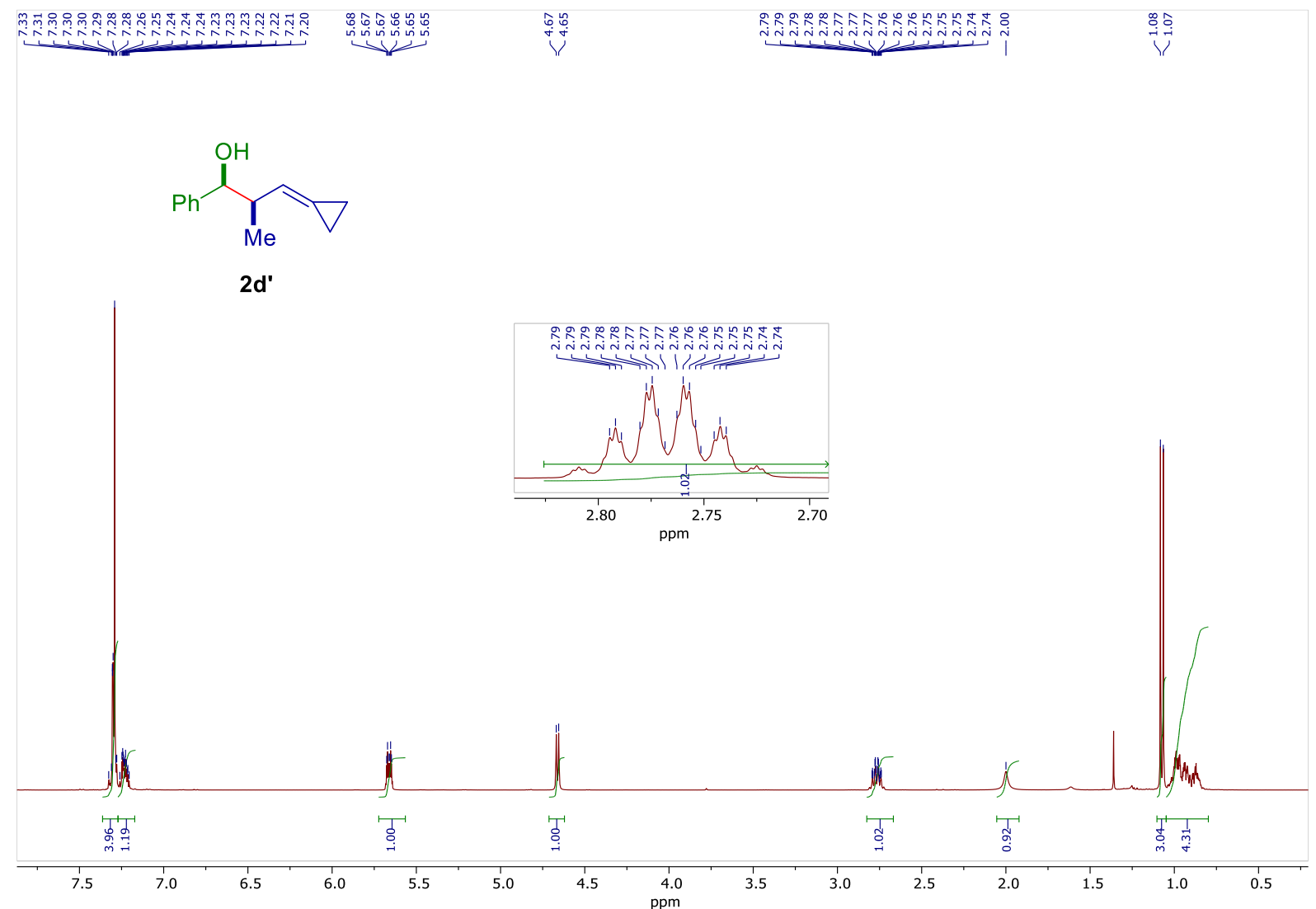

${ }^{13} \mathbf{C}$-NMR (126 MHz, $\left.\mathrm{CDCl}_{3}\right)$ of compound 2d'

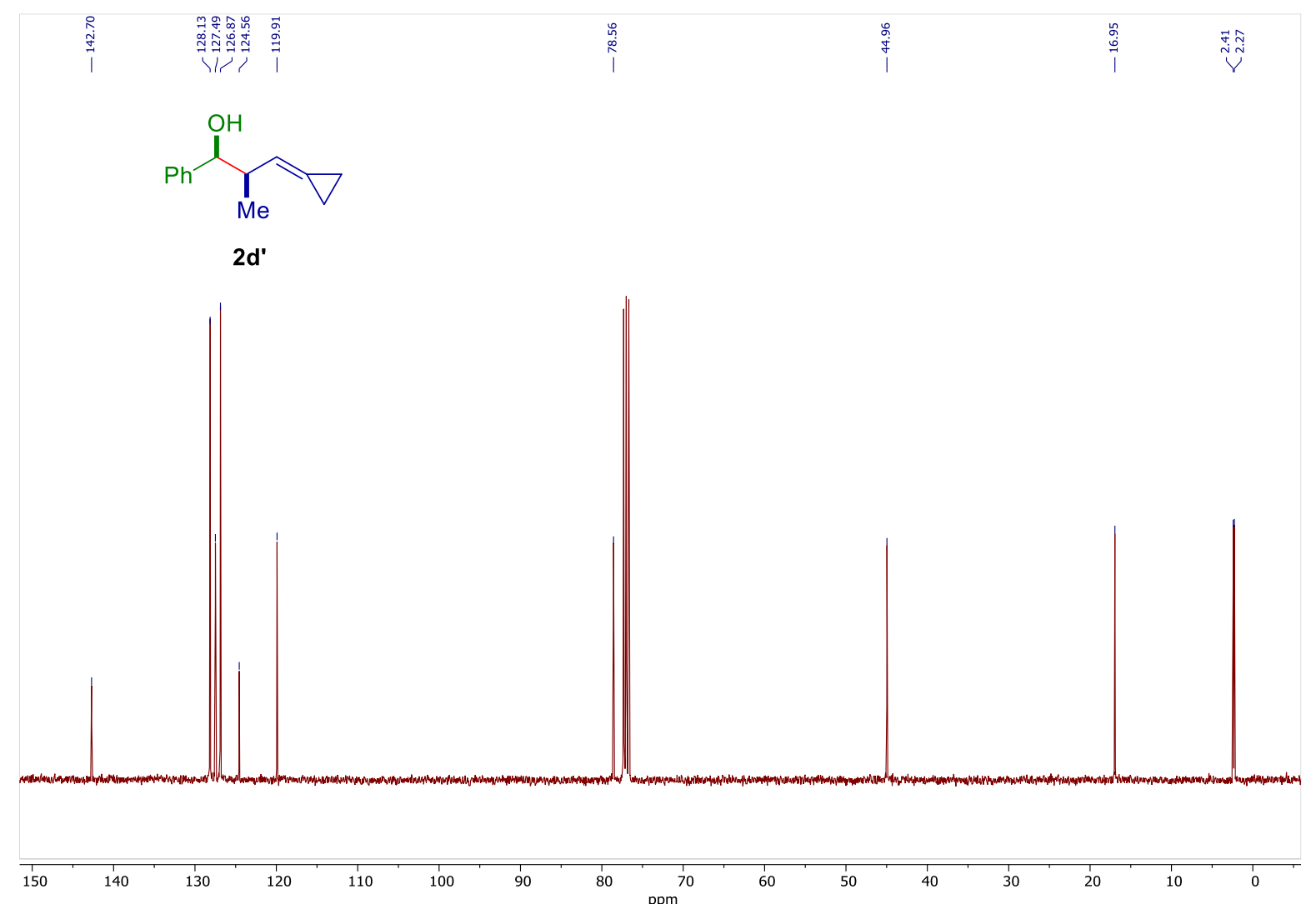


${ }^{1} \mathbf{H}-\mathrm{NMR}\left(500 \mathrm{MHz}, \mathrm{CDCl}_{3}\right)$ of compound 2e see procedure

مِ

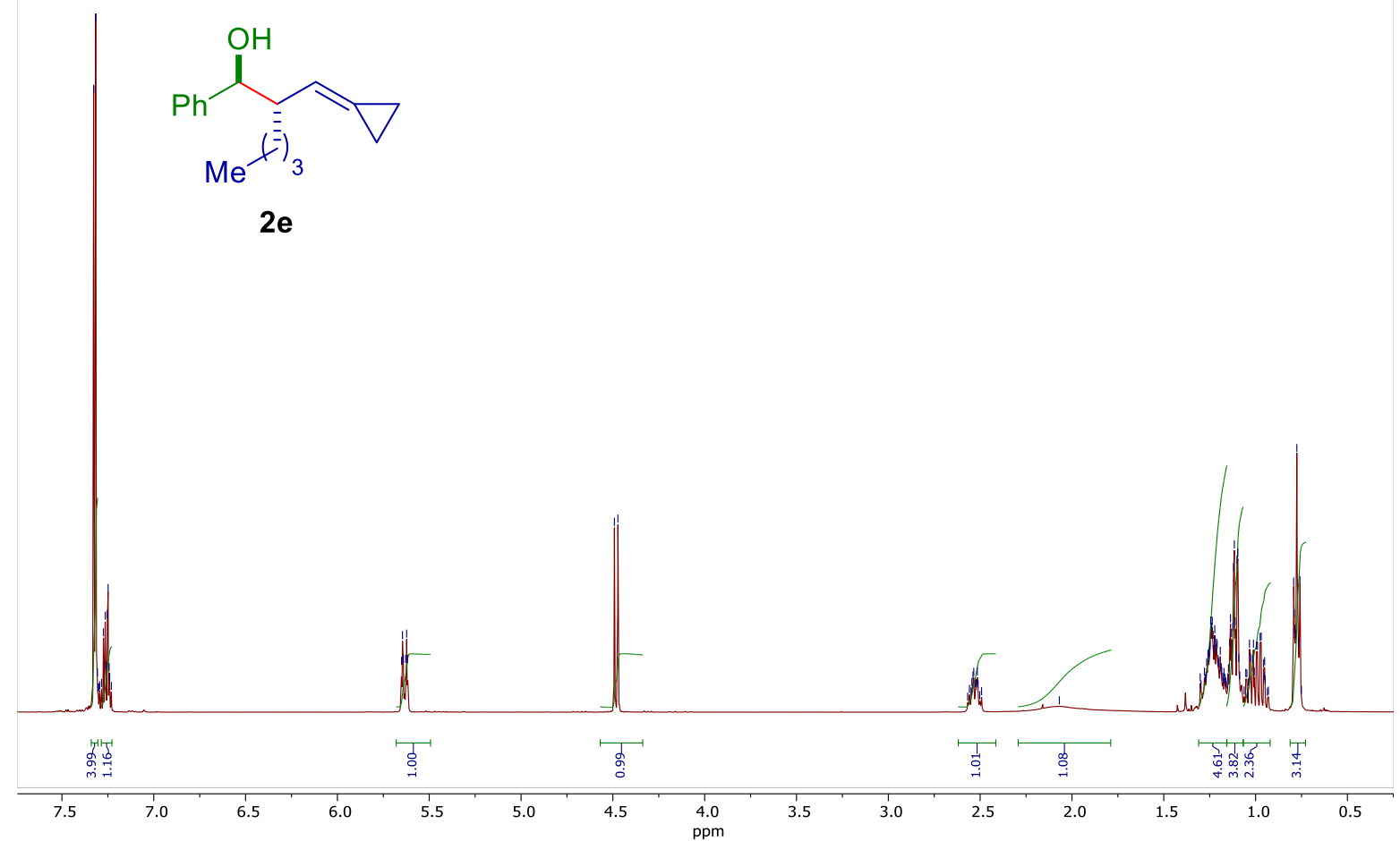

${ }^{13} \mathbf{C}-\mathbf{N M R}\left(126 \mathrm{MHz}, \mathrm{CDCl}_{3}\right)$ of compound $\mathbf{2 e}$

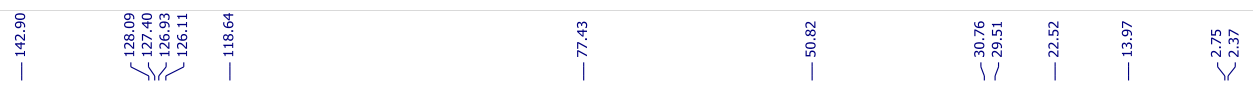<smiles>C[14CH](C=C1CC1)C(O)c1ccccc1</smiles>
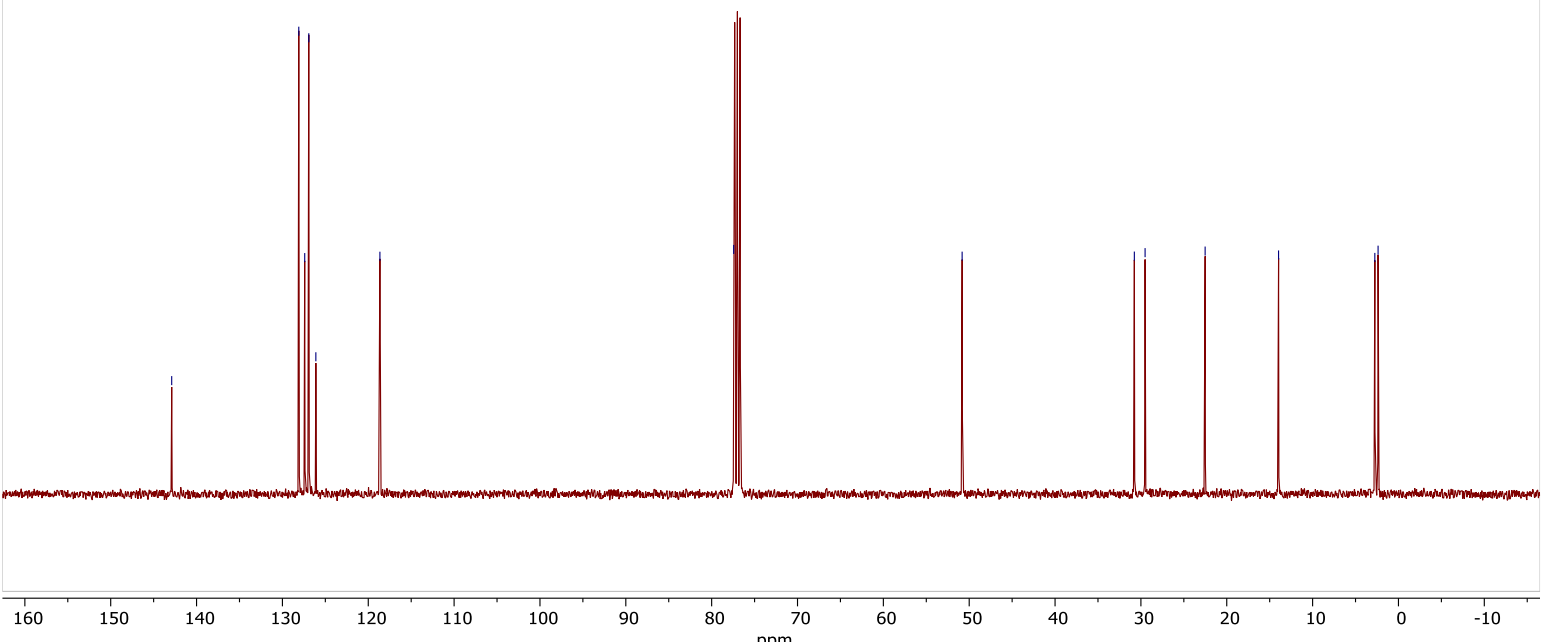
${ }^{1} \mathbf{H}-\mathrm{NMR}\left(500 \mathrm{MHz}, \mathrm{CDCl}_{3}\right)$ of compound $\mathbf{2 f}$ see procedure

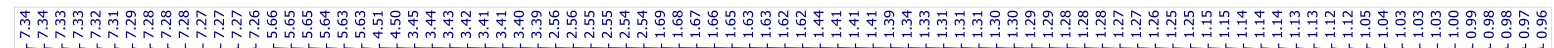

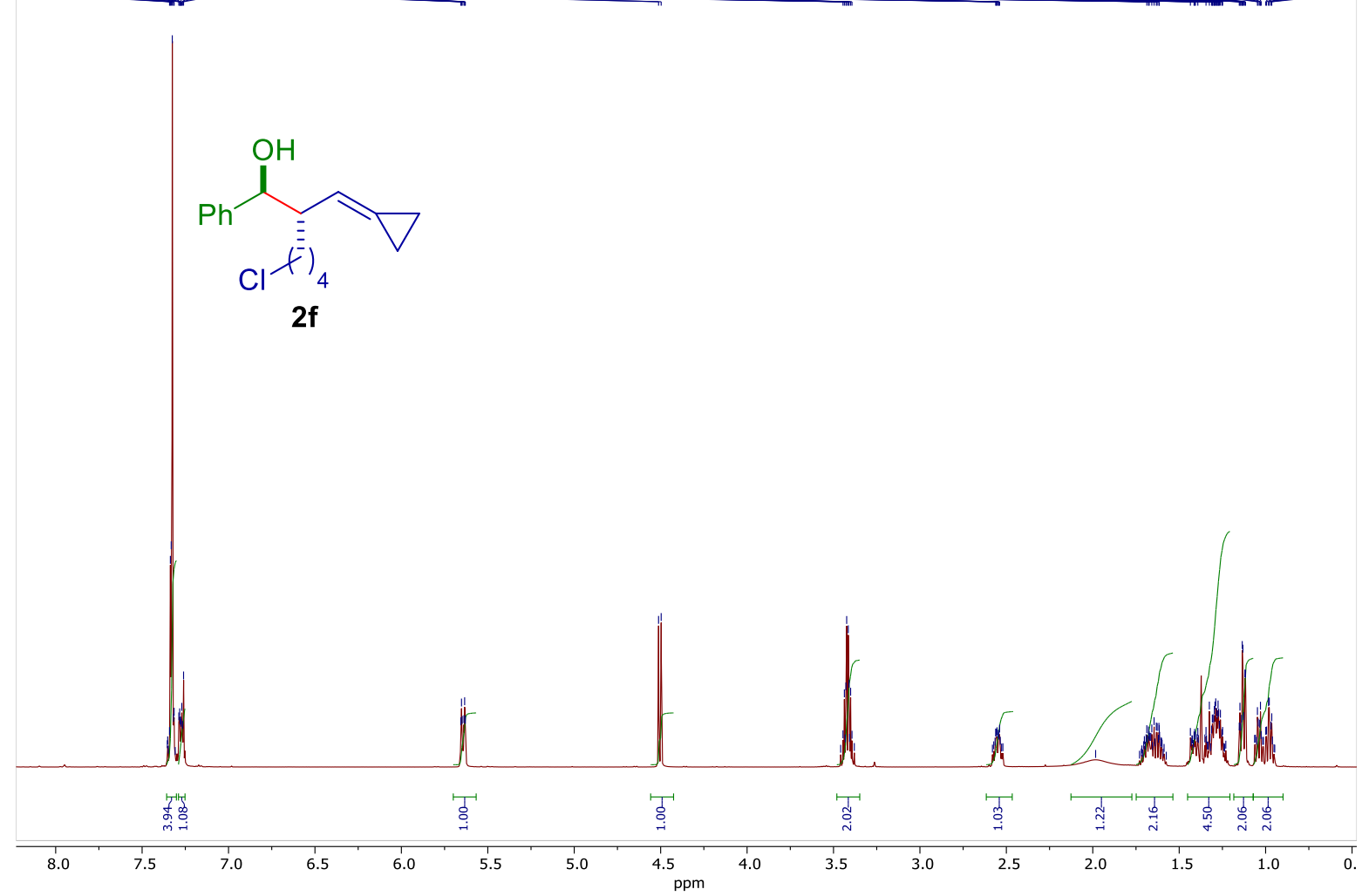

${ }^{13} \mathbf{C}-\mathbf{N M R}\left(126 \mathrm{MHz}, \mathrm{CDCl}_{3}\right)$ of compound $\mathbf{2 f}$

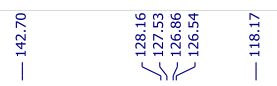

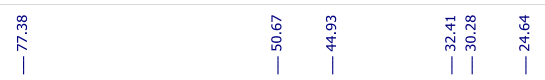

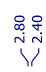<smiles>OC(C=C1CC1)C(C=[Te])C(Cl)c1ccccc1</smiles>
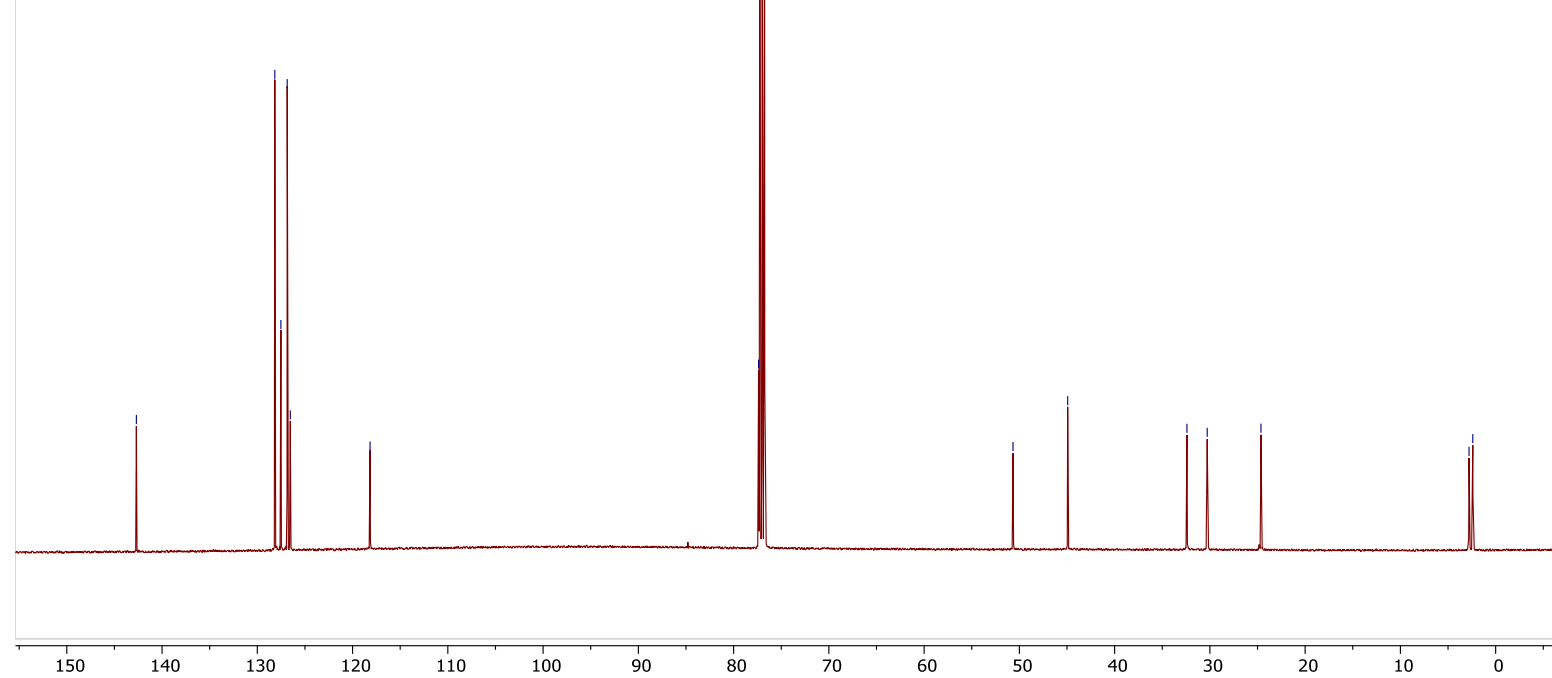
${ }^{1} \mathbf{H}-\mathrm{NMR}\left(500 \mathrm{MHz}, \mathrm{CDCl}_{3}\right)$ of compound $\mathbf{2 g}$ see procedure

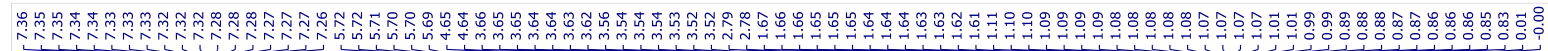<smiles>CCOC(=O)C(CC(C)(C)C)C(O)C=C1CC1</smiles>
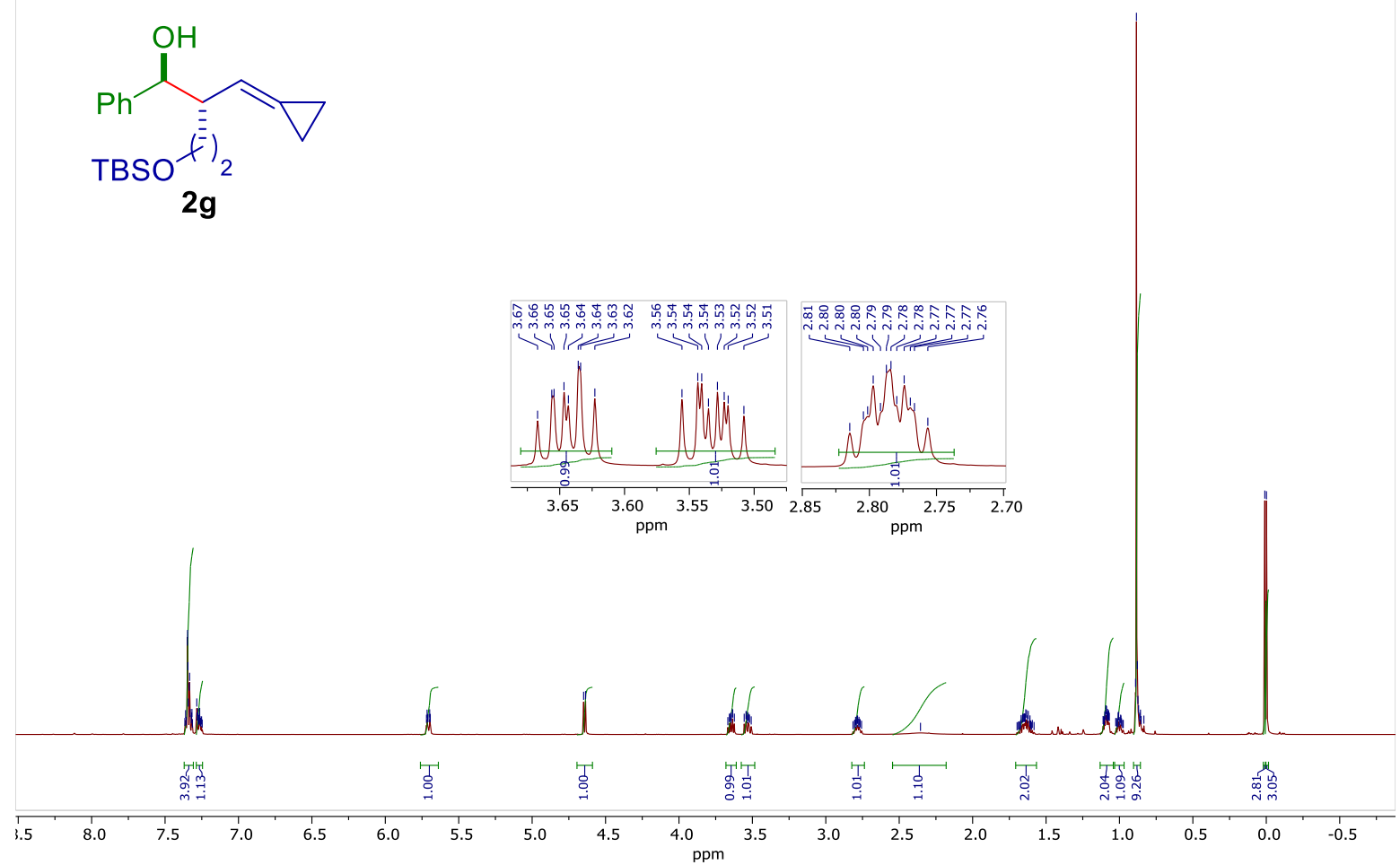

${ }^{13} \mathbf{C}$-NMR $\left(126 \mathrm{MHz}, \mathrm{CDCl}_{3}\right)$ of compound $\mathbf{2 g}$

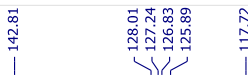

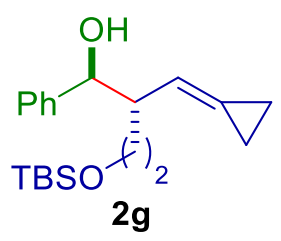

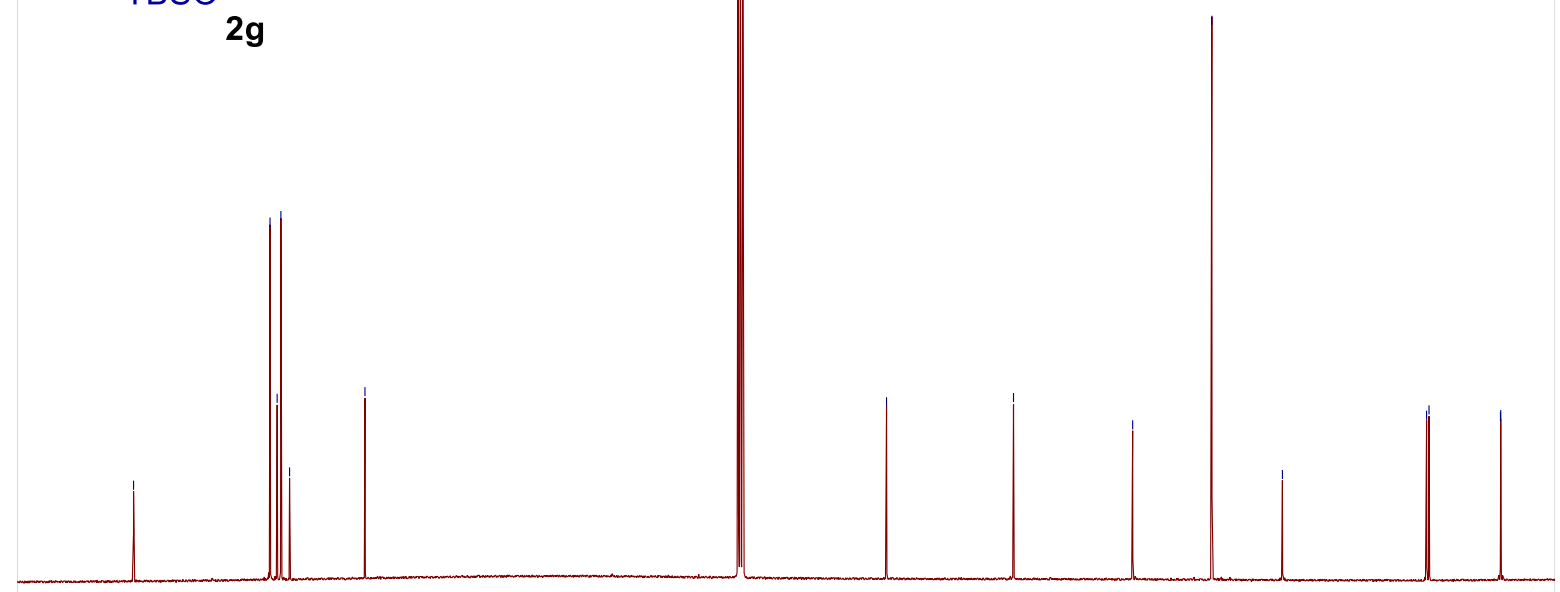


${ }^{1} \mathbf{H}-\mathrm{NMR}\left(500 \mathrm{MHz}, \mathrm{CDCl}_{3}\right)$ of compound $\mathbf{2 h}$ see procedure

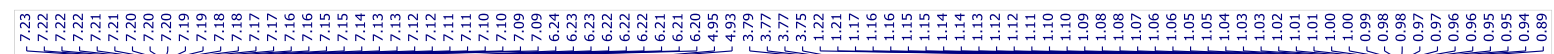<smiles>OC(c1ccccc1)[C@H](C=C1CC1)c1ccccc1</smiles>

$2 \mathrm{~h}$

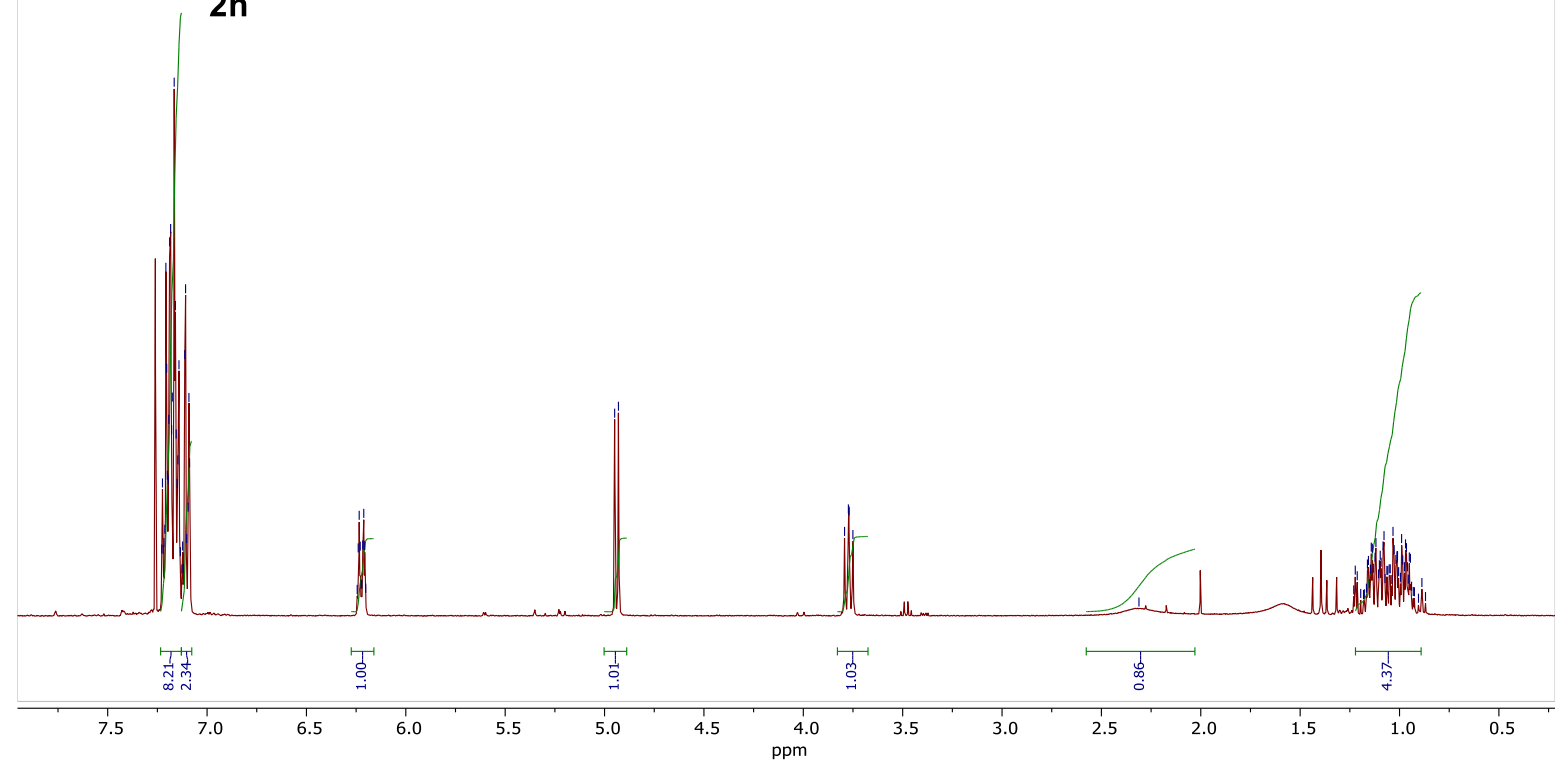

${ }^{13} \mathbf{C}-\mathbf{N M R}\left(126 \mathrm{MHz}, \mathrm{CDCl}_{3}\right)$ of compound $\mathbf{2 h}$

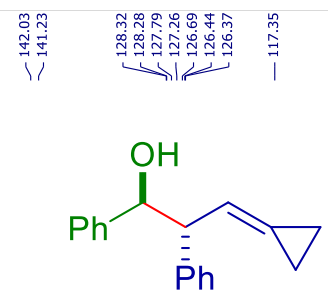

$2 \mathrm{~h}$
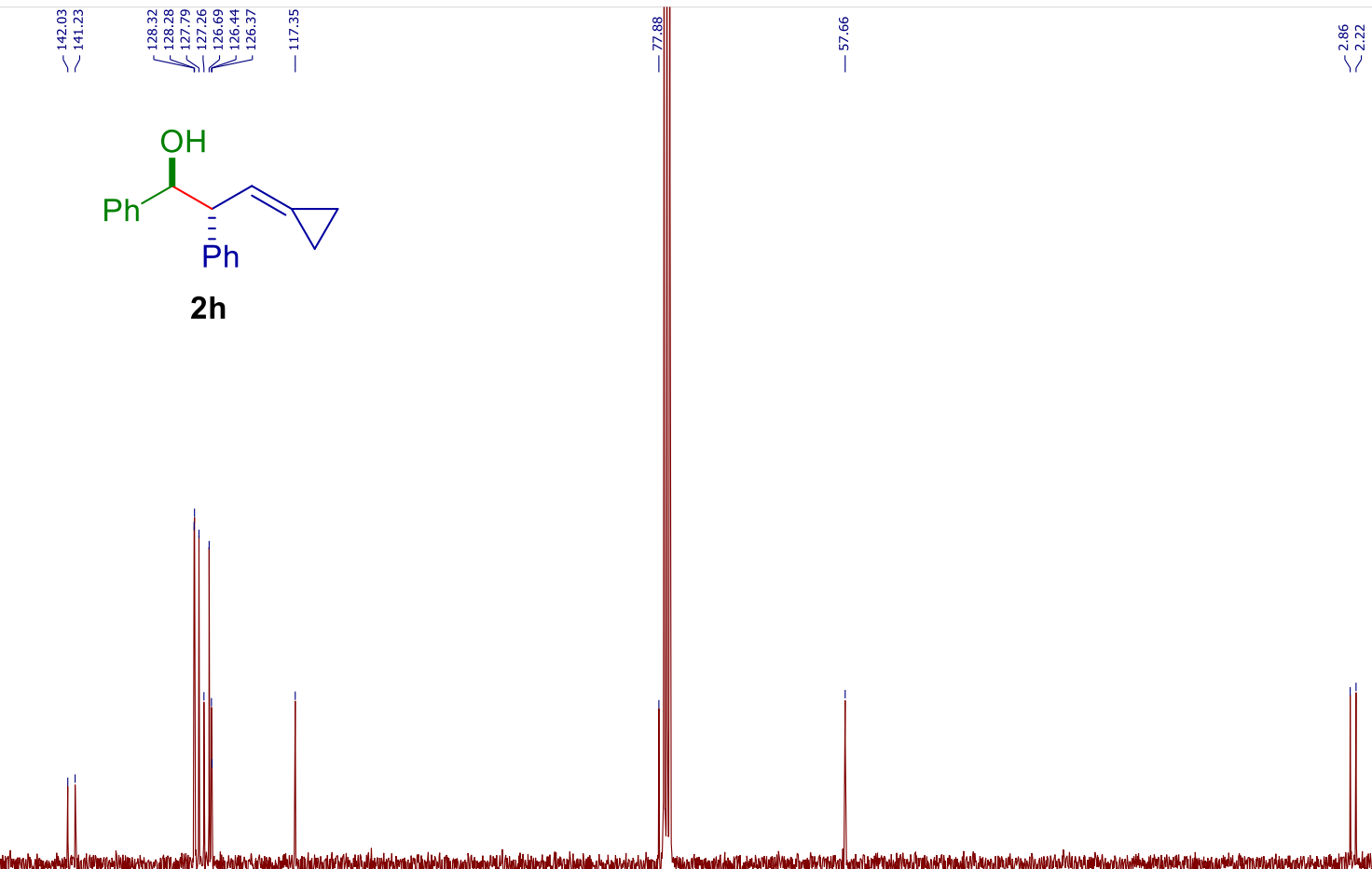
${ }^{\mathbf{1}} \mathbf{H}-\mathrm{NMR}\left(500 \mathrm{MHz}, \mathrm{CDCl}_{3}\right)$ of compound $\mathbf{2 h}$ ' see procedure

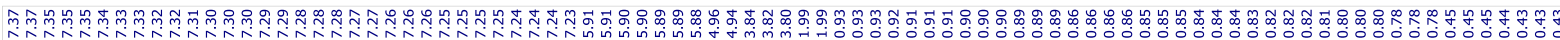

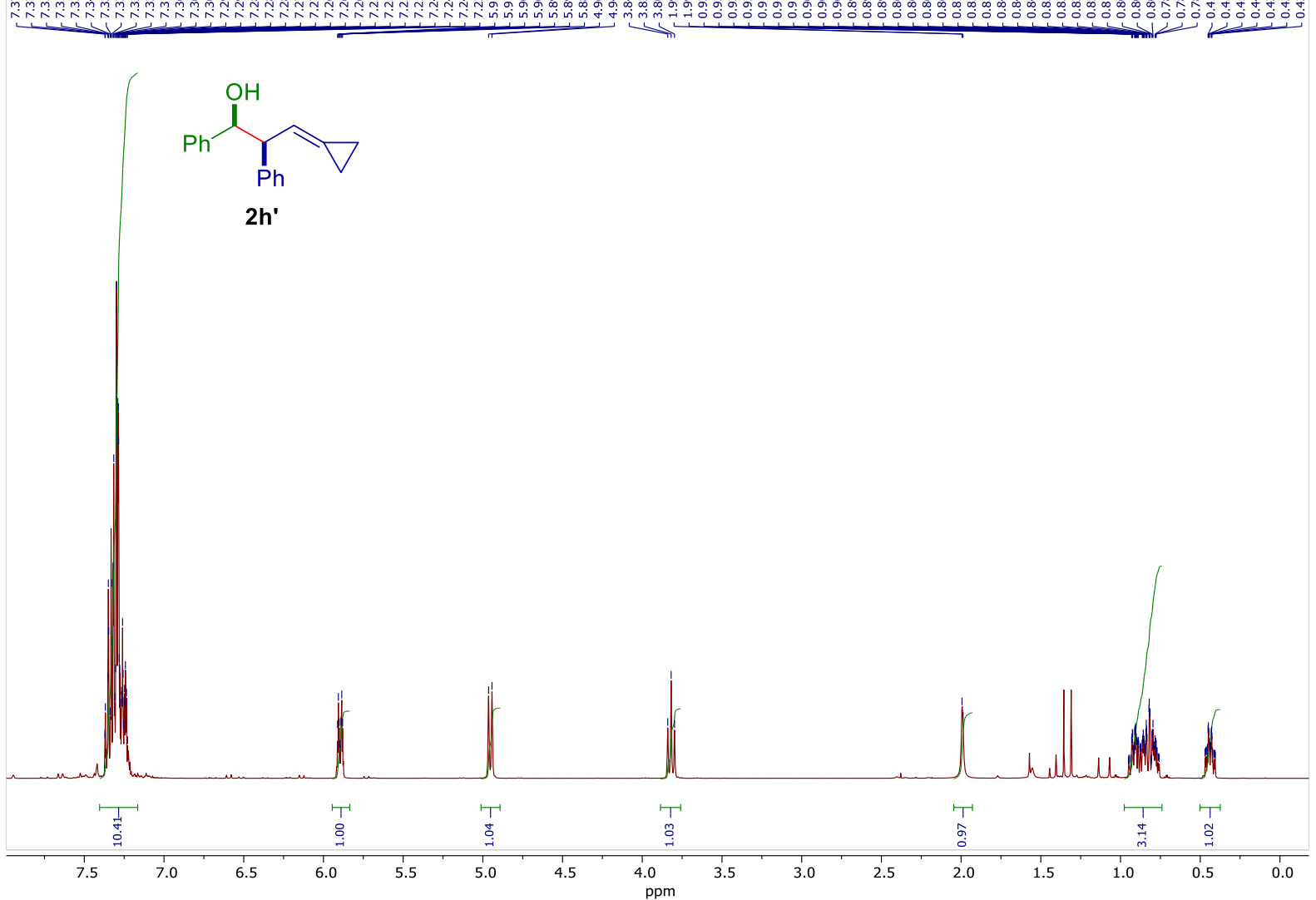

${ }^{13} \mathbf{C}-\mathbf{N M R}\left(126 \mathrm{MHz}, \mathrm{CDCl}_{3}\right)$ of compound $\mathbf{2 h}$

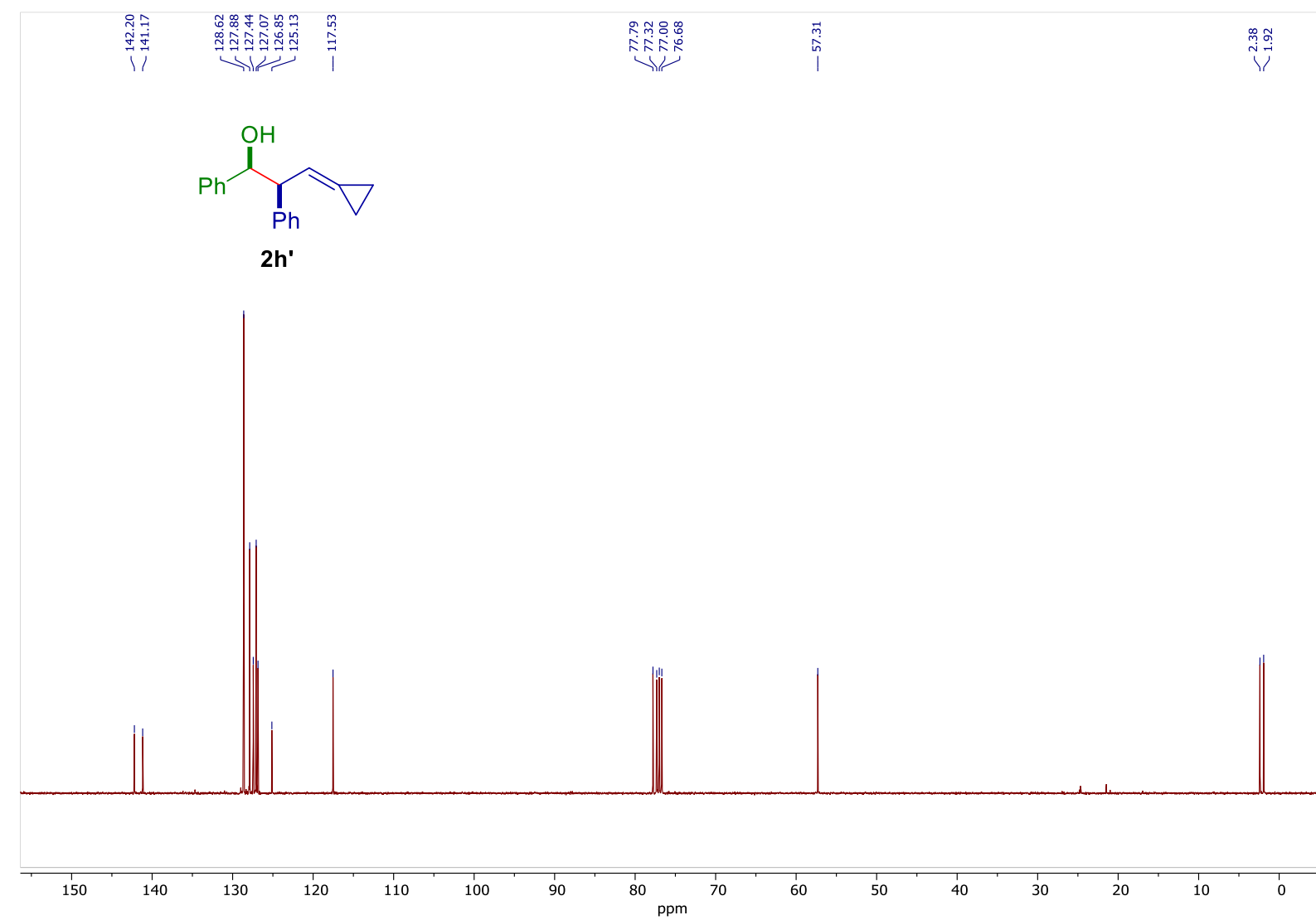


${ }^{\mathbf{1}} \mathbf{H}-\mathrm{NMR}\left(400 \mathrm{MHz}, \mathrm{CDCl}_{3}\right.$ ) of compound $\mathbf{2 i}$ see procedure

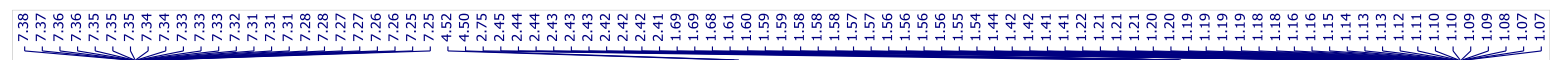<smiles>OC(c1ccccc1)[C@H]1CCCC1=C1CC1</smiles>

2i

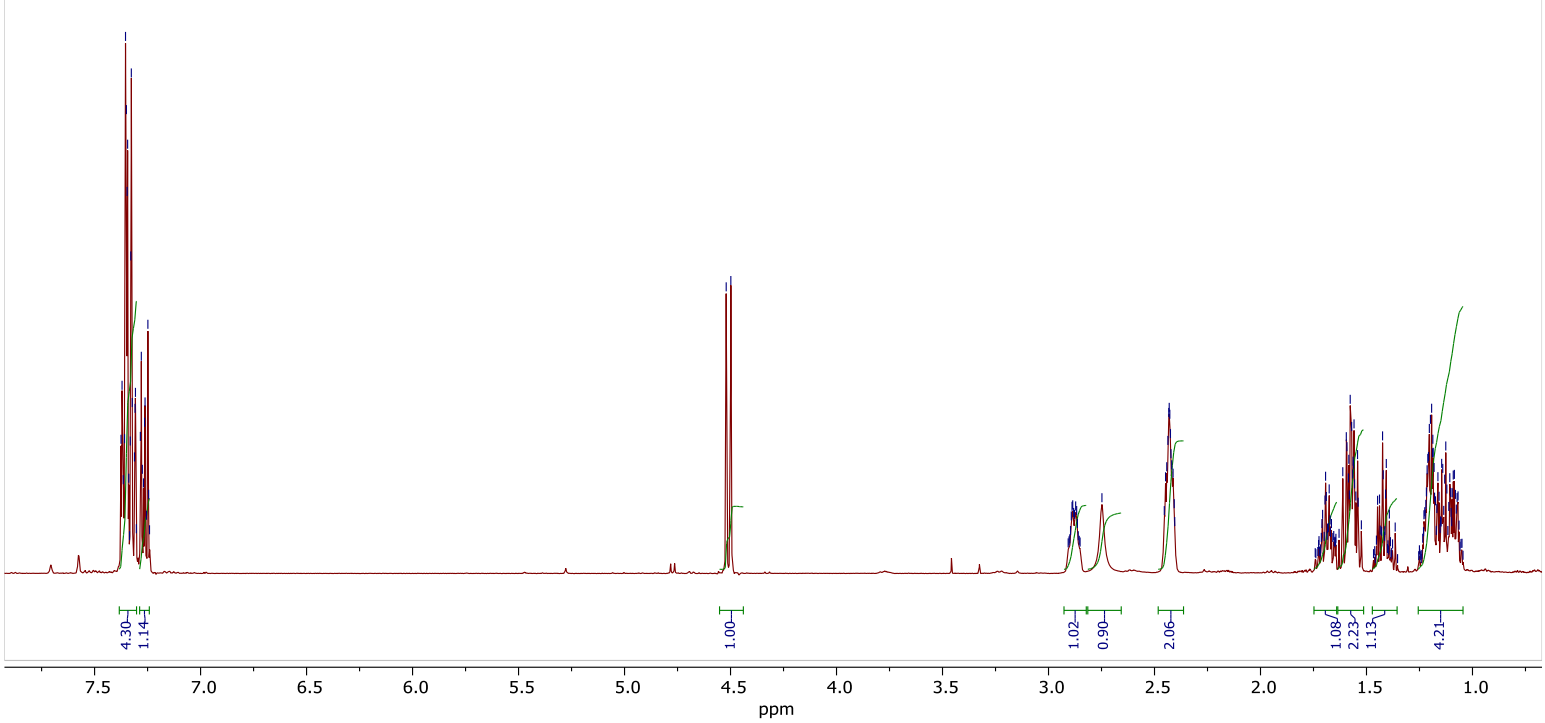

${ }^{13} \mathbf{C}-\mathrm{NMR}\left(101 \mathrm{MHz}, \mathrm{CDCl}_{3}\right)$ of compound $\mathbf{2 i}$

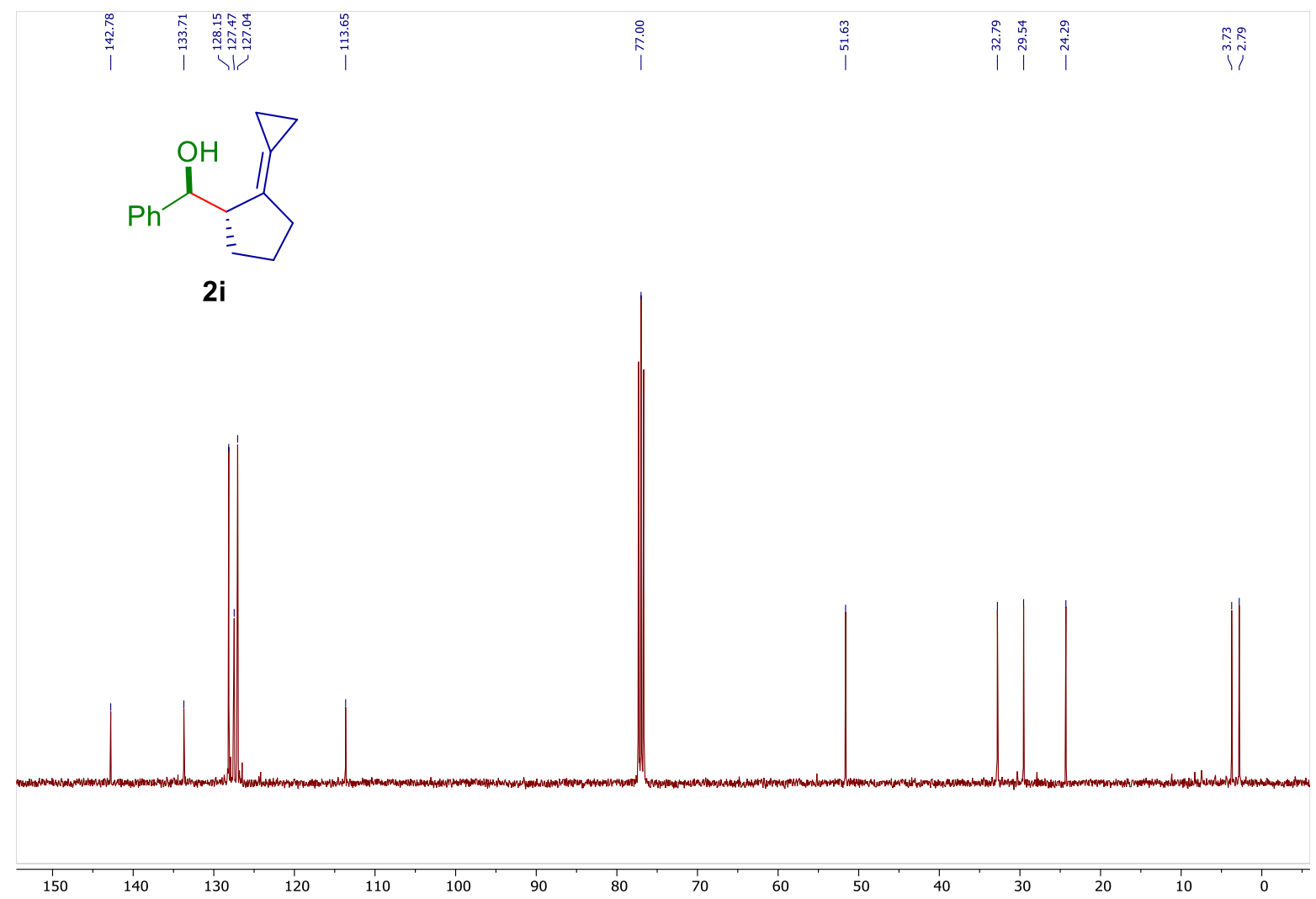


${ }^{\mathbf{1}} \mathbf{H}-\mathrm{NMR}\left(500 \mathrm{MHz}, \mathrm{CDCl}_{3}\right)$ of compound $\mathbf{2} \mathbf{j}$ see procedure

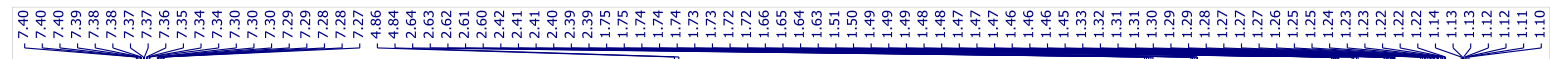

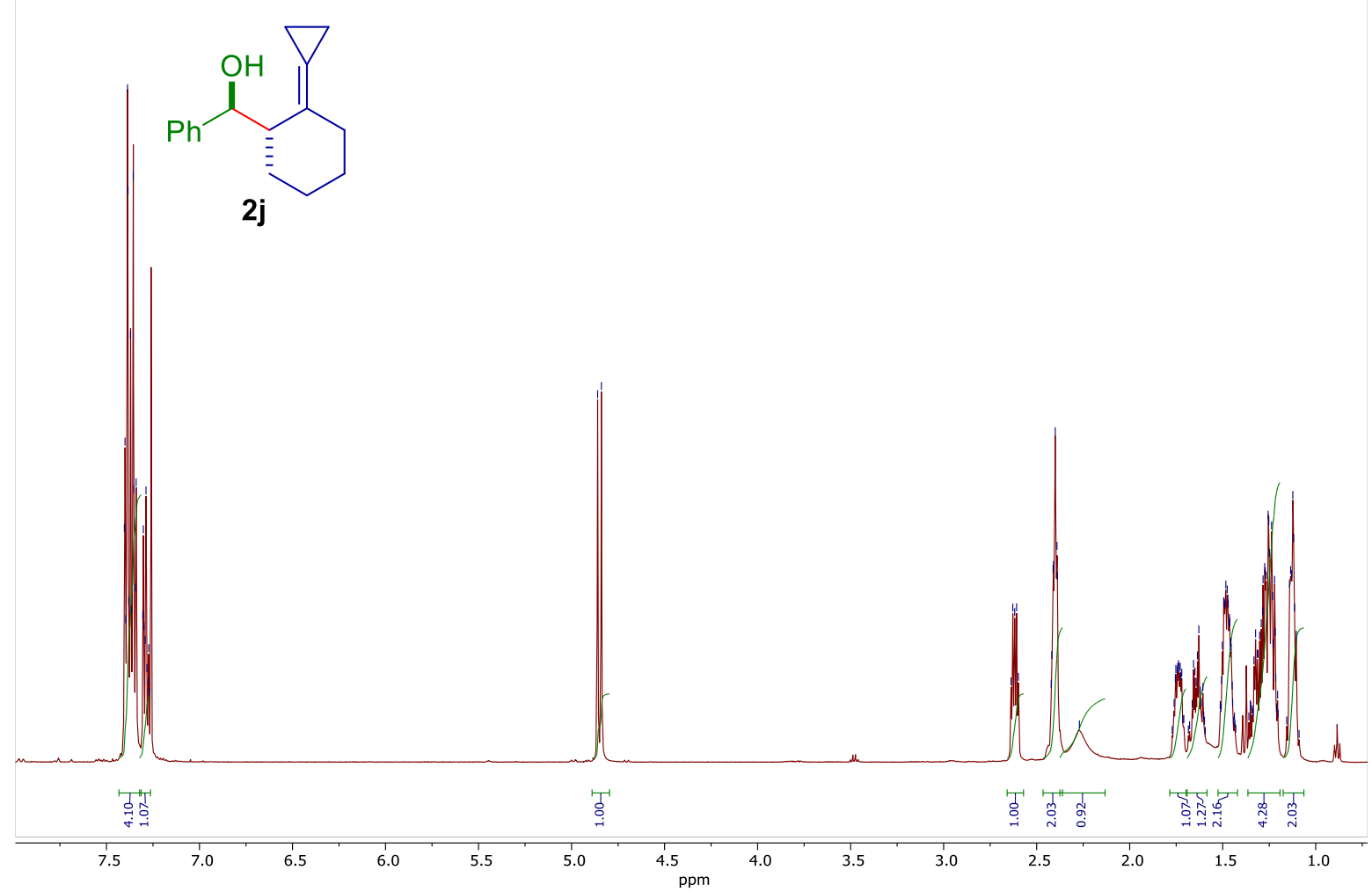

${ }^{13} \mathbf{C}-\mathbf{N M R}\left(126 \mathrm{MHz}, \mathrm{CDCl}_{3}\right)$ of compound $\mathbf{2 j}$

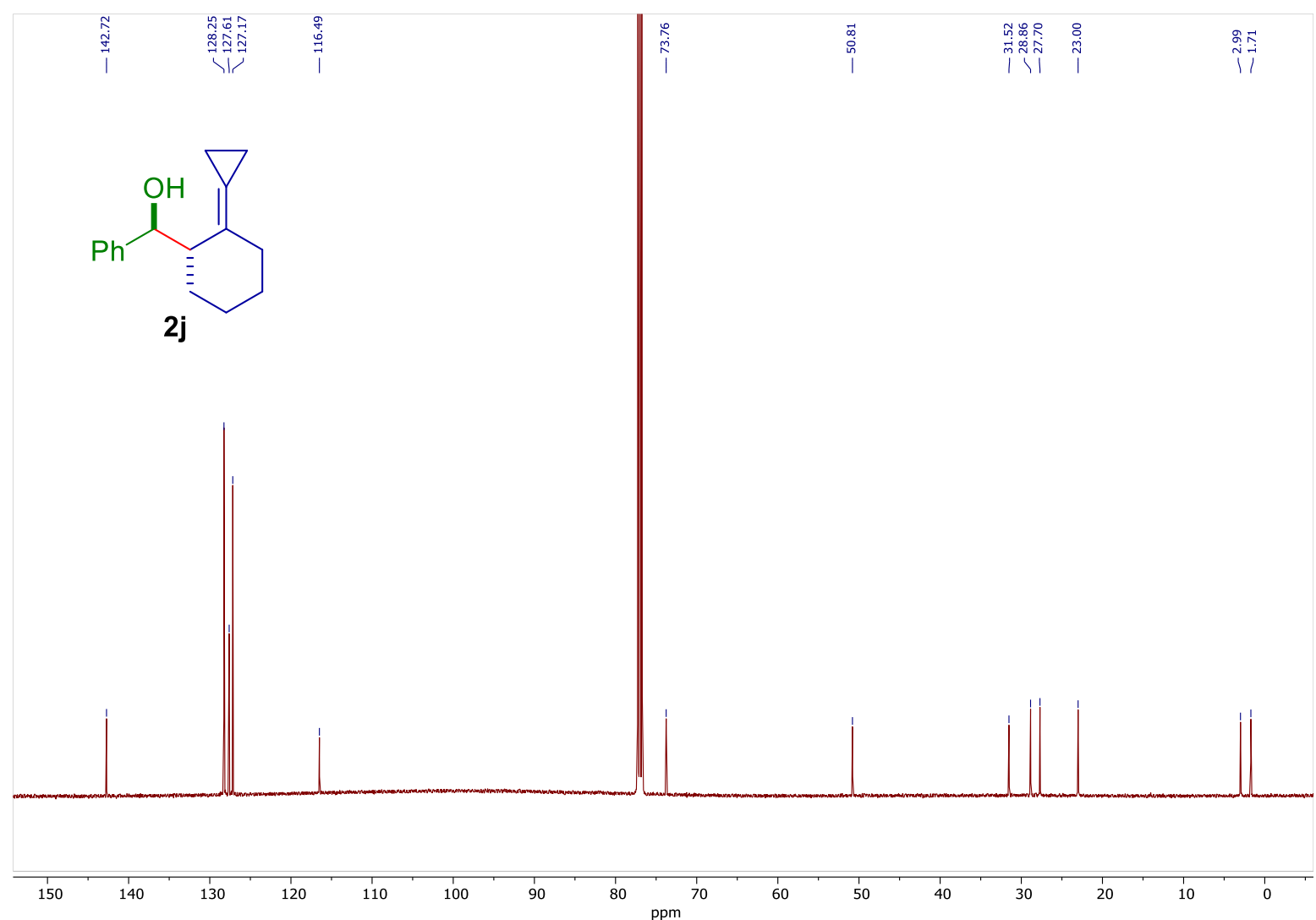


${ }^{1} \mathbf{H}$-NMR $\left(500 \mathrm{MHz}, \mathrm{CDCl}_{3}\right)$ of compound $\mathbf{2 k}$ see procedure

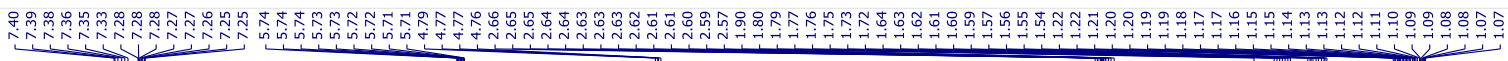<smiles>OC(CC=C1CCCCC1)c1ccccc1</smiles>

2k
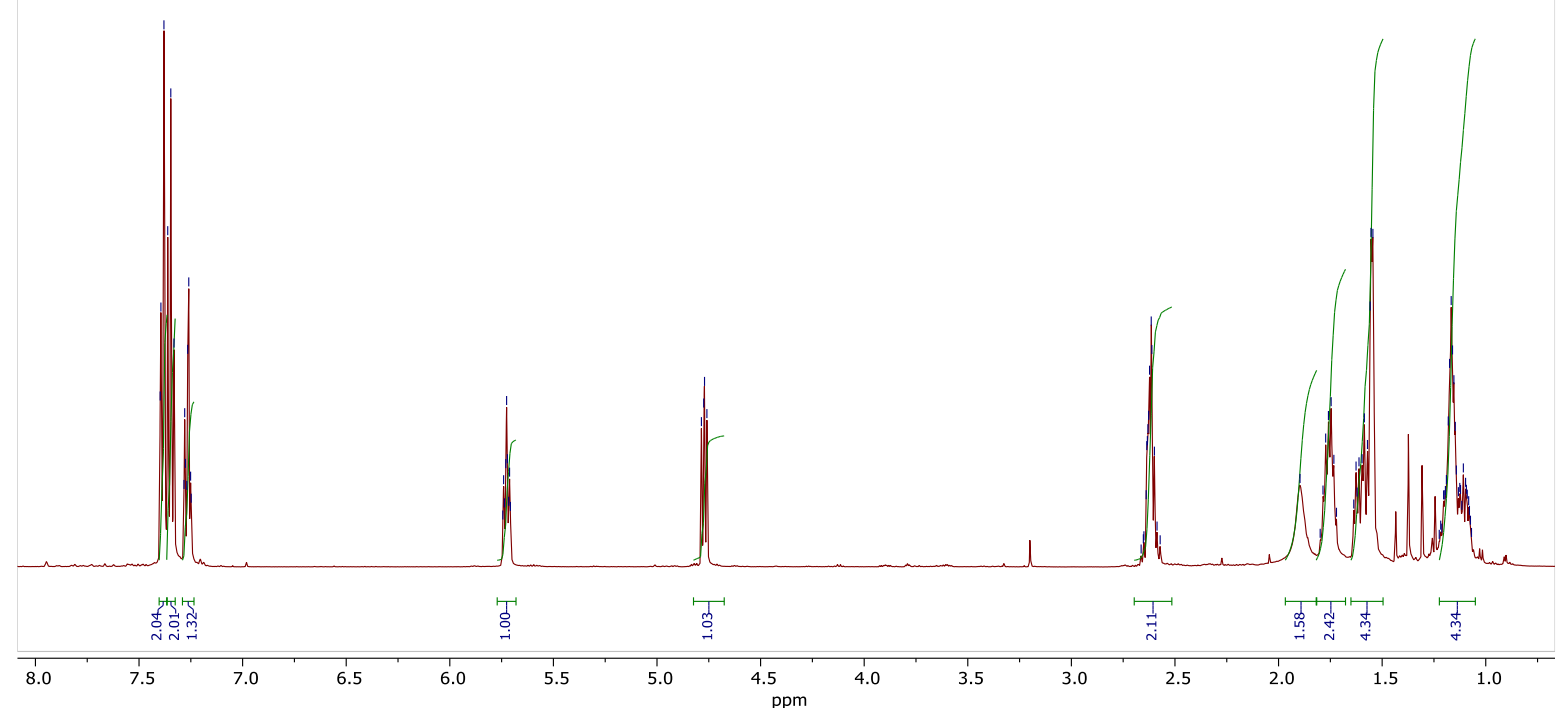

${ }^{13} \mathbf{C}-\mathbf{N M R}\left(126 \mathrm{MHz}, \mathrm{CDCl}_{3}\right)$ of compound $\mathbf{2 k}$

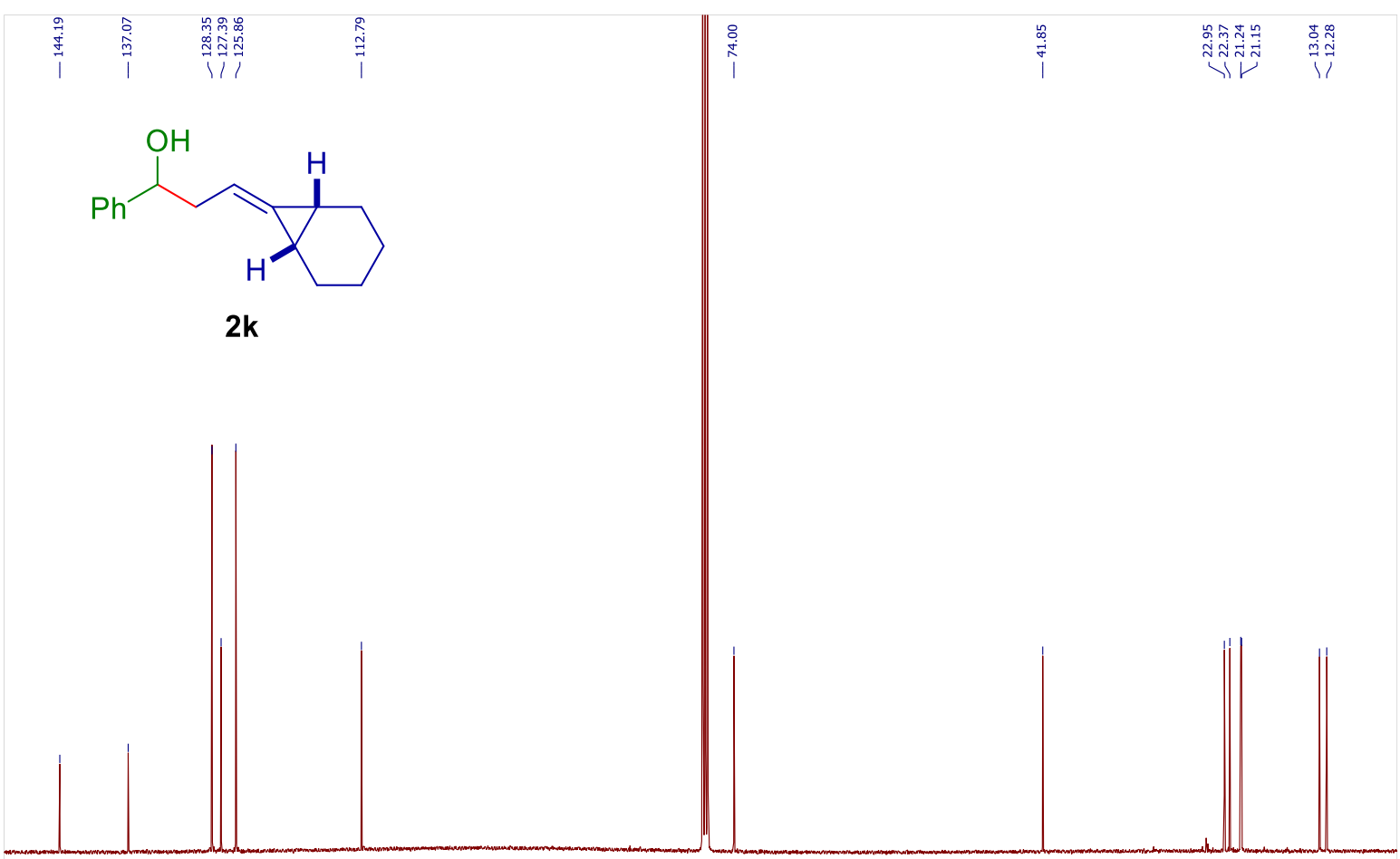

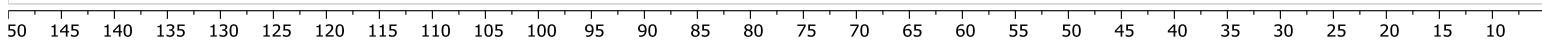


${ }^{1} \mathbf{H}-\mathrm{NMR}\left(500 \mathrm{MHz}, \mathrm{CDCl}_{3}\right)$ of compound $\mathbf{2 l}$ see procedure

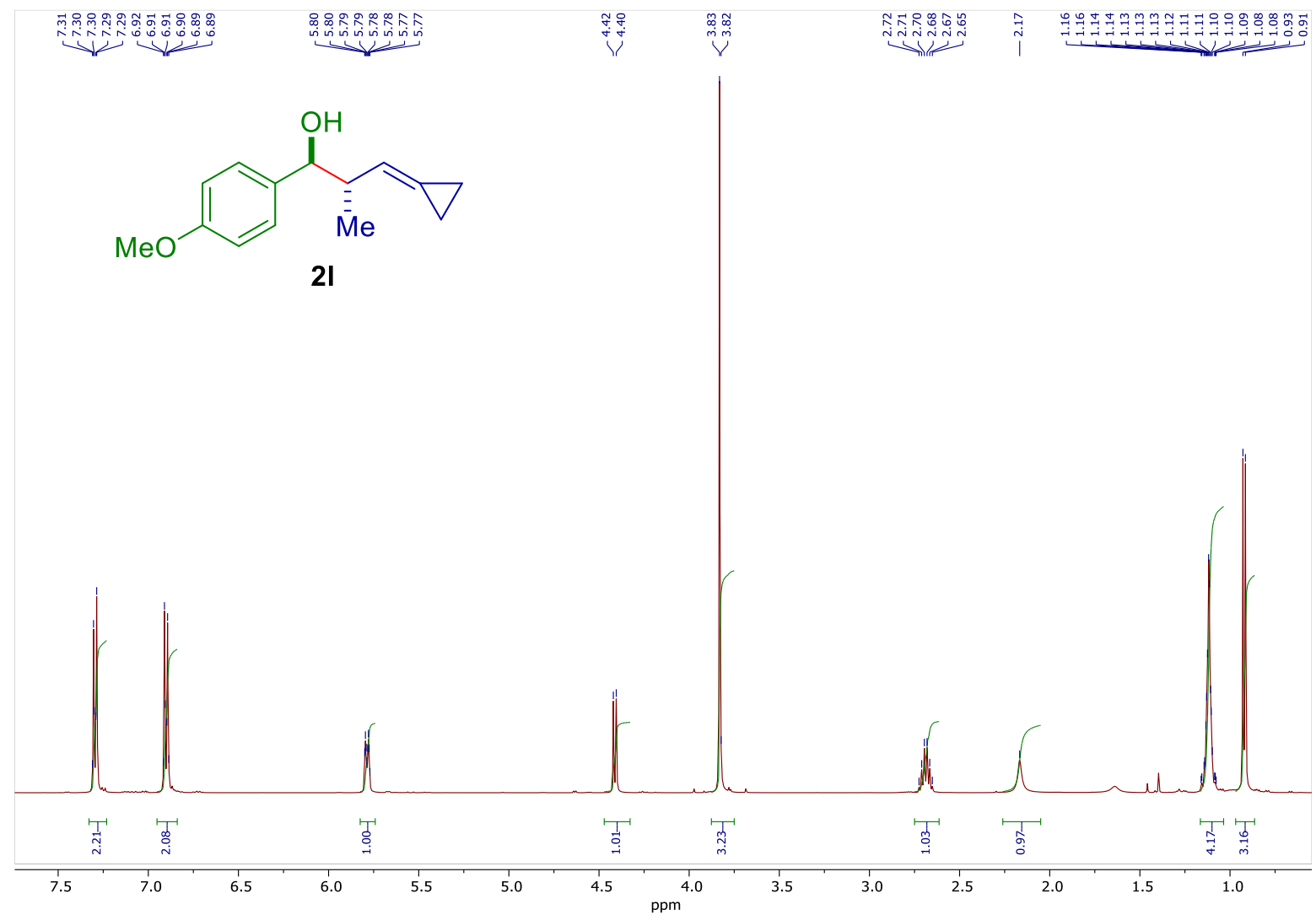

${ }^{13} \mathbf{C}-\mathbf{N M R}\left(126 \mathrm{MHz}, \mathrm{CDCl}_{3}\right)$ of compound $2 \mathbf{I}$

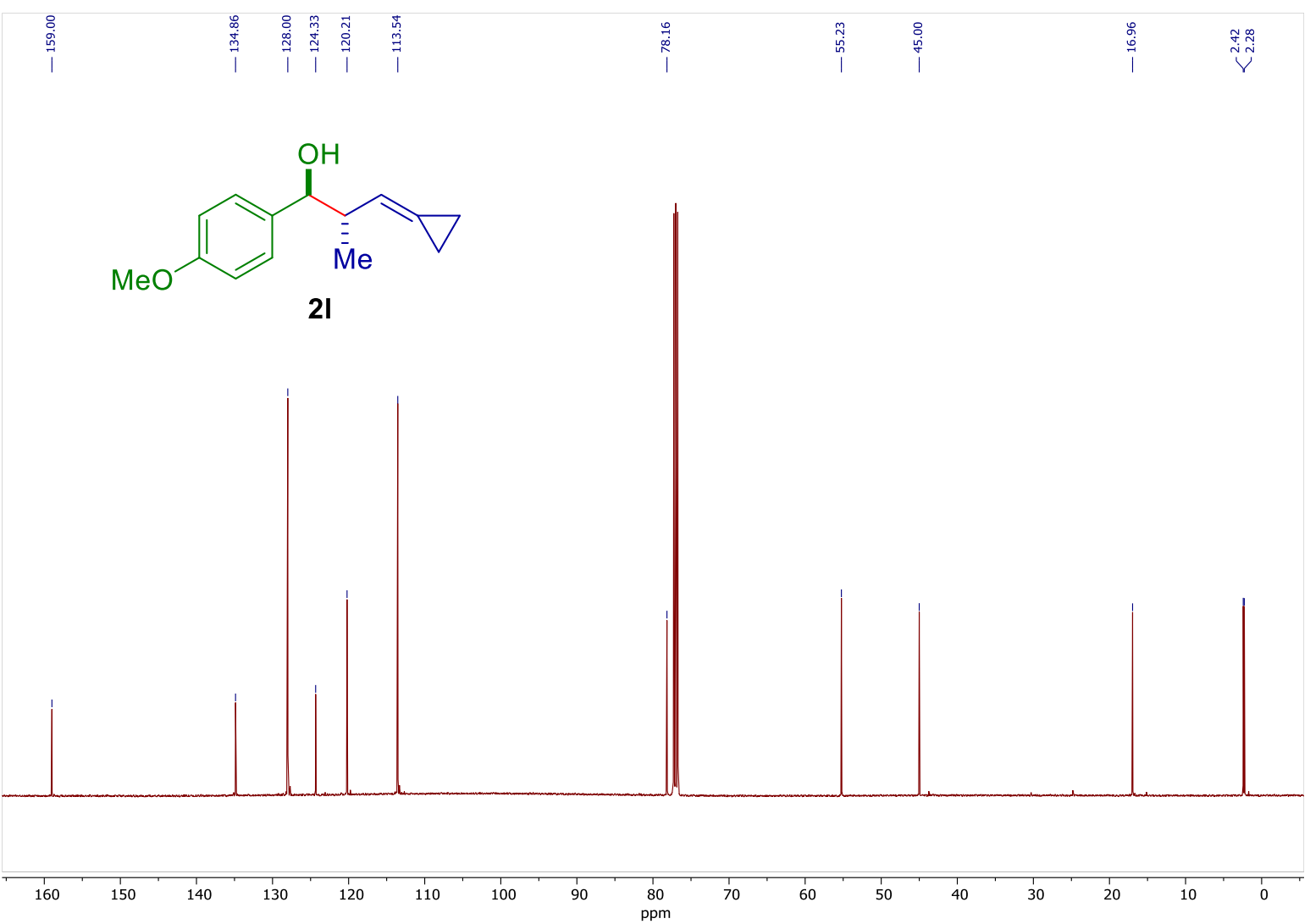


${ }^{1} \mathbf{H}-\mathrm{NMR}\left(500 \mathrm{MHz}, \mathrm{CDCl}_{3}\right)$ of compound $\mathbf{2 m}$ see procedure

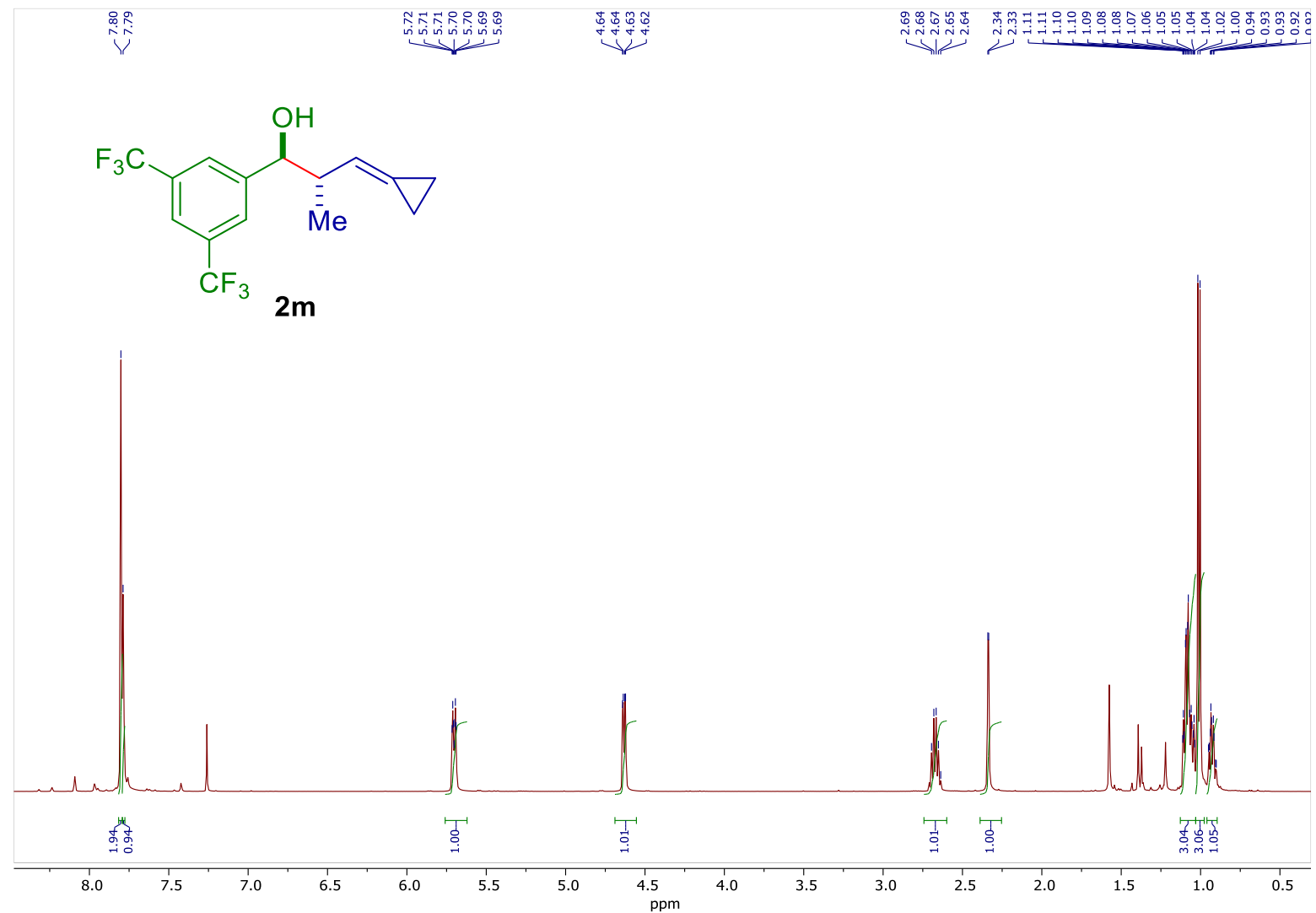

${ }^{13} \mathbf{C}-\mathbf{N M R}\left(126 \mathrm{MHz}, \mathrm{CDCl}_{3}\right)$ of compound $\mathbf{2 m}$

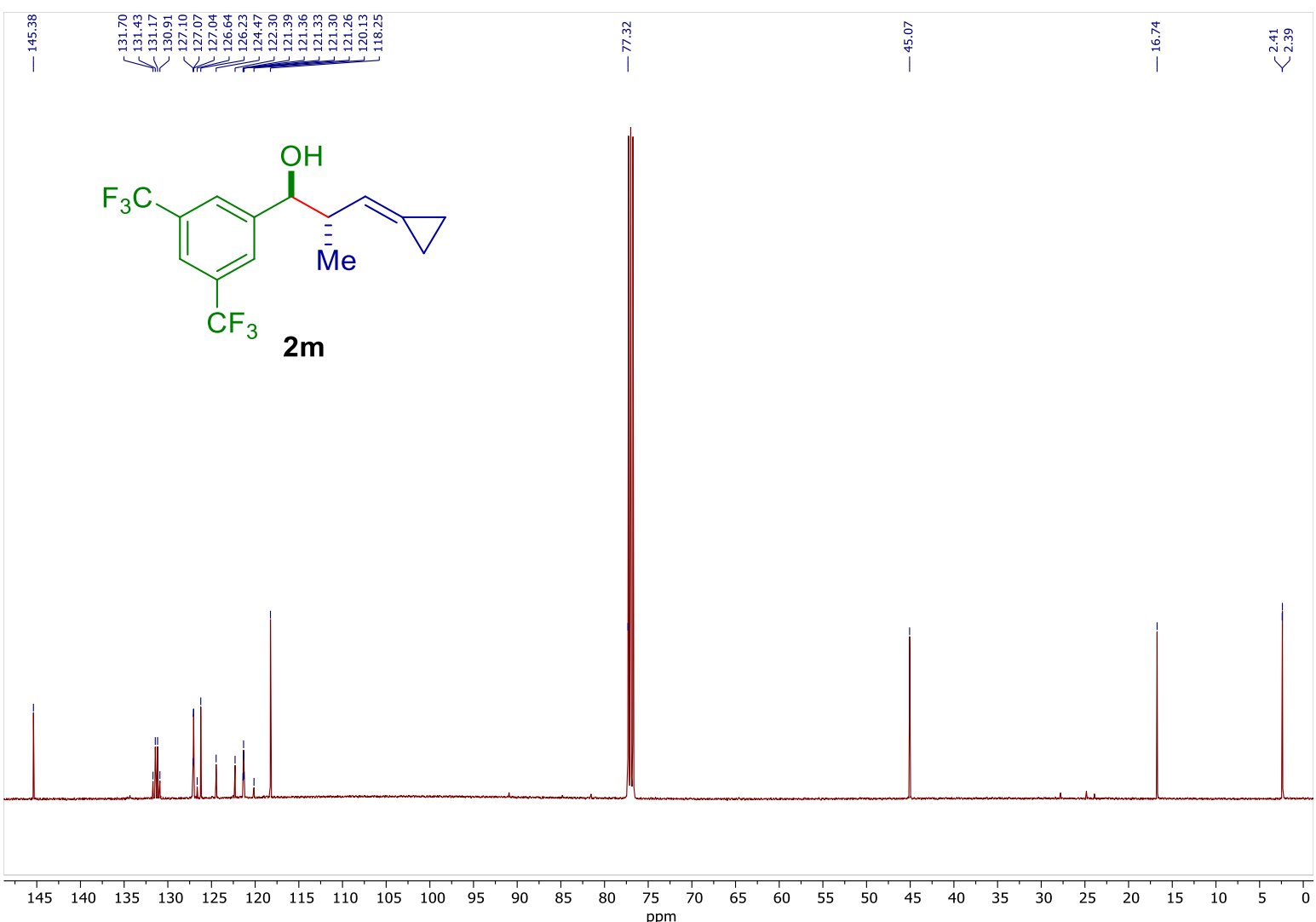


${ }^{1} \mathbf{H}$-NMR $\left(500 \mathrm{MHz}, \mathrm{CDCl}_{3}\right)$ of compound $\mathbf{2 n}$ see procedure

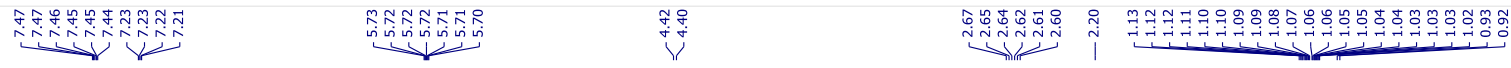<smiles>C[C@H](C=C1CC1)[C@@H](O)c1ccc(Br)cc1</smiles>

2n

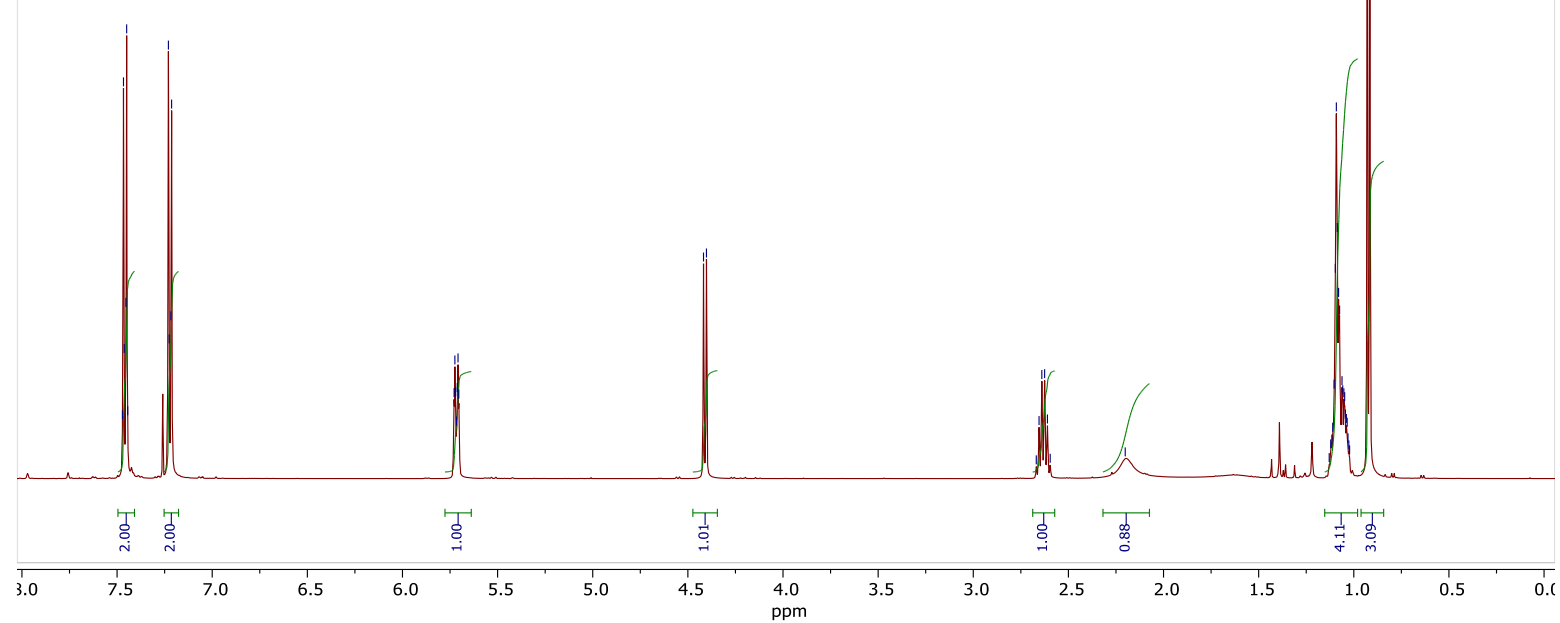

${ }^{13} \mathbf{C}$-NMR $\left(126 \mathrm{MHz}, \mathrm{CDCl}_{3}\right)$ of compound 2n

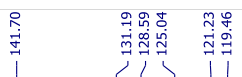

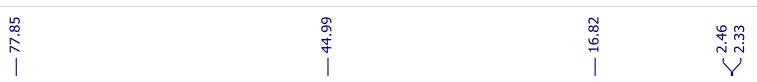<smiles>[M]C(C=C1CC1)C(O)c1ccc(Br)cc1</smiles>

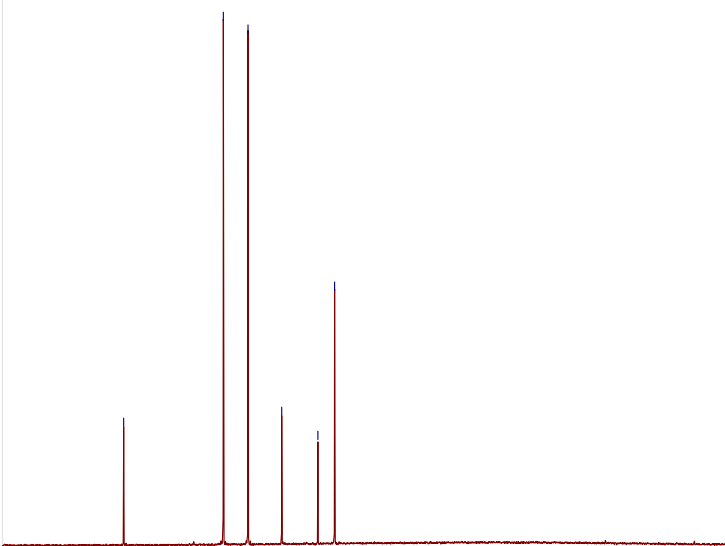

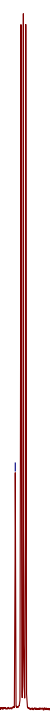

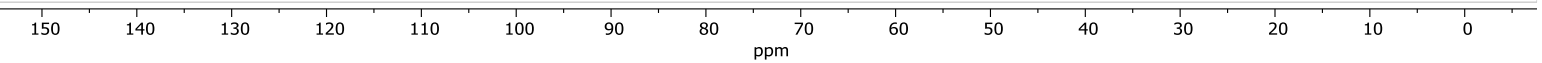


${ }^{1} \mathbf{H}-\mathbf{N M R}\left(500 \mathrm{MHz}, \mathrm{CDCl}_{3}\right)$ of compound $\mathbf{2 o}$ see procedure

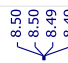

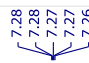

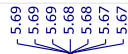

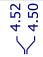

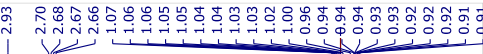<smiles>C[C@@H](C=C1CC1)[C@@H](O)c1ccncc1</smiles>

20

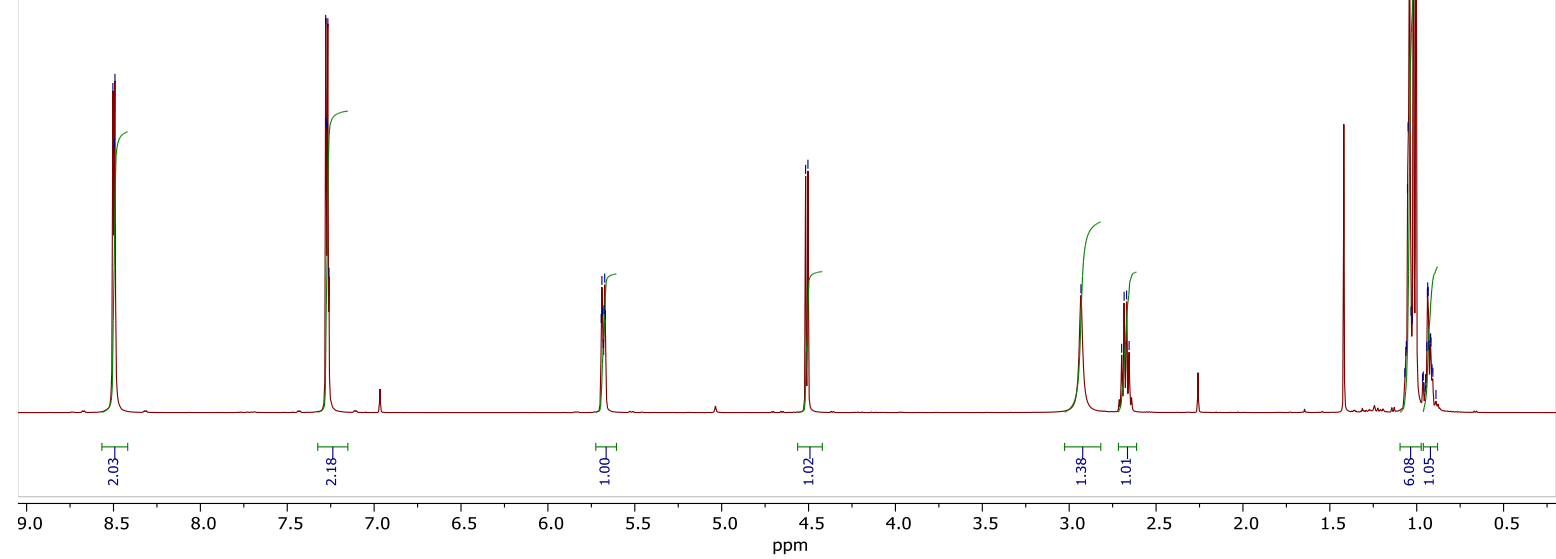

${ }^{13} \mathbf{C}$-NMR (126 MHz, $\left.\mathrm{CDCl}_{3}\right)$ of compound $2 \mathrm{o}$

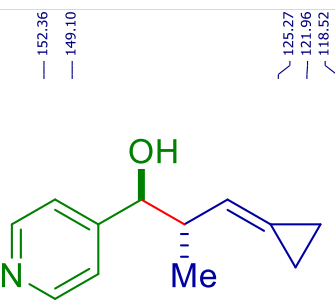

20

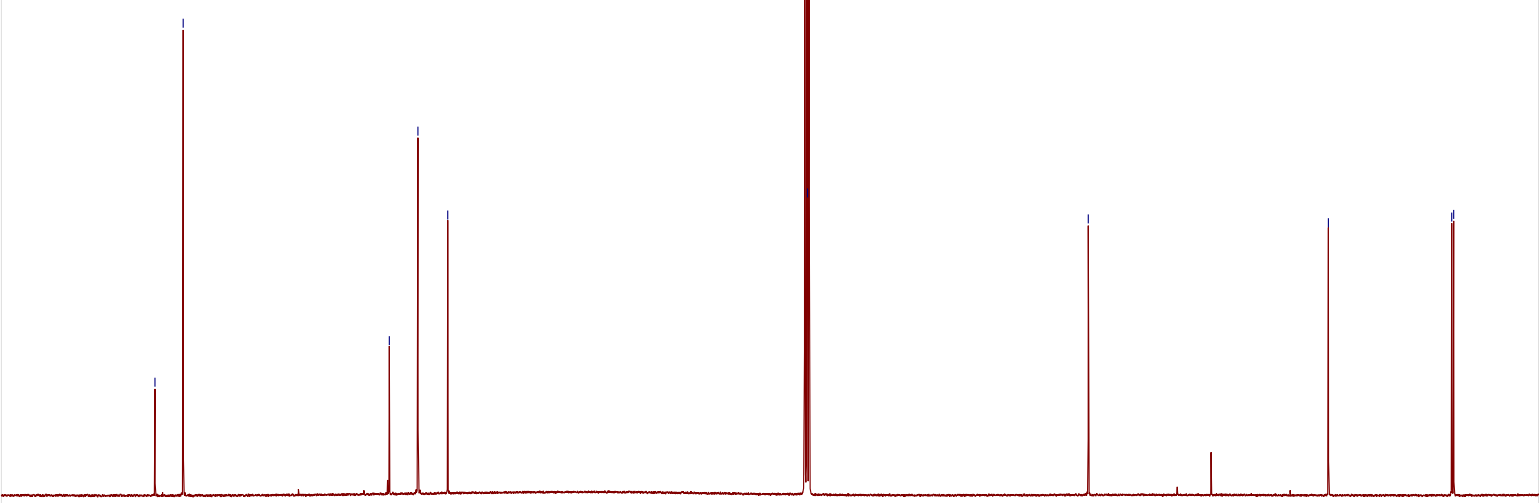

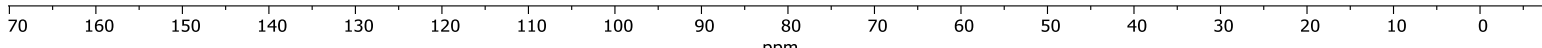


${ }^{\mathbf{1}} \mathbf{H}-\mathrm{NMR}\left(400 \mathrm{MHz}, \mathrm{CDCl}_{3}\right)$ of compound $\mathbf{2 \mathbf { p }}$ see procedure

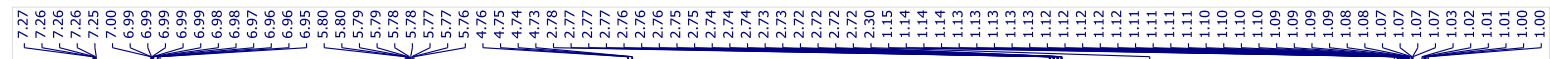<smiles>OC(c1cccs1)[C@H]([Mg])C=C1CC1</smiles>
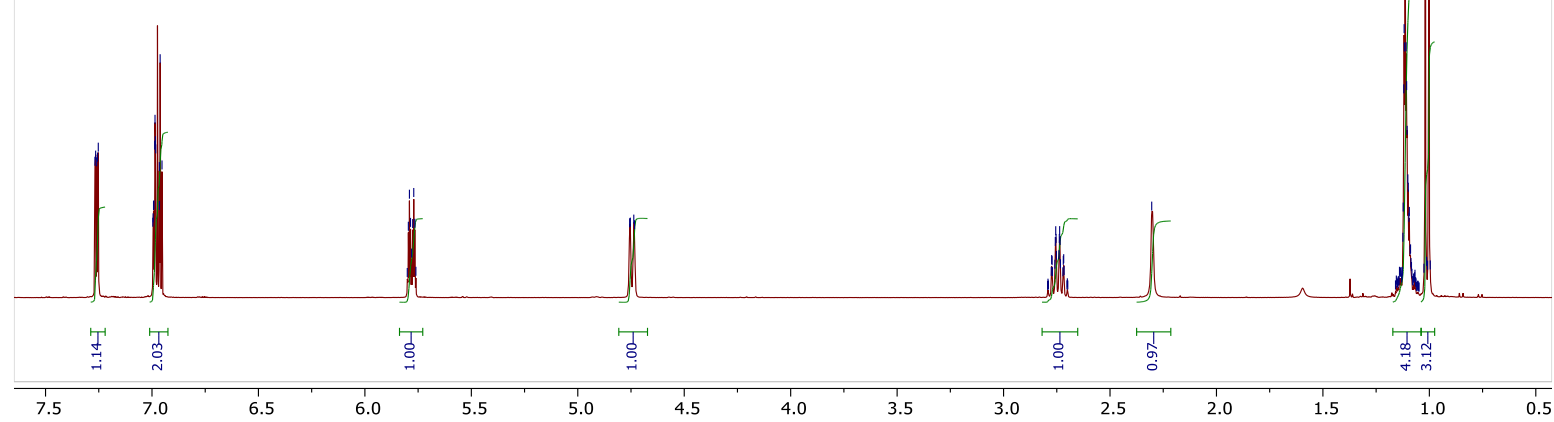

${ }^{13} \mathbf{C}-\mathbf{N M R}\left(101 \mathrm{MHz}, \mathrm{CDCl}_{3}\right)$ of compound 2p
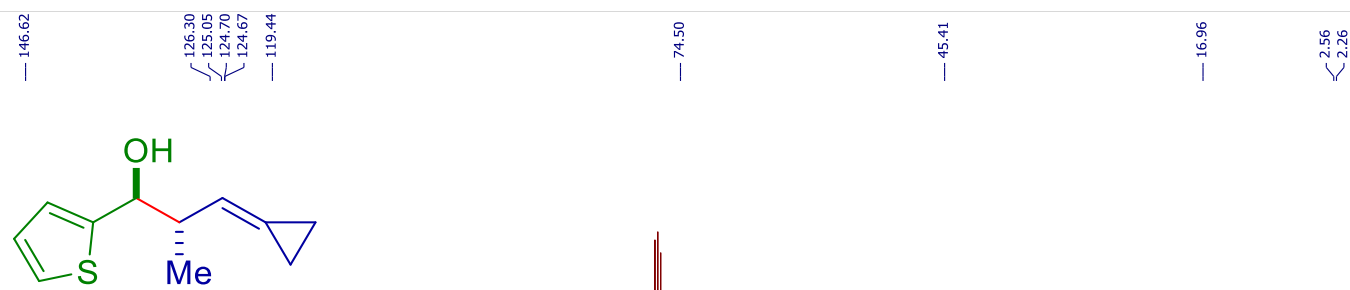

$2 p$
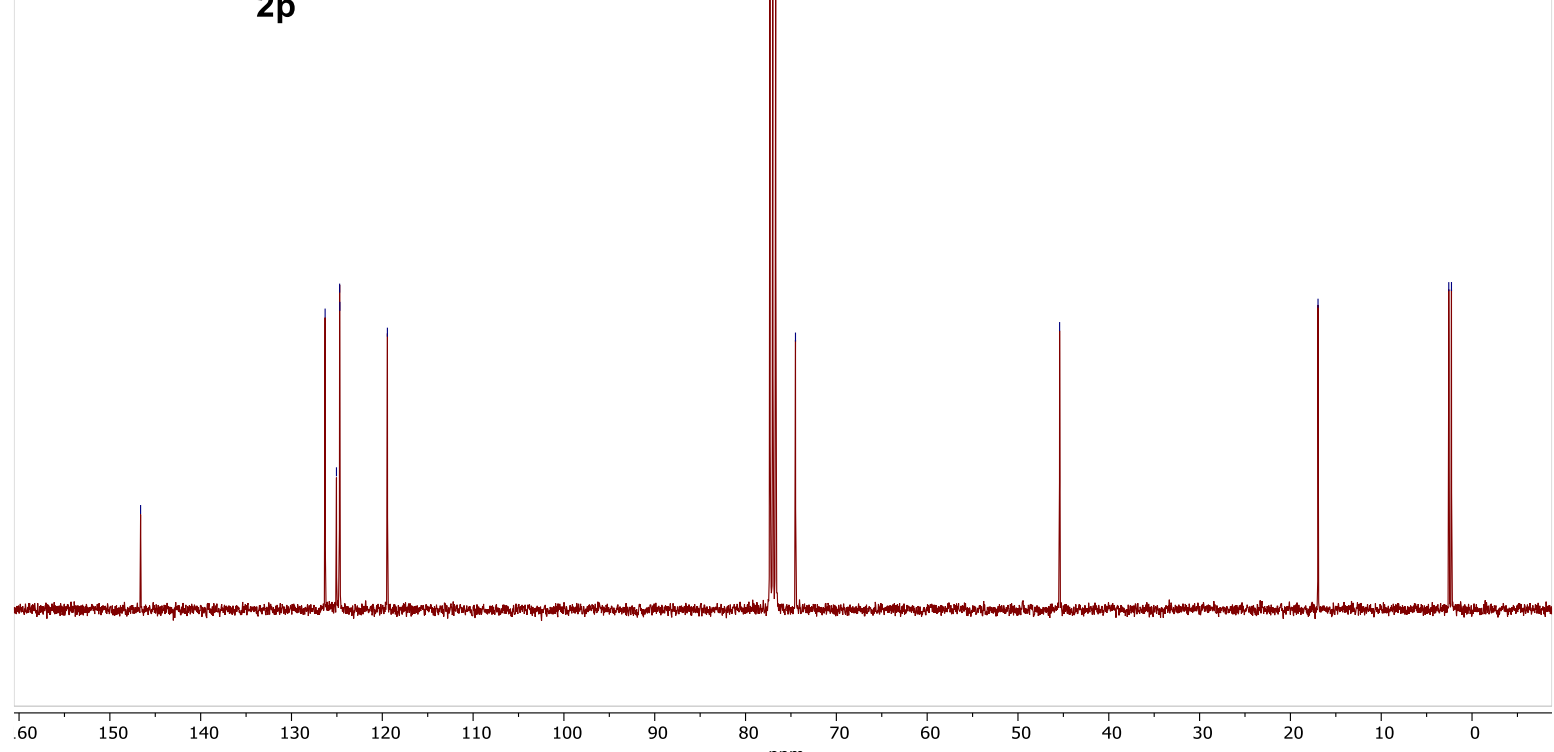
${ }^{1} \mathbf{H}-\mathbf{N M R}\left(400 \mathrm{MHz}, \mathrm{CDCl}_{3}\right)$ of compound $\mathbf{2 q}$ see procedure

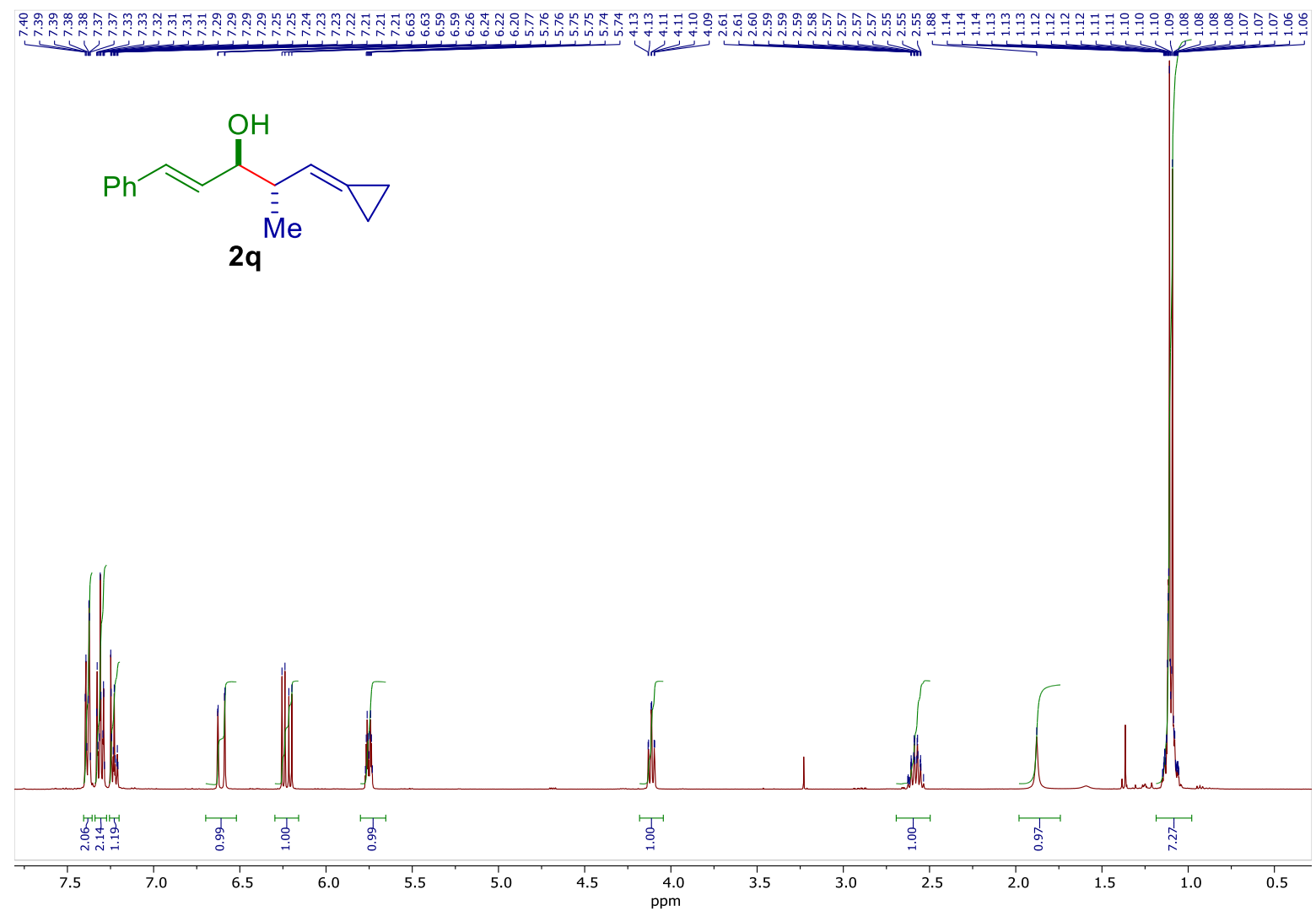

${ }^{13} \mathbf{C}-\mathbf{N M R}\left(101 \mathrm{MHz}, \mathrm{CDCl}_{3}\right)$ of compound $\mathbf{2 q}$

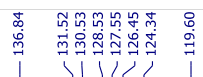

$\stackrel{\overrightarrow{0}}{\stackrel{0}{0}}$

।<smiles>CC(C=C1CC1)[C@@H](O)C=Cc1ccccc1</smiles>

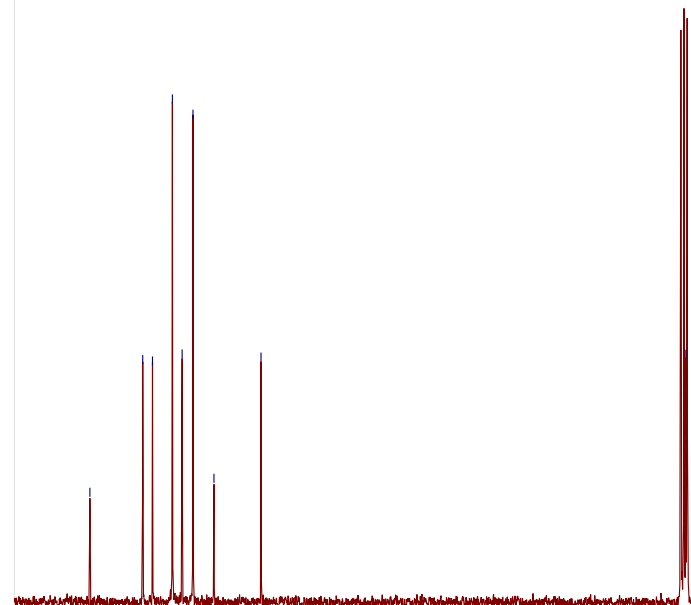

$\begin{array}{lllllllllllllllllllllllllllllllllllllllllll}140 & 135 & 130 & 125 & 120 & 115 & 110 & 105 & 100 & 95 & 90 & 85 & 80 & 75 & 70 & 65 & 60 & 55 & 50 & 45 & 40 & 35 & 30 & 25 & 20 & 15 & 10 & 5 & 0 & -5 & -1\end{array}$ 
${ }^{1} \mathbf{H}-\mathbf{N M R}\left(400 \mathrm{MHz}, \mathrm{CDCl}_{3}\right)$ of compound $2 \mathbf{r}$ see procedure

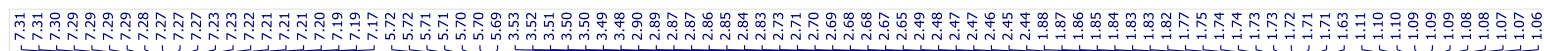<smiles>[Z10]C(C=C1CC1)C(O)CCc1ccccc1</smiles>
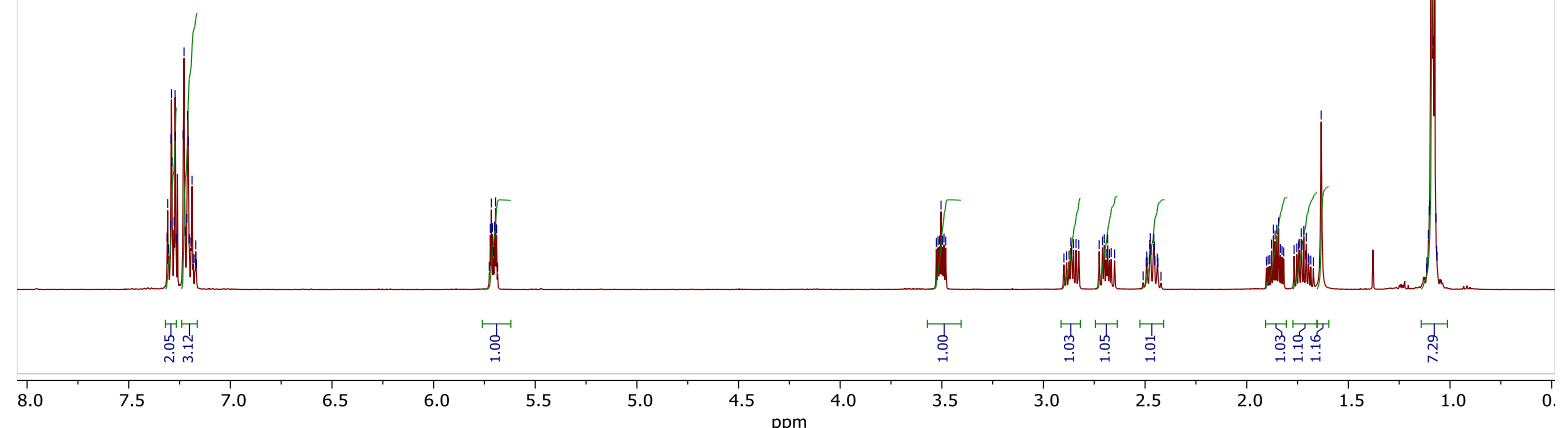

${ }^{13} \mathbf{C}$-NMR (101 MHz, $\left.\mathrm{CDCl}_{3}\right)$ of compound $\mathbf{2 r}$

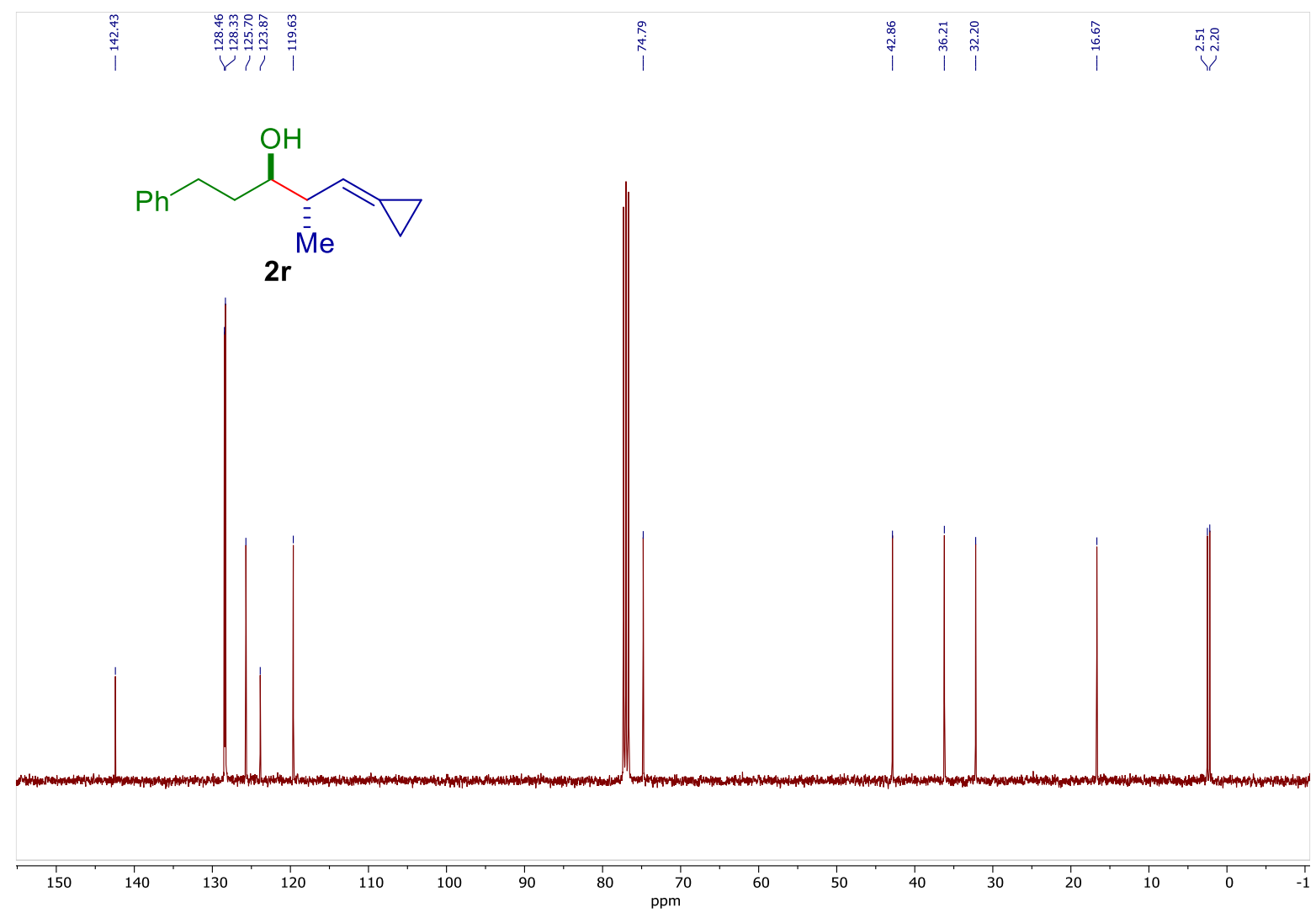


${ }^{\mathbf{1}} \mathbf{H}-\mathrm{NMR}\left(400 \mathrm{MHz}, \mathrm{CDCl}_{3}\right)$ of compound $\mathbf{2 s}$ see procedure

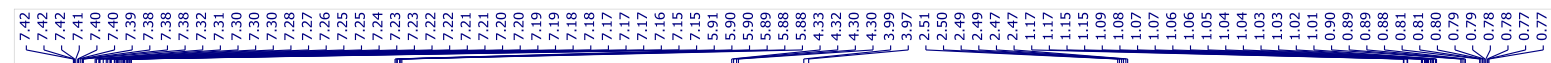

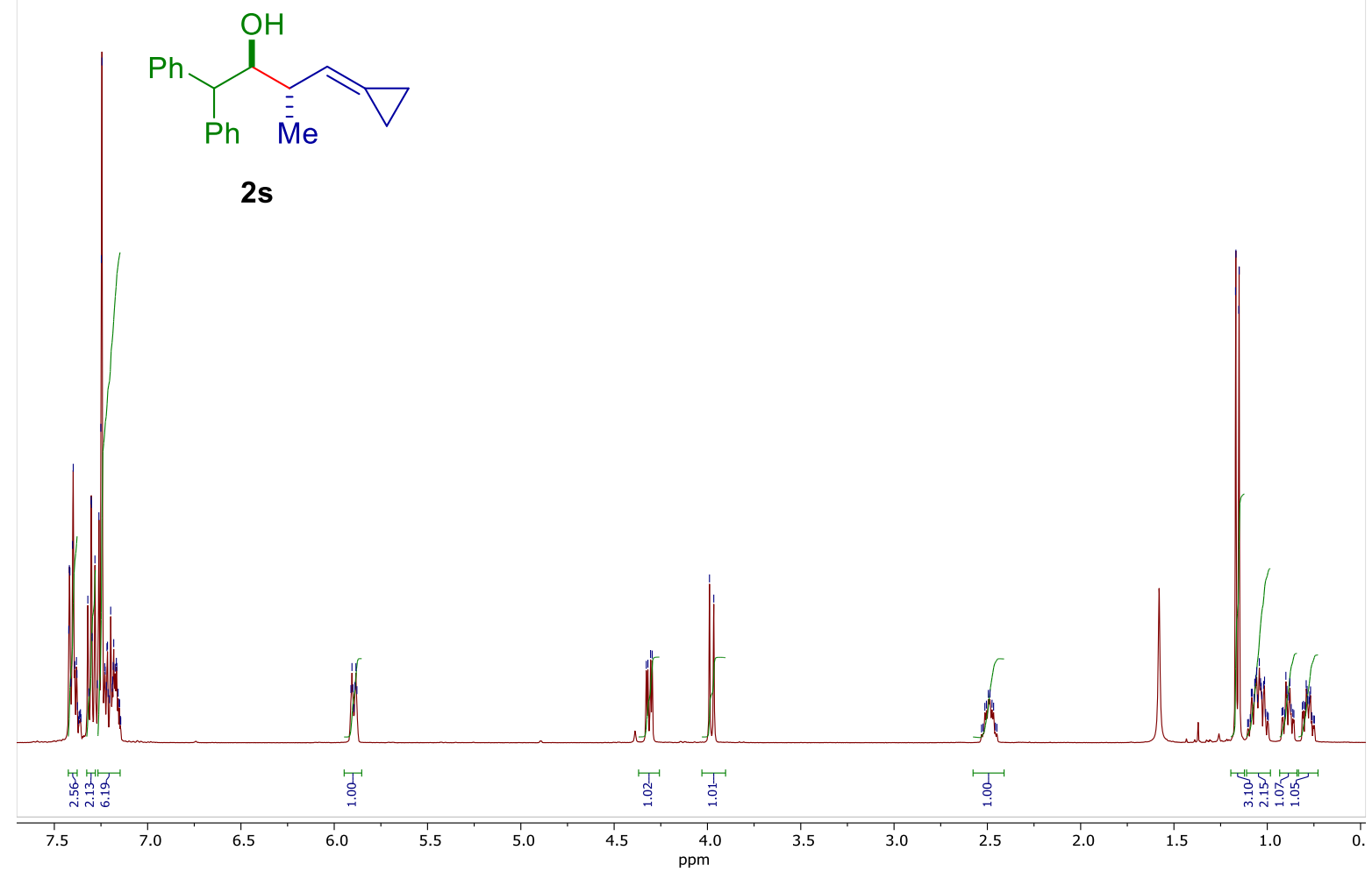

${ }^{13} \mathbf{C}$-NMR (101 MHz, $\left.\mathrm{CDCl}_{3}\right)$ of compound 2s

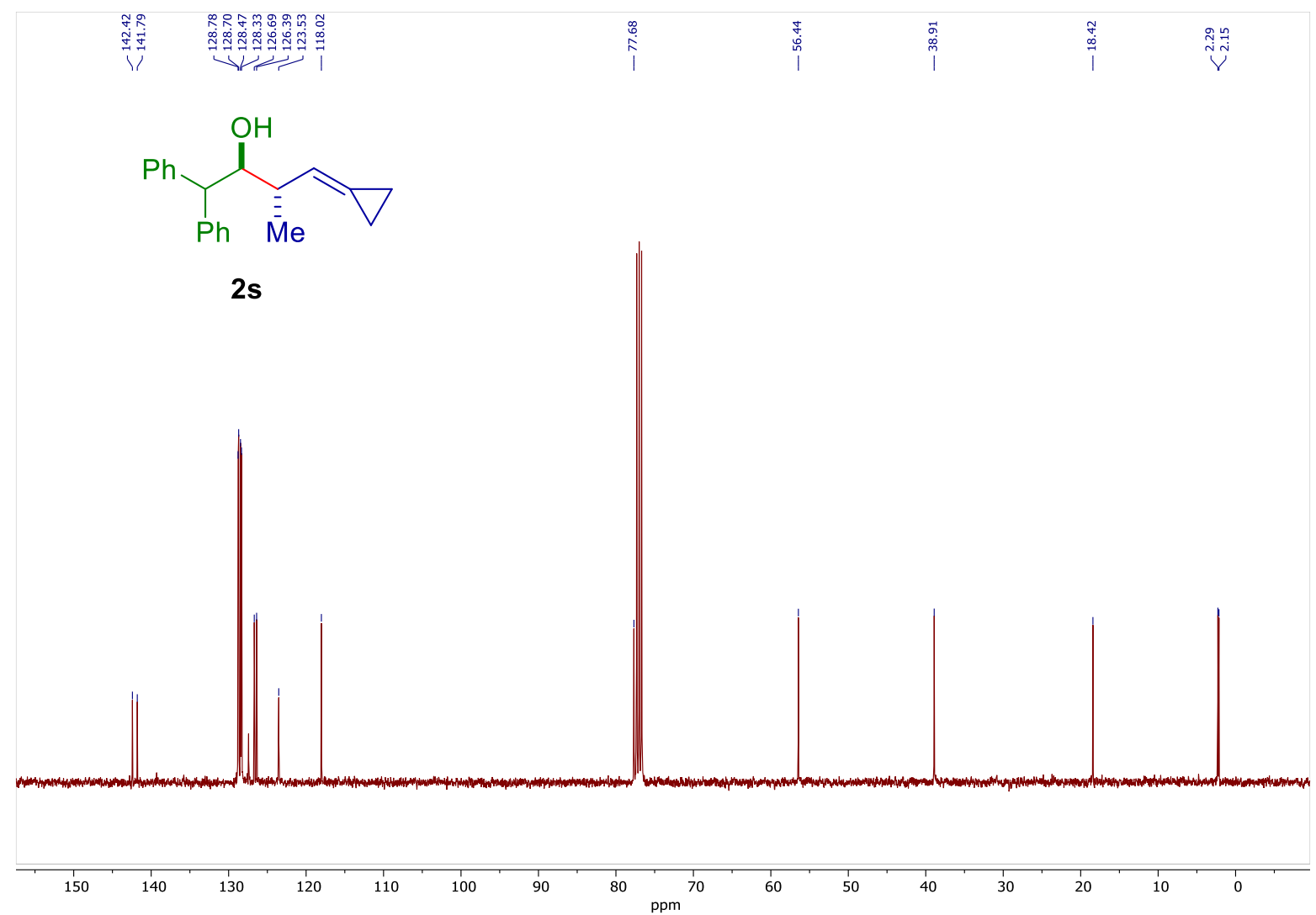


${ }^{1} \mathrm{H}-\mathrm{NMR}\left(500 \mathrm{MHz}, \mathrm{CDCl}_{3}\right)$ of compound $2 \mathrm{t}$ see procedure

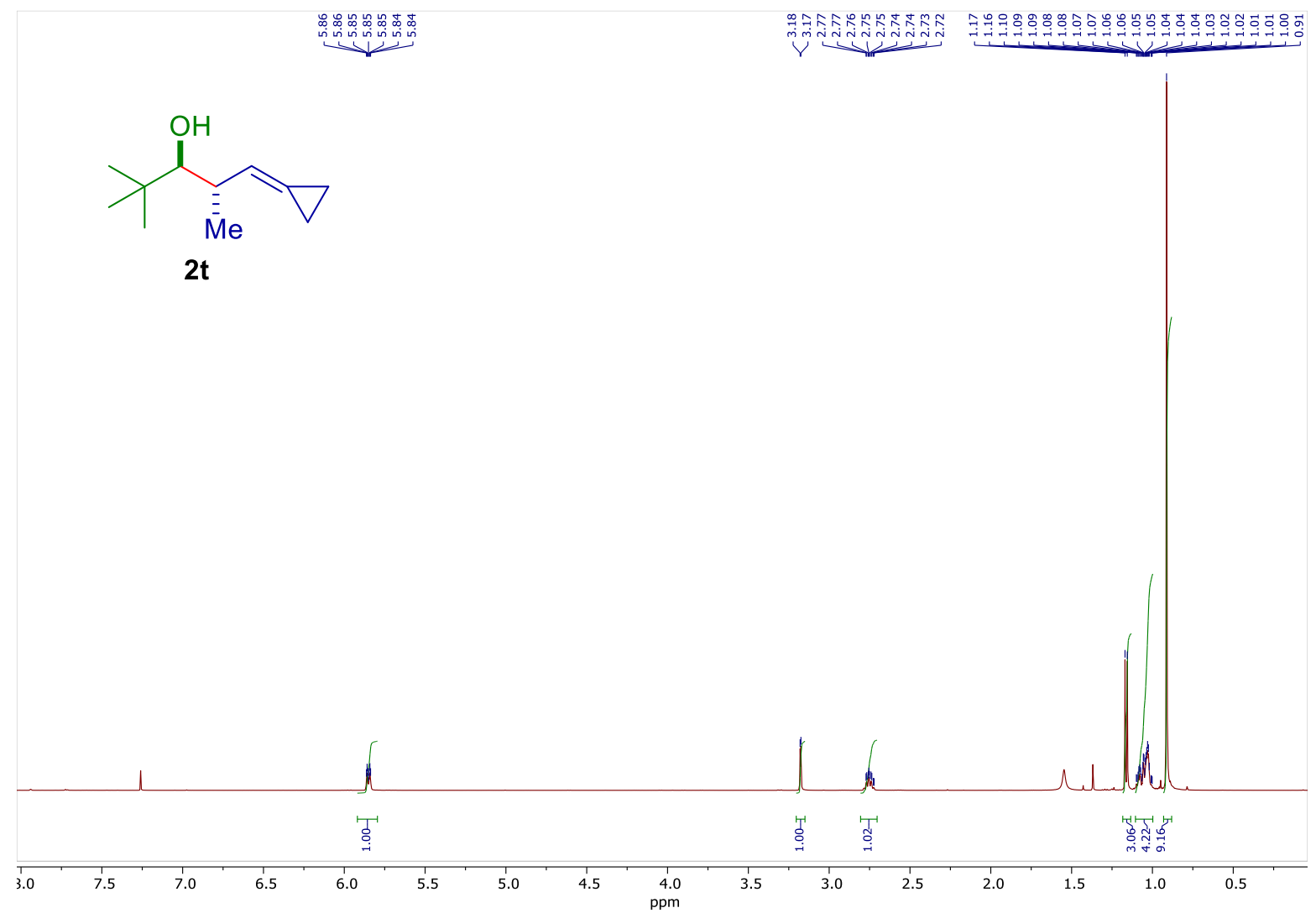

${ }^{13} \mathbf{C}-\mathbf{N M R}\left(126 \mathrm{MHz}, \mathrm{CDCl}_{3}\right)$ of compound $\mathbf{2 t}$
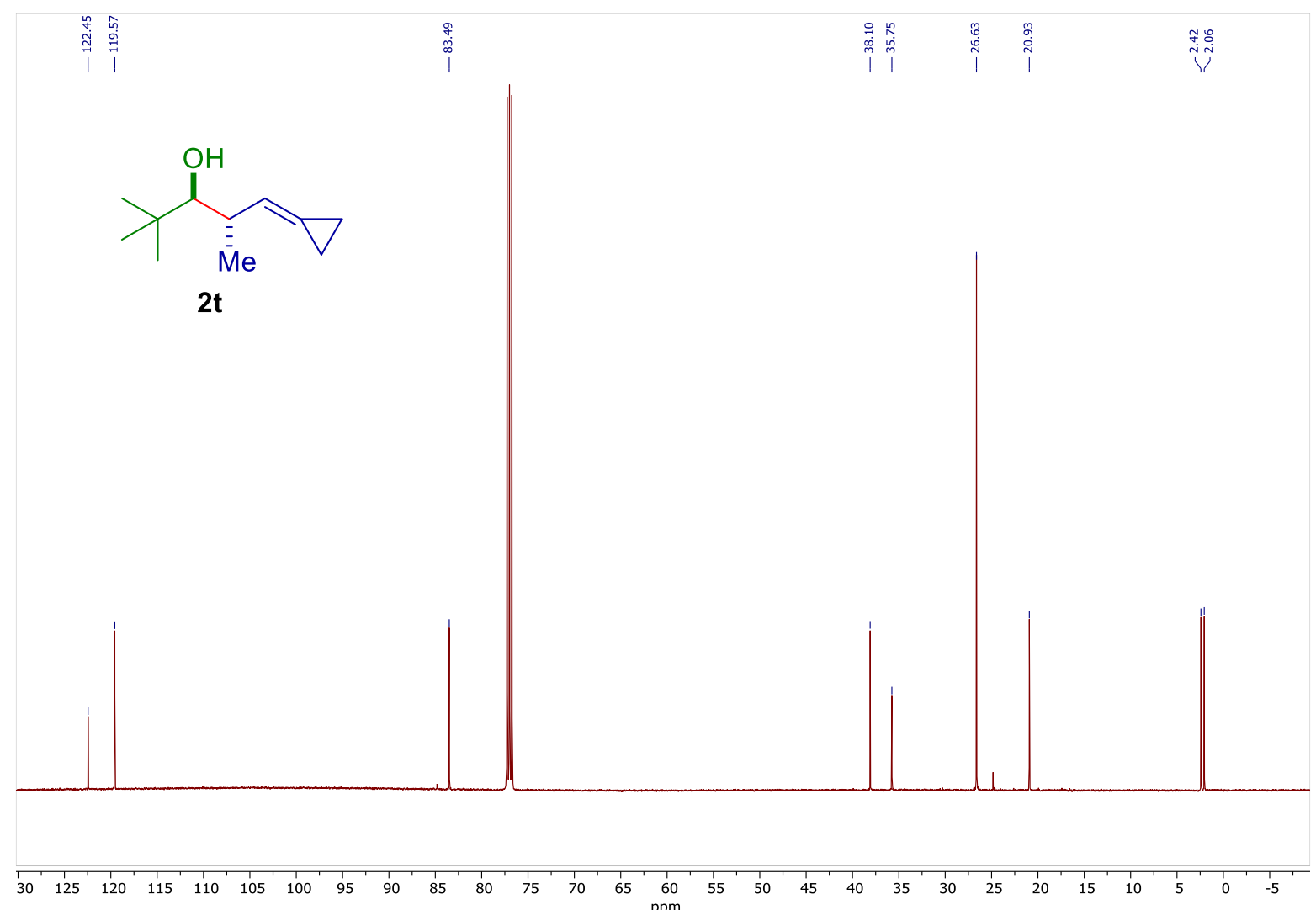
${ }^{1} \mathbf{H}-\mathrm{NMR}\left(400 \mathrm{MHz}, \mathrm{CDCl}_{3}\right)$ of compound 7 a see procedure

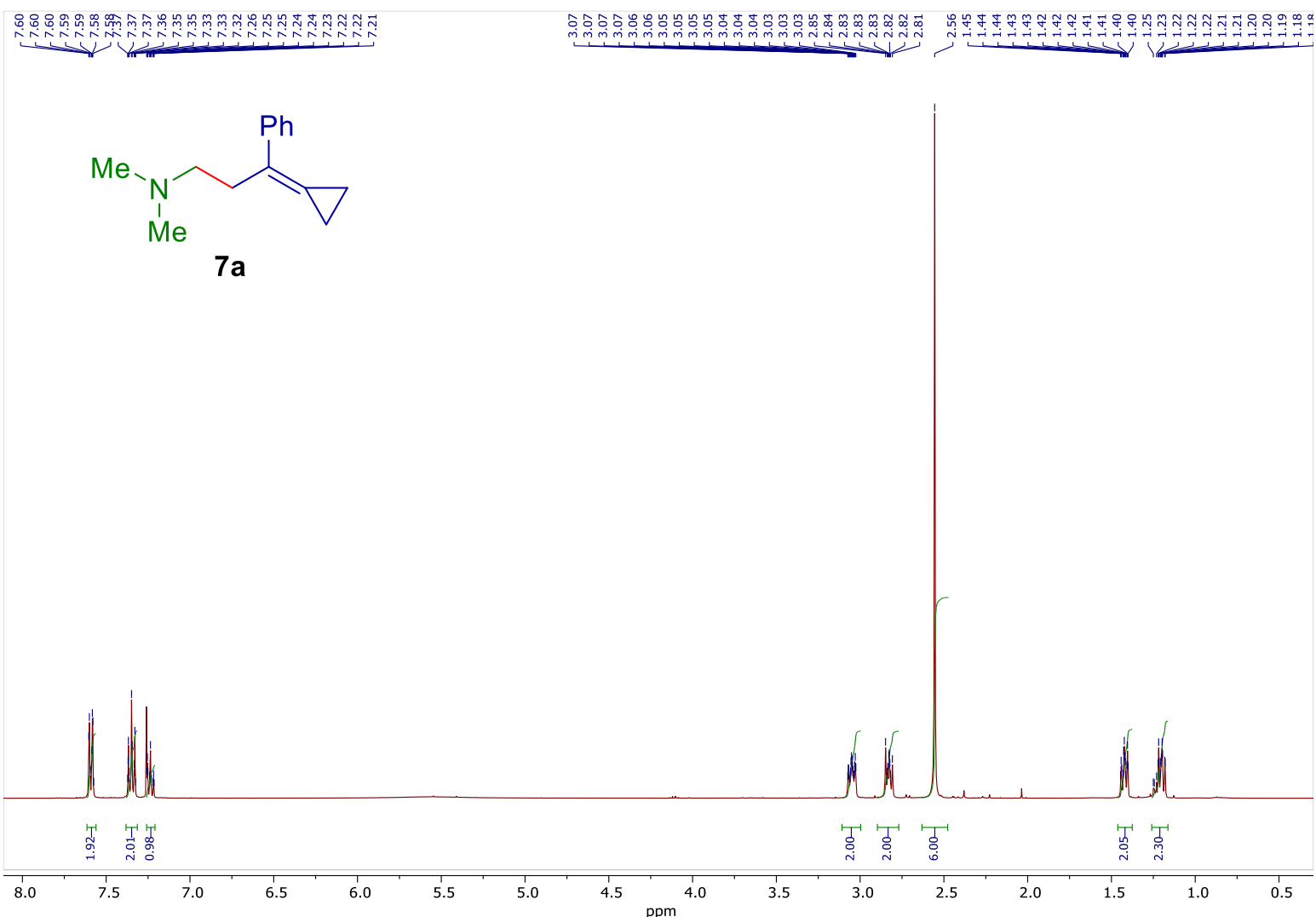

${ }^{13} \mathbf{C}$-NMR (101 MHz, $\left.\mathrm{CDCl}_{3}\right)$ of compound 7a

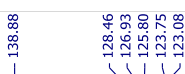

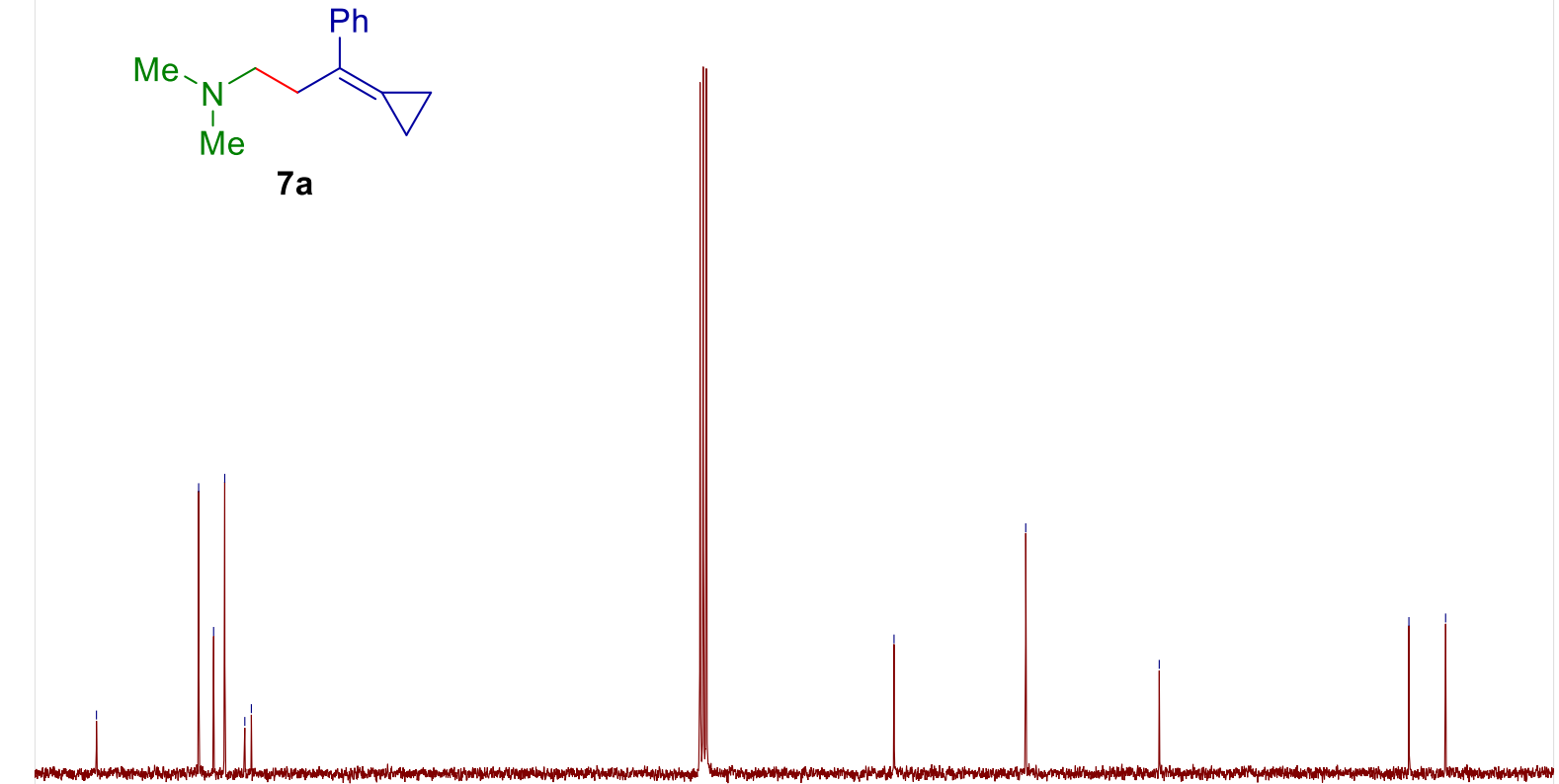

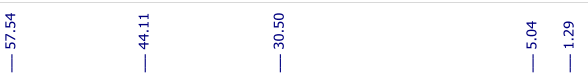

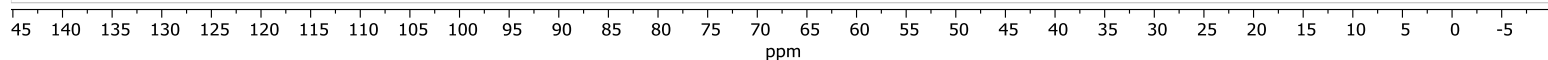


${ }^{1} \mathbf{H}$-NMR $\left(500 \mathrm{MHz}, \mathrm{CDCl}_{3}\right)$ of compound $\mathbf{7 b}$ see procedure

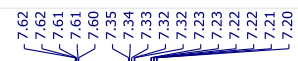

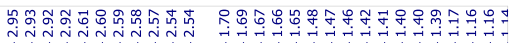<smiles>c1ccc(C(CCN2CCCCC2)=C2CC2)cc1</smiles>

$7 b$

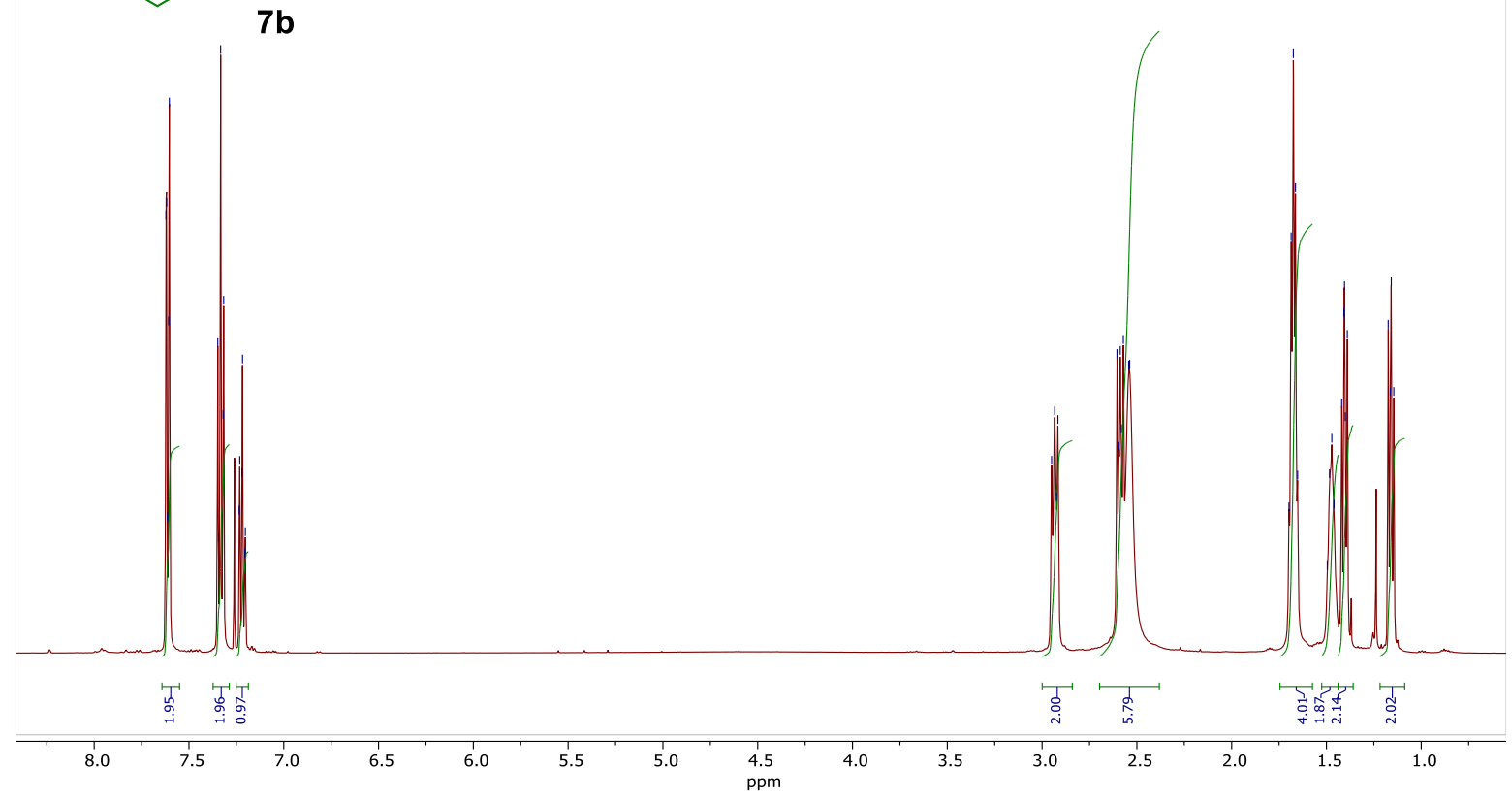

${ }^{13} \mathbf{C}$-NMR (126 MHz, $\mathrm{CDCl}_{3}$ ) of compound $\mathbf{7 b}$

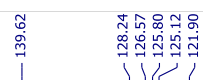

$7 b$<smiles>c1ccc(CCN2CCCCC2)cc1</smiles>

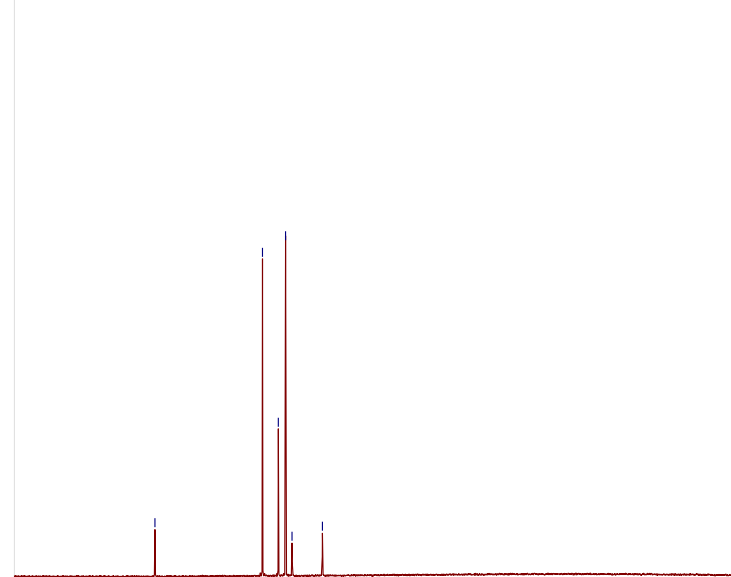

$$
\mid
$$


${ }^{1} \mathbf{H}-\mathrm{NMR}\left(500 \mathrm{MHz}, \mathrm{CDCl}_{3}\right)$ of compound 7c see procedure

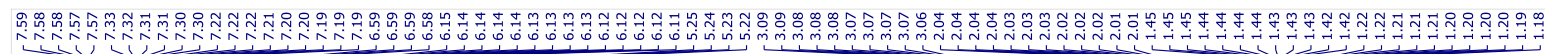<smiles>C1=CC=CC(CC(=C2CC2)c2ccccc2)C=C1</smiles>

7c

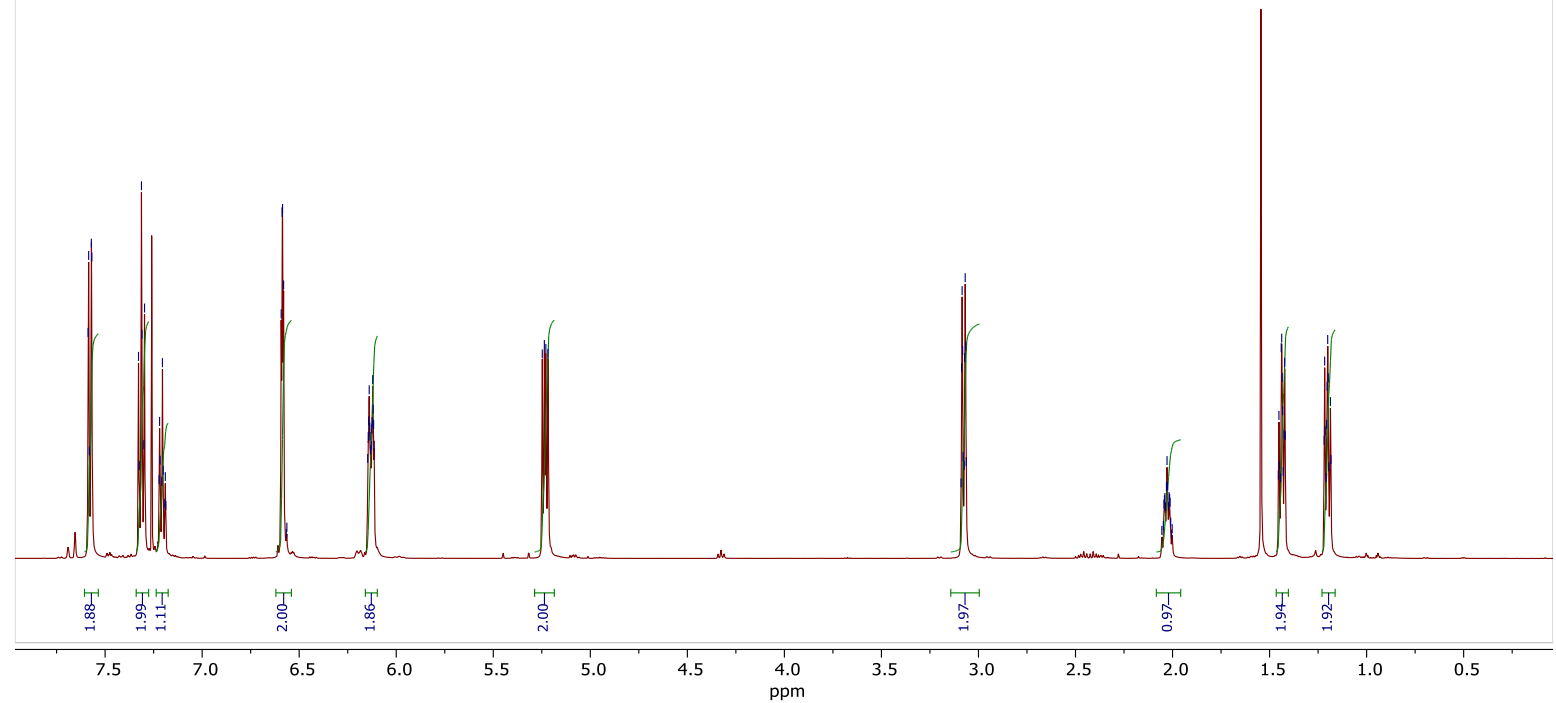

${ }^{13} \mathbf{C}-\mathrm{NMR}\left(126 \mathrm{MHz}, \mathrm{CDCl}_{3}\right)$ of compound 7c

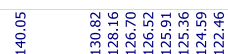

烈

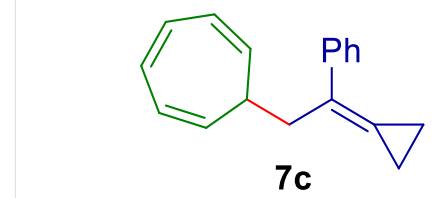

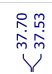

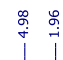
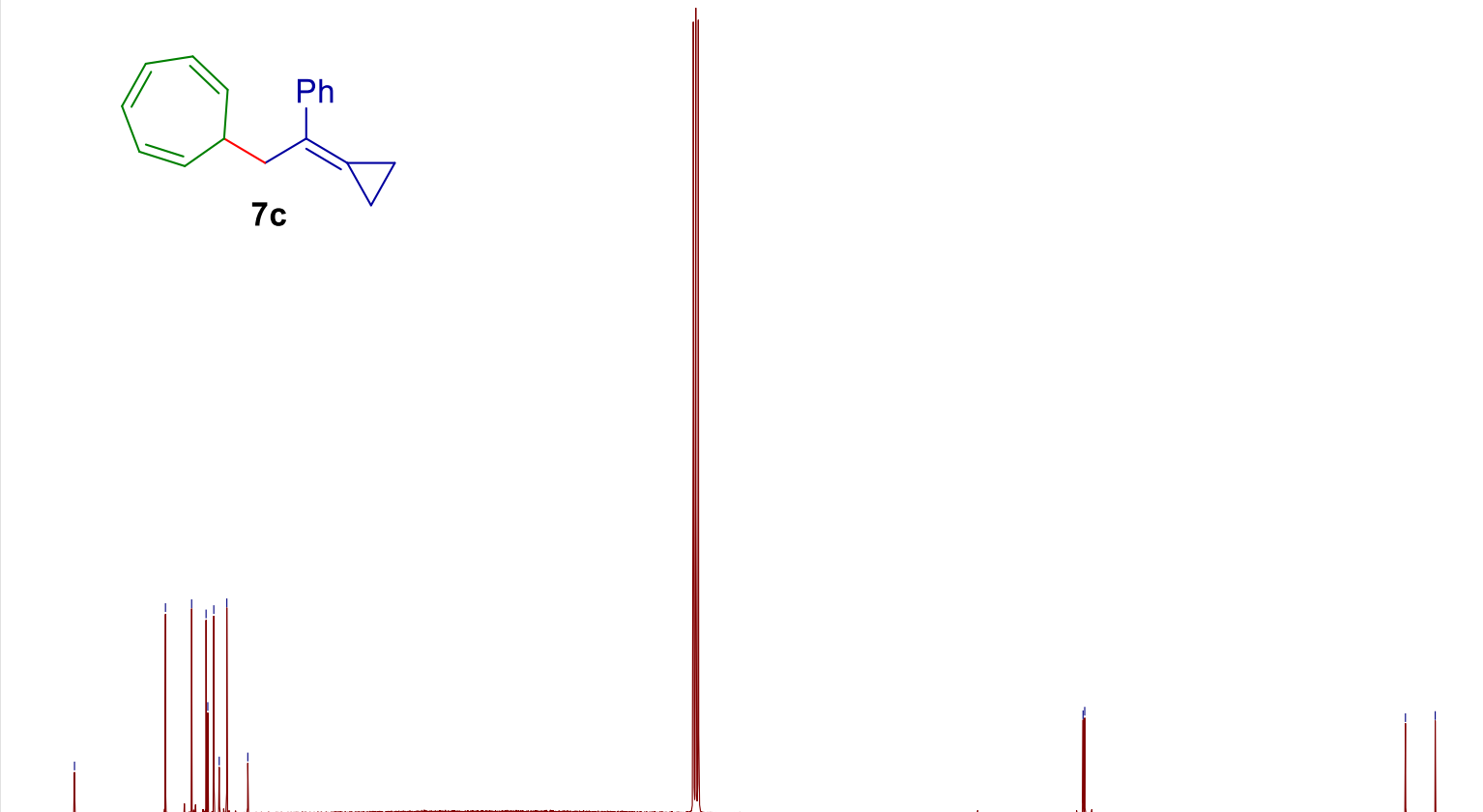

$\begin{array}{lllllllllllllllllllllllllllllllllllllllllllllllll}145 & 140 & 135 & 130 & 125 & 120 & 115 & 110 & 105 & 100 & 95 & 90 & 85 & 80 & 75 & 70 & 65 & 60 & 55 & 50 & 45 & 40 & 35 & 30 & 25 & 20 & 15 & 10 & 5 & 0\end{array}$ 
${ }^{1} \mathbf{H}$-NMR $\left(500 \mathrm{MHz}, \mathrm{CDCl}_{3}\right)$ of compound $\mathbf{7 d}$ see procedure

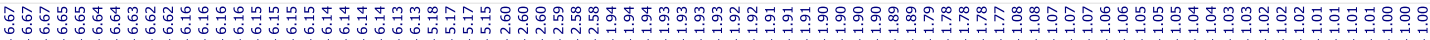<smiles>CC(CC1C=CC=CC=C1)=C1CC1</smiles>
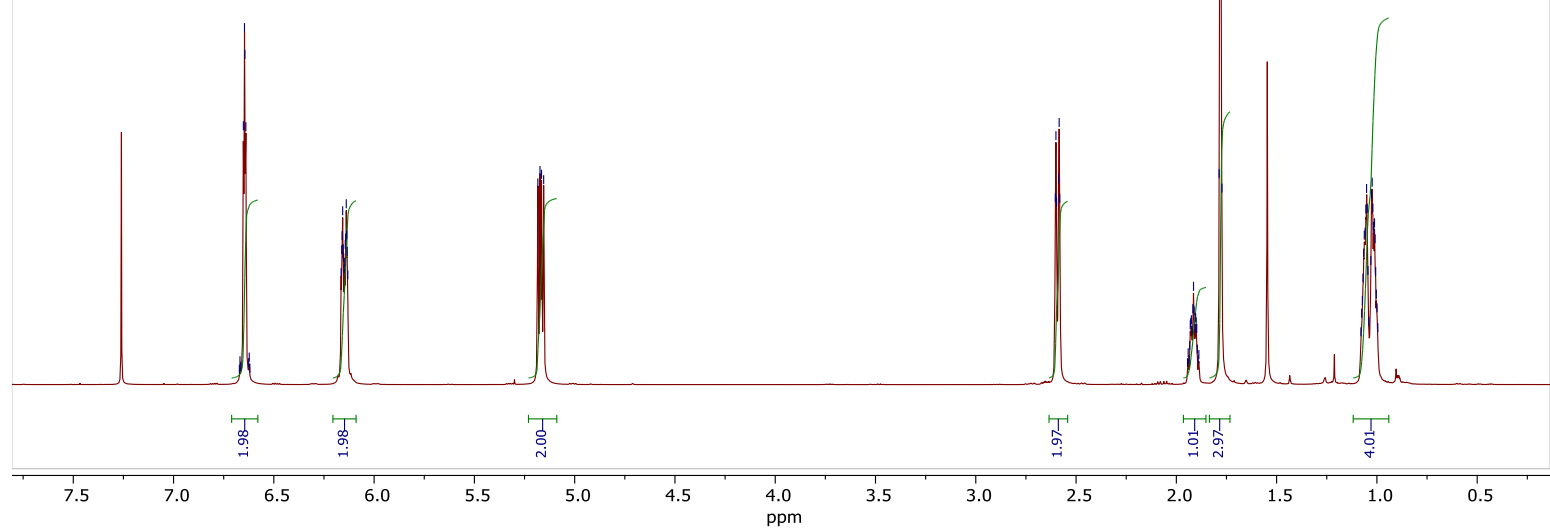

${ }^{13} \mathbf{C}-\mathrm{NMR}\left(126 \mathrm{MHz}, \mathrm{CDCl}_{3}\right)$ of compound 7d
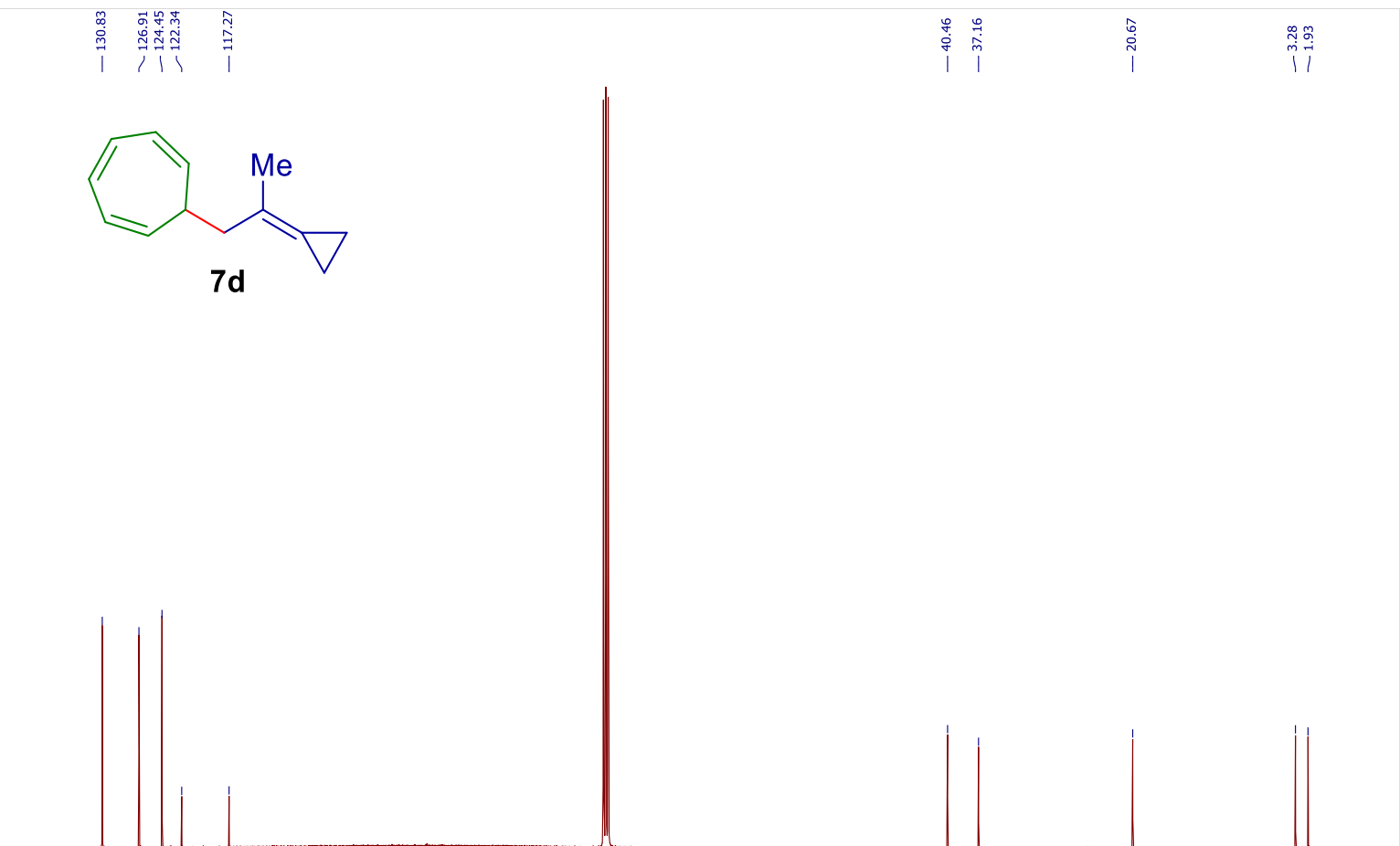

$\begin{array}{llllllllllllllllllllllllllllllllllllllllllllllll}140 & 135 & 130 & 125 & 120 & 115 & 110 & 105 & 100 & 95 & 90 & 85 & 80 & 75 & 70 & 65 & 60 & 55 & 50 & 45 & 40 & 35 & 30 & 25 & 20 & 15 & 10 & 5 & 0 & -5\end{array}$ 
${ }^{1} \mathrm{H}-\mathrm{NMR}\left(500 \mathrm{MHz}, \mathrm{CDCl}_{3}\right)$ of compound $7 \mathbf{f}$ see procedure

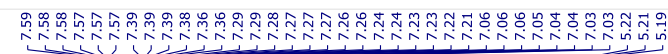

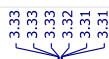

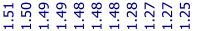<smiles></smiles>
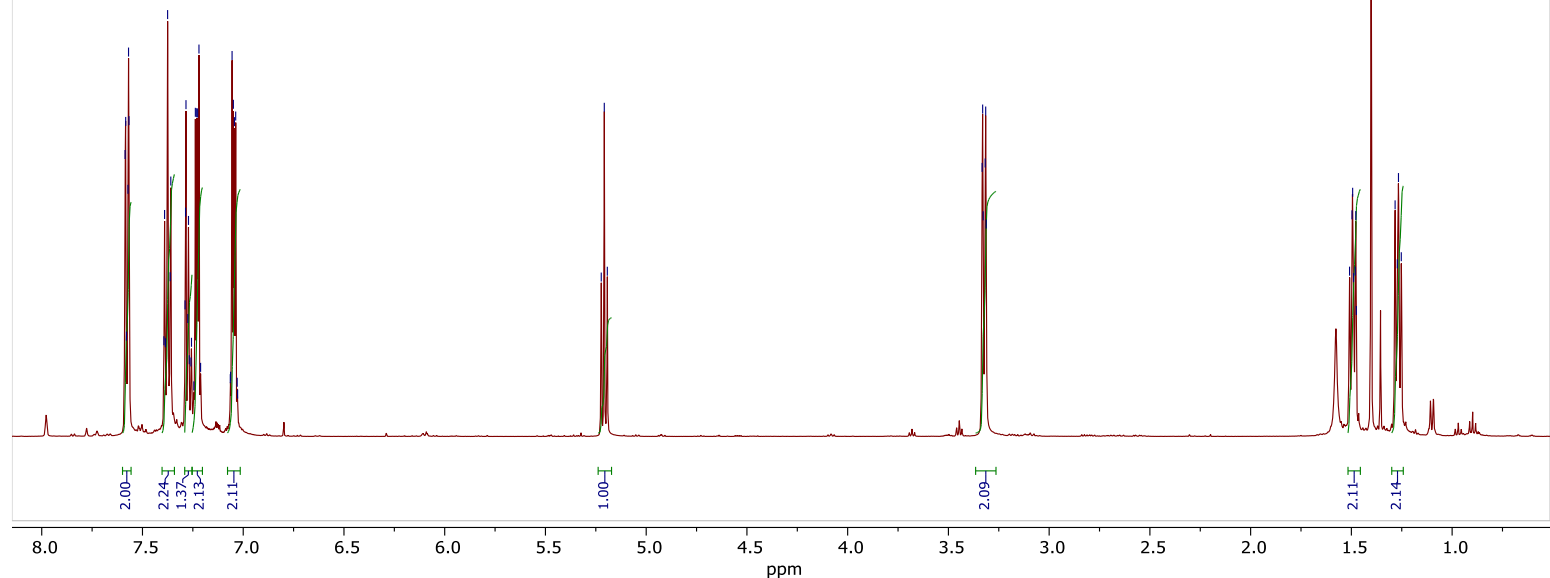

${ }^{13} \mathbf{C}$-NMR (126 MHz, $\left.\mathrm{CDCl}_{3}\right)$ of compound $7 \mathbf{f}$
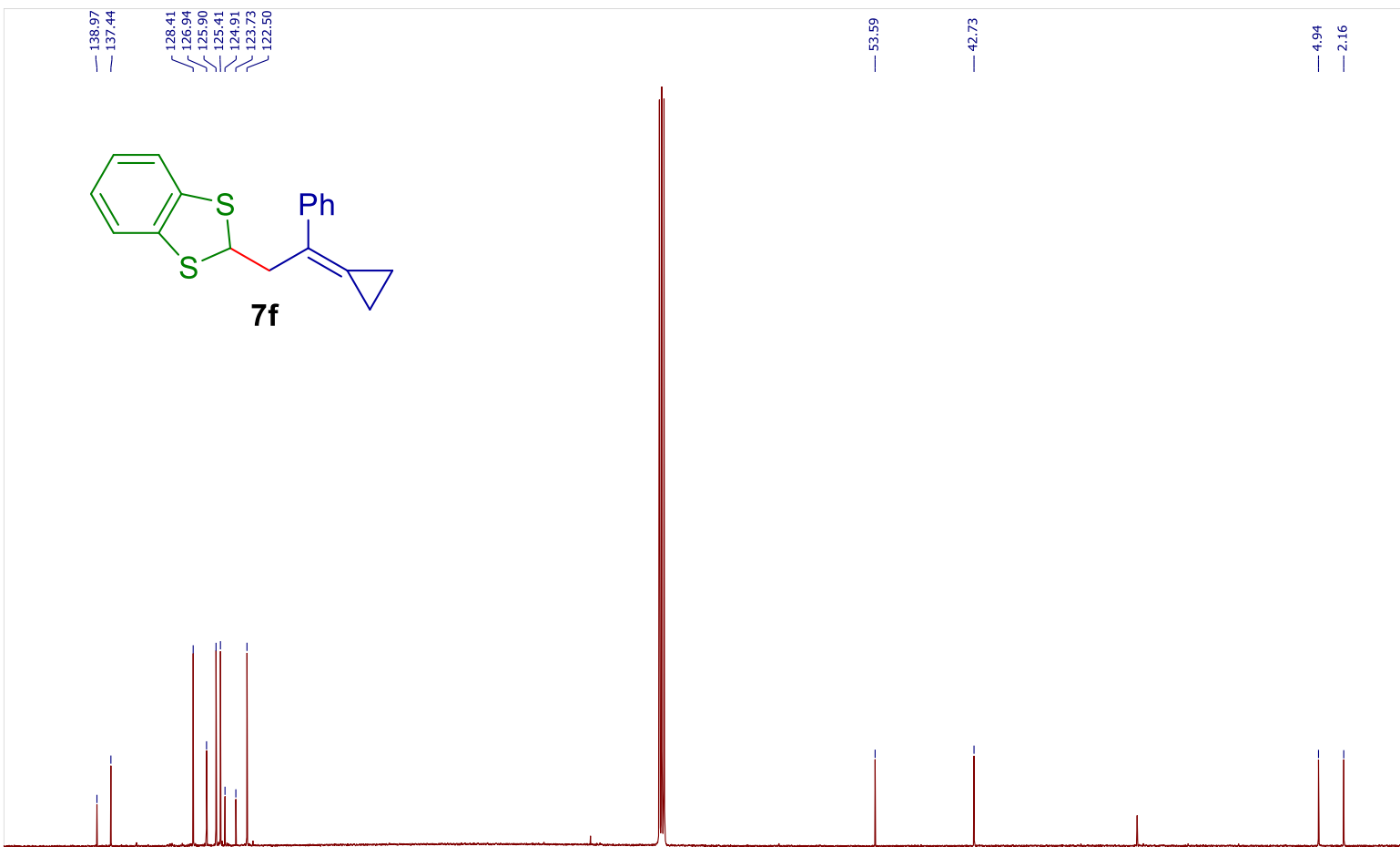

$\begin{array}{llllllllllllllllllllllllllllllll}145 & 140 & 135 & 130 & 125 & 120 & 115 & 110 & 105 & 100 & 95 & 90 & 85 & 80 & 75 & 70 & 65 & 60 & 55 & 50 & 45 & 40 & 35 & 30 & 25 & 20 & 15 & 10 & 5 & 0 & 15 & 1\end{array}$ 
${ }^{1} \mathbf{H}-\mathrm{NMR}\left(500 \mathrm{MHz}, \mathrm{CDCl}_{3}\right)$ of compound $\mathbf{7 g}$ see procedure

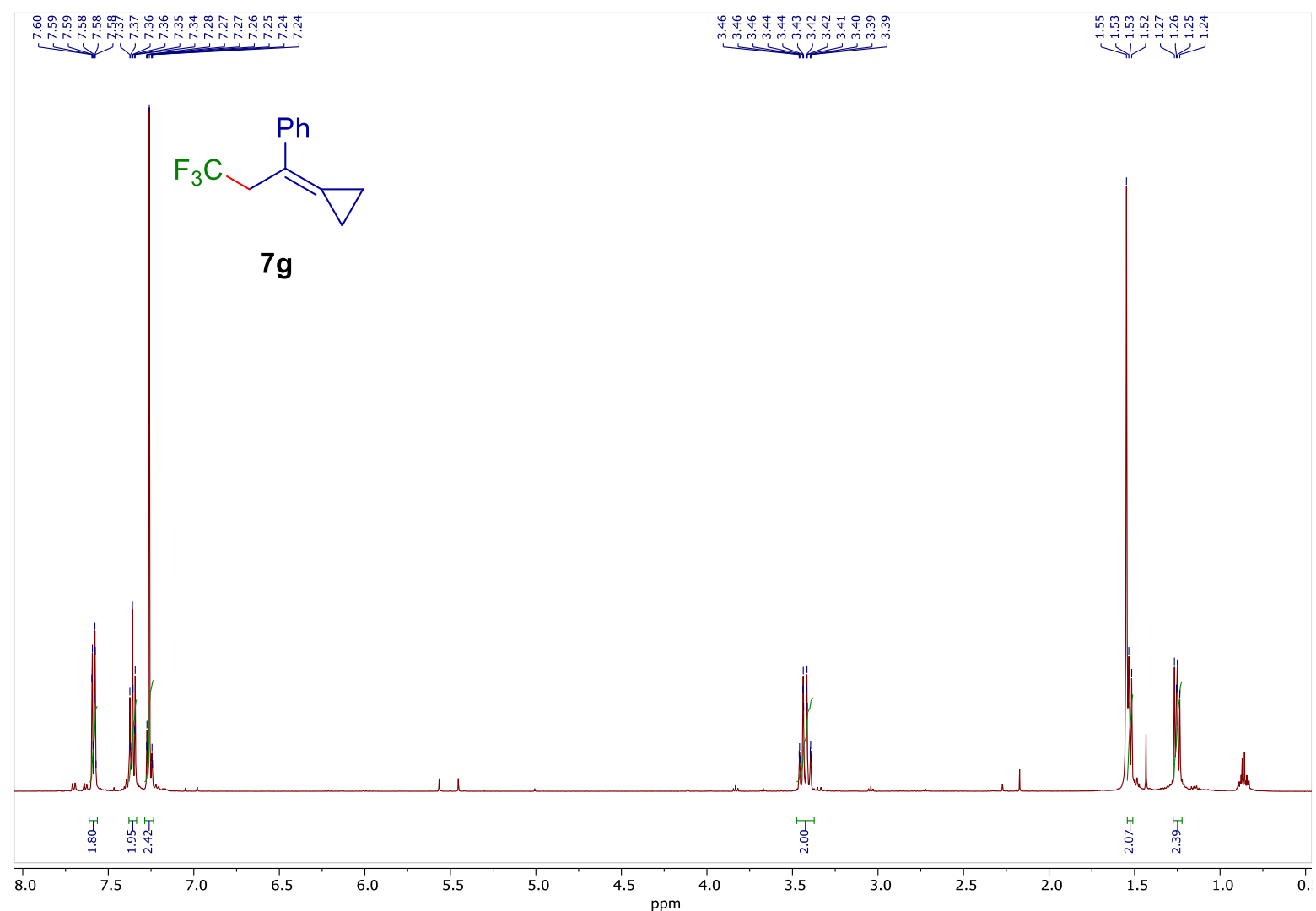

${ }^{13}$ C-NMR (126 MHz, $\mathrm{CDCl}_{3}$ ) of compound 7g

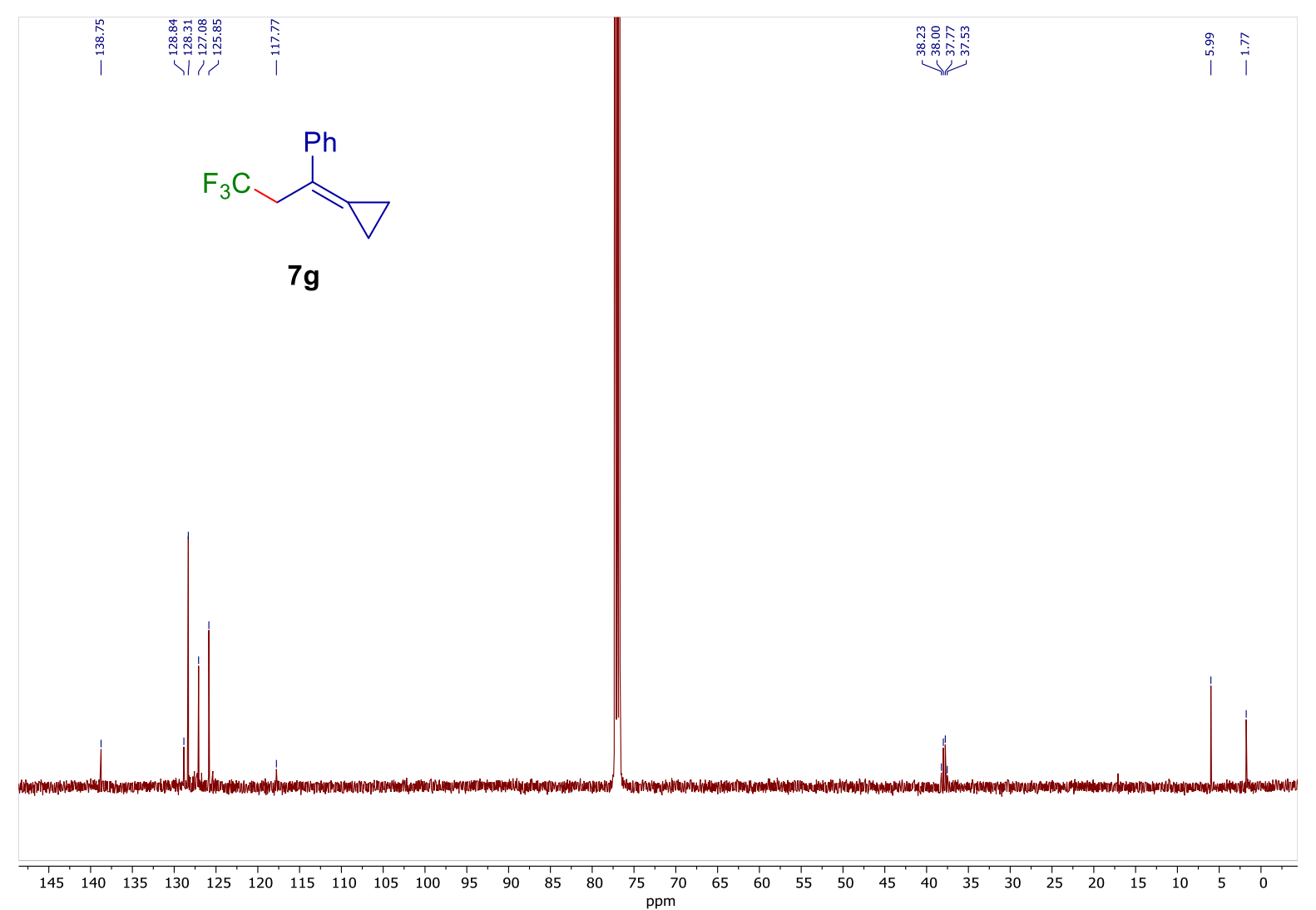


${ }^{1} \mathbf{H}-\mathrm{NMR}\left(500 \mathrm{MHz}, \mathrm{CDCl}_{3}\right)$ of compound $\mathbf{7 i}$ see procedure

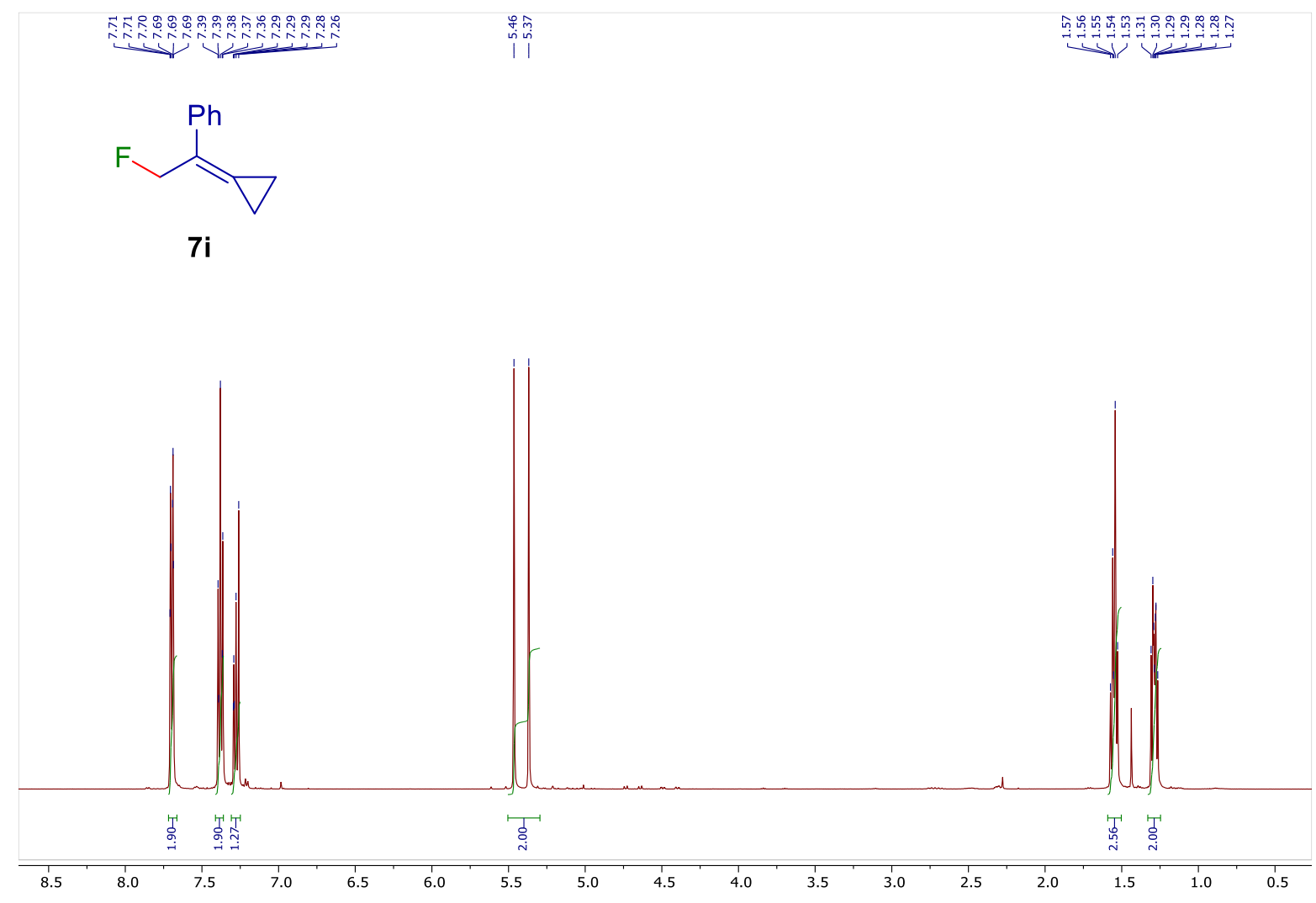

${ }^{13} \mathbf{C}-\mathrm{NMR}\left(126 \mathrm{MHz}, \mathrm{CDCl}_{3}\right)$ of compound $7 \mathbf{i}$

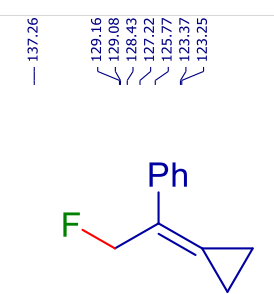

$7 \mathbf{i}$
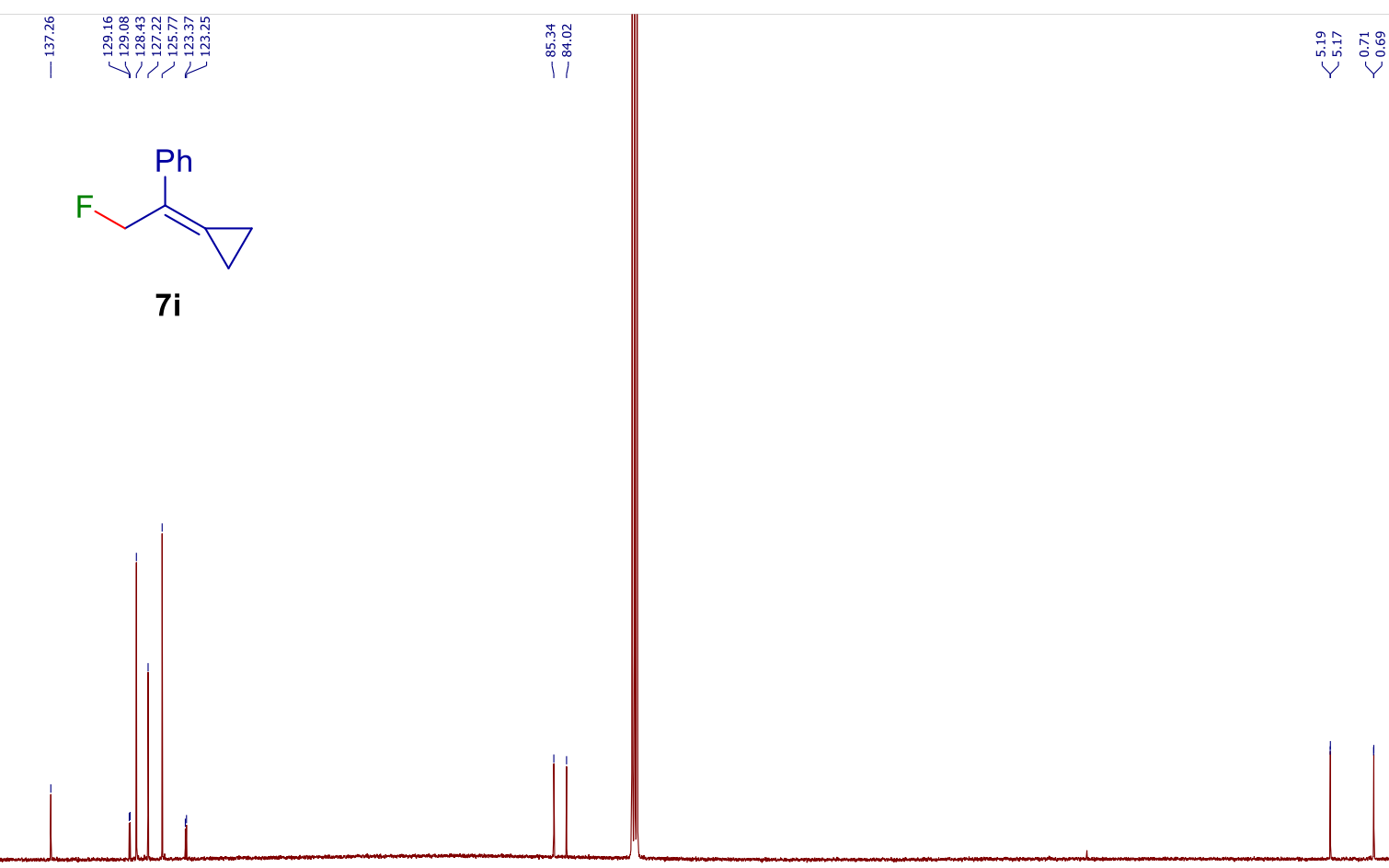

Y Y

$\begin{array}{lllllllllllllllllllllllllllllllllllll}140 & 135 & 130 & 125 & 120 & 115 & 110 & 105 & 100 & 95 & 90 & 85 & 80 & 75 & 70 & 65 & 60 & 55 & 50 & 45 & 40 & 35 & 30 & 25 & 20 & 15 & 10 & 5 & 0\end{array}$ 
${ }^{\mathbf{1}} \mathbf{H}-\mathrm{NMR}\left(500 \mathrm{MHz}, \mathrm{CDCl}_{3}\right)$ of compound $\mathbf{7 j}$ see procedure

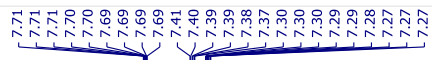<smiles>ClCC(=C1CC1)c1ccccc1</smiles>

$7 \mathbf{j}$

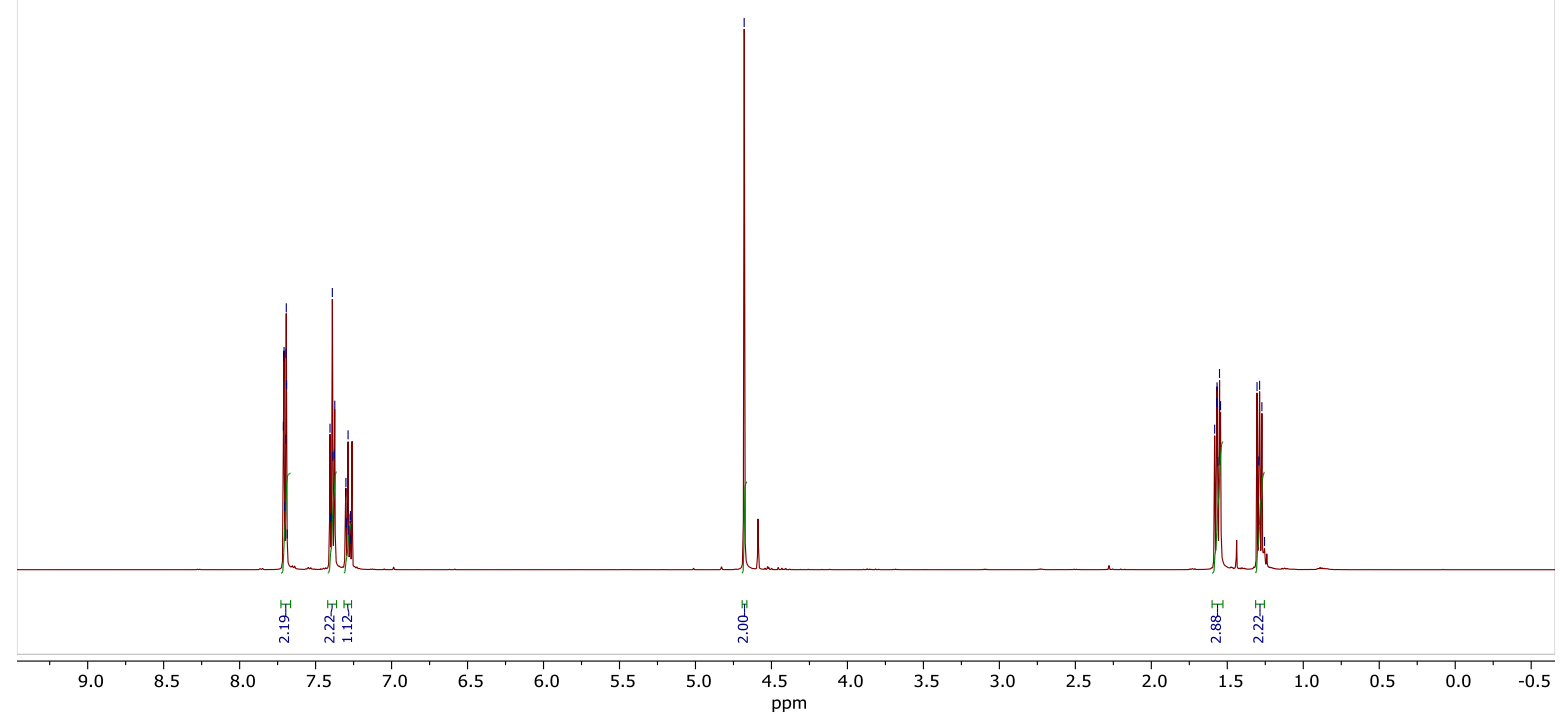

${ }^{13} \mathbf{C}-\mathbf{N M R}\left(126 \mathrm{MHz}, \mathrm{CDCl}_{3}\right)$ of compound $\mathbf{7 j}$

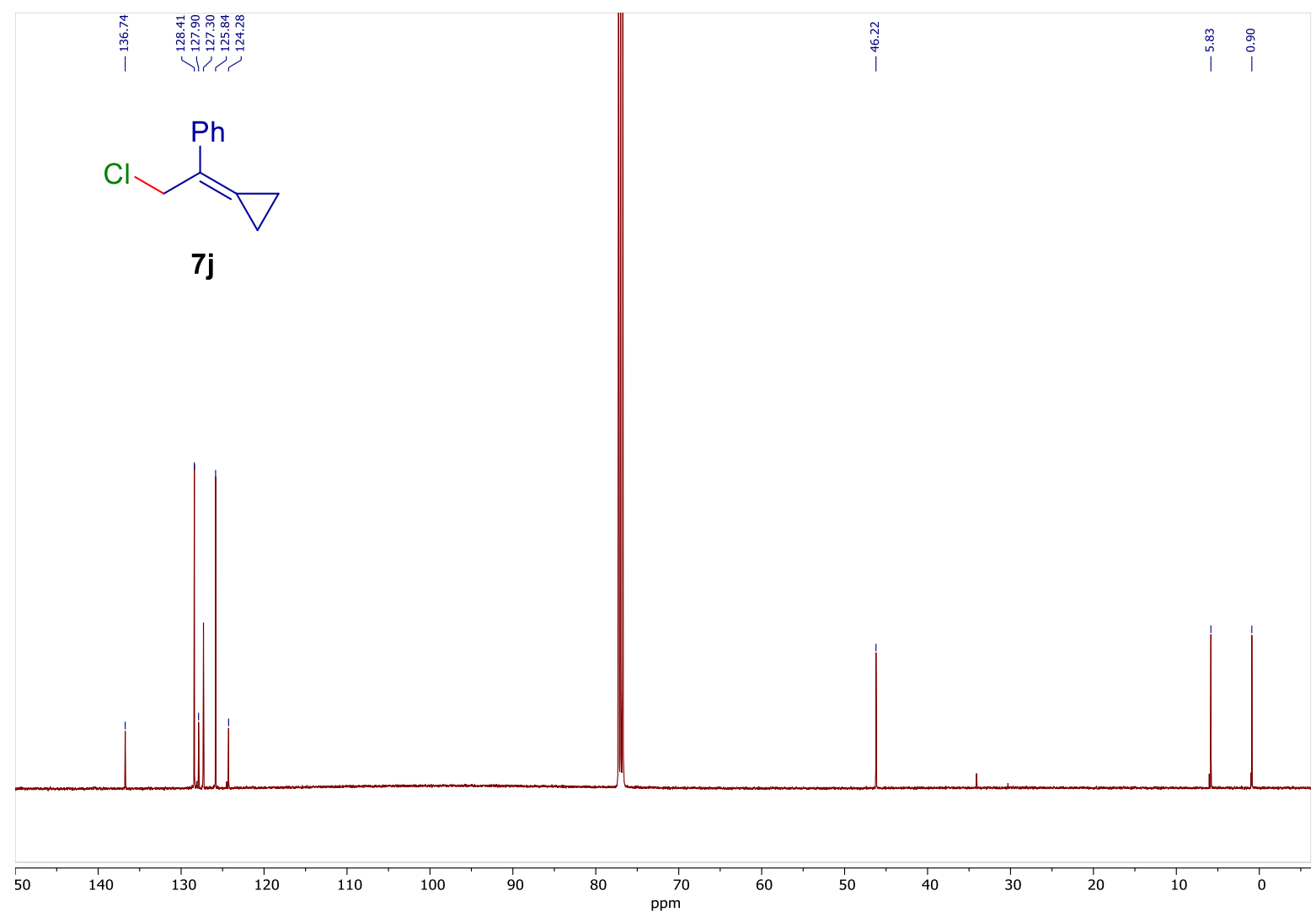


${ }^{1} \mathbf{H}$-NMR $\left(500 \mathrm{MHz}, \mathrm{CDCl}_{3}\right)$ of compound $8 \mathbf{a}$ see procedure

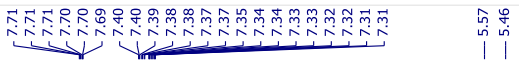<smiles>C=C(c1ccccc1)C1(Br)CC1</smiles>

$8 a$

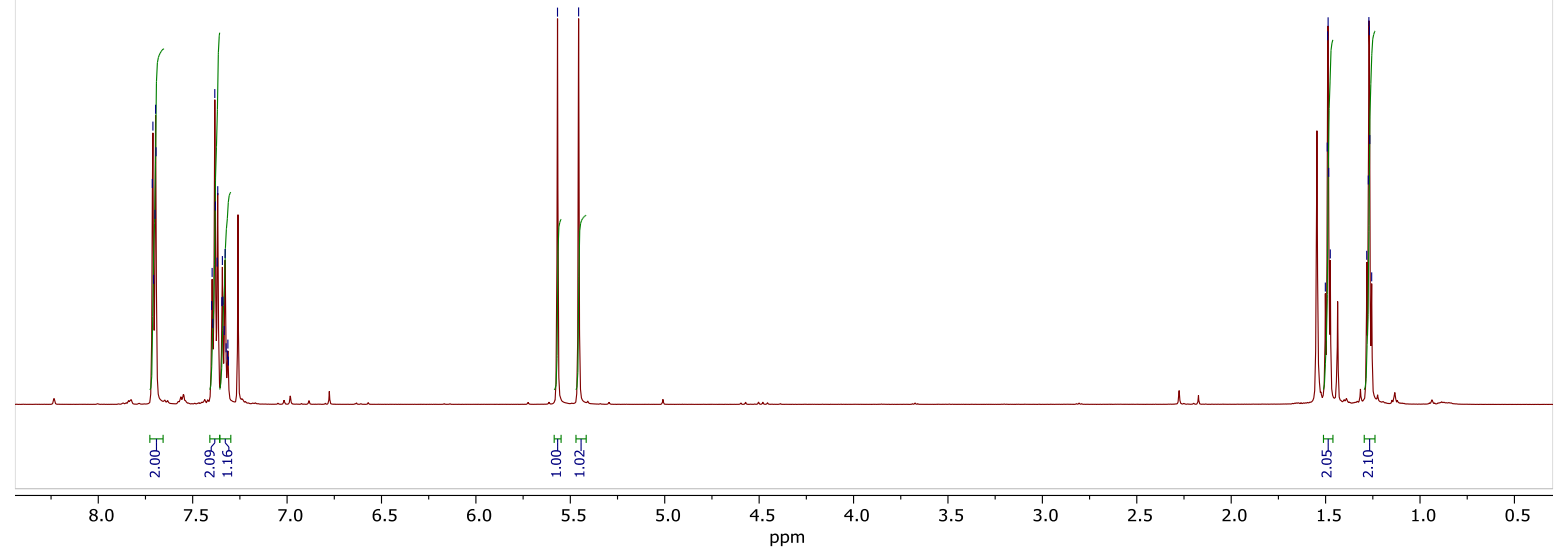

${ }^{13} \mathbf{C}-\mathbf{N M R}\left(126 \mathrm{MHz}, \mathrm{CDCl}_{3}\right)$ of compound 8a

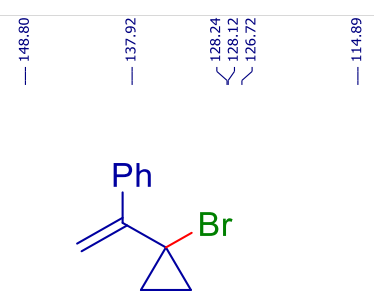

$8 \mathbf{a}$
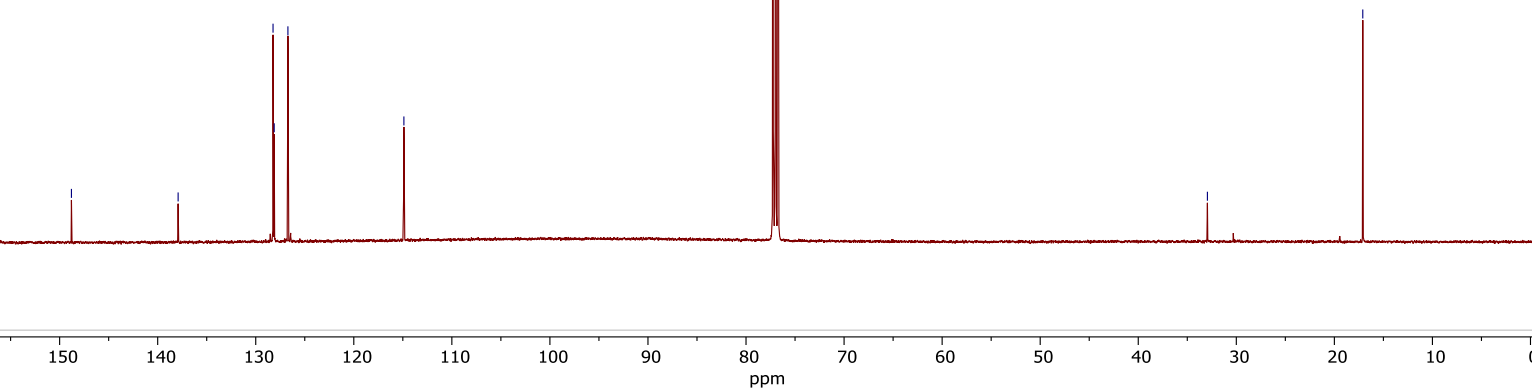
${ }^{1} \mathbf{H}-\mathbf{N M R}\left(500 \mathrm{MHz}, \mathrm{CDCl}_{3}\right)$ of compound $\mathbf{8 b}$ see procedure

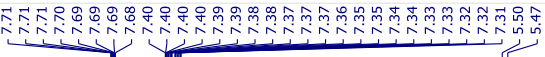<smiles>C=C(c1ccccc1)C1(I)CC1</smiles>

$8 b$

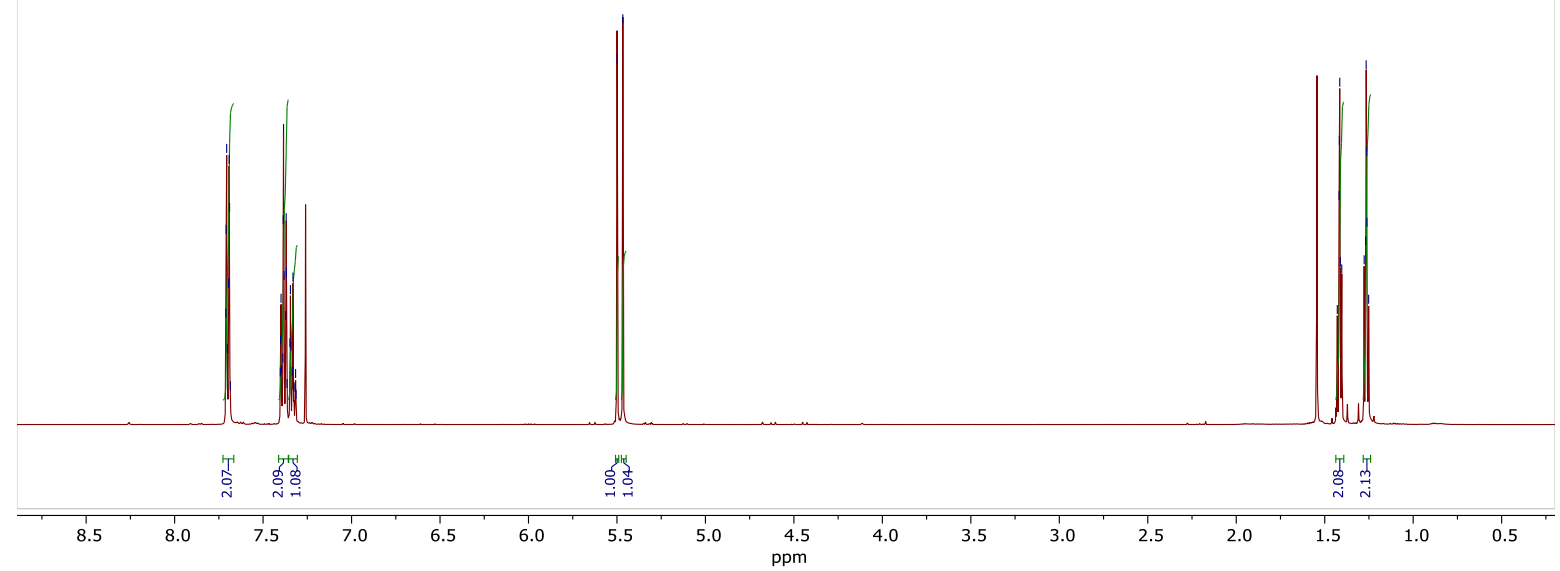

${ }^{13} \mathbf{C}$-NMR (126 MHz, $\left.\mathrm{CDCl}_{3}\right)$ of compound $\mathbf{8 b}$
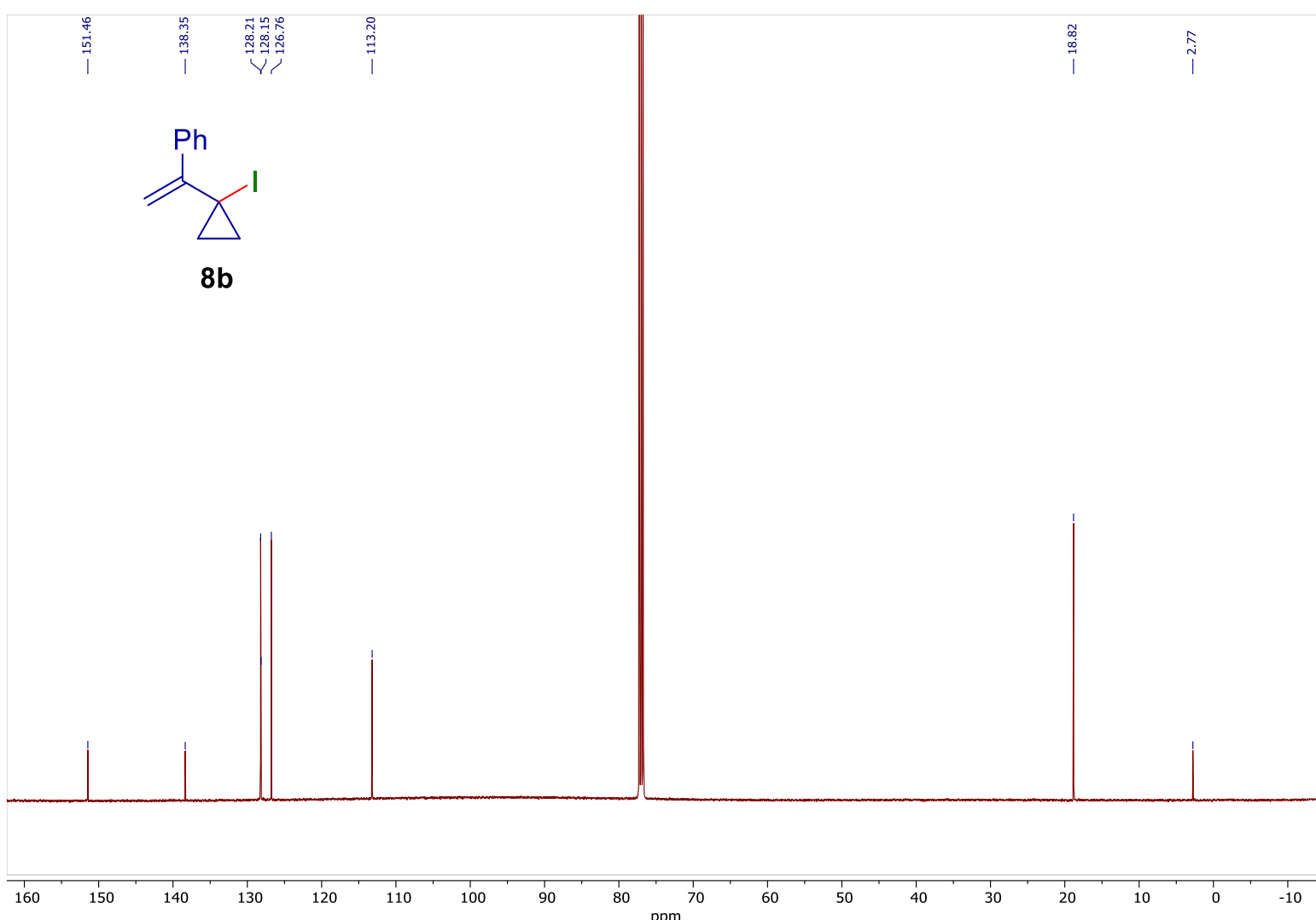
${ }^{1} \mathbf{H}-\mathrm{NMR}\left(400 \mathrm{MHz}, \mathrm{CDCl}_{3}\right)$ of compound $8 \mathbf{c}$ see procedure

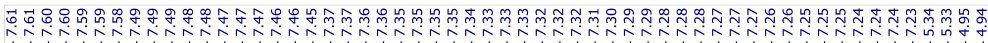

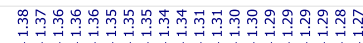<smiles>C=C(c1ccccc1)C1([SbH])CC1</smiles>

$8 c$
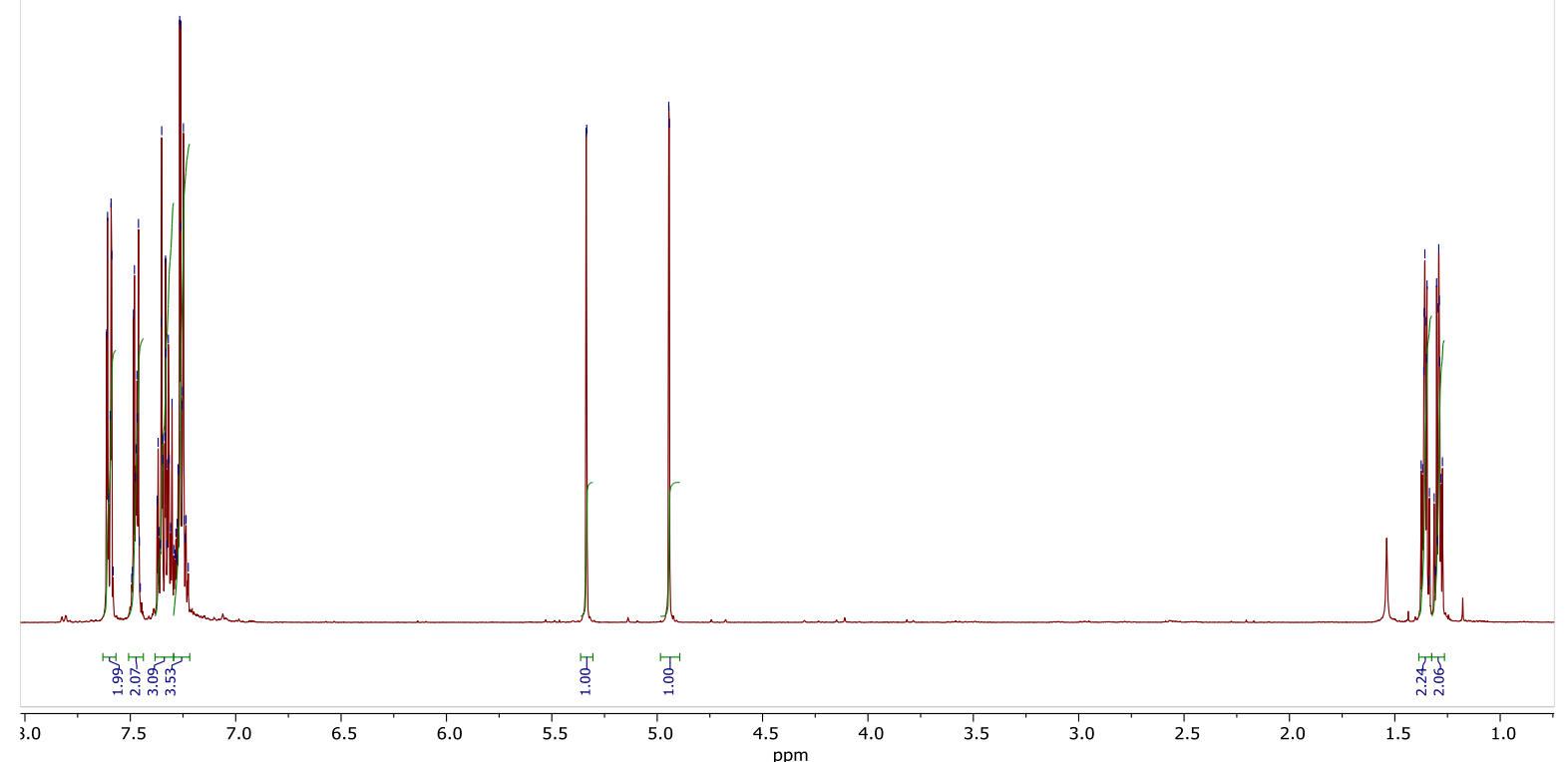

${ }^{13}$ C-NMR (101 MHz, $\left.\mathrm{CDCl}_{3}\right)$ of compound 8c

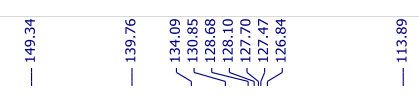<smiles>C=C(c1ccccc1)C1([SbH])CC1</smiles>

$8 \mathrm{c}$

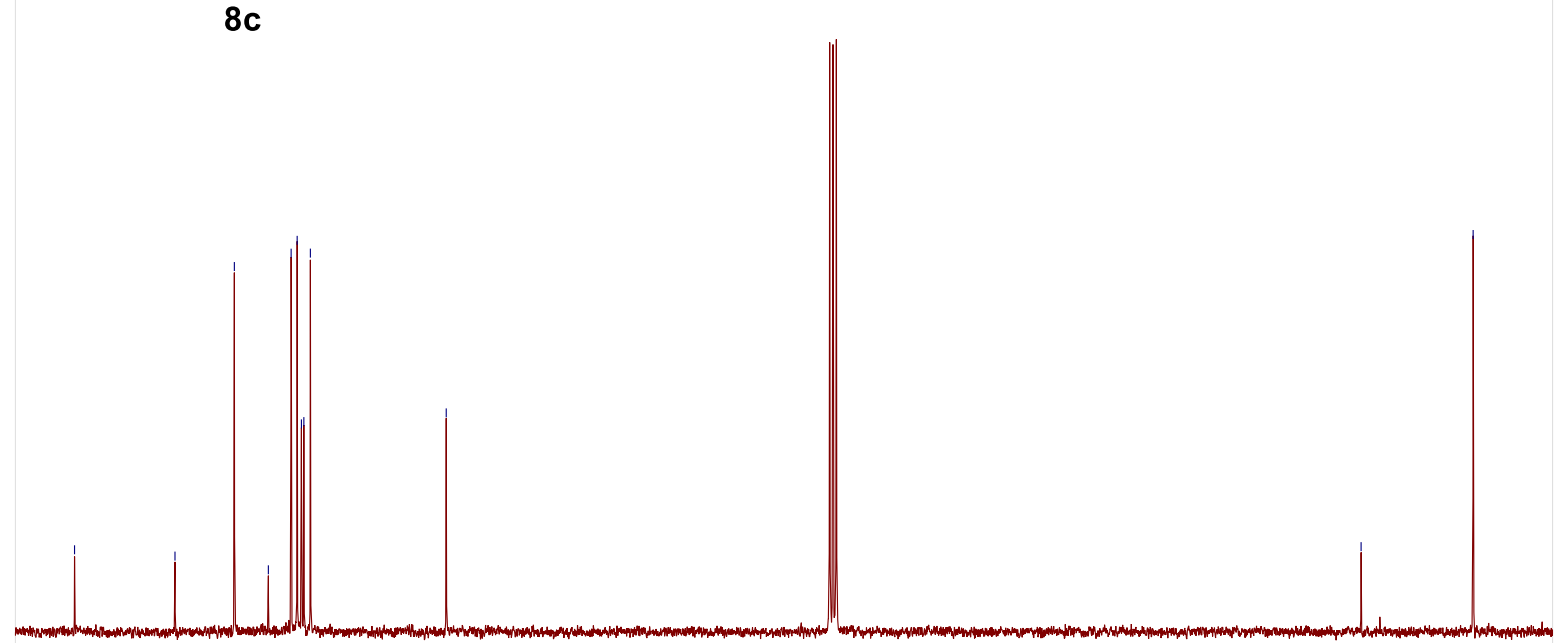

\begin{tabular}{llllllllllllllllllllllllllllllllllllllllll}
\hline 55 & 150 & 145 & 140 & 135 & 130 & 125 & 120 & 115 & 110 & 105 & 100 & 95 & 90 & 85 & 80 & 75 & 70 & 65 & 60 & 55 & 50 & 45 & 40 & 35 & 30 & 25 & 20 & 15 & 10
\end{tabular} 
${ }^{1} \mathbf{H}-\mathbf{N M R}\left(500 \mathrm{MHz}, \mathrm{CDCl}_{3}\right)$ of compound 10a see procedure

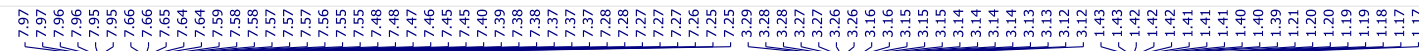<smiles>O=C(CCC(=C1CC1)c1ccccc1)c1ccccc1</smiles>

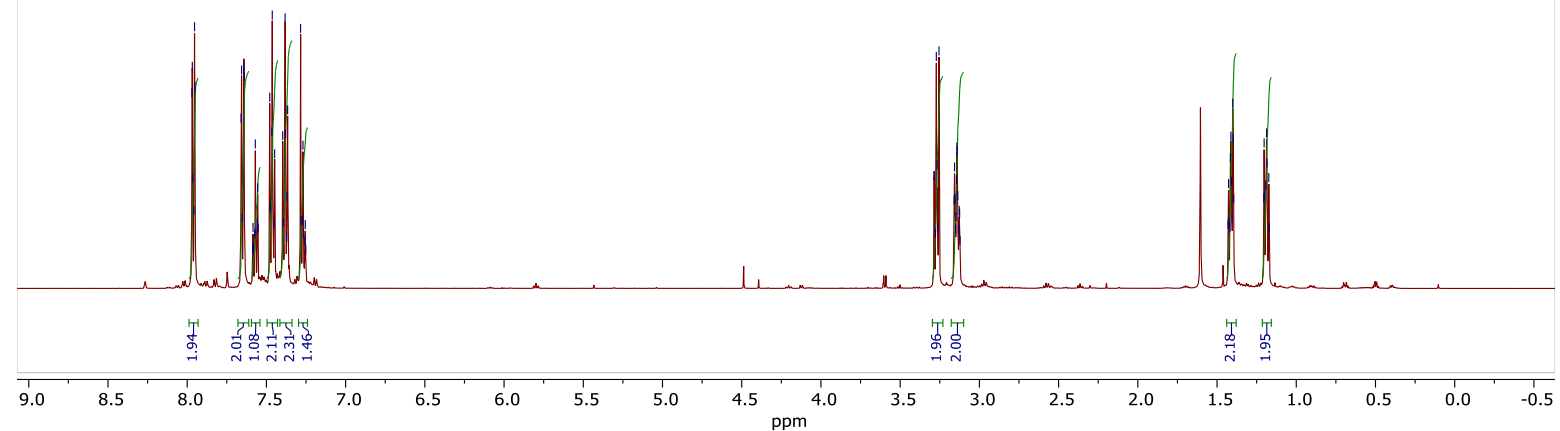

${ }^{13} \mathbf{C}-\mathbf{N M R}\left(126 \mathrm{MHz}, \mathrm{CDCl}_{3}\right)$ of compound 10a

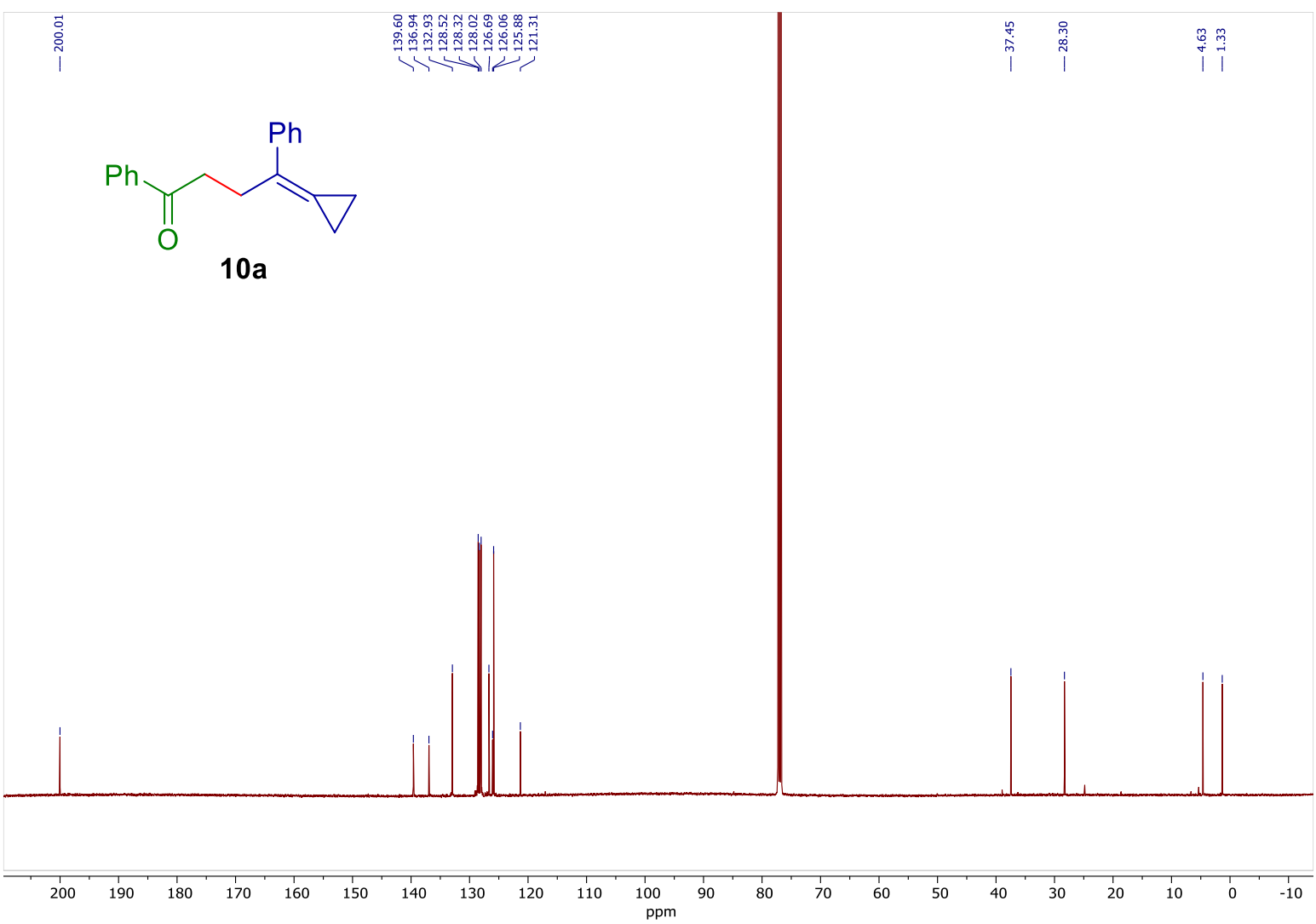


${ }^{1} \mathbf{H}-\mathrm{NMR}\left(500 \mathrm{MHz}, \mathrm{CDCl}_{3}\right)$ of compound $\mathbf{1 0 b}$ see procedure

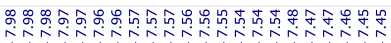

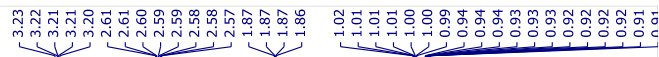<smiles>CC(CCC(=O)c1ccccc1)=C1CC1</smiles>

$10 \mathrm{~b}$

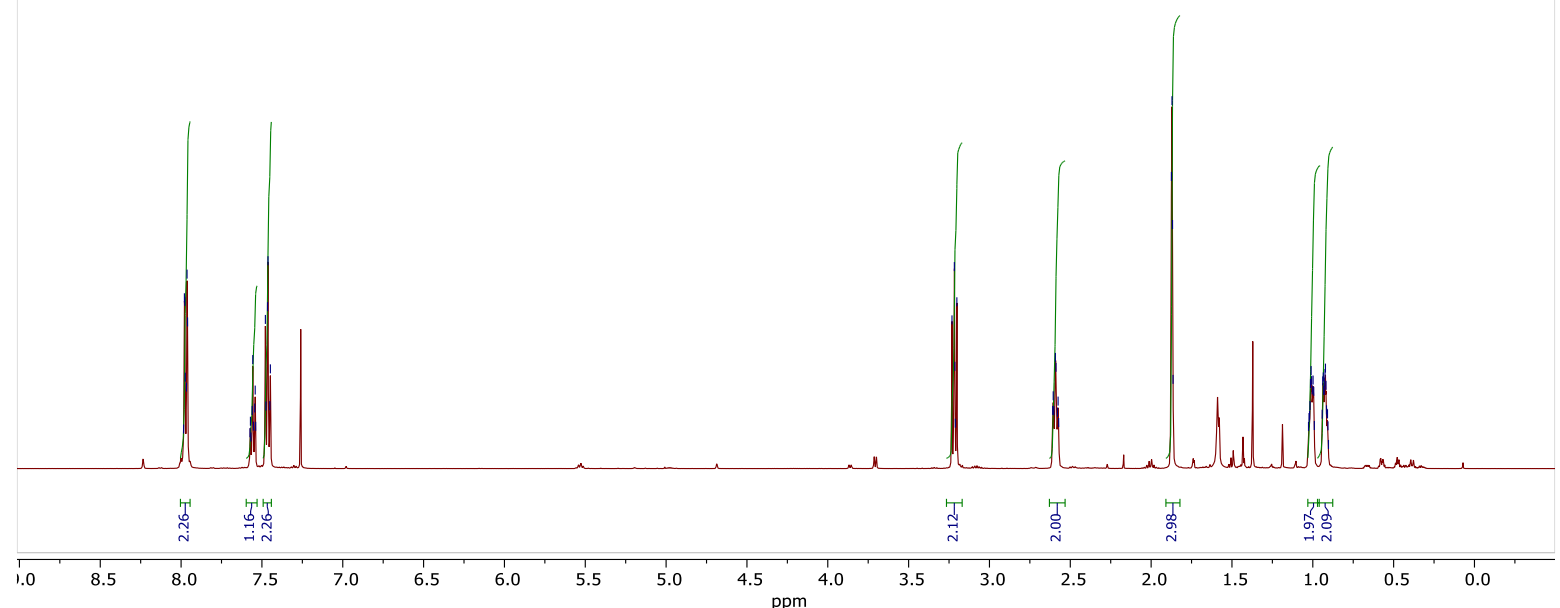

${ }^{13} \mathbf{C}-\mathbf{N M R}\left(126 \mathrm{MHz}, \mathrm{CDCl}_{3}\right)$ of compound $\mathbf{1 0 b}$

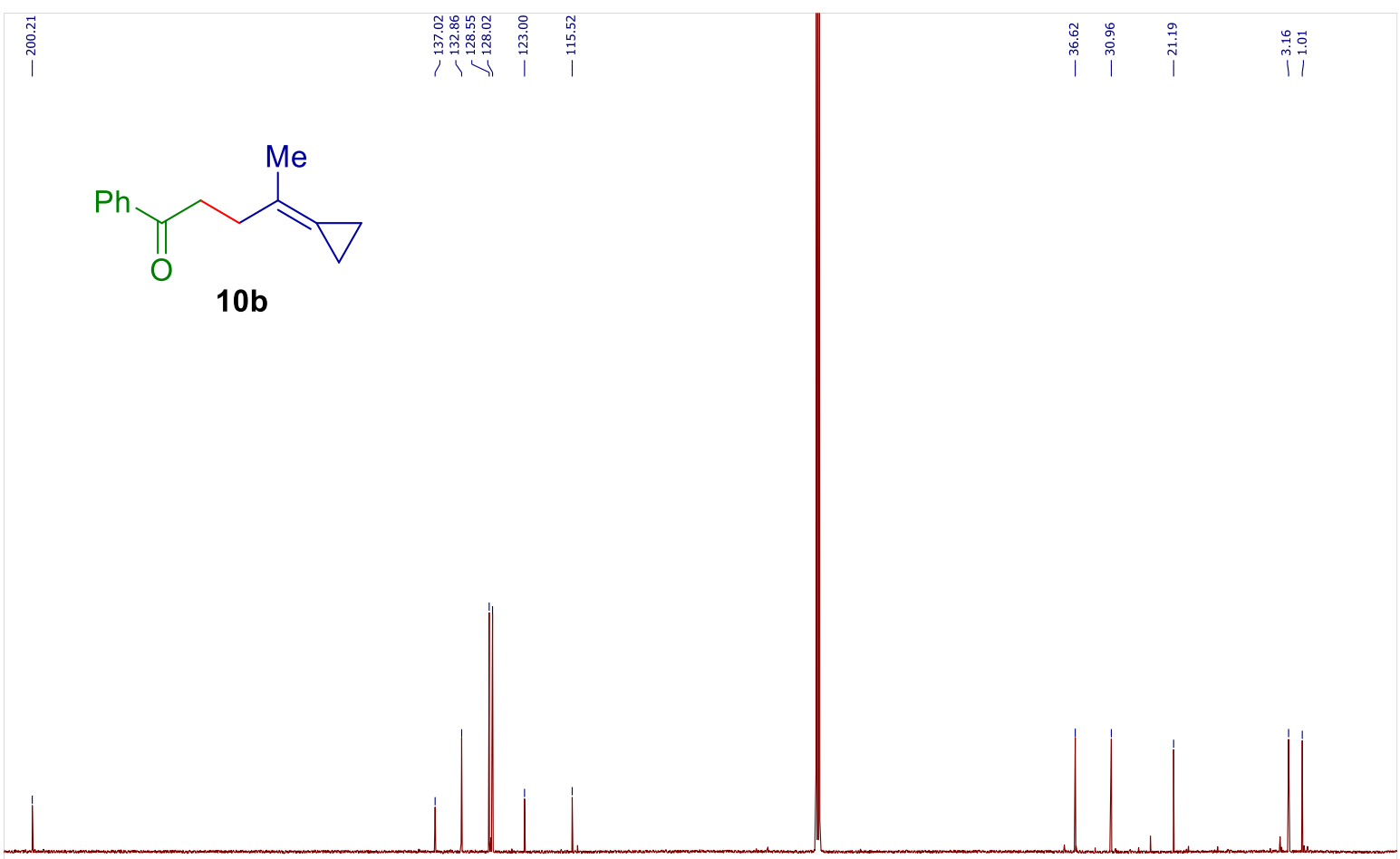

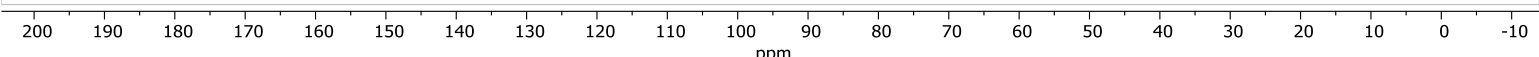


${ }^{1} \mathbf{H}-\mathrm{NMR}\left(500 \mathrm{MHz}, \mathrm{CDCl}_{3}\right)$ of compound $\mathbf{1 0 d}$ see procedure<smiles>N#CCCC(=C1CC1)c1ccccc1</smiles>

$10 d$

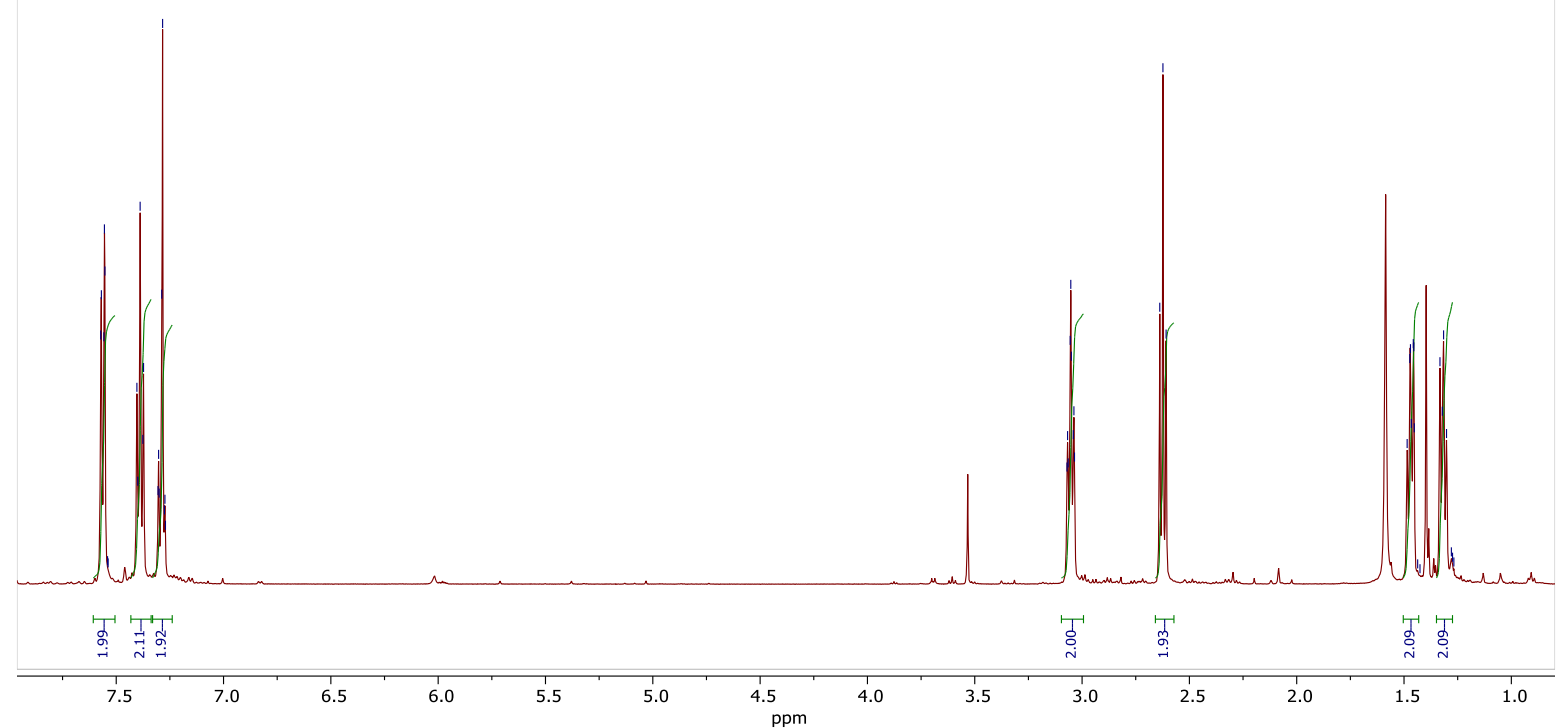

${ }^{13} \mathbf{C}-\mathbf{N M R}\left(126 \mathrm{MHz}, \mathrm{CDCl}_{3}\right)$ of compound 10d

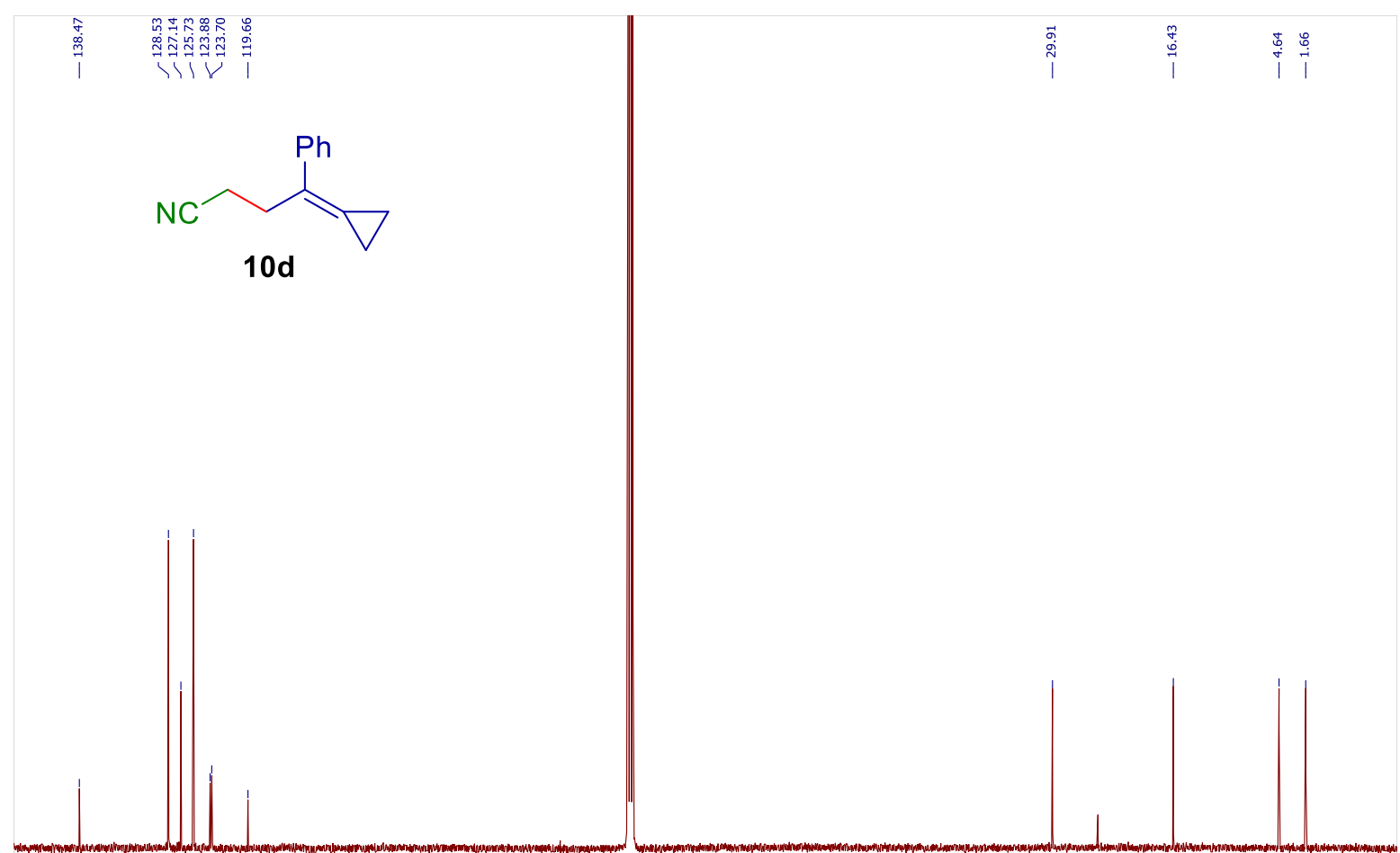

$\begin{array}{llllllllllllllllllllllllllllllllllllllllllllllllllll}145 & 140 & 135 & 130 & 125 & 120 & 115 & 110 & 105 & 100 & 95 & 90 & 85 & 80 & 75 & 70 & 65 & 60 & 55 & 50 & 45 & 40 & 35 & 30 & 25 & 20 & 15 & 10 & 5 & 0 & -5\end{array}$ 
${ }^{1} \mathbf{H}-\mathbf{N M R}\left(500 \mathrm{MHz}, \mathrm{CDCl}_{3}\right)$ of compound 17a see procedure

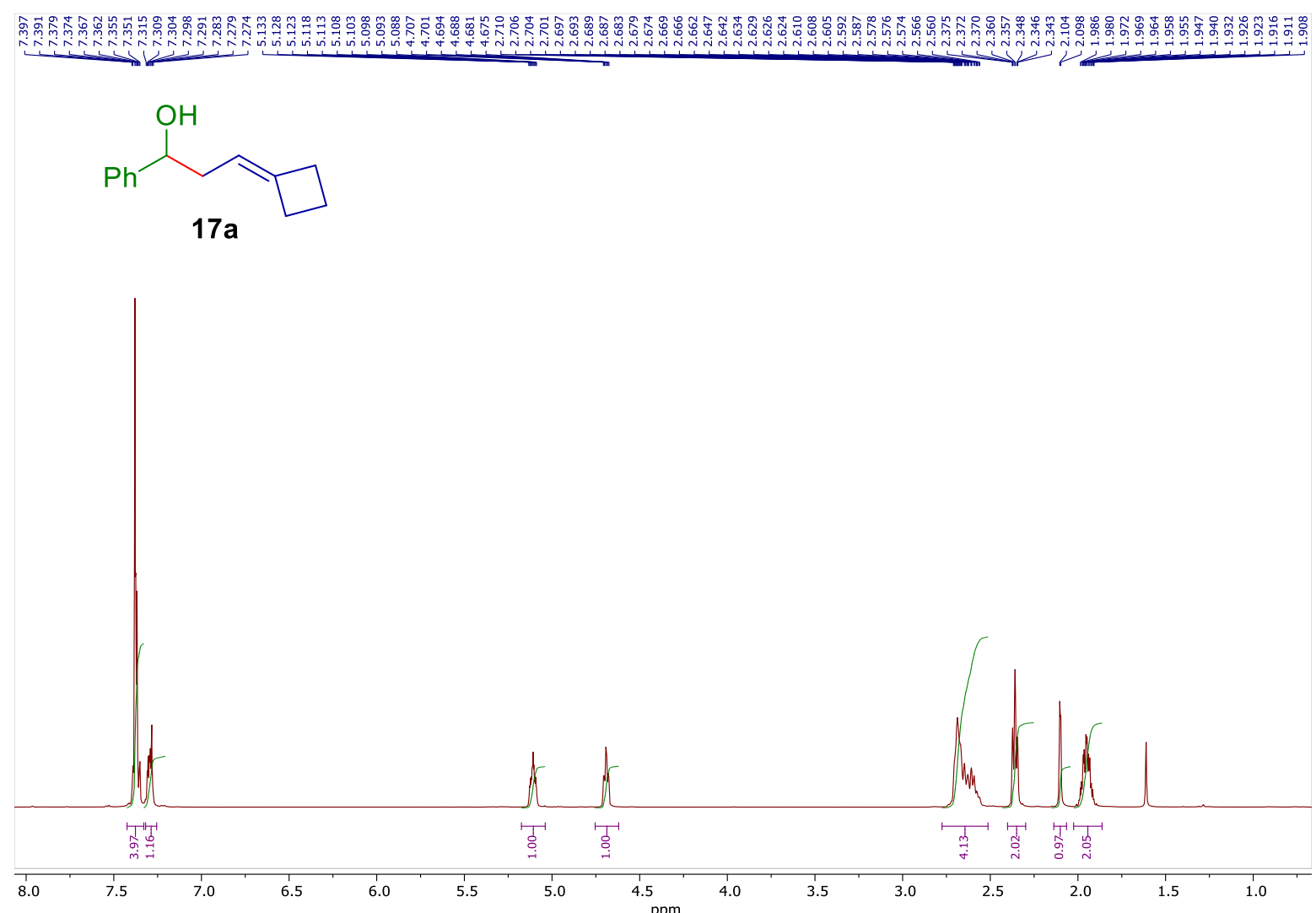

${ }^{13} \mathbf{C}-\mathbf{N M R}\left(126 \mathrm{MHz}, \mathrm{CDCl}_{3}\right)$ of compound $\mathbf{1 7 a}$

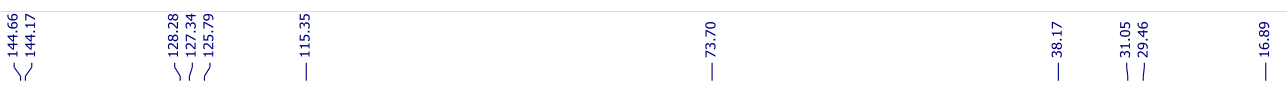<smiles>OC(CC=C1CCC1)c1ccccc1</smiles>

$17 a$

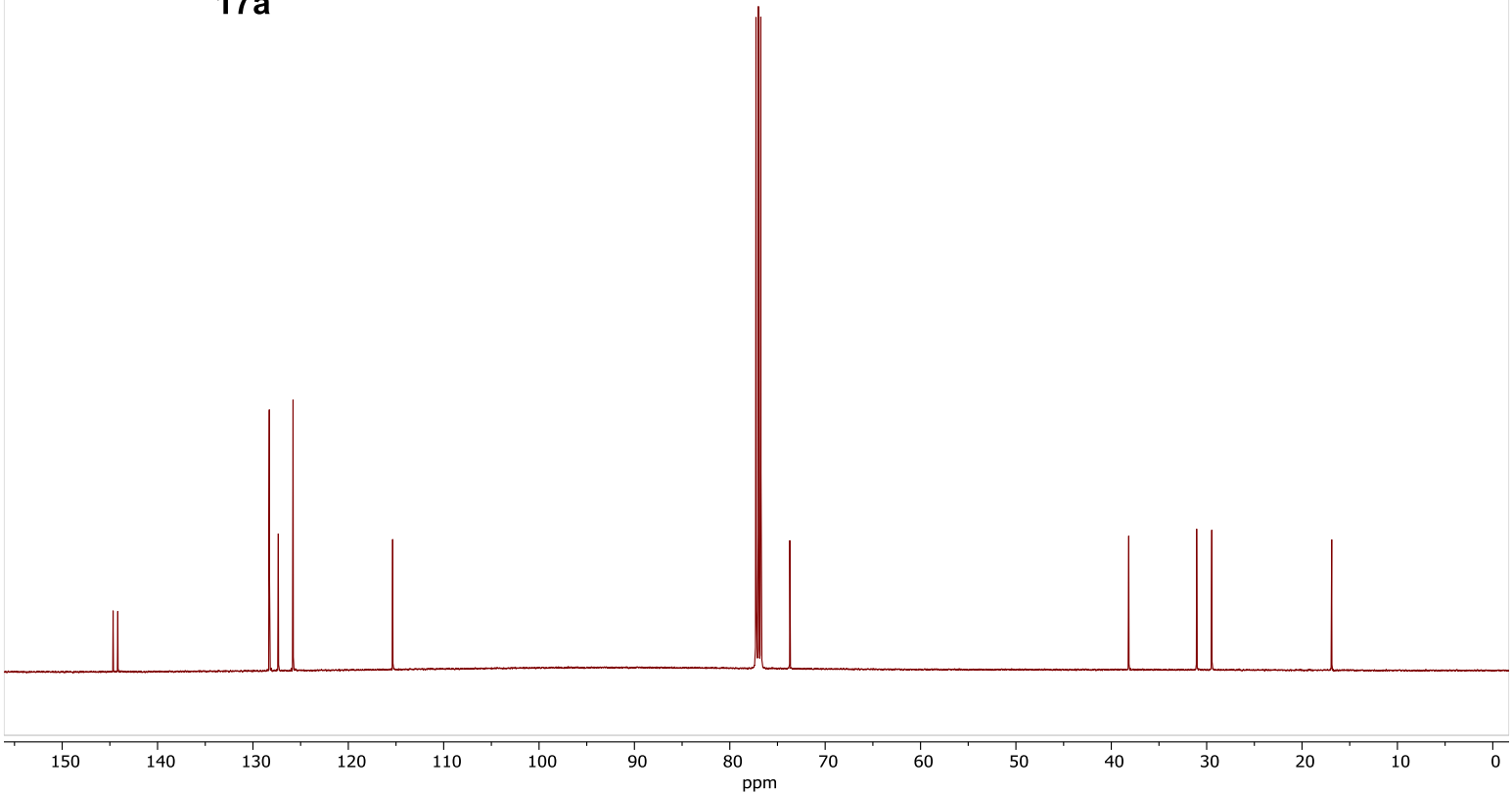


${ }^{1} \mathbf{H}-\mathrm{NMR}\left(500 \mathrm{MHz}, \mathrm{CDCl}_{3}\right)$ of compound $\mathbf{1 7 b}$ see procedure

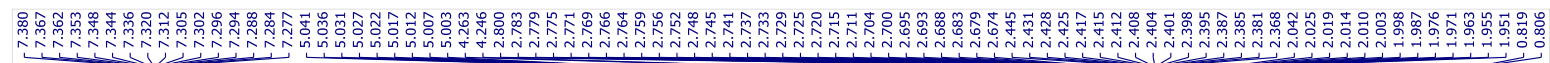<smiles>C[C@H](C=C1CCC1)C(O)c1ccccc1</smiles>

$17 \mathrm{~b}$

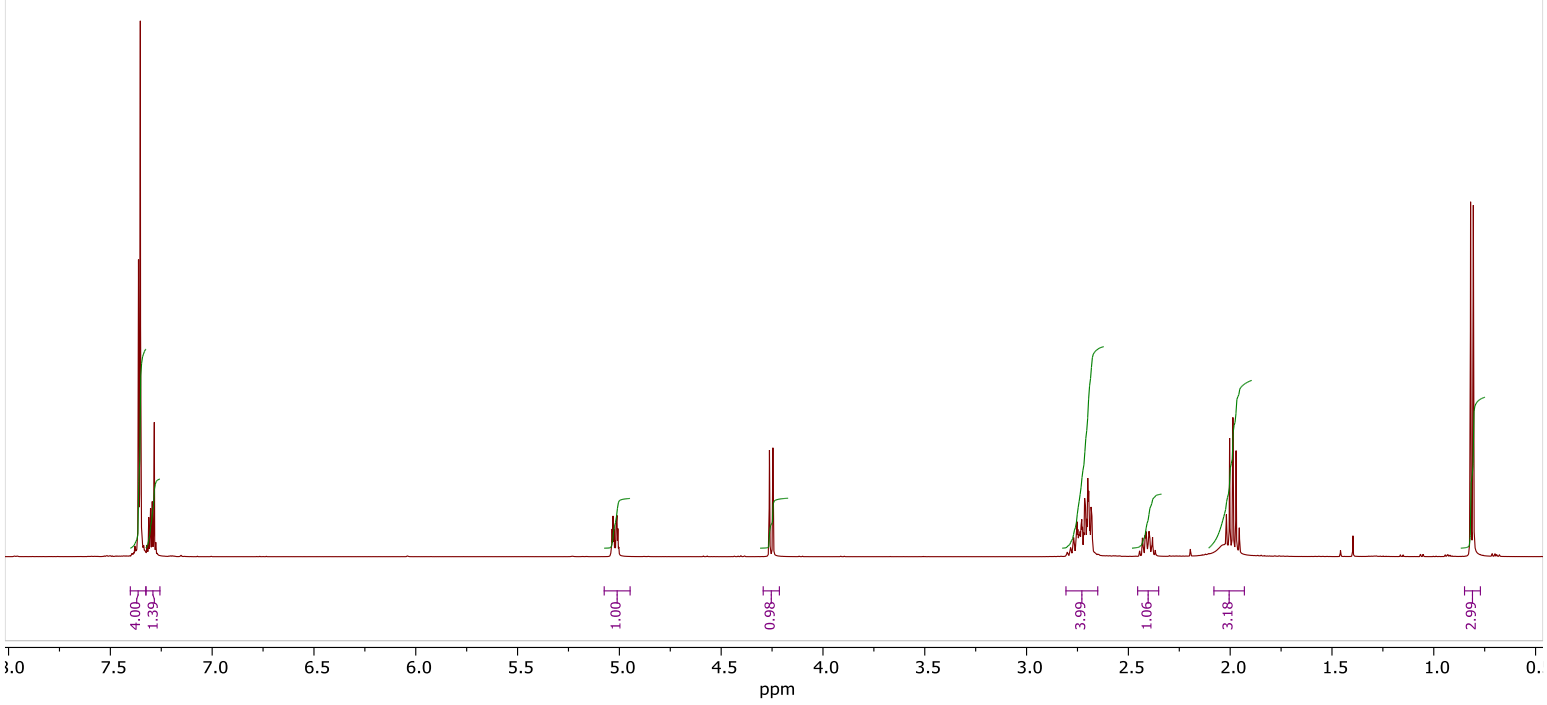

${ }^{13} \mathbf{C}-\mathrm{NMR}\left(126 \mathrm{MHz}, \mathrm{CDCl}_{3}\right)$ of compound $\mathbf{1 7 b}$

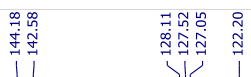

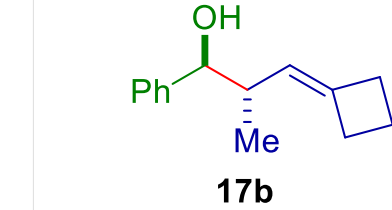

$17 b$
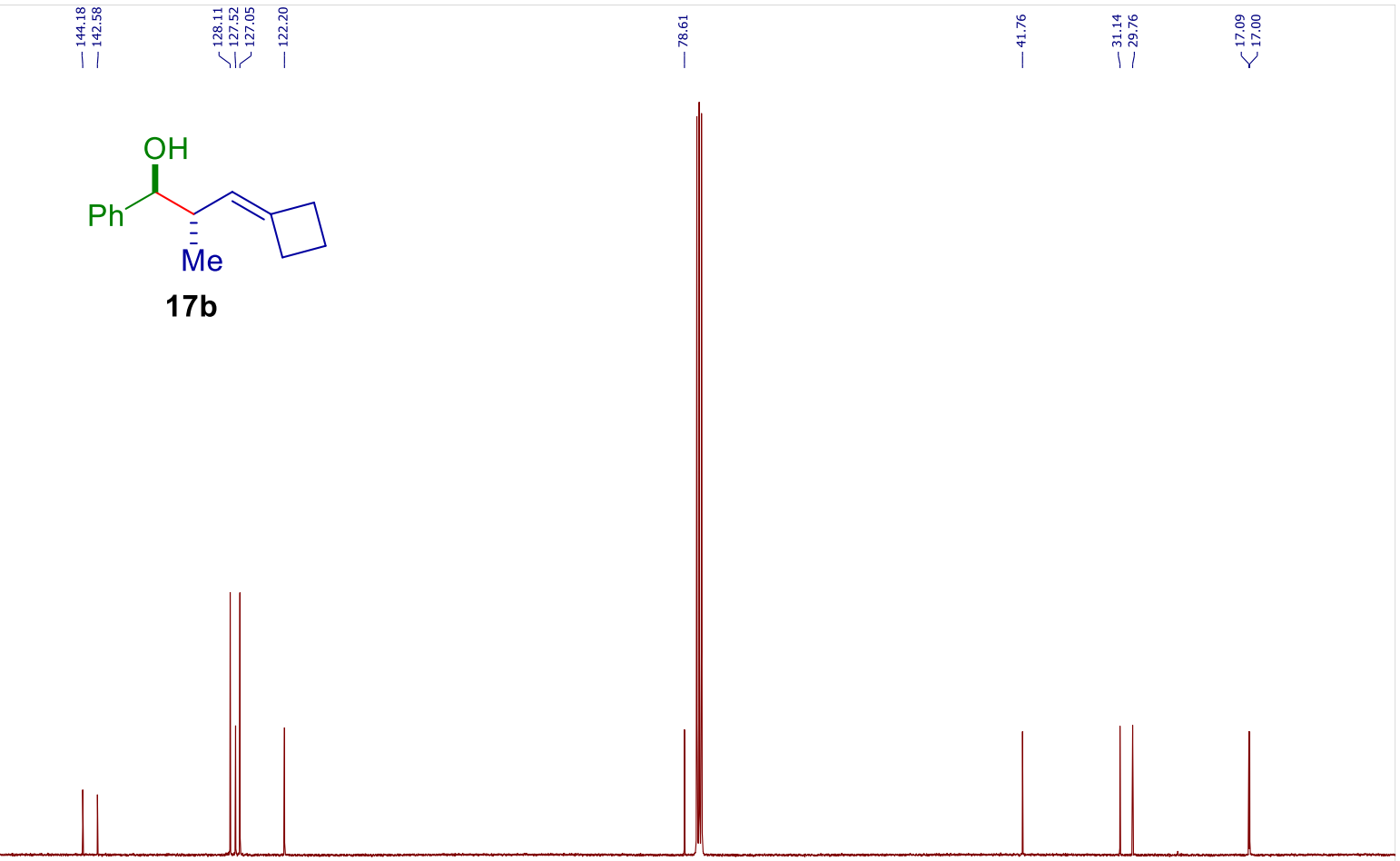

\begin{tabular}{llllllllllllllllllllllllllllllllllllllllllllll}
\hline 150 & 145 & 140 & 135 & 130 & 125 & 120 & 115 & 110 & 105 & 100 & 95 & 90 & 85 & 80 & 75 & 70 & 65 & 60 & 55 & 50 & 45 & 40 & 35 & 30 & 25 & 20 & 15 & 10 & 5
\end{tabular} 
${ }^{1} \mathbf{H}-\mathrm{NMR}\left(500 \mathrm{MHz}, \mathrm{CDCl}_{3}\right)$ of compound $17 \mathbf{c}$ see procedure

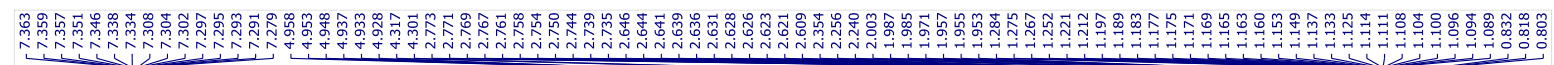

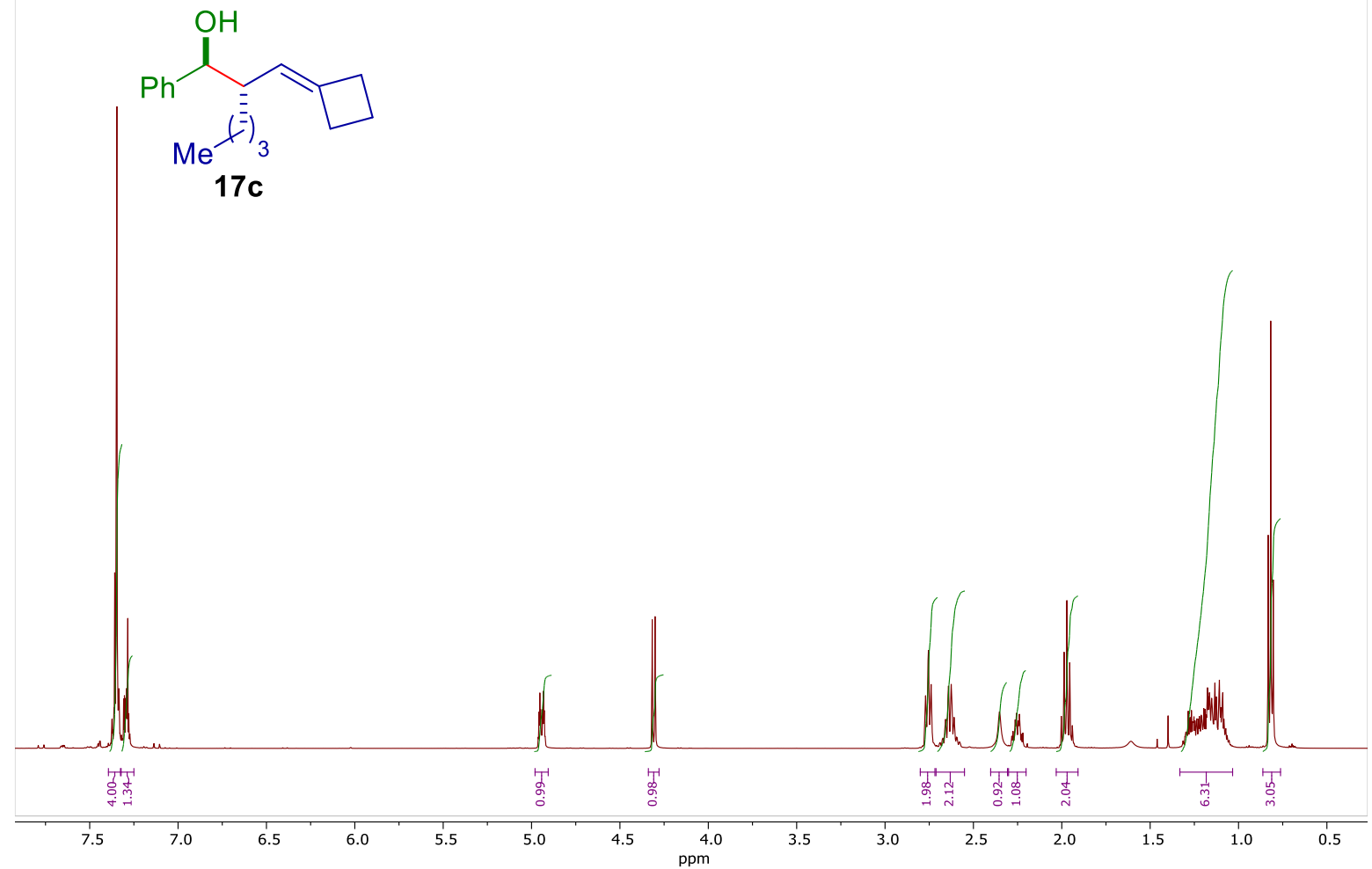

${ }^{13} \mathbf{C}$ - NMR (126 MHz, $\left.\mathrm{CDCl}_{3}\right)$ of compound 17c

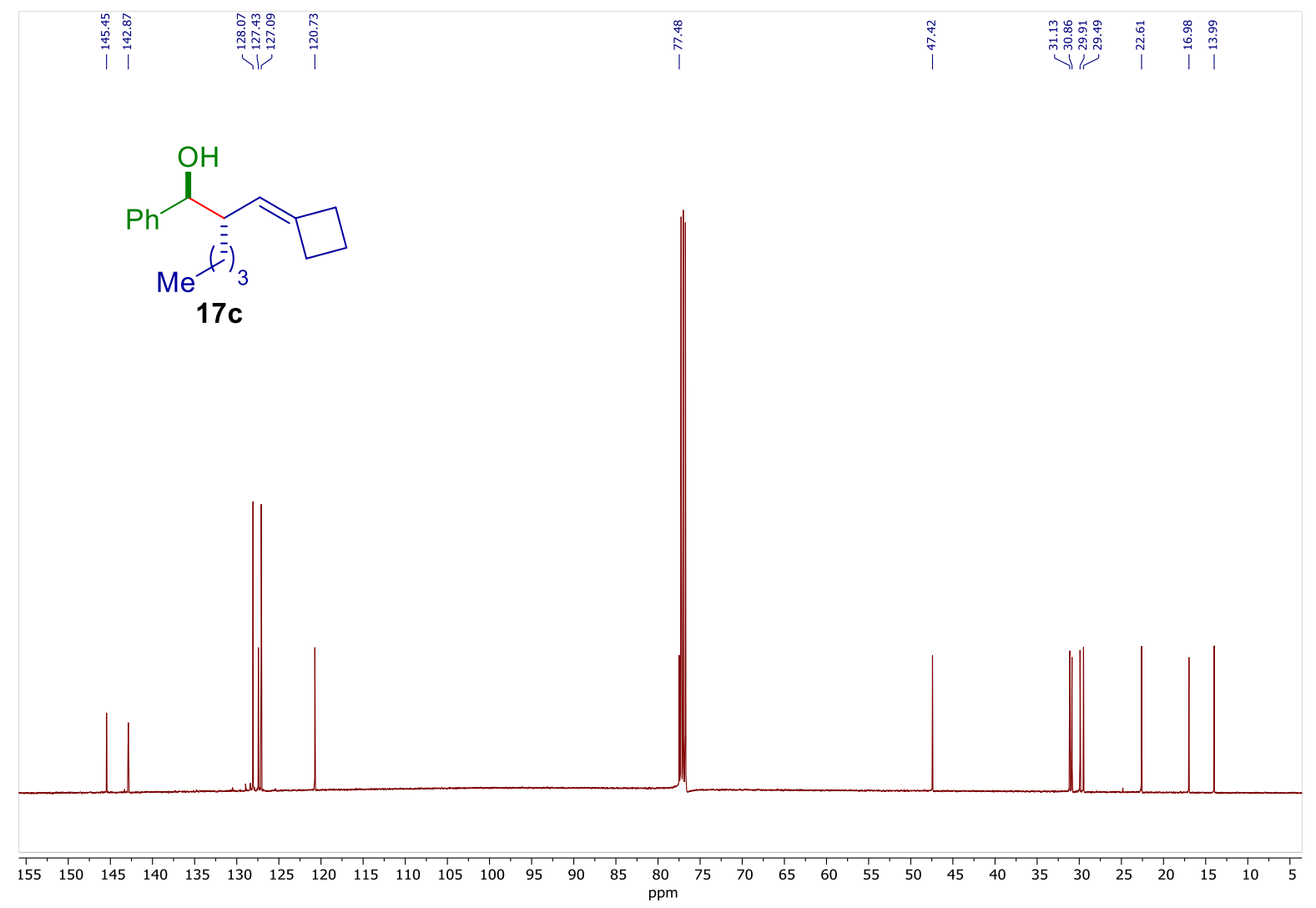


${ }^{1} \mathbf{H}-\mathrm{NMR}\left(500 \mathrm{MHz}, \mathrm{CDCl}_{3}\right)$ of compound $\mathbf{1 7 d}$ see procedure

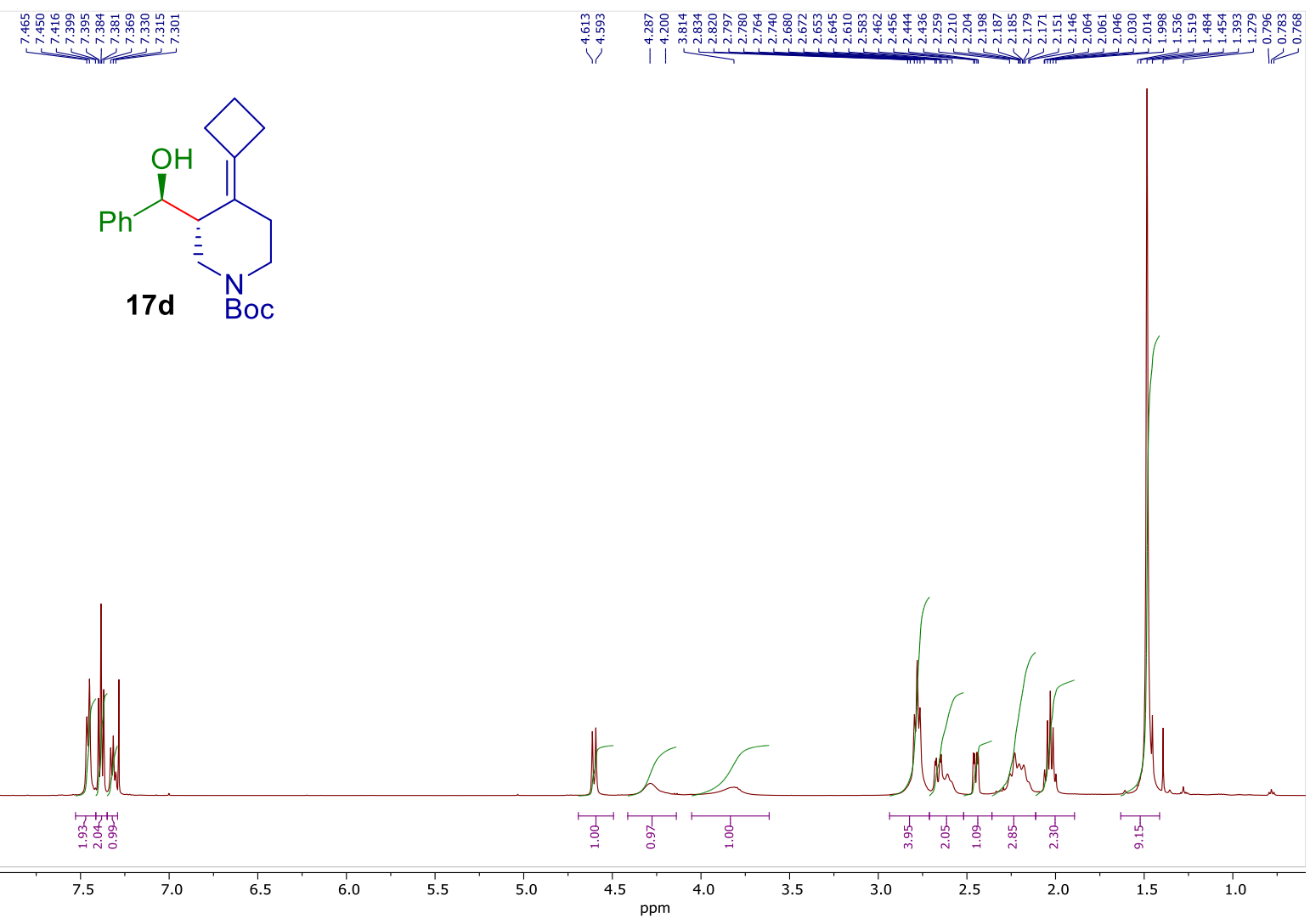

${ }^{13} \mathbf{C}$ - NMR (126 MHz, $\left.\mathrm{CDCl}_{3}\right)$ of compound 17d

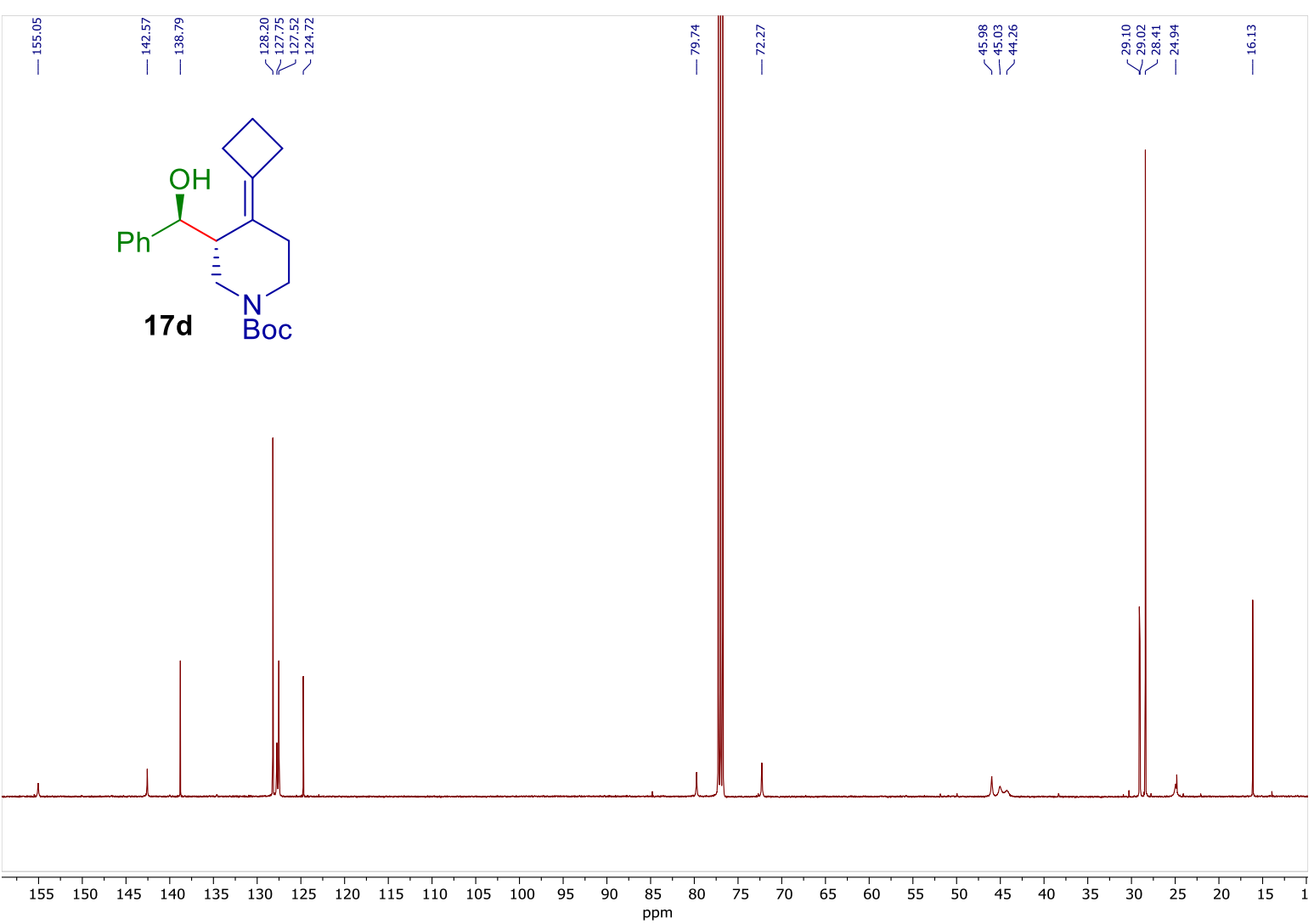


${ }^{1} \mathbf{H}-\mathrm{NMR}\left(500 \mathrm{MHz}, \mathrm{CDCl}_{3}\right)$ of compound $17 \mathrm{e}$ see procedure

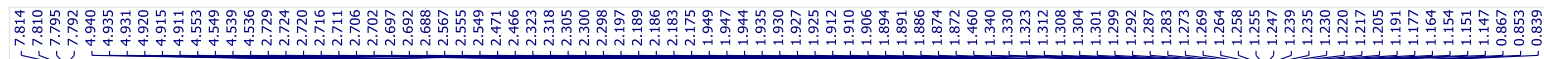<smiles>C[C@@H](C=C1CCC1)C(O)c1cc(C(F)(F)F)cc(C(F)(F)F)c1</smiles>

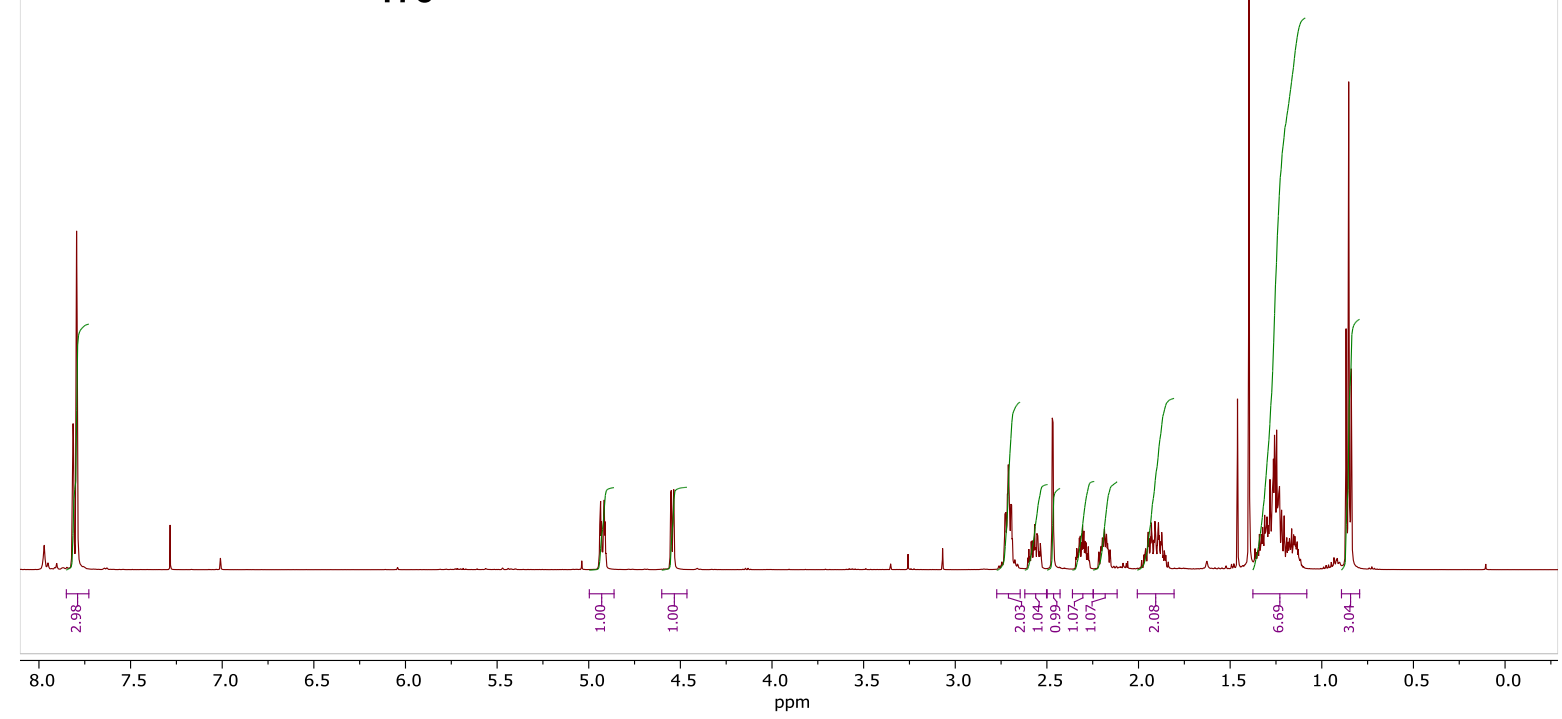

${ }^{13} \mathbf{C}$ - NMR (126 MHz, $\left.\mathrm{CDCl}_{3}\right)$ of compound 17e
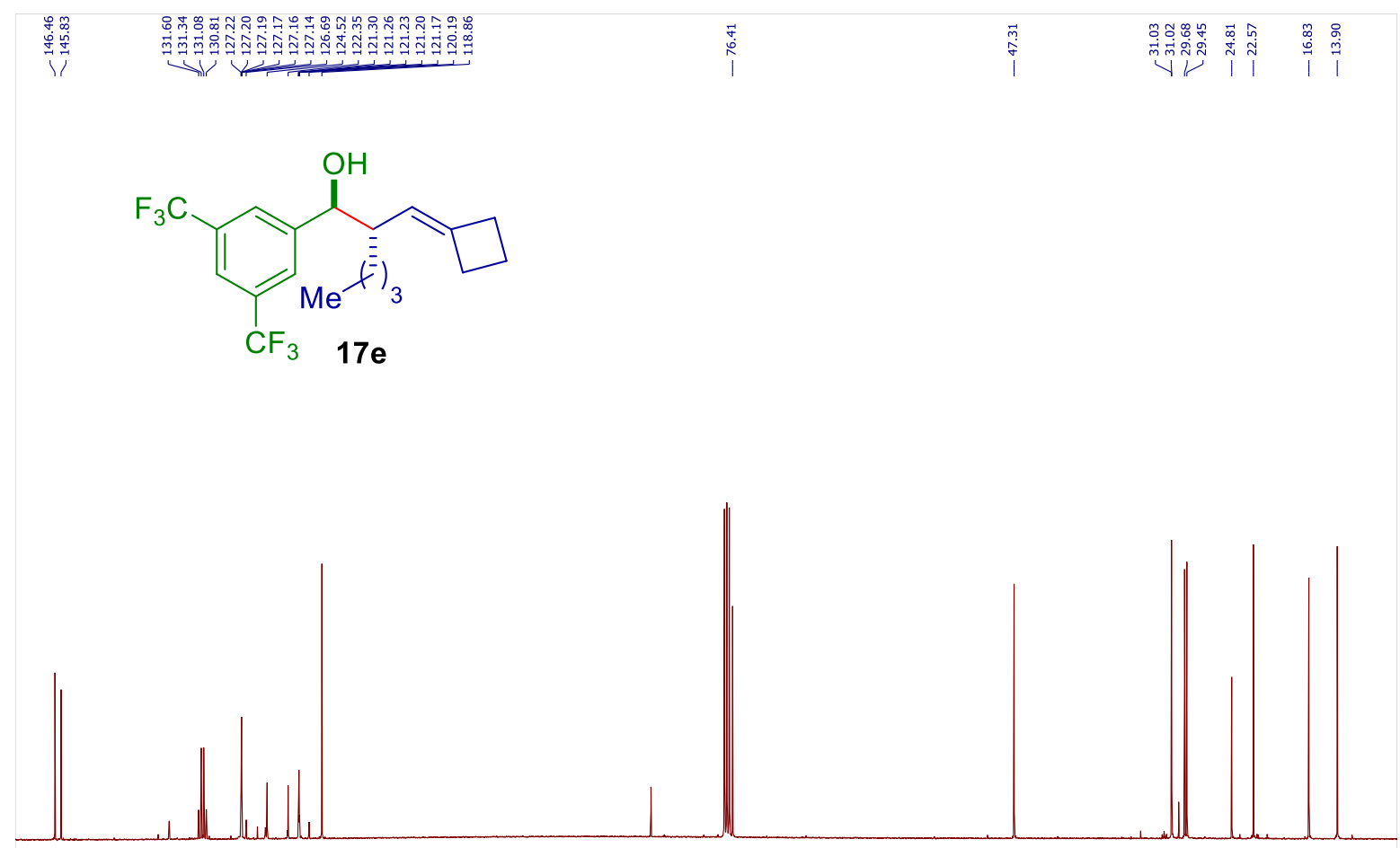

\begin{tabular}{lllllllllllllllllllllllllllllllllllllllll}
\hline 50 & 145 & 140 & 135 & 130 & 125 & 120 & 115 & 110 & 105 & 100 & 95 & 90 & 85 & 80 & 75 & 70 & 65 & 60 & 55 & 50 & 45 & 40 & 35 & 30 & 25 & 20 & 15 & 10
\end{tabular} 
${ }^{1} \mathbf{H}-\mathrm{NMR}\left(500 \mathrm{MHz}, \mathrm{CDCl}_{3}\right)$ of compound $\mathbf{1 7 f}$ see procedure

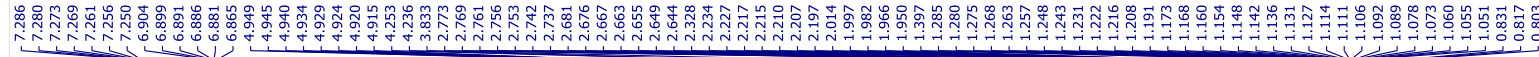<smiles>COc1ccc(C(O)C(C=C2CCC2)C(C)C(C)(C)C)cc1</smiles>

$17 f$

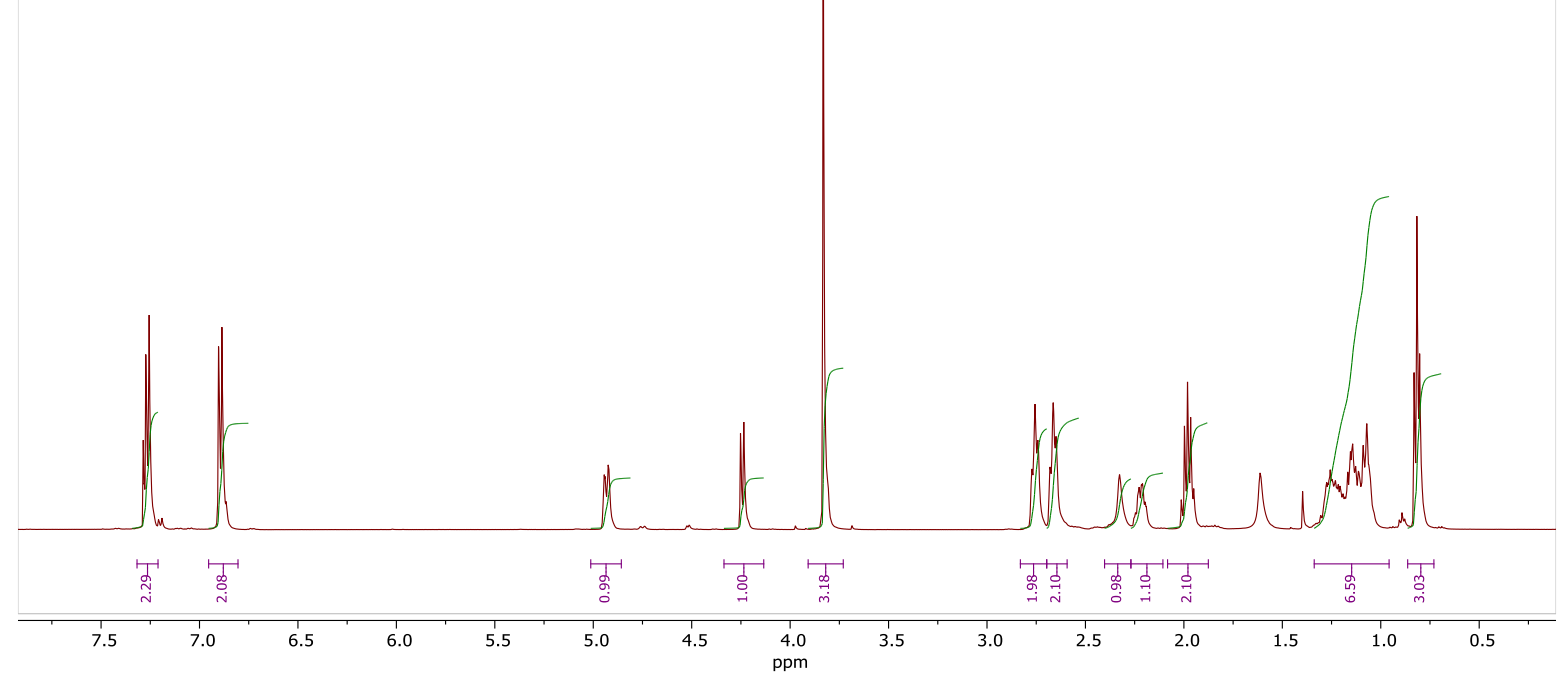

${ }^{13} \mathbf{C}-\mathbf{N M R}\left(126 \mathrm{MHz}, \mathrm{CDCl}_{3}\right)$ of compound $\mathbf{1 7 f}$

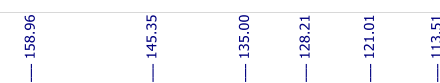<smiles>COc1ccc(C(O)C(C)C=C2CCC2)cc1</smiles>

$17 f$

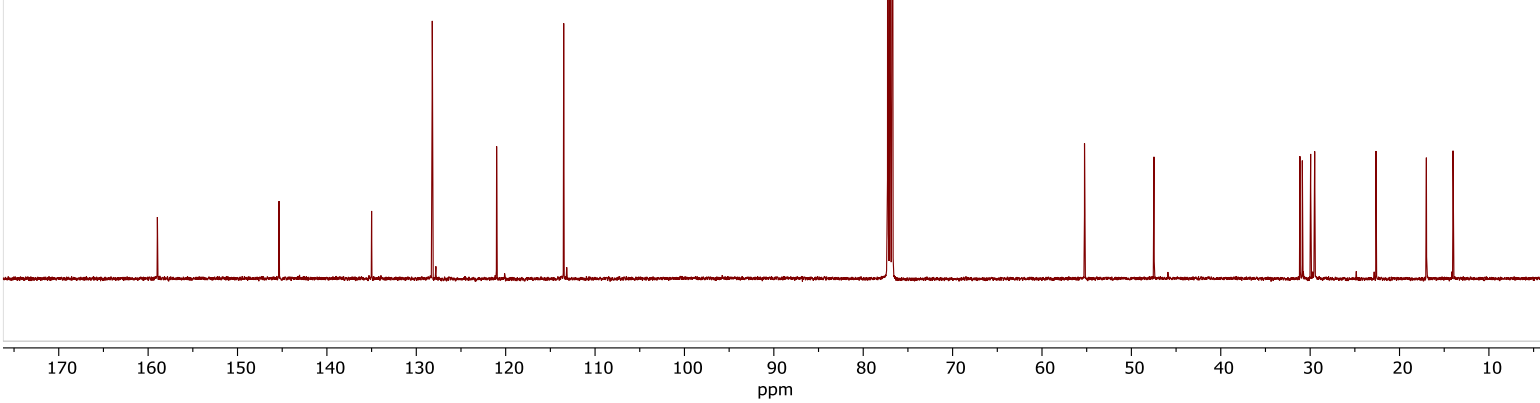


${ }^{1} \mathbf{H}-\mathrm{NMR}\left(500 \mathrm{MHz}, \mathrm{CDCl}_{3}\right)$ of compound $\mathbf{1 7 g}$ see procedure

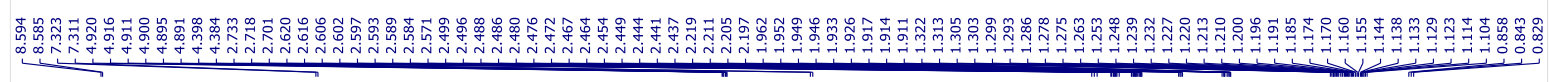

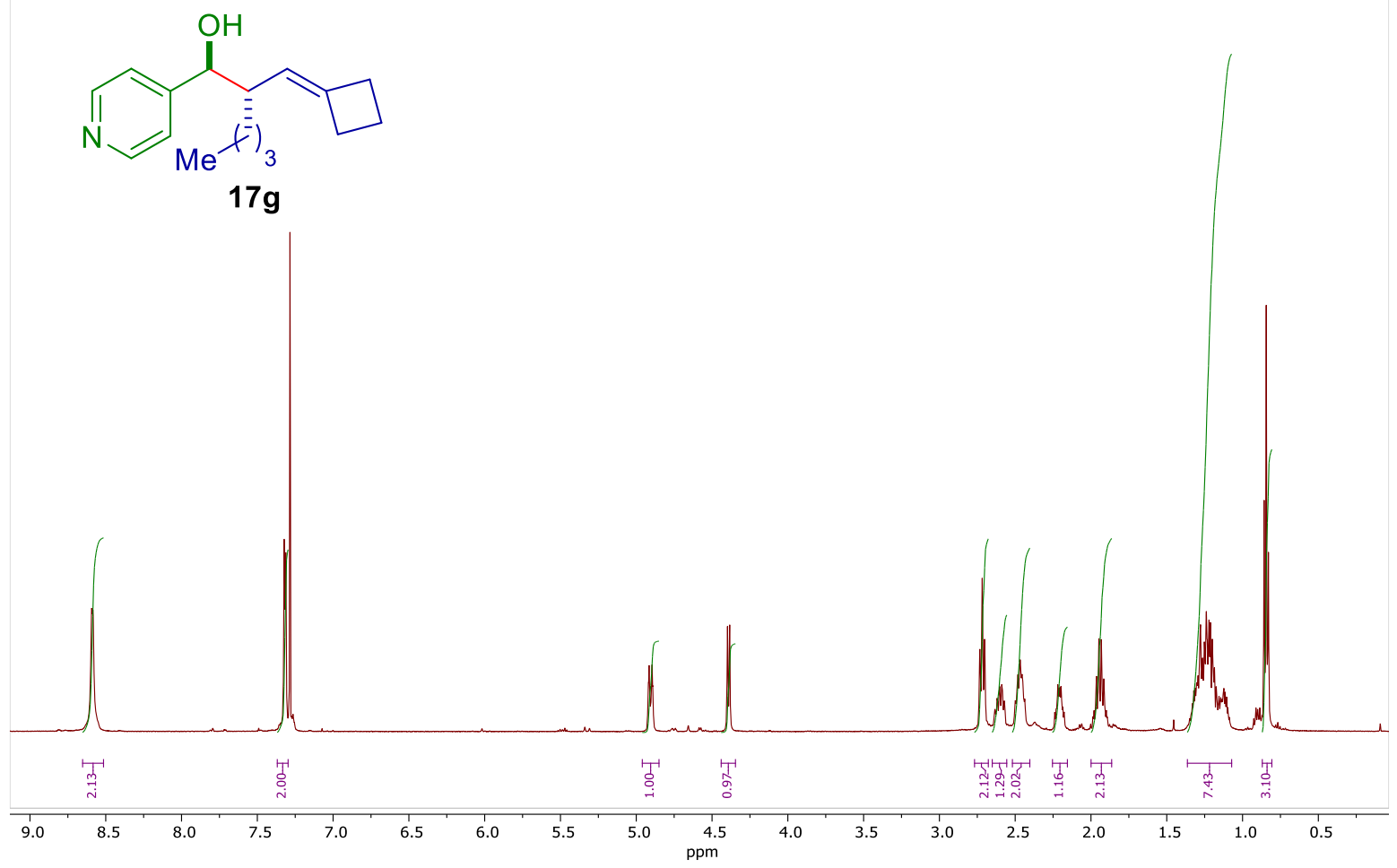

${ }^{\mathbf{1 3}} \mathbf{C}$ - NMR (126 MHz, $\left.\mathrm{CDCl}_{3}\right)$ of compound $\mathbf{1 7 g}$

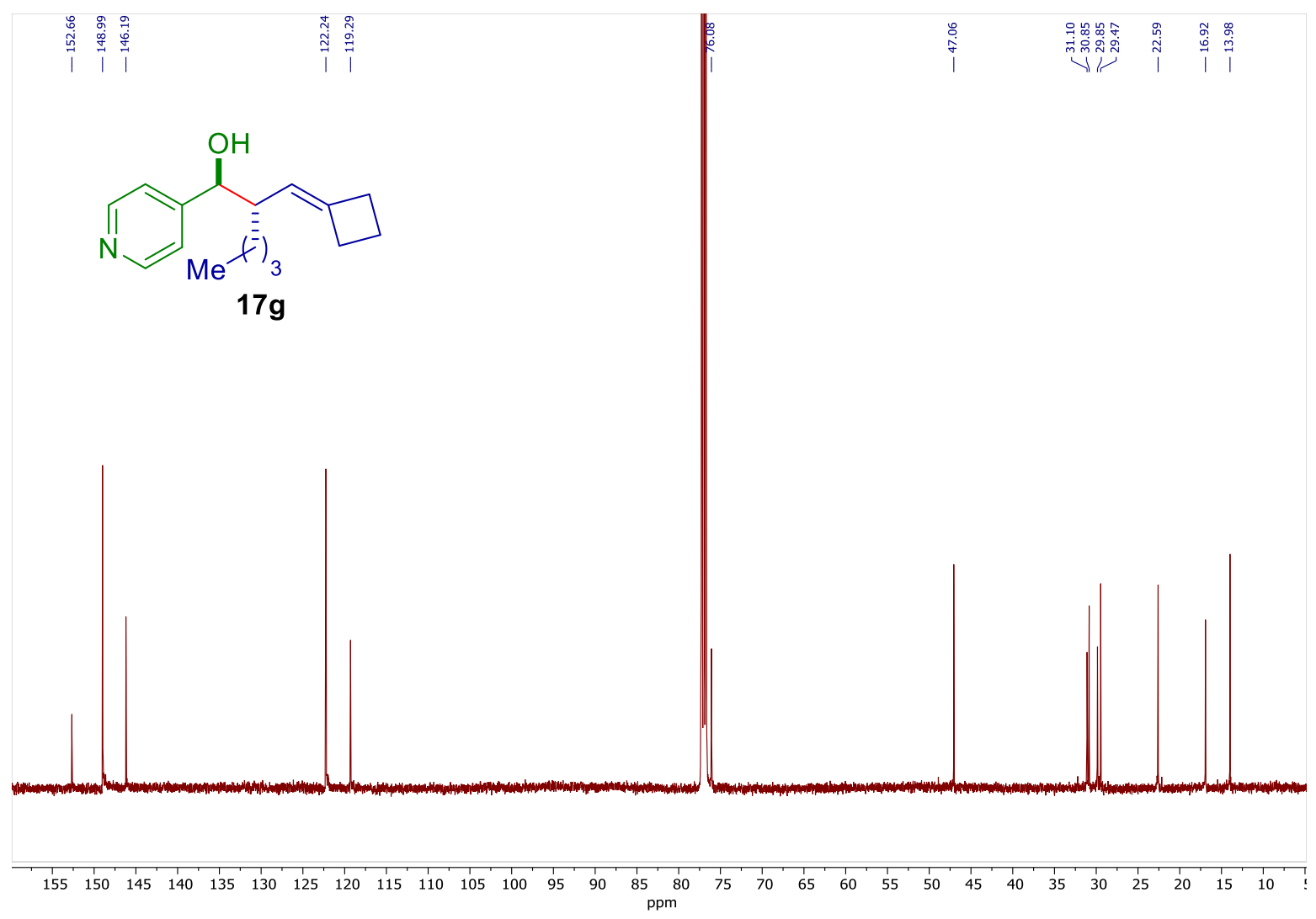

S151 
${ }^{1} \mathbf{H}-\mathrm{NMR}\left(500 \mathrm{MHz}, \mathrm{CDCl}_{3}\right)$ of compound $\mathbf{1 7 h}$ see procedure

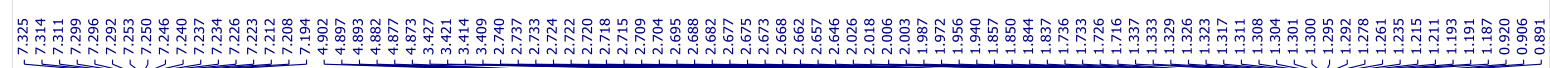<smiles>C[14CH2][C@@H](C=C1CCC1)[C@@H](O)CCc1ccccc1</smiles>

$17 \mathrm{~h}$
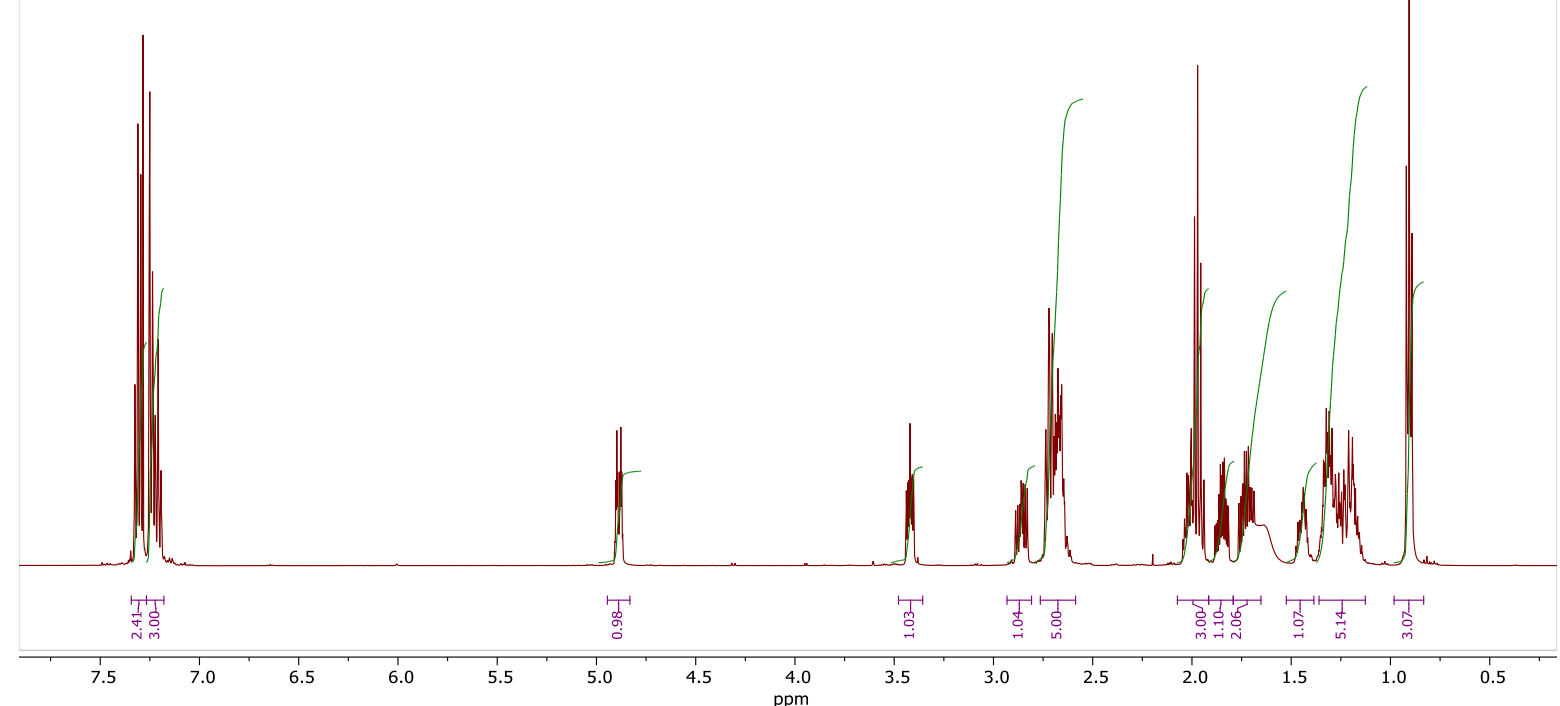

${ }^{13} \mathbf{C}$ - NMR (126 MHz, $\left.\mathrm{CDCl}_{3}\right)$ of compound $\mathbf{1 7 h}$

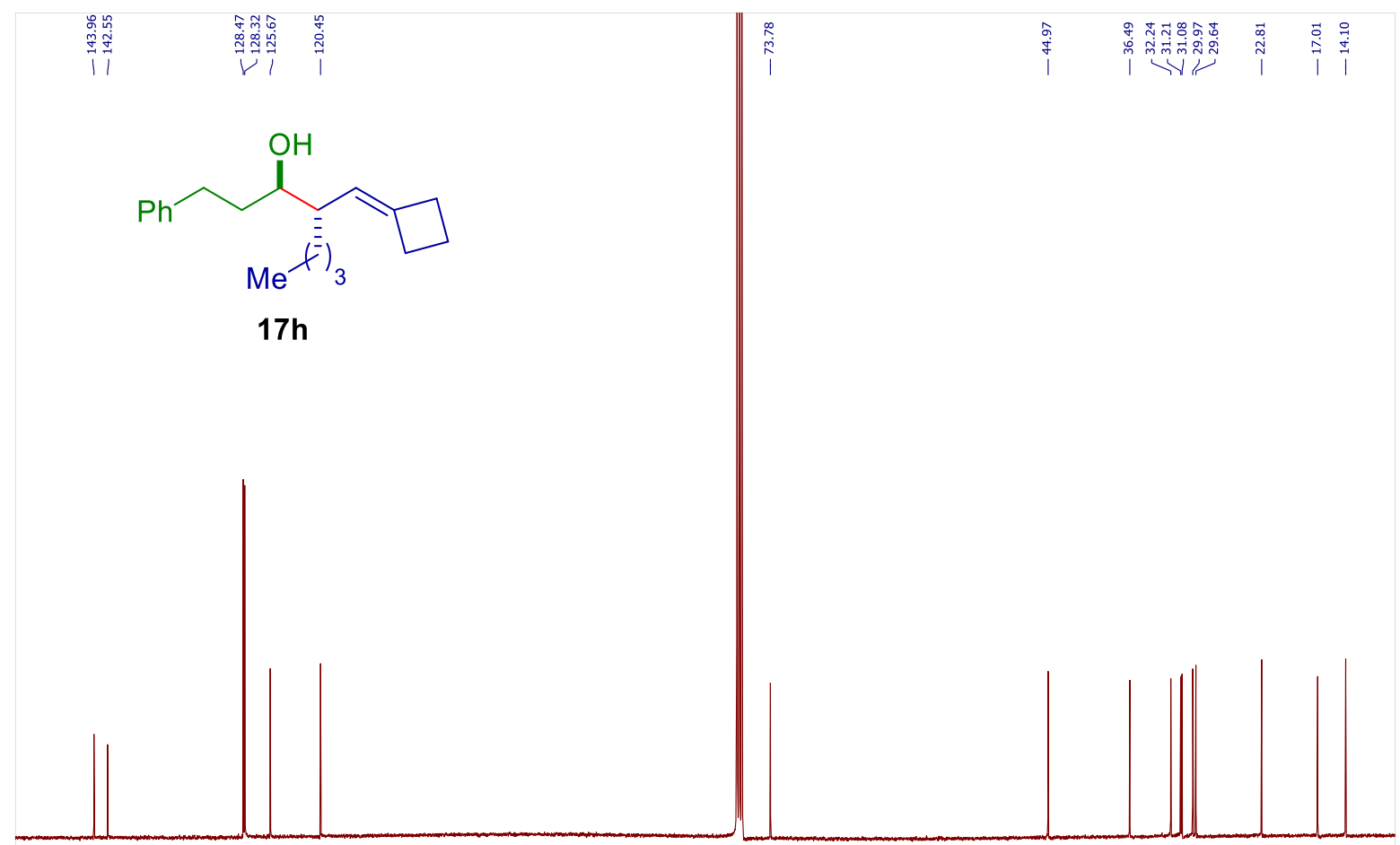

\begin{tabular}{lllllllllllllllllllllllllllllllll}
\hline 150 & 145 & 140 & 135 & 130 & 125 & 120 & 115 & 110 & 105 & 100 & 95 & 90 & 85 & 80 & 75 & 70 & 65 & 60 & 55 & 50 & 45 & 40 & 35 & 30 & 25 & 20 & 15 & 10
\end{tabular} 
${ }^{1} \mathbf{H}-\mathbf{N M R}\left(500 \mathrm{MHz}, \mathrm{CDCl}_{3}\right)$ of compound 18a see procedure

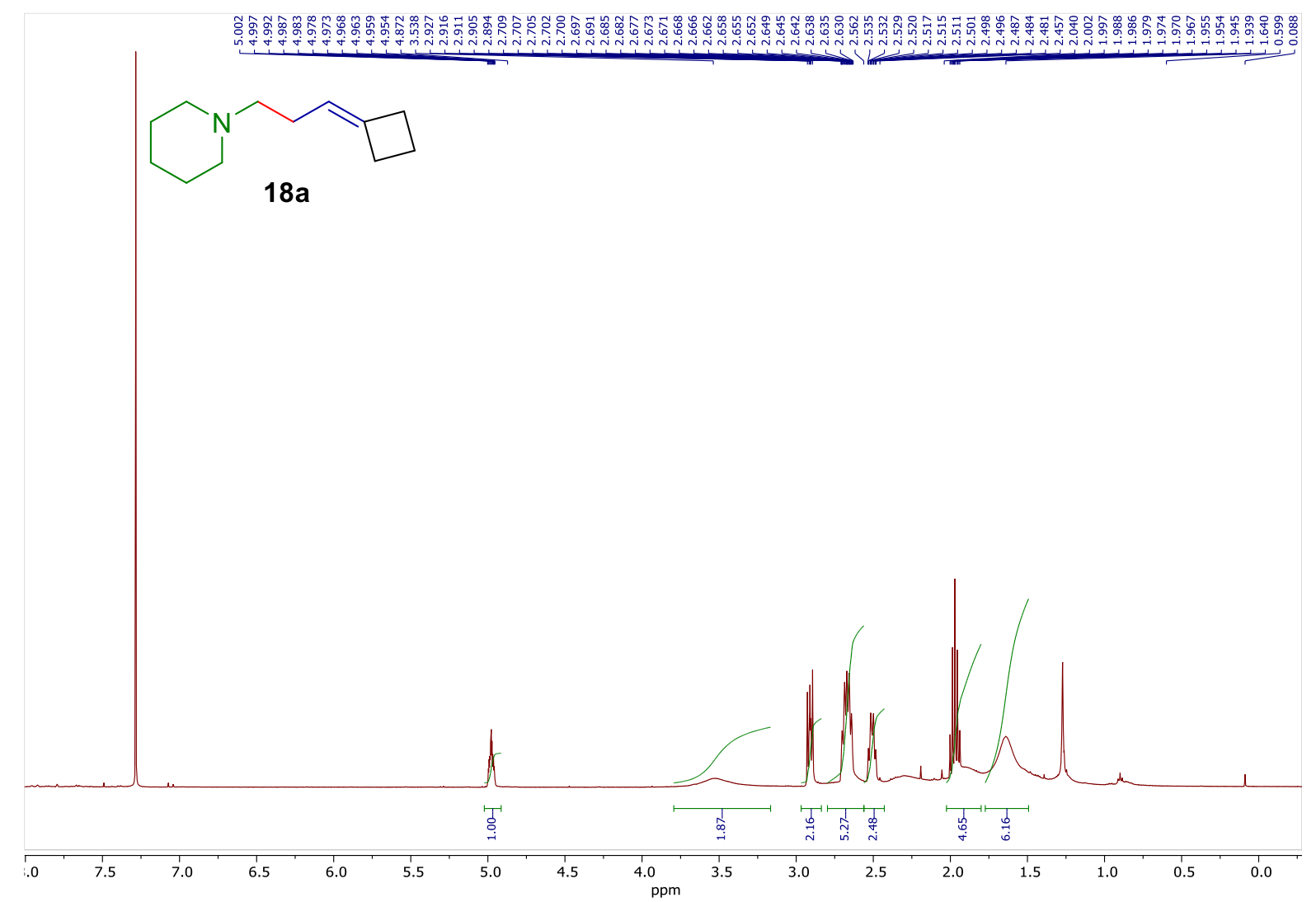

${ }^{13} \mathbf{C}$ - NMR $\left(126 \mathrm{MHz}, \mathrm{CDCl}_{3}\right)$ of compound $\mathbf{1 8 a}$

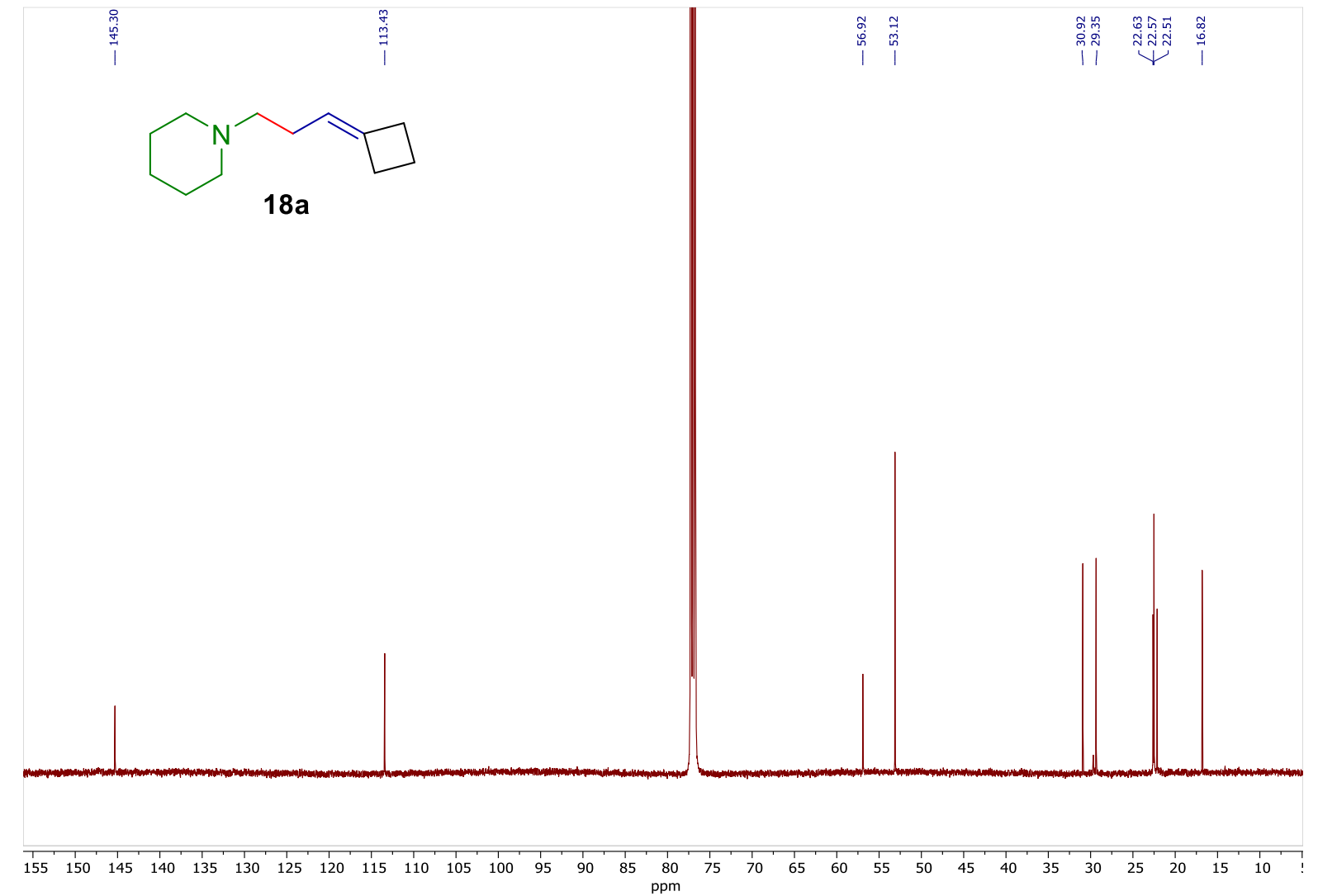


${ }^{\mathbf{1}} \mathrm{H}-\mathrm{NMR}\left(500 \mathrm{MHz}, \mathrm{CDCl}_{3}\right)$ of compound $\mathbf{1 8 b}$ see procedure

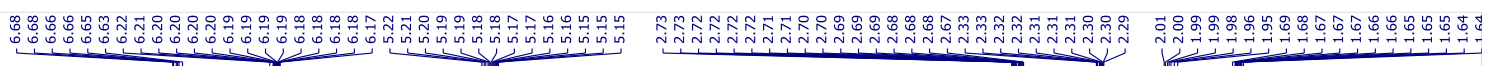

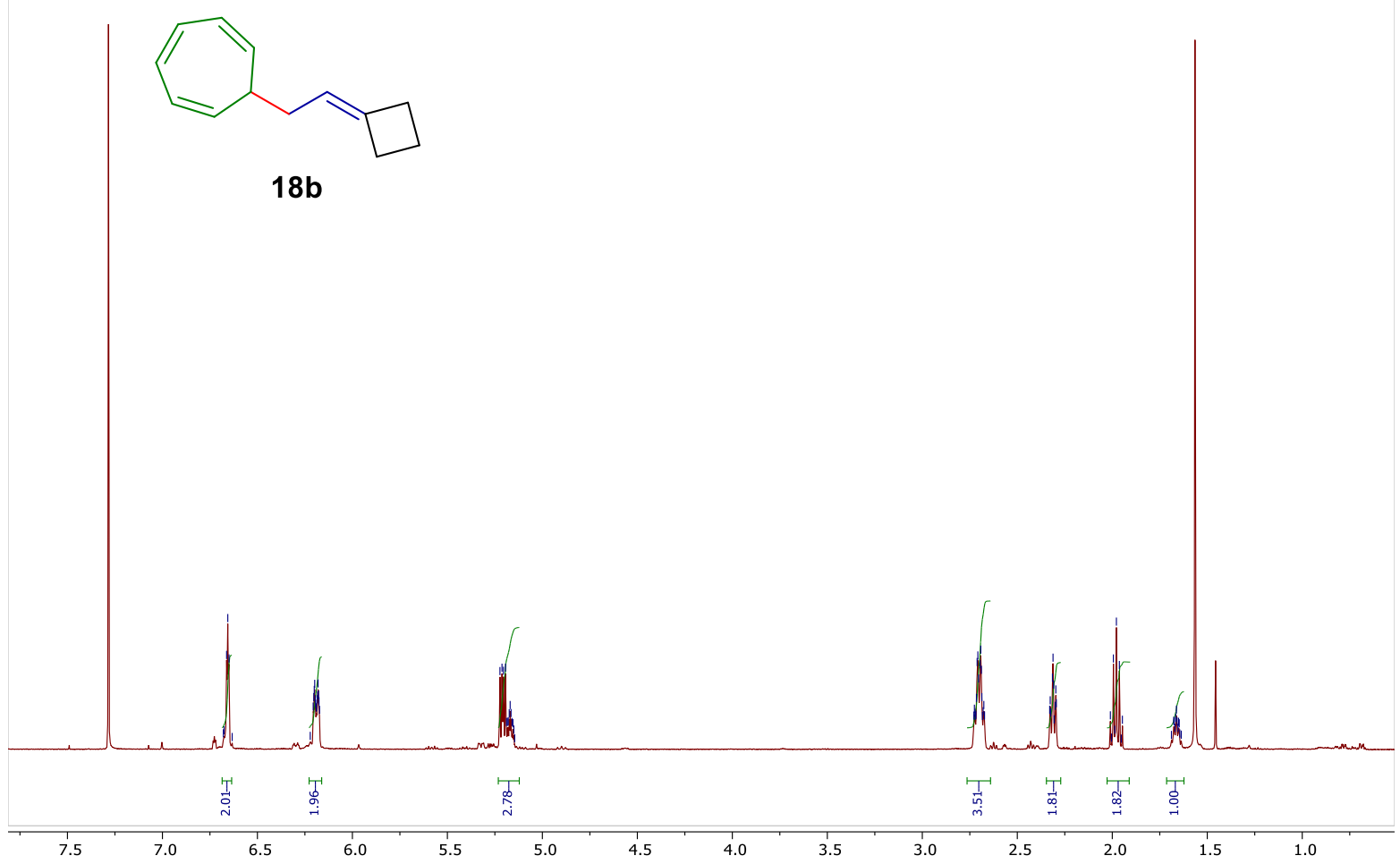

${ }^{13} \mathbf{C}$ - NMR (126 MHz, $\left.\mathrm{CDCl}_{3}\right)$ of compound $\mathbf{1 8 b}$

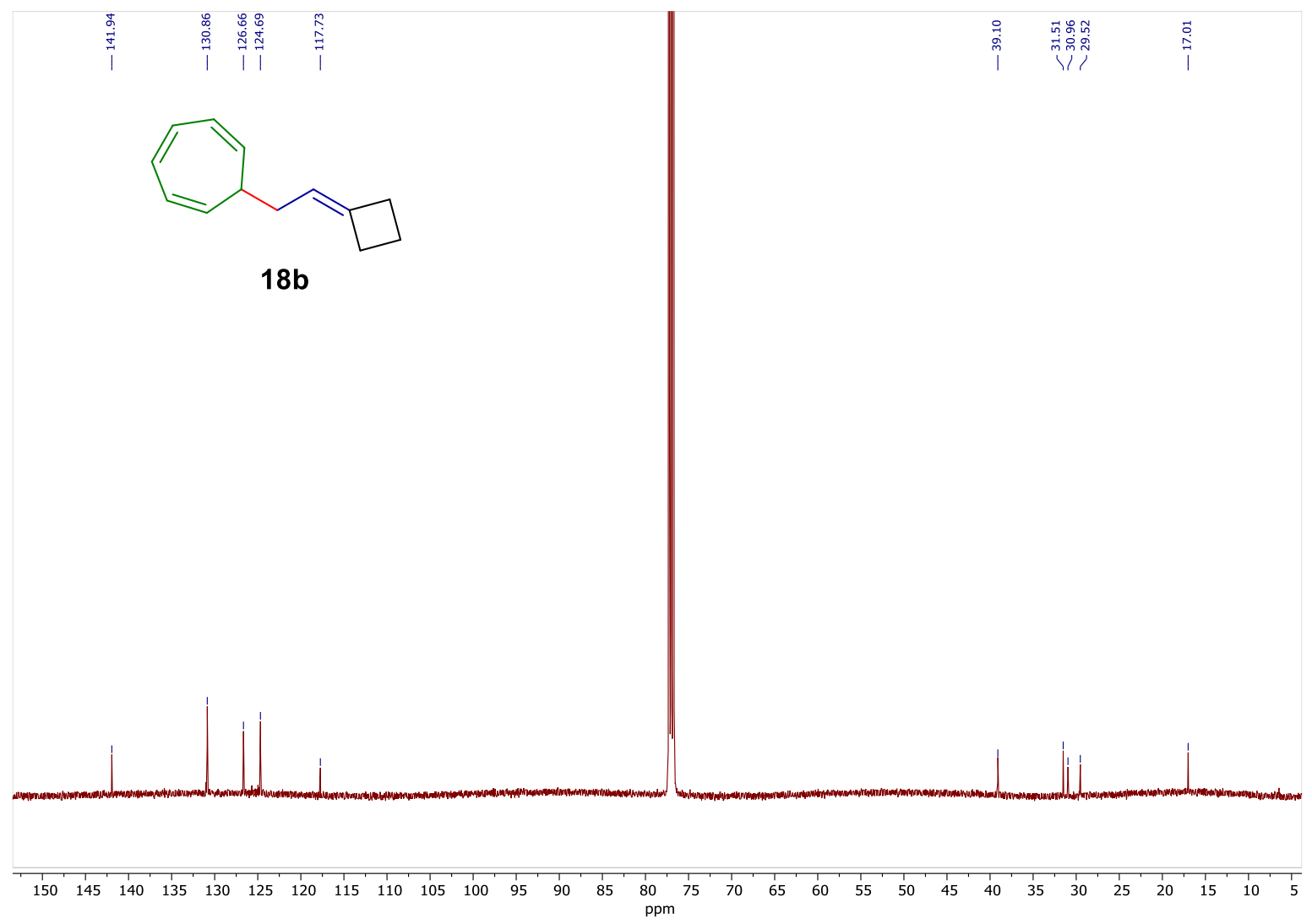

S154 
${ }^{1} \mathbf{H}-\mathbf{N M R}\left(500 \mathrm{MHz}, \mathrm{CDCl}_{3}\right)$ of compound $18 \mathrm{c}$ see procedure

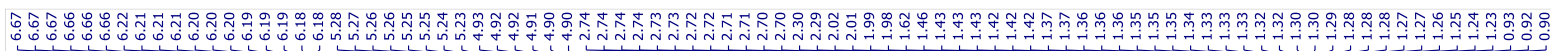
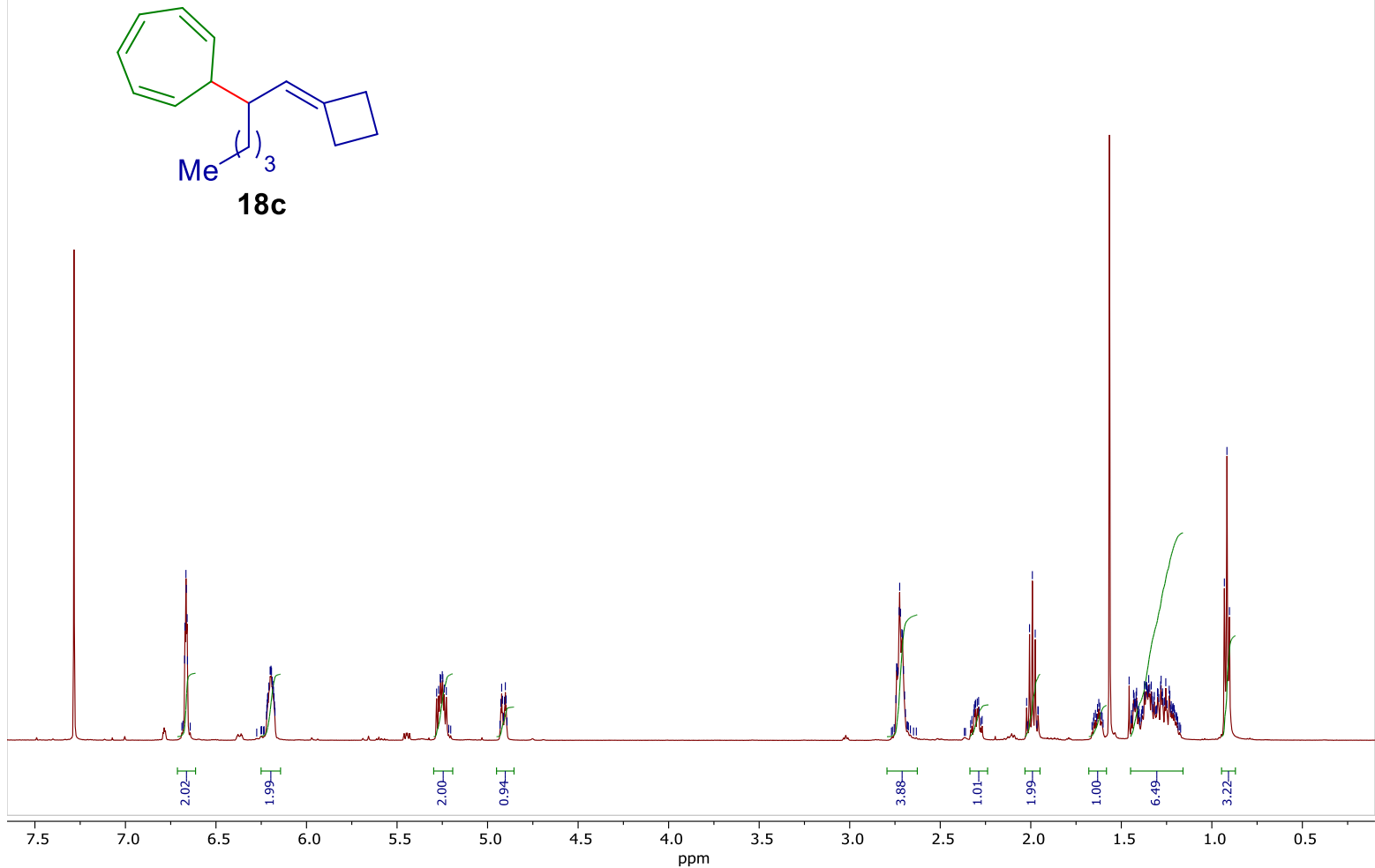

${ }^{13} \mathbf{C}$ - NMR (126 MHz, $\left.\mathrm{CDCl}_{3}\right)$ of compound $\mathbf{1 8 c}$

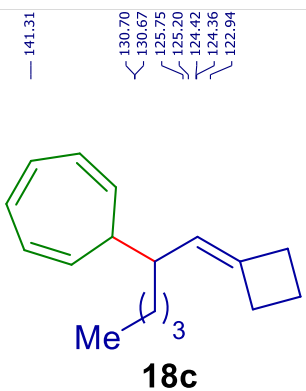

। ।

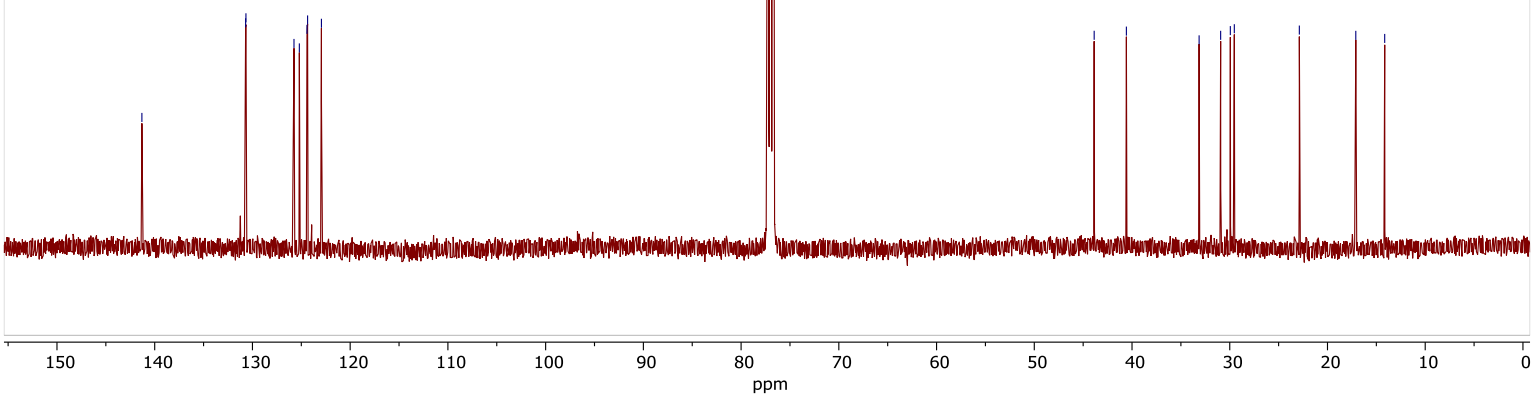


${ }^{1} \mathbf{H}-\mathrm{NMR}\left(500 \mathrm{MHz}, \mathrm{CDCl}_{3}\right)$ of compound $\mathbf{1 8 d}$ see procedure

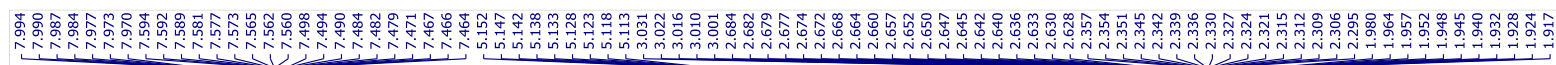<smiles>CC(C)(C)CCCC(=O)c1ccccc1</smiles>

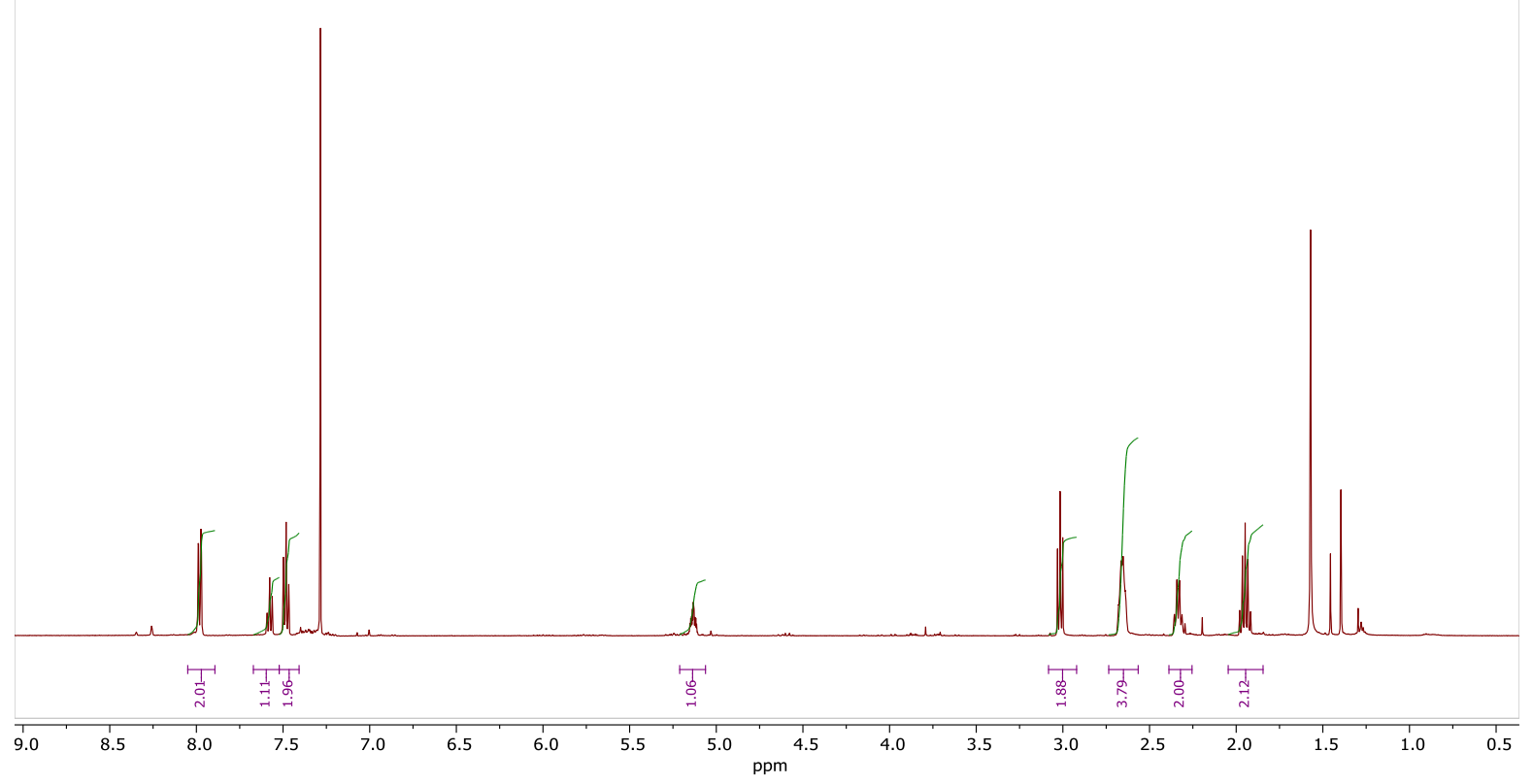

${ }^{13} \mathbf{C}$ - NMR (126 MHz, $\left.\mathrm{CDCl}_{3}\right)$ of compound 18d

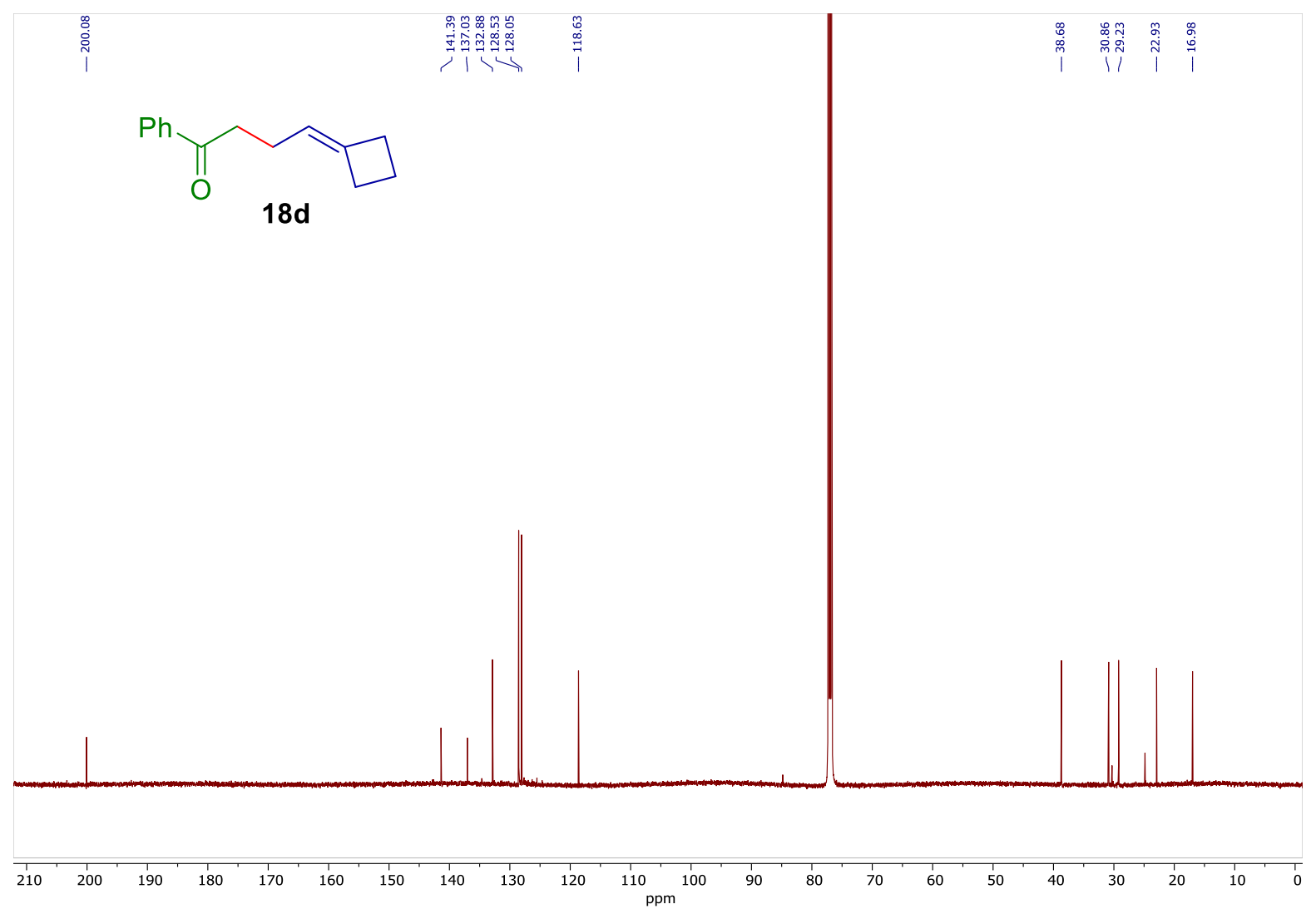


H-NMR $\left(500 \mathrm{MHz}, \mathrm{CDCl}_{3}\right.$ ) of compound 20 see procedure

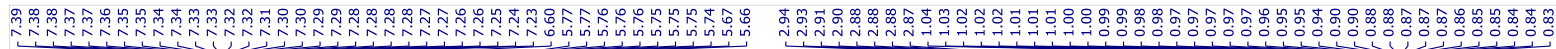

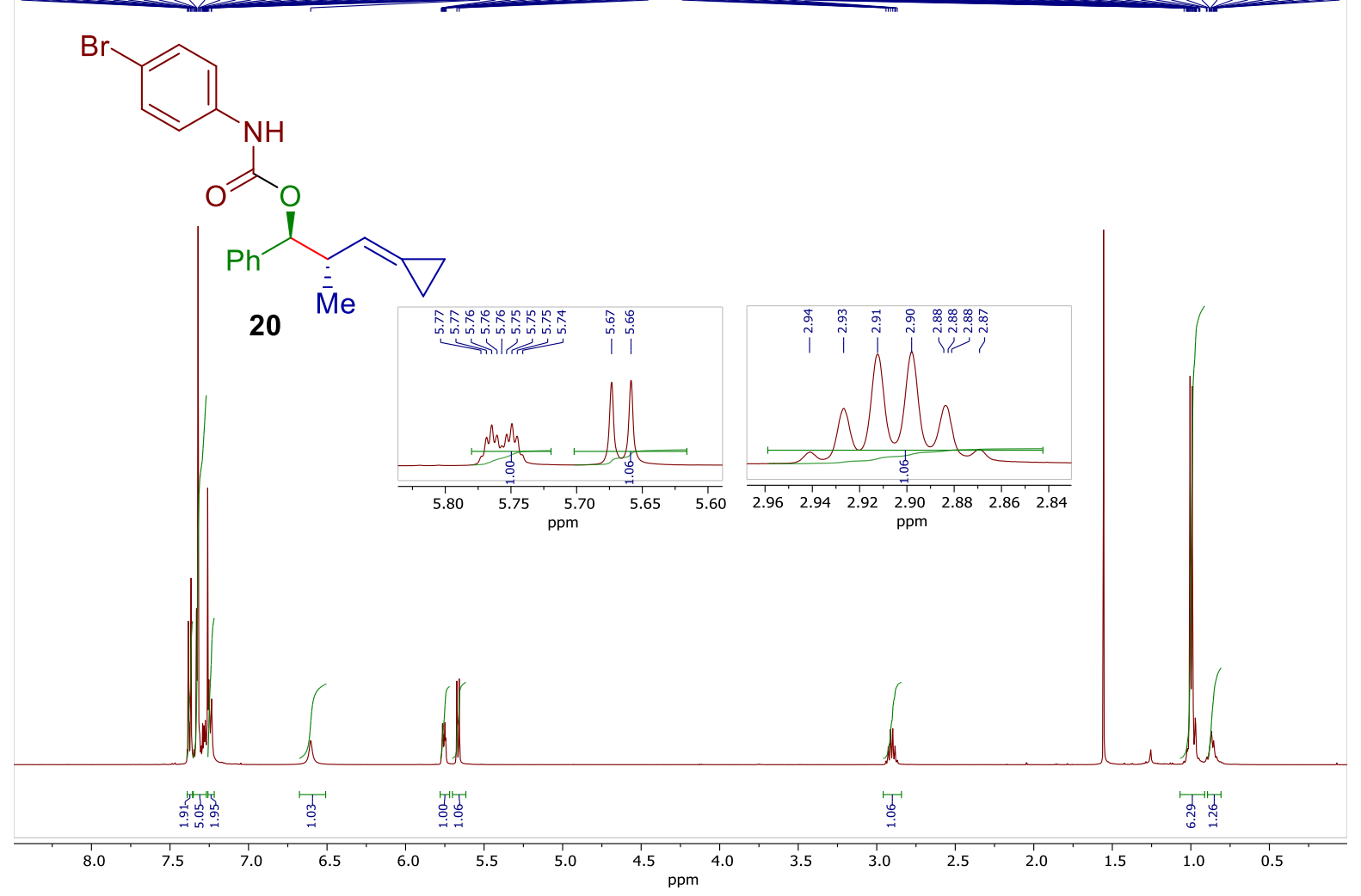

${ }^{13} \mathbf{C}$ - NMR (126 MHz, $\left.\mathrm{CDCl}_{3}\right)$ of compound 20

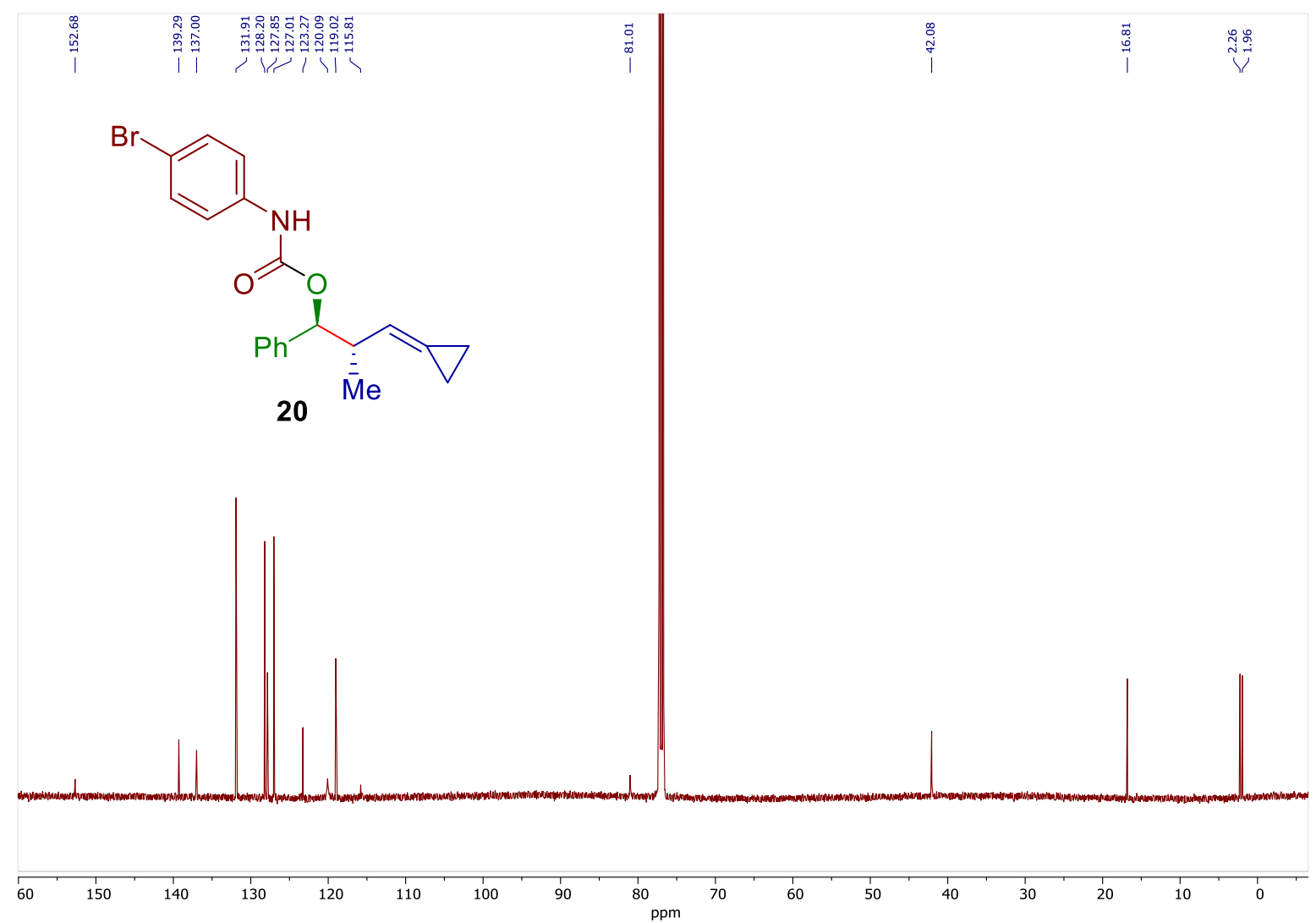

